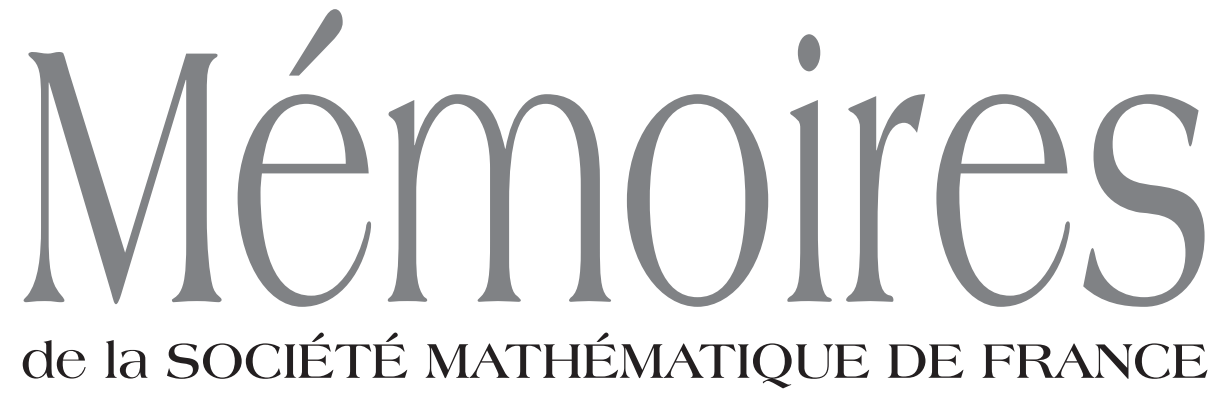

Numéro $125 / 126$

CHAMPS DE HURWITZ

Nouvelle série

J. BERTIN \& M. ROMAGNY

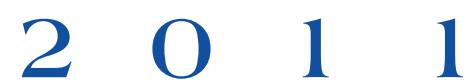




\section{Comité de rédaction}

\author{
Jean BARGE \\ Emmanuel BREUILLARD \\ Gérard BESSON \\ Antoine CHAMBERT-LOIR \\ Jean-François DAT \\ Raphaël KRIKORIAN (dir.) \\ Charles FAVRE \\ Daniel HUYBRECHTS \\ Yves LE JAN \\ Laure SAINT-RAYMOND \\ Wilhem SCHLAG
}

\section{Diffusion}

Maison de la SMF

Case 916 - Luminy

13288 Marseille Cedex 9

France

smf@smf .univ-mrs.fr
Hindustan Book Agency

O-131, The Shopping Mall

Arjun Marg, DLF Phase 1

Gurgaon 122002, Haryana

Inde
AMS

P.O. Box 6248

Providence RI 02940

USA

WWw . ams . org

\section{Tarifs}

Vente au numéro : $50 €(\$ 75)$

Abonnement Europe : $255 €$, hors Europe : $290 €(\$ 435)$

Des conditions spéciales sont accordées aux membres de la SMF.

\section{Secrétariat : Nathalie Christiaën}

Mémoires de la SMF

Société Mathématique de France

Institut Henri Poincaré, 11, rue Pierre et Marie Curie

75231 Paris Cedex 05, France

Tél : (33) 0144276799 • Fax : (33) 0140469096

revues@smf.ens.fr • http://smf.emath.fr/

(C) Société Mathématique de France 2011

Tous droits réservés (article L 122-4 du Code de la propriété intellectuelle). Toute représentation ou reproduction intégrale ou partielle faite sans le consentement de l'éditeur est illicite. Cette représentation ou reproduction par quelque procédé que ce soit constituerait une contrefaçon sanctionnée par les articles L 335-2 et suivants du CPI.

ISSN 0249-633-X

ISBN 978-85629-333-1

Directrice de la publication : Aline BONAMI 


\title{
CHAMPS DE HURWITZ
}

\author{
José Bertin \\ Matthieu Romagny
}

Société Mathématique de France 2011

Publié avec le concours du Centre National de la Recherche Scientifique 
J. Bertin

Institut Fourier, Université Grenoble 1, France.

E-mail : jose.bertin@ujf-grenoble.fr

M. Romagny

Institut de Mathématiques de Jussieu, Université Paris 6, France.

E-mail : romagny@math.jussieu.fr

Classification mathématique par sujets (2000). - 14H10, 14H30, 14H37, 14A20, 14C17; 11G20, 11G30, 14C40.

Mots-clefs. - Courbe algébrique, revêtement, revêtement galoisien, champ de Hurwitz, compactification, structure de niveau, Riemann-Hurwitz, revêtement cyclique, classes tautologiques.

Nous remercions les organisateurs de la semaine thématique Groupes de Galois arithmétiques et différentiels au CIRM (Luminy) en Mars 2004, durant laquelle plusieurs exposés furent consacrés aux espaces de Hurwitz, et qui a servi de point de départ à la présente rédaction. Même si la gestation de ce travail a été lente, et peut-être à cause de cela, nous avons bénéficié d'incitations et sollicitations répétées de nombreux collègues. Nous tenons à les remercier tous pour leur patience et leur bienveillance. Enfin, nous remercions les rapporteurs, dont les nombreuses remarques ont permis d'améliorer grandement la qualité de ce travail. 


\title{
CHAMPS DE HURWITZ
}

\author{
José Bertin, Matthieu Romagny
}

Résumé. - Dans ce travail, nous effectuons une étude détaillée des champs de Hurwitz et de leurs espaces de modules, tant dans le cas galoisien que dans le cas non galoisien, avec une attention particulière portée aux correspondances entre ces espaces de modules. Nous comparons notre construction à celles proposées par Abramovich-Corti-Vistoli, Harris-Mumford, et Mochizuki-Wewers. Nous mettons en application nos résultats pour revisiter des exemples classiques, notamment les champs de courbes stables munies d'une structure de niveau arbitraire, et les champs de revêtements cycliques modérément ramifiés. Dans une deuxième partie, nous mettons en évidence des fibrés tautologiques et des classes de cohomologie qui vivent naturellement sur les champs de Hurwitz, et nous donnons des relations universelles, dont un analogue supérieur de la formule de Riemann-Hurwitz, entre ces classes. Nous donnons des applications au champ des revêtements cycliques de la droite projective, avec un intérêt particulier pour des relations du type de la relation de Cornalba-Harris et pour les intégrales de Hodge cycliques, notamment hyperelliptiques.

Abstract (Hurwitz stacks). - In this work, we give a thorough study of Hurwitz stacks and associated Hurwitz moduli spaces, both in the Galois and the non Galois case, with particular attention to correspondances between these moduli spaces. We compare our construction to those proposed by Abramovich-Corti-Vistoli, HarrisMumford, and Mochizuki-Wewers. We apply our results to revisit some classical examples, particularly the stacks of stable curves equipped with an arbitrary level structure, and the stacks of tamely ramified cyclic covers. In a second part we exhibit some tautological bundles and cohomology classes naturally living on Hurwitz stacks, and give some universal relations, in particular a higher analogue of the RiemannHurwitz formula, between these classes. Applications are given to the stack of cyclic covers of the projective line, with special attention to Cornalba-Harris type relations and to cyclic, in particular hyperelliptic, Hodge integrals. 



\section{TABLE DES MATIÈRES}

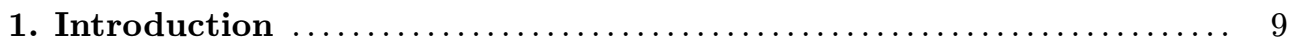

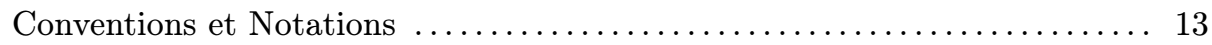

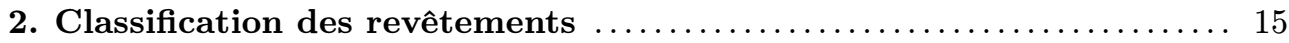

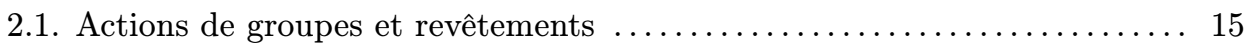

2.1.1. Revêtements, $G$-courbes, $G$-revêtements $\ldots \ldots \ldots \ldots \ldots \ldots \ldots \ldots \ldots \ldots \ldots \ldots \ldots$

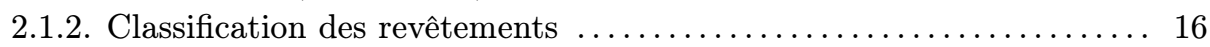

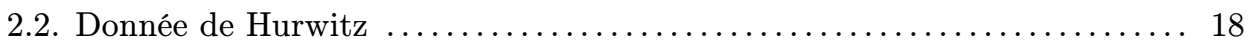



2.2.2. Opérations sur les données de Hurwitz ..................... 19

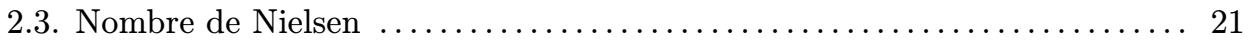

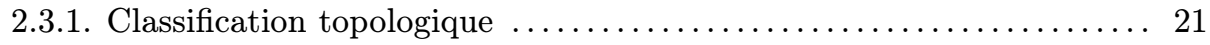

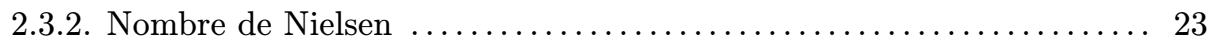

3. Familles de $G$-courbes lisses et Théorème de Chevalley-Weil f.... 29

3.1. Géométrie du diviseur de branchement $\ldots \ldots \ldots \ldots \ldots \ldots \ldots \ldots \ldots \ldots 29$



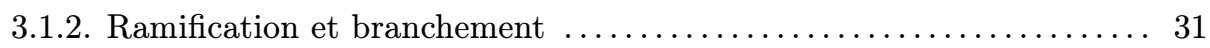

3.2. Inversion de la formule de Chevalley-Weil $\ldots \ldots \ldots \ldots \ldots \ldots \ldots \ldots \ldots \ldots$

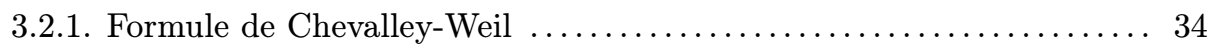

3.2.2. Inversion des relations de Chevalley-Weil $\ldots \ldots \ldots \ldots \ldots \ldots \ldots \ldots . \ldots 6$

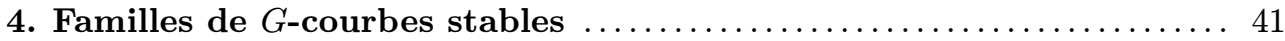

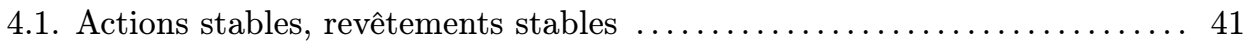

4.1.1. Courbes stables et stables marquées $\ldots \ldots \ldots \ldots \ldots \ldots \ldots \ldots \ldots \ldots . \ldots 1$

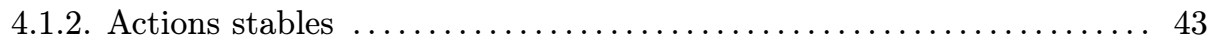

4.1.3. Collision des points de branchement : étude locale .............. 49

4.1.4. Géométrie du quotient par une action stable .................. 51





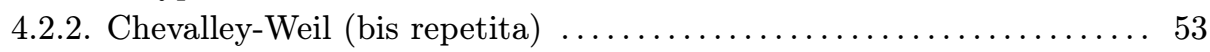

4.3. Courbes stables marquées et actions de groupes $\ldots \ldots \ldots \ldots \ldots \ldots \ldots \ldots 57$

5. Déformations des revêtements modérément ramifiés $\ldots \ldots \ldots \ldots \ldots 61$ 
5.1. Déformations équivariantes des courbes $\ldots \ldots \ldots \ldots \ldots \ldots \ldots \ldots \ldots \ldots \ldots \ldots \ldots$

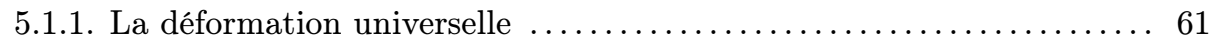



5.1.3. Déformations équivariantes versus déformations du diviseur de

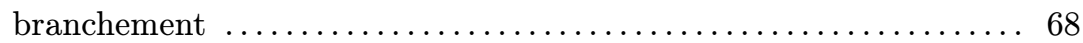

5.2. Modèle stable marqué d'un $G$-revêtement $\ldots \ldots \ldots \ldots \ldots \ldots \ldots \ldots \ldots$

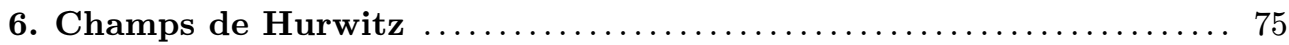

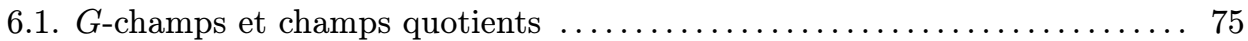

6.1.1. Quotient d'un champ par une action de groupe $\ldots \ldots \ldots \ldots \ldots \ldots . \ldots 6$

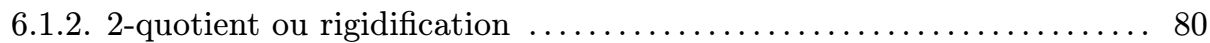

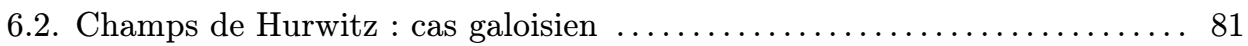

6.2.1. Définition des champs de Hurwitz .................... 81

6.2.2. Fonctorialité des champs de Hurwitz $\ldots \ldots \ldots \ldots \ldots \ldots \ldots \ldots \ldots \ldots$

6.3. Compactification stable du champ de Hurwitz (I) $\ldots \ldots \ldots \ldots \ldots \ldots \ldots 84$

6.4. Compactification du schéma de Hurwitz : Gieseker-Mumford . . ....... 86

6.5. Compactification stable du champ de Hurwitz (II) $\ldots \ldots \ldots \ldots \ldots \ldots . \ldots 8$

6.5.1. Le champ de Hurwitz versus Harris-Mumford . . . . . . . . . . . 88



6.6. Champs de Hurwitz : cas non galoisien $\ldots \ldots \ldots \ldots \ldots \ldots \ldots \ldots \ldots \ldots$

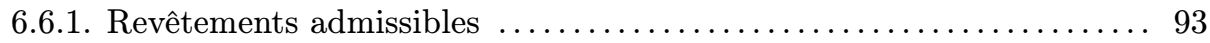

6.6.2. Clôture galoisienne : cas des courbes lisses ............... 94

6.6.3. Clôture galoisienne : courbes stables $\ldots \ldots \ldots \ldots \ldots \ldots \ldots \ldots \ldots \ldots$

6.6.4. Le morphisme discriminant : cas général $\ldots \ldots \ldots \ldots \ldots \ldots \ldots \ldots \ldots \ldots$



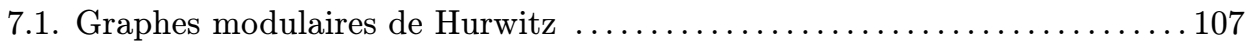

7.2. Graphes de groupes et revêtements de graphes $\ldots \ldots \ldots \ldots \ldots \ldots \ldots \ldots \ldots$

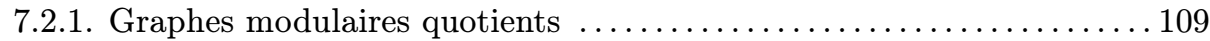

7.2.2. Un exemple : le bord du schéma de Hurwitz « classique » . . . . . . 113

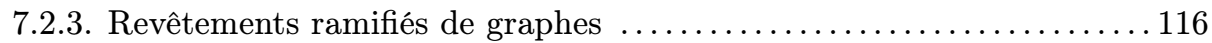

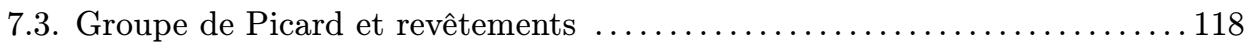

7.3.1. Sous-groupes de décomposition et d'inertie $\ldots \ldots \ldots \ldots \ldots \ldots \ldots \ldots 118$

7.3.2. Faisceaux sans torsion de rang 1 et revêtements stables . .......... 121

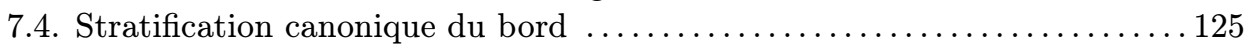



7.4.2. Type topologique d'un point du bord $\ldots \ldots \ldots \ldots \ldots \ldots \ldots \ldots \ldots \ldots \ldots$

8. Structures de niveau sur les courbes stables $\ldots \ldots \ldots \ldots \ldots \ldots \ldots \ldots$

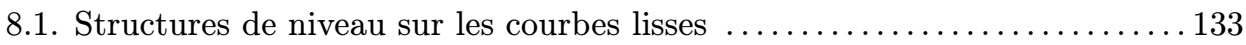

8.2. Structures de niveau sur les courbes stables $\ldots \ldots \ldots \ldots \ldots \ldots \ldots \ldots \ldots \ldots$

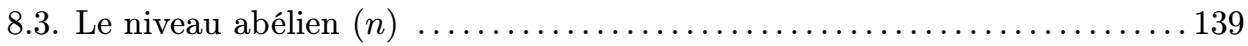

8.3.1. Groupes de décomposition et d'inertie ..................... 139

8.3.2. Composantes irréductibles $\mathrm{du}$ bord $\ldots \ldots \ldots \ldots \ldots \ldots \ldots \ldots \ldots \ldots \ldots \ldots \ldots$ 


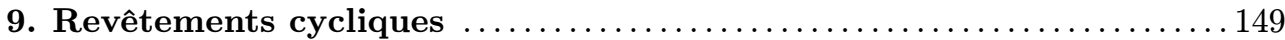

9.1. Revêtements cycliques versus racines d'un faisceau inversible . ......... 149

9.1.1. Racines et quasi-racines d'un faisceau inversible . ............. 149

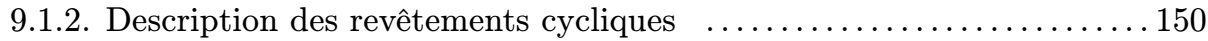

9.2. Revêtements cycliques stables ............................ 153

9.2.1. Revêtements cycliques stables et quasi-racines d'un faisceau

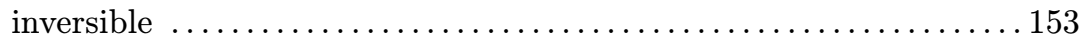

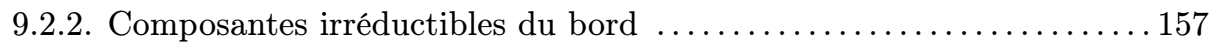

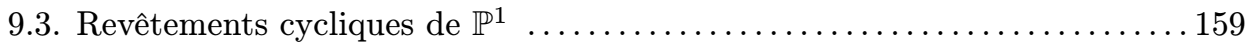

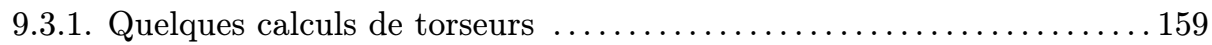

9.3.2. Interlude : Géométrie du discriminant des formes binaires . . . . . 162

9.3.3. Le champ des revêtements cycliques de la droite projective . . . . . 166

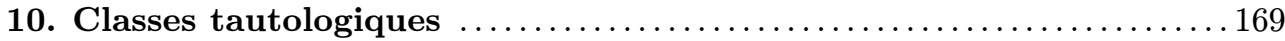

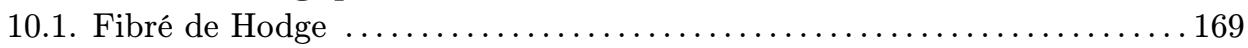

10.1.1. G-fibrés vectoriels sur le champ de Hurwitz . . . . . . . . . . . 169

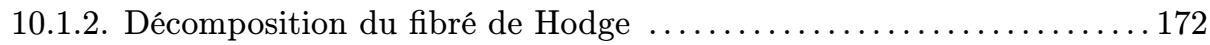

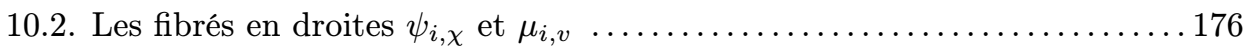

10.3. Relations de Riemann-Hurwitz d'ordre supérieur $\ldots \ldots \ldots \ldots \ldots \ldots \ldots \ldots$



10.3.2. Relations de Riemann-Hurwitz d'ordre supérieur . ............ 181

10.3.3. Faisceaux inversibles associés aux composantes du bord . . . . . . . 184

10.4. Appliquer le théorème de Riemann-Roch .................... 188

10.4.1. Mise en place du théorème de Grothendieck-Riemann-Roch (GRR) 188

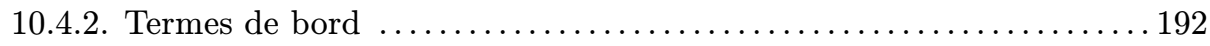

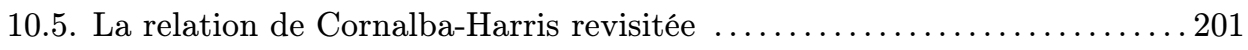

10.5.1. Les faisceaux inversibles $\psi$ et $\mu$ dans le cas cyclique $\ldots \ldots \ldots \ldots \ldots 201$



10.5.3. Intégrales de Hodge-Hurwitz hyperelliptiques $\ldots \ldots \ldots \ldots \ldots \ldots . \ldots 206$

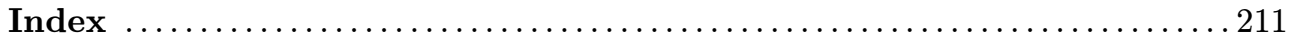

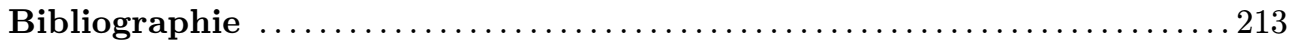





\section{CHAPITRE 1}

\section{INTRODUCTION}

L'objectif de ce travail est l'étude systématique des champs de revêtements entre courbes algébriques selon la direction initiée par M. Fried dans son approche du problème de Galois inverse [46], [47], [48]. Rappelons que, classiquement [49], par espace de Hurwitz, on entend la variété algébrique $H_{g, d}$ paramétrant les revêtements $\pi: C \longrightarrow \mathbb{P}^{1}$ de degré $d$ de la droite projective, par une courbe lisse de genre $g$, et définis sur un corps algébriquement clos $k$. Pour éviter les complications liées aux caractéristiques positives, nous ferons toujours l'hypothèse que le groupe de monodromie, c'est-à-dire le groupe de Galois de la clôture galoisienne, a un ordre premier à la caractéristique de $k$ (si celle-ci est non nulle). Ces espaces (ou mieux les champs associés $\mathcal{H}_{g, d}$ ), et leurs compactifications naturelles $\bar{H}_{g, d}$ et $\overline{\mathcal{H}}_{g, d}$, jouent un rôle significatif dans de nombreuses questions. Citons d'abord la formulation géométrique du problème de Galois inverse par Fried, mais aussi sa généralisation des tours modulaires [47]. Du côté géométrique, mentionnons les récents et profonds développements autour de l'étude asymptotique des nombres de Hurwitz, en d'autres termes la théorie de Gromov-Witten sur $\mathbb{P}^{1}$, développée par OkounkovPandharipande [84], Graber-Vakil [52], et Ekedahl-Lando-Shapiro-Vainshtein [40]. Signalons aussi l'étude, parallèle à certains égards, de l'espace des modules des courbes avec structure de spin (dans un sens généralisé) par Jarvis [59], [58], [60] et Chiodo [22]. On notera enfin que les champs de Hurwitz de dimension 1 sont présents dans l'étude des courbes de Teichmüller par Eskin-Kontsevich-Zorich [43], McMullen et d'autres. Un point de vue différent, celui des applications stables de Kontsevich $C \longrightarrow \mathbb{P}^{1}$ [71], voir aussi Manin [76], a été proposé comme une approche alternative pour aborder ce genre de questions, du moins lorsque la base est rigide. Il n'est pas utilisé dans notre travail.

Il doit être noté que des constructions partielles de l'espace de Hurwitz sont présentes dans la littérature, d'abord par Fulton [49] dans le cas des revêtements simples de la droite projective, puis dans une logique différente par Fried [46]. On notera cependant que l'espace modulaire considéré par Fried, et qui concerne la base $\mathbb{P}^{1}$, n'est pas identique au nôtre, bien que directement lié; le champ de Hurwitz est le champ quotient de celui de Fried par PGL $(2, k)$. La comparaison entre la construction 
de Fried et la présente construction est analysée par Dèbes [26], voir aussi CadoretTamagawa [19], Emsalem [42]. Une approche plus systématique de la construction du schéma de Hurwitz, ainsi que d'une compactification naturelle par adjonction des revêtements admissibles, a sa source dans le travail de Harris-Mumford [56], et dans les raffinements proposés par Mochizuki [77], Wewers [100], [99]. Lorsque la base est $\mathbb{P}^{1}$, on consultera Diaz [33] pour une discussion de la compactification de Natazon-Turaev d'esprit assez différent.

On gagne en souplesse en considérant, plus généralement, les champs $\mathscr{H}_{g, h, d}$ classifiant les revêtements $\pi: C \longrightarrow D$ définis sur un corps algébriquement clos $k$, de degré $d$, d'une courbe de genre $h$ par une courbe de genre $g$. Les champs de Hurwitz seront ainsi vus comme correspondances entre les espaces de modules de courbes $\mathcal{M}_{g}$ et $\mathcal{M}_{h}$. C'est cette position qui a été la motivation principale pour entreprendre le présent travail, point de vue mis en évidence par Looijenga [75].

Notre objectif est de proposer une construction uniforme, puis une étude détaillée, du champ $\mathscr{H}_{g, h, d}$, de sa compactification naturelle $\overline{\mathscr{H}}_{g, h, d}$ par adjonction des revêtements stables, et de leurs espaces de modules $H_{g, h, d}$ et $\bar{H}_{g, h, d}$. Si $h=0$, c'est la situation du début; si $d=1$, le champ $\mathscr{H}_{g, g, d}$ n'est autre que le champ classifiant des courbes $\mathcal{M}_{g}$. On observera qu'en général $\mathcal{H}_{g, h, d}$ n'est pas connexe, mais ses composantes connexes sont irréductibles, propriété qu'il partage avec tout champ localement noethérien et normal. On réunit de manière grossière certaines composantes en fixant le type de la ramification, appelé donnée de Hurwitz ou donnée de ramification et notée le plus souvent par la lettre $\xi$ (section 2.2). Le champ de Hurwitz $\mathcal{H}_{g, h, \xi}$ qui en découle n'est pas connexe en général. Le nombre de composantes connexes (ou irréductibles) est le nombre de Nielsen (définition 2.3.6).

Comme on va le voir, l'utilisation des champs de Hurwitz est d'une extrême souplesse, car il est possible de jouer avec les paramètres : groupe, donnée de ramification, marquage partiel ou total par le diviseur de ramification. Du fait de sa définition, le champ $\mathcal{H}_{g, h, \xi}$ (resp. $\overline{\mathcal{H}}_{g, G, \xi}$ ) est la source d'un morphisme naturel et important appelé discriminant qui associe à un revêtement $C \longrightarrow D$ sa base marquée par les points de branchement $\left(D,\left(\left\{Q_{j}\right\}\right)\right.$. Notant $r$ le nombre de points de branchement et $\mathcal{M}_{h, r}$ le champ des courbes de genre $h$ avec $r$ points marqués, ceci définit un morphisme $\mathcal{H}_{g, h, \xi} \longrightarrow \mathcal{M}_{h, r}$. Extrêmement important est le fait que ce morphisme se prolonge aux compactifications stables en un morphisme $\overline{\mathcal{H}}_{g, h, \xi} \longrightarrow \overline{\mathcal{M}}_{h, r}$. On observera qu'un revêtement stable est essentiellement stable marqué par les points de ramification (avec une subtilité liée à la coalescence éventuelle des points de ramification d'indice 2). De cette manière, les champs de Hurwitz apparaissent comme des revêtements ramifiés de $\mathcal{M}_{h, r}$, resp. $\overline{\mathcal{M}}_{h, r}$. On notera que ces espaces contiennent comme cas particuliers les espaces modulaires de courbes avec structure de niveau. C'est d'ailleurs un point de vue assez fructueux, exploité dans Abramovich-Corti-Vistoli [1], de voir un revêtement comme la donnée d'une sorte de structure de niveau (non abélien) sur la base, et réciproquement. 
La construction de la compactification stable du champ de Hurwitz, modelée sur celle de $\overline{\mathcal{M}}_{h, r}$ pour $\mathcal{M}_{h, r}$, est le sujet de la section 6. L'idée, maintenant classique, revient à ajouter à $\mathscr{H}_{g, h, \xi}$ les revêtements dits « stables », qui doivent être distingués des revêtements admissibles introduits dans Harris-Mumford [55], [56], et des applications stables de Kontsevich [71]. Contrairement aux applications stables, un revêtement stable est un objet qui, dans le cadre des revêtements, provient par spécialisation d'un revêtement entre courbes lisses, ou d'une autre manière est tel que le revêtement générique dans sa déformation universelle est un revêtement entre courbes lisses. Dans le cas galoisien, un point du bord est représenté par une courbe stable marquée par les points de ramification (ce qui interdit à ceux-ci à se localiser en des points doubles) et munie d'une action du groupe de Galois satisfaisant une condition de stabilité. On notera que les points doubles qui peuvent avoir un stabilisateur non trivial, alors cyclique, ne sont pas considérés comme des points de ramification, par contre ils interviennent dans la déformation universelle de $C \longrightarrow D$ (voir la section 5 pour des détails). Il y a une définition alternative au concept de stabilité, qui revient à ignorer le marquage par le diviseur de ramification. En effet, les points de ramification ne sont pas mobiles : ils sont assignés par l'action du groupe de Galois. Bien que sans importance pour les espaces de modules, les deux définitions peuvent conduire à des champs distincts (voir 6.5). On notera qu'un modèle de cette construction était déja visible dans la compactification des revêtements doubles utilisée par Beauville [8].

Dans le cas non galoisien, un revêtement stable sera défini (section 6) comme un revêtement qui étale-localement admet une clôture galoisienne (voir 6.6). Ces uniformisations galoisiennes locales doivent satisfaire à une condition de cohérence. Un revêtement stable est admissible, mais la réciproque n'est pas vraie : les champs correspondants diffèrent. Plus précisément, le champ de Hurwitz est la normalisation du champ de Harris-Mumford, et c'en est donc une désingularisation. On doit noter qu'on arrive essentiellement au même résultat en enrichissant un revêtement admissible d'une log-structure, construction étudiée par Mochizuki [77] et Wewers [99]. Cette construction diffère légèrement de la nôtre. C'est d'ailleurs une vertu essentielle des champs de Hurwitz, ou de ceux qui en dérivent, que de proposer des compactifications lisses aux divers problèmes de modules posés par les revêtements. Cela sera vérifié pour le champ de structures de niveau $\mathcal{M}_{g}(G)$ classifiant les $G$-revêtements principaux de base une courbe lisse de genre $g(g \geqslant 2)$. Nous décrirons (section 8) une compactification lisse $\bar{M}_{g}(G)$ de $\mathcal{M}_{g}(G)$, comme champ classifiant les $G$-revêtements principaux dégénérés stables. En comparaison de celle donnée par Deligne et Mumford dans [30], le léger coût à payer pour avoir la lissité est que $\overline{\mathcal{M}}_{g}(G)$ n'est pas représentable, même si le niveau domine le niveau abélien $(n), n \geqslant 3$. Un objectif similaire au nôtre a été atteint, par des méthodes et points de vue différents, par Abramovich, Vistoli et leurs coauteurs [3], [1], [2]. Les objets qu'ils décrivent sous le vocable de «balanced twisted stable maps » constituent les points d'un champ de Deligne-Mumford lisse et propre, et qui n'est pas autre 
chose que le champ quotient (voir 6.5) :

$$
\overline{\mathcal{M}}_{g^{\prime}, b}(B G)=\bigsqcup_{[\xi]} \overline{\mathcal{H}}_{g, G, \xi} \rrbracket Z(G)
$$

où la somme porte sur les données de Hurwitz et $Z(G)$ désigne le centre de $G$. Le point de vue des revêtements adopté ici rend les constructions et opérations sur ces champs très naturelles. Cela s'applique à l'extension $G$-équivariante des théories topologiques des champs par Jarvis et al. [62], [63], Natanzon [82].

Décrivons brièvement le contenu du présent travail. Dans la section 2 , on introduit les définitions de base, donnée de Hurwitz, ou de ramification, ou d'holonomie autour des points de branchement, et les opérations naturelles supportées par ces données, comme la restriction et l'induction. La description topologique de l'espace de Hurwitz est présentée, pour clarification, et conduit à la définition du nombre de Nielsen, introduit par Fried [46].

Dans la section 3, nous étudions les familles de courbes lisses munies de l'action d'un groupe fini, à donnée de Hurwitz fixée, c'est-à-dire les familles de $G$-revêtements galoisiens. Le résultat principal de cette section est un théorème d'inversion de la formule bien connue de Chevalley-Weil (Théorème 3.2.2). On montre que la donnée de Hurwitz est totalement déterminée par la connaissance des représentations de $G$ dans les espaces $H^{0}\left(C, \omega_{C}^{\otimes m}\right)$, en fait par un nombre fini d'entre elles. Dans la section 4, on étudie de la même manière les familles $G$-équivariantes de courbes stables marquées. L'inversion des relations de Chevalley-Weil est l'outil combinatoire crucial pour analyser le problème de la collision des points de ramification, phénomène qui peut intervenir dans certains cas, et qui correspond à la présence de points doubles à isotropie diédrale (théorème 4.2.2). L'objectif principal de la section 4 est de clarifier le comportement des points de ramification par spécialisation et déformation. On montre en particulier qu'une $G$-courbe stable est essentiellement marquée par les points de ramification, la seule complication venant éventuellement des points de ramification d'indice 2 .

Dans la section 5, nous étudions la déformation verselle d'un revêtement galoisien modérément ramifié $\pi: C \longrightarrow D$ de diviseur de branchement $B$. Dans le cas lisse, il est bien connu que les déformations d'un revêtement correspondent bijectivement à celles de la base équipée du diviseur de branchement. Dans le cas stable, le résultat essentiel (théorème 5.1.5) décrit l'écart entre la déformation verselle équivariante de $C$ et la déformation verselle de la base $D$ marquée par les points de branchement.

Dans la section 6, on construit les champs de Hurwitz et leurs compactifications stables. On montre que la projectivité des espaces $\bar{H}_{g, h, \xi}$ peut s'obtenir par preuve similaire à celle donnée par Gieseker et Mumford pour la projectivité de $\bar{M}_{g}$. Cette approche permet en fait d'obtenir de manière uniforme la projectivité des $\bar{M}_{g, n}$, par une preuve qui réduit essentiellement tout au cas de $\bar{M}_{g}$. La raison est bien sûr le fait que les points marqués sont implicites dans une action : ce sont les points de branchement, ou de ramification. Le théorème 6.5.1 compare notre construction et celle de Harris-Mumford [56]. On montre, d'abord dans le cas galoisien puis dans 
le cas non galoisien, que le champ de Hurwitz (ou l'espace modulaire de Hurwitz) étudié dans le présent travail est une désingularisation de celui, non normal, introduit dans [56].

Dans la section 7, on étudie la combinatoire du bord, qui se résume à la version équivariante de la description habituelle du bord de $\bar{M}_{g, n}$. Un rôle clé est joué dans cette section par la théorie de Bass des revêtements de graphes [7]; voir en particulier le théorème 7.2.7. En guise de test, dans la section 8 , on applique la construction des espaces de Hurwitz à la description modulaire du bord des espaces de modules de courbes avec structure de niveau $G$, abélien ou non. Rappelons que la construction usuelle de la compactification $\bar{M}_{g}(G)$, qui procède de manière indirecte (voir Deligne-Mumford [30]), ne conduit pas à une description modulaire précise des points $\mathrm{du}$ bord. Ici, ils apparaissent comme des revêtements principaux stablement dégénérés de groupe $G$. C'est aussi le point de vue récent développé par Abramovich, Corti et Vistoli [1]. On retrouve et clarifie quelques résultats de Boggi-Pikaart [16], van Geemen-Oort [50].

Dans les sections 9 et 10, on introduit les classes tautologiques qui vivent dans le groupe de Chow d'un champ de Hurwitz, particulièrement dans le cas du champ classifiant les revêtements cycliques (voir aussi Arsie-Vistoli [5]). On met en évidence quelques relations universelles entre ces éléments, de manière analogue au travail de Jarvis sur les courbes à spin [60]. Ces relations contiennent les relations de CornalbaHarris dans le cas hyperelliptique. On observe ensuite que la relation de RiemannHurwitz ordinaire s'étend à un ordre supérieur, sous la forme d'un relation simple entre classes kappa (théorème 10.3.4). Comme application, on calcule certaines intégrales de Hodge sur le champ des courbes hyperelliptiques suggérées par le travail de Bene [11]. Finalement (section 10.4), en suivant Chiodo [22], Coates-Givental [23], Tseng [96] et bien sûr le travail précurseur de Mumford [80], on montre comment appliquer le théorème de Riemann-Roch au revêtement universel, dans le cas des revêtements cycliques, pour mettre en évidence des relations entre les différentes classes introduites auparavant. Des idées similaires, mais avec des objectifs différents apparaissent dans le travail récent et indépendant de Jarvis-Kimura [64].

Les résultats du présent travail ont été annoncés en partie, et sous une forme restreinte, dans la note [13]. Ils s'appuient occasionnellement (sections 6 à 8) sur la thèse du second auteur (voir [90]).

Conventions et Notations. - Fixons tout d'abord quelques conventions et notations communes à la totalité de l'article. Les notations à caractère local seront précisées en début de chaque section.

Toutes les courbes sur un corps de base $k$ seront projectives, géométriquement réduites, connexes, éventuellement singulières avec pour singularités éventuelles des points doubles ordinaires. Dans la terminologie standard, ce sont donc des courbes nodales ou préstables. Pour une telle courbe, le module dualisant $\omega_{C}$ est un faisceau inversible. Le genre $g$ de la courbe $C$ est l'entier $g(C)=\operatorname{dim}_{k} H^{1}\left(C, \vartheta_{C}\right)=$ $\operatorname{dim}_{k} H^{0}\left(C, \omega_{C}\right)$. 
Si $E$ est un ensemble fini, son cardinal sera noté Card $E,|E|$ ou \#E.

Si $G$ est un groupe fini, on notera $\operatorname{Aut}(G)$ le groupe des automorphismes de $G$ et $\operatorname{Out}(G)$ le groupe des automorphismes extérieurs. Pour $s \in G$, on désignera par [[s]] la classe de conjugaison de $s$.

Lorsque la base est un corps $k$, les représentations de $G$ seront à valeurs dans des $k$-espaces vectoriels, et on supposera de manière permanente que, si la caractéristique de $k$ est $p>0$, alors $p$ ne divise pas l'ordre de $G$. Le groupe des caractères (multiplicatifs) $\operatorname{Hom}\left(G, k^{*}\right)$ sera noté $\hat{G}$. Nous noterons $\operatorname{Irrep}(G)$ l'ensemble des représentations (ou caractères) irréductibles de $G$, une même lettre désignant souvent une représentation $V$ ou son caractère $\chi_{V}: G \longrightarrow k^{*}$. La notation $(\cdot, \cdot)_{G}$ désignera le produit scalaire de deux caractères. Finalement, $R(G)$ est l'anneau des représentations (ou des caractères) de $G$. Si $\chi \in \hat{G}$, on notera $V_{\chi}$ l'espace vectoriel qui supporte la représentation de caractère irréductible $\chi$.

Pour tout réel $x$, on notera $[x]$ sa partie entière et $\langle x\rangle=x-[x]$ sa partie fractionnaire.

Nous utiliserons le symbole $\sqcup$ pour noter les sommes disjointes. 


\section{CHAPITRE 2}

\section{CLASSIFICATION DES REVÊTEMENTS}

Dans cette section, les définitions de base concernant la classification des revêtements sont introduites. Pour faire honneur à l'approche classique du problème de la classification et pour faciliter la comparaison avec le point de vue topologique, nous nous plaçons dans le cas de courbes lisses ; les définitions qui le nécessitent seront étendues au cas des courbes nodales dans les sections ultérieures. Nous étudions les invariants fondamentaux : donnée de Hurwitz et représentations de Hurwitz attachées aux revêtements galoisiens.

On fixe un corps algébriquement clos $k$ de caractéristique $p \geqslant 0$, et toutes les courbes sont des courbes lisses sur $k$. Si $p>0$, tous les groupes de monodromie qui apparaissent sont supposés d'ordre premier à $p$.

\subsection{Actions de groupes et revêtements}

\subsubsection{Revêtements, $G$-courbes, $G$-revêtements}

DÉFInItion 2.1.1. - Un revêtement entre deux courbes $C$ et $D$ sur le corps $k$ est un morphisme $\pi: C \longrightarrow D$ fini, surjectif et génériquement étale.

Un automorphisme du revêtement est un automorphisme $\sigma$ de $C$ tel que $\pi \sigma=\pi$. On note $\operatorname{Aut}(\pi)$ le groupe des automorphismes. Le revêtement est galoisien si l'ordre de $\operatorname{Aut}(\pi)$ est égal au degré de $\pi$, ou de manière équivalente si $C / \operatorname{Aut}(\pi) \cong D$. Tout revêtement possède une clôture galoisienne $\tilde{\pi}: \tilde{C} \longrightarrow C$ unique à isomorphisme non unique près ; c'est un revêtement tel que le composé $\pi \circ \tilde{\pi}$ est un revêtement galoisien et par lequel se factorise tout revêtement de $C$ vérifiant cette propriété. Le groupe de Galois d'une clôture galoisienne est appelé groupe de monodromie de $\pi: C \longrightarrow D$.

On associe à un revêtement le diviseur de ramification $\mathfrak{R} \subset C$, dont la multiplicité en un point $x \in X$ est égale à la longueur du $\theta_{C, x}$-module $\left(\Omega_{C / D}^{1}\right)_{x}$ des différentielles relatives, et le diviseur de branchement $\mathfrak{B}=\pi_{*}(\mathfrak{R}) \subset D$. On appelle indice de ramification en un point $x \in C$ l'indice de ramification de l'extension d'anneaux locaux $\theta_{D, y} \subset \theta_{C, x}$ où $y=\pi(x)$. Les points du support du diviseur de ramification sont appelés points de ramification, ce sont exactement les points vérifiant $e_{x}>1$. 
L'hypothèse que l'ordre du groupe de monodromie $G$ des revêtements est premier à la caractéristique $p$ a des conséquences très fortes. Comme l'indice de ramification $e_{x}$ d'un point $x \in \mathfrak{R}$ divise $|G|$, tous les revêtements que nous considérons sont modérément ramifiés. Cette restriction importante permet d'écarter les complications liées à la ramification sauvage, qui ne manqueraient pas de survenir par spécialisation. De plus, dans cette situation, la multiplicité de $\mathfrak{R}$ en $x$ est égale à $e_{x}-1$, de sorte que $\mathfrak{R}=\sum_{x \in C}\left(e_{x}-1\right) x$. La formule de Riemann-Hurwitz pour le revêtement $\pi: C \longrightarrow D$ de degré $d$ s'écrit donc :

$$
2 g(C)-2=d(2 g(D)-2)+\sum_{x \in C}\left(e_{x}-1\right) .
$$

Elle s'obtient en prenant les degrés dans l'égalité de faisceaux $\omega_{C}=\pi^{*}\left(\omega_{D}\right) \otimes \vartheta_{C}(\Re)$, où l'on a noté $\omega_{C}$ et $\omega_{D}$ les faisceaux dualisants de $C$ et $D$, isomorphes aux faisceaux de 1-formes différentielles.

Fixons maintenant un groupe fini $G$, d'ordre premier à $p=\operatorname{car}(k)$ si $p>0$.

DÉfinition 2.1.2. - Une $G$-courbe est une paire $(C, \phi)$ où $C$ est une courbe et $\phi$ est une action fidèle du groupe $G$ sur $C$.

Dans la suite, on omettra le plus souvent la lettre $\phi$ spécifiant l'action; l'action sera alors notée de manière abrégée $(s, x) \mapsto s x$. Une $G$-courbe sera souvent notée $(C, G)$ plutôt que $(C, \phi)$. Il est bien connu que la courbe quotient $C / G$ existe et est encore lisse. Le morphisme quotient $\pi: C \longrightarrow C / G$ est un revêtement galoisien de groupe $G$.

Dans cette situation, un point de ramification est un point $x \in C$ dont le stabilisateur $G_{x}$ est non trivial, il est alors cyclique d'ordre égal à l'indice de ramification $e_{x}$. Les orbites des points de ramification seront appelées des orbites spéciales, et les autres, des orbites non spéciales (la terminologie d'orbite régulière, synonyme de non spéciale, se rencontre aussi).

Si $(C, G)$ est une $G$-courbe, l'action de $G$ s'étend à $\omega_{C}^{\otimes k}$, définissant une $G$-linéarisation de ce dernier (voir Mumford [81], chap. 1, §3). Les représentations de $G$ dans les espaces vectoriels $H^{0}\left(C, \omega_{C}^{\otimes k}\right)$ sont les représentations de Hurwitz. On y reviendra en détail dans la section 3.2.

Une dernière notion de revêtement est fréquemment utilisée dans la littérature :

DÉfinition 2.1.3. - Un G-revêtement est la donnée d'une G-courbe $C$ et d'un revêtement galoisien $\pi: C \longrightarrow D$ tel que $G=\operatorname{Aut}(\pi)$.

2.1.2. Classification des revêtements. - Il y a plusieurs approches à la classification des revêtements, selon que la base est rigide (c'est-à-dire, que les automorphismes la fixent) ou pas, et que l'on prenne en compte les automorphismes du revêtement ou pas, voir notamment Dèbes [26], Fried [46], Mochizuki [77], Wajnryb [98], Wewers [99]. Par équivalence de revêtements, on doit comprendre la définition suivante. 
DÉfinition 2.1.4. - Deux revêtements $\pi: C \longrightarrow D$ et $\pi^{\prime}: C^{\prime} \longrightarrow D^{\prime}$ sont dits équivalents s'il existe des isomorphismes $f: C \stackrel{\sim}{\longrightarrow} C^{\prime}$ et $h: D \stackrel{\sim}{\longrightarrow} D^{\prime}$ tels que $\pi^{\prime} f=h \pi$, formant ainsi un diagramme commutatif :

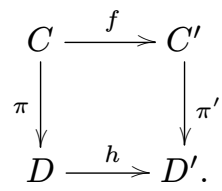

En ce qui concerne les $G$-courbes, la définition naturelle d'équivalence est la suivante.

DÉFInItion 2.1.5. - Un G-isomorphisme entre deux $G$-courbes $(C, \phi)$ et $\left(C^{\prime}, \phi^{\prime}\right)$ est un isomorphisme $f: C \longrightarrow C^{\prime}$ qui est équivariant, c'est-à-dire tel que pour tout $x \in C$ et tout $s \in G$, on a $f(\phi(s)) . x=\phi^{\prime}(s) . f(x)$. On dit que $C$ et $C^{\prime}$ sont $G$-équivalentes, ou en abrégé équivalentes, s'il existe un $G$-isomorphisme $f: C \longrightarrow C^{\prime}$. On parlera alors d'une classe de G-équivalence.

Enfin, la notion naturelle d'équivalence entre deux $G$-revêtements est l'existence d'isomorphismes $f, h$ comme dans la définition 2.1.4 tels que de plus $f$ est un $G$-isomorphisme. On dit alors que les $G$-revêtements sont $G$-équivalents (équivalents en abrégé). On voit facilement que cette définition n'est rien d'autre que la $G$-équivalence entre $(C, G)$ et $\left(C^{\prime}, G\right)$ au sens que l'on vient d'introduire. Cette définition correspond à l'équivalence faible dans Cadoret-Tamagawa [19].

Parallèlement à cette dernière définition, si $D=D^{\prime}$, c'est-à-dire si la base est rigide, on peut choisir comme relation d'équivalence la relation stricte, respectivement $G$-stricte. Cela revient à imposer la condition $h=$ id dans la définition 2.1.4, ce que l'on représente par un diagramme

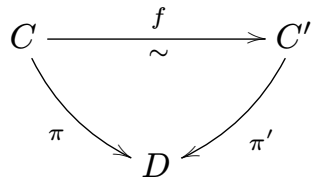

et, respectivement, à $f$ d'être $G$-équivariant. Le groupe $\operatorname{Aut}(\pi)$ des automorphismes d'un revêtement est le groupe des automorphismes stricts. L'équivalence stricte est aussi celle utilisée par Dèbes [26], Fried [46], Fulton [49], la base étant $\mathbb{P}^{1}$. Cette définition restrictive intervient de manière indirecte lors de l'étude des fibres du morphisme discriminant (section 6.5).

Dans le cas galoisien, c'est-à-dire pour les $G$-revêtements, on a donc à notre disposition deux manières, distinctes, d'identifier deux revêtements, selon que l'on exige que $f$ soit $G$-équivariant, ou non, c'est-à-dire la $G$-équivalence, ou l'équivalence simple. Cela correspond classiquement pour les courbes modulaires elliptiques, exemples de schémas de Hurwitz, à la distinction entre les courbes modulaires 
$X_{0}(N)$ et $X_{1}(N)$. Le cas des revêtements non galoisiens sera traité en détail dans la section 6.6, en se ramenant au cas galoisien par passage à la clôture galoisienne.

\subsection{Donnée de Hurwitz}

On désigne encore par $G$ un groupe fini d'ordre premier à la caractéristique $p$ du corps algébriquement clos $k$, si $p>0$.

2.2.1. Donnée de Hurwitz : Définition. - Soit $C$ une $G$-courbe, donc munie d'une action fidèle du groupe fini $G$. Si $x \in C$ est un point de ramification, c'està-dire à isotropie non triviale $G_{x} \neq 1$, et si l'on désigne par $\mathfrak{m}_{x}$ l'idéal maximal de l'anneau local $\theta_{C, x}$, on a une représentation naturelle

$$
\chi_{x}: G_{x} \longrightarrow G L\left(\mathfrak{m}_{x} / \mathfrak{m}_{x}^{2}\right)=k^{*}
$$

du groupe cyclique $G_{x}$ dans l'espace cotangent $T_{x}^{*}=\mathfrak{m}_{x} / \mathfrak{m}_{x}^{2}$ en $x$ (et, en dualisant, dans l'espace tangent). Ceci nous amène à considérer l'ensemble des couples $(H, \chi)$, où $H$ est un sous-groupe cyclique et $\chi$ un caractère de $H$. Ici, le caractère $\chi_{x}$ de $G_{x}$ est primitif, c'est-à-dire d'ordre $e_{x}=\left|G_{x}\right|$. Introduisons la définition suivante : nous dirons que les couples $(H, \chi)$ et $\left(H^{\prime}, \chi^{\prime}\right)$ sont conjugués, et nous écrirons alors $(H, \chi) \sim\left(H^{\prime}, \chi^{\prime}\right)$, s'il existe $s \in G$ tel que

$$
H^{\prime}=s H s^{-1} \quad \text { et } \quad \chi^{\prime}={ }^{s} \chi,
$$

où l'on a noté ${ }^{s} \chi$ le caractère de $H^{\prime}$ défini par ${ }^{s} \chi\left(t^{\prime}\right)=\chi\left(s^{-1} t^{\prime} s\right)$ pour tout $t^{\prime} \in H^{\prime}$. Il est manifeste que si $y=s x$, alors $\left(G_{y}, \chi_{y}\right) \sim\left(G_{x}, \chi_{x}\right)$. Ceci permet de définir le $G$-type d'une orbite spéciale, disons l'orbite de $x$, comme étant la classe de conjugaison de $\left(G_{x}, \chi_{x}\right)$, notée dans la suite $\left[G_{x}, \chi_{x}\right]$. Ainsi, nous parlerons d'une orbite de type $[H, \chi]$. D'une manière plus précise, nous dirons qu'un point fixe $x$ est d'holonomie $(H, \chi)$ si $G_{x}=H$ et $\chi_{x}=\chi$. Décomposons l'ensemble des points de ramification $F$ en une réunion des orbites spéciales $F=F_{1} \cup \cdots \cup F_{b}$, et notons $\left[H_{i}, \chi_{i}\right]$ le type de $F_{i}$. Sous une forme ou une autre, la définition suivante est classique (Fried [46], Lando [72], Serre [93]) : la donnée de Hurwitz (ou de ramification) de l'action de $G$ sur $C$ désigne la liste $\left\{\left[H_{i}, \chi_{i}\right]\right\}$ des types distincts on non, des orbites spéciales. En fait, il s'avère souvent commode de spécifier seulement les types distincts $\left[H_{i}, \chi_{i}\right]$, $1 \leqslant i \leqslant t$, et de préciser par ailleurs la multiplicité de $\left[H_{i}, \chi_{i}\right]$. Pour une formulation plus précise, formons d'abord le groupe abélien libre

$$
R_{+}(G)=\bigoplus_{[H, \chi]} \mathbb{Z}[H, \chi],
$$

la somme directe étant indexée par les classes de conjugaison de couples $[H, \chi]$, où $H$ parcourt les sous-groupes cycliques de $G$ et $\chi: H \longrightarrow k^{*}$ les caractères (non nécessairement primitifs) de $H$. On peut alors définir la notion de donnée de Hurwitz abstraitement, c'est-à-dire indépendamment du lien avec les revêtements. 
DÉFInition 2.2.1. - Une donnée de Hurwitz (ou donnée de ramification) pour le groupe $G$ est un élément du sous-monoïde $\operatorname{Hur}(G) \subset R_{+}(G)$ engendré par les $[H, \chi]$ où $\chi$ est un caractère primitif. En d'autres termes, c'est une combinaison linéaire formelle

$$
\xi=\sum b_{i}\left[H_{i}, \chi_{i}\right]
$$

avec $b_{i} \geqslant 1$ pour tout $i$. L'entier $b=\sum b_{i}$ est appelé le degré de $\xi$, et l'entier $g=$ $|G| \sum_{i} b_{i}\left(1-\left|H_{i}\right|^{-1}\right)$ est appelé le genre de $\xi$.

Il est sous-entendu que le sous-monoïde $\operatorname{Hur}(G) \subset R_{+}(G)$ contient 0. Les $b_{i}$, rangés en ordre décroissant, définissent une partition de $b$, qu'on appelera de manière laconique la partition associée à $\xi$.

Si $C$ est une $G$-courbe comme précédemment, la somme des types $\left[H_{i}, \chi_{i}\right]$, distincts ou non, des orbites spéciales, est une donnée de Hurwitz au sens de la définition 2.2.1. Il est clair que la donnée de Hurwitz ne dépend que de la classe d'équivalence de $(C, G)$. Par exemple, l'action libre a pour donnée de Hurwitz $\xi=0$. L'introduction du groupe $R_{+}(G)$ a pour justification le fait que les opérations sur les données de Hurwitz, détaillées ci-dessous, sont définies de manière naturelle dans $R_{+}(G)$.

2.2.2. Opérations sur les données de Hurwitz. - Il y a plusieurs opérations naturelles de source l'ensemble $\operatorname{Hur}(G)$, en liaison avec les sous-groupes et groupes quotients, naturellement définies sur $R_{+}(G)$. La première est l'induction. Soit $J \subset G$ un sous-groupe, alors toute donnée relative à $J$ définit de manière évidente une donnée de $G$, soit une application $\operatorname{Ind}_{J}^{G}: R_{+}(J) \longrightarrow R_{+}(G)$ :

$$
\xi=\sum b_{i}\left[H_{i}, \chi_{i}\right]_{J} \mapsto \xi=\sum b_{i}\left[H_{i}, \chi_{i}\right]_{G},
$$

l'indice précisant le groupe dans lequel la classe de conjugaison est prise. Si $J_{1}, J_{2}$ sont deux sous-groupes de $G$ et $\xi_{1}, \xi_{2}$ des données relatives à ces deux sous-groupes, on peut ainsi effectuer l'opération d'induction $\operatorname{Ind}_{J_{1}}^{G}\left(\xi_{1}\right)+\operatorname{Ind}_{J_{2}}^{G}\left(\xi_{2}\right)$ conduisant à un élément de $\operatorname{Hur}(G)$.

Il y a deux autres opérations naturelles concernant les données de ramification qui seront utilisées dans la suite, la restriction et le passage au quotient ou corestriction. Soit $J<G$ un sous-groupe ; on définit le morphisme de restriction $\operatorname{Res}_{J}^{G}: \operatorname{Hur}(G) \longrightarrow$ $\operatorname{Hur}(J)$ en étendant par linéarité

$$
\operatorname{Res}_{J}^{G}([H, \chi])=\sum_{J \backslash G / H}\left[J \cap g H g^{-1},{ }^{g} \chi_{\left.\right|_{J \cap g H g^{-1}}}\right]
$$

où la sommation dans le terme de droite porte sur les classes doubles modulo $(J, H)$, et où ${ }^{g} \chi$ désigne le caractère ${ }^{g} \chi(s)=\chi\left(g^{-1} s g\right)$.

Si maintenant $J=G / K$, où $K$ est distingué dans $G$, on définit un morphisme de corestriction, ou de passage au quotient $\operatorname{Cores}_{J}^{G}: \operatorname{Hur}(G) \longrightarrow \operatorname{Hur}(J) \operatorname{par}$

$$
\operatorname{Cores}_{J}^{G}([H, \chi])=\left[H / H \cap K, \chi^{\operatorname{Card}(H \cap K)}\right] .
$$

Les opérations décrites ci-dessus sont analogues à celles définies par exemple, en oubliant les caractères, dans le cadre de l'anneau de Burnside (voir Benson [12], 
chap. 5), et aussi à celles formulées par Snaith [95]. L'interprétation de ces deux opérations au niveau des actions sur les courbes, est expliquée dans l'énoncé ci-dessous, dont la preuve, élémentaire est omise. L'indice 1 signifie qu'on prend la projection sur le sous-groupe $\bigoplus_{H \neq 1} \mathbb{Z}[H, \chi]$. Notons enfin que $R_{+}(G)$ est un anneau commutatif pour la multiplication

$$
[H, \varphi] .[K, \psi]=\sum_{w \in K \backslash G / K}\left[w^{-1} H w \cap K,{ }^{w} \varphi \cdot \psi\right]
$$

et que le sous-monoïde $\operatorname{Hur}(G)$ est stable par la multiplication.

Proposition 2.2.2. - Soit $\xi$ la donnée de Hurwitz de l'action de $G$ sur $C$.

i) Si $J$ est un sous-groupe de $G$, alors $\operatorname{Res}_{J}^{G}(\xi)_{1}$ est la donnée associée à la restriction de l'action à $J$.

ii) Si $J=G / K$ est un quotient de $G$, alors $\operatorname{Cores}_{J}^{G}(\xi)_{1}$ est la donnée associée à l'action de $J=G / K$ sur la courbe $C / K$.

Sous des restrictions évidentes, le degré de $\operatorname{Cores}_{J}^{G}(\xi)$ est égal au degré de $\xi$. Il suffit pour cela que si $\xi=\sum_{i} b_{i}\left[H_{i}, \chi_{i}\right]$, aucun des $H_{i}$ ne soit inclus dans $K$. Cependant, la partition définie par $\operatorname{Cores}_{J}^{G}(\xi)$ peut être de longueur inférieure à celle de $\xi$. Un critère élémentaire qui assure l'égalité des partitions est :

Proposition 2.2.3. - Supposons que $\operatorname{pgcd}\left(|K|,\left|H_{i}\right|\right)=1$ pour tout $i$. Alors, les partitions attachées à $\xi$ et $\operatorname{Cores}_{J}^{G}(\xi)$ sont identiques.

Démonstration. - Il s'agit de prouver que l'application (2) est injective. Si les classes $\left[H_{i}, \chi_{i}\right]$ et $\left[H_{j}, \chi_{j}\right]$ ont même image par l'application de corestriction, cela dit en particulier qu'un conjugué de $H_{i}$ est contenu dans le sous-groupe produit semi-direct $K \rtimes H_{j}$. Quitte à remplacer le couple $\left(H_{i}, \chi_{i}\right)$ par un conjugué, on peut supposer que $H_{i}$ et $H_{j}$ sont deux sections de $K \rtimes H_{j} \longrightarrow J$, et $\chi_{i}=\chi_{j}$ caractère de $K \rtimes H_{j}$. L'hypothèse, i.e la nullité de $\mathrm{H}^{1}\left(H_{i}, K\right)$, assure que ces deux sections sont conjuguées dans $K \rtimes H_{j}$ et donc dans $G$.

Pour utilisation ultérieure, notons qu'il existe une action évidente de $\operatorname{Out}(G)$ sur $\operatorname{Hur}(G)$, définie par

$$
\theta[H, \chi]=\left[\theta(H), \chi \circ \theta^{-1}\right]
$$

Si on considère l'action de $G$ tordue au moyen de $\theta$, c'est-à-dire $(g, x) \mapsto \theta(g) x$, alors $\theta(\xi)$ n'est autre que la donnée de ramification de cette action.

Notons enfin que le groupe $\Gamma=\operatorname{Aut}(k)$ agit de manière naturelle sur le groupe $R_{+}(G)$ et donc sur $\operatorname{Hur}(G)$ selon la règle

$$
\sigma[H, \chi]=[H, \sigma \cdot \chi] .
$$

On peut interpréter la donnée $\sigma(\xi)$ comme celle associée à l'action de $G$ sur la courbe $C^{\sigma}=C \otimes_{k, \sigma} k$ tordue au moyen de $\sigma$. Il peut être utile de signaler une autre manière de décrire une donnée de ramification, voir par exemple Fried [46] ou Serre [93]. Supposons avoir fixé une racine de l'unité $\zeta \in k$ d'ordre $n=\operatorname{Card} G$. Si $\chi$ est un 
caractère primitif du sous-groupe cyclique $H$, et si Card $H=e$, il lui correspond un unique générateur $s$ de $H$ tel que $\chi(s)=\zeta^{n / e}$. Si $s^{\prime}$ correspond de la même manière à $\left(H^{\prime}, \chi^{\prime}\right)$, alors $(H, \chi) \cong\left(H^{\prime}, \chi^{\prime}\right)$ si et seulement si $s^{\prime}$ est conjugué à $s$. De cette manière, une donnée de Hurwitz devient une somme formelle de classes de conjugaison $\xi=\sum_{[[s]]} b_{[[s]]}[[s]]$. On notera que le stabilisateur de $[H, \chi]$ correspondant à $\left[[s]\right.$, pour l'action adjointe de $G$, est le commutant $C(s)=C_{G}(H)$ de $s$ (resp. de $H)$ dans $G$. Les opérations introduites ci-dessus s'explicitent facilement en termes de classes de conjugaison; par exemple,

$$
\operatorname{Res}_{J}^{G}([[s]])=\sum_{g \in J \backslash G / H}\left[\left[g s^{\left[J: g H g^{-1}\right]} g^{-1}\right]\right]
$$

si $H$ est le groupe cyclique de générateur $s$.

On va étendre le formalisme du $G$-type aux diviseurs $G$-invariants. Soit $D=$ $\sum_{i=1}^{d} x_{i}$ un diviseur invariant par $G$, tel que si $i \neq j, x_{i} \neq x_{j}$. Alors $D$ est une somme d'orbites deux à deux distinctes, et si $\left[H_{\alpha}, \chi_{\alpha}\right]$ sont les $G$-types de ces orbites, on appellera l'élément $\sum_{\alpha}\left[H_{\alpha}, \chi_{\alpha}\right]$ le $G$-type de $D$. On prolonge la définition en disant qu'une orbite non spéciale est de type [1] (holonomie locale triviale).

DÉfinition 2.2.4. - Soit une action de $G$ sur la courbe $C$ (lisse ou nodale). On dit que la courbe est marquée par $b+r$ orbites, dont $r$ non spéciales, si on a fait le choix de $b+r$ orbites, contenant les b orbites spéciales, et de $r$ orbites non spéciales, les orbites étant indexées $\theta_{1}, \ldots, \theta_{b+r}$.

Le cas non indexé peut être considéré de la même manière. Il est commode pour la suite d'étendre aux courbes marquées dans le sens précédent, la donnée de Hurwitz qui est attachée à une action de $G$, en ajoutant à la donnée initialement définie le terme $r[1]$.

\subsection{Nombre de Nielsen}

Maintenant, et pour la totalité de cette section, on suppose que $k=\mathbb{C}$. Une courbe est identifiée à une surface de Riemann compacte et connexe.

2.3.1. Classification topologique. - On s'intéresse à la classification équivariante des actions de groupes finis sur les courbes ( $G$-revêtements), maintenant sous le seul aspect topologique, de manière équivalente $C^{\infty}$. On fixe une surface $C^{\infty}$ compacte orientée $C$, de genre $g \geqslant 2$, munie d'une action du groupe fini $G$; on suppose que l'action de $G$ respecte l'orientation. Nous pouvons reformuler dans le cadre $C^{\infty}$ (orienté) l'essentiel de la section 2.2. La première observation est que la donnée de Hurwitz, définie algébriquement, est en fait un invariant $C^{\infty}$ de l'action de $G$ sur la surface $C$. En effet soit $x \in C$ un point fixe de stabilisateur $G_{x}=H$, il en découle une représentation linéaire réelle de degré 2 dans l'espace tangent réel $T_{x}$ de $C$ en $x$. La représentation complexifiée $T_{x} \otimes \mathbb{C}$ est alors somme directe de deux représentations conjuguées de degré 1 . On choisit celle $L$, parmi les deux, de sorte 
que si $\xi \in L-\{0\}$, alors la $\mathbb{R}$-base $(\operatorname{Re}(\xi), \operatorname{Im}(\xi))$ fournit l'orientation de $T_{x}$. Alors, on récupère le caractère local $\chi_{x}$ de la section 2.2 comme celui qui décrit l'action de $H$ sur la droite de direction $\xi$, c'est-à-dire que pour $s \in H$, on a $s \xi=\chi_{x}(s) \xi$.

La définition de l'équivalence des $G$-revêtements (définition 2.1.5) prend maintenant la forme suivante. Soient $C$ et $C^{\prime}$ des surfaces $C^{\infty}$ orientées munies d'une action fidèle de $G$.

DÉFInition 2.3.1. - Les surfaces sont G-équivalentes (en bref, équivalentes), s'il existe un difféomorphisme $G$-équivariant et préservant l'orientation $f: C \stackrel{\sim}{\longrightarrow} C^{\prime}$.

Là encore, cette relation ne doit pas être confondue avec la relation d'équivalence topologique des revêtements associés - dans la situation présente, le revêtement quotient $C \longrightarrow C / G$. Comme dans le cadre algébrique, la relation d'équivalence (2.3.1) s'identifie à l'équivalence au sens des $G$-revêtements formulée dans le contexte $C^{\infty}$ (2.1.5). Ceci étant, il est de nouveau clair que deux actions équivalentes de $G$ sur des surfaces $C$ et $C^{\prime}$ ont des données de Hurwitz identiques. Il est aussi immédiat que, la donnée de Hurwitz et le genre de $C$ étant fixés, alors le genre de $D=C / G$ est déterminé; c'est une conséquence directe de la formule de Riemann-Hurwitz.

Notre objectif maintenant est d'identifier les classes d'équivalence (2.3.1), en d'autres termes les types topologiques d'actions de $G$, groupe fixé, sur les surfaces de genre $g\left(g \geqslant 2\right.$ fixé); on fixe, en plus de $g$, le genre $g^{\prime}$ de la surface quotient $C / G$. La procédure pour conduire cette classification est la suivante.

On fixe tout d'abord une surface $D$, et $\beta \subset D$ un ensemble fini de points (distincts). Rappelons que $\operatorname{Diff}^{+}(D)$ désigne le groupe des $C^{\infty}$-difféomorphismes préservant l'orientation. On suppose en outre avoir fixé une partition $\beta=\beta_{1} \sqcup \cdots \sqcup \beta_{t}$ de $\beta$ en $t$ parties. On pose

$$
\operatorname{Diff}^{+}(D, \beta)=\left\{h \in \operatorname{Diff}^{+}(D), h\left(\beta_{i}\right)=\beta_{i} \quad(i=1, \ldots, t)\right\} .
$$

Il est connu que le groupe $\operatorname{Diff}^{+}(D)$ agit transitivement sur les parties finies numérotées de cardinal fixé, le choix de $\beta$ est donc sans importance. On suppose enfin avoir fixé une donnée de Hurwitz $\xi=\sum_{i=1}^{t} b_{i}\left[H_{i}, \chi_{i}\right]$, les classes $\left[H_{i}, \chi_{i}\right]$ étant deux à deux distinctes. On fait alors l'hypothèse que Card $\beta_{i}=b_{i}$. Fixons enfin un point de base $\star \in U=D-\beta$, et posons $\pi=\pi_{1}(U, \star)$.

DÉFINITION 2.3.2. - Un marquage d'une action de $G$ sur $C$ consiste en le choix d'un morphisme $\phi: C \longrightarrow D$ de classe $C^{\infty}$ qui identifie $D$ avec la surface quotient $C / G$, et qui en plus est tel qu'une orbite spéciale est du type $\left[H_{i}, \chi_{i}\right]$ si et seulement si c'est une fibre $\phi^{-1}(b)$ avec $b \in \beta_{i}$.

Par une définition identique à (2.1.4), une équivalence entre deux actions marquées $(C, \phi),\left(C^{\prime}, \phi^{\prime}\right)$ est donnée par un difféomorphisme $G$-équivariant $f$ : $C \stackrel{\sim}{\longrightarrow} C^{\prime}$ tel que $\phi^{\prime} \circ f=\phi$ (équivalence stricte). Il est clair que $\operatorname{Diff}^{+}(D, \beta)$ agit transitivement sur l'ensemble des classes de marquages de l'action donnée de $G$ sur $C$, mais pas simplement transitivement en général, du fait de l'existence possible d'automorphismes de la base $D$. On associe comme d'habitude à une action marquée 
par $\phi: C \longrightarrow D$ un morphisme de monodromie de $\pi$ dans le groupe des permutations de la fibre $\phi^{-1}(\star)$, qui correspond à une action à droite de $\pi \operatorname{sur} \phi^{-1}(\star)$. Supposons les points de cette fibre numérotés

$$
\phi^{-1}(\star)=\left(\star_{1}, \ldots, \star_{n}\right) .
$$

C'est un fait bien connu que le groupe de monodromie, c'est-à-dire l'image du morphisme $\pi \longrightarrow \mathfrak{S}_{\phi^{-1}(\star)}$, s'identifie, grâce à la numérotation des points de la fibre, avec le commutant de $G$ dans le groupe des permutations $\mathfrak{S}_{n}=\mathfrak{S}_{\phi^{-1}(\star)}$. De cette construction découle un morphisme surjectif

$$
\psi_{\phi}: \pi \longrightarrow G
$$

qui à conjugaison près ne dépend pas de la numérotation des points de $\phi^{-1}(\star)$. Rappelons la définition explicite de $\psi_{\phi}$. On choisit $\star_{1} \in \phi^{-1}(\star)$. L'image par $\psi_{\phi}$ d'un lacet $\alpha$ basé en $\star$ s'obtient en relevant, dans $\phi^{-1}(U), \alpha$ en $\tilde{\alpha}$ d'origine $\star_{1}$. Alors

$$
\psi_{\phi}(\langle\alpha\rangle)=\sigma \in G \Longleftrightarrow \tilde{\alpha}(1)=\sigma\left(\star_{1}\right) \text {. }
$$

Si on fait le choix d' un autre point de base $\star^{\prime} \in U$, et si $l$ est un chemin de $U$ qui joint $\star$ à $\star^{\prime}$, alors l'isomorphisme $l_{\star}: \pi_{1}(U, \star) \longrightarrow \pi_{1}\left(U, \star^{\prime}\right)$ conduit à la relation $\psi_{\phi}=\psi_{\phi}^{\prime} \circ l_{\star}$ à conjugaison près.

Dans la suite, la surjection caractéristique $\psi_{\phi}$ sera comprise à conjugaison près dans $G$, et modulo un changement éventuel du point de base. Le fait suivant, qui fait abstraction de la donnée de Hurwitz, est essentiellement le contenu du résultat élémentaire et bien connu sur la classification topologique (resp. $C^{\infty}$ ) des revêtements. Deux revêtements $\pi: C \longrightarrow D$ et $\pi^{\prime}: C^{\prime} \longrightarrow D$ étales de degré $n$ d'une même courbe $D$ sont topologiquement équivalents si et seulement si les actions de monodromie $\phi, \phi^{\prime}: \pi_{1}(D, \star) \longrightarrow \mathfrak{S}_{n}$ sont conjuguées (voir par exemple Fulton [49], proposition 1.2). Le groupe $\operatorname{Aut}(\pi)$ est le commutant dans $\mathfrak{S}_{n}$ de l'action de monodromie.

LEMme 2.3.3. - La correspondance $(C, \phi) \mapsto \psi_{\phi}$ établit une bijection entre d'une part les classes d'actions marquées de $G$ sur $C$, et d'autre part les classes de conjugaison de surjections $\pi \longrightarrow G$.

2.3.2. Nombre de Nielsen. - Pour poursuivre la classification topologique, fixons une présentation de $\pi=\pi_{1}(U)$ (présentation canonique) :

$$
\pi \cong\left\langle A_{1}, \ldots, A_{g^{\prime}} ; B_{1}, \ldots, B_{g^{\prime}} ; \gamma_{1}, \ldots, \gamma_{b} \quad / \quad \prod_{j=1}^{g^{\prime}}\left[A_{j}, B_{j}\right] \gamma_{1} \ldots \gamma_{b}=1\right\rangle .
$$

Le morphisme $\psi_{\phi}$ est déterminé par les images $a_{j}, b_{j}, \sigma_{k}$ des générateurs $A_{j}, B_{j}, \gamma_{k}$. Pour abréger, nous noterons $(\underline{a}, \underline{b}, \underline{\sigma})$ cette donnée, qui est essentiellement la description du revêtement au sens de Fried [46]. On a donc

$$
G=\left\langle\underline{a}, \underline{b}, \underline{\sigma} / \prod_{j=1}^{g^{\prime}}\left[a_{j}, b_{j}\right] \sigma_{1} \cdots \sigma_{b}=1\right\rangle .
$$


Le lien entre la donnée du $\left(2 g^{\prime}+b\right)$-uplet $(\underline{a}, \underline{b}, \underline{\sigma})$ et la donnée de Hurwitz $\xi$ est le suivant. Soient $C_{1}, \ldots, C_{r}$ les classes de conjugaison distinctes définies par $\sigma_{1}, \ldots, \sigma_{b}$; on suppose que la multiplicité de $C_{i}$ dans cette liste est $b_{i}$. Alors, la donnée de Hurwitz interprétée au moyen de classes de conjugaison (2.2.1) est décrite comme étant $\xi=$ $\sum_{i=1}^{r} b_{i}\left[C_{i}\right]$. D'une autre manière, on peut voir la donnée des $r$ classes distinctes comme un ensemble de $r$ couleurs; le lacet $\sigma_{j}=\psi_{\phi}\left(\gamma_{j}\right)$ étant de couleur $i$ si et seulement si $\sigma_{j} \in C_{i}$. En conséquence, le point de branchement $Q_{j}$ appartient à $\beta_{i}$ si et seulement si $\sigma_{j}=\psi_{\phi}\left(\gamma_{j}\right)$ est de couleur $i$. Dans la suite, nous noterons $\operatorname{Hom}_{\beta}(\pi, G)$ l'ensemble des surjections $\psi: \pi \longrightarrow G$ qui satisfont aux conditions ci-dessus, dictées par la donnée de ramification, donc en résumé :

$$
Q_{j} \in \beta_{i} \Longleftrightarrow \psi\left(\gamma_{j}\right) \in C_{i} .
$$

La classification topologique des actions de $G$ sur les surfaces de genre $g$, à donnée fixée, s'obtient à ce stade par la construction classique suivante. Soit une action de $G$ sur $C$; on choisit un marquage (définition 2.3.2) $\phi: C \longrightarrow D$, ce qui produit une surjection $\psi_{\phi} \in \operatorname{Hom}_{\beta}(\pi, G)$ définie à conjugaison près (lemme 2.3.3). Soit maintenant le mapping class group $\mathbb{M}_{g^{\prime}, b}$, qui est, rappelons-le, le sous-groupe de Out $(\pi)$, composé des automorphismes extérieurs qui permutent les classes de conjugaison des lacets $\left\langle\gamma_{j}\right\rangle$ $(j=1, \ldots, b)$. Il y a un morphisme naturel

$$
\operatorname{Diff}^{+}(B, \beta) \longrightarrow \operatorname{Out}(\pi)
$$

qui se factorise par $\mathbb{M}_{g^{\prime}, b}$. Un résultat fondamental de Nielsen décrit l'image de ce morphisme, qui s'identifie à l'ensemble des automorphismes de $\pi$ qui permutent les classes de conjugaison des $\left\langle\gamma_{j}\right\rangle$ à l'intérieur de chaque partie $\beta_{i}(i=1, \ldots, r)$. Si on considère la surjection canonique $\mathbb{M}_{g^{\prime}, b} \longrightarrow \mathfrak{S}_{b}$, l'image ci-dessus est le groupe noté dans la suite $\mathbb{M}_{g^{\prime},\left(b_{1}, \ldots, b_{r}\right)}$ image réciproque du sous-groupe $\mathfrak{S}_{b_{1}} \times \cdots \times \mathfrak{S}_{b_{r}}$. Il en résulte donc une action de $\mathbb{M}_{g^{\prime},\left(b_{1}, \ldots, b_{r}\right)}$ sur $\operatorname{Hom}_{\beta}(\pi, G) / G$. Le résultat suivant est bien connu :

Proposition 2.3.4. - Fixons une donnée de Hurwitz. Les classes topologiques d'actions de $G$ sur les surfaces de genre $g$ à donnée fixée sont en correspondance bijective avec les classes doubles

$$
\mathbb{M}_{g^{\prime},\left(b_{1}, \ldots, b_{r}\right)} \backslash\left(\operatorname{Hom}_{\beta}(\pi, G) / G\right) .
$$

Démonstration. - La preuve est claire. Il suffit d'éliminer le marquage, ce qui, compte

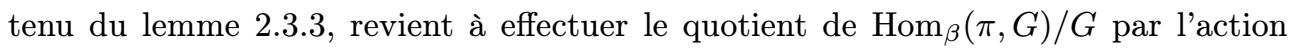
décrite ci-dessus de $\mathbb{M}_{g^{\prime},\left(b_{1}, \ldots, b_{r}\right)}$.

Exemple 2.3.5. - Revêtements de la sphère.

Examinons ce que donne la correspondance ci-dessus dans le cas $g^{\prime}=0$. On a $B=S^{2}$, et le mapping class group est ici le groupe $\mathbb{M}_{0, b}$ image du groupe des tresses à $b$ brins $B(b)$. Le groupe $\pi$ est dans ce cas le groupe libre de rang $b-1$ décrit par la présentation usuelle

$$
\left\langle\gamma_{1}, \ldots, \gamma_{b} / \prod \gamma_{i}=1\right\rangle
$$


Une surjection $\psi \in \operatorname{Hom}_{\beta}(\pi, G)$ est déterminée par le $r$-uplet $\underline{\sigma}=\left(\sigma_{1}, \ldots, \sigma_{b}\right)$ avec $\sigma_{i}=\psi\left(\gamma_{i}\right)(1 \leqslant i \leqslant b)$, ces générateurs étant soumis à la relation $\sigma_{1} \cdots \sigma_{b}=1$. Noter que $G$ est engendré par les $\sigma_{i}(i=1, \ldots, b)$. L'action par conjugaison sur les surjections devient dans cette description la conjugaison diagonale

$$
\underline{\sigma} \mapsto g \underline{\sigma} g^{-1}=\left(g \sigma_{1} g^{-1}, \ldots, g \sigma_{b} g^{-1}\right) .
$$

Si on fixe une donnée de Hurwitz, la restriction qui en découle, c'est-à-dire la couleur assignée aux points de branchement, est $Q_{j} \in \beta_{i} \Longleftrightarrow \sigma_{j} \in C_{i}$. L'action de $\mathbb{M}_{0, b}$ sur $\operatorname{Hom}_{\beta}(\pi, G) / G$ se déduit de l'action standard du groupe des tresses de la sphère, elle est en particulier engendrée par les opérations

$$
S_{i}(\underline{\sigma})=\left(\sigma_{1}, \ldots, \sigma_{i-1}, \sigma_{i} \sigma_{i+1} \sigma_{i}^{-1}, \sigma_{i}, \sigma_{i+2}, \ldots, \sigma_{b}\right) \quad(1 \leqslant i \leqslant b) .
$$

La condition (3) exige de se limiter à l'action du sous-groupe des tresses partiellement colorées $B\left(b_{1}, \ldots, b_{r}\right)$, image réciproque de $\mathfrak{S}_{b_{1}} \times \cdots \times \mathfrak{S}_{b_{r}}$ par la surjection canonique $B(b) \longrightarrow \mathfrak{S}_{b}$.

Dans le cas général, on est conduit à considérer l'ensemble $\mathrm{Ni}(\xi)$ des classes de Nielsen relatives à une donnée de Hurwitz $\xi$ fixée. La définition est la suivante (Dèbes [26], Fried [46]) : on considère les uplets $(\underline{a}, \underline{b}, \underline{\sigma}) \in G^{2 g^{\prime}+b}$ satisfaisant :

$$
\begin{aligned}
& \text { i) } \prod_{i=1}^{g^{\prime}}\left[a_{i}, b_{i}\right] \sigma_{1} \ldots \sigma_{b}=1, \\
& \text { ii) } G=\langle\underline{a}, \underline{b}, \underline{\sigma}\rangle, \\
& \text { iii) } Q_{j} \in \beta_{i} \Leftrightarrow \sigma_{j} \in C_{i} .
\end{aligned}
$$

L'ensemble des classes de Nielsen est alors le quotient

$$
\mathrm{Ni}(\xi)=\left\{(\underline{a}, \underline{b}, \underline{\sigma}) \in G^{2 g^{\prime}+b} \text { satisfaisant les conditions i), ii), iii) }\right\} / \sim
$$

où la relation $\sim$ désigne la conjugaison simultanée par des éléments du normalisateur de $G$ dans le groupe symétrique $\mathfrak{S}_{n}$.

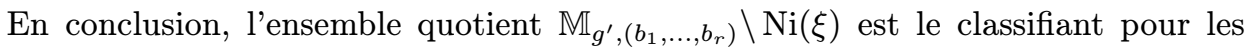
types topologiques d'actions de $G$ sur les surfaces de genre $g$, avec une donnée de Hurwitz fixée $\xi$; c'est un ensemble fini, ce qui conduit à poser :

DÉfinition 2.3.6. - Fixons une donnée de Hurwitz $\xi \in \operatorname{Hur}(G)$. Le nombre de Nielsen $h(\xi)$ est défini comme étant le nombre de types topologiques distincts d'actions de $G$ sur les surfaces de genres $g$, avec une donnée de Hurwitz fixée égale à $\xi$, soit

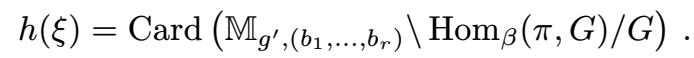

Le fait le plus important pour la suite est que le nombre de Nielsen $^{(1)}$ a une signification géométrique précise : il représente le nombre de composantes connexes, ou irréductibles, de l'espace des modules des revêtements, espace qui sera construit dans

(1) Ce nombre ne doit pas être confondu avec le nombre de Hurwitz $h_{g, G, \xi}=\# \operatorname{Hom}_{\beta}(\pi, G) / G$ (voir Okounkov-Pandharipande [84]). La formule de sommation de Burnside donne $h_{g, G, \xi=}$ $\sum_{[\pi]} \frac{1}{\# \operatorname{Aut}(\pi)}$, la sommation portant sur les classes d'équivalence (strictes) de $G$-revêtements de donnée de ramification fixée, d'une courbe de genre $g^{\prime}$ fixée, ainsi que les points de branchement $\beta$. 
les sections 5 et 6 . Pour le prouver, il faut resituer la classification des revêtements dans son contexte analytique, la théorie de Teichmüller (Earle [36], Edmonds [38]). La discussion qui suit est succincte, et pour plus de détails nous renvoyons aux références précédentes.

On fixe une surface de référence $\Sigma_{g}$ de genre $g \geqslant 2$, ainsi qu'une action fidèle du groupe fini $G$ sur $\Sigma_{g}$. Le couple $\left(\Sigma_{g}, G\right)$ définit donc ce qu'on peut appeler pour abréger, un type topologique. Soit $\operatorname{Conf}\left(\Sigma_{g}\right)$ l'ensemble des structures conformes sur $\Sigma_{g}$. le groupe $\operatorname{Diff}^{+}\left(\Sigma_{g}\right)$ agit naturellement sur $\operatorname{Conf}\left(\Sigma_{g}\right)$, et si $\operatorname{Diff}_{0}\left(\Sigma_{g}\right)$ désigne la composante connexe de l'élément neutre du groupe $\operatorname{Diff}^{+}\left(\Sigma_{g}\right)$, donc le sous-groupe des difféomorphismes homotopes (ou isotopes) à l'identité, le quotient

$$
T_{g}=\operatorname{Conf}\left(\Sigma_{g}\right) / \operatorname{Diff}_{0}\left(\Sigma_{g}\right)
$$

est une des formes de l'espace de Teichmüller [36]. En suivant la construction de Earle (loc. cit.), on est amené à considérer l'ensemble des points fixes de $G$ dans $\operatorname{Conf}\left(\Sigma_{g}\right)$. Soit $Z_{0}(G)$ le centralisateur de $G$ dans $\operatorname{Diff}_{0}\left(\Sigma_{g}\right)$. On peut montrer que, $N(G)$ étant le normalisateur de $G$ dans $\operatorname{Diff}^{+}\left(\Sigma_{g}\right)$, on a $\operatorname{Conf}\left(\Sigma_{g}\right)^{G}=N(G) \cap \operatorname{Diff}_{0}\left(\Sigma_{g}\right)$. L'espace de Teichmüller équivariant, c'est-à-dire celui qui classifie les actions à type topologique fixé, est $T_{g, G}=\operatorname{Conf}\left(\Sigma_{g}\right)^{G} / Z_{0}(G)$.

Sous ces conditions, on montre le résultat fondamental suivant (voir par exemple Earle [36], Natanzon [82]) :

ThÉORÈme 2.3.7. - $T_{g, G}$ est une sous variété fermée et contractile de $T_{g}$, difféomorphe à $\mathbb{R}^{6 g^{\prime}-6+2 b}$.

Ce résultat donne immédiatement le résultat énoncé au dessus, à savoir que l'espace de Teichmüller équivariant, classifiant les actions de $G$ sur les surfaces (de Teichmüller) de genre $g$ à type topologique fixé, est connexe; par suite, l'espace modulaire classifiant les actions à type topologique fixé, étant un quotient du précédent, est aussi connexe. En conclusion, si on fixe seulement la donnée de Hurwitz $\xi$, l'espace modulaire a exactement $h(\xi)$ composantes connexes.

Remarque 2.3.8. - La définition 2.3.6 du nombre de Nielsen $h(\xi)$ montre immédiatement que si $g^{\prime}=0$ et $G$ est abélien, alors $h(\xi)=1$. Dans le cas général, la détermination du nombre de Nielsen est une question connue comme difficile, conduisant à étudier l'action d'un groupe de tresses sur des uplets d'éléments de $G$ (Fried [46], Harris-Graber-Starr [54], Wajnryb [98]). On sait cependant que $h(\xi)=1$ dans plusieurs situations utiles (Edmonds [38], Fried [46], Fulton [49], Wajnryb [98]). Le cas le plus simple, et par ailleurs bien connu, est celui des revêtements simples de degré $n$ de $\mathbb{P}^{1}$, avec $r$ points de branchement [49]. Le résultat est étendu dans [54] à une base de genre $g^{\prime}$ arbitraire, sous l'hypothèse le nombre de points de branchement soit tel que $b \geqslant 2 n$. Il est immédiat que la clôture galoisienne d'un tel revêtement a pour groupe de Galois le groupe symétrique $\mathfrak{S}_{n}$, la donnée de ramification $\xi$ étant égale à $r$ fois la classe de conjugaison des transpositions. On peut prouver que le 
nombre de Nielsen est encore égal à 1 , dans le cas où la ramification est simple sauf au plus en une ou deux fibres, base $\mathbb{P}^{1}$ (voir [98]).

Un autre cas pour lequel la détermination de nombre de Nielsen est aisée est celui des structures de niveau abélien $n \geqslant 3$, voir Deligne-Mumford [30]. Alors $G=(\mathbb{Z} / n \mathbb{Z})^{2 g^{\prime}}$ et $\xi=0$. Dans ce cas, $h$ est égal à l'indice $\left[\mathrm{GL}_{2 g^{\prime}}(\mathbb{Z} / n \mathbb{Z}): \mathrm{Sp}_{2 g^{\prime}}(\mathbb{Z} / n \mathbb{Z})\right]$ (voir section 8).

Supposons maintenant que $G=\mathbb{Z} / n \mathbb{Z}$ et $\xi \neq 0$. La surjection $\psi: \pi \longrightarrow G$ est dans ce cas déterminée par les seules images des lacets d'homologie, du fait que les classes de conjugaison sont réduites à un seul élément; donc la donnée de Hurwitz détermine les éléments $\sigma_{1}, \ldots, \sigma_{b}$, ceux-ci étant soumis à la seule relation $\sigma_{1} \cdots \sigma_{b}=1$. Ne subsiste alors que la condition $G=\langle\underline{a}, \underline{b}, \underline{\sigma}\rangle$, soit $\operatorname{pgcd}\left(a_{j}, b_{k}, \sigma_{l}, n\right)=1$.

Le résultat qui suit est bien connu; il remonte à Nielsen [83]. Un résultat plus général sur le type topologique dans le cas d'un groupe abélien est aussi disponible (Edmonds [38], theorem 3.1).

Proposition 2.3.9. - Si $G$ est cyclique, pour toute donnée de Hurwitz $\xi \neq 0$, on a $h(\xi)=1$; en d'autres termes il y a un seul type topologique, et l'espace modulaire correspondant est connexe.

Exemple 2.3.10. - Revêtements triples de $\mathbb{P}^{1}$.

Considérons le cas $n=3, g^{\prime}=0$, et $r=g+2$. La donnée de ramification s'identifie à une partition (non ordonnée) de $\{1, \ldots, g+2\}$ en deux parties $\Lambda_{1}, \Lambda_{2}$, selon que le caractère local est $\sigma \mapsto j$, ou $j^{2}\left(j^{3}=1\right)$. On a $\left|\Lambda_{1}\right|+2\left|\Lambda_{2}\right| \equiv 0(\bmod 3)$, soit $\left|\Lambda_{1}\right| \equiv\left|\Lambda_{2}\right|(\bmod 3)$. Le nombre de types topologiques, c'est-à-dire de composantes connexes de l'espace de Hurwitz est donc

$$
\frac{1}{2} \sum_{2 l \equiv g-1(\bmod 3)}\left(\begin{array}{c}
g+2 \\
l
\end{array}\right) \text {. }
$$





\section{CHAPITRE 3}

\section{FAMILLES DE $G$-COURBES LISSES ET THÉORĖME DE CHEVALLEY-WEIL}

Dans cette section, on s'intéresse de manière essentielle à la géométrie de l'action d'un groupe $G$ dans une famille de courbes lisses sur une base sur laquelle $|G|$ est une fonction inversible.

\subsection{Géométrie du diviseur de branchement}

Dans toute la section 3.1, on considère une famille de courbes lisses $f: C \longrightarrow S$ de genre $g \geqslant 0$. Le morphisme $f$ est donc projectif, lisse, à fibres connexes. Il est alors bien connu que le foncteur en groupes $T \mapsto \operatorname{Aut}_{T}\left(C \times_{S} T\right)$ défini sur la catégorie des $S$-schémas est représentable par un $S$-schéma $\operatorname{Aut}_{C / S}$, fini et non ramifié si $g \geqslant 2$ (voir [30], théorème 1.11).

3.1.1. Diviseurs de points fixes. - Par définition, une action fidèle du groupe fini $G$ sur $C / S$ est un monomorphisme du schéma en groupes constant $G \times S$ dans le $S$-groupe Aut $_{C / S}$. L'action de $G$ est alors fidèle sur toute fibre de $C \longrightarrow S$; le qualificatif fidèle sera souvent omis. Nous supposons que $|G|$ est inversible dans les anneaux locaux de $S$; dans cette situation, se donner une action fidèle revient à se donner un morphisme injectif de $G$ dans le groupe des $S$-automorphismes de $C$, i.e. le groupe des $S$-points de $\operatorname{Aut}_{C / S}$. En effet, si $\sigma \neq 1$ est un $S$-automorphisme de $C$, l'automorphisme $\sigma_{s}$ induit sur une quelconque fibre $C_{s}$ est distinct de l'identité, comme il découle de la propriété de non ramification du $S$-schéma $\operatorname{Aut}_{C / S}$.

On commence par rassembler quelques propriétés élémentaires relatives aux sousschémas de points fixes. Puisque $|G|$ est inversible dans $\theta_{S}$, les stabilisateurs des points dans les fibres géométriques sont cycliques, et les sous-schémas de points fixes non vides sont donc ceux relatifs aux sous-groupes cycliques. Notons le fait facile suivant :

Proposition 3.1.1. - Pour tout sous-groupe cyclique $H \subset G$, le sous-schéma des points fixes $C^{H}$ de $H$ est un diviseur de Cartier relatif, étale sur $S$. 
Démonstration. - C'est la version relative, pour la propriété étale, du fait bien connu que lorsqu'un groupe réductif opère sur une variété lisse sur un corps, le sous schéma des points fixes est lisse; pour un groupe fini, on renvoie par exemple à Edixhoven [37], prop. 3.4. Dit d'une autre manière, si $P$ est un point fixe de $H$, d'image $s \in S$, on peut alors linéariser (formellement) l'action de $H$ en $P$, donc identifier l'anneau local complété $\hat{\vartheta}_{P}$ à $\hat{\vartheta}_{s}[[T]]$, l'action d'un générateur $\sigma$ de $H$ étant $\sigma(T)=\zeta T$, pour une racine de l'unité convenable $\zeta$. Alors l'équation de $C^{H}$ en $P$ est $\sigma(T)-T=(\zeta-1) T=$ 0 . Le résultat s'ensuit.

Lorsque $S$ est le spectre d'un corps algébriquement clos et $P \in C$ est un point de ramification de stabilisateur $H \neq 1$, on rappelle qu'on attache à $H$ un caractère primitif $\chi_{P}$ de $H(2.2 .1)$.

Lemme 3.1.2. - Supposons que la base $S$ est connexe. Soit un sous-groupe $H \subset G$ stabilisateur d'un point d'une fibre géométrique $C_{s_{0}}$, il est alors le stabilisateur d'un point dans toute fibre géométrique $C_{s}$.

Soit $\left\{H_{1}, \ldots, H_{q}\right\}$ la liste des sous-groupes cycliques de $G$ qui sont les stabilisateurs des points dans une fibre géométrique $C_{s}$, alors cette liste est indépendante de $s$, de même que le nombre de points de $C_{s}$ ayant pour stabilisateur $H_{i}$.

Démonstration. - Il résulte immédiatement de la proposition 3.1.1 que pour un sousgroupe $H$ de $G$, le fait d'avoir un point fixe dans une fibre géométrique $C_{s}$ entraîne que $H$ a un point fixe dans chaque fibre géométrique. De plus le cardinal de $C_{s}^{H}$, qui est le degré de $C^{H}$, est constant sur les fibres géométriques. Soit $\left\{H_{1}, \ldots, H_{q}\right\}$ la liste des sous-groupes $H$ tels que $C^{H} \neq \varnothing$, c'est-à-dire $C_{s}^{H} \neq \varnothing$ pour tout $s$. Posons

$$
\Delta_{i}(s)=\left\{x \in C_{s} \text { tel que } H_{i} \text { est le stabilisateur de } x\right\}
$$

et $r_{i}(s)=\operatorname{Card}\left(\Delta_{i}(s)\right)$. Définissons aussi $r_{i}^{*}(s)=\operatorname{Card} C_{s}^{H_{i}}-r_{i}(s)$, c'est-àdire le nombre de points dont le stabilisateur contient strictement $H_{i}$. Par la proposition 3.1.1, la fonction $r_{i}+r_{i}^{*}$ est constante. Pour prouver que $r_{i}$ est constante, supposons d'abord $H_{i}$ maximal dans la famille $\left\{H_{j}\right\}$; dans ce cas la conclusion est claire. Si tel n'est plus le cas pour $H_{i}$, et si la conclusion est admise pour tout sous-groupe $H_{j}$ tel que $H_{i} \varsubsetneqq H_{j}$, l'égalité $r_{i}^{*}=\sum_{j, H_{i} \varsubsetneqq H_{j}} r_{j}$ montre que $r_{i}^{*}$ est constante, et donc aussi $r_{i}$.

Fixons de nouveau une fibre géométrique $C_{s_{0}}$, et soit $\left\{P_{1}, \ldots, P_{l}\right\}$ la liste des points de $C_{s_{0}}$ qui ont pour stabilisateur $H=H_{i}$; sous l'hypothèse de connexité de la base, le nombre $l$ de ces points est indépendant de la fibre d'après le lemme 3.1.2, ce qui donne un sens à la définition. Pour tout $1 \leqslant j \leqslant l$, on note $\chi_{j}$ le caractère de $H$ attaché à $P_{j}$.

Lemme 3.1.3. - Si $S$ est connexe, l'ensemble $\left\{\chi_{1}, \ldots, \chi_{l}\right\}$ constitué de l caractères (distincts ou non, i.e. comptés avec multiplicité) de $H$ est indépendant de la fibre. 
Démonstration. - Soient $H_{i} \varsubsetneqq H_{j}$ deux sous-groupes de la liste du lemme 3.1.2. Le schéma des points fixes $C^{H_{j}}$ est un sous-schéma fermé de $C^{H_{i}}$, et il est étale sur $S$, par la proposition 3.1.1. L'injection de $C^{H_{j}}$ dans $C^{H_{i}}$ est étale finie sur $S$, et donc ouverte et fermée. Si on pose

$$
Z_{i}=C^{H_{i}}-\bigcup_{H_{i} \varsubsetneqq H_{j}} C^{H_{j}}
$$

il est clair que $Z_{i}$ est fini étale sur $S$, de degré relatif $l$. Considérons sur $Z_{i}$ le faisceau inversible $\mathscr{L}_{i}=\Omega_{C / S}^{1} \otimes \vartheta_{Z_{i}}$. C'est un $\left(\theta_{Z_{i}}, H_{i}\right)$-module, et $\pi_{*}\left(\mathscr{L}_{i}\right)$ est un $\left(\theta_{S}, H_{i}\right)$-module localement libre de rang $l$. Il admet une décomposition en facteurs isotypiques $\pi_{*}\left(\mathscr{L}_{i}\right)=\bigoplus_{\chi \in \hat{H}_{i}} E_{\chi} \otimes 1_{\chi}$, où $1_{\chi}$ désigne la représentation de degré 1 de caractère $\chi$, le module $E_{i}$ étant localement libre. Le nombre de fois que $\chi$ apparaît dans la liste $\left\{\chi_{i}\right\}$ de l'énoncé est égal au rang de $E_{\chi}$. La connexité de $S$ entraîne le résultat.

3.1.2. Ramification et branchement. - Une construction essentielle pour la suite de ce travail est celle des diviseurs de ramification et de branchement, qui sont des généralisations et variantes des notions de 2.1.1. On continue avec une action de $G$ sur la courbe $C / S$. On aura besoin d'introduire le morphisme quotient $\pi: C \longrightarrow C / G$, dont le résultat suivant, bien connu et par ailleurs élémentaire du fait de l'hypothèse de réductivité de $G$, résume les propriétés essentielles :

Proposition 3.1.4. - Soit $f: C \longrightarrow S$ une courbe propre et lisse de base $S$. Soit une action fidèle (3.1.1) de $G$ sur $C / S$. Alors la courbe quotient $D=C / G$ existe, et le morphisme $f$ se factorise par le morphisme quotient $\pi: C \longrightarrow D$ qui est fini plat de rang $|G|$. De plus, la formation de $D$ commute au changement de base. En particulier, au niveau des fibres on a $D_{s}=C_{s} / G(s \in S)$.

Démonstration. - Ces faits sont classiques. Pour la commutation entre quotient et changement de base, voir par exemple Katz-Mazur [67], appendice au chap. 7.

Le morphisme $\pi$ induit un morphisme $\pi^{*}\left(\Omega_{D / S}\right) \longrightarrow \Omega_{C / S}$ entre faisceaux de 1-formes. Au moyen de l'opération Div de Knudsen-Mumford exposée dans [69], chap. II, on peut alors définir le diviseur de ramification et le diviseur de branchement.

DÉfInition 3.1.5. - Soit $C \longrightarrow S$ une $S$-courbe projective lisse munie d'une action fidèle de $G$, comme ci-dessus, et soit $\pi: C \longrightarrow D$ le quotient. Le diviseur de ramification de l'action de $G$ sur $C$ est le diviseur de Cartier relatif $\Re \subset C$ défini par

$$
\mathfrak{R}=\operatorname{Div}\left(\pi^{*}\left(\Omega_{D / S}\right) \longrightarrow \Omega_{C / S}\right) .
$$

Le diviseur de branchement est le diviseur de Cartier relatif image directe $\mathfrak{B}=$ $\pi_{*}(\mathfrak{R}) \subset D$. 
On notera ces diviseurs $\mathfrak{R}_{\pi}$ et $\mathfrak{B}_{\pi}$ s'il est utile de préciser le morphisme $\pi$. Dans notre situation, on peut aussi écrire $\mathfrak{R}=\operatorname{Div}\left(\Omega_{C / D}\right)$ et en conséquence, on a

$$
\vartheta(\Re)=\Omega_{C / S}^{1} \otimes \pi^{*}\left(\Omega_{D / S}^{1}\right)^{-1} .
$$

Pour tout sous-groupe cyclique $H$, notons maintenant $\Delta_{H}$ la composante diviseur de Cartier relatif des points fixes $C^{H}$ formée des points de stabilisateur exactement $H$. On a l'expression classique du diviseur de ramification :

$$
\mathfrak{R}=\sum_{H \neq 1} \varphi(|H|) C^{H}=\sum_{H \neq 1}(|H|-1) \Delta_{H}
$$

où $\varphi$ désigne la fonction d'Euler (voir [88], chap. II, proposition 2.2.5). Cette dernière expression montre que la considération du quotient est accessoire pour définir $\mathfrak{R}$.

Nous allons maintenant définir le diviseur de branchement réduit ou discriminant, qui est un diviseur de Cartier (effectif) relatif $B \subset \mathfrak{B}$. Parmi les sous-groupes de $G$ qui sont des stabilisateurs de points dans les fibres géométriques (lemme 3.1.2), choisissons dans chaque classe de conjugaison un élément, ce qui donne une liste $\left\{H_{1}, \ldots, H_{q}\right\}$. Pour chaque indice $i$, formons le sous-schéma $Z_{i}$ des points de stabilisateur exactement $H_{i}$ (lemme 3.1.3). Rappelons que $Z_{i}$ est fini et étale sur $S$. Soit $N_{i}=N_{G}\left(H_{i}\right)$ le normalisateur de $H_{i}$ dans $G$, et $\bar{N}_{i}=N_{i} / H_{i}$. Le groupe $\bar{N}_{i}$ opère librement sur $Z_{i}$, de sorte que $B_{i}=Z_{i} / \bar{N}_{i}$ est fini étale sur $S$. Ainsi $B_{i} \subset D$ est un diviseur de Cartier relatif de $D / S$, dont les points fermés correspondent aux orbites spéciales de points de $C$ avec isotropie $H_{i}$. Cette remarque élémentaire sera souvent utilisée. Ces considérations mènent à la définition, dans laquelle les notations sont celles en vigueur dans 3.1.2 :

DÉfinition 3.1.6. - Le diviseur de Cartier relatif $B=\sum_{i=1}^{q} B_{i}$ de $D / S$ est appelé diviseur de branchement réduit, ou discriminant, de $(C / S, G)$.

Le discriminant est fini, étale sur $S$, de degré égal au nombre d'orbites spéciales contenues dans chaque fibre, c'est-à-dire le nombre de points de branchement. On le notera $B_{\pi}$ si la précision s'avère utile. Il est facile de relier le diviseur de branchement $\mathfrak{B}$ aux diviseurs $B_{i}$ précédents. En effet, poussant par $\pi$ l'expression $\mathfrak{R}=\sum_{H \neq 1} \varphi(|H|) C^{H}$ (voir ci-dessus), on obtient :

$$
\mathfrak{B}=\sum_{i=1}^{q}\left(|G|-\frac{|G|}{\left|H_{i}\right|}\right) B_{i} .
$$

On peut raffiner les décompositions de $\mathfrak{B}$ et $B$ en invoquant le type d'une orbite spéciale. Notons $\xi$ la donnée de Hurwitz et $\left[H_{1}, \chi_{1}\right], \ldots,\left[H_{k}, \chi_{k}\right]$ les classes deux à deux distinctes qui apparaissent dans $\xi$, de sorte que $\xi=\sum_{i=1}^{k} b_{i}\left[H_{i}, \chi_{i}\right]$ avec $b_{i}>0$. On a alors la décomposition suivante :

Proposition 3.1.7. - Le diviseur de branchement $\mathfrak{B}$ se décompose en une somme $\mathfrak{B}=\sum_{i=1}^{k} T_{\alpha}$ de diviseurs deux à deux disjoints tels que $\operatorname{deg}\left(T_{\alpha}\right)=b_{\alpha}$. Les points 
du support de $T_{\alpha}$ sont les images des fibres singulières de type $\left[H_{\alpha}, \chi_{\alpha}\right]$. La même conclusion vaut pour le discriminant $B$.

Démonstration. - Fixons l'un des sous-groupes $H \in\left\{H_{i}\right\}$, et considérons les différents caractères primitifs $\chi_{1}, \ldots, \chi_{m}$ tels que la classe de $\left(H, \chi_{i}\right)$ apparaisse dans la donnée $\xi$. Notons que $\left(H^{\prime}, \chi^{\prime}\right) \equiv(H, \chi)$ équivaut (voir 2.2) à l'existence de $t \in N=N_{G}(H)$ tel que $\chi^{\prime}(s)=\chi\left(t^{-1} s t\right)$, pour tout $s \in H$. Par réciprocité de Frobenius, cela est aussi équivalent à $\operatorname{Ind}_{H}^{N}(\chi) \cong \operatorname{Ind}_{H}^{N}\left(\chi^{\prime}\right)$. La représentation $\operatorname{Ind}_{H}^{N}(\chi)$ désigne la représentation obtenue par induction de $H$ à $N$ de la représentation de degré 1 de caractère $\chi$. Soit $Z$ le sous-schéma étale sur $S$ associé à $H$ comme dans le lemme 3.1.3. Le faisceau conormal $\mathcal{N}$ de $Z$ dans $C$ coïncide avec $\Omega_{C / S}^{1} \otimes \theta_{Z}$. Il est inversible et muni d'une action de $\bar{N}=N / H$. L'image directe $\pi_{*}(\mathcal{N})$ est alors un faisceau muni d'une action de $\bar{N}$. On peut ainsi le décomposer en facteurs isotypiques

$$
\pi_{*}(\mathcal{N})=\bigoplus_{v \in \operatorname{Irrep}(\bar{N})} \mathcal{L}_{v} \otimes v
$$

où dans cette somme directe, $v$ parcourt les représentations irréductibles de $\bar{N}$, et $\mathscr{L}_{v}$ est un module localement libre. Sur les composantes connexes de $\pi(Z)$, qui sont disjointes deux à deux, le rang de chacun de ces faisceaux est constant et de cela découle que la représentation $\pi_{*}(\mathcal{N})_{y}$ est constante le long des fibres $y \in \pi(Z)$. Cela prouve le résultat.

Remarque 3.1.8. - Soit $(H, \chi)$ une paire constituée d'un sous-groupe cyclique de $G$ et d'un caractère primitif, et notons $\Delta_{(H, \chi)}\left(\right.$ resp. $\left.\Delta_{[H, \chi]}\right)$ le diviseur de $C$, lieu des points fixes d'holonomie exactement $(H, \chi)$ (resp. $(H, \chi)$ ou un conjugué). Soit $C(H, \chi)$ le stabilisateur de $(H, \chi)$ pour l'action adjointe de $G$, qui n'est autre que le commutant $C_{G}(H)$ de $H$ dans $G$. On note que $C_{G}(H) / H$ agit librement sur $\Delta_{(H, \chi)}$, de quotient la composante $B_{[H, \chi]}$ de $B_{\pi}$. On notera par ailleurs la décomposition en une somme disjointe

$$
\Delta_{[H, \chi]}=\sum_{g \in G / C_{G}(H)} e \Delta_{(H, \chi)} .
$$

\subsection{Inversion de la formule de Chevalley-Weil}

Soit $C$ une courbe projective lisse définie sur le corps algébriquement clos $k$, de genre $g \geqslant 2$, munie d'une action de $G$ de donnée de Hurwitz $\xi$. Le faisceau canonique $\omega_{C}=\Omega_{C}$ est un $G$-faisceau inversible, en particulier les espaces vectoriels $H^{0}\left(C, \omega_{C}^{\otimes m}\right)$ sont de manière naturelle des représentations de $G$, appelées dans la suite les représentations de Hurwitz associées à la $G$-courbe $C$, et notées $\operatorname{Hurw}_{m}(C)$, ou Hurw $_{m}$, s'il n'y a pas de doute sur $C$. 
3.2.1. Formule de Chevalley-Weil. - Il y a une connexion étroite entre la structure de ces représentations et la donnée de Hurwitz : pour l'essentiel, elle est donnée par le théorème de Chevalley-Weil (voir [21]), dont le contenu se résume aux relations (5) et (6) ci-dessous. Rappelons tout d'abord la formule de Chevalley-Weil sous la forme donnée par Ellingsrud et Lønsted [41] (voir aussi [62], theorem 6.3, ou [79] pour le cas cyclique). Soit $\mathcal{L}$ un $(\Theta, G)$-module inversible; la trace de Lefschetz de $\mathcal{L}$ est l'élément de l'anneau des représentations $R(G)$ donné par :

$$
L_{G}(\mathscr{L})=\sum_{i=0}^{1}(-1)^{i}\left[H^{i}(C, \mathscr{L})\right]
$$

où $[v]$ désigne la classe dans $R(G)$ d'un $k G$-module $v$. Lorsque l'action de $G$ est libre, on a la formule bien connue (voir [41], theorem 2.4) :

$$
L_{G}(\mathscr{L})=\frac{\chi(\mathscr{L})}{|G|}[k[G]] .
$$

C'est ce résultat qui, lorsque $\mathscr{L}=\omega_{C}$, porte le nom de formule de Chevalley-Weil (pour une action libre). Il donne les multiplicités des facteurs irréductibles des représentations $\operatorname{Hurw}_{m}(C)$. Noter que Morrison et Pinkham ont étudié en détail la représentation $H^{0}\left(C, \omega_{C}\right)$; ils en donnent en particulier une caractérisation lorsque $G$ est cyclique [79].

Indiquons brièvement la preuve de la formule (5). Soit toujours $\pi: C \longrightarrow D$ le quotient de $C$ par $G$. On considère la décomposition du $\left(\Theta_{D}, G\right)$-faisceau $\pi_{*}(\mathscr{L})$ en facteurs isotypiques $\pi_{*}(\mathscr{L})=\bigoplus_{v \in \hat{G}} E_{v} \otimes v$, avec $E_{v}=\pi_{*}^{G}\left(\mathscr{L} \otimes v^{\vee}\right)$. Le faisceau $E_{v}$ est localement libre, car sans torsion, de rang $\operatorname{dim}(v)$. Ceci se voit par exemple en notant qu'au point générique $\pi_{*}(\mathscr{L})$ est la représentation régulière. La relation

$$
L_{G}(\mathscr{L})=\sum_{v \in G^{\vee}} \chi\left(E_{v}\right)[v]
$$

montre que pour obtenir la trace de Lefschetz, il suffit d'expliciter $\chi\left(E_{v}\right)$, du fait du théorème de Riemann-Roch qui donne $\chi\left(E_{v}\right)=\operatorname{deg}\left(E_{v}\right)+\operatorname{dim}(v)\left(1-g^{\prime}\right)$. Notons $\left\{y_{1}, \ldots, y_{r}\right\}$ les points de branchement de $\pi$. Il est clair que le conoyau $Q(\mathcal{L}, v) \mathrm{du}$ morphisme canonique $\pi^{*}\left(E_{v}\right) \longrightarrow \mathscr{L} \otimes v^{\vee}$ a pour support le discriminant, i.e. les points de branchement. Soit un point $x_{i} \in \pi^{-1}\left(y_{i}\right)$ fixé de stabilisateur $H_{i}$, on a

$$
Q(\mathscr{L}, v)_{y_{i}}=\bigoplus_{\pi(x)=y_{i}} Q(\mathscr{L}, v)_{x}=\operatorname{Ind}_{H_{i}}^{G}\left(Q(\mathscr{L}, v)_{x_{i}}\right)
$$

En particulier notant $n=|G|$, si Card $H_{i}=e_{i}$, on a $\operatorname{dim} Q(\mathcal{L}, v)_{y_{i}}=\frac{n}{e_{i}} \operatorname{dim} Q(\mathcal{L}, v)_{x_{i}}$. Pour décrire le $k H_{i}$-module $Q(\mathscr{L}, v)_{x_{i}}$ on peut noter que si $\nu_{i} \in R\left(H_{i}\right)$ est la représentation de degré 1 de $H_{i}$ dans la fibre $\mathscr{L}\left(x_{i}\right)$, on a $\left(\mathscr{L} \otimes v^{\vee}\right)_{x_{i}}=\theta_{x_{i}} \otimes v^{\vee}\left(\nu_{i}\right)$. Le caractère $\chi_{i}$ de la représentation cotangente au point fixe $x_{i}$ de $H_{i}$ étant primitif, on a dans $R\left(H_{i}\right)$ une décomposition

$$
\left[v_{\left.\right|_{H_{i}}}^{\vee}\left(\nu_{i}\right)\right]=\sum_{l=1}^{e_{i}} m_{i l} \chi_{i}^{l} .
$$


Pour expliciter la contribution de $Q(\mathcal{L}, v)_{x_{i}}$, on est ramené à calculer la dimension du conoyau de $\left(\chi_{i}^{l} \otimes \theta_{x_{i}}\right)^{H_{i}} \otimes_{\theta_{y_{i}}} \vartheta_{x_{i}} \longrightarrow \chi_{i}^{l} \otimes \theta_{x_{i}}$, qui est $e_{i}-l$. On en tire immédiatement $\operatorname{dim} Q(\mathcal{L}, v)_{x_{i}}=\sum_{l=1}^{e_{i}-1}\left(e_{i}-l\right) m_{i l}$, d'où le degré de $E_{v}$ :

$$
\operatorname{deg} E_{v}=\frac{\operatorname{dim}(v) \operatorname{deg}(\mathscr{L})}{n}-\sum_{i=1}^{r} \sum_{l=1}^{e_{i}-1} \frac{e_{i}-l}{e_{i}} m_{i l} .
$$

Nous réfèrerons aux relations (5), (6) comme formant le contenu de la formule de Chevalley-Weil.

EXEMPle 3.2.1. - Revêtements simples de $\mathbb{P}^{1}$.

Pour éclairer la relation (6), considérons le cas d'un revêtement simple, avec $m=1$ (un revêtement est simple si le cardinal de la fibre au-dessus d'un point de branchement est le degré diminué de 1). Si $\pi: C \longrightarrow \mathbb{P}^{1}$ est simple, alors il est connu, et facile à vérifier, que le groupe de Galois associé est le groupe symétrique $G=\mathfrak{S}_{n}$, et la donnée de ramification est $r(12)$, où par (12) on désigne la classe de conjugaison des transpositions. Un calcul facile conduit pour $\mathscr{L}=\Omega_{C}$ à

$$
\operatorname{deg}\left(E_{v}\right)=\chi_{v}(1)\left(\frac{r}{2}-2\right)-\frac{r}{4} \chi_{v}((12))
$$

et vu que $\operatorname{deg}\left(E_{v}\right)=\chi_{v}(1)$, on trouve $\chi\left(E_{v}\right)=\left(\frac{r}{4}-1\right) \chi_{v}(1)-\frac{r}{4} \chi_{v}((12))$. Notons que, par les relations d'orthogonalité des caractères, on a

$$
\sum_{v \in \bigotimes_{n}} \chi_{v}((12)) \chi_{v}(1)=0
$$

ce qui assure la consistance des relations de dessus avec l'expression du genre donnée par la relation de Riemann-Hurwitz $g_{C}=\operatorname{dim} H^{0}\left(C, \Omega_{C}\right)=1+n !\left(\frac{r}{4}-1\right)$.

Spécialisons maintenant la relation (6), pour utilisation ultérieure, dans le cas d'un groupe $G$ cyclique (d'ordre $n$ ), et $\mathscr{L}=\omega_{C}^{\otimes m}$. Fixons un générateur $\sigma$ de $G$, et soit $\chi$ la représentation de degré 1 de $G$ donnée par $\chi(\sigma)=\epsilon$, où $\epsilon$ désigne une racine primitive $n$-ième de l'unité fixée. Le stabilisateur $H_{i}$ est alors engendré par $\sigma^{n / e_{i}}$, et le caractère local $\chi_{i}$ est donné par $\chi\left(\sigma^{n / e_{i}}\right)=\epsilon^{n k_{i} / e_{i}}$ pour un certain entier $k_{i}$, avec $0<k_{i}<e_{i}$ et $\left(k_{i}, e_{i}\right)=1$. Soit $\eta_{i}$ défini par $\eta_{i} k_{i} \equiv 1\left(\bmod e_{i}\right)$, alors la restriction de $\chi^{l}$ à $H_{i}$ s'identifie avec $\chi_{i}^{l \eta_{i}}$.

Avec le choix $\mathscr{L}=\omega_{C}^{m}(m \geqslant 1)$, les représentations introduites ci-dessus sont $\nu_{i}=\chi_{i}^{m}$, et si $v=\chi^{l}$, la multiplicité de $\chi_{i}^{j}$ dans la restriction à $H_{i}$ de $v^{\vee}\left(\nu_{i}\right)$ est

$$
m_{i j}=\left\{\begin{array}{ccc}
0 & \text { si } m-l \eta_{i} \not \equiv j & \left(\bmod e_{i}\right) \\
1 & \text { si } m-l \eta_{i} \equiv j & \left(\bmod e_{i}\right)
\end{array}\right.
$$

Adoptons pour la suite la notation suivante : si $\langle x\rangle$ désigne la partie fractionnaire de $x \in \mathbb{R}$, on pose $\langle\langle x\rangle\rangle=1-\langle-x\rangle$. Soit $g^{\prime}$ le genre de $B$; alors avec $v=\chi^{l}$ et en notant 
dans ce cas $E_{l}$ au lieu de $E_{v}$, la relation (6) se spécialise en

$$
\chi\left(E_{l}\right)=\frac{m(2 g-2)}{n}+\left(1-g^{\prime}\right)-\sum_{i=1}^{r}\left(1-\left\langle\left\langle\frac{m-l \eta_{i}}{e_{i}}\right\rangle\right\rangle\right)
$$

et, tenant compte de la formule de Riemann-Roch,

$$
\chi\left(E_{l}\right)=(2 m-1)\left(g^{\prime}-1\right)+m r-\sum_{i=1}^{r}\left(\left\langle\frac{l \eta_{i}-m}{e_{i}}\right\rangle+\frac{m}{e_{i}}\right) .
$$

On obtient en particulier, si $m=1$, que la multiplicité $\mu_{l}$ de $\chi^{l}$ dans $H^{0}\left(C, \omega_{C}\right)$ est

$$
\mu_{l}= \begin{cases}g^{\prime} & \text { si } l=0, \\ g^{\prime}-1+r-\sum_{i=1}^{r}\left(\left\langle\frac{l \eta_{i}-1}{e_{i}}\right\rangle+\frac{1}{e_{i}}\right) & \text { si } l=1, \ldots, n-1,\end{cases}
$$

et pour $l=0$ et $m \geqslant 1$, la multiplicité est

$$
\mu_{0}=(2 m-1)\left(g^{\prime}-1\right)+m r-\sum_{i=1}^{r}\left\lceil\frac{m}{e_{i}}\right\rceil .
$$

3.2.2. Inversion des relations de Chevalley-Weil. - Inverser les relations de Chevalley-Weil (6) revient à montrer que la connaissance des représentations de Hurwitz $H^{0}\left(C, \omega_{C}^{\otimes m}\right)$ permet de retrouver la donnée de Hurwitz $\xi$ de l'action de $G$ (on verra qu'en fait un nombre fini d'entre elles suffisent). Cette remarque tire son utilité du fait que les représentations de Hurwitz se comportent «bien » dans les familles, car les $G$-faisceaux considérés sont localement $\emptyset_{S}[G]$-projectifs ; elle implique la comparaison des représentations associées à des fibres géométriques distinctes d'une courbe relative $C / S$, en particulier les fibres au-dessus d'un point et d'une spécialisation de ce point. Pour clarifier ce que signifie cette comparaison, expliquons la situation lorsque la base est le spectre d'un anneau de valuation discrète.

Soit donc $S=\operatorname{Spec}(R)$, où $R$ est un anneau de valuation discrète complet, de corps des fractions $K$, et de corps résiduel $k$ algébriquement clos, de caractéristiques égales ou inégales. Si la caractéristique de $k$ est $p>0$, on suppose que $|G|$ est premier à $p$. La comparaison des représentations irréductibles de $G$ sur $K$ à celles définies sur $k$ se fait comme d'habitude par l'intermédiaire du triangle cde de Cartan (voir Serre [92]). Sous nos hypothèses sur les caractéristiques, les flèches $c, d, e$ sont des isomorphismes. L'algèbre $k G$ est semi-simple et tout $R G$-module est alors projectif. Si $V_{1}, \ldots, V_{k}$ sont des $R G$-modules projectifs indécomposables qui forment une base de l'anneau des classes $P_{R}(G)$ des $R G$-modules projectifs, alors par extension des scalaires, les $V_{i} \otimes_{R} K$ forment un système complet de $K G$-modules irréductibles; par réduction à $k$, les $V_{i} \otimes k$ conduisent à un système complet de $k G$-modules simples.

Le résultat qui suit est dans l'esprit du théorème d'induction de Brauer (voir Snaith [95]), et à notre connaissance ne semble pas avoir été remarqué. 
ThÉORÈme 3.2.2. - Soit $k$ un corps, et soit une action du groupe fini $G$ sur la $k$-courbe projective lisse $C$. On suppose que $|G|$ est inversible dans $k$. Alors, les $k G$-modules $H^{0}\left(C, \omega_{C}^{\otimes m}\right)$ pour $m \geqslant 1$ déterminent la donnée de Hurwitz de l'action, et un nombre fini d'entre eux suffisent.

Démonstration. - La preuve consiste à faire l'étude d'un point de vue combinatoire de l'action du groupe $G$. Elle découle plus précisément de l'utilisation itérée de la formule d'inversion de Möbius appliquée à l'ensemble ordonné des sous-groupes cycliques de $G$. On procède par étapes, dans la première $G$ est cyclique.

Lemme 3.2.3. - Supposons le groupe $G$ cyclique d'ordre $n$. Soit $N_{k}$ le nombre d'orbites avec $n / k$ éléments. Alors les nombres $N_{k}(k>1, k \mid n)$ sont déterminés par les représentations de Hurwitz.

Démonstration. - Soient $e_{1} \leqslant \cdots \leqslant e_{r}$ les ordres des stabilisateurs des orbites spéciales rangés dans l'ordre croissant. Du fait de (8) et (9), la multiplicité de la représentation triviale dans $H^{0}\left(C, \omega_{C}^{\otimes m}\right)$ est donnée par

$$
\begin{cases}g^{\prime} & \text { si } m=1, \\ 3 g^{\prime}-3+r & \text { si } m=2 .\end{cases}
$$

Pour $m \geqslant 3$, en tenant compte de $r=N_{2}+N_{3}+\cdots$, le résultat est :

$$
\begin{aligned}
5\left(g^{\prime}-1\right)+3 r-\sum_{i=1}^{r}\left(\left\langle-\frac{3}{e_{i}}\right\rangle+\frac{3}{e_{i}}\right) & =5\left(g^{\prime}-1\right)+3 r-\left(2 N_{2}+N_{3}+\cdots\right) \\
& =5\left(g^{\prime}-1\right)+2 r-N_{2},
\end{aligned}
$$

expression qui visiblement détermine $N_{2}$. Supposons maintenant connaître inductivement $N_{2}, \ldots, N_{k-1}$ avec $k \geqslant 3$. Par la relation (7), la multiplicité de la représentation triviale dans le $k G$-module $H^{0}\left(C, \omega_{C}^{k+1}\right)$ est :

$$
\begin{gathered}
(2 k+1)\left(g^{\prime}-1\right)+(k+1) r-\sum_{i=1}^{r}\left(\left\langle-\frac{k+1}{e_{i}}\right\rangle+\frac{k+1}{e_{i}}\right) \\
=(2 k+1)\left(g^{\prime}-1\right)+(k+1) r-\sum_{2 \leqslant d \leqslant k} N_{d}\left(\left\langle-\frac{k+1}{d}\right\rangle+\frac{k+1}{d}\right)+2 N_{k}+N_{k+1}+\cdots
\end{gathered}
$$

ce qui permet de déduire la valeur de $N_{k}$.

Revenons maintenant au cas général. Si $H$ est un sous-groupe cyclique de $G$ de cardinal $h$, on notera pour tout diviseur $k$ de $h, N_{k}(H)$ le nombre de $H$-orbites à $h / k$ éléments. Le lemme 3.1.3 appliqué à la restriction de l'action à $H$, montre que le cardinal Card $\left(C^{H}\right)=N_{h}(H)$ de l'ensemble des points de $C$ fixés par $H$ est déterminé par la seule connaissance des représentations de Hurwitz. Notons $\Delta_{H}$ l'ensemble des points de $C$ dont le stabilisateur est exactement $H$. On a

$$
\Delta_{H}=C^{H}-\bigcup_{K, H \varsubsetneqq K} C^{K} \text {. }
$$


Une orbite spéciale qui contient un point de stabilisateur $H$ coupe $\Delta_{H}$ en $\beta=$ $\left[N_{G}(H): H\right]$ points. La technique usuelle d'inversion de Möbius conduit à :

LEMME 3.2.4. - Considérons des opérations du groupe $G$ sur les courbes lisses $C_{1}$ et $C_{2}$. On suppose que pour tout $m \geqslant 1$, on a un isomorphisme de $k G$-modules

$$
H^{0}\left(C_{1}, \omega_{C_{1}}^{\otimes m}\right) \cong H^{0}\left(C_{2}, \omega_{C_{2}}^{\otimes m}\right) .
$$

Alors, un sous-groupe cyclique $H$ de $G$ est le stabilisateur d'un point de $C_{1}$ si et seulement si $H$ est le stabilisateur d'un point de $C_{2}$.

Démonstration. - On utilise implicitement un argument bien connu, souvent appelé lemme de Brauer ([93], par. 7.1, exer. 1), qui dit que si $X, Y$ sont deux ensembles finis munis d'une action du groupe fini $G$, alors les représentations par permutations de $G$ définies par $X$ et $Y$ sont équivalentes si et seulement si pour tout sous-groupe cyclique $H$ de $G$ on a Card $X^{H}=\operatorname{Card} Y^{H}$. Il découle tout d'abord du lemme 3.2.3, que si $H$ est un sous-groupe cyclique de $G$, alors Card $C_{1}^{H}=\operatorname{Card} C_{2}^{H}$. Posons, pour $i=1,2$ :

$$
f_{i}(H)=\operatorname{Card} C_{i}^{H} \quad \text { et } \quad g_{i}(H)=\operatorname{Card} \Delta_{i}(H)
$$

de sorte que $f_{1}(H)=f_{2}(H)$ pour tout sous-groupe cyclique $H$ de $G$. L'ensemble $\mathscr{C}$ des sous-groupes cycliques de $G$ étant ordonné par l'inclusion, on peut définir la fonction de Möbius correspondante $\mu_{G}: \mathscr{C} \times \mathscr{C} \longrightarrow\{0, \pm 1\}$. Si $\mu$ représente la fonction de Möbius usuelle, on a $\mu_{G}(H, K)=\mu([K: H])$ pour $H, K \in \mathscr{C}, H \subset K$. De (10) on tire immédiatement l'égalité

$$
f_{i}(H)=\sum_{K, H \subset K} g_{i}(K) .
$$

Une simple application de la relation d'inversion de Möbius conduit à la relation

$$
g_{i}(H)=\sum_{K, H \subset K} \mu_{G}(H, K) f_{i}(K) .
$$

Comme $f_{1}=f_{2}$, on a bien $g_{1}=g_{2}$, ce qui est en substance le résultat annoncé.

Preuve du théorème 3.2.2 : On suppose avoir pour tout $m \geqslant 1$ un isomorphisme de $k G$-modules :

$$
H^{0}\left(C_{1}, \omega_{C_{1}}^{m}\right) \cong H^{0}\left(C_{2}, \omega_{C_{2}}^{m}\right) \text {. }
$$

Il s'agit de prouver que les données de Hurwitz $\xi_{1}$ et $\xi_{2}$ attachées à $C_{1}$ et $C_{2}$ sont identiques, c'est-à-dire que la multiplicité dans $\xi_{1}$ et $\xi_{2}$ d'une quelconque classe $[H, \chi]$ est la même. La première observation, qui découle du lemme 3.1.1, est qu'un sousgroupe cyclique $H$ stabilise un point de $C_{1}$ si et seulement si il stabilise un point de $C_{2}$. Il faut donc maintenant prouver, en travaillant avec $C=C_{1}$ ou $C_{2}$, que la classe de conjugaison de $H$ étant fixée, on peut extraire des représentations de Hurwitz de $C$ la multiplicité d'une classe $\left(H_{i}, \chi_{i}\right)$, avec $H_{i}$ conjugué à $H$. Ces classes sont en bijection avec les $N_{G}(H)$-orbites dans $\Delta_{H}$, où $N_{G}(H)$ est comme déjà indiqué le normalisateur 
de $H$, et rappelons-le, $\Delta_{H}=\left\{x \in C, G_{x}=H\right\}$. On est ainsi ramené, par restriction de l'action à $H$, au cas $G=H$ cyclique. Ceci étant, notons

$$
2 \leqslant e_{1} \leqslant \cdots \leqslant e_{r} \leqslant n=\operatorname{Card} G
$$

les ordres des stabilisateurs des orbites spéciales, et $H_{1}, \ldots, H_{r}$ ces stabilisateurs. Extraire la donnée de Hurwitz revient à expliciter, pour chaque $H_{i}$, l'entier $\nu_{i}, 1 \leqslant$ $\nu_{i}<e_{i},\left(\nu_{i}, e_{i}\right)=1$. La multiplicité du caractère $\chi^{l}$ dans la représentation Hurw $_{m}$ étant connue (noter que les entiers $g^{\prime}$ et $r$ sont déterminés), on peut supposer que l'expression

$$
\sum_{i=1}^{r}\left(\left\langle\frac{l \nu_{i}-m}{e_{i}}\right\rangle+\frac{m}{e_{i}}\right)
$$

est déterminée pour tout $m \geqslant 1$, et tout $l$. En particulier, si $m=n$, l'expression $\sum_{i=1}^{r}\left\langle\frac{l \nu_{i}}{e_{i}}\right\rangle$ est déterminée pour tout $l$. Ainsi, l'expression

$$
\sum_{i=1}^{r}\left(\left\langle\frac{l \nu_{i}-m}{e_{i}}\right\rangle-\left\langle\frac{l \nu_{i}}{e_{i}}\right\rangle-\left\langle-\frac{m}{e_{i}}\right\rangle\right)
$$

peut donc être considérée comme acquise pour tout $m \geqslant 1$ et tout $l$. Si $m=a n-1$ $(a \geqslant 1)$, cette dernière expression vaut

$$
\sum_{i=1}^{r}\left(\left\langle\frac{l \nu_{i}+1}{e_{i}}\right\rangle-\left\langle\frac{l \nu_{i}}{e_{i}}\right\rangle-\left\langle-\frac{1}{e_{i}}\right\rangle\right),
$$

mais pour tout $q \in \mathbb{Z},\left\langle\frac{q+1}{e}\right\rangle-\frac{1}{e}-\frac{q}{e}$ vaut -1 si $e \mid q+1$, et 0 sinon. On obtient ainsi que l'expression (14) compte le nombre d'indices $i \in[1, r]$ tels que

$$
l \nu_{i} \equiv-1 \quad\left(\bmod e_{i}\right) .
$$

Pour terminer la preuve, notons que si $n=\operatorname{Card} G=p$ est premier, la conclusion est claire, car alors $H_{1}=\cdots=H_{r}=G$, et le nombre des $\nu_{i}$ tels que $\nu_{i} \equiv q(\bmod p)$ pour tout $q$ premier à $p$. Si maintenant $n$ est quelconque, on peut raisonner par induction croissante sur l'ordre des sous-groupes, et supposer que la donnée relative à tout sousgroupe strict $H \varsubsetneqq G$ est déterminée. Si $\Delta_{G} \neq \varnothing$, la preuve est terminée; dans le cas contraire, il faut pouvoir compter pour tout $q$ premier à $n$, le nombre des $\left\{\nu_{i}\right\}$ égaux à $q$ pour lesquels $e_{i}=n$. Supposons que la liste (13) soit telle que

$$
e_{1} \leqslant \cdots \leqslant e_{t}<e_{t+1}=\cdots=e_{r}=n \quad(0 \leqslant t<r) .
$$

Alors, connaissant les entiers $\nu_{1}, \ldots, \nu_{t}$ et pout tout $l$ premier à $n$ le nombre d'indices $i \in[1, r]$ tels que $l \nu_{i} \equiv-1\left(\bmod e_{i}\right)$, on peut finalement obtenir la totalité de la donnée de Hurwitz, ce qui démontre le théorème.

Il est maintenant clair que si $C / S$ est une courbe (lisse) sur une base connexe, munie d'une action de $G$, alors la donnée de Hurwitz déduite de l'action de $G$ le long des fibres géométriques est constante. Cela découle immédiatement du théorème 3.2 .2 joint au résultat suivant, extension au cas équivariant du résultat classique qui établit 
40 CHAPITRE 3. FAMILlES DE $G$-COURBES LISSES ET THÉORÈME DE CHEVALLEY-WEIL

que sous les hypothèses du présent énoncé, la caractéristique d'Euler-Poincaré $\chi\left(\mathscr{L}_{s}\right)$ est constante.

Proposition 3.2.5. - Sous les hypothèses ci-dessus, soit $\mathscr{L}$ un $\left(\theta_{C}, G\right)$-faisceau localement libre de rang fini. La trace de Lefschetz $L_{G}\left(\mathscr{L}_{s}\right)$ est constante de long des fibres géométriques. En particulier, la représentation $H^{0}\left(C_{s},\left(\omega_{C / S}^{\otimes m}\right)_{s}\right)$ est indépendante de $s \in S$. 


\section{CHAPITRE 4}

\section{FAMILLES DE G-COURBES STABLES}

\subsection{Actions stables, revêtements stables}

Dans cette section, on étend les résultats de la section 3 aux familles de courbes préstables, i.e. nodales. On introduit la propriété la stabilité de l'action d'un groupe fini $G$ sur une courbe préstable, et on propose la définition des revêtements galoisiens stables, respectivement stables marqués. On suppose, comme toujours, que $|G|$ est inversible dans les anneaux locaux des schémas de base qui apparaissent.

4.1.1. Courbes stables et stables marquées. - Les définitions générales en ce qui concerne les courbes préstables et stables marquées sont dans HarrisMorrison [55], Manin [76], ou dans les articles de Deligne-Mumford [30], et Knudsen [68]. Nous suivrons essentiellement la terminologie de Manin [76], chap. V. En particulier, nous utiliserons la terminologie courbe préstable comme équivalente à celle de courbe nodale ou semi-stable.

DÉFinition 4.1.1. - Une courbe préstable de base $S$ est un morphisme propre et plat $f: C \longrightarrow S$ dont les fibres géométriques sont des courbes nodales connexes. Si $S$ est connexe, le genre d'une fibre est constant, c'est le genre de la courbe.

Une courbe préstable est dite stable si les fibres géométriques $\left\{C_{s}\right\}$ sont des courbes stables dans le sens de Deligne-Mumford, i.e. si le groupe des automorphismes $\operatorname{Aut}\left(C_{s}\right)$ est fini.

On sait que la stabilité pour une courbe définie sur un corps algébriquement clos, de genre $g \geqslant 2$, équivaut à propriété suivante : une composante irréductible de $C$, lisse et rationnelle, rencontre les autres composantes en au moins 3 points.

Cette condition s'exprime de manière particulièrement agréable sur le graphe dual classiquement attaché à $C$; cela sera rappelé dans la section 7.1. Puisque, pour une courbe préstable, le morphisme $f: C \longrightarrow S$ est un morphisme localement d'intersection complète, on note que le $\theta_{C}$-module $\Omega_{C / S}^{1}$, vu comme objet de la catégorie dérivée, est quasi-isomorphe au complexe cotangent de $f$, et est un complexe 
parfait. Le faisceau dualisant relatif, localement libre de rang 1, admet la définition commode suivante [76] :

$$
\omega_{C / S}=\operatorname{det}\left(\Omega_{C / S}^{1}\right) .
$$

L'opération det devant être utilisée à plusieurs reprises, rappelons succinctement le résultat de cette construction. Dans [69] le faisceau inversible det (déterminant) est défini en toute généralité pour un complexe parfait de $\theta_{C}$-modules cohérents :

$$
\text { det : } \mathscr{P a r f}_{C} \longrightarrow \operatorname{Pic}_{C} \text {. }
$$

Il se calcule localement, à partir d'un complexe borné de modules localement libres quasi-isomorphe au complexe de départ (loc. cit), par extension $\operatorname{de} \operatorname{det}(E)=\wedge^{d} E$ si $E$ est localement libre de rang $d$. Le point clé est qu'un triangle distingué $F^{\prime} \longrightarrow F \longrightarrow$ $F^{\prime \prime} \longrightarrow F^{\prime}[1]$ conduit à un isomorphisme canonique $\operatorname{det}(F) \cong \operatorname{det}\left(F^{\prime}\right) \otimes \operatorname{det}\left(F^{\prime \prime}\right)$. On notera que si $f: C^{\prime} \longrightarrow C$ est un morphisme, alors $\operatorname{det}\left(L f^{*}(F)\right)=f^{*}(\operatorname{det}(F))$ (loc. cit. p. 40); en particulier la formation du module dualisant est compatible avec les changements de base.

D'une autre manière, si $f: C \longrightarrow S$ est une courbe semi-stable, et si $F$ est un complexe parfait sur $C$, alors le complexe $R f_{*}(F)$ est parfait, conduisant au déterminant $\operatorname{det}\left(R f_{*}(F)\right)$, faisceau inversible sur $S$. Comme ci-dessus $(C=S)$, la formation $\operatorname{de} \operatorname{det}\left(R f_{*}(F)\right)$ est compatible avec un changement de base.

Comme il est bien connu, la définition 4.1.1 se généralise aux courbes stables marquées (Harris-Morrison [55], Knudsen [68], Manin [76], Wewers [99]) :

DÉfinition 4.1.2. - Soit $f: C \longrightarrow S$ une courbe préstable de base $S$. Soit $R \subset C$ un diviseur de Cartier relatif de degré $d \geqslant 1$.

i) On dit que $R$ définit un marquage de $C / S$, ou que $C / S$ est marquée par $R$, si $R$ est étale sur $S$, en particulier de support contenu dans la partie lisse de $f$. On parlera dans la suite de $(C / S, R)$ comme d'une courbe marquée.

ii) La courbe marquée $(C / S, R)$ est dite stable, ou $C / S$ est stable marquée par $R$, si pour tout point géométrique $s \in S$, l'une des propriétés équivalentes suivantes est satisfaite :

1. Le groupe $\operatorname{Aut}\left(C_{s}, R_{s}\right)$ des automorphismes de $C_{s}$ préservant $R_{s}$ est fini.

2. Toute composante irréductible, lisse, de la normalisation de $C_{s}$, qui est rationnelle (resp. de genre 1), contient au moins trois points exceptionnels (resp. un). On appelle point exceptionnel un point qui est soit dans le support de $R$, soit l'origine d'une branche issue d'un point double.

Quitte à effectuer un changement de base étale, on peut supposer que $R$ est une somme de sections disjointes $R=\sum_{i} P_{i}$, avec en tout point géométrique $s \in S$ la condition de stabilité satisfaite, $P_{i}(s)$ est un point non singulier, et $P_{i}(s) \neq P_{j}(s)$ si $i \neq j$. On montre alors qu'une courbe préstable marquée $f: C \longrightarrow S$ est stable si et seulement si le faisceau $\omega_{C / S}(R)$ est relativement ample (voir [76], chap. V, lemma 1.2.1) ; cela peut servir de définition alternative. Rappelons qu'il importe de distinguer deux situations : dans l'une la courbe est marquée par $R$, et dans l'autre 
$R$ est une somme de sections disjointes numérotées, la courbe est alors piquée. On parlera de la suite des $P_{i}$ comme un piquage de $C / S$.

Dans les deux cas, les isomorphismes sont contraints de respecter le marquage, respectivement le piquage. On rencontrera le plus souvent une situation intermédiaire, i.e. un piquage partiel, dans lequel les points marqués ne sont pas numérotés, mais seulement affectés d'une couleur, pas de manière univoque, les isomorphismes devant dans ce cas simplement respecter la couleur. Cela équivaut à numéroter les points par paquets. Dans ce cas $R=R_{1}+\cdots+R_{r}$ est décomposé en une somme de diviseurs disjoints deux à deux, les points de $R_{\alpha}$ étant vus comme les points d'une même holonomie, ou même couleur, disons $\alpha$. Posons $d_{\alpha}=\operatorname{deg}\left(R_{\alpha}\right), d=\operatorname{deg}(R)=\sum_{\alpha} d_{\alpha}$.

On suppose maintenant, comme dans la section précédent dont on conserve les notations, que le groupe fini $G$ agit fidèlement sur $C / S$. Sous les hypothèses fixées au début, le groupe $G$ est réductif, ce qui assure que l'opération de passage au quotient se comporte comme dans le cas lisse, particulièrement les propositions 3.1.1, 3.1.4.

Proposition 4.1.3. - Soit $f: C \longrightarrow S$ une courbe préstable munie d'une action de $G$. Alors la courbe quotient $D=C / G$ est préstable, et sa formation commute aux changements de bases. En particulier, pour tout point géométrique $s \in S, D_{s}=C_{s} / G$.

Démonstration. - Le point essentiel, qui est la commutation du quotient au changement de base, est aisé; voir par exemple Katz-Mazur [67], appendice au chap. 7.

4.1.2. Actions stables. - Il est clair que la conclusion de la proposition 4.1.3 permet d'appliquer sans changement la conclusion de la proposition 3.2.5, avec pour $G$-faisceau le faisceau dualisant relatif $\omega_{C / S}$. Si $S$ est connexe, la représentation de $G$ dans $H^{0}\left(C_{s},\left(\omega_{C / S}\right)_{s}\right)$ est ainsi indépendante de $s \in S$ (voir la remarque préliminaire dans 3.2.2).

Revenons aux conditions de la définition 4.1.2. Soit d'abord $S=\operatorname{Spec}(k)$, et soit $P \in C$ un point double, de sorte que

$$
\hat{\theta}_{C_{s}, P} \cong k[[X, Y]] /(X Y) .
$$

Dans l'anneau local complété, les équations $X=0$ et $Y=0$ définissent les branches formelles en $P$. Supposons que le stabilisateur $G_{P}$ soit non trivial, alors $G_{P}$ opère sur l'ensemble à deux éléments composé par les deux branches. Remarquons aussi qu'on obtient de manière analogue au cas lisse une représentation fidèle de $G_{P}$ dans l'espace cotangent au point $x$, de dimension 2, soit un morphisme injectif $G_{P} \hookrightarrow G L(2, k)$. Cette définition s'applique à tout point double d'une fibre $C_{s} \operatorname{de} C / S$.

La définition suivante trouvera sa motivation ultérieurement; elle est réservée pour le moment aux courbes dépourvues de marquage, et ne porte que sur l'action de $G$ au voisinage des points doubles : 
DÉfinition 4.1.4. - L'action de $G$ sur la courbe préstable $C / S$ est dite stable (certains disent kummérienne [91]), si pour tout point double $P$ d'une fibre géométrique, la représentation de $G_{P}$ dans l'espace cotangent de la fibre en $P$ possède la propriété suivante : pour tout $\sigma \in G_{P}$, on a $\operatorname{det}(\sigma)=1$ si $\sigma$ fixe les branches, et $\operatorname{det}(\sigma)=-1$ si $\sigma$ échange les branches.

Il est clair que la condition de stabilité équivaut à dire que le stabilisateur d'un point double est soit cyclique, soit diédral, et dans ce dernier cas les éléments de déterminant -1 dans la représentation cotangente sont les éléments qui échangent les branches. Plus précisément, soit $H=G_{P}^{0} \triangleleft G_{P}$ le sous-groupe d'indice au plus 2 formé des éléments qui fixent les branches. Ce groupe est cyclique, et l'action de $H$ sur l'espace cotangent en $P$ sur la branche $X=0$ (resp. $Y=0$ ), définit un caractère primitif $\chi_{X}\left(\right.$ resp. $\left.\chi_{Y}\right)$ de $H$. La condition sur le déterminant est alors équivalente à

$$
\chi_{X} \cdot \chi_{Y}=1 .
$$

Si $G_{x}$ est d'ordre 2 , et s'il y a échange des branches par l'élément $\neq 1$ de $G_{P}$, le groupe sera considéré comme diédral. Soit $G$ agissant stablement sur la courbe préstable $C$, définie sur le corps algébriquement clos $k$. Les considérations ci-dessous s'appliqueront donc à une fibre géométrique. On classe les points à isotropie non triviale en trois familles disjointes deux à deux :

(I) points fixes lisses,

(II) points doubles à isotropie cyclique (branches fixées),

(III) points doubles à isotropie diédrale (branches échangées).

Il sera souvent utile d'avoir un modèle local de l'action de $G_{P}$ pour $P \in C_{s}$ de l'un des trois types. Le type I est clair, nous l'omettons. Examinons les types II et III. Soit un point fermé $P \in C$ au-dessus de $s \in S$, d'image $Q \in D$. Rappelons qu'on peut choisir des paramètre locaux le long des branches en $P \in C_{s}$ de sorte que

$$
\widehat{\vartheta}_{P} \cong \widehat{\theta}_{s}[[X, Y]] /(X Y-a)
$$

pour un $a \in \widehat{M}_{s}$. Pour des détails, on renvoie à Bertin-Maugeais [14], Wewers [100], $\S 2.1$, ou Jarvis [58], §5.1. Il est connu que les paramètres $x, y$, images de $X, Y$, sont définis à des substitutions près $(x, y, a) \mapsto(\alpha x, \beta y, \gamma a)$ où $\alpha, \beta \in \theta_{P}^{*}, \gamma=\alpha \beta \in \theta_{s}^{*}$. En particulier, un automorphisme de $\widehat{\vartheta}_{P}$ est déterminé par unique couple $(u, \gamma), u \in \widehat{\vartheta}_{P}^{*}$, $\gamma \in \widehat{\theta}_{Q}^{*},(\gamma-1) a=0$, tel que

$$
x \mapsto u x, \quad y \mapsto \gamma u^{-1} y
$$

si les branches sont fixées, et $x \mapsto u y, y \mapsto \gamma u^{-1} x$ sinon. Supposons $P$ de type II. Cette description, jointe au théorème 90, permet de supposer que l'action de $G_{P}$ se fait par l'intermédiaire de deux caractères opposés $\chi$ et $\chi^{-1}$ d'ordre $e>1$, par

$$
\sigma(x)=\chi(\sigma) x \quad, \quad \sigma(y)=\chi(\sigma)^{-1} y .
$$


Alors,

$$
\widehat{\vartheta}_{Q}=\widehat{\vartheta}_{s}[[U, V]] /\left(U V-a^{e}\right), \quad \text { avec } U=X^{e}, V=Y^{e}
$$

Supposons maintenant $P$ de type III, avec $G_{P} \cong \mathbb{D}_{e}(e \geqslant 1)$. Dans ce cas, l'action du sous-groupe $G_{P}^{0}$ préservant les branches se réduit, sur des paramètres formels le long des branches convenablement choisis, à la forme 17. Si $\tau \in G_{P}-G_{P}^{0}$ échange les branches, on peut adapter les paramètres de sorte que de plus

$$
\tau(x)=y, \tau(y)=x \text {. }
$$

Il est immédiat de voir qu'on a $\widehat{\vartheta}_{Q}=\widehat{\vartheta}_{s}[[T]]$, où $T=X^{e}+Y^{e}$. En particulier, à la différence du type II, $Q$ est un point lisse de $D / S$.

L'observation qui suit est élémentaire, mais néanmoins cruciale. Elle revient à dire que les points de type II ne doivent pas être considérés comme des points de ramification. On verra cependant qu'ils contribuent de manière non triviale à la déformation universelle du revêtement, à la différence des points de ramification. Soit $\omega_{C / S}=\operatorname{det}\left(\Omega_{C / S}^{1}\right)$ le faisceau dualisant de la courbe préstable $f: C \longrightarrow S(15)$. Il admet la description locale suivante [68], [76]. Supposons que localement (pour la topologie étale) sur $U$, on réalise $C$ comme une hypersurface d'équation $\{f=0\}$ dans un schéma lisse $M$ de dimension relative 2 sur $S$. Posant $I=(f)$, il vient une identification canonique

$$
\omega_{C / S}=\operatorname{Hom}_{U}\left(I / I^{2}, \wedge^{2} \Omega^{1}{ }_{M / S} \otimes \theta_{C}\right) .
$$

Il découle de la suite exacte

$$
0 \longrightarrow I / I^{2} \longrightarrow \Omega^{1}{ }_{M / S} \otimes_{\vartheta_{C}} \longrightarrow \Omega^{1}{ }_{C / S} \longrightarrow 0
$$

un morphisme canonique $\Omega_{C / S_{\mid U}} \longrightarrow \omega_{C / S}$ qui est un isomorphisme en dehors du lieu singulier [68]. Rappelons que, si $S=\operatorname{Spec}(k)$ et si $P$ un point double, on a $\widehat{\omega}_{C, P}=\hat{\theta}_{C, P} \cdot \omega$, où $\omega$ est la forme méromorphe qui sur la normalisation de $\mathrm{C}$, vaut $d X / X$ sur la branche $Y=0$, et $-d Y / Y$ sur la branche $X=0$.

Dans le cas d'une base arbitraire, et pour un quotient $\pi: C \longrightarrow D=C / G$ d'une courbe préstable $C$ par une action stable de $G$ (définition 4.1.4), le morphisme naturel $d \pi: \pi^{*}\left(\Omega_{D / S}^{1}\right) \longrightarrow \Omega_{C / S}^{1}$ s'étend canoniquement en un morphisme (noté encore $d \pi$ ), compatible au changement de base :

$$
d \pi: \pi^{*}\left(\omega_{D / S}\right) \longrightarrow \omega_{C / S}
$$

L'existence de $d \pi$ se déduit facilement du théorème de dualité pour un morphisme fini (voir notamment Hartshorne [57], chap. V, prop. 2.4) qui donne

$$
\omega_{C / S}=\mathcal{H}_{\operatorname{com}_{\theta_{D}}}\left(\pi_{*} \theta_{C}, \omega_{D / S}\right),
$$

le faisceau de droite étant compris comme un $\theta_{C}$-module. On va voir que le morphisme (20) est un isomorphisme en dehors des points de type I ou III. Il nous sera utile pour la suite d'en avoir une description locale. Au voisinage d'un point 
double $P$ de type II, d'image $s \in S$, on peut choisir étale-localement des coordonnées le long des branches $(x, y)$, telles que $C$ soit sur ces coordonnées la courbe relative

$$
(x y-a=0) \subset \mathbb{A}_{S}^{2}
$$

et que, d'autre part, l'action du stabilisateur $G_{P}$ (d'ordre $e \geqslant 2$ ) soit donnée par $\sigma(x)=\zeta x, \quad \sigma(y)=\zeta^{-1} y, \sigma$ étant un générateur de $G_{P}$. On en déduit une description locale-étale de $D$ en $Q$ par l'intermédiaire des coordonnées le long des branches $u=x^{e}, v=y^{e}$, soumises à l'équation $u v-a^{e}=0$. Ces descriptions fournissent des générateurs locaux $\omega(x, y)$ et $\omega(u, v)$ pour respectivement $\omega_{C / S}$, et $\omega_{D / S}$. Le morphisme local requis est l'isomorphisme défini par $\pi^{*}(\omega(u, v)) \mapsto \omega(x, y)$. Si $P$ est de type III, comme $Q$ est un point lisse, $d \pi$ est le composé $\pi^{*}\left(\Omega_{D / S, Q}\right) \longrightarrow \Omega_{C / S, P} \longrightarrow$ $\omega_{C / S, P}$. Utilisant la description (19), et relativement à des coordonnées adaptées aux branches en $P$, on on obtient la description locale

$$
d \pi(d t)=e\left(x^{e}-y^{e}\right) \omega(x, y) .
$$

On notera que les faisceaux $\omega_{C / S}$ et $\Omega_{C / S}^{1}$ sont de manière naturelle des $G$-faisceaux, ils seront en permanence pris comme tels. La structure du morphisme (20) peut être précisée :

Lemme 4.1.5. - Soit $f: C \longrightarrow S$ une courbe préstable munie d'une action de $G$. On suppose que $G$ agit fidèlement et stablement sur les fibres géométriques. Soit $\pi$ : $C \longrightarrow D=C / G$ le morphisme de $C$ sur la courbe quotient. Pour tout entier $l \geqslant 1$, on a une suite exacte

$$
0 \longrightarrow \pi^{*}\left(\omega_{D / S}^{\otimes l}\right) \longrightarrow \omega_{C / S}^{\otimes l} \longrightarrow V_{l} \longrightarrow 0
$$

où $V_{l}$ est un $\theta_{C}$-module $S$-plat, dont la formation est compatible avec les changements de base. Le support de $V_{l}$ est fini sur $S$, concentré aux points de type I et III.

Démonstration. - Partant du morphisme $\pi^{*}\left(\omega_{D / S}\right) \longrightarrow \omega_{C / S}$, par passage à la puissance tensorielle $l$-ième, on obtient un morphisme $\pi^{*}\left(\omega_{D / S}^{\otimes l}\right) \longrightarrow \omega_{C / S}^{\otimes l}$. Soit $V_{l}$ le conoyau. Pour décrire ce faisceau, supposons d'abord $S=\operatorname{Spec}(k)$, le corps $k$ étant algébriquement clos. Utilisant la description locale ci-dessus du morphisme $\pi^{*}\left(\omega_{D}^{\otimes l}\right) \longrightarrow \omega_{C}^{\otimes l}$, on entire celle de $V_{l}$. Traitons d'abord le cas $l=1$. En un point lisse $P \in C$, d'image $Q \in D$, soit $t$ un paramètre local, tel que le stabilisateur $G_{P}$ agisse par $\sigma . t=\chi(\sigma) t, \quad\left(\sigma \in G_{P}\right)$, où $\chi \in \widehat{G}_{P}$ est un caractère primitif. Soit $e$ l'ordre de $G_{P}$. Alors $u=t^{e}$ est un paramètre local en $Q$ et le morphisme (20) a pour description locale en $P$

$$
\vartheta_{P} d u \longrightarrow \vartheta_{P} d t, \text { avec } d u=e t^{e-1} d t .
$$

On trouve ainsi $\operatorname{long}\left(V_{P}\right)=e-1$. Si $P$ est de type II, on a vu ci-dessus que (20) est un isomorphisme. 
Il reste le cas où $P$ est de type III. Soit $G_{P} \cong \mathbb{D}_{e}, e \geqslant 1$. Dans ce cas, $Q$ est un point lisse, et si l'on choisit les paramètres le long des branches comme dans (18, alors $t=x^{e}+y^{e}$ est un paramètre en $Q$. La description locale de (20) donne dans ce cas :

$$
\widehat{\vartheta}_{P} d t \longrightarrow \widehat{\vartheta}_{P}\left(\frac{d x}{x},-\frac{d y}{y}\right) \quad \text { avec } \quad d t=\left(e x^{e},-e y^{e}\right)\left(\frac{d x}{x},-\frac{d y}{y}\right) .
$$

En particulier, on voit que $V_{P} \neq 0$, et en fait $\operatorname{long}\left(V_{P}\right)=2 e-1$.

Revenons au morphisme (20). On vient de voir qu'il est injectif sur les fibres, il est donc injectif avec un conoyau $V_{l}$ plat sur $S$, comme il résulte du critère local de platitude, et donc de formation compatible aux changements de base. Le support de $V_{l}$ est ainsi concentré en les points fixes de type I ou de type III.

Dans le cas $l=1$, on posera dans la suite $V=V_{1}$. On conserve les notations du lemme 4.1.5. La suite exacte (22) justifie la définition suivante, dans laquelle Div est le diviseur de Cartier construit par Knudsen-Mumford [69]. Dans notre situation on applique cette opération à un morphisme de complexes parfaits, en fait des faisceaux inversibles $\lambda: M \longrightarrow N$, qui est un isomorphisme sur un ouvert dense. Si $\lambda$ est injectif de conoyau $K$, alors $\operatorname{Div}(M \longrightarrow N)=\operatorname{Div}(0 \longrightarrow K) \stackrel{\text { déf }}{=} \operatorname{Div}(K)$.

DÉFInItion 4.1.6. - Le diviseur de Cartier relatif

$$
\mathfrak{R}=\operatorname{Div} V=\operatorname{Div}\left(\pi^{*}\left(\omega_{D / S}\right) \longrightarrow \omega_{C / S}\right)
$$

est appelé le diviseur de ramification du morphisme $\pi: C \longrightarrow D$.

On peut aussi comprendre $\mathfrak{R}$ comme défini localement par l'idéal différente. On notera que le support de $\mathfrak{R}$ est l'ensemble des points de type I ou de type III. Par ailleurs, de la définition jointe à la suite exacte (22), il vient l'égalité (formule de ramification)

$$
\omega_{C / S} \cong \pi^{*}\left(\omega_{D / S}\right) \otimes \theta(\Re) .
$$

On souhaite relier le diviseur $\mathfrak{R}$ aux sous-schémas de points fixes des sous-groupes cycliques de $G$, comme dans le cas lisse. Soit $H$ un sous-groupe cyclique non trivial de $G$. Ce qui précède justifie la définition d'apparence artificielle suivante :

DÉFInItion 4.1.7. - Soit une action stable de $G$ sur la courbe préstable $C$ définie sur un corps $k$. Soit $p \in C$. On dit que $H$ fixe strictement $p$ si ou bien $p$ est lisse et $H p=p$, ou bien $p$ est un point double, $H p=p$, et tout élément $\sigma \in H, \sigma \neq 1$ inverse les branches.

On observera que du fait de la condition de stabilité, si $H$ fixe strictement le point double $p$, seconde alternative, alors $H$ est d'ordre deux, et l'élément distinct de l'identité de $H$ est une réflexion, i.e. inverse les branches. On étend la définition au cas relatif. La proposition suivante précise la structure du sous-schéma des points fixes d'un sous-groupe cyclique en présence de points doubles. 
Proposition 4.1.8. - Soit $\phi: C \longrightarrow S$ une courbe préstable sur laquelle le groupe $G$ agit stablement, et soit $\pi: C \longrightarrow D=C / G$ le revêtement associé.

1) Pour tout sous-groupe cyclique $H$ de $G$, le sous-schéma $\operatorname{Fix}(H)=C^{H}$ des points fixes de $H$ est réunion disjointe d'une partie horizontale et d'une partie dite de type $I I$ :

$$
\operatorname{Fix}(H)=\operatorname{Fix}(H)_{\text {hor }} \sqcup \operatorname{Fix}(H)_{I I}
$$

où $\operatorname{Fix}(H)_{\text {hor }}$ est un diviseur de Cartier relatif qui a pour support les points fixés strictement par $H$. Le support de $\operatorname{Fix}(H)_{I I}$ est l'ensemble des points doubles de

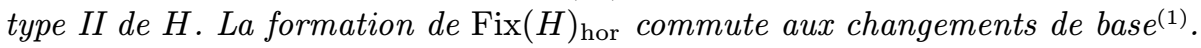

2) Comme dans le cas lisse on a l'égalité de diviseurs, la somme étant étendue aux sous-groupes cycliques non triviaux

$$
\mathfrak{R}=\sum_{H} \varphi(|H|) \operatorname{Fix}(H)_{\text {hor }} .
$$

Démonstration. - 1) Fixons un générateur $\sigma$ de $H$, avec Card $H=e$. Soit $P \in \operatorname{Fix}(H)$ un point fixe de type I, donc non singulier dans sa fibre, et soit $\phi(P)=s \in S$. Il est clair qu'au point $P$ le sous-schéma fermé $\operatorname{Fix}(H)$ est un diviseur de Cartier relatif étale sur $S$. Supposons maintenant $P$ du type II. Quitte à passer aux anneaux locaux complétés, on suppose que $\widehat{\mathscr{\theta}}_{P} \cong \widehat{\mathscr{\theta}}_{s}[[X, Y]] /(X Y-a), \quad\left(a \in \hat{\mathcal{M}}_{s}\right)$, l'action de $H$ étant décrite par $\sigma(x)=\zeta x, \sigma(x)=\zeta^{-1}$, pour une racine primitive d'ordre $e$ convenable de l'unité, et $x, y$ étant les classes de $X, Y$. Il est immédiat que l'idéal du sous-schéma fermé $\operatorname{Fix}(H)$ en $P$ est engendré par les classes de $\sigma(x)-x$ et $\sigma(y)-y$, il est donc égal à $(x, y)$. On voit ainsi qu'en ce point $\operatorname{Fix}(H)$ n'est pas un diviseur de Cartier.

Supposons maintenant le point $P$ de type III, et que $P$ soit un point fixe strict de $H$. Alors $H=\{1, \sigma\}$ est d'ordre deux, et relativement à des paramètres locaux le long des branches, $\sigma(x)=y, \sigma(y)=x$. L'idéal de $\operatorname{Fix}(H)$ est engendré par la classe de $x-y$, en particulier c'est un diviseur de Cartier en ce point. L'anneau local complété de $\operatorname{Fix}(H)$ en $P$ est $\widehat{\mathscr{\vartheta}}_{\mathrm{Fix}(H), P} \cong \widehat{\mathscr{\vartheta}}_{s}[[X]] /\left(X^{2}-a\right)$, avec $a \in \hat{\mathcal{M}}_{s}$. Il n'est pas étale sur $\widehat{\theta}_{s}$.

L'ensemble (ouvert) des points du support de $\operatorname{Fix}(H)$ en lesquels $\operatorname{Fix}(H)$ est un diviseur de Cartier, est égal au support du diviseur de ramification du morphisme quotient $C \longrightarrow D=C / H$ (définition 4.1.6). Finalement $\operatorname{Fix}(H)$ se décompose en une somme disjointe

$$
\operatorname{Fix}(H)=\operatorname{Fix}(H)_{\text {hor }} \sqcup \operatorname{Fix}(H)_{I I}
$$

où $\operatorname{Fix}(H)_{\text {hor }}$ est un diviseur de Cartier relatif supporté par les points fixes stricts de $H$ (4.1.7), et $\operatorname{Fix}(H)_{I I}$ a pour support l'ensemble des points fixes de type II de $H$, ce qui prouve 1).

2) L'égalité (27) a été observée précédemment pour les points fixes lisses dans leur fibre. Reste a vérifier l'égalité en les points de type III. Soit $P$ un tel point, avec un stabilisateur diédral d'ordre $2 e$. Les notations étant comme au-dessus, on a

(1) On suppose comme toujours que $|G|$ est inversible sur $S$. 
observé qu'une équation locale de $\mathfrak{R}$ en $P$, est $x^{e}-y^{e}(21)$. Si le stabilisateur $G_{P}$ a pour générateurs $\sigma$ et $\tau$, avec $\sigma^{e}=\tau^{2}=(\tau \sigma)^{2}=1$, alors l'équation locale en $P$ de $\operatorname{Fix}\left(\tau \sigma^{i}\right)_{\text {hor }}$ est comme obtenu ci-dessus $x-\zeta_{e}^{i} y$. De sorte que le résultat se réduit à la seule égalité $x^{e}-y^{e}=\prod_{i=0}^{e-1}\left(x-\zeta_{e}^{i} y\right)$.

Une conséquence numérique est la suivante :

Corollaire 4.1.9. - Sous les hypothèses de la proposition 4.1.8, supposons la base $S$


$s \in S$, on $a$

$$
d_{H}=\#\left\{P \in \operatorname{Fix}(H)_{\text {hor }}(s), P \text { de type } I\right\}+2 \#\left\{P \in \operatorname{Fix}(H)_{\text {hor }}(s), P \text { de type III }\right\} \text {. }
$$

Démonstration. - On vient de voir que $\operatorname{Fix}(H)_{\text {hor }}$ est étale sur $S$ en les points de type I, et que si $P \in \operatorname{Fix}(H)_{\text {hor }}$ est un point de type III d'image $s$, alors $\operatorname{dim}\left(\widehat{\vartheta}_{\mathrm{Fix}(H)_{\mathrm{hor}}, P} \otimes k(s)\right)=2$. Le résultat en découle.

Toujours dans la même situation, soit $K \subset G$ un sous-groupe cyclique. Si $H \subset K$, il est clair que $\operatorname{Fix}_{\text {hor }}(K) \subset \operatorname{Fix}_{\text {hor }}(H)$ est un sous-schéma ouvert et fermé. Définissons un diviseur de Cartier relatif par

$$
\Delta(H)=\operatorname{Fix}_{\mathrm{hor}}(H)-\bigcup_{K, H \subset K} \operatorname{Fix}_{\mathrm{hor}}(K),
$$

l'union étant étendue aux sous-groupes cycliques. Le support de $\Delta(H)$ est formé des points de stabilisateur strict $H$. On définit le diviseur de ramification réduit de $\pi: C \longrightarrow D=C / G$ comme étant

$$
\mathfrak{R}=\sum_{H} \Delta(H)
$$

où la somme est étendue aux sous-groupes cycliques. Sur la partie lisse, le diviseur $\mathfrak{R}$ est étale sur $S$, mais cela est en défaut sur le lieu des points doubles de type III.

4.1.3. Collision des points de branchement : étude locale. - Les informations fournies par la proposition 4.1.8 indiquent clairement de quelle manière dégénère un revêtement galoisien, c'est-à-dire comment les points de ramification se comportent par spécialisation, en restant bien sûr dans le cadre de la ramification modérée. Entre autres choses, cela permettra ultérieurement de décrire avec précision le bord des champs de Hurwitz. Le contexte est évidemment beaucoup plus favorable que celui qui accepte des dégénérescences en caractéristique $p>0$ divisant l'ordre de $G$ (pour plus de détails sur ces problèmes, on consultera par exemple Raynaud [87]).

La situation suivante donne corps à cette assertion, et permet de comprendre en des termes simples le processus. Fixons comme base $S=\operatorname{Spec}(R)$, où $R$ est un anneau de valuation discrète complet, de corps résiduel $k$ algébriquement clos avec la restriction habituelle sur la caractéristique $p$, si $p>0$, et de corps des fractions $K$. Soit $f: C \longrightarrow$ $S$ une courbe semi-stable, munie d'une action stable du groupe $G$. Supposons la fibre générique $C_{K}$ lisse. Soit $H$ un sous-groupe cyclique de $G$. Si $|H|>2$, alors il ne peut 
fixer strictement que des points lisses, dès lors $\operatorname{Fix}(H)_{\text {hor }}$ est étale sur $S$, donc une somme de sections disjointes de $f$ contenues dans le lieu lisse. Si maintenant $|H|=2$, alors $\operatorname{Fix}(H)_{\text {hor }}$ est une somme de diviseurs disjoints deux à deux

$$
\operatorname{Fix}(H)_{\text {hor }}=\sqcup_{i} P_{i},
$$

l'intersection ensembliste $P_{i} \cap C_{k}$ se réduisant à un point, par suite le degré relatif de $P_{i}$ est $d_{i} \leqslant 2$. Si $d_{i}=1, P_{i}$ est une section contenue dans le lieu lisse. Par contre si $d_{i}=2, P_{i}$ coupe $C_{k}$ en un point double de type III.

La situation est claire : après extension quadratique éventuelle de $K$, les deux points rationnels de $P_{i}$ situés sur la fibre générique, coalescent dans la fibre spéciale. C'est le seul désagrément qui puisse arriver. Si on cherche à faire un décompte des $G$-orbites de points fixes de la fibre générique qui se spécialisent en des points doubles de type III, la question se réduit à celles qui se spécialisent en un point double donné d'isotropie $K \cong \mathbb{D}_{m}$. Cela donne un ensemble de points de cardinal $2 m$, car les sousgroupes de $K$ qui sont stabilisateurs stricts sont au nombre de $m$, donc ces points se répartissent en deux orbites.

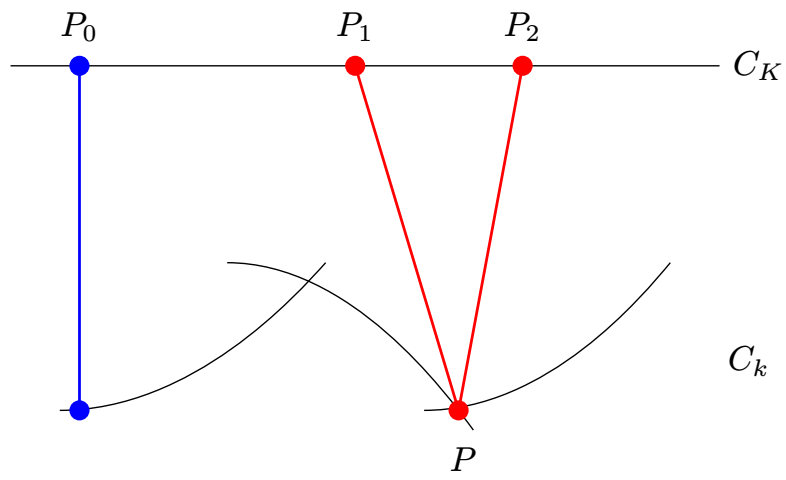

Figure 1. Collision de points de ramification

Exemple 4.1.10. - Dégénérescence en genre 2 .

Les notations étant celles de dessus, soit $R=k[[t]]$. Soit la courbe $C_{K}$, de genre 2 , définie sur $K=k((t))$ par l'équation $y^{2}=x^{2}\left(x^{2}-1\right)^{2}-t^{2}$. Le groupe $G$ est le groupe d'ordre deux engendré par l'involution hyperelliptique $y \mapsto-y, x \mapsto x$. La réduction stable de $C_{K}$ est l'union de deux $\mathbb{P}^{1}$ se coupant en trois points. On voit facilement que les six points fixes sur $C_{K}$, les points de Weierstrass, s'organisent en trois paires $\left(P_{0}^{+}, P_{0}^{-}\right),\left(P_{1}^{+}, P_{1}^{-}\right),\left(P_{-1}^{+}, P_{-1}^{-}\right)$qui coalescent respectivement en les trois points doubles de la fibre spéciale. Dans cet exemple, $\mathfrak{R}=\sum_{i=-1,0,1} P_{i}^{ \pm}$.

L'autre dégénérescence stable de $C_{K}$ est l'union de deux courbes de genre un se coupant transversalement en un point. Dans ce cas, il y a une unique paire de points fixes de $C_{K}$ qui se spécialisent en le point double. 
4.1.4. Géométrie du quotient par une action stable. - Il sera utile pour la suite, la courbe préstable $C$ étant toujours définie sur le corps $k$, de comparer les $G$-faisceaux $\pi^{*}\left(\Omega_{D / k}^{1}\right)$ et $\Omega_{C / k}^{1}$. Les notations précédentes étant toujours en vigueur, rappelons que le foncteur $\pi_{*}^{G}:(G-\operatorname{Coh} C) \longrightarrow(\operatorname{Coh} D)$ transforme un $G$-faisceau (cohérent) $\mathcal{F}$ sur $C$ en le faisceau sur $D$ des sections $G$-invariantes $\pi_{*}(\mathcal{F})^{G}$ de $\pi_{*}(\mathcal{F})$.

Appelons $\theta_{C / S}$ le faisceau tangent relatif, i.e. le dual de $\Omega_{C / S}$. On poursuit cidessous l'étude différentielle des quotients $C \longrightarrow C / G$, où $C$ est une courbe nodale avec action stable de $G$.

Proposition 4.1.11. - $\quad$ 1) Le morphisme $\Omega_{D / k}^{1} \longrightarrow \pi_{*}^{G}\left(\Omega_{C / k}^{1}\right)$ est injectif et le conoyau est concentré aux points images des points doubles de type II.

2) Le morphisme $\pi_{*}^{G}\left(\theta_{C}\right) \longrightarrow \theta_{D}$ est injectif et le conoyau est concentré aux points de $D$ images d'un point de type I, donc lisse, ou d'un point double de type III; en ces points le conoyau est de dimension 1.

En particulier, s'il n'y a pas de point fixe de type III, on a $\pi_{*}^{G}\left(\theta_{C}\right)=\pi_{*}^{G}\left(\theta_{C}(-R)\right)=$ $\theta_{D}(-\Delta)$, où $B=\sum_{j} Q_{j}$ (resp. $R=\sum_{i} P_{i}$ ) désigne le diviseur de branchement (resp. de ramification) réduit.

Démonstration. - Partons de la suite exacte $\pi^{*}\left(\Omega_{D / k}^{1}\right) \longrightarrow \Omega_{C / k}^{1} \longrightarrow \Omega_{C / D}^{1} \longrightarrow 0$. Appliquant le foncteur $\pi_{*}^{G}$, on obtient un morphisme $\Omega_{D / k}^{1} \longrightarrow \pi_{*}^{G}\left(\Omega_{C / k}^{1}\right)$ qui est un isomorphisme en dehors des points de branchement (lisses), et des points doubles. Soit $Q=\pi(P)$ un point de branchement lisse, et soit $e$ l'indice de ramification correspondant. Si on choisit des paramètres locaux $t$ et $u$ en $P$ et $Q$ respectivement, alors comme dans le lemme 4.1.5, le morphisme (20) admet la description locale suivante :

$$
g(u) d u \in\left(\Omega_{B / k}^{1}\right)_{Q} \longmapsto e u g(u) \frac{d t}{t} .
$$

Comme il est clair que $\pi_{*}^{G}\left(\Omega_{C / k}^{1}\right)_{Q}=u \frac{d t}{t} \vartheta_{D, Q}$, on trouve bien que le morphisme (20) est bijectif en un tel point.

Soit maintenant le cas de $P$ de type II, donc un point double avec isotropie cyclique d'ordre $e \geqslant 2$. En utilisant les coordonnées locales le long des branches introduites dans le lemme 4.1.5, on obtient la description suivante pour les deux termes du morphisme (20). Pour le premier de ces termes :

$$
\left(\Omega_{D / k}^{1}\right)_{Q}=\frac{\widehat{\vartheta}_{Q} d u \oplus \widehat{\theta}_{Q} d v}{(u d v+v d u)}
$$

et pour l'autre :

$$
\left(\pi _ { * } ^ { G } \left(\widehat{\left(\Omega_{C / k}^{1}\right)_{Q}}=\left(\frac{\widehat{\vartheta}_{P} d x \oplus \widehat{\theta}_{P} d x}{(x d y+y d x)}\right)^{G_{P}} .\right.\right.
$$

Soit $\sigma$ un générateur de $G_{P}$ agissant par $\sigma x=\zeta x$, et $\sigma y=\zeta^{-1} y$. Un calcul élémentaire conduit à l'expression suivante d'une forme $G_{P}$-invariante :

$$
\omega=\left(\frac{A(u)}{x}, b y\right) d x+\left(0, \frac{D(v)}{y}\right) d y, \quad \text { avec } \quad b \in k, A(0)=D(0)=0 .
$$


Il est clair que le morphisme (20) est injectif en $P$, d'image l'ensemble des 1-formes qui dans l'expression (29) ont le coefficient $b$ nul. En particulier, le conoyau est de dimension 1, comme indiqué. Pour la seconde assertion, en dualisant le morphisme (20) on obtient un morphisme $\pi_{*}^{G}\left(\theta_{C}\right) \longrightarrow \theta_{D}$; la conclusion relève des mêmes calculs locaux qu'au-dessus, que nous omettons.

Remarque 4.1.12. - Si l'action stable du groupe $G$ sur $C / S$ n'a pas de points de type III, alors le diviseur de ramification relève de la définition habituelle. La partie horizontale de $\operatorname{Fix}(H), H$ sous-groupe cyclique, est une somme de diviseurs de Cartier relatifs à supports disjoints $\operatorname{Fix}(H)_{\text {hor }}=\sum_{C \subset H} \Delta_{C}$, en notant $\Delta_{C}$ le lieu des points de stabilisateur exactement $C$. Il est immédiat de retrouver l'expression usuelle du diviseur de ramification

$$
\Re=\sum_{H}(|H|-1) \Delta_{H}
$$

\subsection{Collision des points de ramification}

4.2.1. $G$-type d'un $G$-diviseur. - Soit une action stable de $G$ sur $C$. On suppose que $R \subset C$ est un diviseur de Cartier relatif, étale de degré $N$ sur $S$, de support contenu dans la partie lisse de $f: C \longrightarrow S$, et $G$-stable. Cela permet de parler du $G$-type de la fibre $R_{s}$ en un point géométrique $s \in S$ (section 2.2.1). Tout d'abord, une remarque élémentaire :

Lemme 4.2.1. - Le G-type de $R_{s}$ en un point géométrique $s \in S$ est constant.

Démonstration. - Par changement de base étale, on peut supposer que $R$ est somme de $N$ sections disjointes $\left\{P_{i}\right\}$, soit $R=\sum_{i=1}^{N} P_{i}$. Un élément $\sigma \in G$ agit par permutation sur les $\left\{P_{i}\right\}$. Soit pour tout indice $i, H_{i}$ le stabilisateur de $P_{i}$. Le faisceau conormal le long de la section $P_{i}, \mathcal{N}_{P_{i} / S}^{\vee} \cong P_{i}^{*}\left(\Omega_{C / S}^{1}\right)$ est un $G$-faisceau inversible sur $S$. On peut donc l'écrire $\mathcal{N}_{P_{i} / S}^{\vee} \cong \mathscr{L}_{i} \otimes V_{i}$, pour une certaine représentation $V_{i}$ de degré 1 de $H_{i}$; soit $\chi_{i}$ le caractère de $V_{i}$. Alors le type de $R_{s}$ est $\sum_{i=1}^{N}\left[H_{i}, \chi_{i}\right]$, donc constant le long des fibres.

L'objectif est de compléter la discussion qui suit la proposition 4.1.8, sur la dégénérescence des revêtements. Le résultat principal de cette section est la confirmation que seuls les points de type III, ceux à isotropie diédrale, sont responsables de la collision éventuelle des points de ramification dans une famille de revêtements. Ce comportement, ennuyeux à certains égards, pourra être éliminé par le marquage a priori de la courbe au moyen d'un diviseur contenant les points de ramification. 
4.2.2. Chevalley-Weil (bis repetita). - Soit $f: C \longrightarrow S$ une courbe stable, munie d'une action stable de $G$. Si la courbe est marquée (resp. stable marquée) par un diviseur $R$, celui-ci sera supposé $G$-invariant. La base étant connexe, le $G$-type de $R$ (lemme 4.2.1) est défini. On fixe une donnée de $\operatorname{Hurwitz} \xi \in \mathbf{H u r}(G)$, de manière équivalente, une collection de représentations $\operatorname{Hurw}_{m}(m \geqslant 1)$. Cela signifie que si $G$ agit sur la courbe lisse $C$ avec la donnée $\xi$, alors pour $m \geqslant 1, H^{0}\left(C, \omega_{C}^{\otimes m}\right) \cong \mathrm{Hurw}_{m}$.

Soit maintenant $C$ une $G$-courbe stable (stable et action stable) définie sur $k$, soit $D=C / G$ la courbe quotient. Les points doubles de $D$ sont les images des points doubles de type II de $C$. On suppose avoir pour tout $m \geqslant 1$ :

$$
H^{0}\left(C, \omega_{C}^{\otimes m}\right) \cong \operatorname{Hurw}_{m} .
$$

Dans cette situation, de manière analogue au cas lisse, on souhaite extraire des repésentations $\mathrm{Hurw}_{m}$ la structure combinatoire de l'action. Rappelons que la suite exacte (22) définit un faisceau $V_{l}$ concentré en les points fixes de types I et III. Pour $l \geqslant 2$, la courbe étant stable, donc $H^{1}\left(C, \omega_{C}^{\otimes l}\right)=0([30]$, th. 1.2), on a :

$$
\operatorname{dim}\left(H^{0}\left(C, \omega_{C}^{\otimes l}\right)^{G}\right)=\chi\left(D, \omega_{D}^{\otimes l}\right)+\operatorname{dim}\left(V_{l}^{G}\right)
$$

Si $l=1$, tenant compte de la description de $V_{1}(23),(24)$, on voit que $V_{1}^{G}=0$, donc $H^{0}\left(D, \omega_{D}\right)=H^{0}(C, \omega)^{G}$, en particulier $D$ est de genre $g^{\prime}$.

Pour $l \geqslant 2, \operatorname{dim}\left(V_{l}^{G}\right)$ est la contribution significative à $\operatorname{dim}\left(H^{0}\left(C, \omega_{C}^{\otimes l}\right)^{G}\right)$. Nous sommes en mesure de reprendre les calculs à la Chevalley-Weil de la section 3.2 pour une courbe $G$-stable. Rappelons tout d'abord quelques faits élémentaires sur les représentations irréductibles des groupes diédraux; les représentations sont sur $k$.

On identifie le groupe diédral $\mathbb{D}_{e}(e \geqslant 1)$ d'ordre $2 e$ avec le sous-groupe de $\operatorname{GL}(2, k)$ engendré par les matrices

$$
\tau=\left(\begin{array}{ll}
0 & 1 \\
1 & 0
\end{array}\right) \quad, \quad \sigma=\left(\begin{array}{cc}
\epsilon & 0 \\
0 & \epsilon
\end{array}\right)
$$

où $\epsilon \in k$ est une racine primitive $e$-ième de l'unité. Notons $\psi_{1}$ et $\psi_{1}^{\prime}$ les caractères de degré 1 de $\mathbb{D}_{e}$ définis par $\psi_{1}=1$ et $\psi_{1}^{\prime}(\sigma)=1, \psi_{1}^{\prime}(\tau)=-1$. Pour tout $j \geqslant 0$, soit le caractère $\phi_{j}$ de degré 2 donné par

$$
\phi_{j}(\tau)=0 \quad, \quad \phi_{j}(\sigma)=\epsilon^{j}+\epsilon^{-j}
$$

de sorte que $\phi_{j}$ est le caractère de la représentation décrite par

$$
\tau \mapsto\left(\begin{array}{cc}
0 & 1 \\
1 & 0
\end{array}\right) \quad, \quad \sigma \mapsto\left(\begin{array}{cc}
\epsilon^{j} & 0 \\
0 & \epsilon^{-j}
\end{array}\right) .
$$

On observe la relation de périodicité $\phi_{j e}=\phi_{j}$. D'autre part, $\phi_{j}$ est irréductible sauf dans deux cas : si $j \equiv 0(\bmod e)$, on a $\phi_{0}=\psi_{1}+\psi_{1}^{\prime} ;$ si $e=2 m$ et $j \equiv m(\bmod e)$, on a $\phi_{m}=\phi_{m}^{\prime}+\phi_{m}^{\prime \prime}$ où $\phi_{m}^{\prime}$ et $\phi_{m}^{\prime \prime}$ sont deux caractères de degré 1 donnés par $\phi_{m}^{\prime}(\sigma)=\phi_{m}^{\prime \prime}(\sigma)=-1$ et $\phi_{m}^{\prime}(\tau)=1, \phi_{m}^{\prime \prime}(\tau)=-1$.

Fixons quelques notations supplémentaires. Soit une action stable de $G \operatorname{sur} C$ semistable. 
- On note $b^{\prime}$ le nombre d'orbites (singulières) formées de points de type I, et $\delta$ le nombre d'orbites de points de type III.

Afin d'éviter toute confusion, rappelons la terminologie introduite en ce qui concerne les «stabilisateurs » (définition 4.1.7) en présence d'un point double.

- Le sous-groupe cyclique $H \subset G$ fixe strictement $P \in C$ si

- soit $P$ est lisse et $H \subset G_{P}$

- soit $P$ est un point double, et $H$ est d'ordre deux engendré par une réflexion échangeant les branches en $P$.

- Dans la même veine, $H$ est dit le stabilisateur strict de $P$, si $H=G_{P}$ lorsque $P$ est lisse, sinon si $P$ est un point double, $H$ fixe strictement $P$. Dans le second cas $H$ est « diédral »d'ordre deux.

Noter que la situation courante pour un point double de type III est d'avoir un stabilisateur $G_{P} \cong \mathbb{D}_{l}, l \geqslant 1$. Seuls les $l$ sous-groupes d'ordre 2 de $G_{P}$ contenant une « réflexion» sont des stabilisateurs au sens strict du point $P$. Si $l>1, G_{P}$ tout entier n'est pas considéré comme un stabilisateur, du moins strict, de $P$.

Soit $b$ le degré de la donnée de Hurwitz $\xi \in \operatorname{Hur}(G)$ qui a été fixée. En particulier si $G$ agit avec la donnée $\xi$ sur une courbe lisse, cela spécifie $b$ classes de conjugaison de caractères primitifs de sous-groupes cycliques, donc en particulier une liste de classes de conjugaison de sous-groupes cycliques, distinctes ou non, $\left[H_{1}\right], \ldots,\left[H_{b}\right]$ d'ordres respectifs $e_{1} \leqslant e_{2} \leqslant \cdots \leqslant e_{b}$. Pour tout $e \geqslant 2$ divisant $|G|$, posons

$$
N_{e}=\operatorname{Card}\left\{i \in[1, b], e_{i}=e\right\}
$$

de sorte que $N_{e}$ représente le nombre d'orbites spéciales de cardinal $|G| / e$ dans toute courbe lisse $C$ supportant une action de $G$ du type spécifié. Si $H$ est un sous-groupe, on définit ainsi $N_{e}(H)$.

On retourne à l'action stable de $G$ sur la courbe stable $C$, avec $H^{0}\left(C, \omega_{C}^{\otimes m}\right) \cong$ $\operatorname{Hurw}_{m}(m \geqslant 1)$. Les mêmes conventions de notations s'appliquent à $G$ et à un quelconque sous-groupe $H$ de $G$. Par $N_{e}^{\prime}$, on désigne le nombre de $G$-orbites spéciales de cardinal $|G| / e$ contenues dans la partie lisse de $C$. Ainsi

$$
b^{\prime}=\sum_{2 \leqslant e, e \mid \operatorname{Card}(G)} N_{e}^{\prime} .
$$

Pour tout sous-groupe $H$ de $G$, on a une définition de $b^{\prime}(H), \delta(H), N_{e}^{\prime}(H)$. Le résultat qui suit aide à clarifier la coalescence éventuelle des points de ramification :

ThÉORÈme 4.2.2. - On a les égalités :

i) $b=b^{\prime}+2 \delta$, et

ii) $N_{e}=N_{e}^{\prime}$ si $e \geqslant 3, N_{2}=N_{2}^{\prime}+2 \delta$. 
Démonstration. - L'assertion ii) est plus forte que i), mais on commence néanmoins par prouver i). Choisissons dans chaque orbite spéciale un point $P_{i}\left(1 \leqslant i \leqslant b^{\prime}\right)$, de stabilisateur $H_{i}=G_{P_{i}}$, pour celles de type I, et $Q_{j},(1 \leqslant j \leqslant \delta)$ avec $K_{j}=G_{Q_{j}}$, pour celles formées de points doubles de type III. Posons $\lambda_{i}=\left(V_{l}\right)_{P_{i}}, \mu_{j}=\left(V_{l}\right)_{Q_{j}}$ $(l=1,2, \ldots)$. On a une décomposition de $G$-modules

$$
\Gamma\left(C, V_{l}\right)=\left(\bigoplus_{i} \operatorname{Ind}_{H_{i}}^{G}\left(\lambda_{i}\right)\right) \bigoplus\left(\bigoplus_{j} \operatorname{Ind}_{K_{j}}^{G}\left(\mu_{j}\right)\right),
$$

d'où, par réciprocité de Frobenius :

$$
\operatorname{dim} \Gamma\left(C, V_{l}\right)^{G}=\sum_{i} \operatorname{dim}\left(\lambda_{i}^{H_{i}}\right)+\sum_{j} \operatorname{dim}\left(\mu_{j}^{K_{j}}\right) .
$$

L'analyse de la partie $H_{i}$-invariante de $\lambda_{i}$ relève de la description faite dans la section 3.2 , on trouve de manière analogue :

$$
\operatorname{dim}\left(\lambda_{i}^{H_{i}}\right)=l-1+\left(\left\langle\left\langle\frac{l}{e}\right\rangle\right\rangle-\frac{l}{e}\right) \quad \text { si } e=\operatorname{Card} H_{i} .
$$

Supposons maintenant que $K_{j}=\mathbb{D}_{e}$. On peut linéariser l'action de $K_{j}$ relativement à des coordonnées $x, y$ le long des branches au point $Q_{j}$, et ainsi supposer que les générateurs $\sigma$ et $\tau$ de $K_{j}$ agissent par $\sigma(x)=\epsilon x, \sigma(y)=\epsilon^{-1} y, \tau(x)=y, \tau(y)=x$, avec $\epsilon \in k^{*}$ une racine primitive d'ordre $e$. Le morphisme quotient a pour forme locale au point $Q_{j}:(x, y) \mapsto u=x^{e}+y^{e}$, de sorte que la 1-forme $\omega=\left(\frac{d x}{x},-\frac{d y}{y}\right)$ est une base locale de $\omega_{C}$ en $Q_{j}$. On a $\sigma^{*}(\omega)=\omega$ et $\tau^{*}(\omega)=-\omega$. Du fait que $(d u)^{\otimes l}=\left(x^{e l},(-1)^{l} y^{e l}\right) \omega^{\otimes l}$, le module $\mu_{j}$ admet la description :

$$
\mu_{j}=\frac{\theta_{C, Q_{j}} \omega^{\otimes l}}{\theta_{C, Q_{j}}(d u)^{\otimes l}} \cong\left(\frac{\theta_{C, Q_{j}}}{\left(x^{e l},(-1)^{l} y^{e l}\right)}\right) \omega^{\otimes l} .
$$

Il est aisé de décomposer le $K_{j}$-module $\mu_{j}$ en représentations irréductibles du groupe $K_{j}=\mathbb{D}_{e}$. Le résultat est

$$
\mu_{j}= \begin{cases}\left(2 \psi_{1}+\sum_{i=1}^{e l-1} \phi_{i}\right) \psi_{1}^{\prime} & \text { si } l \text { est impair } \\ \psi_{1}+\psi_{1}^{\prime}+\sum_{i=1}^{e l-1} \phi_{i} & \text { si } l \text { est pair } .\end{cases}
$$

Si $l=2$, ceci conduit à $\operatorname{dim} \gamma_{2}^{G}=b^{\prime}+2 \delta$. Du fait que $\chi\left(B, \omega_{B}^{\otimes 2}\right)=3 g^{\prime}-3$, l'égalité (30) entraîne $b=b^{\prime}+2 \delta$, c'est-à-dire l'assertion 1 ).

Si $l \geqslant 2$, on les mêmes égalités

$$
\begin{aligned}
& \operatorname{dim} V_{l}^{G}=(l-1) b^{\prime}+\sum_{i=1}^{b^{\prime}}\left(\left\langle\left\langle\frac{l}{e_{i}}\right\rangle\right\rangle-\frac{l}{e_{i}}\right)+ \begin{cases}\delta(l-1) & \text { si } l \text { est impair }, \\
\delta l & \text { si } l \text { est pair } .\end{cases} \\
& =(l-1) b^{\prime}+\sum_{2 \leqslant e \mid \operatorname{Card} G} N_{e}\left(\left\langle\left\langle\frac{l}{e}\right\rangle\right\rangle-\frac{l}{e}\right)+ \begin{cases}\delta(l-1) & \text { si } l \text { est impair }, \\
\delta l & \text { si } l \text { est pair } .\end{cases}
\end{aligned}
$$

Pour $l=3$, ces relations donnent $2 b-N_{2}=2 b^{\prime}-N_{2}^{\prime}+2 \delta$, ce qui, tenant compte de l'assertion (1), conduit à $N_{2}=N_{2}^{\prime}+2 \delta$. Avec $l=4$, on obtient de la même manière 
$3 b-N_{2}-N_{3}=3 b^{\prime}-N_{2}^{\prime}-N_{3}^{\prime}+4 \delta$, d'où on tire l'égalité $N_{3}=N_{3}^{\prime}$. En continuant ce raisonnement, on obtient de proche en proche l'égalité $N_{e}=N_{e}^{\prime}$ pour tout $e \geqslant 3$, c'est-à-dire l'assertion (2).

Les arguments combinatoires utilisés pour relier la donnée de Hurwitz aux représentations $\mathrm{Hurw}_{m}$ dans le cas lisse (section 3.2.2), peuvent être repris sans changement dans le cas d'une action stable. Par exemple :

Proposition 4.2.3. - Sous les hypothèses précédentes, les sous-groupes cycliques de $G$ qui sont des stabilisateurs stricts de points (réguliers ou points doubles) de $C$, sont les conjugués des sous-groupes $\left\{H_{1}, \ldots, H_{b}\right\}$ spécifiés par la donnée de Hurwitz.

Démonstration. - On reprend pour l'essentiel la méthode de dénombrement utilisée dans la preuve du théorème 3.2.2. Pour tout sous-groupe cyclique non trivial $H \subset G$, soit

$$
C^{H}=\{x \in C, H \text { fixe strictement } x\} .
$$

Posons :

$$
f(H)= \begin{cases}\operatorname{Card} C^{H} & \text { si } e=\operatorname{Card} H \geqslant 3, \\ N_{2}^{\prime}(H)+2 s(H) & \text { si } e=2 .\end{cases}
$$

Donc $f(H)=$ Card $C^{H}$, chaque point double étant si $e=2$ compté deux fois. On sait que que $f$ est totalement déterminé par les seules représentations de Hurwitz (section 3.2.2). Soit encore $\Delta_{H}=\{x \in C, H$ est un stabilisateur de $x\}$. Rappelons que si $x$ est un point double cela signifie que $e=\operatorname{Card} H=2$, et que $H$ est engendré par une reflexion qui échange les branches en $x$. Il découle des définitions que si $H \neq 1$, on a :

$$
C^{H}=\bigsqcup_{H \subseteq K} \Delta_{K}
$$

On peut poser $C^{H}=\Delta_{H}=\varnothing$ si $H$ n'est pas cyclique. Si $x \in C^{H}$ est un point double, alors Card $H=2$ et $x \in \Delta_{H}$. Si on pose de nouveau $g(H)=$ Card $\Delta_{H}$, un point double étant toujours compté deux fois, on retrouve la relation familière $f(H)=\sum_{H \subseteq K} g(K)$, avec la convention que $f(H)=g(H)=0$ si $H$ n'est pas cyclique. Par un argument similaire à celui utilisé dans le lemme 3.2.4, on obtient par inversion de Möbius

$$
g(H)=\sum_{H \subseteq K} \mu(H, K) f(K)
$$

où $\mu(\cdot, \cdot)$ désigne la fonction de Möbius de l'ensemble ordonné des sous-groupes de $G$. La preuve se poursuit comme dans loc. cit., on trouve que Card $\Delta_{H}$ ne dépend finalement que des $\operatorname{Hurw}_{m}$. Le résultat s'ensuit.

Finalement, de manière équivalente à 3.2.2, il est possible d'extraire directement des représentations $H^{0}\left(C, \omega_{C}^{\otimes m}\right)$ la donnée de ramification $\xi$, sans faire référence à une $G$-courbe lisse qui se spécialise en $C$. La description de la spécialisation d'un $G$-revêtement donnée dans la section 4.1 .3 suggère la définition suivante. 
Notons d'abord que dans un groupe diédral $\mathbb{D}_{m}$, les sous-groupes d'ordre deux, dits de réflexion forment une unique classe de conjugaison, notée $[H]$ si $m$ est impair, et deux classes $\left[H^{\prime}\right] \neq\left[H^{\prime \prime}\right]$ sinon. Si $K \cong \mathbb{D}_{m} \subset G$, la notation $\left[H^{\prime}\right]+\left[H^{\prime \prime}\right] \in R_{+}(G)$ est un raccourci pour la somme des deux classes précédentes, mais prises dans $G$, si $m$ est pair, sinon $2[H]$ si $m$ est impair.

On peut poser :

DÉfinition 4.2.4. - La donnée de Hurwitz de l'action de $G$ sur $C$ est $\xi=\xi_{I}+$ $\xi_{I I}$, où : $\xi_{I}=\sum_{i=1}^{b^{\prime}}\left[H_{i}, \chi_{i}\right]$, somme portant sur les $G$-orbites de type $I$, et $\xi_{I I}=$ $\sum_{j=1}^{\delta}\left(\left[H_{j}^{\prime}\right]+\left[H_{j}^{\prime \prime}\right]\right)$, somme portant sur les $G$-orbites de points fixes de type III.

Que l'on prenne pour donnée de Hurwitz celle de la définition ci-dessus ou celle attachée à une quelconque $G$-courbe lisse qui se spécialise en $C$, on a :

Proposition 4.2.5. - Étant donnée une action stable de $G$ sur la courbe stable $C$, la donnée de ramification (4.2.4) est déterminée par les représentations de Hurwitz $H^{0}\left(C, \omega_{C}^{\otimes m}\right)$.

\subsection{Courbes stables marquées et actions de groupes}

Parallèlement à la stabilité de la courbe $C$ qui supporte l'action de $G$, et de l'action de $G$, on aura à imposer la stabilité de la courbe marquée par un diviseur $G$-invariant $\Delta$, et souvent à rigidifier un peu plus en supposant que $\Delta$ est une somme de points lisses numérotés, ou numérotés par paquets, donc définissant un piquage de $C$ (définition 4.1.2).

Dans toute la suite, nous utiliserons la convention suivante : le diviseur $\Delta$ sera (sauf exception) supposé contenir le diviseur de ramification réduit $R(28)$, on dira pour abréger qu'il contient les points de ramification. Pour être en cohérence avec l'action de $G$, on doit prendre en compte l'holonomie en chaque point $P_{i}$ du diviseur, i.e. la classe $\left[H_{i}, \chi_{i}\right]$. Dans une numérotation des points de $\Delta$, les points de même rang seront contraints d'avoir la même holonomie, i.e. la même couleur. Naturellement, pour être élu diviseur de marquage, le diviseur doit être étale sur la base. Comme on va le voir, le dysfonctionnement découlant de la présence de points à isotropie diédrale pourra être éliminé par rigidification de l'action, i.e. en piquant celle-ci au moyen des points de ramification.

Dans la suite, $C / S$ est une $G$-courbe stable marquée par le diviseur $G$-invariant $\Delta$ (définition 4.1.2). Pour compléter l'étude précédente, limitée au cas stable non marqué, on doit maintenant faire une étude à la Chevalley-Weil du $G$-faisceau $\omega_{C / S}(\Delta)^{\otimes m}$, substitut naturel à $\omega_{C / S}^{\otimes m}$. Il s'agit, $s$ étant un point géométrique, d'extraire des informations des $k G$-modules $H^{0}\left(C_{s}, \omega_{C_{s}}\left(\Delta_{s}\right)^{\otimes m}\right)$. On sait que le faisceau dualisant modifié $\omega_{C / S}(\Delta)$ possède la propriété d'annulation (Knudsen [68], th. 1.8) :

$$
R^{1} f_{*}\left(\omega_{C / S}(\Delta)^{\otimes m}\right)=0 \quad \text { si } m \geqslant 2 .
$$


De plus $f_{*}\left(\omega_{C / S}(\Delta)^{\otimes m}\right)$ est localement libre de rang $(2 g-2+N) m-(g-1)$ si $m \geqslant 1$. D'autre part, la formation du faisceau $\omega_{C / S}(\Delta)^{\otimes m}$ commute aux changements de base. Le lemme suivant découle d'arguments déja utilisés, la preuve ne sera donc pas répétée.

LEMme 4.3.1. - Si $f: C \longrightarrow S$ est une courbe stable marquée par le diviseur $G$-invariant $\Delta=\sum_{i=1}^{N} P_{i}$, avec $S$ connexe, les $k G$-modules

$$
\operatorname{Hurw}_{m}(\Delta)=H^{0}\left(C_{s}, \omega_{C_{s}}\left(\Delta_{s}\right)^{\otimes m}\right)
$$

sont indépendants de la fibre géométrique $C_{s}, s \in S(m \geqslant 1)$.

Dans ce qui suit, on suppose les représentations $\operatorname{Hurw}_{m}(\Delta)$ fixées, ainsi que le $G$-type de $\Delta$. Supposons d'abord la fibre $C_{s}$ lisse. La suite exacte canonique

$$
0 \longrightarrow \omega_{C_{s}}^{\otimes m} \longrightarrow \omega_{C_{s}}\left(\Delta_{s}\right)^{\otimes m} \longrightarrow \bigoplus_{i} N_{P_{i}(s)}^{\otimes m-1} \longrightarrow 0
$$

avec $N_{P_{i}(s)}=T_{C_{s}, P_{i}(s)}^{*}$, montre que le $G$-type de $\Delta$ étant supposé connu, alors les représentations de Hurwitz $\mathrm{Hurw}_{m}$ le sont aussi. Il en est donc de même pour la donnée de ramification, évaluée le long des fibres non singulières (4.2.5). Pour tout sous-groupe cyclique $H$ de $G$, considérons le diviseur de Cartier relatif $\operatorname{Fix}(H)_{\text {hor }}$ (Proposition 4.1.8). Le corollaire de cette proposition montre que le degré $d$ de ce diviseur est égal à $d=d_{1}+2 d_{2}$ avec

$$
\begin{cases}d_{1} & =\text { nombre de points fixes de type I, } \\ d_{2}=\text { nombre de points fixes de type III. }\end{cases}
$$

Ce degré coïncide avec $f(H)$, fonction introduite dans la proposition 4.2.3, de sorte que l'argument combinatoire invoqué dans cette proposition conduit visiblement à un résultat identique. Pour l'énoncer, posons $g(H)=g_{1}(H)+2 g_{2}(H)$, avec

$$
\left\{\begin{array}{l}
g_{1}(H)=\text { nombre de points fixes de type I de stabilisateur } H \\
g_{2}(H)=\text { nombre de points fixes stricts de } H \text { de type III, }
\end{array}\right.
$$

de sorte que $g(H)$ est constant le long des fibres géométriques.

Introduisons quelques notations. Fixons une fibre géométrique notée pour simplifier $C$, les points qui participent au marquage seront notés $P_{i}$, de sorte que $\Delta=\sum_{i} P_{i}$. Soit $b_{1}$ le nombre d'orbites spéciales (points de type I) contenues dans les points marqués $\left\{P_{i}\right\}$ i.e. dans $D$; ce nombre est fixé par le $G$-type de $\Delta$, et indépendant de la fibre. Soit $b_{2}$ le nombre d'orbites spéciales (points de type I) disjointes de $\Delta$; ce nombre dépend de la fibre choisie. On a $b=b_{1}+b_{2}$ si la fibre est non singulière. Soit enfin $\delta$ le nombre d'orbites constituées de points de type III, à isotropie diédrale.

Le résultat suivant étend le théorème 3.2.2 aux courbes stables marquées, fournissant ainsi une explication à la coalescence éventuelle des points de ramification, cela en fonction des conditions initiales, de marquage, et la donnée de ramification. 
Soit $f: C \longrightarrow S$ une courbe stable marquée munie d'une action stable du groupe $G$. On suppose que la base $S$ est connexe et que l'ouvert des points $s \in S$ en lesquels la fibre $C_{s}$ est lisse est dense. Ainsi, une quelconque fibre est spécialisation d'une fibre non singulière, et la donnée de ramification est bien définie. Dire que le $G$-type de $\Delta$ contient la donnée de ramification a alors un sens. Le résultat suivant est une extension immédiate de (4.2.2). Il traduit comment $\Delta$ dégénère si la courbe est une spécialisation équivariante d'une courbe lisse.

Proposition 4.3.2. - Sous les hypothèses précédentes, on a le long des fibres géométriques l'égalité :

$$
b=b_{1}+b_{2}+2 \delta .
$$

Démonstration. - Montrons en premier que pour toute fibre, on a l'égalité relative à la fonction $g$ introduite au-dessus (32) :

$$
b=\frac{1}{|G|} \sum_{H \neq 1}|H| g(H) \text {. }
$$

La somme est limitée aux sous-groupes cycliques. Comme $g(H)$ ne dépend pas de la fibre, il suffit de vérifier cette égalité pour une fibre non singulière, dans ce cas $b$ représente le nombre d'orbites spéciales de la fibre. On peut alors écrire $b=\sum_{H \neq 1} b_{H}$, avec $b_{H}$ égal au nombre de ces orbites telles que le stabilisateur d'un de ses points est dans la classe de conjugaison de $H$. Soit $\omega$ une telle orbite; le nombre de points de $\omega$ de stabilisateur $H$ est égal à $\left|N_{G}(H)\right| /|H|$, d'où :

$$
g(H)=\left|\Delta_{H}\right|=b_{H} \frac{\left|N_{G}(H)\right|}{|H|} .
$$

On peut ainsi écrire (33) sous la forme d'une somme portant maintenant sur les classes de conjugaison notées $\langle H\rangle$ :

$$
b=\sum_{\langle H\rangle \neq 1} g(H) \frac{\left|N_{G}(H)\right|}{|H|}=\frac{1}{|G|} \sum_{H \neq 1}|H| g(H),
$$

la dernière somme portant elle sur les sous-groupes, et non sur les classes de conjugaison. Supposons maintenant la fibre singulière, avec $s$-orbites de type III. En substituant à $g(H)$ l'expression (32), on trouve l'expression

$$
b=\frac{1}{|G|} \sum_{H \neq 1}|H| g_{1}(H)+\frac{2}{|G|} \sum_{H \neq 1}|H| g_{2}(H) \text {. }
$$

Il est clair que le premier terme de la somme de droite est égal à $b_{1}+b_{2}$. Il reste à prouver que le second, qui ne fait intervenir que les sous-groupes d'ordre deux, conduit à :

$$
|G| \delta=2\left(\sum_{H,|H|=2} g_{2}(H)\right) \text {. }
$$


Dans ce but, on écrit $\delta=\sum_{m \geqslant 1} \delta_{m}$, où $\delta_{m}$ est le nombre d'orbites à groupe d'isotropie $\mathbb{D}_{m}$. Soit $\omega$ une orbite à groupe d'isotropie $\mathbb{D}_{m} ;$ notons $g_{2, \omega}(H)$ le nombre de points fixes stricts de $H$ contenus dans $\omega$. Dans la somme $\sum_{H, \text { Card } H=2} g_{2, \omega}(H)$ un point est en fait compté $m$ fois, car $\mathbb{D}_{m}$ contient $m$ réflexions. Cette somme vaut donc $m|\omega|=m(|G| / 2 m)=|G| / 2$ de sorte que

$$
\sum_{H,|H|=2} g_{2}(H)=\sum_{H, m \geqslant 1} \delta_{m} g_{2, \omega_{m}}(H)
$$

où $\omega_{m}$ désigne une orbite avec isotropie $\mathbb{D}_{m}$. En reportant (35) dans la relation qui précède, on obtient immédiatement le résultat.

Corollaire 4.3.3. - Les hypothèses de la proposition 4.3.2 étant conservées, supposons en outre que le G-type du marquage $\left\{P_{i}\right\}$ contienne la donnée de ramification, en abrégé $\Delta$ contient le diviseur de ramification. Alors, pour toute fibre géométrique, on a $\delta=0$. En d'autres termes, il n'y a pas d'orbite à isotropie diédrale.

Démonstration. - En effet, on a $b=b_{1}$ de sorte que $b_{2}=\delta=0 \mathrm{du}$ fait de la relation (33).

Dans la suite, la stabilité d'une courbe préstable marquée $\left(C,\left\{P_{i}\right\}\right)$ munie d'une action de $G$, comme au-dessus sera prise dans le sens étendu suivant (comparer avec 4.1.4) :

DÉfinition 4.3.4. - La G-courbe $\left(C,\left\{P_{i}\right\}\right)$ marquée par un diviseur $G$-invariant est $G$-stable marquée (stable marquée pour abréger) si

i) elle est stable marquée, abstraction faite de l'action de $G$,

ii) le diviseur de marquage contenant le diviseur de ramification,

iii) l'action de $G$ est stable (4.1.4). 


\section{CHAPITRE 5}

\section{DÉFORMATIONS DES REVÊTEMENTS MODÉRÉMENT RAMIFIÉS}

\subsection{Déformations équivariantes des courbes}

5.1.1. La déformation universelle. - On décrit la déformation formelle universelle d'un revêtement galoisien modérément ramifié $\pi: C \longrightarrow B=C / G$. Suivant nos notations habituelles, on notera $D$ la courbe quotient et $R=\sum_{i=1}^{N} P_{i}$ un diviseur de $C$ qui marque $C$ (4.1.2), qu'on ne confondra pas avec le diviseur de ramification réduit, même si le choix pour $R$ du diviseur de ramification sera fréquent. Dans cette section les hypothèses sont plus générales. Dans le présent contexte la théorie des déformations se simplifie de manière substantielle (comparer avec BertinMézard [15]). Les seules contributions locales à la déformation verselle viennent des points doubles et des points marqués, points de ramification exceptés, car la ramification étant modérée, ceux-ci n'apportent aucune contribution au foncteur des déformations. On comparera ensuite la déformation formelle verselle équivariante de la courbe marquée $C$, à la déformation formelle verselle de la base $D$ marquée par les images des points exceptionnels $P_{i}$ de $C$.

On fixe une courbe stable marquée $\left(C, P_{1}, \ldots, P_{N}\right)$ munie d'une action du groupe $G$. Il n'est pas nécessaire à ce stade de préciser la manière dont les points marqués $P_{i}$ sont traités, c'est-à-dire, ordonnés, ordonnés par paquets, ou pas. Le diviseur $R=\sum_{i} P_{i}$ est supposé $G$-invariant, contenant les points de ramification (voir section 4.3). Dans ces conditions on sait qu'une déformation formelle verselle existe, et qu'elle est universelle du fait de l'absence d'obstructions. Pour une discussion plus précise, voir BertinMézard [15].

On peut présenter le foncteur des déformations de la manière suivante, qui sera utile pour la suite. Soit une courbe stable marquée $\left(C, \sum_{i=1}^{N} P_{i}\right)$, en raccourci $\left(C,\left\{P_{i}\right\}\right)$. Oubliant l'action de $G$, soit un relèvement de $\left(C,\left\{P_{i}\right\}\right)$ à une $k$-algèbre locale artinienne $A$ de corps résiduel $k$, ou à une $W(k)$-algèbre si la caractéristique $p$ de $k$ est positive). Cela signifie qu'on a une courbe $\mathscr{C}$ au-dessus de $A$, plate sur $A$, marquée par des sections que nous noterons encore $\left\{P_{i}\right\}_{i=1, \ldots, N}$ (4.1.2). La donnée 
contient aussi un isomorphisme

$$
j: C \longrightarrow \mathscr{C} \otimes k
$$

tel que $P_{i} \circ j$ soit le point marqué initial $P_{i}$ de $C$, ceci pour tout indice $i, 1 \leqslant i \leqslant N$. La courbe $C$ étant supposée stable marquée, et donc sans automorphisme infinitésimal, si l'action de $G$ se relève à $\mathscr{C}$, ce relèvement est alors unique. Deux relèvements sont dits équivalents s'ils sont isomorphes par un isomorphisme qui relève l'identité. On notera $\mathbf{D}(A)$ l'ensemble des déformations, i.e. des classes de relèvements de $\left(C,\left\{P_{i}\right\}\right)$ à $A$; une classe étant notée $\left[\left(\mathscr{C},\left\{P_{i}\right\}, j\right)\right]$. Comme d'habitude, on étend le foncteur des déformations à tout anneau local noethérien complet qui est une $k$-algèbre ( resp. une $W(k)$-algèbre). Le groupe $G$ opère sur le foncteur des déformations de la manière évidente suivante. L'action de $\sigma \in G$ sur $\left[\mathscr{C},\left\{P_{i}\right\}, j\right]$ est

$$
\sigma\left[\mathscr{C},\left\{P_{i}\right\}, j\right]=\left[\mathscr{C},\left\{P_{i}\right\}, j \circ \sigma^{-1}\right] .
$$

Avec ces définitions, la déformation $\left[\mathscr{C},\left\{P_{i}\right\}, j\right]$ est un point fixe de l'action de $G$ si et seulement s'il existe un $A$-automorphisme $\Sigma$ de la courbe marquée $\left(\mathscr{C},\left\{P_{i}\right\}\right)$ qui relève $\sigma$, c'est-à-dire tel que $\Sigma \circ j=j \circ \sigma$. Ce relèvement est unique et définit ainsi un relèvement de l'action de $G$ à $\left(\mathscr{C},\left\{P_{i}\right\}\right)$. On observe donc que le foncteur des déformations équivariantes $\mathbf{D}_{G}$ est exactement le foncteur des points fixes relativement à l'action (36) de $G$ sur le foncteur des déformations $\mathbf{D}$. Il est bien connu que le foncteur des déformations $\mathbf{D}$ de la courbe $C$ est effectivement proreprésentable et formellement lisse (Deligne-Mumford [30], Knudsen [68]). Si $g$ désigne le genre de $C$, la déformation universelle de la courbe pointée $\left(\mathscr{C},\left\{P_{i}\right\}\right)$ a donc pour base le spectre d'une algèbre de séries formelles en $3 g-3+N$ variables

$$
R_{v e r}=W(k)\left[\left[t_{1}, \cdots t_{3 g-3+N}\right]\right] .
$$

La théorie cohomologique qui gouverne le foncteur $\mathbf{D}$ est $\operatorname{Ext}_{\vartheta_{C}}^{\bullet}\left(\Omega_{C}^{1}, \theta_{C}\left(-\sum_{i} P_{i}\right)\right)$. On sait que $\operatorname{Ext}_{\vartheta_{C}}^{2}\left(\Omega_{C}^{1}, \theta_{C}\left(-\sum_{i} P_{i}\right)\right)=0$, et que l'espace tangent s'identifie canoniquement à

$$
D(k[\epsilon])=\operatorname{Ext}_{\theta_{C}}^{1}\left(\Omega_{C}^{1}, \theta_{C}\left(-\sum_{i} P_{i}\right)\right) .
$$

Dans le cas équivariant, le foncteur $\mathbf{D}_{G}$ des classes de déformations équivariantes étant le foncteur des points fixes, et comme par ailleurs l'ordre de $G$ est inversible dans $k$, la théorie cohomologique qui gouverne $\mathbf{D}_{G}$ est particulièrement simple (comparer avec [15], section 3.1), c'est le groupe des Ext équivariants

$$
\operatorname{Ext}_{\vartheta_{C}, G}\left(\Omega_{C}^{1}, \theta_{C}\left(-\sum_{i} P_{i}\right)\right) \cong \operatorname{Ext}_{\vartheta_{C}}^{\bullet},\left(\Omega_{C}^{1}, \theta_{C}\left(-\sum_{i} P_{i}\right)\right)^{G}
$$

On a ainsi $\operatorname{Ext}_{\vartheta_{C}, G}^{2}\left(\Omega_{C}^{1}, \theta_{C}\left(-\sum_{i} P_{i}\right)\right)=0$, il n'y a donc pas d'obstructions au relèvement infinitésimal, et l'espace tangent est

$$
\mathbf{D}_{G}(k[\epsilon]) \cong \operatorname{Ext}_{\theta_{C}}^{1}\left(\Omega_{C}^{1}, \theta_{C}\left(-\sum_{i} P_{i}\right)\right)^{G}
$$


En conséquence, la déformation universelle $G$-équivariante se déduit de la déformation universelle $\mathscr{C} \longrightarrow \operatorname{Spec}\left(R_{\text {univ }}\right)=\mathscr{B}$ de $\left(C,\left\{P_{i}\right\}\right)$ privée de l'action de $G$, simplement par restriction au sous-schéma fermé $\mathscr{B}^{G}$ des points fixes, donc $\mathscr{C}^{G}=\mathscr{C} \times{ }_{\mathscr{B}} \mathscr{B}^{G}$.

L'absence d'obstruction pour le problème équivariant signifie que $\mathscr{B}^{G}$ est formellement lisse, donc est aussi le spectre d'une algèbre de séries formelles. Pour être plus précis, rappelons la forme du discriminant dans la déformation universelle $\mathscr{C} \longrightarrow \mathscr{B}$ de $C \longrightarrow B$ (voir [30]). Notons pour cela $x_{1}, \ldots, x_{k}$ les points doubles de $C$. Par localisation en $x_{i}$, on obtient un morphisme

$$
\operatorname{Ext}_{\vartheta_{C}}^{1}\left(\Omega_{C}^{1}, \theta_{C}\left(-\sum_{i} P_{i}\right)\right) \longrightarrow \prod_{i=1}^{k} \operatorname{Ext}_{\widehat{\vartheta}_{x_{i}}}^{1}\left(\widehat{\Omega}_{C, x_{i}}^{1}, \widehat{\vartheta}_{x_{i}}\right)
$$

qui représente l'application linéaire tangente au morphisme de localisation en le $k$-uplet des points doubles $x_{i}$. Cette application est surjective, et pour tout $i$ on a

$$
\operatorname{dim} \operatorname{Ext} \widehat{\widehat{\theta}}_{x_{i}}^{1}\left(\widehat{\Omega}_{C, x_{i}}^{1}, \widehat{\vartheta}_{x_{i}}\right)=1 .
$$

Choisissons des coordonnées $t_{1}, \ldots, t_{3 g-3+N}$ sur la base $\mathcal{B}$, numérotées de telle sorte que si $1 \leqslant i \leqslant k, t_{i}$ soit le paramètre induit par la déformation verselle du point double $x_{i}$. Le discriminant de la déformation universelle, c'est-à-dire le lieu paramétrant les fibres singulières, a donc pour équation

$$
t_{1} \cdots t_{k}=0 \text {. }
$$

Si on retourne à la situation équivariante, on voit que l'action de $G$ est lissifiable, si et seulement si la fibre générique de la déformation universelle $\mathscr{C}^{G} \longrightarrow \mathscr{B}^{G}$ est lisse, donc si $\mathscr{B}^{G}$ n'est pas inclus dans le lieu discriminant (39). La traduction de cette condition donne le résultat suivant (comparer avec Ekedahl [39]) :

THÉORÈme 5.1.1. - L'action de $G$ sur la courbe stable marquée $\left(C, \sum_{i=1}^{N} P_{i}\right)$ admet une déformation équivariante lisse si et seulement si l'action de $G$ est stable (4.1.4).

Démonstration. - Notons que les points marqués sont sans effet sur la stabilité de l'action, vu que celle-ci ne porte que sur l'isotropie aux points doubles. On peut donc les ignorer de manière provisoire. Soit $\mathscr{I}$ l'idéal de $\mathscr{B}^{G}$ dans l'anneau $R_{\text {univ }}=$ $k\left[\left[t_{1}, \ldots, t_{3 b-3+N}\right]\right]=\Theta_{\mathscr{B}}$, et soit $\mathcal{M}$ l'idéal maximal de $R_{\text {univ }}$. Posons par ailleurs

$$
W_{i}=\operatorname{Ext}_{\widehat{\vartheta}_{x_{i}}}^{1}\left(\widehat{\Omega_{C, x_{i}}^{1}}, \widehat{\vartheta}_{x_{i}}\right)^{*} \quad, \quad V=\operatorname{Ext}_{\vartheta_{C}}^{1}\left(\Omega_{C}^{1}, \theta_{C}\left(-\sum_{i} P_{i}\right)\right)^{*}
$$

et observons que la classe de $t_{i}$ peut être vue comme un élément de l'espace cotangent de $R_{\text {univ }}$, identifié à $V$. L'application (38) transposée donne un morphisme $G$-équivariant

$$
W=\prod_{i=1}^{k} W_{i} \longrightarrow V
$$


Décomposons l'ensemble des points doubles $\left\{x_{1}, \ldots, x_{k}\right\}$ en $G$-orbites : dans la $j$-ième orbite, on choisit un point $x_{i_{j}}$ noté $y_{j}$ de stabilisateur $G_{y_{j}}$ noté $H_{j}$. On a donc :

$$
\left\{x_{1}, \ldots, x_{k}\right\}=\bigsqcup_{j=1}^{s} G y_{j} \quad \text { et } \bigoplus_{x_{i} \in G y_{j}} W_{i}=\operatorname{Ind}_{H_{j}}^{G}\left(W_{i_{j}}\right)
$$

Soit $\chi_{j} \in \hat{H}_{j}$ le caractère attaché à l'action de $H_{j}$ sur $W_{i_{j}}$. Soit la décomposition du $G$-module $V$ en $V=V^{G} \oplus V_{G}$, les notations étant les notations usuelles, alors l'idéal I est engendré par $V_{G}$. Dès lors, la condition à vérifier est la suivante : pour tout indice $i$, on a $t_{i} \notin V_{G}$. Ceci est équivalent à $\operatorname{Ind}_{H_{j}}^{G}\left(W_{i_{j}}\right)^{G} \neq 0$, ou encore, par réciprocité de Frobenius, à $W_{i_{j}}^{H_{j}} \neq 0$. Finalement, la condition s'exprime par $\chi_{j}=1$ pour $1 \leqslant j \leqslant s$. Pour en faire la traduction, il est commode de passer à l'anneau local complété $\theta=\widehat{\vartheta}_{y_{j}}$ du point double $y_{j}$. On a la résolution libre de $\Omega_{\vartheta}^{1}$ :

$$
0 \longrightarrow I / I^{2} \longrightarrow \vartheta d x \oplus \theta d y \longrightarrow \Omega_{\vartheta}^{1} \longrightarrow 0
$$

où $I=(x y)$ et la flèche de gauche est définie par $x y \mapsto x d y+y d x(\bmod I)$. On en tire l'identification standard

$$
\operatorname{Ext}_{\theta}^{1}\left(\Omega_{\theta}^{1}, \theta\right)=\operatorname{Hom}\left(I / I^{2}, \theta\right) / \operatorname{Hom}(\theta d x \oplus \theta d y, \theta) .
$$

L'application $\phi: I / I^{2} \longrightarrow \vartheta, \phi(x y)=1$ est un élément non nul de cet espace vectoriel. Soit $\sigma \in H=H_{j}$; si $\sigma$ fixe les branches, on peut supposer que les coordonnées sont choisies de telle sorte que

$$
\sigma(x)=\alpha x+\cdots, \quad \sigma(y)=\beta y+\cdots
$$

On a $\sigma(\phi)=\alpha \beta \phi$, de sorte que la condition s'exprime alors par $\alpha \beta=\operatorname{det}(d \sigma)=1$. S'il y a échange des branches, on peut dans ce cas supposer que $\sigma(x)=\alpha y+\cdots, \sigma(y)=$ $\beta x+\cdots$, de sorte que dans ce cas la condition est encore $\alpha \beta=1, \operatorname{soit} \operatorname{det}(d \sigma)=-1$. On retrouve bien de la sorte la condition de stabilité de la définition 4.1.4.

Dans la suite, nous noterons $\mathscr{C} \longrightarrow \mathcal{B}$ la déformation universelle équivariante de la courbe stable marquée $\left(C,\left\{P_{i}\right\}\right)$, oubliant à partir de maintenant l'exposant $G$ dans les notations. On pourra supposer que les points $\left(P_{1}, \ldots, P_{s}\right)(s \leqslant N)$ sont les points de ramification. La base notée $\mathscr{B}$ est donc le spectre d'une algèbre de séries formelles sur $k$, ou $W(k)$ selon les cas. Si le nombre d'orbites de points doubles est $d$, alors on peut choisir des variables $t_{1}, \ldots, t_{M}$, de sorte que $t_{1}, \ldots, t_{d}$ correspondent aux paramètres de déformation des points doubles. L'équation du discriminant est alors $t_{1} \cdots t_{d}=0$. Il est facile de voir que l'espace tangent s'insère dans une suite exacte :

$$
0 \longrightarrow H_{G}^{1}\left(C, \Theta_{C}\left(-\sum_{j} P_{j}\right)\right) \longrightarrow \operatorname{Ext}_{\vartheta_{C}, G}^{1}\left(\Omega_{C}^{1}, \vartheta_{C}\left(-\sum_{j} P_{j}\right)\right) \longrightarrow
$$

$$
\longrightarrow \prod_{i=1}^{d} \operatorname{Ext}_{\widehat{\theta}_{x_{i}}}^{1}\left(\widehat{\Omega}_{C, x_{i}}^{1}, \widehat{\vartheta}_{x_{i}}\right) \longrightarrow 0
$$


où $\Theta$ désigne le faisceau tangent et on a fait le choix d'un point $x_{i}$ dans chaque orbite de points singuliers, et $H_{G}^{1}(\cdot)$ désigne la cohomologie équivariante ([15], section 3). Comme d'habitude, on note $\pi_{*}^{G}$ le foncteur $\mathcal{F} \mapsto \pi_{*}(\mathcal{F})^{G}$; on prouve facilement que

$$
H_{G}^{1}\left(C, \Theta_{C}\left(-\sum_{j} P_{j}\right)\right) \cong H^{1}\left(D, \pi_{*}^{G}\left(\Theta_{C}\left(-\sum P_{j}\right)\right)\right)
$$

notant toujours $\pi: C \longrightarrow D=C / G$ le morphisme quotient. On observera que dans le terme de droite de la suite exacte (40), le stabilisateur $G_{x_{j}}$ est omis en exposant, du fait que par la condition de stabilité, il agit trivialement. On en tire

$$
\operatorname{dim}(\mathscr{B})=M=3 g^{\prime}-3+b+r
$$

où $g^{\prime}$ est le genre de $C / G, b$ le degré de la donnée de ramification de l'action de $G$, et $r$ le nombre d'orbites de points marqués à isotropie triviale.

Une manière d'obtenir ce résultat est de calculer $\operatorname{dim} H^{1}\left(C / G, \pi_{*}^{G}\left(\Theta_{C}\left(-\sum P_{j}\right)\right)\right)$. Brièvement, si $C$ est une courbe préstable de composantes normalisées $C_{\alpha}(1 \leqslant \alpha \leqslant d)$, et si $i_{\alpha}: C_{\alpha} \longrightarrow C$ est le morphisme canonique, on vérifie facilement que $\Theta_{C}=$ $\bigoplus_{\alpha} i_{\alpha, *}\left(\theta_{C_{\alpha}}\left(-\sum p_{j}\right)\right.$, les points $p_{j}$ étant les origines des branches sur la composante $C_{\alpha}$. Cela ramène le calcul à un simple calcul de dimension sur une courbe lisse. Pour toute composante $D_{\beta}$ de $C / G$, appelons $v_{\beta}$ le nombre des images des origines des branches de type III, et $l_{\beta}$ le nombre de points de branchement situés sur $D_{\beta}$. La conclusion vient de la relation $b=\sum_{\beta} v_{\beta}+\sum_{\beta} l_{\beta}$, identique à la relation $b=b^{\prime}+2 \delta$ (théorème 4.2.2).

Si $r=0$, donc si le diviseur de marquage est exactement le diviseur de ramification (réduit), alors on voit que la dimension de l'espace tangent est identique à celle correspondante au problème sans marquage. Comme application du théorème 5.1.1, notons le résultat suivant :

Proposition 5.1.2. - Soit $A$ un anneau de valuation discrète complet de corps résiduel $k$ algébriquement clos, et de corps des fractions $K$. Soit $\mathscr{C} \longrightarrow \operatorname{Spec}(A)$ une courbe stable marquée par les points $\left\{P_{i}\right\}_{i=1}^{N}$, de fibre générique lisse. On suppose la fibre générique $\mathscr{C}_{K}$ munie d'une action du groupe $G$. Alors l'action de $G$ s'étend de manière unique en une action sur $\left(\mathscr{C},\left\{P_{i}\right\}\right)$, et cette action est stable.

Démonstration. - Du fait de l'unicité du modèle stable marqué $\mathscr{C}$ de $\mathscr{C}_{K}$, l'action de $G$ se prolonge de manière unique à $C$, de sorte que $G$ s'étend en un groupe d'automorphismes de $\mathscr{C} \longrightarrow \operatorname{Spec}(A)$. Le seul point non évident est que l'action de $G$ sur la fibre spéciale $C=\mathscr{C} \otimes k$ est stable. Pour le voir, soit $\Sigma \longrightarrow \operatorname{Spec}\left(R_{\text {univ }}\right)$ la déformation universelle équivariante de la courbe marquée $C$. L'absence d'automorphismes infinitésimaux justifie l'existence d'un morphisme unique $u: R_{\text {univ }} \longrightarrow A$ tel que $\mathscr{C} \cong \Sigma \otimes_{R_{\text {univ }}} A$. Soit $x=x_{i}$ un quelconque point double de $C$, alors l'anneau local complété de $\mathscr{C}$ en $x$ est de la forme

$$
\widehat{\vartheta}_{\mathscr{C}, x} \cong A[[X, Y]] /(X Y-a), \quad\left(a \in \mathcal{M}_{A}\right) \text {. }
$$


La lissité de $\mathscr{C}_{K}$ exige que $a \neq 0$. Il en découle que $x$ doit se déformer dans la déformation universelle de $C$, donc que le paramètre $t=t_{i}$ qui mesure la déformation de $x$ dans la déformation universelle non équivariante, doit avoir une restriction non nulle à $\operatorname{Spec}(R)$. La preuve du théorème 5.1.1 montre que cette condition, exprimée en chaque point double, revient à dire que l'action de $G$ est stable.

5.1.2. Stabilité de la courbe quotient. - On conserve les notations de la section précédente. Soit toujours $\left(C,\left\{P_{i}\right\}_{1 \leqslant i \leqslant N}\right)$ une courbe stable marquée, équipée d'une action stable du groupe $G$. En particulier, le diviseur $R=\sum_{i} P_{i}$ est $G$-invariant. On suppose que les stabilisateurs des points doubles ne sont pas diédraux, excluant ainsi les points fixes du type III. On verra comment ramener la situation générale à cette situation particulière. Soit le quotient $\pi: C \longrightarrow D=C / G$, de sorte que $D$ est préstable (proposition 4.1.3), marquée par les images $\left\{Q_{j}\right\}_{1 \leqslant j \leqslant r} \operatorname{des}\left\{P_{i}\right\}$. Rappelons que les points de ramification, qui sont tous lisses par hypothèse, sont inclus dans l'ensemble des points marqués. Il n'y a aucune perte de généralité à supposer cela, car les points de ramification sont des points marqués d'office. On a donc $b \leqslant r, b$ étant le nombre de points de branchement. Notons la remarque élémentaire suivante :

\section{Proposition 5.1.3. - Sous les hypothèses précédentes :}

i) la courbe quotient $\left(D,\left\{Q_{j}\right\}_{1 \leqslant j \leqslant r}\right)$ est stable marquée.

ii) Réciproquement, si $P_{1}, \ldots, P_{m}$ sont les points de $C$ d'images $Q_{1}, \ldots, Q_{r}$, alors la stabilité de $\left(D,\left\{Q_{j}\right\}_{1 \leqslant j \leqslant r}\right)$ implique la stabilité de $\left(C,\left\{P_{i}\right\}_{1 \leqslant i \leqslant m}\right)$.

Démonstration. - i) Soit $\mathcal{E}=\left\{Q_{j}\right\}_{1 \leqslant j \leqslant r}$, l'ensemble des points exceptionnels. On doit vérifier la condition de stabilité (4.1.1), donc si $\Delta$ est une composante de $D$, $\Delta \cong \mathbb{P}^{1}$, alors

$$
\text { (\# points doubles de } D \in \Delta)+\#(\Delta \cap \mathcal{E}) \geqslant 3 \text {. }
$$

Soit $\Gamma$ une composante de $C$ d'image $\Delta$. Comme l'action de $G$ est supposée sans inversion de branches, $\Gamma$ est non singulière. Notons $H$ le stabilisateur de $\Gamma$. Si $Q \in \Delta$ est un point double de $\Sigma$, nécessairement $Q$ est l'image d'un point double $P \in C$, situé sur $\Gamma$. Si $P^{\prime}$ est un second point double d'image $Q$, il y a un élément $g \in G$ tel que $g P=P^{\prime}$. Mais $\Delta$ étant non singulière, on doit avoir $g \in H$ et ainsi les points doubles de $\Sigma$ situés sur $\Gamma$ correspondent bijectivement aux $H$-orbites de points doubles de $C$ situés sur $\Gamma$. Distinguons plusieurs cas :

1) Le genre $h$ de $\Gamma$ est non nul. Soit $t \geqslant 1$ le nombre de points de branchement du revêtement $\Gamma \longrightarrow \Delta=\Gamma / H$. Notons $e_{1}, \ldots, e_{t}$ les indices de ramification respectifs. Si Card $H=N$, la formule de Riemann-Hurwitz donne

$$
2 h-2=-2 N+N\left(\sum_{i=1}^{t}\left(1-\frac{1}{e_{i}}\right)\right) \text {. }
$$

En particulier $(2 h-2) / N=t-2-\sum_{i=1}^{t} 1 / e_{i} \geqslant 0$, donc $t \geqslant 3$ et la condition de stabilité est satisfaite. 
2) Le genre $h$ est nul. On peut supposer que, avec les notations précédentes, $t<3$. Si $t=2$, alors $H$ est cyclique et opère sur $\Gamma=\mathbb{P}^{1}$ avec deux points fixes. La courbe $C$ étant stable marquée, $\Gamma$ contient au moins un point parmi les points doubles de $C$, ou parmi les points $\left\{P_{i}\right\}$ hors les points de ramification. La condition de stabilité est encore satisfaite. Reste pour conclure à examiner les deux cas : $\Delta$ est une courbe rationnelle avec un point double, et $\Delta$ est non singulière de genre un. Dans ces deux cas, la conclusion, immédiate, est laissée sans vérification.

ii) La preuve reprend les mêmes arguments que le point i), nous l'omettons.

À ce stade, il est sans doute nécessaire de clarifier le statut des points de ramification parmi les points marqués. Rappelons que, par convention, la courbe $C$ est marquée par un diviseur $G$-invariant $R$ supposé contenir les points de ramification. On peut donc légitimement demander de quelle manière ces points participent effectivement à la stabilisation de la courbe.

LEMme 5.1.4. - Soit, comme ci-dessus, une courbe stable marquée $\left(C,\left\{P_{i}\right\}_{i=1}^{N}\right)$ supportant une action stable de $G$ telle que les points de ramification sont les $m$ premiers points marqués $(m \leqslant N)$. Pour toute composante irréductible $\Gamma$ de $C$, notons $H$ son stabilisateur, $\mu$ le nombre de points doubles de $C$ situés sur $\Gamma$ et $\eta$ le nombre des $P_{i}(m+1 \leqslant i \leqslant N)$ situés sur $\Gamma$. Alors, la courbe $\left(C,\left\{P_{i}\right\}_{i=m+1}^{N}\right)$ est stable marquée seulement si $C$ n'a pas de composante non singulière rationnelle $\Gamma$ telle que

i) $H \neq 1, \mu=1, \eta=0$, ou

ii) $H \neq 1$ est diédral, $\mu=2, \eta=0$.

Démonstration. - Il est clair que la non stabilité est conséquence de l'existence de composantes $\Gamma=\mathbb{P}^{1}$, telles que $\mu+\eta \leqslant 2$. On peut exclure le cas trivial $\mu=0$, et supposer $\mu \geqslant 1$.

Supposons $\mu=1$. La stabilité initiale de la courbe permet d'exclure le cas $H=1$, $\eta \leqslant 1$. Si maintenant $H$ est non trivial, il est nécessairement cyclique et si $p \in \Gamma$ est le seul point double de $C$ sur $\Gamma$, on a $H p=p$, et $H$ doit avoir un second point fixe $p^{\prime} \in \Gamma$. La stabilité est mise en défaut que si $\eta=0$ car dans le cas contraire, $H$ étant non trivial, on a $\eta \geqslant 2$.

Supposons maintenant $\mu=2$ et $\eta=0$. Soient $p, q$ les deux points doubles de $C$ situés sur $\Gamma$. Si $p \notin H q$, alors $H p=p$, et $H q=q$, et $H$ est alors cyclique. Ce cas est exclu par la stabilité initiale. Si maintenant $q \in H p$, le sous-groupe $H$ a une orbite à deux éléments sur $\Gamma$, il est donc en vertu de la nature des groupes finis de transformations homographiques, cyclique ou diédral. Si $H$ est cyclique, alors $|H|=2$ par la stabilité de l'action, et ce cas rentre dans le cas diédral. Donc finalement, le seul cas pour lequel les points de ramification contribuent à la stabilité initiale de la courbe est le cas $H=\mathbb{D}_{l}, l \geqslant 1, \mu=2$, et $\eta=0$, ce qui prouve le lemme. 
Dans le cas $N=m$, donc si le marquage initial $\left\{P_{i}\right\}$ est exactement l'ensemble des points de ramification, alors seul le point ii) du lemme 5.1.4 peut se produire; dans ce cas, après stabilisation de la courbe (Knudsen [68], Manin [76]), on tombe sur un point double à isotropie diédrale.

\subsubsection{Déformations équivariantes versus déformations du diviseur de} branchement. - On souhaite comparer les déformations universelles respectives de la courbe marquée $\left(C,\left\{P_{i}\right\}_{1 \leqslant i \leqslant N}\right)$ munie de l'action de $G$, avec celles de la base $\left(D,\left\{Q_{j}\right\}_{1 \leqslant j \leqslant r}\right)$. Les points doubles de la base $D$, qui correspondent aux $G$-orbites de points doubles de $C$, contribuent individuellement par un paramètre indépendant, cela dans les deux déformations universelles. Notons $\left\{y_{1}, \ldots, y_{d}\right\}$ les points doubles de $D$; soit alors $e_{i} \geqslant 1$ l'ordre du stabilisateur, cyclique par hypothèse, d'un point au-dessus de $y_{i}$. Le passage au quotient par $G$ commute avec un changement de base arbitraire (voir propositions 3.1.4 et 4.1.3), il est alors clair que le morphisme $\pi: C \longrightarrow D=C / G$ induit un morphisme entre les foncteurs de déformations

$$
d \pi: D_{\left(C, G,\left\{P_{i}\right\}\right)} \longrightarrow D_{\left(D,\left\{Q_{j}\right\}\right)} .
$$

On a finalement le résultat important suivant, qui peut être comparé avec l'interprétation en termes de log-structures donnée dans Jarvis [58], Mochizuki [77], Wewers [99]. Il dit en substance que pour avoir un espace de déformations formelles d'un revêtement non galoisien formellement lisse, il faut déformer simultanément la clôture galoisienne.

THÉORÈme 5.1.5. - On peut choisir des systèmes de coordonnées

$$
\left(t_{1}, \ldots, t_{d}, \ldots, t_{3 g^{\prime}-3+r}\right) \text { et }\left(\tau_{1}, \ldots, \tau_{d}, \ldots, \tau_{3 g^{\prime}-3+r}\right)
$$

sur les bases des déformations universelles de $\left(C, G,\left\{P_{i}\right\}\right)$ et $\left(D,\left\{Q_{j}\right\}\right)$ respectivement, de sorte que relativement à ces coordonnées le morphisme di est donné par :

$$
\left.d \pi^{*}: W(k)\left[\left[\tau_{1}, \ldots, \tau_{d}, \ldots, \tau_{3 g^{\prime}-3+r}\right]\right] \longrightarrow W(k)\left[\left[t_{1}, \ldots, t_{d}, \ldots, t_{3 g^{\prime}-3+r}\right)\right]\right]
$$

avec $(d \pi)^{*}\left(\tau_{i}\right)=t^{e_{i}}$ si $1 \leqslant i \leqslant d$, et $(d \pi)^{*}\left(\tau_{i}\right)=t_{i}$ si $i>d$.

Démonstration. - On sait (voir la suite exacte (40)) que la base de la déformation équivariante universelle admet d'une part une contribution venant des déformations localement triviales autour des points doubles, c'est-à-dire au niveau de l'espace tangent le sous-espace vectoriel $H_{G}^{1}\left(C, \Theta_{C}\left(-\sum_{i} P_{i}\right)\right)$, et d'autre part une contribution de dimension 1 apportée par chaque orbite de points doubles. L'action du groupe étant stable et sans isotropie diédrale, la proposition 4.1.11 montre que la première contribution apparaît de manière identique dans le foncteur des déformations de $\left(D,\left\{Q_{j}\right\}\right)$. Seule la contribution des points doubles diffère dans les deux foncteurs. 
Au niveau des espaces tangents, cela correspond au diagramme suivant :



Cela ramène à comparer par le principe local-global ([15], théorème 3.3.4) les foncteurs de déformations locaux, c'est-à-dire pour un point double. Soit maintenant $x \in C$ un point double d'image $y \in B$. On suppose que le stabilisateur de $x$ est $H \cong \mathbb{Z} / e \mathbb{Z}$, et que $\sigma$ en est un générateur, qui agit le long des branches au point $x$, relativement à des paramètres convenablement choisis, par

$$
\sigma X=\zeta X \quad, \quad \sigma Y=\zeta^{-1} Y
$$

où $\zeta$ est une racine primitive $e$-ième de l'unité. La déformation verselle équivariante du point $x$ est, du fait de la stabilité de l'action (voir la preuve du théorème 5.1.1), représentée par $k[[X, Y, \lambda]] /(X Y-\lambda)$ (on remplace $k$ par l'anneau des vecteurs de Witt $W(k)$ dans le cas d'inégales caractéristiques). De manière analogue, la déformation universelle du point double $y$ est représentée par $k[[U, V, \mu]] /(U V-\mu)$, où $U=X^{e}, V=Y^{e}$. Le morphisme $d \pi$ correspond alors à

$$
k[[U, V, \mu]] /(U V-\lambda) \longrightarrow k[[X, Y, \lambda]] /(X Y-\lambda)
$$

avec $d \pi(U)=X^{e}, d \pi(V)=Y^{e}, d \pi(\mu)=\lambda^{e}$. Le résultat en découle.

\subsection{Modèle stable marqué d'un $G$-revêtement}

On a vu que deux définitions de la stabilité d'un $G$-revêtement $\pi: C \longrightarrow D=C / G$ sont en concurrence. La première définition revient à imposer la stabilité de $C$, jointe à celle de l'action (4.1.4). La seconde, plus flexible à certains égards, revient à demander à côté de la stabilité de l'action, la stabilité de $C$ marquée maintenant par un diviseur $G$-invariant contenant les points de ramification. Le cas non galoisien sera traité à part dans la section 6.6.

Avec l'une ou l'autre de ces deux définitions, on est en mesure de formuler un théorème de réduction stable. Fixons un anneau de valuation discrète complet $A$, de corps des fractions $K$, et de corps résiduel $k$, supposé algébriquement clos. Donnons nous un revêtement $\pi: C_{K} \longrightarrow D_{K}$ entre courbes lisses et connexes. Si la caractéristique de $k$ est $p>0$, on suppose que $p$ ne divise pas l'ordre du groupe de monodromie. Sous ces conditions, on s'attend à ce que le revêtement donné, défini sur le corps $K$, se prolonge de manière unique, après extension finie éventuelle de $A$, en un revêtement stable (resp. stable marqué) $\pi: \mathscr{C} \longrightarrow \mathscr{D}$. On notera cependant qu'en général, on ne peut pas prendre pour $\mathscr{C}$ et $\mathscr{D}$ les modèles stables respectifs. Ce phénomène sera clarifié lorsqu'on aura remarqué que la seule difficulté provient des isotropies diédrales (éventuelles) dans la clôture galoisienne. La définition suivante 
est essentiellement la notion de stabilité utilisée par Harris-Mumford [56] pour compactifier le schéma de Hurwitz « classique»

DÉFinition 5.2.1. - Soit un revêtement entre courbes préstables $\pi: C \longrightarrow D=$ $C / G$, galoisien de groupe $G$, au-dessus de la base $S$. Il est dit stable, ou stable marqué (ou HM-stable), si d'une part l'action de $G$ est stable (définition 4.1.4), et si d'autre part la courbe $C / S$ est stable marquée par les points de ramification.

Noter que, la courbe étant marquée par les points de ramification (définition 4.3.4), cela signifie en particulier que les points de ramification sont lisses, en conséquence il n'y a pas de point à isotropie diédrale (points de type III) (corollaire 4.3.3). Sous ces hypothèses, on sait aussi que la courbe quotient $D=C / G$ est stable marquée par les points de branchement (proposition 5.1.4). La stabilité étant prise dans l'un ou l'autre des deux sens (définitions 4.1 .4 et 4.3.4), la variante du théorème de réduction stable s'énonce alors ainsi :

Proposition 5.2.2. - Soit $S=\operatorname{Spec}(A)$ le spectre d'un anneau de valuation discrète complet, de corps des fractions $K$, et de corps résiduel $k$. Soit $\pi: C_{K} \longrightarrow D_{K}$ un $G$-revêtement galoisien entre courbes lisses connexes définies sur $K$, avec $|G|$ inversible dans A. Alors, après extension finie éventuelle de A, le G-revêtement $\pi$ admet un modèle stable unique $\pi: \mathscr{C} \longrightarrow \mathscr{D}$.

Démonstration. - C'est simplement une reformulation de la proposition 5.1.2. En effet, celle-ci entraîne comme conséquence directe du théorème de réduction stable (resp. stable marquée), que si on considère le modèle stable (resp. stable marqué) $\mathscr{C}$ de $C_{K}$, alors l'action de $G$ s'étend stablement à $\mathscr{C}$.

On notera que, sauf si la donnée de Hurwitz impose des contraintes particulières, on ne peut exclure a priori les points doubles à isotropie diédrale dans la réduction stable non marquée d'un revêtement. Pour éliminer ces points, il faut stabiliser la courbe $\mathscr{C}$ au moyen des points de ramification, c'est-à-dire déployer ceux qui se sont localisés en les points doubles de type III de la fibre spéciale, et ainsi aboutir au modèle stable marqué. Rappelons cette procédure bien connue.

Soit $C=\mathscr{C}_{k}$ la fibre spéciale; soit aussi $P \in C$ un point double de $C$ d'épaisseur $k \geqslant 1$, donc $\widehat{\mathscr{\theta}}_{\mathscr{C}_{P}} \cong A[[X, Y]] /(X Y-a)$, avec $a \in \mathcal{M}_{A}$ de valuation $k$. Si $k \geqslant 2$, $P$ est un point singulier de la surface normale $\mathscr{C}$, de type $A_{k-1}$. On résout cette singularité par $[k / 2]$ éclatements successifs de l'idéal maximal (voir par exemple [30]). Le graphe de la fibre exceptionnelle correspondante est une chaîne de $k-1$ droites projectives, chaque brin étant d'auto-intersection -2 (voir figure 1).

Pour rendre la procédure d'éclatement équivariante à chaque étape, on éclate en fait chacun des points de l'orbite de $P$. Soit $H \cong \mathbb{D}_{m}$ le stabilisateur de $P$, et supposons ce groupe engendré par l'élément $\sigma$ d'ordre $m$ et par l'involution $\tau$, agissant sur les branches en $P$ par $\sigma x=\zeta x, \sigma y=\zeta^{-1} y$ et $\tau x=y$, pour une certaine racine $m$-ième de l'unité. Distinguons deux cas selon la parité de $k$. 


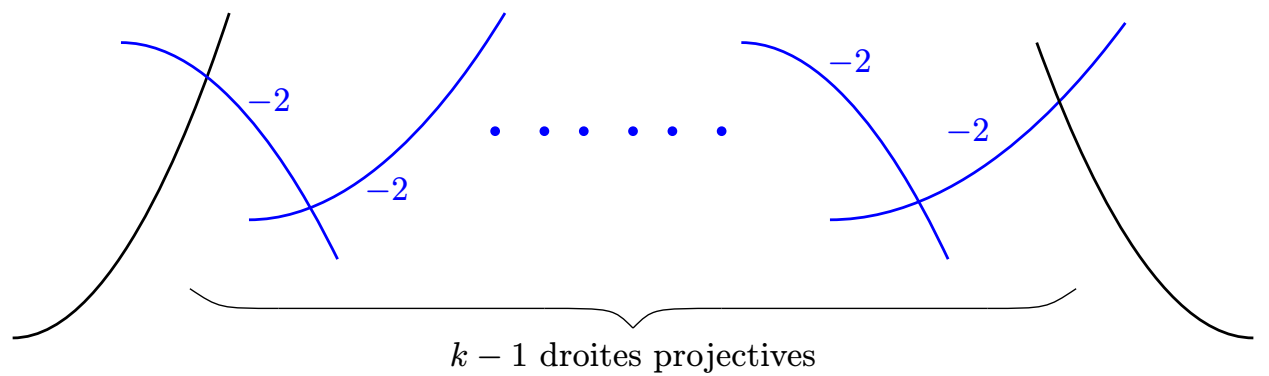

Figure 1. Graphe de la résolution de la singularité $A_{k-1}$

- $k$ pair. Si $k \geqslant 4$, l'éclatement des points de l'orbite de $P$ produit au-dessus de chacun de ces points une paire de courbes rationnelles se coupant en un point de type $A_{k-3}$. Il est facile de voir que ce point reste à isotropie $\mathbb{D}_{m}$, les transformées strictes des branches en $P$ étant par contre séparées et coupant respectivement les deux composantes du lieu exceptionnel en deux points à isotropie cyclique. Noter que la stabilité de l'action est préservée. Après $(k / 2)-1$ éclatements ponctuels, on arrive finalement à un point d'épaisseur 2 , et d'isotropie diédrale $\mathbb{D}_{m}$ (voir figure 2 ).
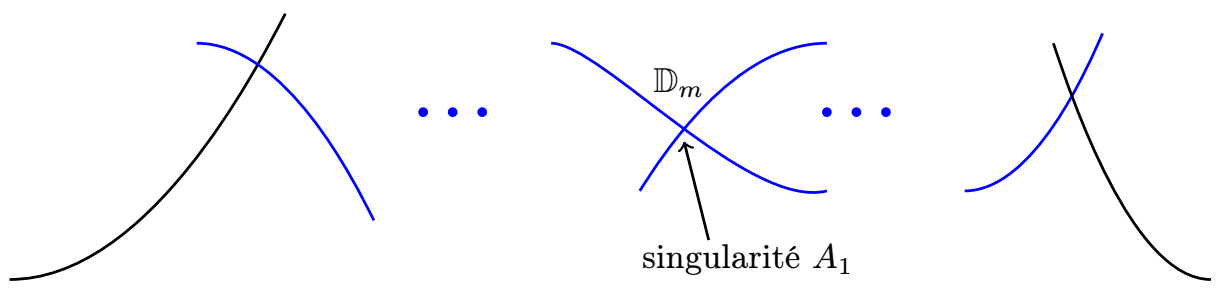

Figure 2. Résolution équivariante ( $k$ pair) : persistance d'un point à isotropie diédrale au cours des $(k / 2)-1$ premiers éclatements

Un dernier éclatement produit une courbe $E=\mathbb{P}^{1}$, et résout la singularité $A_{k-1}$ initiale (voir figure 3).

Il est immédiat à ce stade que les stabilisateurs sont tous cycliques, l'action de $H$ sur la composante $E$, de paramètre $t$, étant l'action standard $\sigma t=\zeta t, \tau t=t^{-1}$. La courbe obtenue n'est pas stable marquée; pour obtenir cette condition, on doit contracter les deux chaînes de longueur $(k / 2)-1$ de part et d'autre de $E$, conduisant à une situation où les points $P_{1}$ et $P_{2}$ sont des points singuliers de type $A_{k / 2}$. Les points fixes de $H$ sur la composante $E$ stabilisent maintenant la courbe (figure 4 ). 


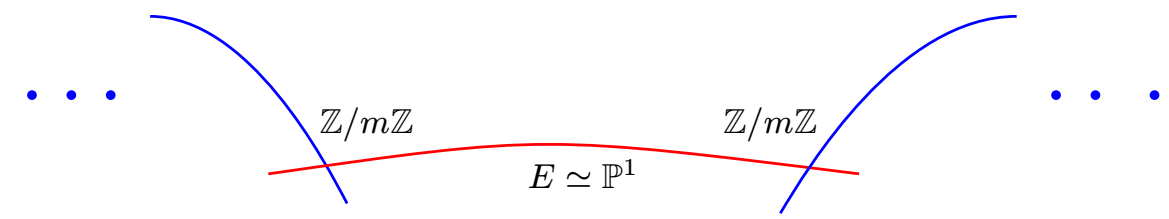

Figure 3. Résolution équivariante ( $k$ pair) : éclatement final de la singularité d'épaisseur 2 à isotropie diédrale

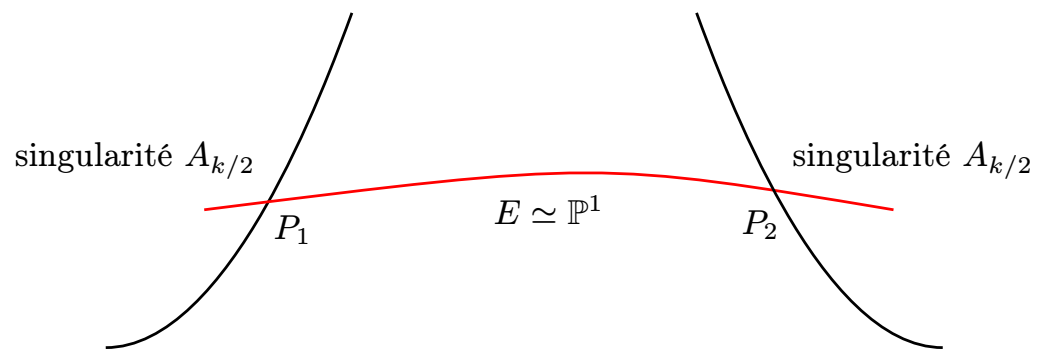

Figure 4. Résolution équivariante ( $k$ pair) : contraction des deux chaînes de part et d'autre de $E$

- $k$ impair. Dans ce cas, on résout le point singulier par une suite de $[k / 2]$ éclatements. On doit noter que le point d'intersection des deux brins qui proviennent du dernier éclatement est encore à isotropie diédrale $H=\mathbb{D}_{m}$, au contraire du cas $k$ pair (voir figure 5).
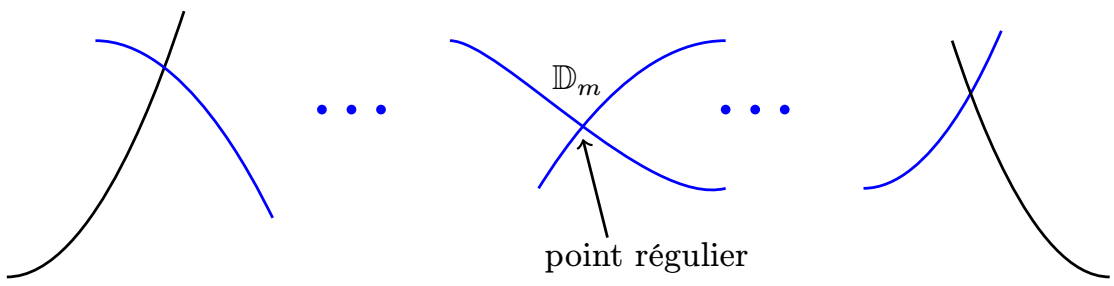

Figure 5. Résolution équivariante ( $k$ impair) : persistance d'un point à isotropie diédrale dans la résolution

L'éclatement de ce dernier point (régulier) conduit à une composante exceptionnelle rationnelle $E$. Supposons le point donné par l'équation $x y-z=0$; on peut prendre pour paramètre sur $E, t=x / y$, l'action de $H$ étant alors donnée par $\sigma t=\zeta^{2} t$, $\tau t=t^{-1}$. La stabilité de l'action impose $m$ impair, ce qui nous ramène à une situation 
identique à la précédente. Par contraction des deux chaînes de longueur $[k / 2]$ de part et d'autre de la courbe centrale, on arrive au modèle stable marqué. La construction montre clairement l'unicité du modèle stable marqué.

Remarque 5.2.3. - On conserve les hypothèses de la proposition 5.2.2. Soit $\pi$ : $C_{K} \longrightarrow D_{K}$ un revêtement galoisien défini sur $K$, de groupe de Galois $G$.

1 - Il est facile de voir que si $C_{K}$ admet un modèle stable $\mathscr{C}$ sur $A$, alors $D_{K}$ possède un modèle stable $\mathscr{D}$ sur $A$ et le morphisme $\pi$ se prolonge en un morphisme, non nécessairement fini, $\mathscr{C} \longrightarrow \mathscr{D}$. Soit en effet le modèle stable $\mathscr{C}^{\prime} \longrightarrow \mathscr{D}^{\prime} \mathrm{du}$ revêtement $\pi: C_{K} \longrightarrow D_{K}(5.1 .2)$; la preuve donnée montre en fait qu'un tel modèle existe sur $A$. La courbe quotient $\mathscr{D}^{\prime}$ est alors stable marquée par les points de branchement. Le procédé de stabilisation de Knudsen [68] conduit à un morphisme $\mathscr{D}^{\prime} \longrightarrow \mathscr{D}$, où $\mathscr{D}$ est le modèle stable de $D$, donc défini sur $A$. Il est par ailleurs clair que le morphisme composé $\mathscr{C}^{\prime} \longrightarrow \mathscr{D}^{\prime} \longrightarrow \mathscr{D}$ factorise par la stabilisation $\mathscr{C}$ de $\mathscr{C}^{\prime}$. On notera que même si $\mathscr{C}^{\prime}=\mathscr{C}$, en général $\mathscr{D}^{\prime} \neq \mathscr{D}$; l'égalité n'est réalisée que si le revêtement générique est étale.

2 - Sous les mêmes hypothèses, supposons maintenant que $D_{K}$ possède un modèle lisse sur $A$ i.e. $D_{K}$ a bonne réduction sur $A$, et soit $B \subset D$ un diviseur de Cartier relatif étale sur $A$. On suppose que $B_{K}$ est le diviseur de branchement de $\pi: C_{K} \longrightarrow B_{K}$. Alors sous ces conditions, $C_{K}$ a bonne réduction sur $A$, plus précisément, $\mathscr{C}$ étant le modèle lisse de $C_{K}$ sur $A$, le revêtement générique se prolonge en un revêtement $\pi: \mathscr{C} \longrightarrow \mathscr{D}$ de lieu de branchement $B$.

On prouve cela comme suit : soit une extension finie galoisienne $L / K$ de groupe de Galois $I$, relativement à laquelle $C_{K}$, équipée de l'action de $G$, acquiert un modèle stable marqué $\mathscr{C}$. La courbe quotient $D=C / G$ est alors le modèle stable sur la normalisation de $A$ de la courbe $D_{K}$ marquée par les points de branchement. La lissité de $D_{k}$ implique celle de $C_{k}$. Reste à prouver que $C$ est en fait définie sur $A$. Pour cela notons que le théorème 5.1.5 montre que le foncteur des déformations $G$-équivariantes infinitésimales de $C_{k}$ est isomorphe à celui de $\left(D_{k}, B_{k}\right)$. Donc, si $\sigma \in I$, le modèle tordu $\mathscr{C}^{\sigma}$ est une déformation formelle de $C_{k}$ de quotient $\mathcal{B}$ et de discriminant $B$. On a donc un isomorphisme unique $\mathscr{C}^{\sigma} \cong \mathscr{C}$, donc en fait une donnée de descente, ce qui permet de descendre le modèle $\mathscr{C}$ à $A$.

On déduit facilement de cette remarque le théorème de $\mathrm{S}$. Beckmann [9] qui montre que le corps des modules d'un revêtement $C \longrightarrow D$ défini sur la clôture algébrique de $K$, et dont la base est définie sur $K$, a bonne réduction sur $K$, et est non ramifié en $A$ si les points de branchement ne coalescent pas dans la fibre spéciale. 



\section{CHAPITRE 6}

\section{CHAMPS DE HURWITZ}

Dans cette section, on définit le champ de Hurwitz qui classifie les revêtements, galoisiens ou non, entre courbes lisses de genres fixés, et à monodromie fixée, et on construit ensuite le champ compactifié par addition des revêtements stables. Le cas non galoisien est ramené au cas galoisien par un argument de clôture galoisienne.

Une difficulté courante dans les problèmes modulaires est que les objets ont parfois des automorphismes permanents; dans le cas présent, les éléments du centre du groupe $G$ donnent des automorphismes des revêtements. Il est naturel de chercher à les éliminer pour arriver à un champ classifiant plus proche d'un schéma. De cette manière, le champ de Hurwitz est relié au champ $\overline{\mathcal{M}}_{g^{\prime}, n}(B G)$ introduit par Abramovich, Corti et Vistoli [1] par :

$$
\bigsqcup_{[\xi]} \overline{\mathcal{H}}_{g, G, \xi} \rrbracket Z(G)=\overline{\mathcal{M}}_{g^{\prime}, n}(B G) .
$$

Bien que des résultats généraux assurent, une fois les champs définis, l'existence des espaces grossiers de modules, nous en donnerons une construction projective en suivant la construction de $\bar{M}_{g}$ par Gieseker [51]. Il est amusant de noter que la projectivité des espaces de Hurwitz conduit, si on le désire, à une preuve uniforme de la projectivité des $\bar{M}_{g, n}$, preuve qui se réduit essentiellement à celle de $\bar{M}_{g}$.

\subsection{G-champs et champs quotients}

Pour débuter, on rassemble une série de définitions et constructions sur les champs algébriques nécessaires dans la suite. Le cadre naturel pour les résultats que nous présenterons est celui des champs algébriques au sens d'Artin (au sens de Laumon et Moret-Bailly [73]), munis d'actions de schémas en groupes séparés, plats et de présentation finie. Cependant, pour les besoins de ce mémoire, il est suffisant de considérer des champs algébriques de Deligne-Mumford munis d'actions de groupes constants finis. Dans toute la section 6.1, nous nous placerons donc dans ce cadre simplifié, qui présente par ailleurs l'avantage (essentiellement psychologique) de 
pouvoir traiter les groupes comme des ensembles d'éléments, ce qui simplifie les écritures des différents cocycles amenés à apparaître.

Nous utiliserons librement la terminologie en vigueur dans la littérature sur les champs, en particulier celle de 2-morphisme, et de diagramme 2-commutatif (resp. 2-cartésien), et renvoyons à [73]. En ce qui concerne les opérations dans une 2-catégorie, les détails sont dans Romagny [90]. On rappelle que $B G$ désigne le champ classifiant du groupe $G$.

6.1.1. Quotient d'un champ par une action de groupe. - On fixe un schéma de base $S$. Les champs $\mathcal{M}, \mathcal{N}$ (etc.) qui apparaissent sont des $S$-champs algébriques de Deligne-Mumford, et les groupes $G, H$ (etc.) sont des groupes finis. Nous notons $m: G \times G \longrightarrow G$ la multiplication de $G$, et $e: \operatorname{Spec}(k) \longrightarrow G$ l'élément neutre. Les morphismes identiques sont notés id ou simplement 1 .

Dans ce qui suit, par souci de légèreté, nous épargnons au lecteur la recopie de certaines conditions d'associativité qui sont aussi lourdes que naturelles, et renvoyons alors à [90]. La définition d'un $G$-champ, qui découle de l'action d'un groupe sur une catégorie, est la suivante.

DÉfinition 6.1.1. - i) Une action de $G$ sur $\mathcal{M}$ est un triplet $(\mu, \alpha, \mathfrak{a})$ formé d'un morphisme $\mu: G \times \mathcal{M} \longrightarrow \mathcal{M}$ et de 2-isomorphismes $\alpha$, a rendant 2 -commutatifs les diagrammes :
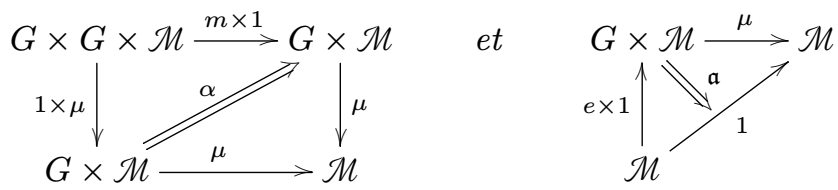

et satisfaisant à des relations d'associativité naturelles ([90], def. 1.3 (i)). L'action est dite stricte si $\alpha$ et $\mathfrak{a}$ sont des identités.

ii) Un morphisme de $G$-champs $(\mathcal{M}, \mu, \alpha, \mathfrak{a}) \longrightarrow\left(\mathcal{M}^{\prime}, \mu^{\prime}, \alpha^{\prime}, \mathfrak{a}^{\prime}\right)$ est une paire $(f, \sigma)$, ò̀ $f: \mathcal{M} \longrightarrow \mathcal{M}^{\prime}$ un morphisme de champs et $\sigma: \mu^{\prime} .(1 \times f) \longrightarrow f . \mu$ est un 2-morphisme vérifiant des relations de compatibilité naturelles avec $\alpha, \alpha^{\prime}$ et $\mathfrak{a}, \mathfrak{a}^{\prime}$ (loc. cit. (ii)).

On montre qu'il existe un foncteur de «strictification » qui envoie un $G$-champ sur un $G$-champ strict qui lui est isomorphe ([90], prop. 1.5). Dans la pratique, ceci permet de se ramener à des actions strictes lorsque certaines constructions conduisent à des actions non strictes. Dans la suite, si $x \in \mathcal{M}(U)$, on écrira $g x$ pour l'image $\mu(g, x)$, et aussi $g \varphi: g x \stackrel{\sim}{\longrightarrow} g x^{\prime}$ pour l'image de $\varphi: x \stackrel{\sim}{\longrightarrow} x^{\prime}$ par $g$.

DÉfinition 6.1.2. - Un G-isomorphisme entre deux G-champs $\mathcal{M}$ et $\mathcal{N}$ est un $G$-morphisme $(f, \sigma): \mathcal{M} \longrightarrow \mathcal{N}$ qui est un isomorphisme de champs, i.e. une équivalence de catégories. 
Si $f: \mathcal{M} \longrightarrow \mathcal{N}$ est un $G$-isomorphisme, il existe un quasi-inverse qui est un $G$-morphisme ([90], remark 1.4). Une action de $G$ sur $\mathcal{M}$ est triviale si l'identité de $\mathcal{M}$ peut être prolongée en un $G$-isomorphisme entre $\mathcal{M}$ et le champ $\mathcal{M}$ muni de l'action strictement triviale (i.e. l'action stricte pour laquelle $\mu$ est la seconde projection). Par exemple, soit $\mathcal{M}$ un $G$-champ, et soit $H \triangleleft G$ un sous-groupe distingué. Si la restriction de l'action à $H$ est triviale, la trivialisation étant compatible à l'action de $G$ sur $H$ par automorphismes intérieurs, il y a une action induite de $G / H$ sur $\mathcal{M}$. On peut par exemple passer par une transversale de $G$ modulo $H$. On notera que l'action construite peut ne pas être stricte.

Si $X$ est un $G$-schéma et $\mathcal{M}$ est un $G$-champ strict, un $G$-morphisme $X \longrightarrow \mathcal{M}$ est déterminé par un objet $x \in \mathcal{M}(X)$ qui est $G$-linéarisé, c'est-à-dire équipé d'une famille d'isomorphismes $\sigma_{g}^{x}: g x \stackrel{\sim}{\longrightarrow} g^{*} x$, pour $g \in G$ variable, contraints par une relation de cocycle

$$
\sigma_{g h}^{x}=\sigma_{g}^{h x} \circ g\left(\sigma_{h}^{x}\right) .
$$

Soulignons que dans les écritures précédentes, l'objet $g x$ est l'image de $x$ sous l'action de $g$ sur $\mathcal{M}(X)$, et l'objet $g^{*} x$ est l'image de $x$ par le changement de base $g: X \longrightarrow X$.

Les $G$-champs forment une 2-catégorie. Il est aisé de voir que si $p: \mathcal{M} \longrightarrow \mathcal{Y}$ et $q: \mathcal{N} \longrightarrow \mathcal{Y}$ sont $G$-équivariants, alors le produit fibré $\mathcal{M} \times_{p, y, q} \mathcal{N}$ supporte une action canonique de $G$.

Le champ quotient $\mathcal{M} G$ du $G$-champ $\mathcal{M}$ par l'action de $G$ est caractérisé par une propriété universelle dans la 2-catégorie des $G$-champs ([90], définition 2.3).

DÉFINITION 6.1.3. - Un champ quotient d'un G-champ $\mathcal{M}$ est un morphisme $\pi$ : $\mathcal{M} \mathcal{M} / G$ de but un champ $\mathcal{M} / G$, qui est un $G$-morphisme si on munit $\mathcal{M} / G$ de l'action strictement triviale, et qui est universel au sens des 2-catégories pour les $G$-morphismes de $\mathcal{M}$ vers un champ avec action triviale de $G$.

On sait que si $\mathcal{M}$ est représentable, $\mathcal{M} / G$ existe et s'interprète comme un champ de $G$-torseurs. La même description, que nous rappelons maintenant, produit un champ quotient également lorsque $\mathcal{M}$ n'est pas représentable. Les sections de $\mathcal{M} / G$ au-dessus de $U$ sont les diagrammes

$$
\mathcal{M} \stackrel{f}{\longleftarrow} E \stackrel{p}{\longrightarrow} U
$$

où $p: E \longrightarrow U$ est un $G$-torseur, et le morphisme $f: E \longrightarrow \mathcal{M}$ est $G$-équivariant. Les

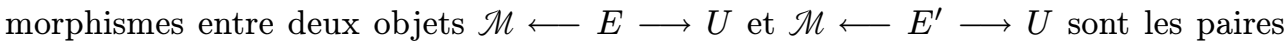
$(u, \Phi)$ composées d'un morphisme de $G$-torseurs $u: E \longrightarrow E^{\prime}$ et d'un 2-morphisme $\Phi: f \stackrel{\sim}{\longrightarrow} f^{\prime} u$. Les objets qui définissent $f$ et $f^{\prime}$ étant notés $x \in \mathcal{M}(E)$ et $x^{\prime} \in \mathcal{M}\left(E^{\prime}\right)$, le 2-morphisme $\Phi$ est déterminé par $\phi: x \stackrel{\sim}{\longrightarrow} u^{*}\left(x^{\prime}\right)$. La compatibilité aux actions de $G$ se traduit par la relation de cobord

$$
\sigma_{g}^{u^{*} x^{\prime}} \circ g \phi=g^{*} \phi \circ \sigma_{g}^{x} .
$$

Pour résumer, on a ([90] théorème 4.1) : 
ThÉORÈme 6.1.4. - Soit $G$ un groupe fini et $\mathcal{M}$ un $G$-champ algébrique de DeligneMumford.

i) Il existe un champ quotient $\mathcal{M} / G$, qui est un champ algébrique de Deligne-Mumford.

ii) Le morphisme $\mathcal{M} \longrightarrow \mathcal{M} / G$ est un $G$-torseur, et s'insère dans un carré 2 -cartésien

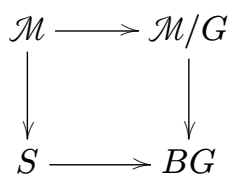

iii) Si M a un espace grossier des modules $M$, alors $G$ agit sur $M$, et l'espace algébrique $M / G$ est un espace grossier des modules pour $M / G$.

Dans (42) le morphisme $\mathcal{M} G \longrightarrow B G$ est celui qui à un objet $\mathcal{M} \longleftarrow E \longrightarrow U$ du champ $M / G$ associe le $G$-torseur $E \longrightarrow U$, objet de $B G$ au-dessus de $U$. Par exemple, pour l'action triviale de $G$ sur $\mathcal{M}$, le champ quotient est $\mathcal{M} B G$. Soit $H$ un sous-groupe distingué de $G$; il y a une action naturelle non stricte en général de $G / H$ sur $\mathcal{M} / H$, d'où on tire une équivalence

$$
(\mathcal{M} / H) /(G / H) \stackrel{\sim}{\longrightarrow} \mathcal{M} / G .
$$

Soit $\mathcal{M}$ un $G$-champ, et soit $H \triangleleft G$ un sous-groupe distingué. Si la restriction de l'action à $H$ est triviale, la trivialisation de l'action étant compatible à l'action de $G$ sur $H$ par automorphismes intérieurs, condition qu'on formalise au niveau des sections par

$$
\tau_{g h g^{-1}}^{g x}=g\left(\tau_{h}^{x}\right) \quad(g \in G, h \in H)
$$

il y a alors une action induite de $G / H$ sur $\mathcal{M}$. On notera cependant que l'action construite peut ne pas être stricte. On construit une telle action en choisissant une transversale $G / H \rightarrow G$. A équivalence près, i.e. 2-isomorphisme près, le résultat ne dépend pas de ce choix. Cela contient en particulier le fait que $G / H$ agit sur le champ $B H$, la restriction à $H$ de l'action naturelle de $G$ sur $B H$, étant clairement triviale. On peut voir l'existence de l'action induite de $G / H$ comme une propriété "d'exactitude" de la suite $B_{0} H \rightarrow B_{0} G \rightarrow B_{0}(G / H)$. Noter qu'une telle construction s'applique plus simplement à l'action du groupe $G$ sur un groupoïde $\mathfrak{G}$, qui si la restriction à $H$ est triviale (dans le sens ci-dessus, donc non strictement triviale), et la trivialisation est invariante par conjugaison par les éléments de $G$, alors il y a une action induite de $G / H$ sur $\mathfrak{G}$, bien définie à un 2-isomorphisme près. De telles situations se rencontrerons dans la suite.

EXemple 6.1.5. - Les champs de courbes $n$-marquées $\mathcal{M}_{g, n}$ et $\overline{\mathcal{M}}_{g, n}$ sont munis d'une action du groupe symétrique $\mathfrak{S}_{n}$ par permutation des points marqués. Le champ quotient $\mathcal{M}_{g, n} / \mathfrak{S}_{n}\left(\right.$ resp. $\left.\overline{\mathcal{M}}_{g, n} / \mathfrak{S}_{n}\right)$ est naturellement isomorphe au champ $\mathcal{M}_{g,(n)}$ (resp. $\left.\overline{\mathcal{M}}_{g,(n)}\right)$ classifiant les courbes lisses (resp. stables) de genre $g$ munies d'un diviseur de Cartier relatif étale de degré $n$ (resp. inclus dans le lieu lisse). 
$\mathrm{Du}$ fait de la propriété universelle dont jouit le quotient, tout $G$-morphisme $f: \mathcal{M} \longrightarrow \mathcal{N}$ induit un morphisme $\bar{f}: \mathcal{M} / G \longrightarrow \mathcal{N} / G$ et on a un diagramme 2-commutatif



Observons d'autre part que si $P \longrightarrow \mathcal{M}$ est un atlas, alors le morphisme composé $P \longrightarrow \mathcal{M} \longrightarrow \mathcal{M} / G$ définit un atlas de $\mathcal{M} / G$. En sens inverse, si $Q \longrightarrow \mathcal{M} / G$ est un atlas de $\mathcal{M} / G$, alors la projection $q: Q \times \mathcal{M} G \mathcal{M} \longrightarrow \mathcal{M}$ définit un $G$-atlas, i.e. un atlas muni d'une action libre de $G$, le morphisme $q$ étant $G$-équivariant. Curieusement, on aura à prêter attention à des $G$-champs pour lesquels l'action de $G$ est triviale (et néanmoins très intéressante). On a la caractérisation immédiate suivante :

Proposition 6.1.6. - Soit $\mathcal{M}$ un G-champ. Il y a équivalence entre :

i) l'action de $G$ est triviale,

ii) tout $G$-atlas $q: U \rightarrow \mathcal{M}$ se factorise (dans le sens des 2-catégories) par $U / G$,

iii) il existe un atlas $p: V \rightarrow \mathcal{M}$ qui est un $G$-morphisme, le groupe $G$ agissant trivialement sur $V$.

Démonstration. - i) $\Rightarrow$ ii) soit $\mathcal{M}_{\text {st }}$ le champ muni de l'action strictement triviale de $G$, de sorte que l'identité $\imath: \mathcal{M} \rightarrow \mathcal{M}_{s t}$ est un $G$-morphisme. Le morphisme $\imath q$ correspond à une section $x \in \mathcal{M}(U)$ qui est $G$-linéarisée. Une telle $G$-linéarisation n'est rien d'autre qu'une donnée de descente relativement au morphisme étale $U \rightarrow U / G$. Ainsi $x$ se descend à $U / G$, ce qui correspond à ii).

ii) $\Rightarrow$ iii) est clair.

iii) $\Rightarrow$ i) Il s'agit de prouver que toute section $a \in \mathcal{M}(S)$ admet une $G$-linéarisation canonique. C'est le cas pour la section $y \in \mathcal{M}(V)$ définissant $p$. Si on forme le carré 2-cartésien

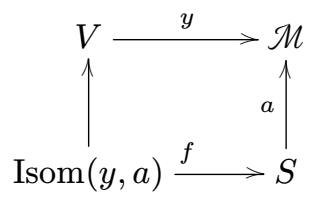

il est clair que la section $f^{*}(a)$ hérite d'une $G$-linéarisation de $y$, et que par descente par le morphisme étale $f$, on en obtient une sur $a$. Ceci répond à la question.

La catégorie des faisceaux cohérents sur le champ quotient $\mathcal{M} G$ s'identifie canoniquement à la catégorie des $G$-faisceaux cohérents sur $\mathcal{M}$. Un $G$-faisceau cohérent sur $\mathcal{M}$ se définit comme la donnée pour tout $G$-atlas $P \longrightarrow \mathcal{M}$, d'un $G$-faisceau $\mathcal{F}$ sur $P$, avec la compatibilité usuelle si $P \longrightarrow P^{\prime} \longrightarrow \mathcal{M}$ est un $G$-atlas qui domine un autre $G$-atlas.

Si on suppose l'action de $G$ sur $\mathcal{M}$ libre, c'est-à-dire libre sur les objets et donc sur les flèches, on décrit alors le champ quotient comme suit. Soit le préchamp dont les 
objets sont les classes modulo $G$ d'objets de $\mathcal{M}$, et les morphismes entre deux objets $\bar{x}$ et $\bar{y}$ sont les classes dans le quotient $\left(\sqcup_{s, t \in G} \operatorname{Hom}(s x, t y)\right) / G$. La composition se définit de manière évidente. Cela définit un préfaisceau, de sorte que le faisceau des morphismes de $\bar{x}$ vers $\bar{y}$ est le faisceau associé, c'est-à-dire le faisceau somme

$$
\operatorname{Hom}(\bar{x}, \bar{y})=\underset{s, t \in G}{\sqcup} \operatorname{Hom}(s x, t y) / G=\underset{s \in G}{\sqcup} \operatorname{Hom}(x, s y) .
$$

Il y a une obstruction à l'effectivité d'une donnée de descente d'objets au-dessus de $S$, qui réside dans $H^{1}(S, G)$. En effet, soit $\left(\bar{x}_{i}, \sigma_{i, j}\right)$ un objet donné localement sur un recouvrement $\left(S_{i} \longrightarrow S\right)$. Il existe donc un élément $\theta_{i, j} \in G$ tel que $\sigma_{i, j}: x_{i} \stackrel{\sim}{\longrightarrow}$ $\theta_{i, j}\left(x_{j}\right)$. L'action étant supposée libre, on a $\theta_{i, j} \theta_{j, k}=\theta_{i, k}$ et la condition de cocycle se résume à

$$
\theta_{i, j}\left(\sigma_{j, k}\right) \circ \sigma_{i, j}=\sigma_{i, k} .
$$

La classe d'obstruction est la classe $\left[\theta_{i, j}\right] \in H^{1}(S, G)$. Le champ quotient $\mathcal{M} / G$ s'identifie au champ associé (Laumon et Moret-Bailly [73], lemme 3.2) au préchamp que nous venons de décrire. L'identification des deux constructions est immédiate.

6.1.2. 2-quotient ou rigidification. - La seconde opération que nous utiliserons est le 2-quotient (ou $G$-rigidification) $\mathcal{M} \rrbracket G$, notation distinguant cette opération $\mathrm{du}$ 1-quotient défini ci-dessus. Les références sont Abramovich-Corti-Vistoli [1], section 5.1, et [90], section 5. Dans cette construction, on suppose que pour tout objet $x$ de $\mathcal{M}$, on a une inclusion

$$
i_{x}: G \hookrightarrow \operatorname{Aut}(x)
$$

où, par commodité, on notera $g$ à la place de $i_{x}(g)$. On fait deux hypothèses sur ces plongements $i_{x}$. La première est qu'ils sont compatibles au changement de base : pour tout $x \in \mathcal{M}(S)$ et pour tout morphisme $f: T \longrightarrow S$, on a $f^{*}(g)=g$. Notons que pour tout couple d'objets $x, y \in \mathcal{M}(U)$, le groupe $G$ opère des deux côtés sur $\operatorname{Hom}(x, y)$. La deuxième hypothèse est que pour tout couple d'objets $x, y$ et pour tout $u \in \operatorname{Hom}(x, y)$, on a $u^{-1} G u=G$. Il est alors clair que la composition des morphismes passe au quotient par $G$.

On a $([\mathbf{1}]$, theorem 5.1 .5 et $[\mathbf{9 0}]$, théorème 5.1$)$ :

Proposition 6.1.7. - Soit $\mathcal{M}$ un S-champ algébrique (de Deligne-Mumford), et soit $G$ un groupe fini qui admet un plongement $G \hookrightarrow \operatorname{Aut}(x)$ pour tout objet $x$, avec les conditions ci-dessus. Alors, il existe un champ algébrique (de Deligne-Mumford) noté $\mathcal{M} \rrbracket G$ et un morphisme $f: \mathcal{M} \longrightarrow \mathcal{M} \rrbracket G$, avec la propriété universelle suivante : tout morphisme de source $\mathcal{M}$ qui envoie les éléments de $G$ sur l'identité se factorise par $M \rrbracket G$, de manière unique. De plus :

i) le morphisme $\mathcal{M} \longrightarrow \mathcal{M} \rrbracket G$ est une gerbe étale de lien $G$, de degré $1 /|G|$,

ii) si $\mathcal{M}$ est propre, $\mathcal{M} \rrbracket G$ l'est également, et si $M$ est un espace des modules grossier de $\mathcal{M}$, c'est aussi l'espace des modules de $\mathcal{M} \rrbracket G$. 
L'exemple le plus simple est pour $G$ abélien, $B G \sqcap G=\operatorname{Spec}(k)$. Un objet $x \in$ $(\mathcal{M} \rrbracket G)(S)$ est entièrement déterminé par la donnée d'un recouvrement $\left(S_{i} \longrightarrow S\right)_{i}$, d'objets $x_{i} \in \mathcal{M}\left(S_{i}\right)$, et d'une donnée de descente $\sigma_{j, i}: x_{i \mid S_{i, j}} \stackrel{\sim}{\longrightarrow} x_{j \mid S_{i, j}}$ à un élément de $G$ près, c'est-à-dire que pour tout triplet $i, j, k$ on a :

$$
\left.\sigma_{k, i}^{-1} \sigma_{k, j} \sigma_{j, i}\right|_{i, j, k} \in G \text {. }
$$

Si $G \neq 1$, le morphisme $\mathcal{M} \longrightarrow \mathcal{M} \rrbracket G$ n'est pas représentable.

Soient enfin $\mathcal{M}$ un $G$-champ et $H \triangleleft G$ un sous-groupe distingué. Si $H$ agit trivialement sur $\mathcal{M}$, alors on peut former d'une part le double quotient $(\mathcal{M} / G) \square H$, et d'autre part $\mathcal{M}(G / H)$. Il n'est pas difficile d'observer l'identification

$$
(\mathcal{M} / G) \sqcap H \cong \mathcal{M} /(G / H) \text {. }
$$

En fait, $\mathcal{M} / G=(\mathcal{M} H) /(G / H)([\mathbf{9 0}]$, remark 2.3), mais $\mathcal{M} H=\mathcal{M} \times B H$, donc

$$
(\mathcal{M} / G) \sqcap H=\mathcal{M} /(G / H) \times B H \square H=\mathcal{M} /(G / H) .
$$

\subsection{Champs de Hurwitz : cas galoisien}

Un champ de Hurwitz classifie les revêtements $\pi: C \longrightarrow D$ entre courbes projectives lisses de genres respectifs, $g$ et $h$ fixés, et de degré fixé $d$. La relation de Riemann-Hurwitz relie $g, h$ et $d$ par

$$
2 g-2=d(2 h-2)+r
$$

où $r$ est le degré du diviseur de ramification. Si on fixe $D$, et les $b$ points de branchement, il n'y a qu'un nombre fini de revêtements de degré fixé, ainsi la dimension du champ de Hurwitz est $3 h-3+b$. Si $r>0$, on voit que cette dimension est maximum lorsque $r$ est minimum, donc si la ramification est simple. Si $h=0$, i.e. $D=\mathbb{P}^{1}$, c'est la situation classique étudiée par Fulton [49], et plus récemment par Fried [46], [48], Harris-Mumford [56], Mochizuki [77] et Wewers [100], [99].

6.2.1. Définition des champs de Hurwitz. - On suppose ici les revêtements galoisiens de groupe $G$; le cas non galoisien sera abordé dans la suite. Comme cela a été défini dans les sections 2.1 et 2.2 , on fixe une donnée de ramification $\xi=$ $\sum_{i=1}^{r} b_{i}\left[H_{i}, \chi_{i}\right] \in \operatorname{Hur}(G)$ de degré $b=\sum b_{i}$ qui représente le nombre de points de branchement. Le degré du diviseur de branchement est alors

$$
B=|G|\left(\sum_{i} b_{i}\left(1-\frac{1}{\left|H_{i}\right|}\right)\right) \text {. }
$$

Nous allons construire le champ de Hurwitz $\mathscr{H}_{h, G, \xi}$, puis décrire sa compactification stable $\overline{\mathcal{H}}_{h, G, \xi}$. Le résultat est un champ, non connexe en général, mais lisse sur $\mathbb{Z}[1 /|G|]$. Il en résultera que les composantes connexes sont identiques aux composantes irréductibles. Le nombre de composantes connexes de $\mathcal{H}_{h, G, \xi}$, qui est aussi celui de $\overline{\mathcal{H}}_{h, G, \xi}$, est le nombre de Nielsen $h(\xi)$ introduit dans la définition 2.3.6. 
Rappelons que la définition retenue pour classifier les revêtements en général (galoisiens ou non), par opposition avec les $G$-revêtements est l'équivalence (2.1.5). Fixons dorénavant le groupe $G$, ainsi qu'une donnée de Hurwitz afférente $\xi \in \mathbf{H u r}(G)$. La définition suivante sera ultérieurement renforcée par le marquage au moyen du diviseur de ramification.

DÉfinition 6.2.1. - i) Le champ de Hurwitz $\mathscr{H}_{g, G, \xi}$ est le champ sur $\mathbb{Z}[1 /|G|]$ défini comme suit. Ses sections au-dessus d'un schéma $S$ sont les $G$-revêtements $\pi: C \longrightarrow$ $D$, où les courbes $C$ et $D$ sont lisses, propres sur $S$, à fibres géométriques connexes, $C$ est de genre $g$, et la donnée de ramification de $\pi$ dans les fibres est égale à $\xi$. Les morphismes sont les paires d'isomorphismes $(f, h)$ formant un carré commutatif

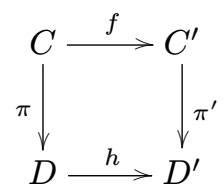

tels que $f$ est G-équivariant (voir définition 2.1.4).

ii) Le champ de Hurwitz $\overline{\mathcal{H}}_{g, G, \xi}$ a pour objets au-dessus du $\mathbb{Z}[1 /|G|]$-schéma $S$ les $G$-revêtements $\pi: C \longrightarrow D$, où $C$ est une $G$-courbe stable sur $S$ (voir 4.1.4) de genre $g$ et la donnée de ramification de $\pi$ dans les fibres est égale à $\xi$. Les morphismes sont définis de la même manière que dans i).

Notons que $\mathcal{H}_{g, G, \xi}$ (resp. $\overline{\mathcal{H}}_{g, G, \xi}$ ) n'est a priori qu'une catégorie fibrée en groupoïdes au-dessus de la catégorie des $\mathbb{Z}[1 /|G|]$-schémas. Comme nous le verrons plus loin, ces groupoïdes sont des champs ${ }^{(1)}$ de Deligne-Mumford lisses (DeligneMumford [28], Moret-Bailly [78]), et $\mathcal{H}_{g, G, \xi}$ est un sous-champ ouvert de $\overline{\mathcal{H}}_{g, G, \xi}$, ce dernier étant propre sur $\mathbb{Z}[1 /|G|]$. Noter aussi que pour un $G$-revêtement lisse $\pi: C \longrightarrow D$, le groupe des automorphismes $\operatorname{Aut}(\pi)$ est égal au centre $Z(G)$. D'une manière un peu différente, on a observé que la considération d'un $G$-revêtement $\pi: C \longrightarrow D$, se réduit à la seule donnée de la courbe $C$ munie de l'action de $G$. En d'autres termes, le foncteur $C \mapsto(C \longrightarrow C / G)$ établit une équivalence entre le champ dont les objets au-dessus de $S$, sont les $S$-courbes lisses (resp. stables) munies d'une action de $G$ (resp. action stable) de donnée $\xi$ le long des fibre géométriques, et le champ $\mathscr{H}_{g, G, \xi}\left(\operatorname{resp} . \overline{\mathcal{H}}_{g, G, \xi}\right)$.

Comme cela a été vérifié dans le théorème 3.2 .2 et la proposition 4.2 .5 , fixer la ramification équivaut à fixer les représentations de Hurwitz, ce qui donne lieu à une définition équivalente à la définition initiale, plus commode au moins dans le cadre

(1) Pour l'application initiée par Fried [46] des variétés modulaires de Hurwitz au problème de Galois inverse, le groupoïde des $G$-revêtements $C \longrightarrow \mathbb{P}^{1}$ a pour morphismes les isomorphismes stricts, voir 2.1.2. Cela définit un champ de Deligne-Mumford $\mathcal{H}_{g, G, \xi}^{s t}$ forme équivariante du champ des applications à la Kontsevich [71]. Il y a une action évidente de PGL(2) $=\operatorname{Aut}\left(\mathbb{P}^{1}\right)$ sur $\mathcal{H}_{g, G, \xi}^{s t}$ par reparamétrisation de $\mathbb{P}^{1}$. Il est facile de voir que le morphisme naturel $\mathcal{H}_{g, G, \xi}^{s t} \longrightarrow \mathcal{H}_{g, G, \xi}$ est un PGL(2)-torseur, de sorte que $\mathscr{H}_{g, G, \xi}=\left[\mathscr{H}_{g, G, \xi}^{s t} / \mathrm{PGL}(2)\right]$. 
des familles de courbes stables. Dans cette interprétation, les morphismes sont les isomorphismes $G$-équivariants, et l'isomorphisme inverse est simplement l'oubli de la base $D \cong C / G$. Dans la suite, $\mathcal{H}_{g, G, \xi}$ désignera l'une ou l'autre des deux définitions équivalentes précédentes.

6.2.2. Fonctorialité des champs de Hurwitz. - Un champ de Hurwitz est la source, ou le but, de foncteurs naturels que nous allons préciser. Soit $H \subset G$ un sous-groupe. Les opérations sur les données de Hurwitz définies dans la section 2.2 .2 (proposition 2.2.2) apparaissent dans le présent contexte comme des morphismes de champs. Par exemple, la restriction de l'action de $G$ au sous-groupe $H$, soit $(C / S, G) \mapsto(C / S, H)$, définit un morphisme

$$
\overline{\mathscr{H}}_{g, G, \xi} \longrightarrow \overline{\mathscr{H}}_{g, H, \operatorname{Res}_{H}^{G}(\xi)} \text {. }
$$

Un second morphisme correspond au passage au quotient par un sous-groupe distingué $H \triangleleft G$. Précisément, en envoyant une $G$-courbe $(C, G)$ sur la $H$-courbe $(C / H, G / H)$, ce qui est justifié par la proposition 3.1.4, on définit un morphisme

$$
\mathcal{H}_{g, G, \xi} \longrightarrow \mathcal{H}_{g^{\prime}, G / H, \operatorname{Cores}_{H}^{G}(\xi)} \text {. }
$$

Il n'y a pas a priori d'extension naturelle directe du morphisme (43) à $\overline{\mathcal{H}}_{g, G, \xi}$, car la courbe préstable quotient peut ne pas être stable. Cela justifie la nécessité d'un renforcement du concept de stabilité. Notons les deux cas particuliers $H=1$, correspondant à l'oubli de l'action de $G$, et $H=G$. Dans ce second cas, on associe à un revêtement $\pi: C \longrightarrow D$ la base $D$, marquée par les points de branchement, ou plus généralement un ensemble de points les contenant.

Remarque 6.2.2. - L'oubli de l'action de $G$ définit un morphisme $\overline{\mathcal{H}}_{g, G, \xi} \longrightarrow \overline{\mathcal{M}}_{g}$, $(\pi: C \longrightarrow D) \mapsto C$. Il est facile de voir qu'il est représentable et fini. On note qu'il y a une action libre naturelle, par torsion de l'action, du groupe $\operatorname{Aut}_{\xi}(G)$ (stabilisateur de $\xi$ ) sur le champ $\overline{\mathscr{H}}_{g, G, \xi}$.

Il est nécessaire d'étendre la définition 6.2.1 aux $G$-courbes stables marquées, ce qui conduit à la définition des champs de Hurwitz versus Harris-Mumford :

DÉfinition 6.2.3. - Soit $\eta \in \mathbf{H u r}(G)$ spécifiant le G-type d'un diviseur. On note $\overline{\mathcal{H}}_{g, G, \xi, \eta}$ le champ (de Harris-Mumford ${ }^{(2)}$ ) dont les objets sont les courbes munies d'une action stable de $G$, et qui sont stables marquées par un diviseur $G$-invariant de $G$-type fixé $\eta$, la donnée de ramification étant fixée égale à $\xi$.

Dans la suite, on supposera que $\xi \leqslant \eta$, en d'autres termes que les points de ramification sont inclus dans les points marqués. Rappelons que cela implique (corollaire 4.3.3) que les stabilisateurs des points doubles sont maintenant cycliques, ces points ne devant pas être considérés comme des points de ramification. Si la base

(2) Même si la notation le suggère, ce champ n'est pas le champ défini par Harris et Mumford classifiant les revêtements admissibles, voir ci-après pour des précisions. 
est $\mathbb{P}^{1}$, et si la ramification est simple, on retrouve essentiellement le champ introduit par Harris et Mumford [56]. Pour être plus précis, comme nous le verrons ci-après, $\overline{\mathcal{H}}_{g, G, \xi, \eta}$ en est une désingularisation.

\subsection{Compactification stable du champ de Hurwitz (I)}

Le résultat suivant est certainement bien connu, faute d'une référence précise nous en donnons une preuve détaillée.

ThÉORÈme 6.3.1. - $\overline{\mathcal{H}}_{g, G, \xi}$ (resp. $\overline{\mathcal{H}}_{g, G, \xi, \eta}$ ) est un champ de Deligne-Mumford lisse et propre sur $\mathbb{Z}[1 /|G|]$.

Démonstration. - Rappelons que le contenu de ce résultat se réduit essentiellement à la vérification de deux choses :

a) si $C_{1}$ et $C_{2}$ sont deux objets quelconques de l'un des champs considérés audessus d'un $\mathbb{Z}[1 /|G|]$-schéma $S$, alors le foncteur $\operatorname{Isom}_{S, G}\left(C_{1}, C_{2}\right)$ qui à $T / S$

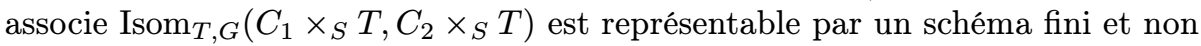
ramifié sur $S$,

b) il existe un atlas $X \longrightarrow \overline{\mathcal{H}}_{g, G, \xi}$, ce qui signifie un morphisme étale surjectif du champ représenté par $X$ sur le champ de Hurwitz sus-mentionné.

La preuve de a) est claire car le foncteur $\operatorname{Isom}_{S, G}\left(C_{1}, C_{2}\right)$ est visiblement un sousfoncteur fermé $\mathrm{du}$ foncteur non équivariant $\operatorname{Isom}_{S}\left(C_{1}, C_{2}\right)$; le résultat découle alors $\mathrm{du}$ résultat classique sur ce dernier ([30], Theorem 1.11). Avant de procéder à une vérification plus détaillée de l'assertion b), on peut observer que par le procédé d'adjonction de points marqués (section 2.2.2), on peut se limiter sans perte de généralité au cas $\xi=\eta$. Pour simplifier les notations, les champs seront simplement notés $\overline{\mathscr{H}}_{g, G, \xi}$ et $\overline{\mathscr{H}}_{g, G, \xi}$ (on omet l'indice $\eta$ ). Par ailleurs, une fois la condition a) vérifiée, on sait qu'il suffit dans b) de trouver un atlas lisse et surjectif pour $\overline{\mathcal{H}}_{g, G, \xi}$ (voir Laumon et Moret-Bailly [73], théorème 8.1). On obtiendra un tel atlas $X$ en associant à un point de $\overline{\mathscr{H}}_{g, G, \xi}$ un point de Hilbert convenable, selon la méthode de Gieseker-Mumford (Gieseker [51]).

Rappelons les points essentiels de cette construction, en traitant en premier le cas lisse, donc en nous concentrant sur le champ $\mathscr{H}_{g, G, \xi}$. Fixons un entier $m \geqslant 3$, dont le choix sera précisé ultérieurement, ainsi que le $G$-module (de Hurwitz) $V=V_{m}=$ $H^{0}\left(C, \omega_{c}^{\otimes m}\right)$; soit enfin $\operatorname{dim}(V)=n+1$. Considérons le schéma de Hilbert noté Hilb qui classifie les courbes de genre $g$ et de degré $m(2 g-2)$ dans $\mathbb{P}(V)=\mathbb{P}^{n}$, c'est-à-dire de polynôme de Hilbert

$$
P(\nu)=(2 m \nu-1)(g-1) .
$$

On peut fixer pour schéma de base $\mathbb{Z}[1 /|G|, \zeta]$, la racine de l'unité $\zeta$ convenablement choisie, assurant l'existence de $V$ comme $G$-module. Il vient une action naturelle du groupe $\operatorname{PGL}(n)$ sur Hilb, ainsi que par restriction, sur la courbe universelle $Z \subset$ 
$\mathbb{P}^{n} \times$ Hilb. Si $C$ est une telle courbe, définie par exemple sur le corps algébriquement clos $k$, on a pour $\nu \gg 0$, une surjection

$$
H^{0}\left(\mathbb{P}^{n}, \Theta_{\mathbb{P}^{n}}(\nu)\right) \longrightarrow H^{0}\left(C, \oslash_{C}(\nu)\right)
$$

définissant le $\nu^{e}$ point de Hilbert, qui par définition est le point de l'espace projectif $\mathbb{P}^{N}=\mathbb{P}\left(\bigwedge^{P(\nu)} H^{0}\left(\mathbb{P}^{n}, \vartheta_{\mathbb{P}^{n}}(\nu)\right)\right)$ représenté par la surjection

$$
\bigwedge^{P(\nu)} H^{0}\left(\mathbb{P}^{n}, \vartheta_{\mathbb{P}^{n}}(\nu)\right) \longrightarrow \bigwedge^{P(\nu)} H^{0}\left(C, \vartheta_{C}(\nu)\right) .
$$

Il y a une version équivariante de cette construction, dans laquelle les courbes plongées $m$-canoniquement dans $\mathbb{P}^{n}$, non dégénérées i.e. $H^{0}\left(\mathbb{P}^{n}, \Theta_{\mathbb{P}^{n}}(1)\right) \cong H^{0}\left(C, \vartheta_{C}(1)\right)$ et invariantes par l'action de $G$. La courbe $C$ hérite alors canoniquement d'une action de $G$. Ces courbes correspondent dans l'interprétation par les points de Hilbert, aux surjections (44) équivariantes. Soit $\pi: \Sigma \longrightarrow K$ la courbe universelle de GiesekerMumford [51]; le groupe $G$ agit sur $\Sigma$ et $K$, soit alors $H=K^{G}$ le sous-schéma des points fixes. Comme $K$ est lisse et $|G|$ inversible dans le faisceau structural, il en est de même de $H$ (voir Edixhoven [37], prop. 3.4); alors, si on note $\Gamma=\pi^{-1}(H)$, le morphisme $\Gamma \longrightarrow H$ obtenu par restriction est la courbe universelle cherchée. On notera que la lissité de $H$ se déduit aussi des résultats sur la structure des déformations équivariantes. En conclusion, $H$ conduit à un morphisme représentable et lisse $H \longrightarrow$ $\mathcal{H}_{g, G, \xi}$, ce qui prouve b).

Pour terminer la preuve, il suffit de montrer que la procédure précédente s'étend à $\overline{\mathscr{H}}_{g, G, \xi}$, resp. à $\overline{\mathcal{H}}_{g, G, \xi}$. On donne quelques détails pour le premier cas, les modifications de routine pour traiter le second sont omises; notons, pour rassurer le lecteur, qu'en corollaire, les deux champs seront identifiés. Soit encore, conservant les notations de dessus, $V=V_{m}$ la représentation de Hurwitz de degré $m$, de rang $n+1=(2 m-1)(g-1)$, et $\mathbb{P}^{n}=\mathbb{P}(V)$. Maintenant, rappelons qu'un résultat fondamental de Gieseker et Mumford assure l'existence d'un $m \gg 0$, puis d'un $\nu \gg 0$, de sorte que le $\nu$-ième point de Hilbert de toute courbe connexe lisse (définie sur un corps algébriquement clos $k$ ) soit GIT-stable pour l'action du groupe linéaire. Une fois $\nu$ fixé, on peut considérer la courbe universelle $Z \longrightarrow H$ de base le schéma de Hilbert. Soit alors $H^{s s}$ l'ensemble des points de $H$ dont le $\nu$-ième point de Hilbert est semi-stable, et $W$ l'ensemble des points $h \in H^{s s}$ tels que la courbe $Z_{h}$ est connexe et plongée $m$-canoniquement dans $\mathbb{P}^{n}$. Il est prouvé dans [51] que $W$ est un sous-schéma fermé de $H^{s s}$, et que si $h \in H$, la courbe $Z_{h}$ est réduite avec seulement des points doubles ordinaires. Rappelons que l'espace modulaire grossier classifiant les courbes stables de genre $g \geqslant 2$ s'identifie au quotient géométrique $\bar{M}_{g}=W / / S L(n)$. De manière identique a ce qui a été fait au-dessus, on doit traiter la situation équivariante, ce qui amène d'abord à former le sous-schéma des points fixes $T=W^{G}$; l'action de $G$ est l'action naturelle décrite dans la section 6.1. Le schéma $T$ est lisse, du fait de la lissité de $W$, ou si on préfère par la théorie des déformations équivariantes de la section 5. Les composantes connexes $\left\{T_{\alpha}\right\}$ de $T$ sont alors irréductibles, et le long d'une telle composante, les représentations de Hurwitz 
$H^{0}\left(C_{h}, \omega_{C_{h}}^{\otimes t}\right)$ sont constantes; noter que pour $t=m$, cela résulte des hypothèses. Si on travaille avec les objets du champ $\overline{\mathcal{H}}_{g, G, \xi}$, le même argument que celui utilisé au-dessus nous assure que le $G$-type du diviseur des points marqués est constant le long des $T_{\alpha}$. Parmi les composantes $\left\{T_{\alpha}\right\}$, on ne retient que celles dont le point générique définit une courbe lisse, et le long desquelles la donnée de Hurwitz, et éventuellement le $G$-type du marquage sont constants égaux à $\xi$ et $\nu$ respectivement ; la réunion de ces composantes est encore notée $T$. La restriction à $T$ de la famille universelle fournit un morphisme représentable surjectif $T \longrightarrow \overline{\mathcal{H}}_{g, G, \xi}$, prouvant que $\overline{\mathcal{H}}_{g, G, \xi}$ est un champ algébrique, de Deligne-Mumford. Notons pour conclure que la propreté dans l'un ou l'autre des deux cas résulte du théorème de réduction stable sous sa forme équivariante (proposition 5.1.2).

Remarque 6.3.2. $-\mathrm{Si} k=\mathbb{C}$, le type topologique de l'action sépare les composantes connexes. On invoque la version équivariante de la théorie de Teichmüller [38], de laquelle on déduit que deux points de $H$ qui correspondent à des actions topologiquement conjuguées peuvent être joints par un arc. Ainsi Card $\pi_{0}(H)$ est égal à $h(\xi)$, le nombre de Nielsen.

\subsection{Compactification du schéma de Hurwitz : Gieseker-Mumford}

On se concentre dorénavant sur les espaces de modules $\bar{H}_{g, G, \xi}$ (resp. $\overline{H M}_{g, G, \xi}$ ) associés aux champs $\overline{\mathscr{H}}_{g, G, \xi}$ (resp. $\overline{\mathscr{H}}_{g, G, \xi}$ ). La construction GIT esquissée cidessus (section 6.3) conduit à la projectivité des schémas modulaires de Hurwitz, et en corollaire à la projectivité des $\bar{M}_{g, n}$. On remarquera que finalement tout est réduit à une et une seule construction, la projectivité de $\bar{M}_{g}$.

Identifions $G$ à son image dans $G L(V)$, et notons $A$ (resp. $B$ ) le centralisateur (resp. le normalisateur) de $G$. Si $V=\bigoplus_{i} n_{i} V_{i}$ est la décomposition de $V$ en représentations irréductibles deux à deux distinctes, alors $A \cong \prod_{i} G L\left(n_{i}\right)$. Notons que $A$ opère sur $H$ en fixant individuellement les composantes connexes. La construction de $H_{g, G, \xi}$ (resp. de $\left.\bar{H}_{g, G, \xi}\right)$ résulte de la proposition suivante :

Proposition 6.4.1. - Si m est assez grand, les quotients géométriques $H / A$ et $\bar{H} / A$ existent, et de plus on a :

$$
\left.H_{g, G, \xi}=H / A \quad, \quad \bar{H}_{g, G, \xi}=\bar{H} / A\right) .
$$

Démonstration. - C'est une simple adaptation à la situation équivariante présente des arguments de Mumford et Gieseker [51]. Pour plus de lisibilité, regardons en premier l'espace modulaire $H_{g, G, \xi}$. On observe qu'une courbe $C \subset \mathbb{P}^{n}$, lisse et connexe, de genre $g \geqslant 2$, est Chow-stable ou Hilbert-stable, si son degré est grand par rapport à $g$. La stabilité relative d'abord au groupe linéaire entraîne a fortiori celle relative à $A$. Pour que la preuve de la proposition soit complète, on doit tout d'abord noter la validité des deux points suivants : 
- Toute action de $G$ sur $C$, du type indiqué, apparaît dans une fibre de la courbe universelle.

- Si $C_{1}$ et $C_{2}$ sont deux fibres $G$-isomorphes, alors l'isomorphisme est induit par un élément de $A$. En fait, si $\pi: C \longrightarrow S$ est un objet de $\mathcal{H}_{g, G, \xi}$, on prouve que localement pour la topologie de Zariski sur $S, C / S$ appartient à $\operatorname{Hom}(S, H) / \operatorname{Hom}(S, A)$. Pour cela, on considère le faisceau $\mathcal{E}=\pi_{*}\left(\omega_{C / S}^{\otimes m}\right) ;$ on a clairement $R^{1} \pi_{*}\left(\omega_{C / S}^{\otimes m}\right)=0$, et $\mathcal{E}$ est localement libre, muni d'une action de $G$. On peut alors décomposer $\mathcal{E}$ en facteurs isotypiques

$$
\mathcal{E}=\bigoplus_{V_{i} \in \operatorname{Irrep}(G)} \mathcal{E}_{i} \otimes V_{i}
$$

Il suffit alors de noter que les modules de covariants $\mathcal{E}_{i}$ sont localement libres, et que $V=\bigoplus r g\left(\varepsilon_{i}\right) V_{i}$.

On peut réaliser de la même manière l'espace modulaire grossier $\bar{H}_{g, G, \xi}$, prouvant ainsi que c'est un schéma projectif, et que $H_{g, G, \xi}$ s'identifie à un ouvert partout dense de $\bar{H}_{g, G, \xi}$; en particulier les deux schémas ont le même nombre de composantes connexes, nombre égal au nombre de Nielsen $h(\xi)$ (définition 2.3.6, théorème 2.3.7). Conservant les notations du début de section, on prouve comme ci-dessus que le quotient géométrique $T / / A$ existe et que finalement $\bar{H}_{g, G, \xi}=T / / A$. On doit dans une seconde étape s'assurer que toute courbe stable $C$ munie d'une action stable de $G$ de donnée de ramification prescrite, apparaît dans le bord. On sait, du fait de la structure de la déformation universelle de $(C, G)$ (section 5.1) qu'on peut étendre de manière équivariante $C$ en une courbe stable $\pi: X \longrightarrow S=\operatorname{Spec}(R)$, l'anneau $R$ étant de valuation discrète et complet, de sorte que si on note $a$ le point fermé, et $b$ le point générique, alors $\pi^{-1}(a) \cong C$ et la courbe $\pi^{-1}(b)$ est lisse. Cette courbe définit un morphisme classifiant $f: \operatorname{Spec}(R) \longrightarrow H^{G}$ avec $f(b) \in W$. L'argument de Gieseker ([51], theorem 2.0.2) montre qu'il existe une courbe appartenant à la $A$-orbite de $X$ et qui correspond à un morphisme classifiant $\operatorname{Spec}(R) \longrightarrow \sqcup T_{\alpha}$. Les fibres au dessus du point fermé sont ainsi dans une même $A$-orbite. Cela étant, la projectivité de $\bar{H}_{g, G, \xi}$ découle bien évidemment de la construction de cet espace modulaire comme quotient. On peut aussi, la propreté étant conséquence du théorème de réduction stable sous sa forme équivariante, s'appuyer sur la projectivité connue de $\bar{M}_{g}$ et utiliser le morphisme oubli de l'action de $G$ :

$$
\bar{H}_{g, G, \xi} \longrightarrow \bar{M}_{g}
$$

qui est alors propre et quasi-fini, donc fini.

Proposition 6.4.2. - Soit $\xi$ une donnée de Hurwitz attachée au groupe $G$, et soit $h(\xi)$ le nombre de Nielsen correspondant. Si $k$ est un corps algébriquement clos de caractéristique $p \geqslant 0$, et $(p,|G|)=1$ si $p>0$, alors

$$
h(\xi)=\operatorname{Card} \pi_{0}\left(\bar{H}_{g, G, \xi}\right)=\operatorname{Card} \pi_{0}\left(H_{g, G, \xi}\right) .
$$


Démonstration. - On reprend la construction de la proposition 6.4.1, en suivant [51], maintenant sur un schéma de base $S=\operatorname{Spec}(R)$, où $R$ est un anneau de valuation discrète complet, de corps des fractions $K$ de caractéristique zéro, et de corps résiduel $k$. Il est connu que la théorie GIT s'étend à ce cadre (Seshadri [94]) . Si $G$ agit stablement sur une courbe stable définie sur $k$, on sait qu'on peut relever la courbe, ainsi que l'action de $G$, à $R$, cela à donnée de ramification constante. Notons $a$ (resp. $b$ ) le point fermé (resp. le point générique) de $S$. Considérons le morphisme quotient

$$
\sqcup_{\alpha} T_{\alpha} \longrightarrow \bar{H}_{g, G, \xi}=\left(\sqcup_{\alpha} T_{\alpha}\right) / / A
$$

Rappelons que chaque composante connexe $T_{\alpha}$ est lisse sur $S$. Si on prend la fibre en $a$ (resp. $b$ ), on a par un résultat de Seshadri (voir [94], partie II, section 2) :

$$
\left.\left(T_{\alpha} / / A\right)_{a}=\left(T_{\alpha}\right)_{a} / / A \quad \text { (resp. }\right) \quad\left(T_{\alpha} / / A\right)_{b}=\left(T_{\alpha}\right)_{b} / / A .
$$

Le théorème de connexion de Zariski montre que $\left(T_{\alpha} / / A\right)_{a}$ est connexe; il s'ensuit que $\left(T_{\alpha}\right)_{a}$ est connexe, donc irréductible, car lisse. On en déduit que $\left(T_{\alpha}\right)_{a} / / A=\left(\bar{H}_{g, G, \xi}\right)_{\alpha}$ est irréductible, d'où le résultat annoncé.

Corollaire 6.4.3. - Sous les conditions précédentes, si $G$ est un groupe cyclique, alors pour tout corps algébriquement clos $k$ de caractéristique ne divisant pas $|G|$, l'espace de Hurwitz $\bar{H}_{g, G, \xi}$ est irréductible.

Démonstration. - En effet, sous ces hypothèses $h(\xi)=1$ (proposition 2.3.9).

\subsection{Compactification stable du champ de Hurwitz (II)}

6.5.1. Le champ de Hurwitz versus Harris-Mumford. - La construction du schéma de Hurwitz au moyen d'un quotient géométrique conduit, avec des modifications mineures, à la construction des espaces modulaires $H M_{g, G, \xi}$ (resp. $\left.\overline{H M}_{g, G, \xi}\right)$. Rappelons que dans le cas Harris-Mumford, les objets du champ sont les $G$-courbes stables marquées par les points de ramification, ou plus généralement marquées par un diviseur $G$-invariant les contenant. On va prouver ci-après que les espaces de modules $\bar{H}_{g, G, \xi}$ et $\overline{H M}_{g, G, \xi}$ sont en fait isomorphes, bien qu'en général cela ne soit pas le cas pour les champs. Rappelons au préalable les constructions de Knudsen [68], [76] :

- Soit $\left(C^{\prime},\left\{y_{j}\right\}\right)$ une courbe préstable marquée, connexe. Parmi tous les morphismes surjectifs $\left(C^{\prime},\left\{y_{j}\right\}\right) \longrightarrow\left(C,\left\{x_{i}\right\}\right)$ de but une courbe stable marquée, les $\left(y_{j}\right)$ s'envoyant surjectivement sur les $\left(x_{i}\right)$, il y a si l'ensemble des $\left\{y_{j}\right\}$ est non vide un morphisme minimal. Il s'obtient en contractant en un point les composantes instables (de genre 0) contenues dans $C^{\prime}$. On montre que ce morphisme est défini par une puissance convenable du faisceau inversible $\omega_{C^{\prime}}\left(\sum y_{j}\right)$. Il est clair que si $G$ agit sur $C^{\prime}$ en permutant les $y_{j}$, alors l'action de $G$ descend à $C$, de sorte que le morphisme commute à l'action de $G$. On applique cette procédure lorsque l'oubli de certains points marqués d'une courbe stable 
$\left(C^{\prime},\left\{y_{j}\right\}\right)$ détruit la stabilité. Les sections oubliées induisent des sections de $C$, éventuellement non disjointes et passant par des points doubles. Important pour la suite est le fait que cette opération de contraction (ou de stabilisation) est compatible aux changements de base, donc définit, après oubli du marquage par les points de ramification, un morphisme

$$
\text { st }: \overline{\mathscr{H} M}_{g, G, \xi} \longrightarrow \overline{\mathcal{H}}_{g, G, \xi} \text {. }
$$

- Il y a un foncteur en sens inverse ([68], cor. 2.6). Ce morphisme, qui revient à éclater certains points doubles, transforme une courbe stable marquée par un ensemble de $n$ sections, à laquelle on ajoute une section supplémentaire, en une courbe stable marquée par $n+1$ sections, la $(n+1)$-ième étant la section additionnelle.

Ces deux opérations conjointes montrent que le morphisme $\bar{M}_{g, n+1} \longrightarrow \bar{M}_{g, n}$ d'oubli de $(n+1)$-ième section représente la courbe universelle $n$-piquée. Le résultat suivant clarifie en partie la situation.

THÉORÈme 6.5.1. - Le morphisme (45) est un isomorphisme au-dessus du souschamp ouvert partout dense $\mathcal{H} M_{g, G, \xi}$ sur son image; il induit un isomorphisme d'espaces modulaires grossiers : $\overline{H M}_{g, G, \xi} \stackrel{\sim}{\longrightarrow} \bar{H}_{g, G, \xi}$.

Démonstration. - Soit $\pi^{\prime}: C^{\prime} \longrightarrow S$ une $G$-courbe stable marquée par les points de ramification. Rappelons que cela impose en particulier que pour tout sous-groupe cyclique $H$ de $G$, le diviseur de Cartier relatif $\operatorname{Fix}(H)_{\text {hor }}$ (proposition 4.1.8) est une somme de sections disjointes. Par oubli du marquage, suivi d'une contraction éventuelle des composantes instables, on obtient une $G$-courbe stable $\pi: C \longrightarrow S$. Le morphisme $\pi^{\prime}$ se factorise en $\pi^{\prime}=\pi \gamma$, où $\gamma: C^{\prime} \longrightarrow C$ contracte les orbites de composantes lisses rationnelles à isotropie diédrale (lemme 5.1.4). l'image d'une telle composante $E$ est un point double à isotropie diédrale $\mathbb{D}_{m}(m \geqslant 1)$, si $m$ est le nombre de sections de points fixes qui rencontrent $E$. Ces points sont les points fixes des réflexions de $\mathbb{D}_{m}$. Notons ainsi que $C^{\prime} \cong C$ sauf si $C$ possède des fibres avec des points doubles à isotropie diédrale. Comme expliqué ci-dessus, on peut reconstruire $C^{\prime}$ en partant de $C$ munie des images des sections de points fixes, en séparant les sections de points fixes qui coalescent en les points d'isotropie diédrale. Le morphisme 'st' est clairement un isomorphisme au-dessus du sous-champ ouvert formé des revêtement de courbes lisses. Ce n'est cependant pas un isomorphisme en général. Si $C^{\prime} \longrightarrow S$ est la stabilisation de $C \longrightarrow S$ et si $Q \in C_{s}$ est un point double à isotropie diédrale $H=\mathbb{D}_{m}$, pour une réflexion $\tau \in H$, on a noté que le diviseur des points fixes (horizontal) est de manière spécifique la réunion de deux sections.

Soit $\hat{\theta}_{Q}=\hat{\theta}_{s}[[x, y]] /(x y-a)$ l'anneau local complété de $C$ en $Q$. On suppose que $\tau(x)=y$ et $\tau(y)=x$. Si l'une des sections de points fixes est $x=b, y=c$, alors $b=c$ et $a=b^{2}$. Si on se place sur $S=\operatorname{Spec}(k[\epsilon]), \epsilon^{2}=0$, on voit que si $C^{\prime}$ et $C$ sont définies sur un corps algébriquement clos $k$, alors l'application st au niveau des espaces tangents des déformations universelles respectives n'est pas surjective. En 
fait les déformations qui sont dans l'image sont topologiquement triviales en les points doubles à isotropie diédrale.

$\mathrm{Si}$, pour tout sous-groupe cyclique $H$ d'ordre deux, le diviseur $\operatorname{Fix}(H)_{\text {hor }}$ est une somme de deux sections, alors on peut inverser 'st'. L'action de $G$ sur les composantes exceptionnelles se réduit à l'action d'un groupe diédral $\mathbb{D}_{m}$. On notera que 'st' est un monomorphisme car il est clair que $\operatorname{Aut}_{G}(C) \cong \operatorname{Aut}_{G}(\operatorname{st}(C))$. Finalement le fait qu'au niveau des espaces modulaires grossiers 'st' est un isomorphisme est clair, car il est propre, birationnel et bijectif entre variétés normales.

Dans la suite, nous travaillerons avec le champ $\overline{\mathscr{H} M}_{g, G, \xi}$, qui pour alléger les notations sera noté $\overline{\mathscr{H}}_{g, G, \xi}$. Rappelons que si $H \triangleleft G$, on peut réaliser $\overline{\mathscr{H}}_{g, G, \xi}$ comme une correspondance entre $\overline{\mathcal{H}}_{g, H, \operatorname{Res}_{H}^{G}(\xi)}$ et $\overline{\mathcal{H}}_{g^{\prime}, G / H, \text { Cores }_{H}^{G}(\xi)}$. Prenant $H=1$ et $H=G$, on obtient les deux morphismes fondamentaux

$$
\overline{\mathcal{M}}_{g, r} \stackrel{\imath}{\longleftarrow} \overline{\mathcal{H}}_{g, G, \xi} \stackrel{\delta}{\longrightarrow} \overline{\mathcal{M}}_{g^{\prime}, b}
$$

où $r$ désigne le nombre de points de ramification, $b$ le nombre de points de branchement, et $g^{\prime}$ le genre de la base. Le morphisme de gauche est l'oubli de l'action de $G$ et $\delta$ est le passage au quotient par $G$. Le morphisme $\delta$ sera appelé le morphisme discriminant. Son existence est justifiée par la proposition 5.1.3, qui assure que si $\pi: C \longrightarrow S$ est un objet de $\overline{\mathcal{H}}_{g, G, \xi}$, alors la courbe quotient $C / G \longrightarrow S$, marquée par le diviseur des points de branchement (resp. piquée par les points de branchement) est stable. En résumé :

Proposition 6.5.2. - i) Le morphisme $\imath: \overline{\mathcal{H}}_{g, G, \xi} \longrightarrow \overline{\mathcal{M}}_{g,(r)}$ est fini (représentable) et non ramifié; c'est une immersion régulière locale au sens de Vistoli ([97], definition 1.20).

ii) Le morphisme discriminant $\delta: \overline{\mathcal{H}}_{g, G, \xi} \longrightarrow \overline{\mathcal{M}}_{g^{\prime}, b}$ est propre, quasi-fini et plat, non représentable si le centre $Z(G)$ est non trivial.

iii) Le morphisme discriminant $\delta$ induit un morphisme fini surjectif entre espaces de modules grossiers.

Démonstration. - i) Si $C \longrightarrow S$ est une courbe stable marquée, la catégorie fibre de $\imath$ en $C$ a pour objets au-dessus de $T \longrightarrow S$ les plongements $G \hookrightarrow \operatorname{Aut}_{T}\left(C \times_{S} T\right)$. Ces plongements correspondent aux $T$-points du produit $|G|$-uple

$$
\operatorname{Aut}_{S}(C) \times{ }_{S} \cdots \times \times_{S} \operatorname{Aut}_{S}(C)
$$

dont l'image est disjointe de la diagonale et stable par la multiplication de $\operatorname{Aut}_{S}(C)$. Du fait que $\operatorname{Aut}_{S}(C)$ est fini non ramifié sur $S$, le fait que $\imath$ le soit aussi est clair. Comme les champs sont lisses, $\imath$ est une immersion locale régulière dans le sens de Vistoli, i.e. représentable non ramifié et localement d'intersection complète.

ii) La propreté de $\delta$ découle du critère de réduction stable pour les revêtements (proposition 5.1.2). La platitude découle du théorème 5.1.5 donnant la structure locale de $\pi$. Le caractère étale de $\delta$ (au-dessus de $\mathcal{M}_{g^{\prime}, b}$ ) découle aussi du théorème 5.1.5. Le dernier point est clair. 
Remarque 6.5.3. - La définition des revêtements stables donnée ci-dessus permet de retrouver d'une manière uniforme les objets étudiés par Abramovich, Corti et Vistoli [1], sous le terme de balanced twisted stable maps. Les objets étudiés par ces auteurs sont les morphismes $\pi: D \longrightarrow B G$ où la source $D$, est la courbe $D$ munie de sa structure d'orbifold, i.e. donnée par $D=C / G$. Dans cette structure, on ignore les automorphismes induits par le centre de $G$. En conséquence, on a, avec les notations de [1] :

$$
\bigsqcup_{[\xi]} \overline{\mathcal{H}}_{g, G, \xi} \sqcap Z(G) \stackrel{\sim}{\longrightarrow} \overline{\mathcal{M}}_{g^{\prime}, b}(B G)
$$

6.5.2. Le revêtement « universel ». - On continue l'exploration des aspects fonctoriels des champs de Hurwitz. Soit $[H, \chi]$ une classe de conjugaison apparaissant dans la donnée de ramification $\xi$. Soit $\Delta_{(H, \chi)}$ la composante de $\Delta_{H}$, lieu des points fixes d'holonomie exactement égale à $(H, \chi)$. Il y a une action libre de $C_{G}(H) / H$ sur $\Delta_{(H, \chi)}$, le quotient étant la composante $B_{[H, \chi]}$ de $B$, définissant un fibré principal $\Delta_{(H, \chi)} \longrightarrow B_{[H, \chi]}$. Supposons que $B$ soit somme de sections $Q_{1}, \ldots, Q_{b}$ ( $D$ est piquée) contenant les points de branchement. Par image réciproque, la section $Q_{i}$ d'holonomie notée $\left(H_{i}, \chi_{i}\right)$ définit un fibré principal de base $S$, et de groupe $C_{G}\left(H_{i}\right) / H_{i}$. Cela fournit un foncteur d'évaluation en $Q_{i}$ (voir Jarvis-Kaufmann-Kimura [61]) :

$$
e v_{Q_{i}}: \overline{\mathscr{H}}_{g, G, \xi} \longrightarrow B\left(C_{G}\left(H_{i}\right) / H_{i}\right) \text {. }
$$

Soit $\operatorname{Spec}(k) \longrightarrow B\left(C\left(H_{i}\right) / H_{i}\right)$ l'atlas correspondant au fibré principal trivial, alors le 2-produit fibré $\mathcal{H}_{g, G, \xi} \times_{B\left(C\left(H_{i}\right) / H_{i}\right)} \operatorname{Spec}(k)$ a pour objets les revêtements $\pi: C \longrightarrow D$ équipés d'un point $P: S \longrightarrow C$ qui est un point de ramification d'holonomie $\left(H_{i}, \chi_{i}\right)$. Soit $W_{\chi}$ une représentation irréductible de $C\left(H_{i}\right) / H_{i}$ de caractère $\chi$. Si l'on considère la décomposition isotypique $\pi_{*}\left(\theta_{\Delta_{i}}\right)=\bigoplus_{\eta \in C_{G} \widehat{\left(H_{i}\right) / H_{i}}} W_{\eta} \otimes L_{\eta}$, alors le faisceau des sections de $e v_{Q_{i}}^{*}\left(W_{\chi}\right)$ est $L_{i}$.

Soit $\imath: \overline{\mathcal{H}}_{g, G, \xi} \longrightarrow \overline{\mathcal{M}}_{g,(r)}$ le morphisme oubli ${ }^{(3)}$ de l'action de $G$, et considérons $\overline{\mathscr{C}}_{g,(r)}=\overline{\mathcal{M}}_{g,(r), 1} \longrightarrow \overline{\mathcal{M}}_{g,(r)}$ la courbe universelle au-dessus de $\overline{\mathcal{M}}_{g,(r)}$ [68]. Rappelons que les parenthèses informent que le marquage est par paquets. Une section de $\overline{\mathscr{C}}_{g,(r)}$ au-dessus de $S$ est la donnée d'une courbe $r$-marquée $q: C \longrightarrow S$ équipée d'une section supplémentaire $P: S \longrightarrow C$. Les morphismes sont les morphismes qui fixent la section additionnelle. Il n'est pas nécessaire d'exiger que la section $P$ soit disjointe des points doubles ou des points marqués (loc. cit.). Définissons un champ $\overline{\mathscr{C}}_{g, G, \xi}$ par le carré 2-cartésien

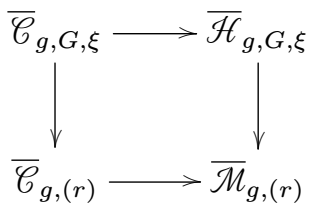

(3) Rappelons que dans $\overline{\mathcal{M}}_{g,(r)}$ le marquage est par paquets, déterminés par la donnée $\xi$. 
Une section de $\overline{\mathscr{C}}_{g, G, \xi}$ au-dessus de $S$ est la donnée d'un diagramme

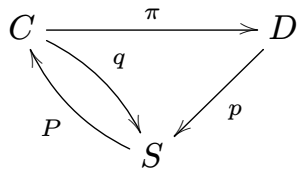

dans lequel $\pi: C \longrightarrow D$ est un $G$-revêtement de base $S$ et $P: S \longrightarrow C$ une section de $q: C \longrightarrow S$. Le groupe $G$ agit (dans le sens de la section 6.1) sur le champ $\overline{\mathscr{C}}_{g, G, \xi}$ par $g(C \stackrel{\pi}{\longrightarrow} D, P)=(C \stackrel{\pi}{\longrightarrow} D, g \circ P)$. On peut demander que $C$ soit stable marquée non seulement par les orbites sélectionnées par la donnée de ramification, mais aussi par l'orbite additionnelle de $P$. Cela revient à considérer la donnée modifiée $\xi+[1]$ (voir section 2.2), et le champ associé $\overline{\mathcal{H}}_{g, G, \xi+[1]}^{\prime}$ dont les objets sont les revêtements avec un point sélectionné dans la dernière orbite. La procédure de stabilisation de Knudsen donne alors un isomorphisme

$$
\overline{\mathscr{C}}_{g, G, \xi} \stackrel{\sim}{\longrightarrow} \overline{\mathcal{H}}_{g, G, \xi+[1]}^{\prime} .
$$

Soit le morphisme $S \longrightarrow \overline{\mathcal{H}}_{g, G, \xi}$ défini par le $G$-revêtement $\pi: C \longrightarrow D$ au-dessus de $S$. Il est clair qu'on a une identification de $G$-champs $\overline{\mathscr{C}}_{g, G, \xi} \times_{\overline{\mathscr{H}}_{g, G, \xi}} S \cong C$, justifiant le fait que $\overline{\mathscr{C}}_{g, G, \xi} \longrightarrow \overline{\mathcal{H}}_{g, G, \xi}$ est la $G$-courbe universelle au-dessus de $\overline{\mathcal{H}}_{g, G, \xi}$. Le 1-morphisme de but la courbe universelle au-dessus de $\overline{\mathscr{C}}_{g^{\prime}, b}$

$$
\Delta: \overline{\mathscr{C}}_{g, G, \xi} \longrightarrow \overline{\mathscr{C}}_{g^{\prime}, b} \quad, \quad(C \stackrel{\pi}{\longrightarrow} D, P) \mapsto(D, Q=\pi P)
$$

fait office de revêtement universel.

Soit $A: U \longrightarrow \overline{\mathcal{H}}_{g, G, \xi}$ un atlas, et notons $\pi: C_{U} \longrightarrow U$ le $G$-revêtement définissant $A$. Noter que $U$ n'est pas connexe en général. La seconde projection $p_{2}: C_{U} \times_{U} C_{U} \longrightarrow$ $C_{U}$ définit un $G$-revêtement, le groupe $G$ agissant sur le facteur de gauche dans le produit fibré. La diagonale $\Delta: C_{U} \longrightarrow C_{U} \times_{U} C_{U}$, vue comme section de $p_{2}$, fait de $p_{2}: C_{U} \times_{U} C_{U} \longrightarrow C_{U}$ un objet de $\overline{\mathscr{C}}_{g, G, \xi}$, donc conduit à un morphisme $C_{U} \longrightarrow \overline{\mathcal{H}}_{g, G, \xi}$ rendant le carré suivant 2-cartésien :

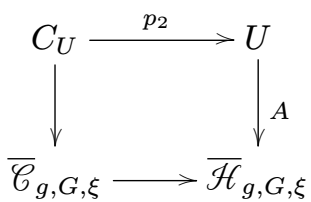

En particulier, le morphisme $C_{U} \longrightarrow \overline{\mathscr{C}}_{g, G, \xi}$ définit un atlas de $\overline{\mathscr{C}}_{g, G, \xi}$. Pour faire le lien avec la base, notons le diagramme 2-commutatif

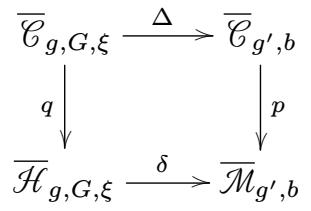


qui conduit à un morphisme $\psi: \overline{\mathscr{C}}_{g, G, \xi} \longrightarrow \overline{\mathcal{H}}_{g, G, \xi} \times \overline{\mathcal{M}}_{g^{\prime}, b} \overline{\mathscr{C}}_{g^{\prime}, b}$. Le morphisme $\Delta$ n'est pas le quotient de $\overline{\mathscr{C}}_{g, G, \xi}$ par $G$, mais on a cependant :

Proposition 6.5.4. - Le morphisme $\psi: \overline{\mathscr{C}}_{g, G, \xi} \longrightarrow \overline{\mathscr{D}}_{g, G, \xi}=\overline{\mathscr{H}}_{g, G, \xi} \times{\overline{M_{g^{\prime}, b}}}_{\overline{\mathscr{C}}_{g^{\prime}, b} \text { est }}$ fini de degré $|G|$. Le champ $\overline{\mathscr{D}}_{g, G, \xi}$ est normal et a pour désingularisation le quotient $\left[\overline{\mathscr{C}}_{g, G, \xi} / G\right]$.

Démonstration. - En effet, le champ fibre de $\psi$ en le $G$-revêtement $\pi: C \longrightarrow D$ de base $S$, et muni de la section supplémentaire $Q: S \longrightarrow D$, est clairement représenté par le schéma $\pi^{-1}(Q) \subset C$. Pour le second point, notons que, avec les notations de la section 5.1.3, une carte locale pour $\bar{D}_{g, G, \xi}$ en le point $(C \longrightarrow D, Q)$, où $Q$ est un point double de $D$ de paramètre de déformation $\tau_{1}$, et $e_{1}$ étant l'ordre de l'isotropie, est le spectre de $k\left[\left[t_{1}, \ldots, u, v\right]\right] /\left(u v-t_{1}^{e_{1}}\right)$.

Noter que les sections universelles $\left\{Q_{\alpha}\right\}_{\alpha=1}^{b}$ font de $\overline{\mathscr{D}}_{g, G, \xi}=\overline{\mathscr{H}}_{g, G, \xi} \times \overline{\mathcal{M}}_{g^{\prime}, b} \overline{\mathscr{C}}_{g^{\prime}, b}$ une courbe $b$-marquée de base $\overline{\mathcal{H}}_{g, G, \xi}$. Suite à l'identification (49), il vient un morphisme additionnel d'évaluation analogue à (47) :

$$
\text { ev }: \overline{\mathscr{C}}_{g, G, \xi}=\overline{\mathcal{H}}_{g, G, \xi+[1]}^{\prime} \longrightarrow B G \text {. }
$$

Du fait de ce morphisme, dans la section 10, on associera à toute représentation $V$ de $G$ un faisceau cohérent $R q_{*} e v^{*}(V)$ sur $\overline{\mathscr{H}}_{g, G, \xi}$.

\subsection{Champs de Hurwitz : cas non galoisien}

Dans cette section, on construit le champ de Hurwitz classifiant les revêtements non galoisiens stables à monodromie fixée. Nous le comparons au champ des revêtements admissibles [55], [56], [77], [100], [99]. Il est nécessaire en préliminaire d'effectuer une étude "en famille" de la monodromie et de la clôture galoisienne, de sorte que la définition du champ de Hurwitz lui-même doit attendre la section 6.6.2.

6.6.1. Revêtements admissibles. - Le point clé dans la construction du champ de Hurwitz classifiant les revêtements galoisiens est le fait de considérer la base $D$ d'un $G$-revêtement, comme une courbe marquée par les points de branchement (lisses), qui en conséquence ne sont pas autorisés à se rencontrer par spécialisation. Les revêtements appelés admissibles par Harris-Mumford (loc.cit. p. 57) satisfont à cette condition, mais cependant ont une «mauvaise » théorie des déformations, en particulier la base de la déformation verselle n'est en général pas lisse, même pas normale. Ce défaut a été corrigé par Mochizuki [77] et Wewers [100], en proposant d'enrichir un tel revêtement d'une log-structure, ce qui a pour effet de rétablir la lissité du foncteur des déformations.

Dans la construction qui suit, on procède en sens inverse. Un revêtement stable sera décrit, du moins étale-localement, comme quotient d'une clôture galoisienne, qui est un $G$-revêtement stable marqué par les points de branchement. Fixer une collection de 
clôtures galoisiennes locales ajoute une structure supplémentaire qui cependant n'est pas équivalente à celle fournie par une log-structure. C'est une structure plus faible. Rappelons la définition des revêtements admissibles (Harris-Morrison [55], chap. 3, section G, Harris-Mumford [56] § 4, ou Wewers [100], section 2.3) :

DÉfinition 6.6.1. - Soit une courbe stable marquée $D \longrightarrow S$, de genre $g^{\prime}$, avec $S$ connexe, le marquage étant défini par un diviseur de Cartier relatif $B / S$, étale de degré b. Un revêtement admissible $\pi: C \longrightarrow D$ au-dessus de $S$, de base $D / S$, est défini par:

i) Une courbe préstable $C / S$, munie de la donnée d'un morphisme fini surjectif $\pi: C \longrightarrow D$, et d'un diviseur de Cartier relatif $R \subset C$, étale sur $S$, et disjoint du lieu de non lissité, tel que $\pi(R)=B$. On fait l'hypothèse que $\pi$ est (modérement) ramifié exactement le long de $R$. On suppose en outre que la monodromie le long des fibres géométriques est constante, égale à celle fixée initialement.

ii) (structure locale aux points doubles) Si $Q \in D_{s}$ est un point double d'une fibre géométrique, on suppose que localement pour la topologie étale la structure du morphisme $\pi$ en $P \in \pi^{-1}(Q)$ est décrite $\operatorname{par}^{(4)}: \pi_{P}^{*}: \hat{\theta}_{D, Q} \longrightarrow \hat{\theta}_{C, P}$ avec $\hat{\theta}_{C, P}=$ $\vartheta_{s}[[x, y]] /(x y-t), \hat{\theta}_{D, Q}=\hat{\vartheta}_{s}[[u, v]] /(u v-\tau)$, ò̀ $t \in \hat{\mathcal{M}}_{P}, \tau \in \hat{\mathcal{M}}_{Q}$, et pour un certain $d \geqslant 1, u=x^{d}, v=y^{d}\left(\right.$ donc $\left.\tau=t^{d}\right)$.

Étale-localement, on peut préciser le sens de l'hypothèse $\pi$ est ramifié le long de $R$. On peut en effet supposer que $B=\sum_{j} Q_{j}$ est une somme de sections disjointes, et de même $R=\sum_{i, j} P_{i, j}$, où les $\left(P_{i, j}\right)_{i}$ sont les points d'image $Q_{j}$. On suppose alors que, l'indice de ramification le long de $P_{i}$ étant $e_{i, j}$, on a $\pi^{-1} Q_{j}=\sum_{i} e_{i, j} P_{i, j}$. Il reste à expliquer la condition sur la monodromie dans la définition 6.6.1, condition qui n'est pas a priori définie le long des fibres singulières. On passe par une clôture galoisienne.

6.6.2. Clôture galoisienne : cas des courbes lisses. - L'opération fondamentale pour la suite est l'opération de clôture galoisienne dans le contexte d'une famille de courbes lisses ou préstables. Pour une famille de courbes, même lisses, cette opération exige quelques précisions.

Dans ce qui suit, on fixe un corps algébriquement clos $k$. Soit $\pi: C \longrightarrow D$ un revêtement entre courbes lisses sur $k$, de points de branchement $Q_{1}, \ldots, Q_{b}$. L'hypothèse sur la monodromie, assertion (ii) de la définition 6.6.1, revient à fixer en premier le groupe de Galois d'une clôture galoisienne de $\pi: C \longrightarrow D$, c'est-à-dire le groupe de Galois $G$ de l'extension $k(C) / k(D)$. Soit $\phi: Z \longrightarrow D$ une $G$-clôture galoisienne de $\pi$. Cela signifie d'abord que le revêtement $\phi: Z \longrightarrow D$ est galoisien, et que le groupe des automorphismes de $\pi: Z \longrightarrow D$ est identifié à $G$. Il y a d'autre part une factorisation (non unique) de $\phi$ en $\phi=\pi h: Z \stackrel{h}{\longrightarrow} C \stackrel{\pi}{\longrightarrow} D$. La classe de

(4) On peut exprimer la structure locale en disant que le système de coordonnées $(u, v)$ en $Q$ admet une racine $e$-ième $(x, y)$ dans $\hat{\theta}_{C, P}[\mathbf{9 9}]$. On vérifie facilement en utilisant le lemme de Hensel que tout système de coordonnées en $Q$ admet une racine $e$-ième. 
conjugaison du sous-groupe $H=\operatorname{Aut}(h) \subset G$ est par contre bien définie. Comme on a une clôture galoisienne, l'égalité suivante est vérifiée :

$$
\bigcap_{s \in G} s H s^{-1}=1
$$

Si $\phi^{\prime}: Z^{\prime} \longrightarrow D$ est une autre clôture galoisienne, et si $\phi^{\prime}=\pi h^{\prime}$, la théorie de Galois, ou théorie du groupe fondamental, nous assure qu'il y a un isomorphisme $\psi: Z \stackrel{\sim}{\longrightarrow} Z^{\prime}$, qui n'est pas nécessairement un $G$-isomorphisme, tel que $h^{\prime} \psi=h$.

Soit $\xi \in \operatorname{Hur}(G)$ la donnée de ramification du $G$-revêtement galoisien $\phi: Z \longrightarrow D$. Nous définissons la monodromie de $\pi: C \longrightarrow D$ de la manière suivante :

DÉFINITION 6.6.2. - Un type de monodromie est un triplet $m=(G, H, \xi)$ composé de

i) un groupe fini $G$,

ii) un sous-groupe $H \subset G$ tel que $\bigcap_{s \in G} s H s^{-1}=1$, et

iii) une donnée de ramification $\xi \in \operatorname{Hur}(G)$.

Le revêtement $\pi: C \longrightarrow D$ défini sur $k$ est dit à monodromie $m=(G, H, \xi)$, s'il existe une $G$-clôture galoisienne $\phi=\pi h: Z \longrightarrow C \longrightarrow D$, telle $\operatorname{Aut}(h)=H$, de donnée de ramification $\xi$. On parlera alors de $m$-clôture galoisienne.

Posons (voir section 2.2.2) :

$$
\operatorname{Aut}(m)=\{\theta \in \operatorname{Aut}(G) ; \theta(H)=H, \theta(\xi)=\xi\} .
$$

Soit $Z(G)$ le centre de $G$. Du fait de la relation (51), on a $H \cap Z(G)=1$, et il s'ensuit que $H$ se réalise par automorphismes intérieurs comme sous-groupe distingué $H \triangleleft \operatorname{Aut}(m)$. On posera :

$$
\Delta(m)=\operatorname{Aut}(m) / H .
$$

Noter que $\bar{N}_{G}(H)=N_{G}(H) / Z(G)$ est un sous-groupe distingué de Aut $(m)$ contenant $H$. Fixons un type de monodromie $m$, et un revêtement $\pi: C \longrightarrow D$ défini sur $k$.

Deux $m$-clôtures galoisiennes $(Z, h, \phi)$ et $\left(Z^{\prime}, h^{\prime}, \phi^{\prime}\right)$ sont isomorphes s'il existe un $C$-isomorphisme $G$-équivariant $f: Z \stackrel{\sim}{\longrightarrow} Z^{\prime}$ qui est l'identité sur $C$. D'une autre manière, on sait que pour deux telles clôtures, il existe un isomorphisme $f: Z \stackrel{\sim}{\longrightarrow} Z^{\prime}$ tel que $h^{\prime} f=h$. Cela définit un automorphisme $\theta$ de $G$ unique tel que $f: Z \stackrel{\sim}{\longrightarrow} Z^{\prime \theta}$ est $G$-équivariant. Notons que cela force à avoir $\theta \in \operatorname{Aut}(m)$.

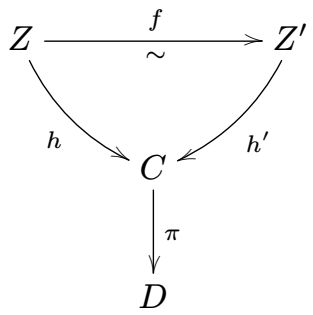


Soit $G(\pi)$ le groupoïde des $m$-clôtures galoisiennes de $\pi: C \longrightarrow D$. Le groupe $\operatorname{Aut}(m)$ agit à droite sur $G(\pi)$ par torsion de l'action. On a la remarque évidente :

LEMME 6.6.3. - $G(\pi)$ est un groupoïde équivalent à $\Delta(m)$.

Démonstration. - Fixons $Z \in G(\pi)$. Identifions $\Delta(m)$ avec un système de représentants dans Aut $(m)$. Il suffit de prouver que pour un quelconque $Z^{\prime} \in G(\pi)$ il y a un unique $\theta \in \Delta(m)$, et un unique isomorphisme $Z^{\theta} \stackrel{\sim}{\rightarrow} Z^{\prime}$. Il y a certainement un isomorphisme $f: Z \longrightarrow Z^{\prime}$ tel que $h^{\prime} f=h$, et alors un unique $\theta \in \operatorname{Aut}(m)$ tel que $f: Z \stackrel{\sim}{\longrightarrow} Z^{\prime \theta}$ est $G$-équivariant. Pour $\theta$ donné, $f$ est unique car $Z(G) \cap H=1$. Noter que s'il existe un isomorphisme $Z^{\theta} \cong Z^{\theta^{\prime}}$, alors $\theta^{\prime}=\theta \in \Delta(m)$. D'une autre manière, l'action de $\operatorname{Aut}(m)$ sur $G(\pi)$ est triviale sur $H$, donc induit une action (au sens des groupoïdes) de $\Delta(m)$, et $G(\pi) / \Delta(m)$ est réduit à un point.

Notons que $\theta \in \operatorname{Aut}(m)$ fixe la classe d'équivalence du $G$-revêtement galoisien $\phi: Z \longrightarrow D$ si et seulement si $\theta \in \bar{N}_{G}(H)$ (automorphisme intérieur), conduisant en accord avec la théorie de Galois au fait que $N_{G}(H) / H$ s'identifie au groupe des automorphismes de $\pi: C \longrightarrow D$.

La définition d'une clôture galoisienne s'étend aux revêtements entre courbes lisses, de base quelconque. On conserve le triplet $m=(G, H, \xi)$.

DÉFinition 6.6.4. - Soit $\pi: C \longrightarrow D$ un revêtement entre $S$-courbes lisses. Une $m$-clôture galoisienne de $\pi$ est la donnée d'un $G$-revêtement galoisien $\phi: Z \longrightarrow D$ de base $S$ et d'une factorisation $\phi: Z \stackrel{h}{\longrightarrow} C \stackrel{\pi}{\longrightarrow} D$ tels que Aut $(h)=H$ et que la monodromie le long des fibres géométriques de $\phi$ est fixe égale à $m$.

Le $G$-revêtement $\pi: C \longrightarrow D$ est dit à monodromie $m=(G, H, \xi)$ si la monodromie est constante égale à $m$ le long des fibres géométriques, par exemple s'il existe une $m$-clôture galoisienne.

DÉFInition 6.6.5. - Soient $g, g^{\prime}$ des entiers et $m=(G, H, \xi)$ un type de monodromie (définition 6.6.2). Le champ de revêtements non galoisiens $\mathcal{H}_{g, g^{\prime}, m}$ est le champ sur $\mathbb{Z}[1 /|G|]$ défini comme suit. Ses sections au-dessus d'un schéma $S$ sont les revêtements $\pi: C \longrightarrow D$ entre courbes lisses, propres sur $S$, à fibres géométriques connexes de genres $g$ et $g^{\prime}$, et la monodromie de $\pi$ dans les fibres est égale à $m$. Les morphismes sont les paires d'isomorphismes $(f, h)$ formant un carré commutatif

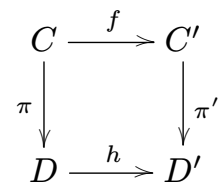


Par les arguments habituels, le champ $\mathcal{H}_{g, g^{\prime}, m}$ est un champ de Deligne-Mumford, sauf si $g=g^{\prime}=0$ et $b=2$. C'est essentiellement une conséquence de la théorie du groupe fondamental modéré, comme noté dans [56], ou de manière plus détaillée dans [99]. Si $\phi: Z \longrightarrow D=Z / G$ est un $G$-revêtement galoisien de donnée de Hurwitz $\xi$, le revêtement $C=Z / H \longrightarrow D$ est à monodromie $m$, et $\phi$ en est une $m$-clôture galoisienne. Cela définit un morphisme de champs

$$
\imath: \mathscr{H}_{g, G, \xi} \longrightarrow \mathcal{H}_{g^{\prime}, g, m}
$$

Le groupe $\operatorname{Aut}(m)$ agit sur le champ $\mathcal{H}_{g, G, \xi}$ par torsion de l'action. Comme l'action du sous-groupe distingué $H$ est triviale dans le sens de la section 6.1.1, il en résulte une action de $\Delta(m)$, cependant non stricte.

THÉORÈME 6.6.6. - Le morphisme (53) est représentable. Il identifie $\mathcal{H}_{g^{\prime}, g, m}$ au champ quotient $\bar{\imath}: \mathcal{H}_{g^{\prime}, g, m} \stackrel{\sim}{\longrightarrow}\left[\mathcal{H}_{g, G, \xi} / \Delta(m)\right]$.

Démonstration. - On supprime, pour alléger, le préfixe $m$. Montrons d'abord que $\imath$ est un épimorphisme, i.e. localement pour la topologie étale $\pi$ admet une clôture galoisienne. C'est en fait une conséquence de la théorie du groupe fondamental (voir [30] par exemple). On peut préférer un argument de déformation, essentiellement équivalent, que l'on développe comme suit. Soit $\pi: C_{s} \longrightarrow D_{s}$ une fibre géométrique définie sur une clôture algébrique de $k(s)$. Soient $\left(Q_{j}\right)_{1 \leqslant j \leqslant b}$ les points de branchement. Comme les courbes sont lisses, on sait que le foncteur des déformations formelles de $\pi_{s}: C_{s} \longrightarrow D_{s}$ est isomorphe à celui de la courbe marquée $\left(D_{s}, B=\sum_{j=1}^{b} Q_{j}\right)$, voir Wewers [100]. Soit alors $h: Z \longrightarrow C_{s}$ une clôture galoisienne de $\pi: C_{s} \longrightarrow D_{s}$. Considérons la déformation universelle $Z \longrightarrow \mathscr{D}$ du $G$-revêtement $\phi: Z \longrightarrow D_{s}$, et soit $\mathscr{B} \subset \mathscr{D}$ le diviseur de branchement réduit. On sait (voir théorème 5.1.5) que $(\mathscr{D}, \mathcal{B})$ est la déformation universelle de la courbe marquée $\left(D_{s}, B_{s}\right)$, et que la base de cette déformation est formellement lisse. On sait par ailleurs que le foncteur des déformations du revêtement $\pi_{s}$ peut être identifié à celui de la base marquée par les points de branchement [100]. Il s'ensuit que $Z / H \longrightarrow \mathscr{D}$ est la déformation universelle de $\pi$, et $Z \longrightarrow Z / H$ en est une clôture galoisienne. Des arguments classiques d'algébrisation montrent que cette clôture galoisienne existe sur un voisinage étale du point $s \in S$.

Prouvons la représentabilité de $\imath$. Il suffit de prouver que le morphisme diagonal est un monomorphisme ([73], prop. 4.4). En d'autres termes, pour tout $G$-revêtement galoisien $\varphi: Z \longrightarrow D$ de base $S$ et tout $\alpha \in \operatorname{Aut}(Z)$ tel que $\imath(\alpha)=1$, on doit montrer que $\alpha=1$. Or, le groupe $\operatorname{Aut}_{D}(Z)$ des automorphismes de $Z$ qui induisent l'identité sur $D$ est le groupe constant $H \times S$. En effet, ce groupe est fini non ramifié sur $S$ et contient $H \times S$. Comme sur les fibres géométriques il y a égalité, ces deux groupes sont égaux. Si de plus $\alpha$ est un $G$-isomorphisme, alors $\alpha \in H \cap Z(G)=1$.

Passons à la seconde assertion. Du fait de la propriété universelle du quotient, le morphisme $\imath$ se factorise en

$$
\mathcal{H}_{g, G, \xi} \longrightarrow\left[\mathcal{H}_{g, G, \xi} / \operatorname{Aut}(m)\right] \longrightarrow \mathcal{H}_{g^{\prime}, g, m}
$$


Pour tout objet $Z \longrightarrow D \in \mathcal{H}_{g, G, \xi}$, et tout $\sigma \in H$, on a $\sigma: Z \cong Z^{\sigma}$, donc $H \subset$ Aut $[Z]$, en notant $[Z]$ l'image de $Z$ dans le quotient. Il s'ensuit une factorisation de $\imath$ :

$$
\bar{\imath}:\left[\mathcal{H}_{g, G, \xi} / \Delta(m)\right]=\left[\mathcal{H}_{g, G, \xi} / \operatorname{Aut}(m)\right] / \| H \longrightarrow \mathcal{H}_{g^{\prime}, g, m}
$$

qui est un épimorphisme. Pour conclure que $\bar{\imath}$ est un isomorphisme, il suffit de prouver que c'est un monomorphisme. Le champ de gauche étant un quotient, on est ramené à prouver que si $\pi: C \longrightarrow D$ et $\pi^{\prime}: C^{\prime} \longrightarrow D^{\prime}$ sont deux revêtement de base connexe $S$ et si $\phi: Z \longrightarrow C \longrightarrow D, \phi^{\prime}: Z^{\prime} \longrightarrow C^{\prime} \longrightarrow D^{\prime}$ sont deux clôtures galoisiennes, alors on a un isomorphisme de schémas

$$
\bigsqcup_{\theta \in \Delta(m)} \operatorname{Isom}_{G}\left(Z^{\theta}, Z^{\prime}\right) \cong \operatorname{Isom}\left((C \longrightarrow D),\left(C^{\prime} \longrightarrow D^{\prime}\right)\right) .
$$

Dans la somme de gauche, $\theta \in \Delta(m)$ parcourt un système de représentants de Aut $(m)$ modulo $H$. Le $S$-schéma $\operatorname{Isom}_{G, C}\left(Z^{\theta}, Z^{\prime}\right)$ a pour points

$$
\operatorname{Isom}_{G, C}\left(Z^{\theta}, Z^{\prime}\right)(T)=\left\{f: Z^{\theta} \times_{S} T \stackrel{\sim}{\longrightarrow} Z^{\prime} \times_{S} T, h^{\prime} f=h\right\} .
$$

Il est clair que $\operatorname{Isom}_{G}\left(Z^{\theta}, Z^{\prime}\right)$ qui est un sous-schéma fermé de $\operatorname{Isom}\left(Z, Z^{\prime}\right)$, est fini et non ramifié sur $S$, de sorte que les deux membres de (54) sont finis non ramifiés sur $S$. Le lemme 6.6.3 montre que sur les fibres géométriques le morphisme est bijectif. Pour conclure qu'on a bien un isomorphisme, il suffit de prouver que si $C=C^{\prime}$, le sous-schéma fibre au-dessus de l'identité de $C / S$, qui n'est autre que

$$
\bigsqcup_{\theta \in \Delta(m)} \operatorname{Isom}_{G, C}\left(Z^{\theta}, Z^{\prime}\right)
$$

est isomorphe à $S$ (qui, on le rappelle, est connexe). Il est clair qu'il y a un $\theta$ unique tel que le morphisme $\operatorname{Isom}_{G, C}\left(Z^{\theta}, Z^{\prime}\right) \longrightarrow S$ est fini, non ramifié bijectif. Ce morphisme est en fait étale, donc un isomorphisme : cette propriété est locale sur $S$, et devient claire si on passe à la déformation universelle comme dans la première partie, car elle est lisse, donc réduite.

Remarque 6.6.7. - Pour un revêtement de base $S$, l'obstruction à l'existence d'une $G$-clôture galoisienne s'identifie à une classe dans $H^{1}(S, \Delta(m))$ (voir section 6.1.1).

6.6.3. Clôture galoisienne : courbes stables. - Soit $m=(G, H, \xi)$ un type de monodromie. La discussion précédente suggère qu'un revêtement stable de base $D$ défini au-dessus du schéma de base $S$, de monodromie $m$, est un revêtement obtenu par factorisation

$$
\pi: C=Z / H \longrightarrow D=Z / G
$$

d'un $G$-revêtement galoisien $\phi: Z \longrightarrow D$ à donnée de ramification $\xi$. Le $G$-revêtement galoisien $\phi: Z \longrightarrow D$, clôture galoisienne de $\pi: C \longrightarrow D$, doit être vu comme un élément structurel du revêtement stable $\pi: C \longrightarrow D$. Noter que dans cette définition, la courbe $Z$ étant stable marquée par les points de ramification du revêtement $Z \longrightarrow$ $Z / G$, la courbe $D$ est en conséquence stable marquée par les points de branchement de $\pi: C \longrightarrow D$, et $C$ est stable marquée par les préimages des points de branchement de 
$Z \longrightarrow D$, ces points pouvant ne pas être des points de ramification de $\pi$. D'autre part le revêtement $Z \longrightarrow C$ est un $H$-revêtement galoisien stable marqué par un diviseur $H$-invariant qui en général contient strictement les seuls points de ramification de $Z \longrightarrow C$.

Il est clair qu'un revêtement stable est admissible. Du fait de l'impossibilité de recoller les uniformisations galoisiennes locales, un revêtement stable ne sera de la forme (55) que localement pour la topologie fppf (ou étale).

DÉfinition 6.6.8. - Soit $m=(G, H, \xi)$ un type de monodromie. Un revêtement stable au-dessus de la base $S$, de monodromie $m$, est une section au-dessus de $S$ du champ quotient

$$
\left[\overline{\mathcal{H}}_{g, G, \xi} / \Delta(m)\right]=\left[\overline{\mathscr{H}}_{g, G, \xi} / \operatorname{Aut}(m)\right] / \| H .
$$

Le champ de Hurwitz compactifié $\overline{\mathcal{H}}_{g, g^{\prime}, m}$ paramétrant les revêtements stables de monodromie $m$, entre courbes stables marquées de genres respectifs $g, g^{\prime}$ est par définition le champ quotient

$$
\overline{\mathcal{H}}_{g, g^{\prime}, m}=\left[\overline{\mathcal{H}}_{g, G, \xi} / \Delta(m)\right] .
$$

Soit un revêtement stable $\pi: C \longrightarrow D$ défini sur le corps $k$. On suppose qu'il dérive d'un revêtement galoisien stable $\tilde{\pi}: \tilde{C} \longrightarrow D$. Nous allons comparer la déformation formelle verselle du revêtement admissible $\pi: C \longrightarrow D$ à la déformation verselle de sa clôture galoisienne $\phi: \tilde{C} \longrightarrow D$, qui est aussi celle du revêtement stable $\pi$, c'est-à-dire enrichi par l'uniformisation galoisienne

$$
\phi: \tilde{C} \stackrel{h}{\longrightarrow} C \stackrel{\pi}{\longrightarrow} D .
$$

Notons $Q_{1}, \ldots, Q_{r}$ les points doubles de $D$, et $\left\{Q_{\alpha, j}\right\}$ les points de $C$ au-dessus de $Q_{\alpha}$. Soit $\tilde{Q}_{\alpha}$ un quelconque point de $\tilde{C}$ au-dessus de $Q_{\alpha}$. On notera que par définition les $Q_{\alpha, j}$ sont les points doubles de $C$. Notons $d_{\alpha}$ l'ordre du stabilisateur de $\tilde{Q}_{\alpha}$ et $d_{\alpha, j}$ l'indice «de ramification » décrivant la structure locale (définition 6.6.1 ii)) de $\pi$ en $Q_{\alpha, j}$. On a le résultat suivant dont la preuve est en tout point analogue au cas lisse :

LEMme 6.6.9. - Sous les hypothèses qui précèdent, on a $d_{\alpha}=\operatorname{ppcm}_{j} d_{\alpha, j}$.

Démonstration. - Le résultat découle de la cyclicité de $I=G_{\tilde{Q}_{\alpha}}$, et de la condition (51). On a d'abord clairement $d_{\alpha, j} \mid q_{\alpha}$ pour tout $j$, de sorte que ppcm $\left\{d_{\alpha, j}\right\}_{j}$ divise $d_{\alpha}$. Par ailleurs, la fibre $\tilde{\pi}^{-1}\left(Q_{\alpha}\right)=G \cdot \tilde{Q}_{\alpha} \cong G / I$ est une réunion de $H$-orbites $\theta_{1}, \ldots, \theta_{r_{\alpha}}$, où $\theta_{j}$ est la $H$-orbite des points doubles de $\tilde{C}$ qui sont au-dessus de $Q_{\alpha, j}$. Soit $\delta_{j}$ l'indice de ramification relativement à $H$ des points de $\theta_{j}$; on a donc $\delta_{j}=d_{\alpha} / d_{\alpha, j}$. Le résultat se ramène en fait à prouver que $\operatorname{pgcd}\left(\delta_{1}, \ldots, \delta_{r_{\alpha}}\right)=1$. Or, si $K$ est le sous-groupe de $I$ d'ordre égal à ce pgcd, on voit tout de suite que $K \subset \bigcap_{s \in G} s H s^{-1}=1$. D'où la conclusion. 
D'après Harris-Mumford ([56], p. 62), la base de la déformation universelle du revêtement admissible $\pi: C \longrightarrow D$ est :

$$
R_{v e r}=W(k)\left[\left[\tau_{1}, \ldots, \tau_{r}, \ldots, \tau_{3 g^{\prime}-3+b},\left\{\tau_{\alpha, j}\right\}\right]\right] / \mathscr{I}
$$

où $I$ désigne l'idéal engendré par les relations $\tau_{\alpha}=\tau_{\alpha, j}^{d_{\alpha, j}}\left(\alpha=1, \ldots, r, j=1, \ldots, r_{\alpha}\right)$. Dans cette description, $\tau_{\alpha}$ représente le paramètre de déformation du point double $Q_{\alpha} \in D$, et $\tau_{\alpha, j}$ celui de $Q_{\alpha, j}$; on sait par ailleurs que la base de la déformation universelle de la courbe marquée $\left(D,\left\{Q_{\alpha}\right\}\right)$ est $W(k)\left[\left[\tau_{1}, \ldots, \tau_{3 g^{\prime}-3+b}\right]\right]$. L'anneau $R_{v e r}$ est réduit, mais en général pas intégralement clos dans son anneau total des fractions. La structure de $R_{v e r}$ peut être précisée comme suit :

Proposition 6.6.10. - Posons $N=3 g^{\prime}-3+b$. Pour tout entier $d$ premier à $p$, soit $\mu_{d} \subset W$ le groupe des racines d-ièmes de l'unité.

i) L'anneau $R_{\text {ver }}$ est réduit, ses idéaux premiers minimaux sont les noyaux $\mathscr{P}_{\zeta}$ des morphismes

$$
\varphi_{\zeta}: R_{v e r} \longrightarrow W\left[\left[t_{1}, \ldots, t_{N}\right]\right] \quad, \quad \tau_{i} \mapsto t_{i}^{d_{i}} \quad, \quad \tau_{i, j} \mapsto \zeta_{i, j} t_{i}^{r_{i, j}}
$$

ò̀ $\zeta=\left\{\zeta_{i, j}\right\} \in \prod_{i, j} \mu_{d_{i, j}}$ et $r_{i, j}=d_{i} / d_{i, j}$. On a $\mathscr{P}_{\zeta}=\mathscr{P}_{\zeta^{\prime}}$ si et seulement si $\zeta_{i, j}^{\prime}=$ $\epsilon_{i}^{r_{i, j}} \zeta_{i, j}$ pour des racines de l'unité $\epsilon_{i} \in \mu_{d_{i}}$. En particulier, le nombre des idéaux premiers minimaux est

$$
N_{\pi}=\prod_{i}\left(\frac{\prod_{j} d_{i, j}}{d_{i}}\right) .
$$

ii) La fermeture intégrale de $R_{\text {ver }}$ dans son anneau total des fractions est le produit de $N_{\pi}$ copies de $W\left[\left[t_{1}, \ldots, t_{N}\right]\right]$.

Démonstration. - Posons $R=W\left[\left[t_{1}, \ldots, t_{N}\right]\right]$. Si $\zeta^{\prime}$ et $\zeta$ sont reliés comme indiqué, il est clair que $\mathscr{P}_{\zeta}=\mathscr{P}_{\zeta^{\prime}}$ car si $\psi$ est l'automorphisme de $R$ tel que $\psi\left(t_{i}\right)=\epsilon_{i} t_{i}$, alors $\varphi_{\zeta^{\prime}}=\psi \varphi_{\zeta}$. Montrons que si $\varphi=\prod \varphi_{\zeta}$ alors $\operatorname{ker} \varphi=0$. On peut se limiter à $N=1$, ce qui permet de supprimer l'indice $i$, et de supposer que $1 \leqslant j \leqslant m$; on note alors simplement $r_{j}=r_{1, j}$. Posons $\mu=\prod \mu_{d_{j}}$, et $\tau=\tau_{1}$. L'algèbre $R_{v e r}$ a pour base sur $W[[\tau]]$ les monômes $\prod_{j} \tau_{j}^{\alpha_{j}}, \alpha_{j}<d_{j}$. De la sorte, un élément du noyau est un

$$
\xi=\sum_{k, \alpha=\alpha_{1}, \ldots, \alpha_{m}} a_{\alpha, k} \prod_{j} \tau_{j}^{\alpha_{j}}
$$

tel que pour $l \geqslant 0$ on a

$$
\sum_{k, \alpha=\alpha_{1}, \ldots, \alpha_{m}} a_{\alpha, k} \prod_{j} \zeta_{j}^{\alpha_{j}}=0
$$

la somme étant étendue aux couples $(k, \alpha)$ tels que $k d+\sum_{j} \alpha_{j} r_{j}=l$. Si on regarde $\prod_{j} \zeta_{j}^{\alpha_{j}}$ comme définissant un caractère sur le groupe $\mu$, le théorème d'indépendance des caractères conduit à $a_{\alpha, k}=0$.

Observons que $R$ est la fermeture intégrale de l'image de $\varphi_{\zeta}$. Du fait que $r_{1}, \ldots, r_{m}$ sont premiers entre eux, il existe avec $e_{j} \in \mathbb{Z}$, et une relation $\sum_{j} e_{j} r_{j}=1$, par suite la relation $t=\prod_{j} \tau_{j}^{e_{j}}$. Le résultat est alors clair. De cela on tire le fait que si $\varphi_{\zeta}$ 
et $\varphi_{\zeta^{\prime}}$ ont un noyau identique, alors il y a un $W$-automorphisme $\gamma$ de $R$ tel que $\varphi_{\zeta^{\prime}}=\gamma \varphi_{\zeta}$. Alors nécessairement $\gamma(t)=\epsilon t$ pour une certaine racine $d$-ième de l'unité. Cela termine la preuve de la proposition.

La structure de l'anneau des déformations $R_{v e r}$ explique la non unicité d'une clôture galoisienne pour un revêtement stable $\pi: C \longrightarrow D$ défini sur $k$.

ThÉORÈme 6.6.11. - Un revêtement admissible $\pi: C \longrightarrow D$ au-dessus de $k$ admet une clôture galoisienne, i.e. peut être stabilisé. Le nombre de classes de clôtures galoisiennes (sous l'action de $\Delta(m)$ ) est

$$
N_{\pi}=\prod_{i}\left(\frac{\prod_{j} d_{i, j}}{d_{i}}\right),
$$

c'est-à-dire le nombre de facteurs intègres de la fermeture intégrale de $R_{v e r}$ (correspondant aux branches de $\operatorname{Spec}\left(R_{\text {ver }}\right)$ ). Dans cette correspondance, les facteurs (isomorphes à $\left.W\left[\left[t_{1}, \ldots, t_{N}\right]\right]\right)$ correspondent bijectivement aux anneaux de déformation des clôtures galoisiennes respectives.

Démonstration. - Admettons d'abord le premier point. Soit une G-clôture galoisienne $Z \longrightarrow C \longrightarrow D$ de $\pi$, de déformation universelle $\mathcal{Z} \longrightarrow \mathscr{D}$. Par le théorème 5.1 .1 , on sait que la base de cette déformation s'identifie à $\operatorname{Spec}\left(R_{Z}\right)$, avec

$$
R_{Z}=W(k)\left[\left[t_{1}, \ldots, t_{r}, \ldots, t_{3 g^{\prime}-3+b}\right]\right] .
$$

Posons $N=3 g^{\prime}-3+b$. Si $R=W\left[\left[\tau_{1}, \ldots, \tau_{N}\right]\right]$ représente la base de la déformation universelle $\mathscr{D}$ de $D$ marquée par les points de branchement, on peut supposer que le morphisme discriminant $R \longrightarrow R_{Z}$ est donné par $\tau_{\alpha}=t_{\alpha}^{d \alpha}$ si $\alpha=1, \ldots, r$, et $\tau_{\alpha}=t_{\alpha}$ si $\alpha>r, o^{6} u d_{\alpha}$ a la signification ci-dessus.

La considération de la déformation $Z / H$ de $C$ conduit à une factorisation $R \longrightarrow$ $R_{v e r} \stackrel{\varphi}{\longrightarrow} R_{Z}$. On peut supposer que $\varphi$ est donné par

$$
\tau_{\alpha, j}=t_{\alpha}^{d_{\alpha} / d_{\alpha, j}} \quad\left(\alpha=1, \ldots, r ; j=1, \ldots, r_{\alpha}\right) .
$$

Soit une autre clôture galoisienne $Z^{\prime} \longrightarrow C \longrightarrow D$, telle que pour un $\theta \in \operatorname{Aut}(m)$, on a un isomorphisme de clôtures $f: Z \stackrel{\sim}{\longrightarrow} Z^{\prime \theta}$. Si $\imath^{\prime}: Z^{\prime} \hookrightarrow Z^{\prime}$ est le plongement canonique de $Z^{\prime}$, la considération de $\left(Z^{\prime}, \imath^{\prime} f\right)$ comme une autre déformation universelle de $Z$, permet d'étendre $f$ en un isomorphisme de diagrammes




Passant au quotient par $H$, il s'ensuit un diagramme commutatif

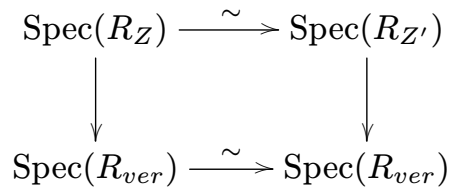

Cela montre que les deux clôtures galoisiennes conduisent à la même classe de morphisme $R_{v e r} \longrightarrow W\left[\left[t_{1}, \ldots, t_{N}\right]\right]$, donc au même facteur de la fermeture intégrale.

Prouvons la réciproque. Cela revient à supposer qu'on a comme donnée les diagrammes commutatifs de dessus, excepté l'isomorphisme $f: \mathcal{Z} \stackrel{\sim}{\longrightarrow} \mathcal{Z}^{\prime}$. On peut se ramener à $R_{Z}=R_{Z^{\prime}}$. De la sorte, on a deux $G$-revêtements $Z$ et $Z^{\prime}$ au-dessus d'une même base $R_{Z}$, avec $Z / H \cong \mathscr{C} \otimes R \cong Z^{\prime}$. On sait que pour les clôtures galoisiennes induites au-dessus de la fibre générique $\nu$ (lisse) $\operatorname{de} \operatorname{Spec}\left(R_{Z}\right)$, il y a $\theta \in \operatorname{Aut}(m)$ tel que $Z_{\nu} \cong Z_{\nu}^{\prime}{ }^{\theta}$. Les courbes étant stables marquées, cet isomorphisme se prolonge en un isomorphisme global $Z \cong Z^{\prime \theta}$. Par restriction à la fibre spéciale, on a obtient un isomorphisme $Z \cong Z^{\prime \theta}$ de clôtures galoisiennes de $\pi$. Pour terminer la preuve, reste à prouver que tout revêtement admissible $\pi: \bar{C} \longrightarrow \bar{D}$ admet une clôture galoisienne, i.e. peut être stabilisé.

On peut dans ce but supposer qu'on a une courbe stable marquée $D \longrightarrow S=$ $\operatorname{Spec}(R)$, où $R$ est un anneau de valuation discrète complet, de corps résiduel $k$, le marquage étant défini par un diviseur étale $B \longrightarrow S$, et de fibre spéciale $\bar{D}$. On suppose que la fibre générique est lisse. Il y a des obstructions à trouver un revêtement admissible $C \longrightarrow D$, relevant $\bar{C} \longrightarrow \bar{D}$. Par contre on peut trouver un tel revêtement après extension finie de $R$. Quitte à agrandir $R$, faisons le choix d'un tel relèvement $C \longrightarrow D$. Rappelons que si $B=\sum_{j} Q_{j}$, cela implique l'existence de points $P_{i, j}$ au-dessus de $Q_{j}$ en lesquels est concentrée la ramification. La courbe $C$ n'est pas forcément stable marquée par les $P_{i, j}$, mais elle l'est par l'ensemble de toutes les préimages des $Q_{j}$. Soit $\tilde{C}_{K} \longrightarrow C_{K} \longrightarrow D_{K}$ une clôture galoisienne de $C_{K} \longrightarrow D_{K}$, les groupes de Galois respectifs étant $G$ et $H$. Quitte à agrandir si nécessaire $R$, soit $\tilde{C} \longrightarrow D$ le modèle stable marqué. Du fait que $\tilde{C} / H$ est stable marquée par les préimages des $Q_{j}$, on a $\tilde{C} / H=C$, il en découle une factorisation

$$
\tilde{C} \longrightarrow C \longrightarrow D \text { et } C=\tilde{C} / H \text {. }
$$

Si on prend les fibres spéciales, ceci montre que $\bar{C}$ possède une clôture galoisienne, en d'autres termes, qu'on peut enrichir le revêtement admissible $\pi: \bar{C} \longrightarrow \bar{D}$ en un revêtement stable.

Il y a un morphisme naturel de source le champ de Hurwitz $\overline{\mathcal{H}}_{g, g^{\prime}, m}$ de but le champ $\overline{\mathscr{H}}_{g, g^{\prime}, m}^{\mathrm{adm}}$ des revêtements admissibles au sens de Harris-Mumford [56]. On a le résultat (comparer avec l'approche de [1] ) :

Proposition 6.6.12. - Le champ $\overline{\mathscr{H}}_{g, g^{\prime}, m}$ est une désingularisation, ainsi que la normalisation, de $\overline{\mathcal{H}}_{g, g^{\prime}, m}^{\mathrm{adm}}$. 
Le champ $\overline{\mathcal{H}}_{g, g^{\prime}, m}$ (classifiant les revêtements stables) n'est pas en général isomorphe au champ, plus fin des revêtements log-admissibles (comparer avec Wewers [99]). Si la base $S$ d'un revêtement stable est arbitraire, on ne peut en général décrire un tel revêtement comme un quotient $\pi: Z / H \longrightarrow Z / G$, partant d'un $G$-revêtement stable. On peut cependant, partant de la définition abstraite (56), donc d'un objet de $\overline{\mathscr{H}}_{g, g^{\prime}, m}(S)$, donner un contenu plus visible à la structure d'un tel revêtement. Les définitions de la section 6.1.1 relatives aux quotients disent qu'un objet de ce champ peut être décrit comme d'une part la donnée d'un recouvrement $\left(S_{i} \longrightarrow S\right)$ de $S$, et ensuite pour tout $i$, d'un $G$-revêtement $Z_{i} \longrightarrow Z_{i} / G$ de base $S_{i}$, l'action de $G \operatorname{sur} Z_{i}$ étant fixée à une torsion près par un élément $\operatorname{Aut}(m)$. L'objet inclut en plus une donnée de descente, qui en posant $S_{i, j}=S_{i} \times{ }_{S} S_{j}$ se ramène à une collection d'isomorphismes : $\sigma_{j, i}: Z_{i \mid S_{i, j}} \stackrel{\sim}{\longrightarrow} Z_{j \mid S_{i, j}}^{\theta_{j, i}}$ qui vérifient après restriction à $S_{i, j, k}$ la condition de cocycle

$$
\sigma_{k, j}^{\theta_{j, i}} \circ \sigma_{j, i}=\sigma_{k, i}, \quad \theta_{k, j} \theta_{j, i}=\theta_{k, i} .
$$

L'égalité (58) est à prendre à un automorphisme interieur près par un élément $h_{i, j, k} \in$ $H$. En particulier, passant aux quotients par $G$ et $H$, et faisant abstraction de la torsion de l'action, on obtient un diagramme



où les isomorphismes horizontaux définissent des données de descente sur les courbes définies localement par $\left(Z_{i} / H\right)$, et $\left(Z_{i} / G\right)$. De la sorte, on récupère par descente à $S$ un revêtement admissible $C \longrightarrow D$, ainsi qu'un revêtement $Z \longrightarrow D$, qui est localement un $G$-revêtement galoisien. Pour installer une action globale de $G$, il est nécessaire de passer à un recouvrement étale de $S$, alors dans ce cas $C=Z / G$ et $D=Z / G$.

6.6.4. Le morphisme discriminant : cas général. - Il a été observé (section 3.1) que le discriminant d'un $G$-revêtement galoisien $\pi: C \longrightarrow D$, entre courbes lisses de base $S$, pouvait s'exprimer comme une somme avec multiplicités de diviseurs de Cartier relatifs étales sur $S$. Ces diviseurs sont les images des diviseurs $\Delta_{H}$ (étales sur $S$ ), lieux des points fixes de stabilisateur exactement $H$.

Pour tout sous-groupe cyclique $K$ de $G$, le diviseur des points fixes de $K$ est $\operatorname{Fix}(K)=\sum_{K \subset C} \Delta_{C}$. Ce fait s'étend à un $G$-revêtement galoisien stable (remarque 4.1.6), en se limitant alors à la partie horizontale $\operatorname{Fix}_{\text {hor }}(K)$ (proposition 4.1.8). Dans le cas général des revêtements non galoisiens, la définition 55 permet de valider les constructions. Soit un revêtement de base $S$, et degré $d$, que l'on peut sans perte de généralité supposer de la forme $\pi: C=Z / H \longrightarrow D=Z / G$ où 
$\phi: Z \longrightarrow D=Z / G$ est une clôture galoisienne au-dessus de $S$. Soit le diviseur de ramification $\mathfrak{R}_{\pi}(4.1 .6)$, défini par

$$
\mathfrak{R}_{\pi}=\operatorname{Div}\left(\pi^{*}\left(\omega_{D / S}\right) \longrightarrow \omega_{C / S}\right) .
$$

Le diviseur de branchement est par définition $\mathfrak{B}_{\pi}=\pi_{*}\left(\mathfrak{R}_{\pi}\right)$. Notons $[K]$ la $H$-classe de conjugaison du sous-groupe cyclique $K$.

Proposition 6.6.13. - i) Le diviseur de ramification du revêtement $\pi: C \longrightarrow D$ de base $S$ a pour expression

$$
\mathfrak{R}_{\pi}=\sum_{[K]}\left(\frac{|K|}{|H \cap K|}-1\right) R_{[K]}
$$

pour des diviseurs de Cartier relatifs $R_{[K]}$ disjoints et étales sur $S$. Le terme $\frac{|K|}{|H \cap K|}$ représente un indice de ramification.

ii) Le diviseur de branchement est $\mathfrak{B}_{\pi}=\sum_{[K] \nsubseteq H}\left(d-e_{H, K}\right) B_{[K]}$, pour certains entiers $e_{H, K}$ qui représentent des indices de ramification, et $B_{[K]}$ un diviseur de Cartier relatif étale sur $S$.

Démonstration. - i) On a clairement $\mathfrak{R}_{\phi}=p^{*}\left(\mathfrak{R}_{\pi}\right)+\mathfrak{R}_{p}$. Utilisant l'expression du diviseur de ramification dans le cas galoisien, c'est-à-dire $\mathfrak{R}_{\phi}=\sum_{K}(|K|-1) \Delta_{K}$, et l'expression analogue pour $R_{p}$, on obtient tout de suite

$$
p^{*}\left(\mathfrak{R}_{\pi}\right)=\mathfrak{R}_{\phi}-\mathfrak{R}_{p}=\sum_{K, K \not \subset H}(|K|-|H \cap K|) \Delta_{K} .
$$

D'autre part, les diviseurs $\Delta_{K}, \Delta_{K^{\prime}}$ ont une même image dans $C$ si et seulement si $K^{\prime}$ est conjugué à $K$ par un élément de $H$. Le nombre de ces conjugués est $[H$ : $\left.N_{G}(K) \cap H\right]$, et le degré de $\Delta_{K}$ sur son image $R_{[K]}$ est $\left[N_{G}(K) \cap H: K \cap H\right]$. Comme $p_{*} p^{*}\left(\Re_{\pi}\right)=|H| R_{\pi}$, il vient

$$
|H| \Re_{\pi}=\sum_{K, K \nsubseteq H}(|K|-|H \cap K|) p_{*}\left(\Delta_{K}\right)=|H| \sum_{[K]}\left(\frac{|K|}{|H \cap K|}-1\right) R_{[K]} .
$$

ii) L'expression du diviseur de branchement s'en déduit immédiatement.

EXemple 6.6.14. - Soit le cas du champ de Hurwitz classique classifiant les revêtements simples de degré $n \geqslant 3$ de $\mathbb{P}^{1}$, avec $b=2 g+2 n-2$ points de branchement. Le groupe de monodromie est $G=\mathfrak{S}_{n}$, et $H=\mathfrak{S}_{n-1}$ (comme dans Fulton [49]). Dans ce cas $\Delta(m)=\operatorname{Aut}(m) / H=1$. La compactification lisse du champ de HurwitzFulton $\overline{\mathscr{H}}_{g, \mathfrak{S}_{n}, b(12)} / \mathfrak{S}_{n-1}$ s'identifie au champ classifiant les clôtures galoisiennes à groupe de galois $\mathfrak{S}_{n}$. Il est distinct du champ de Harris-Mumford.

Soit de nouveau $m=(G, H, \xi)$ un type de monodromie, $b$ étant le degré du diviseur de branchement. Comme dans le cas galoisien, le champ de Hurwitz $\overline{\mathcal{H}}_{g, g^{\prime}, m}$ est source du morphisme discriminant

$$
\delta: \overline{\mathcal{H}}_{g, g^{\prime}, m} \longrightarrow \overline{\mathcal{M}}_{g^{\prime}, b}
$$


tel que $\delta(\pi: C \longrightarrow D)=\left(D, B=\sum B_{[K]}\right)$. Le morphisme (60) est plat, propre, quasi-fini et génériquement étale, i.e étale sur un sous-champ ouvert partout dense. On peut voir $\delta$ comme un revêtement entre champs de Deligne-Mumford lisses. Sous ces conditions il n'est pas difficile de définir le diviseur de ramification du morphisme de champs $\delta$.

Proposition 6.6.15. - Soit un revêtement $\delta: \mathscr{H} \longrightarrow \mathcal{M}$ entre champs de DeligneMumford lisses sur $k$ (un morphisme propre quasi-fini, génériquement étale). Il existe un diviseur de Cartier effectif $\mathscr{R} \subset \mathcal{H}$ (le diviseur de ramification), tel que

$$
\omega_{\mathcal{H}} \cong \delta^{*}\left(\omega_{\mathcal{M}}\right) \otimes \theta(\mathscr{R}) \text {. }
$$

Démonstration. - Le champ $\mathcal{H}$ (resp. $\mathcal{M}$ ) étant lisse, de dimension $N$ disons, le faisceau localement libre $\Omega_{\mathcal{H} / k}^{1}$ (de rang $N$ ) est défini en tant que faisceau sur le site étale, de même pour le faisceau $\Omega_{\mathcal{M} / k}^{1}$. Soit $q: V \longrightarrow \mathcal{M}$ un atlas. Formons le 2-produit fibré

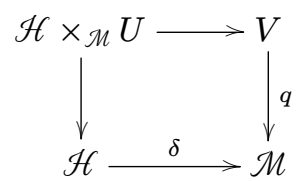

avec flèches verticales étales surjectives. Si $U \longrightarrow \mathscr{H} \times \mathcal{M} U$ est un atlas, de sorte que le morphisme composé $p: U \longrightarrow \mathscr{H}$ est un atlas de $\mathscr{H}$, on obtient le carré 2-commutatif

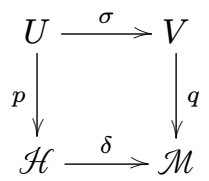

Le morphisme $\sigma: U \longrightarrow V$ est quasi-fini, génériquement étale, par conséquent le conoyau $\Omega_{U / V}^{1}$ de $d \sigma: \sigma^{*}\left(\Omega_{V / k}^{1}\right) \longrightarrow \Omega_{U / k}^{1}$ est de torsion. Noter que $\sigma^{*}$ est une injection. On peut alors former le diviseur $R=\operatorname{Div}\left(\omega_{V / U}\right)$, qui est un diviseur de Cartier effectif de $U$ sur $V$, définissant de manière évidente un diviseur de cartier $\mathscr{R}$ sur $\mathscr{H}$. Dans la situation de (60), si $C \longrightarrow D$ est un revêtement avec $r$ points doubles sur $D$, on décrit la structure locale de $\mathscr{R}$ comme suit. Soit $k\left[\left[t_{1}, \ldots, t_{r}, \ldots, t_{N}\right]\right] \hookrightarrow$ $k\left[\left[\tau_{1}, \ldots, \tau_{r}, \ldots, \tau_{N}\right]\right]$ le morphisme induit entre les bases des déformations universelles respectives, avec $t_{i}=\tau^{e_{i}}$ si $1 \leqslant i \leqslant r$, et $t_{j}=\tau_{j}$ si $j>r$. Il est alors clair que l'équation locale de $\mathcal{R}$ est $\prod_{i, e_{i}>1} \tau_{i}^{e_{i}-1}$. 



\section{CHAPITRE 7}

\section{GRAPHES ET REVÊTEMENTS}

Dans cette section, le champ de Hurwitz $\overline{\mathcal{H}}_{g, G, \xi}$, respectivement l'espace modulaire de Hurwitz $\bar{H}_{g, G, \xi}$, est étudié d'un point de vue combinatoire. Rappelons (définition 6.2.3) que les points du bord $\partial \overline{\mathscr{H}}_{g, G, \xi}=\overline{\mathcal{H}}_{g, G, \xi}-\mathscr{H}_{g, G, \xi}$ (resp. $\partial \bar{H}_{g, G, \xi}$ ) sont représentés par les courbes stables marquées munies d'une action stable de $G$ (resp. par les classes à isomorphisme $G$-équivariant près).

\subsection{Graphes modulaires de Hurwitz}

La combinatoire d'une courbe préstable se décrit commodément à l'aide du graphe dual, appelé aussi graphe modulaire, voir Manin [76], chap. III, § 2. Fixons les notations relatives aux graphes utilisées ci-après.

DÉfinition 7.1.1. - Un graphe (fini) $\Gamma$ est la donnée de

i) un ensemble $\mathcal{F}$ (de demi-arêtes, ou d'arêtes orientées) muni d'une involution $\tau: \mathcal{F} \longrightarrow \mathcal{F}$

ii) un ensemble $\mathcal{V}$ de sommets et d'une application bord (ou incidence) $: \partial: \mathcal{F} \longrightarrow$ V. Si $e \in \mathcal{F}, \partial(e)$ est le sommet (initial) de e.

Les orbites à deux éléments de $\tau$ constituent les arêtes géométriques du graphe, tandis que les points fixes de $\tau$ correspondent aux «pattes» du graphe. En modifiant légèrement la terminologie, on a une partition

$$
\mathcal{F}=\mathscr{E} \sqcup \mathscr{L}
$$

où $\mathscr{E}$ (resp. $\mathscr{L}$ ) désigne l'ensemble des arêtes orientées (resp. des pattes). La valence d'un sommet $c$ est le nombre $v(c)$ des demi-arêtes incidentes à ce sommet. Si on ignore l'involution $\tau$, on obtient l'ensemble des drapeaux $\mathcal{F} l(\Gamma)$, c'est-à-dire des couples $(v, e) \in \mathcal{V} \times \mathcal{F}, \partial(e)=v$.

Un graphe modulaire est un graphe $\Gamma$ comme ci-dessus enrichi par la donnée d'une application $g: \mathcal{V} \longrightarrow \mathbb{Z}_{\geqslant 0}$. Le graphe modulaire est dit stable si pour tout sommet $c$ tel que $g(c)=0$ (resp. $g(c)=1$ ), on a $v(c) \geqslant 3$ (resp. $v(c) \geqslant 1$ ). Le graphe modulaire $\Gamma$ est marqué si les pattes sont indexées. 
Un isomorphisme $\Gamma \stackrel{\sim}{\longrightarrow} \Gamma^{\prime}$ est la donnée d'un couple de bijections $f: \mathcal{F} \longrightarrow \mathcal{F}^{\prime}$, $h: V \longrightarrow V^{\prime}$, tel que $h \partial=\partial f$, et $f \tau=\tau^{\prime} f$, pour les graphes, avec en plus la condition $g^{\prime} h=g$ pour les graphes modulaires ${ }^{(1)}$. Ainsi, si $\Gamma$ est un graphe (modulaire), on peut définir le groupe Aut $(\Gamma)$, et parler d'une action du groupe fini $G$ sur le graphe (resp. graphe modulaire) $\Gamma$. Sous ces conditions, il n'est pas difficile de définir le graphe (resp. graphe modulaire) quotient $\Gamma / G$. Il est défini par les ensembles $\mathcal{F} / G, V / G$, et les applications déduites de $\tau, \partial$, par passage au quotient. Il y a un morphisme de graphes $\Gamma \longrightarrow \Gamma / G$, induit par le passage au quotient dans les ensembles $\mathcal{F}$ et $V$. Dans la suite, on notera par la même lettre, dans la mesure où cela n'induit pas de confusion, un graphe modulaire et le graphe (ordinaire) sous-jacent.

On définit le graphe modulaire $\Gamma(C)$ associé à une courbe préstable marquée $(C, P)$ comme suit. L'ensemble $P$ des pattes est formé des piqûres, ou points marqués, de $C$. Soit $\left\{C_{i}\right\}_{i \in I}$ l'ensemble des composantes irréductibles de $C$, ou ce qui est la même chose l'ensemble des composantes $\left\{\tilde{C}_{i}\right\}$ de la normalisation $\tilde{C}$ de $C$. Soit aussi $\left\{b_{\alpha}\right\}_{\alpha \in \Lambda}$ la réunion de l'ensemble des branches (points de $\tilde{C}$ ) en les différents points doubles de $C$ et de l'ensemble des points marqués. De manière équivalente, c'est l'ensemble des points de $\tilde{C}$ qui se projettent sur un point double ou un point marqué. Le graphe (dit dual) $\Gamma(C)$ est alors défini par :

i) $\mathcal{F}=\Lambda, V=I$,

ii) $\tau\left(b_{\alpha}\right)=b_{\beta}$ si et seulement si $b_{\alpha} \neq b_{\beta}$ sont les deux branches d'un même point double, ou alors $\tau\left(b_{\alpha}\right)=b_{\alpha}$, et dans ce cas $b_{\alpha}$ est un point marqué,

iii) $\partial b_{\alpha}=C_{i}$ si et seulement si $b_{\alpha}$ est une branche d'un point double, ou bien un point marqué situé sur la composante $C_{i}$; dans ce cas $g\left(C_{i}\right)$ est le genre géométrique de $C_{i}$ (le genre de $\tilde{C}_{i}$ ).

De manière alternative, on peut voir les demi-arêtes comme des arêtes orientées. Ceci revient à dire que les arêtes géométriques sont dédoublées, en une arête orientée et l'arête opposée. Dans cette description, les piqûres ou pattes du graphe sont vues comme des arêtes orientées, mais dépourvues d'arêtes opposées. L'involution $\tau$, que nous noterons aussi $a \longrightarrow \bar{a}$, renverse de fait l'orientation des arêtes, et fixe les pattes. Pour une composante $C_{i}$, on note $l_{i}$ le nombre des branches d'origine $C_{i}$, et $h_{i}$ le nombre des piqûres d'origine $C_{i}$. De sorte que la condition de stabilité s'écrit de manière équivalente, si $g_{i}=g\left(C_{i}\right)$ est le genre :

$$
2 g_{i}-2+h_{i}+l_{i}>0 \quad(i=1, \ldots, s=\operatorname{Card} I) .
$$

Rappelons aussi la relation bien connue qui donne le genre arithmétique de la courbe $C$, supposée connexe, en fonction des paramètres contenus dans le graphe

(1) Si $f(e) \neq \tau(e)$ pour toute demi-arête $e$, l'automorphisme $f$ est dit sans inversion. Nous ne considérerons dans la suite que des automorphismes sans inversion. Noter qu'il est possible de définir plus généralement un morphisme de graphes $\Gamma \longrightarrow \Delta$ : c'est la donnée d'un couple d'applications $f: \mathcal{F}_{\Gamma} \longrightarrow \mathcal{F}_{\Delta}, h: V_{\Gamma} \longrightarrow V_{\Delta}$ avec les relations de commutation analogues, voir Bass [7]. 
dual, c'est-à-dire le nombre $d$ d'arêtes et le nombre $s$ de sommets ([76], prop. III.2.6) :

$$
g=\sum_{i} g_{i}+\operatorname{dim} H_{1}(\Gamma)=\sum_{i} g_{i}+d-s+1 .
$$

Revenons maintenant à la situation d'origine, donc $C$ est une $G$-courbe stable. Il est clair que $G$ agit de manière naturelle sur le graphe modulaire $\Gamma=\Gamma(C)$. Du fait de la stabilité de l'action, $G$ agit sans inversion, i.e. pour toute branche $a$, et tout $\sigma \in G$, $\sigma \neq 1$, on a $\sigma(a) \neq \bar{a}$. Il n'est pas difficile d'implémenter sur $\Gamma$ la donnée de Hurwitz. Plus précisément, on attache à chaque élément de $\mathcal{F}$ une décoration matérialisée par un couple $(H, \chi)$, où $H$ est l'holonomie i.e. le stabilisateur (cyclique) soit d'un point non-singulier, soit d'une branche, et $\chi$ est le caractère de l'action de $H$ sur l'espace tangent du point distingué. De plus, si l'arête $e$ est décorée par $(H, \chi)$, alors l'arête opposée $\bar{e} \neq e$ est décorée par $\left(H, \chi^{-1}\right)$. Si on ne regarde que les classes de conjugaison des décorations, qui de fait sont constantes sur les $G$-orbites, on retrouve la donnée de Hurwitz. En résumé, appelons graphe modulaire de Hurwitz un graphe modulaire muni d'une action sans inversion d'un groupe fini $G$ qui satisfait aux conditions précédentes. Pour une arête, ou patte, décorée par le couple $(H, \chi)$, on parlera de ce couple comme étant l'holonomie; l'holonomie en ge est $\left(g \mathrm{Hg}^{-1},{ }^{g} \chi\right)$. Enfin, il y a une notion évidente d'isomorphisme de graphes modulaires de Hurwitz.

DÉFInITION 7.1.2. - On appelle graphe modulaire de Hurwitz associé au revêtement galoisien stable $C \longrightarrow D$, le graphe modulaire $\Gamma=\Gamma(C)$ muni de l'action de $G$, et décoré par les caractères locaux attachés aux orbites de points de ramification (points spéciaux) et branches de $C$. Le graphe est marqué si les orbites de pattes sont indexées.

Soit $\Gamma$ un graphe modulaire de Hurwitz attaché à $(g, G, \xi)$. Un G-revêtement $\pi$ : $C \longrightarrow D$ est dit de type $\Gamma$, si $\Gamma(C) \cong \Gamma$.

On utilisera une notation identique pour le graphe modulaire de Hurwitz du revêtement $\pi: C \longrightarrow D$ et le graphe modulaire de la courbe $C$. Les graphes seront comme les revêtements, marqués.

\subsection{Graphes de groupes et revêtements de graphes}

Utilisant la théorie de Bass des revêtements de graphes (voir [7]), on peut décrire un graphe $\Gamma$, partant du graphe quotient $\Delta=\Gamma / G$, et d'un morphisme de monodromie.

7.2.1. Graphes modulaires quotients. - Soit $(C, R)$ une courbe stable marquée, définie sur $k$, munie d'une action stable de $G$ (définition 6.2.3). Rappelons que $C$ est marquée par un diviseur $G$-invariant $R$ contenant les points de ramification. Soit $\Gamma=\Gamma(C)$ le graphe modulaire de Hurwitz attaché à $C$ (définition 7.1.2). Notons pour débuter la remarque élémentaire suivante :

Proposition 7.2.1. - Le graphe quotient $\Delta=\Gamma / G$ possède une structure canonique de graphe modulaire de genre $g^{\prime}$, ̀̀ b piqûres. Il s'identifie au graphe modulaire de la courbe stable marquée $D=C / G$. 
Démonstration. - Par définition du graphe combinatoire quotient [7], on a :

$$
V(\Delta)=V(\Gamma) / G \text { et } \mathcal{F}(\Delta)=\mathscr{F}(\Gamma) / G .
$$

L'action de $G$ commute avec l'involution $\tau_{\Gamma}$, celle-ci induit donc une involution sur l'ensemble quotient, définissant ainsi la structure du graphe quotient $\Delta$. Noter aussi que les pattes (points fixes de $\tau_{\Delta}$ ) du quotient, sont les images des pattes de $\Gamma$. Pour finir de décrire $\Delta$ comme graphe modulaire, il faut assigner une valeur $g_{\alpha}^{\prime}$ pondérant le sommet $v_{\alpha}^{\prime}$ associé à la composante $D_{\alpha}$ de $D$. Par convention, $g_{\alpha}^{\prime}$ est le genre (géométrique) de $D_{\alpha}$. Il est clair que $g_{\alpha}^{\prime}$ s'exprime en fonction des seules données de $\Gamma$. Soit une composante $C_{i}$ de $C$, d'image $D_{\alpha}$, et soit $G_{i}$ le stabilisateur de $C_{i}$, i.e. du sommet $v_{i}$ de $\Gamma$. Donc $D_{\alpha}=C_{i} / G_{i}$. L'expression cherchée est la formule de Riemann-Hurwitz, appliquée au revêtement $\tilde{C}_{i} \longrightarrow \tilde{D}_{\alpha}$ :

$$
2 g_{i}-2=\left|G_{i}\right|\left(2 g_{\alpha}^{\prime}-2\right)+\sum_{\lambda} B_{\lambda}
$$

où les $B_{\lambda}$ sont les contributions des points de branchement, contributions dépendantes de la seule combinatoire du graphe de modulaire de Hurwitz $\Gamma$. Il est facile de prouver que l'on définit ainsi une structure de graphe modulaire (quotient) sur $\Delta$. Le dernier point est clair.

Pour éviter toute confusion avec d'autres définitions de revêtements de graphes ${ }^{(2)}$, insistons sur le fait que l'action de $G$ sur les sommets et arêtes n'est (dans cette définition) pas nécessairement libre, et le quotient $\Gamma \longrightarrow \Gamma / G$ est donc à prendre dans le sens d'un revêtement ramifié. Avant de discuter en détail le cas général, il peut être utile de traiter deux cas particuliers importants; ce sont les graphes modulaires de Hurwitz qui sont associés aux composantes de codimension $1 \mathrm{du}$ bord de $\overline{\mathcal{H}}_{g, G, \xi}$, ou de l'espace modulaire grossier de même nom. Ce sont les deux cas pour lesquels $\Delta$ n'a qu'une seule arête géométrique, $\Delta$ est donc soit un segment, soit une boucle.

Avant d'entreprendre de développer ces exemples, faisons une observation sur le cas où $\Delta$ est un arbre. Il est connu que sous cette hypothèse, la projection $\Gamma \longrightarrow \Delta$ admet une section [7]; pour simplifier, nous noterons par la même lettre $\Delta$ l'image d'une section supposée choisie. Notant toujours $V$ l'ensemble des sommets, et $\mathscr{F}$ l'ensemble des arêtes orientées, nous noterons pour simplifier $a \in \Gamma$ au lieu de $a \in \mathcal{F}$, même chose pour les sommets; la notation est analogue pour $\Delta$. On a alors les identifications

$$
\mathcal{F}=\bigsqcup_{a \in \Delta} G / H_{a} \quad \text { et } \quad \mathcal{V}=\bigsqcup_{v \in \Delta} G / G_{v} .
$$

On note $G_{v}$ le stabilisateur du sommet $v$, et $H_{a}$ le stabilisateur de l'arête (orientée, ou géométrique) $a$. Décrivons la relation d'incidence. Si $e$ est une arête d'extrémités $g_{1} v_{1}$ et $g_{2} v_{2}$, il existe alors $a \in \Delta$ tel que $e=g a \in G a$; alors $g^{-1} g_{1} v_{1}=v_{1}$ et $g^{-1} g_{2} v_{2}=v_{2}$. Cela montre que l'ensemble des arêtes d'extrémités $g_{1} v_{1}$ et $g_{2} v_{2}$ est en

(2) On peut exiger, ce qui n'est pas le cas dans notre définition, la propriété de relèvement des chemins (revêtements non ramifiés). 
bijection naturelle avec les classes $g H_{a}, a$ étant l'unique arête de $\Delta$ joignant $v_{1}$ à $v_{2}$, qui vérifient l'inclusion $g H_{a} \subset g_{1} G_{v_{1}} \cap g_{2} G_{v_{2}}$.

Exemple 7.2.2. - L'étoile (la courbe correspondante est un « peigne »).

Détaillons le cas particulier où $\Delta$ est une étoile à $k \geqslant 3$ branches. La courbe correspondante à ce graphe dual est appelée un «peigne » en raison de sa topologie (voir figure 1). Notons ainsi les sommets de $\Delta$ :

- $v_{0}$ est la racine de l'étoile, qui est un sommet de valence $k$

- $v_{1}, \ldots, v_{k}$ sont les sommets terminaux des $k$ branches.

On doit installer sur $\Delta$ une structure de graphe modulaire, et implémenter la donnée de Hurwitz. Pour la structure de graphe modulaire, on pose $g_{0}=g_{1}=\cdots=$ $g_{k}=0$. La donnée de Hurwitz dans cet exemple est du type Harbater-Mumford (voir Dèbes [26], Dèbes-Emsalem [27]) c'est-à-dire de la forme

$$
\xi=\sum_{i=1}^{k}\left(\left[H_{i}, \chi_{i}\right]+\left[H_{i}, \chi_{i}^{-1}\right]\right) .
$$

La forme spécifique de la donnée de Hurwitz impose à chaque sommet $v_{1}, \ldots, v_{k}$ d'être la source de deux pattes. En effet, si $C_{i}$ est une composante de $C$ au-dessus du brin d'indice $i$ de $D$, alors $C_{i} \longrightarrow \mathbb{P}^{1}$ est ramifié en trois points au plus. La forme de la donnée de ramification impose que le nombre de points de branchement est 2 , le point double étant d'isotropie triviale.



Figure 1. Le «peigne»

Pour décrire la structure de graphe de Hurwitz sur $\Gamma$, choisissons un sommet $s_{i}$ au-dessus de $v_{i}(i=0, \ldots, k)$, et posons $H_{i}=G_{s_{i}}$; notons que nécessairement $H_{0}=1$. Observons aussi que $G$ est en conséquence engendré par la réunion des $H_{i}$. On obtient finalement la description suivante de $\Gamma$ en termes de $G$ et des $H_{i}$ :

$$
V_{\Gamma}=\left(\bigsqcup_{i=1}^{k} G / H_{i}\right) \bigsqcup G, \quad \mathcal{F}_{\Gamma}=\bigsqcup_{1}^{2 k} G
$$


Notons que le genre $g$ de $C$ est donné par

$$
g=1+k \operatorname{Card} G-\sum_{i=1}^{k}\left[G: H_{i}\right] .
$$

EXEMPLE 7.2.3. - Le segment.

Dans cet exemple, $\Delta$ est un segment de sommets $v_{1}$ et $v_{2}$, et d'arêtes orientées $e$ et $\bar{e}$, avec $e$ pointant vers $v_{2}$. On relève $\Delta$ en un segment de $\Gamma$ de sommets $s_{1}$ et $s_{2}$, de stabilisateurs respectifs $G_{1}$ et $G_{2}$. L'arête orientée $a$ relève $e$ : soit $H$ le stabilisateur de $a$, alors $H \subset G_{1} \cap G_{2}$, et la connexité impose que $G_{1} \cup G_{2}$ engendre $G$. La description du graphe (ordinaire) $\Gamma$ est aisée : on a

$$
\mathcal{F}_{\Gamma}=G / H \times\{ \pm 1\}, \quad V_{\Gamma}=G / G_{1} \bigsqcup G / G_{2} .
$$

Dans ce modèle, une arête géométrique est représentée par un élément de $G / H$; l'arête $g H$ joint les sommets $g_{1} G_{1}$ et $g_{2} G_{2}$ si et seulement si $g H \subset g_{1} G_{1} \cap g_{2} G_{2}$. L'involution est donnée par $\tau(g H, \epsilon)=(g H,-\epsilon)$. D'une manière plus géométrique, supposons que les composantes $C_{1}$ et $C_{2}$ correspondent à $s_{1}$ et $s_{2}$. Posons

$$
\operatorname{Ind}_{G_{1}}^{G}\left(C_{1}\right)=C_{1} \times G_{1} G .
$$

Cela signifie qu'on effectue le quotient de $C_{1} \times G$ par l'action de $G_{1}: \alpha(x, g)=$ $\left(\alpha x, g \alpha^{-1}\right)$. Le résultat est une courbe avec $\left[G: G_{1}\right]$ composantes irréductibles disjointes, toutes identiques à $C_{1}$, et munie de l'action de $G$ donnée par $g[x, h]=$ $[x, g h]$. La courbe $C$ est alors de la forme

$$
C=\operatorname{Ind}_{G_{1}}^{G}\left(C_{1}\right) \bigvee \operatorname{Ind}_{G_{2}}^{G}\left(C_{2}\right),
$$

expression dans laquelle le symbole $\vee$ signifie que l'on recolle les deux facteurs en identifiant des paires de points, selon la combinatoire des arêtes de $\Gamma$. Les points doubles forment une unique $G$-orbite; soit $p \in C_{1} \cap C_{2}$ l'un d'eux, d'isotropie $H$. Si $H$ agit sur la branche $C_{1}$ par le caractère $\chi$, et sur la branche $C_{2}$ par $\chi^{-1}$ (stabilité oblige), alors la donnée de Hurwitz $\xi$ de l'action de $G$ sur $C$ s'obtient par $\xi=\xi_{1}+\xi_{2}$, où l'on suppose que la donnée définie par l'action de $G_{1}$ sur $C_{1}$ est $\tilde{\xi}_{1}=\xi_{1}+[H, \chi]$, et celle définie par l'action de $G_{2}$ sur $C_{2}$ est $\tilde{\xi}_{2}=\xi_{2}+\left[H, \chi^{-1}\right]$. La combinatoire est ainsi totalement contenue dans la donnée $\left(G_{1}, G_{2}, H, \chi, \xi_{1}, \xi_{2}\right)$. Noter qu'il n'est pas nécessaire de s'assurer que le genre $g$ de $C$ est celui fixé initialement. En effet, ceci résulte de la donnée du genre $g^{\prime}=g_{1}^{\prime}+g_{2}^{\prime}$ de la base $D=C_{1} / G_{1} \vee C_{2} / G_{2}$ joint aux informations contenues dans la donnée de Hurwitz. Si l'on note $g_{i}$ étant le genre de $C_{i}$, on a donc l'égalité :

$$
g=\left[G: G_{1}\right] g_{1}+\left[G: G_{2}\right] g_{2}+[G: H]-\left[G: G_{1}\right]-\left[G: G_{2}\right]+1 .
$$

EXEMPLE 7.2.4. - La boucle.

Si $\Delta$ est une boucle, alors $G$ est une «extension HNN ». On distingue deux cas selon que $\Gamma$ a ou n'a pas de boucle. Traitons d'abord le second cas :

- On suppose que $\Gamma$ ne contient pas de boucle, et que $\Delta=\Gamma / G$ se réduit à une boucle. Alors $\Gamma$ est défini par la donnée (à conjugaison près) par un triplet $\left(G_{0}, H, g_{0}\right)$ 
formé de deux sous-groupes $H$ et $G_{0}$ de $G, H$ étant cyclique, et $H \subset G_{0} \subset G$, et d'un élément $g_{0} \in G, g_{0} \notin G_{0}$. On impose à cette donnée les conditions $g_{0}^{-1} H g_{0} \subset G_{0}$ et $G_{0} \cup\left\{g_{0}\right\}$ engendre $G$.

En effet, soit une arête $a$ de $\Gamma$ de sommets $x \neq y$. Soit $H=G_{a}, G_{0}=G_{x}$. Il y a $g_{0} \in G-G_{0}$ tel que $g_{0}(x)=y$. Alors $H \subset G_{0} \cap g_{0} G_{0} g_{0}^{-1}$. Les arêtes issues de $x$ forment deux orbites sous $G_{0}$, l'orbite de $a$, et l'orbite de $g_{0}^{-1}(a)$. Il est facile de voir qu'on reconstruit le graphe $\Gamma$ en posant :

$$
\mathcal{F}_{\Gamma}=(G / H) \times\{ \pm 1\} \quad, \quad V_{\Gamma}=G / G_{0} .
$$

L'involution $\tau$ est comme dans l'exemple 7.2.3 la multiplication par -1 sur le facteur de droite. L'incidence est donnée par

$$
(\alpha H,+1) \longrightarrow\left(\alpha G_{0}, \alpha g_{0} G_{0}\right) \quad, \quad(\alpha H,-1) \longrightarrow\left(\alpha g_{0} G_{0}, \alpha G_{0}\right),
$$

en particulier $\operatorname{dim} H_{1}(\Gamma)=[G: H]-\left[G: G_{0}\right]+1$. Par exemple, si $G_{0}=1$, alors $G$ est cyclique engendré par $g_{0}$, et $\Gamma$ est un $n$-circuit, avec $n=$ Card $G$. Par construction il y a dans $\Gamma$ une seule orbite de sommets, soit $v$ l'un d'entre eux et soit $G_{0}$ le stabilisateur de $v$. Si $C_{0}$ est la composante représentée par $v$, la donnée de Hurwitz définie par l'action de $G_{0}$ sur $C_{0}$ est de la forme

$$
\tilde{\xi}=\xi+[H, \chi]+\left[g_{0}^{-1} H g_{0},,_{0} \chi^{-1}\right] \in R_{+}\left(G_{0}\right) .
$$

L'hypothèse de stabilité de l'action impose aux caractères locaux en $\chi$ en $v$ et $\chi^{0}$ en $g_{0}^{-1} v$ d'être reliés par

$$
\chi^{0}(s)=\chi^{-1}\left(g_{0} s g_{0}^{-1}\right) .
$$

- Il reste le cas où $\Gamma$ contient une boucle; il est facile de voir que dans ce cas $\Gamma$ n'a qu'un seul sommet, et que toutes les arêtes sont des boucles, i.e. $\Gamma$ est une fleur à $m \geqslant 1$ pétales.

7.2.2. Un exemple : le bord du schéma de Hurwitz « classique ». - Pour illustrer la description ci-dessus des composantes irréductibles de $\overline{\mathcal{H}}_{g, G, \xi}$, revenons au schéma de Hurwitz classique $\overline{\mathscr{H}}_{g, d}$ qui classifie les revêtements simples $\pi: C \longrightarrow$ $D=\mathbb{P}^{1}$ de degré $d$, et $C$ de genre $g$ (exemple 3.2.1). Si $b$ est le nombre de points de branchement on a $b=2 g+2 d-2$. On sait que le groupe de monodromie est le groupe symétrique $G \cong \mathfrak{S}_{d}$, et que la donnée de ramification de la clôture galoisienne $\tilde{C} \longrightarrow D$ est $\xi=b[(12)]$, i.e. $b$ fois la classe de conjugaison des transpositions. (On renvoie à 4.2.4 et 6.6.3 pour les notions de donnée de ramification et de cloture galoisienne d'un revêtement de courbes stables.) On a $C=\tilde{C} / \mathfrak{S}_{d-1}$. Dans la suite, on note $G=\mathfrak{S}_{d}$.

Soit un point du bord de $\overline{\mathcal{M}}_{g, G, \xi}$ qui est un revêtement simple stable

$$
\tilde{C} \longrightarrow C=\tilde{C} / \mathfrak{S}_{d-1} \longrightarrow D
$$

où $D=D_{1} \cup D_{2}$ est la réunion de deux $\mathbb{P}^{1}$ d'intersection $Q, b_{1}$ des points de branchement étant sur $D_{1}$, et $b_{2}=b-b_{1}$ étant sur $D_{2}$. On est dans le cas du segment. On reprend les notations de l'exemple 7.2.3. On considère un couple $\tilde{C}_{1}, \tilde{C}_{2}$ de composantes de $\tilde{C}$, l'image de $\tilde{C}_{i}$ étant $D_{i} \subset D$. On suppose que $\tilde{P} \in \tilde{C}_{1} \cap \tilde{C}_{2}$ est un point double. Soit enfin $G_{i}$ le groupe de Galois de $\tilde{C}_{i} \longrightarrow D_{i}$, et $H$ le stabilisateur 
de $\tilde{P}$. Rappelons que le graphe dual de $\tilde{C}$ est bipartite, les sommets étant répartis en l'orbite de $\tilde{C}_{1}$, et l'orbite de $\tilde{C}_{2}$.

Si $I \subset[1, d]$ est une partie non vide, soit $\mathfrak{S}_{I} \subset G=\mathfrak{S}_{d}$ le sous-groupe des permutations de $I$, i.e. les permutations de $[1, d]$ qui induisent l'identité sur $[1, d]-I$.

Proposition 7.2.5. - $\quad$ i) Il existe deux partitions $\mathscr{J}=\left(I_{\alpha}\right)_{1 \leqslant \alpha \leqslant r}, \mathcal{J}=\left(J_{\lambda}\right)_{1 \leqslant \lambda \leqslant s}$ de $I=[1, d]$, telles que ${ }^{(3)} \operatorname{Inf}(\mathcal{J}, \mathcal{g})=\{I\}$, et avec $G_{1}=\mathfrak{S}_{g}, G_{2}=\mathfrak{S}_{g}$.

ii) Les composantes irréductibles de $C$ qui s'appliquent sur $D_{1}$ sont en bijection avec les parties $I_{\alpha}$ de I. Si $C_{\alpha}$ correspond à $I_{\alpha}$, le degré de $C_{\alpha}$ sur $D_{1}$ est $d_{\alpha}=\left|I_{\alpha}\right|(1 \leqslant \alpha \leqslant r)$; conclusion identique pour les composantes $C_{\lambda}$ de $C$ d'image $D_{2}$.

iii) Les points doubles de $C$ sont en bijection avec les orbites de $H$ dans I. Si $\Delta$ est une telle orbite, le point double correspondant étant noté $P_{\Delta}$, soit $(\alpha, \lambda)$ l'unique couple tel que $\Delta \subset I_{\alpha} \cap J_{\lambda}$, alors $P_{\Delta} \in C_{\alpha} \cap C_{\lambda}$. Le revêtement $C_{\alpha} \longrightarrow D_{1}$ admet $b_{\alpha}$ points de branchement simples en dehors de l'origine $Q_{1} d u$ point double, et si $e=|\Delta|>1$, un point de branchement en $Q_{1}$, la monodromie au-dessus de ce point étant décrite par l'image de $H$ dans $\mathfrak{S}_{I_{\alpha}}$. De manière précise, les points de ramification de $C_{\alpha}$ au-dessus de $Q_{1}$ sont en bijection avec les orbites de $H$ dans $I_{\alpha}$, les indices de ramification étant les longueurs de ces orbites.

iv) Si $g=0$, les orbites de $H$ sont les $I_{\alpha} \cap J_{\lambda} \neq \varnothing$.

Démonstration. - i) On a $C=\tilde{C} / \mathfrak{S}_{d-1}$, avec par convention $\mathfrak{S}_{d-1}=\left\{\sigma \in \mathfrak{S}_{d}, \sigma(d)=\right.$ $d\}$. La donnée de ramification est préservée par spécialisation, elle ainsi lisible sur $\tilde{C}$, ce qui signifie que les stabilisateurs des points au-dessus des $b$-points de branchement, $b_{1} \geqslant 2$ sur $D_{1}, \quad b_{2} \geqslant 2$ sur $D_{2}$ sont d'ordre deux engendrés par des transpositions. Il n'y a pas de perte de généralité à supposer que $G_{1} \nsubseteq \mathfrak{S}_{d-1}$, ceci du fait que l'une des courbes de l'orbite de $\tilde{C}_{1}$ est ramifiée en au moins un point de $D_{1}$ autre que l'origine du point double. En particulier le sous-groupe $G_{1}$ est un sous-groupe de $G=\mathfrak{S}_{d}$ engendré par des transpositions, car c'est le groupe de Galois du revêtement $\tilde{C}_{1} \longrightarrow D_{1}=\mathbb{P}^{1}$, et que la donnée de ramification est sauf au plus en un point

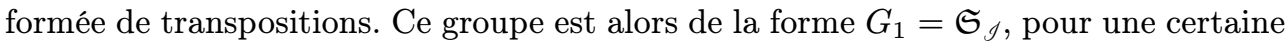
partition $\mathscr{I}=\left(I_{\alpha}\right)_{1 \leqslant \alpha \leqslant r}$ de $I$. On peut supposer quitte à renuméroter que $d \in I_{1}$, et $d_{1}>1$. Notons que pour tout point $x \in \tilde{C}_{1}, G x=G_{1} x$. Pour la même raison $\tilde{C}_{2}=\mathfrak{S}_{\mathrm{g}}$ pour une partition $\mathcal{J}=\left(J_{\lambda}\right)_{1 \leqslant \lambda \leqslant s}$. Le fait que $G_{1} \cup G_{2}$ engendre $G$ correspond à la condition indiquée sur le couple $\mathscr{I}, \mathcal{J}$. On a, avec un petit abus d'écriture

$$
G_{1} \cap \mathfrak{S}_{d-1}=\mathfrak{S}_{d_{1}-1} \subset \mathfrak{S}_{d_{1}}
$$

On voit ainsi que le revêtement $\tilde{C}_{1} \rightarrow D_{1}$ se factorise en

$$
\tilde{C}_{1} \longrightarrow C_{1}^{*}=C_{1} /\left(\prod_{\alpha=2}^{r} \mathfrak{S}_{I_{\alpha}}\right) \longrightarrow C_{1} \longrightarrow D_{1}
$$

(3) il n'existe pas de partition non triviale $I=A \sqcup B$ telle que pour tout $\alpha$, soit $I_{\alpha} \subset A$, soit $I_{\alpha} \subset B$, même chose pour les $J_{\lambda}$. 
Le revêtement galoisien $C_{1}^{*} \rightarrow D_{1}$ de groupe $\mathfrak{S}_{d_{1}}$ est la clôture galoisienne de $C_{1} \rightarrow$ $D_{1}$. La donnée de ramification de $\tilde{C}_{1}^{*} \rightarrow D_{1}$ en dehors de la fibre au-dessus de $Q$ est formée de transpositions, ce qui montre que le revêtement $C_{1} \rightarrow D_{1}$ est de degré $d_{1}$, et de ramification simple de dehors du point $Q$.

ii) Identifions les composantes de $C$ d'image $D_{1}$. Soient $g \tilde{C}_{1}, h \tilde{C}_{1}$ deux composantes de $\tilde{C}$ au-dessus de $D_{1}$. Elles ont même image dans $C$ si et seulement si il existe $s \in \mathfrak{S}_{d-1}$ tel que $h \tilde{C}_{1}=\xi\left(g \tilde{C}_{1}\right)$, soit $h^{-1} s g \in G_{1}$, c'est à dire $h^{-1} \in G_{1} \backslash \mathfrak{S}_{d} / \mathfrak{S}_{d-1}$. En conclusion, les composantes de $D$ d'image $D_{1}$ sont en bijection avec les classes doubles $G_{1} \backslash \mathfrak{S}_{d} / \mathfrak{S}_{d-1}$, et donc avec les parties $I_{\alpha}$ de $I$. On a observé ci-dessus que le degré sur $D_{1}$ de la composante attachée à $I_{\alpha}$ est $d_{\alpha}$. Conclusion identique pour les composantes au-dessus de $D_{2}$.

iii) Passons aux points doubles de $C$. Ils sont en bijection avec les classes doubles

$$
H \backslash \mathfrak{S}_{d} / \mathfrak{S}_{d-1}
$$

c'est à dire avec les orbites de $H$ (cyclique) dans $I=[1, d]$. Comme $H \subset G_{1} \subset G_{2}$, si $\Delta$ est une orbite de $H$, il existe un couple unique $(\alpha, \lambda)$ tel que

$$
\Delta \subset I_{\alpha} \cap J_{\Lambda}
$$

L'identification des composantes de $C$ avec les $I_{\alpha}$ (resp. $J_{\lambda}$ ) montre que les orbites de $H$ contenues dans $I_{\alpha} \cap J_{\lambda}$ correspondent bijectivement aux points doubles de $C_{\alpha} \cap C_{\lambda}$, soit

$$
P_{\delta} \in C_{\alpha} \cap C_{\lambda} \Longleftrightarrow \Delta \subset I_{\alpha} \cap J_{\lambda}
$$

Il résulte de i), ii) et (64) que le revêtement $C_{\alpha} \longrightarrow D_{1}=\mathbb{P}^{1}$, de degré $d_{\alpha}$, a $b_{1}$ points de branchement simples distincts de l'origine du point double, et au-dessus de l'origine $Q_{1}$ du point double $Q$ sur $D_{1}$, un branchement dont le type est décrit par le nombre d'orbites $\ell_{\alpha}$ de $H$ dans $I_{\alpha}$. Le nombre de ces orbites est le nombre de points de ramification au-dessus de $Q_{1}$, le cardinal est l'indice de ramification. La relation de Riemann-Hurwitz donne $b_{\alpha}$ en fonction du genre $g_{\alpha}$ de $C_{\alpha}$

$$
b_{\alpha}=2 g_{\alpha}-2+d_{\alpha}+\ell_{\alpha}
$$

Relation analogue pour le degré $f_{\lambda}$ de $C_{\lambda} \rightarrow D_{2}$. Notons la description du graphe dual de $C$ qui est quotient du graphe de $\tilde{C}$ par $\mathfrak{S}_{d-1}$. Il a pour sommets l'ensemble des classes doubles

$$
\mathfrak{S}_{d_{1}} \backslash \mathfrak{S}_{d} / \mathfrak{S}_{d-1} \sqcup \mathfrak{S}_{d_{2}} \backslash \mathfrak{S}_{d} / \mathfrak{S}_{d-1}
$$

et pour ensemble d'arêtes géométriques les classes doubles $H \backslash \mathfrak{S}_{d} / \mathfrak{S}_{d-1}$. Le genre $g$ de $C$ est ainsi donné par la relation usuelle, notant $\ell$ le nombre d'orbites de $H$ dans $I$

$$
g=\sum_{\alpha} g_{\alpha}+\sum_{\lambda} g_{\lambda}+\ell-r-s+1
$$

iv) Supposons $g=0$. Dans ce cas $g_{\alpha}=g_{\lambda}=0$, et $\ell=r+s-1$. Comme le graphe de sommets $[1, r] \sqcup[1, s]$, et d'arêtes les couples $(\alpha, \lambda)$ tels que $I_{\alpha} \cap J_{\lambda} \neq \varnothing$ est connexe, car $C$ est connexe, on voit que l'égalité $\ell=r+s-1$ impose aux $I_{\alpha} \cap J_{\lambda} \neq \varnothing$ d'être les orbites de $H$. 
Si $g=0$, notons le cas particulier $\left|I_{2}\right|=\cdots=\left|I_{r}\right|=\left|J_{2}\right|=\cdots\left|J_{s}\right|=1$, et $\left|I_{1}\right|>1,\left|J_{1}\right|>1$. Dans ce cas il y a une unique composante de degré $d_{1}>1$ sur $D_{1}$, et une unique composante de degré $>1$ sur $D_{2}$. Ces deux composantes se coupent en un point double dont l'holonomie le long des deux branches est cyclique. La proposition précédente permet d'avoir une idée précise sur la forme des revêtements simples stables d'une courbe de genre zéro qui sont réunion de deux $\mathbb{P}^{1}$. En particulier, on a une description des composantes irréductibles du bord de $\overline{\mathcal{H}}_{g, d}$ qui dominent une composante donnée $\Delta_{b_{1}, b_{2}}$ de $\bar{M}_{0, b}$. Les résultats des sections 9 et 10 permettront d'expliciter le diviseur $\delta^{*}\left(\Delta_{b_{1}, b_{2}}\right)$ comme combinaison linéaire des composantes précédentes. Cette description, jointe aux résultats de la section 10, suggère une interprétation en terme de dégénérescence de revêtements des relations de récursion entre nombres de Hurwitz, les relations "cut and join" de Goulden-Jackson, chose que nous ne développerons pas, voir cependant [84] pour des informations et des références.

7.2.3. Revêtements ramifiés de graphes. - Pour reconstruire le graphe $\Gamma$, partant du quotient $\Delta$ et d'une donnée additionnelle définie ci-après, on compense le fait que l'action de $G$ n'est pas libre par une structure de graphe de groupes sur le graphe quotient $\Delta$ (théorie de Bass-Serre, voir [7] et les références contenues dedans).

Rappelons qu'un graphe de groupes $\Delta$ (tous les graphes sont finis) est la donnée d'un graphe $\Delta$, tel qu'à tout sommet $v$ (resp. toute arête orientée $e$ ) est associé un groupe fini $G_{v}$ (resp. un groupe $H_{e}$ ); si $e$ pointe vers $v$, on a un morphisme injectif $\partial_{e}^{1}: H_{e} \longrightarrow G_{v}$, cette donnée étant telle que $H_{e}=H_{\bar{e}}$. On pose $\partial_{e}^{0}=\partial_{\bar{e}}^{1}$. On définit alors le groupe fondamental d'un graphe de groupes $\Delta$ de la manière suivante (loc. cit.) :

DÉFInition 7.2.6. - Soit $F_{\Delta}$ le groupe libre de base l'ensemble des arêtes orientées $\mathcal{F}_{\Delta} ;$ choisissons un arbre maximal $T \subset \Delta$. Le groupe fondamental $\pi_{1}(\Delta ; T)$ est défini comme étant le quotient du produit libre $F_{\Delta} \star\left(\star_{v} G_{v}\right)$ par le sous-groupe distingué engendré par les relations

$$
e=1 \quad(e \in T) \quad ; \quad e \bar{e}=1 \quad(e \in \Delta) \quad ; \quad e \partial_{e}^{1}(h) \bar{e}=\partial_{e}^{0}(h) \quad\left(e \in \Delta, h \in H_{e}\right) .
$$

Le groupe $\pi_{1}(\Delta ; T)$ ne dépend pas, à isomorphisme près, du choix de $T$. On a la description alternative suivante du groupe fondamental [7]; appelons chemin de $\Delta$ une suite

$$
\gamma=\left(g_{0}, e_{1}, g_{1}, \ldots, e_{n}, g_{n}\right)
$$

où $\left(e_{1}, \ldots, e_{n}\right)$ est un chemin « ordinaire », $a_{i}$ désignant le sommet terminal de $e_{i}$ (donc aussi le sommet initial de $e_{i+1}$ si $i<n$ ), $a_{0}$ étant le sommet initial de $e_{1}$, on a $g_{i} \in G_{a_{i}},(0 \leqslant i \leqslant n)$. Si $a_{0}=a_{n}, \gamma$ est un lacet en $v=a_{0}$. Ceci étant, on forme le groupe des chemins

$$
\pi(\Delta)=\left[\left(\star_{v} G_{v}\right) \star F_{\Delta}\right] / R
$$


où $R$ désigne le sous-groupe distingué engendré par les relations

$$
e \bar{e}=1(e \in \Delta), e \partial_{e}^{1}(h) \bar{e}=\partial_{e}^{0}(h) \quad\left(e \in \Delta, h \in H_{e}\right) .
$$

Si $\gamma$ est le chemin (67), on pose

$$
|\gamma|=g_{0} e_{1} g_{1} \ldots e_{n} g_{n} \in \pi(\Delta) \text {. }
$$

Lorsque $\gamma$ est un lacet en $v=a_{0}$, les éléments (68) forment un sous-groupe noté $\pi_{1}(\Delta, v)$. On montre alors [7] que la surjection $\pi(\Delta) \longrightarrow \pi_{1}(\Delta, T)$, restreinte au sous-groupe $\pi_{1}(\Delta, v)$ est un isomorphisme $\pi_{1}(\Delta, v) \cong \pi_{1}(\Delta, T)$.

Formons le graphe quotient $\Delta=\Gamma / G$, et soit $p: \Gamma \longrightarrow \Delta$ le morphisme quotient. On sait munir $\Delta$ d'une structure de graphe de groupes, structure qui capture les informations sur l'action de $G$, i.e. définissant sur $\Delta$ une structure « orbifold» [7]. Disons seulement, et cela sera suffisant pour la suite, que si $s$ est un sommet de $\Delta$, alors le groupe $G_{s}$ est essentiellement le stabilisateur d'un sommet $v \in \Gamma$ tel que $p(v)=s$; définition analogue pour $H_{e}$ si $e$ est une arête de $\Delta$. La construction nécessite le choix de sections de $p$ au niveau des sommets et arêtes. On notera dans la suite par $\Gamma / / G$ le graphe $\Delta$ muni de la structure de graphe de groupes quotient. On a besoin du résultat suivant de Bass-Serre (Bass [7], theorem 3.6) :

THÉORÈme 7.2.7. - Il existe une surjection naturelle $\psi: \pi_{1}(\Gamma / / G) \longrightarrow G$, appelée morphisme de monodromie, telle que $\operatorname{ker} \psi \cong \pi_{1}(\Gamma)$ (groupe fondamental du graphe ordinaire quotient).

La définition de $\psi$ mime la définition usuelle de l'action de monodromie. Noter que par simplicité, dans les $\pi_{1}$ on ne fait pas référence aux sommets de base. Le théorème 7.2.7 dit qu'on peut reconstruire le graphe $\Gamma$ muni de l'action de $G$, partant de la structure de graphe de groupes sur $\Delta=\Gamma / / G$, et du morphisme de monodromie, comme dans le cas classique. Le graphe $\Gamma$ apparaît comme le quotient du revêtement universel de $\Gamma / / G$ par $\pi_{1}(\Gamma)$

DÉFInItion 7.2.8. - Sous les hypothèses précédentes, notons $D$ le sous-groupe distingué de $G$ engendré par les stabilisateurs des sommets (sous-groupe de décomposition), et notons I le sous-groupe distingué engendré par les stabilisateurs des arêtes (sous-groupe d'inertie).

On peut préciser la structure du groupe fondamental ordinaire de $\Delta=\Gamma / G$ comme suit :

Proposition 7.2.9. - Il y a une surjection naturelle $\phi: \pi_{1}(\Gamma / G) \longrightarrow G / D$, qui conduit à une suite exacte

$$
\pi_{1}(\Gamma) \longrightarrow \pi_{1}(\Gamma / G) \longrightarrow G / D \longrightarrow 1
$$


Démonstration. - Définissons d'abord le morphisme $\phi$. Soit $a_{0} \in \Gamma$ un sommet, et posons $\bar{a}_{0}=p\left(a_{0}\right)$. Si $\bar{\gamma}=\left(\bar{e}_{1}, \ldots, \bar{e}_{n}\right)$ représente un lacet de $\Delta$ basé en $\bar{a}_{0}$, on peut le relever en un lacet $\gamma=\left(e_{1}, \ldots, e_{n}\right)$ d'origine $a_{0}$. Soit $s$ le sommet terminal de l'arête $e_{n}$; alors $p(s)=p\left(a_{0}\right)$. Il y a donc $g \in G$ tel que $s=g a_{0}$. On doit vérifier que la classe de $g$ modulo $D$ ne dépend pas du relèvement choisi. Soit en effet $\left(e_{1}^{\prime}, \ldots, e_{n}^{\prime}\right)$ un autre choix de relèvement, de sorte qu'il y a $g_{1} \in G$ avec $e_{1}^{\prime}=g_{1} e_{1}$. L'action de $G$ étant sans inversion, $g_{1} a_{0}=a_{0}$, donc $g_{1} \in D$. Le relèvement $\left(e_{1}, g_{1} e_{2}^{\prime}, \ldots, g_{1} e_{n}^{\prime}\right)$ diffère de $\left(e_{1}, \ldots, e_{n}\right)$ par au plus $(n-1)$ arêtes. Par une récurrence immédiate, on peut supposer que si $s^{\prime}$ est le sommet terminal de $e_{n}^{\prime}$, alors pour le sommet terminal de $g_{1} e_{n}^{\prime}$, on a $g_{1} s^{\prime} \in g D a_{0}$. On en tire immédiatement que $s^{\prime} \in g D a_{0}$, et donc $g$ et $g^{\prime}$ sont dans la même classe modulo $D$. Le morphisme $\phi$ assigne à $\gamma$, la classe modulo $D$ de l'élément $g$ ci-dessus. La vérification du fait que le morphisme $\phi$ est bien défini est évidente.

On sait par ailleurs que si $R$ est le sous-groupe distingué de $G$ engendré par les sous-groupes $G_{s}, s \in \Gamma / / G$, alors $\pi_{1}(\Gamma / / G) / R \cong \pi_{1}(\Gamma / G)$. La construction montre qu'en fait $\phi$ coïncide avec le morphisme déduit de $\psi$ par passage au quotient par les groupes $R$ et $D$ respectivement. Cela a pour traduction un diagramme commutatif :

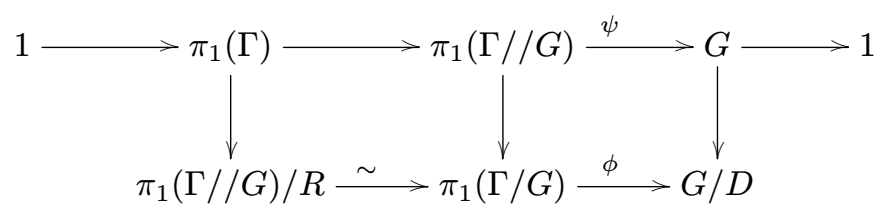

De ce diagramme, on tire un morphisme composé $\theta: \pi_{1}(\Gamma) \longrightarrow \pi_{1}(\Gamma / G)$ qui n'est pas autre chose que le morphisme canonique déduit de $p$, et aussi l'égalité $\psi(R)=D$. La conclusion vient alors d'une chasse au diagramme immédiate.

Passant à l'homologie, la suite exacte (70) donne immédiatement comme corollaire : Corollaire 7.2.10. - Sous les hypothèses précédentes, on a une suite exacte canonique :

$$
H_{1}(\Gamma, \mathbb{Z}) \longrightarrow H_{1}(\Gamma / G, \mathbb{Z}) \longrightarrow(G / D)_{\mathrm{ab}} \longrightarrow 1
$$

où $(G / D)_{\text {ab }}$ désigne l'abélianisé du groupe $G / D$.

\subsection{Groupe de Picard et revêtements}

7.3.1. Sous-groupes de décomposition et d'inertie. - La proposition 7.2.9 admet une interprétation géométrique simple lorsque $\Gamma$ est le graphe dual (modulaire) d'une courbe préstable, sur laquelle le groupe $G$ agit stablement. Soit C une telle courbe définie sur $k$ corps algébriquement clos, de composantes irréductibles $C_{1} \ldots, C_{s}$, de sorte que $s$ représente le nombre de sommets de $\Gamma$. Soit la normalisation $\tilde{C}$ de $C$ :

$$
\tilde{C}=\bigsqcup_{i} \tilde{C}_{i} \longrightarrow C
$$


On considère $\tilde{C}$ comme munie de l'action induite de $G$, et de même pour $\Gamma$. On note $G_{i}$ le stabilisateur de $C_{i}$; si $p$ est un point double, on notera $G_{p}$ son stabilisateur (cyclique). Soient, comme dans la section précédente, les sous-groupes distingués de décomposition $D$ et d'inertie $I$. On notera pour simplifier $H^{1}(\Gamma)$ le groupe $H^{1}(\Gamma, \mathbb{Z})$. Rappelons tout d'abord la description bien connue du groupe de Picard de $C$; on le décrit au moyen de la suite exacte suivante (voir [30], lemma 1.17) :

$$
1 \longrightarrow H^{1}(\Gamma) \otimes k^{*} \longrightarrow \operatorname{Pic}(C) \longrightarrow \operatorname{Pic}(\tilde{C})=\prod_{i} \operatorname{Pic}\left(\tilde{C}_{i}\right) \longrightarrow 1
$$

Considérons la courbe quotient $D=C / G$, qui est préstable, et soit $\pi: C \longrightarrow D=$ $C / G$ le morphisme quotient. Une suite exacte analogue à celle utilisée pour décrire $\operatorname{Pic}(C)$, appliquée à $\operatorname{Pic}(C / G)$, conduit à un diagramme commutatif

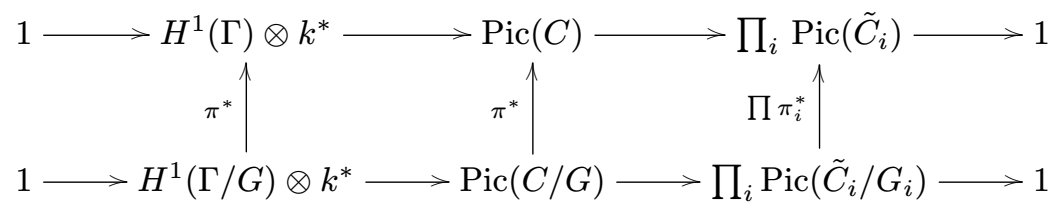

dans lequel les flèches verticales sont induites par les morphismes quotients respectifs, notés sans distinction $\pi$. On peut noter que la flèche verticale $\pi^{*}$ de gauche est celle qui dérive du morphisme de graphes $\pi: \Gamma \longrightarrow \Gamma / G$.

On va préciser le contenu du diagramme (72) en étudiant la suite exacte formée par les noyaux des flèches verticales. Rappelons que si $A$ est un groupe fini (de cardinal toujours supposé premier à la caractéristique de $k$ en ce qui nous concerne), on note $\hat{A}=\operatorname{Hom}\left(A, k^{*}\right)$ le groupe des caractères à valeurs dans $k$. Le résultat suivant est important pour la suite :

ThÉORÈme 7.3.1. - Le sous-groupe d'inertie de $\left(\tilde{C}_{i}, G_{i}\right)$ étant noté $I_{i}$, la suite exacte des noyaux du diagramme (72) s'identifie canoniquement $\grave{a}$ :

$$
1 \longrightarrow \widehat{G / D} \longrightarrow \widehat{G / I} \longrightarrow \prod_{i} \widehat{G_{i} / I_{i}}
$$

Démonstration. - La preuve utilise la structure naturelle de $G$-faisceau sur $D=C / G$ supportée par $\pi_{*}\left(\theta_{C}\right)$. Du fait de la présence de points doubles (éventuels) avec un groupe d'isotropie non trivial, ce faisceau n'est pas en général localement libre, il est seulement de type fini et sans torsion. Cependant, comme la ramification est modérée, on a toujours une décomposition en facteurs isotypiques (voir section 3.2) :

$$
\pi_{*}\left(\theta_{C}\right)=\bigoplus_{\chi \in \operatorname{Irrep}(G)} \mathscr{L}_{\chi} \otimes V_{\chi},
$$

où $V_{\chi}$ désigne l'espace supportant la représentation irréductible de caractère $\chi$, et $\mathscr{L}_{\chi}$ étant un faisceau sans torsion donné par $\mathscr{L}_{\chi}=\pi_{*}^{G}\left(\theta_{C} \otimes \check{V}_{\chi}\right)$. Analysons plus en détail cette décomposition au voisinage d'un point double $q=\pi(p)$ de $D=C / G$, le point double $p$ étant de stabilisateur $H$. Après localisation et complétion, on est amené 
à décomposer $\pi_{*}\left(\widehat{\vartheta}_{C}\right)_{q}$ en tant que $\left(\widehat{\vartheta}_{D, q}, G\right)$-module. La décomposition suivante est immédiate :

$$
\pi_{*}\left(\widehat{\theta}_{C}\right)_{q} \cong \operatorname{Ind}_{H}^{G}\left(\widehat{\theta}_{C, p}\right) \text {. }
$$

On est de la sorte ramené à supposer que $\widehat{\theta}_{C, p}=k[[x, y]] /(x y)$, et que l'action sur les deux branches d'un générateur $\sigma \in H$ de $H$, se réduit à la forme usuelle $\sigma(x)=\zeta x$, $\sigma(y)=\zeta^{-1} y$ pour une certaine racine de l'unité $\zeta$ d'ordre $e=|H|$. Ainsi, si on pose $u=x^{e}, v=y^{e}$, on a $\widehat{\vartheta}_{D, q}=k[[u, v]] /(u v)$. La décomposition cherchée est alors totalement explicite :

$$
\widehat{\vartheta}_{C, p} \cong \widehat{\vartheta}_{D, q} \bigoplus\left(\bigoplus_{j=1}^{e-1}\left(x^{j}, y^{e-j}\right) \widetilde{\widehat{\vartheta}_{D, q}}\right)
$$

où le symbole tilde désigne la normalisation. Notons $\mu_{j}$ la représentation de degré 1 de caractère $\mu_{j}(\sigma)=\zeta^{j}$, de sorte que la décomposition (75) équivaut à

$$
\widehat{\theta}_{C, p} \cong \widehat{\theta}_{D, q} \bigoplus\left(\bigoplus_{j=1}^{e-1} \mu_{j} \otimes \widehat{\widehat{\vartheta}}_{D, q}\right)
$$

donnant finalement

$$
\pi_{*}\left(\widehat{\vartheta}_{C}\right)_{q} \cong \widehat{\vartheta}_{D, q} \otimes \operatorname{Ind}_{H}^{G}\left(1_{H}\right) \bigoplus\left(\bigoplus_{j=1}^{e-1} \widetilde{\widehat{\mathscr{\theta}}_{D, q}} \otimes \operatorname{Ind}_{H}^{G}\left(\mu_{j}\right)\right) .
$$

De cette décomposition on extrait le fait que le facteur $\mathscr{L}_{\chi}$ est sans torsion de rang égal au degré de $\chi$. Par réciprocité de Frobenius, un caractère irréductible de $G$ apparaît dans $\operatorname{Ind}_{H}^{G}\left(\mu_{j}\right)$ que si la restriction de $\chi$ à $H$ contient $\mu_{j}$. On voit ainsi que $\mathscr{L}_{\chi}$ est localement libre en $q=\pi(p)$ si et seulement si la restriction de $\chi$ à $H$ est le caractère trivial $^{(4)}$. En particulier, $\mathscr{L}_{\chi}$ est localement libre si et seulement si $\chi$ est non ramifié, au sens de la définition suivante :

DÉFInItion 7.3.2. - Le caractère $\chi$ est dit non ramifié si $\chi(I)=1$, i.e. si $\chi$ est trivial sur le sous-groupe d'inertie.

Pour terminer la preuve, on montre d'abord que le noyau de $\pi^{*}: \operatorname{Pic}(C / G) \longrightarrow$ $\operatorname{Pic}(C)$ s'identifie canoniquement à $\widehat{G / I}$. C'est un fait bien connu, du moins dans le cas lisse. Adaptons la démonstration aux conditions de la présente situation. Soit $\mathscr{L}$ un élément du noyau, de sorte que $\pi^{*}(\mathscr{L}) \cong \theta_{C}$ définit une $G$-linéarisation de $\theta_{C}$. La courbe $C$ étant réduite et connexe, une telle linéarisation est définie par un caractère $\chi$ de $G$. Soit alors $\theta_{C}(\chi)$ le faisceau structural de $C$ ainsi $G$-linéarisé. Sous ces conditions, on a

$$
\mathscr{L} \cong \pi_{*}^{G} \pi^{*}(\mathscr{L}) \cong \pi_{*}\left(\theta_{C}(\chi)\right)=\mathscr{L}_{\chi}
$$

Noter qu'alors $\chi$ est non ramifié. Inversement, si $\chi$ est un tel caractère, on a un morphisme canonique $\pi^{*}\left(\mathscr{L}_{\chi}\right) \longrightarrow \mathscr{\theta}_{C}$ entre faisceaux inversibles qui est un

(4) La décomposition (76) montre en fait que $\left(\mathscr{L}_{\chi}\right)_{q} \cong \widehat{\vartheta}_{D, q}^{r} \oplus\left(\widetilde{\widehat{\vartheta}_{D, q}}\right)^{s}$, avec $r=\langle 1, \chi\rangle_{H}$. 
isomorphisme sur un ouvert dense, donc un isomorphisme. Si $\chi$ est non ramifié, cela prouve que le facteur isotypique $\mathscr{L}_{\chi}$ de la décomposition (74) est un faisceau inversible qui est dans le noyau de $\pi^{*}$. Sous une forme explicite, l'identification cherchée est $\chi \in \widehat{G / I} \rightsquigarrow \mathscr{L}_{\chi}$. Le même argument appliqué aux composantes $\tilde{C}_{i}$ de la normalisation donne

$$
\operatorname{ker}\left(\pi^{*}: \operatorname{Pic}\left(\tilde{C}_{i} / G_{i}\right) \longrightarrow \operatorname{Pic}\left(\tilde{C}_{i}\right)\right) \cong \widehat{G_{i} / I_{i}}
$$

Le résultat découle alors, après identification, de la suite exacte des noyaux.

Corollaire 7.3.3. - Le facteur isotypique $\mathscr{L}_{\chi}$ de la décomposition (74) est

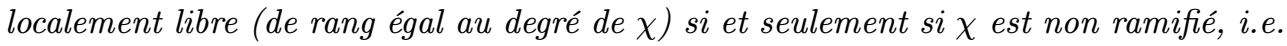
$\chi(I)=1$.

REMARQUE 7.3.4. - Le corollaire précédent apporte une réponse équivalente à l'alternative qu'on rencontre usuellement, lorsque dans une compactification, un faisceau inversible dégénère par spécialisation en un point du bord. Pour comparaison avec l'étude du bord de l'espace modulaire des courbes à spin, problème au parallélisme frappant, la correspondance avec la terminologie employée par Jarvis (voir [60]) est

$$
\chi \text { est }\left\{\begin{array}{l}
\text { non ramifié si } \chi \text { est RR (Ramond-Ramond), } \\
\text { ramifié si } \chi \text { est NS (Neveu-Schwarz). }
\end{array}\right.
$$

7.3.2. Faisceaux sans torsion de rang 1 et revêtements stables. - Soit $p$ : $D \longrightarrow S$ une courbe préstable. Rappelons la définition :

DÉFInITION 7.3.5. - Un faisceau cohérent $E$ sur $D$ est dit sans torsion de rang $n$ relativement à $S$ si $E$ est plat sur $S$, et si sur chaque fibre $D_{s}$, le faisceau induit $E_{s}$ est sans torsion de rang $n$.

Si $E$ est sans torsion, $E$ est localement libre sur l'ouvert de lissité de $p$. Appelons point singulier de $E$ un point en lequel $E$ n'est pas libre et notons $\operatorname{Sing}(E)$ l'ensemble de ces points. Un théorème de Faltings ([45] theorem 3.5, voir aussi [59] section 2.2) précise la structure locale de $E$ en un point de $\operatorname{Sing}(E)$, donc en un point de $C$ qui est un point double de sa fibre. Pour décrire le résultat, on peut choisir des coordonnées locales le long des branches, et supposer que $E$ est un module sans torsion de rang $n$ sur l'anneau local noethérien complet $A=R[[x, y]] /(x y-\pi)$, où $R=\hat{\theta}_{s}$ et $\pi \in \mathcal{M}_{R}$. Si l'on note $\mathfrak{a}=A \otimes k$, alors on sait que $E \otimes k$ est une somme directe de copies de $\mathfrak{a}$ et de sa normalisation $\tilde{\mathfrak{a}}$ :

$$
E \otimes k \cong \mathfrak{a}^{r} \oplus \tilde{\mathfrak{a}}^{s} \quad(r+s=n) .
$$

Comme indiqué dans [45], pour décrire la structure locale de $E$, on peut se limiter au cas $n=s$. La construction d'une déformation verselle de $\tilde{\mathfrak{a}}^{n}$ se fait comme suit (noter que seul le cas $n=1$ sera en fait utilisé). Supposons que $P, Q \in \mathrm{M}_{n}(R)$ sont deux 
matrices carrées de taille $n$ telles que $P \cdot Q=Q \cdot P=\pi 1_{n}$, où $1_{n}$ désigne la matrice identité. Définissons deux matrices carrées de taille $2 n$, à coefficients dans $A$, par

$$
\Phi=\left(\begin{array}{cc}
x .1 & P \\
Q & y .1
\end{array}\right) \quad, \quad \Psi=\left(\begin{array}{cc}
y .1 & -P \\
-Q & x .1
\end{array}\right)
$$

Alors $\Phi \Psi=\Psi \Phi=0$, et $E(P, Q)=\operatorname{ker} \Psi=\operatorname{im} \Phi$ est sans torsion de rang $n$, de réduction $\tilde{\mathfrak{a}}^{n}$. Réciproquement, tout module sans torsion de rang $n$, de réduction $\tilde{\mathfrak{a}}^{n}$, est isomorphe à un $E(P, Q)$. On peut prouver (loc. cit.) que si $P \equiv Q \equiv 0\left(\bmod \mathcal{M}_{R}\right)$, alors le couple $(P, Q)$ est déterminé à la transformation suivante près :

$$
P^{\prime}=U P V^{-1} \quad, \quad Q^{\prime}=V Q U^{-1} \quad\left(U, V \in \mathrm{GL}_{n}(R)\right) .
$$

Revenons à un $G$-revêtement galoisien stable $\pi: C \longrightarrow D$ au-dessus de la base $S=\operatorname{Spec}(R)$, l'anneau $R$ étant local noethérien complet. Soit $P \in C$ un point double de la fibre spéciale, et soit $Q=\pi(P)$.

Il s'agit de décrire selon les termes de la construction de Faltings le module sans torsion $E=\left(\widehat{\pi_{*}\left(\theta_{C}\right)}\right)_{Q}$, de rang $n=|G|$, et ses facteurs isotypiques $\mathscr{L}_{\chi}$. Soient $x, y$ des coordonnées locales le long des branches en $P$, telles qu'un générateur $\tau$ du stabilisateur $H$ de $P$ agisse par $\tau(x)=\zeta x, \tau(y)=\zeta^{-1} y$, pour une certaine racine $e$-ième de l'unité $\zeta$. On peut supposer que $A=\widehat{\theta_{C}, P}=R[[x, y]] /(x y-\pi)$ où $\pi \in \mathcal{M}_{R}$, en conséquence

$$
B=A^{H}=\widehat{\theta_{D}, Q}=R[[u, v]] /\left(u v-\pi^{e}\right)
$$

avec $u=x^{e}, v=y^{e}$. Un élément $\xi \in A$ admet une écriture unique de la forme

$$
\xi=f(x)+g(y) \quad, \quad f(x) \in R[[x]] \quad, \quad g(y) \in y R[[y]] .
$$

Il est facile de voir que le facteur isotypique de caractère $\zeta^{\alpha}$ de $A$, est le sous$B$-module $\mathscr{L}_{\alpha}$ formé par les éléments de la forme

$$
x^{\alpha} f(u)+y^{e-\alpha} g(v) \quad(f \in R[[u]], g \in R[[v]]) .
$$

Il est aisé d'identifier ce module; on note dans la suite $\beta=e-\alpha$.

LEMME 7.3.6. - Le module $\mathscr{L}_{\alpha}$ est le conoyau de l'application $B^{2} \longrightarrow B^{2}$ définie par la matrice $\Phi=\left(\begin{array}{cc}u & \pi^{\alpha} \\ \pi^{\beta} & v\end{array}\right)$; il est en particulier sans torsion de rang 1 , et $\mathscr{L}_{\alpha} \cong$ $E\left(\pi^{\alpha}, \pi^{\beta}\right)$.

Démonstration. - Soit l'application $\Psi: B^{2} \longrightarrow A$ définie par la matrice $\left(y^{\beta},-x^{\alpha}\right)$. On a clairement $\Psi . \Phi=0$. Montrons que $\operatorname{ker} \Psi=\operatorname{im} \Phi$. Soit $(f, g) \in B^{2}$ tel que $y^{\beta} f-x^{\alpha} g=0$. Ecrivons $f$ et $g$ sous la forme

$$
\begin{aligned}
& f=f_{+}(u)+a_{0}+f_{-}(v) \\
& g=g_{+}(u)+b_{0}+g_{-}(v)
\end{aligned}
$$


les séries $f_{+}, f_{-}, g_{+}, g_{-}$étant sans terme constant, et $a_{0}, b_{0} \in R$. Un calcul élémentaire montre que l'équation $y^{\beta} f-x^{\alpha} g=0$ implique les deux égalités :

$$
\begin{aligned}
& g_{+}(u)=\frac{\pi^{\beta}}{u} f_{+}(u)-b_{0} \\
& f_{-}(v)=\frac{\pi^{\alpha}}{v} g_{-}(v)-a_{0}
\end{aligned}
$$

En posant $\phi(u)=\frac{f_{+}(u)}{u}$ et $\psi(v)=\frac{g_{-}(v)}{v}$, on obtient bien :

$$
\left(\begin{array}{l}
f \\
g
\end{array}\right)=\left(\begin{array}{cc}
u & \pi^{\alpha} \\
\pi^{\beta} & v
\end{array}\right)\left(\begin{array}{l}
\phi(u) \\
\psi(v)
\end{array}\right)
$$

En utilisant les notations de dessus, notons que

$$
\widehat{\vartheta}_{C, P}=\widehat{\vartheta}_{D, Q} \bigoplus\left(\bigoplus_{\alpha=1}^{e-1} E\left(\pi^{\alpha}, \pi^{\beta}\right) \otimes W_{\alpha}\right)
$$

où $W_{\alpha}$ désigne la représentation de degré 1 de $H$ telle que $\tau \in H$ agisse par la multiplication par $\zeta^{\alpha}$. D'où, par induction de $H$ à $G$ :

$$
\widehat{\pi_{*}\left(\vartheta_{C}\right)_{Q}}=\widehat{\vartheta}_{D, Q} \otimes \operatorname{Ind}_{H}^{G}(1) \bigoplus\left(\bigoplus_{\alpha=1}^{e-1} E\left(\pi^{\alpha}, \pi^{\beta}\right) \otimes \operatorname{Ind}_{H}^{G}\left(W_{\alpha}\right)\right) .
$$

Pour tout caractère irréductible $\chi$ de $G$, soit $n_{\alpha, \chi}$ la multiplicité de $W_{\alpha}$ dans la restriction de $\chi$ à $H$. Le facteur isotypique $\mathscr{L}_{\chi}$ admet finalement la structure suivante en le point double $Q \in D$ :

Proposition 7.3.7. - La structure du module sans torsion $\left(\widehat{\mathscr{L}}_{\chi}\right)_{Q}$ est donnée par :

$$
\left(\widehat{\mathscr{L}}_{\chi}\right)_{Q} \cong \bigoplus_{\alpha=0}^{e-1} E\left(\pi^{\alpha}, \pi^{\beta}\right)^{n_{\alpha, \chi}}
$$

On notera que la situation considérée correspond à une matrice $P$ diagonale. Le cas $G$ abélien sera détaillé dans la section 8 . Le fait que les faisceaux $\mathcal{L}_{\chi}$ peuvent être sans torsion de rang 1, non inversibles, peut être contourné par la construction suivante, inspirée par la procédure de stabilisation de Knudsen [68] (voir aussi [58], $3.1 .2)$.

Soit une courbe préstable $D \longrightarrow S$, et soit $\mathscr{L}$ un $\vartheta_{D}$-module sans torsion de rang 1 relativement à $S$. On note $\operatorname{Sym}^{\bullet}(\mathscr{L})$ l'algèbre symétrique de $\mathcal{L}$. Soit le $D$-schéma

$$
\rho: \tilde{D}=\operatorname{Proj}(\operatorname{Sym} \bullet(\mathscr{L})) \longrightarrow D \text {. }
$$

Si $\mathscr{L}$ est localement libre, alors $\tilde{D}=D$. Si $\mathscr{L}$ est sans torsion de rang 1 de lieu singulier $\operatorname{Sing}(\mathscr{L})$ non vide, la structure locale de $\rho$ se décrit comme suit. Soit le morphisme

$$
\rho: \tilde{D}=\operatorname{Proj}(A[\xi, v] /(v x-p \xi, v q-y \xi)) \longrightarrow D=\operatorname{Spec}(B) .
$$


Le schéma $\tilde{D}$ est un sous-schéma fermé de $D \times \mathbb{P}^{1}$, en particulier il est recouvert par les deux ouverts affines

$$
U_{1}=\operatorname{Spec}(A[s] /(s x-p, s q-y)) \quad\left(s=\frac{v}{\xi}\right)
$$

et

$$
U_{2}=\operatorname{Spec}(A[t] /(x-p t, q-y t)) \quad\left(t=\frac{\xi}{v}\right) .
$$

Sur $U_{1} \cap U_{2}$, on a $s t=1$. Soit dans $D$ le lieu singulier $S:=(x=y=0)=\operatorname{Spec}(R /(\pi))$. Posons $\tilde{S}=\rho^{-1}(S)$.

Proposition 7.3.8. - $\quad$ i) En dehors de $\tilde{S}$, $\rho$ est un isomorphisme, $\rho: \tilde{D}-\tilde{S} \cong$ $D-S$.

ii) Le lieu exceptionnel $\tilde{S}$ est réunion d'une partie horizontale $E$ de codimension un, qui est une fibration en $\mathbb{P}^{1}$ au-dessus d'un sous-schéma fermé de $S$, et de deux parties 《verticales 》

$$
Z_{0}=\operatorname{Spec}(R /(p)) \quad, \quad Z_{\infty}=\operatorname{Spec}(R /(q)) .
$$

L'anneau local complété dans $\tilde{D}$ du point $(x=y=s=0) \in Z_{0}$ est $R[[s, x]] /(s x-$ $p)$, et le point $(x=y=t=0) \in Z_{\infty}$ a pour anneau local complété $R[[t, y]] /(t y-$ $q)$. En particulier $\tilde{D} \rightarrow S$ est une courbe préstable.

iii) Le lieu singulier de $\tilde{D}$ est $Z=Z_{0} \sqcup Z_{\infty}$.

iv) Le faisceau $\rho^{*}(\mathcal{L})$ admet une quotient localement libre de rang un.

Démonstration. - i) Au-dessus de $x \neq 0$, on a $A[\xi, v]\left[x^{-1}\right] /(v x-p \xi, v q-y \xi)=$ $A\left[\xi, x^{-1}\right] /(v q-y \xi)=A\left[\xi, x^{-1}\right]$ car dans $A\left[\xi, x^{-1}\right]$ on a $x(v q-y \xi)=0$ donc $v q-y \xi=0$. On a alors $\operatorname{Proj}\left(A\left[\xi, x^{-1}\right]\right)=\operatorname{Spec}\left(A\left[x^{-1}\right]\right)$.

ii) On a $U_{1} \cap \tilde{S}=\operatorname{Spec}(A[s] /(p, y, s q))=\operatorname{Spec}(R /(p)[s] /(s q))$. Ce sous-schéma fermé est l'union de deux parties, d'une part le fermé $q=0$, qui donne la fibration en droites

$$
E \cap U_{1}=\operatorname{Spec}(R /(p, q)[s])
$$

et d'autre part le fermé $Z_{0}: s=0$, donc $Z_{0}=\operatorname{Spec}(A /(p))$. Sur la carte $U_{2}$, on a de la même manière $E \cap U_{2}=\operatorname{Spec}(R /(p, q)[t])$ et $Z_{\infty}=\operatorname{Spec}(R /(q))$. Donc $E=$ $\operatorname{Spec}(R /(p, q)) \times \mathbb{P}^{1}$ est une fibration en $\mathbb{P}^{1} \operatorname{sur} \operatorname{Spec}(R /(p, q))$.

Supposons $S$ lisse, i.e. $R=k\left[\left[\tau=t_{1}, t_{2}, \ldots, t_{N}\right]\right]$, avec $\pi=\tau^{e}, p=\tau^{\alpha}, q=\tau^{\beta}$, $\alpha, \beta \geqslant 1, \alpha+\beta=e$. Alors $\tilde{S}=\operatorname{Spec}\left(R /\left(\tau^{e}\right)\right)$ mais $S_{\text {red }}=\operatorname{Spec}(R /(\tau))$ est lisse. On voit aussi que $E$ n'est pas lisse, mais que $E_{\text {red }}$ qui est un fibré en $\mathbb{P}^{1}$ sur $S_{\text {red }}$ est lisse, ainsi $E_{\text {red }}$ définit un diviseur de Weil dans $\tilde{D}$. Les sous-schémas fermés $Z_{0}, Z_{\infty}$ lieu des points doubles, sont des épaississement des sections $s=0$ (resp. $\infty$ ).

iii) Soit $z_{0} \in Z_{0}$ le point d'idéal $(s, \tau)$, alors $\hat{\vartheta}_{\tilde{D}, z_{0}}=R[[s, x]] /(s x-p)$ exhibe une singularité $A_{\beta-1}$. Le groupe de Picard en ce point est cyclique d'ordre $\alpha$, et, de manière similaire, il est cyclique d'ordre $\beta$ au point correspondant $z_{\infty} \in Z_{\infty}$. On voit ainsi que $E_{\text {red }}$ est un diviseur de Cartier si et seulement si $\operatorname{ppcm}(\alpha, \beta)$ divise $e$. Finalement $Z=Z_{0} \sqcup Z_{\infty}$ est le lieu singulier de $\tilde{D}$. 
iv) Clair car la construction fournit un morphisme canonique surjectif $\rho^{*}(\mathscr{L}) \rightarrow$ $\theta_{\tilde{D}}(1)$.

On remarquera que la décomposition $x y=\pi=p q$ correspond à une section de la courbe

$$
q: D=\operatorname{Spec}(R[[x, y]] /(x y-\pi)) \longrightarrow D=\operatorname{Spec}(R) .
$$

Le passage de $D$ à $\tilde{D}$ est exactement l'opération de stabilisation de Knudsen de cette section qui en $x=y=0$ passe par le point double.

Le faisceau inversible $\Theta_{\tilde{D}}(1)$ sur $\tilde{D}$ résout les singularités de $\mathscr{L}$ dans le sens naturel suivant (Jarvis [58], [60]) :

Proposition 7.3.9. - Le schéma $\tilde{D}$ est une $S$-courbe préstable, et

i) On $a \rho_{*}\left(\theta_{\tilde{D}}\right)=\theta_{D}$ et $\rho_{*}\left(\theta_{\tilde{D}}(1)\right)=\mathscr{L}$,

ii) $S i j>0$ et $n \geqslant 0, R^{j} \rho_{*}(\theta(n))=0$,

iii) La formation de $\tilde{D}$ ainsi que de $\rho_{*}\left(\Theta_{\tilde{D}}(1)\right)$ commute à tout changement de base. De plus $\omega_{\tilde{D} / S}=\rho^{*}\left(\omega_{D / S}\right)$, de sorte que la restriction de $\omega_{\tilde{D} / S}$ à toute composante exceptionnelle est triviale.

Démonstration. - La preuve est contenue dans les références précédentes, particulièrement ([58], lemma 3.1.4, [60], theorem 3.3.9). Au voisinage d'un point double du type NS, $\tilde{D} \longrightarrow D$ est comme indiqué au-dessus le morphisme de stabilisation de Knudsen, de sorte que les propriétés de $\tilde{D} \longrightarrow S$ découlent de ([68], theorem 2.4).

\subsection{Stratification canonique du bord}

7.4.1. Stratification du bord. - Une propriété appréciable de $\bar{M}_{g, n}$ est que le bord $\partial \overline{\mathcal{M}}_{g, n}$, sous-champ fermé lieu des courbes stables à points doubles, admet une stratification naturelle par le type combinatoire d'une courbe stable marquée (resp. piquée) ([76], chap. III, section 2.7). Pour rappeler comment cela fonctionne, fixons un graphe modulaire $\Gamma$ de genre $g$, avec $n$ pattes (voir section 7.1). Pour fixer les idées on suppose le graphe marqué, les pattes sont alors numérotées de 1 à $n$. Soit pour tout sommet $v \in V, h_{v}$ (resp. $l_{v}$ ) le nombre de pattes (resp. d'arêtes) incidentes en $v$. On définit un morphisme

$$
\beta_{\Gamma}: \prod_{v \in V} \bar{M}_{g_{v}, h_{v}+l_{v}} \longrightarrow \overline{\mathcal{M}}_{g, n}
$$

de la manière suivante. On commence par numéroter l'ensemble $V$, soit $V=$ $\left\{v_{1}, \ldots, v_{c}\right\}$, et aussi pour tout sommet $v=v_{i}, e_{1}^{v}, \ldots, e_{l_{v}}^{v}$ l'ensemble des arêtes d'origine $v$. Noter que le graphe étant marqué, les pattes incidentes à $v$ sont ordonnées par l'ordre induit de $[1, n]$, soit $p_{1}^{v}, \ldots, p_{h_{v}}^{v}$ cette liste ordonnée. La donnée d'un objet $\left(\left(C_{i}, x_{1}^{i}, \ldots x_{h_{i}+l_{i}}^{i}\right)_{v_{i} \in V}\right)$ au-dessus de la base $S$ de $\prod_{v \in V} \overline{\mathcal{M}}_{g_{v}, h_{v}+l_{v}}$ peut 
s'interpreter comme un «plongement», ou réalisation de l'ensemble des drapeaux $\mathcal{F l}(\Gamma)$ :

$$
\imath: \mathcal{F} l(\Gamma) \hookrightarrow \bigsqcup_{v \in V} C_{v}=\bigsqcup_{v_{i} \in V} C_{i}
$$

Cela signifie que $\imath$ identifie les arêtes de $\Gamma$ avec l'ensemble des points spéciaux de la somme disjointe $\bigsqcup_{v \in V} C_{v}$. Par convention les pattes d'origine $v$ ont pour images (ordonnées) $x_{1}^{v}, \ldots, x_{h_{v}}^{v}$, et les arêtes d'origine $v$, ont pour images (ordonnées) $x_{h_{v}+1}^{v}, \ldots, x_{h_{v}+l_{v}}^{v}$. Réciproquement, un plongement (79) conduit à un objet du champ $\prod_{v_{i} \in V} \bar{M}_{g_{v}, h_{v}+l_{v}}$.

Pour en donner la description, rappelons brièvement la définition de l'opération de recollement (clutching morphism) le long d'une paire de sections (Knudsen [68], Manin [76], chap. V). La construction se résume ainsi : soit $C^{\prime}$ une courbe préstable de base $S$, dont on ne suppose pas a priori les fibres géométriques connexes. Soient deux sections $s_{1}, s_{2}: S \longrightarrow C^{\prime}$, dont les images sont formées de points non singuliers le long des fibres. Il existe une courbe préstable $C^{\prime}$, un morphisme fini $p: C \longrightarrow C^{\prime}$ tel que $p s_{1}=p s_{2}$, le couple $\left(C^{\prime}, p\right)$ étant universel en un sens évident. En particulier, il est défini à un isomorphisme canonique près. Une fibre géométrique étant donnée, si la fibre image de $C^{\prime}$ est connexe, deux cas se présentent :

- La fibre est irréductible, dans ce cas on crée dans la fibre correspondante de $C^{\prime}$ une boucle, on a : $g^{\prime}=g+1$.

- La fibre de $C$ est somme disjointe de deux courbes connexes $C_{1}$ et $C_{2}$, avec $s_{i} \in C_{i}(i=1,2)$. Dans ce cas, la fibre (connexe) de $C^{\prime}$ a pour genre $g^{\prime}=g_{1}+g_{2}$.

Revenant à notre construction, par identification des sections $x_{i}^{v}, x_{j}^{w}$ associées à un couple d'arêtes opposées, le clutching morphism, on forme la courbe $C=$ $\bigsqcup_{v \in V} C_{v} / \Gamma \in \bar{M}_{g, n}(S)$. Observons qu'il en découle une identification bien définie $\Gamma=\Gamma(C)$. On note aussi qu'il y a une action évidente (à droite) de $\operatorname{Aut}(\Gamma)$ (en fait, de $\operatorname{Aut}(\mathcal{F} l(\Gamma)))$ sur de tels plongements, i.e. $\sigma \cdot\left(\left\{C_{v}\right\}_{v}, \imath\right)=\left(\left\{C_{v}\right\}, \imath . \sigma\right)$. Alors, le morphisme $\beta_{\Gamma}$ définit un $\operatorname{Aut}(\Gamma)$-torseur sur son image ${ }^{(5)} \overline{\mathcal{M}}_{g, n}(\Gamma)$, qui est en conséquence le quotient

$$
\prod_{v \in V} \bar{M}_{g_{v}, h_{v}+l_{v}} / \operatorname{Aut}(\Gamma)
$$

Soit maintenant $(\Gamma, G)$ un graphe modulaire de Hurwitz (définition 7.1.2). On considère le sous-champ localement fermé $\mathscr{H}_{g, G, \xi}(\Gamma)$ de $\overline{\mathscr{H}}_{g, G, \xi}$ dont les objets sont les $G$-courbes stables de type combinatoire fixé $\Gamma$, i.e. dont les fibres géométriques sont de type $\Gamma$. Si $\Gamma=\varnothing, \mathscr{H}_{g, G, \xi}(\Gamma)=\mathscr{H}_{g, G, \xi}$. Les sous-champs (resp. sous-schémas) localement fermés $\mathscr{H}_{g, G, \xi}(\Gamma)$ (resp. les espaces de modules grossiers $H_{g, G, \xi}(\Gamma)$ ) forment une stratification de $\overline{\mathcal{H}}_{g, G, \xi}$ (resp. $\bar{H}_{g, G, \xi}$ ), analogue à la stratification

(5) Le morphisme $\beta_{\Gamma}$ est fini, en particulier représentable [68], de sorte que son image, en tant que sous-champ fermé de $\overline{\mathcal{M}}_{g, n}$ est définie. 
de $\bar{M}_{g, n}$ par le type combinatoire des courbes stables marquées [76]. On a la décomposition

$$
\overline{\mathcal{H}}_{g, G, \xi}=\bigsqcup_{\Gamma} \mathcal{H}_{g, G, \xi}(\Gamma) .
$$

Le morphisme discriminant $\delta: \overline{\mathcal{H}}_{g, G, \xi} \longrightarrow \overline{\mathcal{M}}_{g^{\prime}, b}$ qui associe à un revêtement la base marquée par les points de branchement est visiblement compatible aux stratifications. En posant $\Delta=\Gamma / G$, on traduit cela par le fait que $\delta$ induit un morphisme

$$
\mathcal{H}_{g, G, \xi}(\Gamma) \longrightarrow \mathcal{M}_{g^{\prime}, b}(\Delta) \text {. }
$$

De manière similaire, notons $r$ le nombre de points de branchement (points marqués sur la base) et $\imath: \overline{\mathscr{H}}_{g, G, \xi} \longrightarrow \overline{\mathcal{M}}_{g, r}$ le morphisme d'oubli de l'action de $G$. On a alors clairement $\mathscr{H}_{g, G, \xi}(\Gamma)=\imath^{-1}\left(\mathcal{M}_{g, r}(\Gamma)\right)$, où dans le terme de droite, $\Gamma$ est le graphe modulaire, allégé de la donnée de Hurwitz.

Avant de préciser la structure de la strate $\mathcal{H}_{g, G, \xi}(\Gamma)$ des revêtements de type combinatoire $\Gamma$, revenons à un graphe modulaire de Hurwitz $\Gamma$. Soit $v \in \Gamma$ un sommet et notons $s t(v)$ l'étoile de $v$. Les arêtes éléments de $s t(v)$, décorées par l'holonomie $(H, \chi)$, sont de deux sortes. D'une part les arêtes orientées d'origine un sommet $v$ et d'extrémité $w, v=w$ étant possible (boucle), et d'autre part les arêtes monovalentes associées aux pattes issues de $v$. Faisons tout d'abord le choix d'un système de représentants $v_{1}, \ldots, v_{k}$ pour l'action de $G$ sur $V$, ensemble des sommets de $\Gamma$, i.e. une section de $V \longrightarrow V / G$. Soit $G_{i}$ le stabilisateur de $v_{i}$, et notons $\mathrm{st}_{i}=\mathcal{E}_{i} \bigsqcup \mathcal{L}_{i}$ l'étoile de $v_{i}$, union de l'ensemble $\mathcal{E}_{i}$ des arêtes (décorées) issues de $v_{i}$ et de l'ensemble $\mathscr{L}_{i}$ des pattes d'origine $v_{i}$. Si $\mathcal{F}=\mathscr{L} \bigsqcup \mathcal{E}$, du point de vue des $G$-ensembles, on a :

$$
\mathscr{L}=\bigcup_{i} \operatorname{Ind}_{G_{i}}^{G} \mathscr{L}_{i} \quad, \quad \mathcal{E}=\bigcup_{i} G \times{ }^{G_{i}} \mathcal{E}_{i} .
$$

Pour avoir l'analogue équivariant de (80), il nous faut un plongement équivariant $\mathcal{F} l(\Gamma)$ dans ce qui remplace le second membre de (79). Comme seules les orbites de points spéciaux sont indexées, il est nous faut à ce stade choisir un marquage plus strict pour un revêtement galoisien. Outre un système de représentants des sommets, on va choisir pour chaque classe de conjugaison d'holonomie décorant une $G_{i}$-orbite de pattes ou arêtes d'origine $v_{i}$, un représentant d'holonomie $\left(H_{i, \alpha}, \chi_{i, \alpha}\right)$ pour les arêtes, et $\left(H_{i, \lambda}, \chi_{i, \lambda}\right)$ pour les orbites de pattes. Ceci étant, on fait ensuite le choix d'un point $e_{i, \alpha}$ (resp. $l_{i, \lambda}$ ) dans chaque orbite d'arêtes qui a pour holonomie le représentant choisi. D'une autre manière on se donne des sections de $\mathcal{E}_{i} \longrightarrow \mathcal{E}_{i} / G_{i}, \mathscr{L}_{i} \longrightarrow \mathscr{L}_{i} / G_{i}$ avec holonomie prescrite.

Soit maintenant un revêtement $\pi: C \longrightarrow D$ de type combinatoire $\Gamma$, et $\widetilde{C}=\bigsqcup_{v} \widetilde{C}_{v}$ la normalisation de $C$. La composante $\widetilde{C}_{v}$ munie de l'action du stabilisateur $G_{v}$ a pour donnée de Hurwitz

$$
\tilde{\xi}_{v}=\xi_{v}+\eta_{v}
$$

où $\xi_{v}$ représente la contribution des points de ramification de $C$, et $\eta_{v}$ est la contribution des arêtes orientées d'origine $v$, branches des points doubles. Ceci nous 
conduit à voir $\widetilde{C}_{v}$ comme un objet du $\mathscr{H}_{g_{v}, G_{v}, \xi_{v}, \eta_{v}}$ classifiant les courbes de genre $g_{v}$, munies d'une action de $G_{v}$ de donnée de Hurwitz $\tilde{\xi}_{v}=\xi_{v}+\eta_{v}$. Dans cette définition les courbes sont marquées par un diviseur $G_{v}$-invariant, ce qui signifie que seules les $G_{v}$-orbites de points spéciaux sont numérotées.

Avec la normalisation fixée sur le graphe modulaire de Hurwitz $\Gamma$, soit un objet

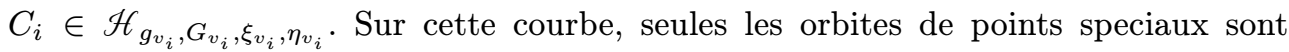
numérotées. Pour tout $i$, et dans chaque orbite de tels points, on fait comme cela a été fait pour le graphe, le choix d'un point $x_{i, \alpha}$ (resp. $y_{i, \lambda}$ ) dans chaque $G_{i}$-orbite d'holonomie celle sélectionnée au départ. On a donc pour $1 \leqslant \alpha \leqslant h_{i}, x_{i, \alpha}$ d'holonomie $\left(H_{i, \alpha}, \chi_{i, \alpha}\right)$, et si $1 \leqslant \lambda \leqslant l_{i}$ le point $x_{i, h_{i}+\lambda}=y_{i, \lambda}$ d'holonomie $\left(H_{i, \lambda}, \chi_{i, \lambda}\right)$. Pour tout $1 \leqslant i \leqslant k$, notons alors $\mathcal{H}_{g_{v_{i}}, G_{v_{i}}, \xi_{v_{i}}, \eta_{v_{i}}}$ le champ de Hurwitz modifié, résultat de ce marquage plus strict. Noter que l'oubli de ce choix supplémentaire conduit à un morphisme

$$
\mathcal{H}_{g_{v_{i}}, G_{v_{i}}, \xi_{v_{i}}, \eta_{v_{i}}} \longrightarrow \mathcal{H}_{g_{v_{i}}, G_{v_{i}}, \xi_{v_{i}}, \eta_{v_{i}}}
$$

de degré $\prod_{\alpha, \lambda}\left|Z_{G_{i}}\left(H_{i, \alpha}\right) / H_{i, \alpha}\right|\left|Z_{G_{i}}\left(H_{i, \lambda}\right) / H_{i, \lambda}\right|$ où $Z_{G}(H)$ désigne le centralisateur de $H$ dans $G$. Ce morphisme est un quotient par un produit de groupes symétriques convenables.

On définit de même le champ $\mathscr{H}_{g, G, \xi}^{*}(\Gamma)$, avec le morphisme $\mathcal{H}_{g, G, \xi}^{*}(\Gamma) \longrightarrow$ $\mathcal{H}_{g, G, \xi}^{*}(\Gamma)$ de degré $\prod_{i, \lambda}\left|Z_{G_{i}}\left(H_{i, \lambda}\right) / H_{i, \lambda}\right|$. On peut formuler l'analogue de $(78)$ :

Proposition 7.4.1. - Il y a un morphisme naturel

$$
\xi_{\Gamma}: \prod_{i=1}^{k} \mathcal{H}_{g_{v_{i}}, G_{v_{i}}, \xi_{v_{i}}, \eta_{v_{i}}}^{*} \longrightarrow \mathcal{H}_{g, G, \xi}^{*}(\Gamma)
$$

qui identifie le terme de droite au quotient de la source par le groupe $\operatorname{Aut}_{G}(\Gamma)$. Ce morphisme s'insère dans un diagramme

$$
\prod_{i=1}^{k} \mathcal{H}_{g_{v_{i}}, G_{v_{i}}, \xi_{v_{i}}, \eta_{v_{i}}} \longleftarrow \prod_{i=1}^{k} \mathcal{H}_{g_{v_{i}}, G_{v_{i}}, \xi_{v_{i}}, \eta_{v_{i}}}^{*} \stackrel{\xi_{\Gamma}}{\longrightarrow} \mathcal{H}_{g, G, \xi}^{*}(\Gamma) \longrightarrow \mathcal{H}_{g, G, \xi}(\Gamma) .
$$

Démonstration. - Il faut tout d'abord définir le morphisme $\xi_{\Gamma}$. Cela revient à préciser la procédure d'identification des branches par paires. Cette procédure doit être $G$-équivariante. Elle revient comme dans le cas $G=1$ ci-dessus, à plonger de manière équivariante l'ensemble $\mathcal{F} l(\Gamma)$ sur l'ensemble correspondant des points spéciaux de $\bigsqcup_{i} \operatorname{Ind}_{G_{v_{i}}}^{G}\left(C_{i}\right)$, puis à transporter l'involution canonique. Il est clair que les bijections entre points sélectionnés s'étendent en un plongement $G$-équivariant

$$
\mathcal{F} l(\Gamma) \hookrightarrow \tilde{C}=\bigsqcup_{i} \operatorname{Ind}_{G_{v_{i}}}^{G}\left(C_{i}\right)
$$

typiquement $g e_{i, \alpha} \longrightarrow g x_{i, \alpha}$. On peut alors transporter l'involution $\tau: a \longrightarrow \bar{a}$ sur l'image dans le terme de droite, donnant de la sorte un procédé d'identification par paires des points distingués de $\tilde{C}$. Par recollement le long de ces paires de sections, 
on obtient une $G$-courbe stable marquée connexe de donnée de ramification $\xi$. De manière plus concise, on peut écrire :

$$
C=\bigvee_{i} \operatorname{Ind}_{G_{v_{i}}}^{G}\left(C_{i}\right) \in \mathcal{H}_{g, G, \xi}(\Gamma) .
$$

On pose alors $\xi_{\Gamma}\left(\left\{C_{i}\right\}\right)=C$. Cette construction définit le foncteur $\xi_{\Gamma}$. Le reste est conséquence des remarques qui précèdent l'énoncé.

Notons que la codimension de $\mathscr{H}_{g, G, \xi}(\Gamma)$ est Card $\mathcal{F}_{\Gamma / G}$, et que la construction est définie en fait au niveau des champs compactifiés.

Les revêtements sont marqués par les points de branchement réels ou virtuels. Supposons que la donnée de ramification $\xi$ soit telle que l'un des sous-groupe $H_{i}$ soit trivial, par exemple $H_{b+1}=1$, signifiant que le point marqué $Q_{b+1}$ n'est pas un point de branchement au sens strict. On a donc $\xi=\xi^{\prime}+[1]$, avec les notations de la section 2. Par contraction (stabilisation) on peut effacer ce point.

Proposition 7.4.2. - L'effacement du point marqué (de «branchement ») d'indice $b+1$ définit un morphisme de champs $\rho: \overline{\mathcal{H}}_{g, G, \xi} \longrightarrow \overline{\mathcal{H}}_{g, G, \xi^{\prime}}$.

Démonstration. - Soit un revêtement stable $\pi: C \longrightarrow D$ de base $S$. L'oubli du point de branchement $Q_{b+1}$, suivi d'une stabilisation, donne un morphisme $\psi: D \longrightarrow$ $D^{\prime}$. Soit d'autre part l'oubli suivi d'une stabilisation des points de l'orbite régulière $\pi^{-1}\left(Q_{b+1}\right)$, conduisant à un morphisme $\phi: C \longrightarrow C^{\prime}$. Par le caractère universel de cette opération, l'action de $G$ se descend à $C^{\prime}$, et le morphisme $\psi \pi$ factorise par $C^{\prime}$. Soit $\pi^{\prime}: C^{\prime} \longrightarrow D^{\prime}$ cette factorisation. Le morphisme $\pi^{\prime}$ factorise en $C^{\prime} \longrightarrow C^{\prime} / G \longrightarrow D^{\prime}$ et $\psi$ en $D=C / G \longrightarrow C^{\prime} / G \longrightarrow D^{\prime}$. Comme la courbe $C^{\prime} / G$ est stable marquée par les images des points $Q_{1}, \ldots, Q_{b}$, il y a un morphisme $D^{\prime} \longrightarrow C^{\prime} / G$ qui est clairement l'inverse de $C^{\prime} / G \longrightarrow D^{\prime}$. Donc $D^{\prime}=C^{\prime} / G$. Le morphisme $\rho$ est ainsi donné par $\rho(C \longrightarrow D)=\left(C^{\prime} \longrightarrow D^{\prime}\right)$.

On reprend les notations et hypothèses de la section 7.2.1. Considérons donc deux sous-groupes $G_{1}, G_{2}$ de $G$, et un sous-groupe cyclique $H \subset G_{1} \cap G_{2}$. Soient des données de ramification

$$
\xi_{1}^{*}=\xi_{1}+[H, \chi] \in R_{+}\left(G_{1}\right) \quad \text { et } \quad \xi_{2}^{*}=\xi_{2}+\left[H, \chi^{-1}\right] \in R_{+}\left(G_{2}\right) .
$$

Posons $\xi=\operatorname{Ind}_{G_{1}}^{G} \xi_{1}+\operatorname{Ind}_{G_{2}}^{G} \xi_{2} \in R_{+}(G)$. Par recollement le long de deux sections, on obtient :

Proposition 7.4.3. - Il existe un morphisme de recollement le long de deux sections d'holonomie opposées :

$$
\rho: \overline{\mathcal{H}}_{g_{1}, G_{1}, \xi_{1}^{*}} \times \overline{\mathcal{H}}_{g_{2}, G_{2}, \xi_{2}^{*}} \longrightarrow \overline{\mathcal{H}}_{g_{1}+g_{2}, G, \xi} \cdot
$$

Si $G=G_{1}=G_{2}$, et si $\xi^{*}=\xi+[H, \chi]+\left[H, \chi^{-1}\right]$ (Harbater-Mumford), par recollement des deux sections, on a le morphisme analogue

$$
\rho: \overline{\mathscr{H}}_{g-1, G, \xi^{*}} \longrightarrow \overline{\mathscr{H}}_{g, G, \xi} \text {. }
$$


Démonstration. - Soit $C_{i} \longrightarrow D_{i}(i=1,2)$ un point du terme de gauche, et soient $Q_{i} \in D_{i}$ les points de branchement d'holonomie $\left[H, \chi_{1}=\chi\right]$ et $\left[H, \chi_{2}=\chi^{-1}\right]$. Soit $P_{i} \in C_{i}$ un point d'holonomie exacte $\left(H, \chi_{i}\right)$. Par identification des paires de points $\left(g P_{1}, g P_{2}\right)$, on donne un sens au revêtement

$$
C=\operatorname{Ind}_{G_{1}}^{G} C_{1} \bigvee \operatorname{Ind}_{G_{2}}^{G} C_{2} \longrightarrow D=D_{1} \bigvee D_{2}
$$

Cette construction est bien définie à isomorphisme unique près. Elle définit le morphisme cherché. Une construction similaire fonctionne dans le cas d'une donnée de ramification du type Harbater-Mumford $\xi^{*}=\xi+[H, \chi]+\left[H, \chi^{-1}\right]$, et donne le second morphisme.

Soit le morphisme discriminant $\delta: \overline{\mathcal{H}}_{g, G, \xi} \longrightarrow \overline{\mathcal{M}}_{g^{\prime}, b}$. On sait que ce morphisme définit un revêtement entre champs de Deligne-Mumford (proposition 6.5.2), dont on peut préciser le diviseur de ramification. Le résultat est une simple traduction de la proposition 6.6.13.

Proposition 7.4.4. - Le diviseur de ramification du morphisme discriminant $\delta$ : $\overline{\mathcal{H}}_{g, G, \xi} \longrightarrow \overline{\mathcal{M}}_{g^{\prime}, b}$ est

$$
\mathfrak{R}=\sum_{\Delta=\mathrm{NS}}(|H|-1) \Delta,
$$

la somme étant étendue aux composantes $\mathrm{NS} d u$ bord, i.e. celles telles que $H \neq 1$.

7.4.2. Type topologique d'un point du bord. - Le corps de base est maintenant $k=\mathbb{C}$. Soient $H_{1}, \ldots, H_{h(\xi)}$ les composantes irréductibles de $H_{g, G, \xi}$, où $h(\xi)$ est le nombre de Nielsen. Le schéma des modules compactifié est somme disjointe des « compactifications » $\bar{H}_{\alpha}$ avec $\alpha=1, \ldots, h(\xi)$ (voir proposition 6.4.2). Si $C \longrightarrow D$ représente un point du bord, on peut légitimement chercher à quelle composante ce point appartient, c'est-à-dire trouver un procédé pour lire sur $C \longrightarrow D$ le nom de la composante à laquelle le point est rattaché. Si $C$ est lisse, le type topologique qui fixe la composante à laquelle ce point appartient, est entièrement donné par le morphisme de monodromie $\psi: \pi_{1}(D-\beta) \longrightarrow G$ (proposition 2.3.3). En fait, une réponse similaire peut être donnée dans le cas singulier. Elle découle directement des résultats de Asada, Matsumoto et Oda [6]. Supposons donc la courbe $C$ singulière, et considérons la déformation universelle équivariante, ici prise dans un sens analytique

$$
\pi: \mathscr{C} \longrightarrow D^{3 g^{\prime}-3+b} \quad(D=\{z \in \mathbb{C},|z|<1\}),
$$

avec par conséquent un isomorphisme (équivariant) $\pi^{-1}(0)=C$. Si $t \in D$ est en dehors du discriminant, la fibre $\mathscr{C}_{t}$ est non-singulière, et indique donc le type topologique de la composante qui contient $C$. En fait, et c'est le point que nous allons retenir, le type topologique peut être lu directement $\operatorname{sur} C$. La raison est que le morphisme de monodromie associé à la fibre générale $\mathscr{C}_{t}$ peut être construit directement à partir de données lisibles sur $C$. C'est essentiellement le résultat de ([6], theorem 2.1), appelé théorème de Seifert-van Kampen. Il est nécessaire dans un tel énoncé de remplacer le groupe fondamental ordinaire par le groupe fondamental d'un graphe de groupes. 
Résumons la construction de ([6], def. 2.5). Soit $\Gamma$ le graphe modulaire défini par $C$, et $\Delta=\Gamma / G$ le graphe quotient. Il y a sur $\Delta$ une structure de graphe de groupes plus riche que celle utilisée dans la section 7.2 , permettant de reconstruire non seulement $\Gamma$ mais aussi $C$.

Notons $S_{v}(v \in \Delta)$ les composantes irréductibles normalisées de $D=C / G$, et notons $D_{v} \subset S_{v}$ les points qui ont pour images dans $D$ un point double, donc les origines des branches. Notons aussi $\beta_{v}$ l'ensemble des points marqués portés par la composante $S_{v}$. Ces points contiennent donc l'ensemble des points de ramification. On note alors $\pi_{v}=\pi_{1}\left(S_{v}-\left(\beta_{v} \cup D_{v}\right), *_{v}\right)$ le groupe fondamental de la courbe $S_{v}$ privée des points exceptionnels, relativement à un point de base $*_{v}$. La structure de graphe de groupes portée par $\Delta$ est définie ainsi : tout d'abord, on associe à $v$ le groupe $\pi_{v}$. Soit $(e, \bar{e})$ une arête qui pointe vers $v$, et $\bar{e}$ pointant vers $w$. On pose alors $H_{e}=H_{\bar{e}}=\mathbb{Z}$, en fixant un générateur privilégié $t_{e}$ tel que $t_{e}+t_{\bar{e}}=0$. On définit ensuite

$$
\partial_{e}^{1}(1)=\alpha(e) \quad, \quad \partial_{e}^{0}(1)=\alpha(\bar{e}),
$$

expression dans laquelle on note $\alpha(e)$ et $\alpha(\bar{e})$, des lacets de «pointe » autour des origines $p_{v}$ et $p_{w}$ des branches définies par le point double $a$. La classe de conjugaison d'un tel lacet de pointe est bien définie, ce qui assure que le graphe de groupes est bien défini à isomorphisme près. Notons $\Delta_{\pi}$ ce graphe de groupes, cela pour éviter toute confusion avec la définition de la section 7.2. Rappelons le résultat ([6], théorème 2.1), de spécialisation du groupe fondamental :

Proposition 7.4.5. - Le groupe fondamental $\pi_{1}\left(\Delta_{\pi}\right)$ du graphe de groupes $\Delta_{\pi}$ est isomorphe au groupe fondamental d'une fibre générique $\mathscr{C}_{t}$ de la déformation universelle de $C$; en outre on peut choisir l'isomorphisme de sorte qu'il préserve les classes de lacets autour des piqûres (points marqués).

La preuve de la proposition 7.4.5, pour laquelle on réfère à [6], fournit en outre un algorithme pour expliciter un système de générateurs canoniques de « groupe de surface » pour le groupe fondamental. Pour lire directement sur le revêtement $\pi: C \longrightarrow D$ le nom de la composante qui contient de point du bord, on voit qu'il suffit de pouvoir reconstruire le morphisme de monodromie, au niveau de $D$, donc sous la forme

$$
\psi: \pi_{1}\left(\Delta_{\pi}\right) \longrightarrow G,
$$

les images des lacets autour des piqûres étant soumises aux contraintes imposées par la donnée de ramification. En résumé :

Proposition 7.4.6. - La correspondance explicitée dans 2.3.3 reste valable en les points du bord de l'espace de Hurwitz. De manière précise, le morphisme de monodromie permet de reconstruire le revêtement $\pi: C \longrightarrow D$. La classe double (4) définit le type topologique, i.e. le nom de la composante du schéma de Hurwitz qui contient $\pi: C \longrightarrow D$. 
Démonstration. - La donnée de $\psi$ permet de construire le revêtement $\pi: C \longrightarrow D$. En bref, la restriction de $\psi$ à $\pi_{v}$ donne un $G$-revêtement (non connexe peut être) $C_{v} \longrightarrow D$. Sur la courbe $C_{v}$ il y a une collection d'orbites exceptionnelles (les origines des branches) qui autorisent que l'on recolle les $C_{v}$ pour obtenir $C$. Noter que d'une autre manière on récupère le graphe de groupes du théorème 7.2 .7 , et donc $\Gamma$. On prend pour $G_{v}$ (resp. $H_{a}$ ) l'image par $\psi$ de $\pi_{v}$ (resp. $H_{a}$ ). La construction de BassSerre permet de conclure. 


\section{CHAPITRE 8}

\section{STRUCTURES DE NIVEAU SUR LES COURBES STABLES}

\subsection{Structures de niveau sur les courbes lisses}

Les conventions sur le corps de base $k$ sont toujours en vigueur, en particulier si $G$ est un groupe fini, on suppose que $|G| \neq 0 \in k$. Soit $C$ une courbe algébrique projective et lisse définie sur $k$, de genre $g \geqslant 1$. On travaille avec un groupe fini $G$, qu'on suppose être quotient du groupe fondamental $\pi_{1}(C)$ d'une courbe lisse projective de genre $g$, groupe fondamental algébrique et modéré si $k$ est arbitraire, ou topologique si $k=\mathbb{C}$. Il sera commode de noter $\pi_{g}$ le groupe décrit par générateurs et relations

$$
\pi_{g}=\left\langle A_{1}, \ldots, A_{g}, B_{1}, \ldots, B_{g} \mid \prod_{j=1}^{g}\left[A_{j}, B_{j}\right]\right\rangle
$$

de sorte que $\pi_{1}(C) \cong \pi_{g}$.

DÉFInition 8.1.1. - Une structure de niveau $G$ sur $C$ est la donnée d'une surjection extérieure $\phi: \pi_{1}(C) \longrightarrow G$ ([30] définition $\left.5.6 ;[\mathbf{1 8}]\right)$.

On exigera en fait un peu plus sur $G$, à savoir que $G$ est un quotient caractéristique (quotient par un sous-groupe caractéristique) de $\pi_{g}$. Le niveau $G^{\prime}$ est dit dominer le niveau $G$ si la surjection $\phi: \pi_{1}(C) \longrightarrow G$ factorise par $G^{\prime}$. Le niveau abélien $(n)$ correspond à $G=(\mathbb{Z} / n \mathbb{Z})^{2 g}$. Une structure de niveau $(n)$ sur la courbe lisse $C$ revient à spécifier un isomorphisme

$$
\alpha: \operatorname{Pic}(C)[n] \stackrel{\sim}{\longrightarrow}(\mathbb{Z} / n \mathbb{Z})^{2 g}
$$

ou $H^{1}(C, \mathbb{Z} / n \mathbb{Z}) \stackrel{\sim}{\longrightarrow}(\mathbb{Z} / n \mathbb{Z})^{2 g}$ si $k=\mathbb{C}$.

DÉFInition 8.1.2. - Notons $\pi_{g}$ le groupe fondamental d'une surface de Riemann compacte de genre $g \geqslant 2$. Un niveau $G$ est géométrique si le noyau d'une surjection $\phi: \pi_{g} \longrightarrow G$ est indépendant de $\phi$. 
Les niveaux abéliens sont géométriques, ainsi que les niveaux diédraux de Brylinski [18], voir aussi [74]. Le niveau diédral d'ordre $m \geqslant 2$ est défini par

$$
G=\pi_{g} /\left[\pi_{g}^{(2)}, \pi_{g}^{(2)}\right] \pi_{g}^{(2 m)},
$$

où $[-,-]$ désigne le sous-groupe des commutateurs et $(-)^{(k)}$ le sous-groupe engendré par les puissances d'ordre $k$. On se limite dans la suite aux seuls niveaux géométriques.

La définition d'une structure de niveau $G$ s'étend, avec l'aide de la théorie du groupe fondamental modéré de Grothendieck, aux familles de courbes lisses ([16], [18], [30], section 5). Rappelons brièvement la construction. Soit $\pi: C \longrightarrow S$ une courbe lisse au-dessus de la base $S$, et de genre $g \geqslant 2$ pour simplifier. Le cas $g=1$ entre dans ce cadre, mais avec quelques précautions supplémentaires.

Soit une section $s: S \longrightarrow C$; une telle section existe localement pour la topologie étale. Soit $\mathbb{L}$ un ensemble de nombres premiers contenant les caractéristiques des corps résiduels de $\theta_{S}$. Le théorème de spécialisation pour le groupe fondamental permet de définir $\pi_{1}^{\mathbb{L}}(C / S, s)$, un pro-objet dans la catégorie des faisceaux en groupes finis, localement constants, d'ordres premiers à $\mathbb{L}$. La formation de ce faisceau commute à tout changement de base. Cela permet de définir sur $S_{e t}$ le faisceau localement constant $\mathcal{H}_{\mathrm{om}^{\text {ext }}}\left(\pi_{1}(C / S, s)^{\mathbb{L}}, G\right)$ des homomorphismes extérieurs, c'est-à-dire le quotient de $\mathscr{H} \operatorname{com}\left(\pi_{1}(C / S, s)^{\mathbb{L}}, G\right)$ par l'action par automorphismes intérieurs de $\pi_{1}(C / S, s)^{\mathbb{L}}$. Il ne dépend plus de $s$, ce qui permet de le noter $\mathcal{H}_{\text {om }}{ }^{\text {ext }}\left(\pi_{1}(C / S)^{\mathbb{L}}, G\right)$. Alors, la définition précise d'une structure de niveau $G$ sur $C / S$ est la suivante (voir [30], définition 5.6) :

DÉFInition 8.1.3. - Une G-structure de niveau sur une $S$-courbe lisse $C / S$ est la donnée d'une surjection extérieure $\phi: \pi_{1}^{\mathbb{L}}(C / S)^{\mathbb{L}} \longrightarrow G$, c'est-à-dire la donnée d'une section globale $\phi \in \Gamma\left(S, \mathcal{H}\right.$ om $\left.^{\text {ext }}\left(\pi_{1}^{\mathbb{L}}(C / S)^{\mathbb{L}}, G\right)\right)$.

Une structure de niveau ${ }^{(1)} \phi$ n'est donc définie que localement pour la topologie étale par un homomorphisme du $\pi_{1}$ dans $G$. La catégorie des courbes lisses de genre $g \geqslant 2$, équipées d'une structure de niveau $G$, définit un champ algébrique ${ }_{G} \mathcal{M}_{g}$. On a le résultat suivant $([\mathbf{1 8}]$, theorem $2.3 .2,[\mathbf{3 0}]$, lemma 5.7) :

THÉORÈme 8.1.4. - i) Le champ ${ }_{G} \mathcal{M}_{g}$ est un champ algébrique de Deligne-Mumford sur $\mathbb{Z}[1 /|G|]$.

ii) Le morphisme ${ }_{G} \mathcal{M}_{g} \longrightarrow \mathcal{M}_{g}$ d'oubli de la structure de niveau est fini, étale et surjectif.

iii) Le nombre de composantes connexes des fibres de ${ }_{G} \mathcal{M}_{g}$ est constant.

iv) Si le niveau $G$ domine un niveau $(n), n \geqslant 3$, alors ${ }_{G} \mathcal{M}_{g}$ est représentable. De plus, dans ce cas le champ $\mathcal{M}_{g}(G):=\mathscr{H}_{g}(G) \sqcap Z(G)$ est isomorphe à ${ }_{G} \mathcal{M}_{g}$ et le morphisme naturel $\mathcal{M}_{g}(G) \longrightarrow \mathcal{M}_{g}$ est un $\operatorname{Out}(G)$-torseur.

(1) Ou structure de Teichmüller de niveau $G$ dans [18], [30]. 
La preuve de iii) nécessite une compactification de ${ }_{G} \mathcal{M}_{g}$ ([30], cor. 5.11). Faute d'une définition modulaire raisonnable du champ compactifié ${ }_{G} \overline{\mathcal{M}}_{g}$, la procédure usuellement retenue est de prendre pour définition du champ compactifié ${ }_{G} \overline{\mathcal{M}}_{g}$ la normalisation de $\overline{\mathcal{M}}_{g}[1 /|G|]$ dans ${ }_{G} \mathcal{M}_{g}$ (loc.cit. section 5). Seul le point iv) du théorème 8.1.4 demande une vérification. Débutons par un lemme élémentaire :

Lemme 8.1.5. - $\quad$ i) Soient $\pi_{i}: \Sigma_{i} \longrightarrow C$ deux G-revêtements principaux d'une courbe lisse $C$ sur une base $S$ connexe. Alors il existe $\theta \in \operatorname{Aut}(G)$, d'image unique dans $\operatorname{Out}(G)$, tel que le morphisme $\operatorname{Isom}_{C, G}\left(\Sigma_{1}^{\theta}, \Sigma_{2}\right) \longrightarrow S$ soit un $Z(G)$-torseur.

ii) $S i \pi: \Sigma \longrightarrow C$ est un $G$-torseur de base $S$, alors $\operatorname{Aut}_{C}(\Sigma)=G$ et $\operatorname{Aut}_{C, G}(\Sigma)=$ $Z(G)$.

Démonstration. - Nous conservons les notations du théorème 6.6.6, dont en particulier la définition du foncteur $\operatorname{Isom}_{C, G}\left(\Sigma_{1}^{\theta}, \Sigma_{2}\right)$. Des arguments identiques à ceux utilisés dans le preuve de ce théorème, conduisent au fait que pour un certain $\theta \in \operatorname{Aut}(G)$, le

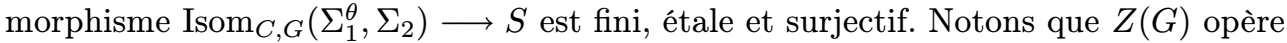
naturellement sur $\operatorname{Isom}_{C, G}\left(\Sigma_{1}^{\theta}, \Sigma_{2}\right)$. Comme cette action est simplement transitive sur les fibres géométriques, on conclut que $\operatorname{Isom}_{C, G}\left(\Sigma_{1}^{\theta}, \Sigma_{2}\right) / Z(G)=S$.

Le point ii) se traite de la même manière, en observant que la courbe $\pi: \Sigma \longrightarrow S$ étant lisse, $\operatorname{Aut}_{C}(\Sigma)$ est étale sur $S$, et égal à $G$ sur les fibres géométriques, donc égal à $G$. Notons que cela prouve que dans i), l'automorphisme $\theta$ a une image bien déterminée dans $\operatorname{Out}(G)$, d'où le lemme.

Démonstration $d u$ point iv) de (8.1.4). - On peut invoquer la théorie du groupe fondamental à la manière de Boggi-Pikaart [16], Deligne-Mumford [30]. On peut aussi, de manière plus directe, noter que si on forme le champ algébrique $\mathcal{N}_{C / S}(G)=$ $\mathcal{H}_{g}(G) \times \mathcal{M}_{g} S$ défini par le morphisme $S \longrightarrow \mathcal{M}_{g}$, i.e. par la section $C / S \in \mathcal{M}_{g}(S)$, alors le groupoïde des objets au-dessus du $S$-schéma $T$ a pour objets les $G$-fibrés principaux de base $C \times_{S} T$. Le lemme 8.1.5 dit que le champ $\mathcal{N}_{C / S}(G) \sqcap Z(G)$ est représentable, et que c'est un $\operatorname{Out}(G)$-torseur de base $S$. Noter qu'il n'y a pas d'action stricte de $\operatorname{Out}(G)$ sur $\mathcal{M}_{g}(G)$, seulement une action faible; mais le lemme dit que ce champ est isomorphe au champ formé des courbes de genre $g$ munies d'une structure de niveau $G$, qui, lui, possède une action stricte de $\operatorname{Out}(G)$.

Le point de vue des schémas de Hurwitz, le point iv), conduit de fait à renverser la définition 8.1.3, et nous amène à considérer une structure de niveau comme dérivant d'un $G$-fibré principal de base $C$. Cela suggère qu'on doit voir le champ ${ }_{G} \bar{M}_{g}$ comme une sorte de compactification de $B G$, le champ classifiant du groupe fini $G$ (on pourra comparer avec le point de vue voisin mais plus sophistiqué de Abramovich-CortiVistoli [1]).

EXEMPLE 8.1.6. - Le niveau abélien.

Considérons le niveau abélien $(n)$, donc $G=(\mathbb{Z} / n \mathbb{Z})^{2 g}$, et notons $\mathcal{M}_{g}(n)$ le champ correspondant. La correspondance entre les deux définitions est aisée à expliciter. Si $\pi$ : 
$\Sigma \longrightarrow C$ est un $G$-revêtement principal, l'algèbre $\pi_{*}\left(\theta_{\Sigma}\right)$ se décompose canoniquement en une somme directe de faisceaux inversibles (sous-faisceaux propres)

$$
\pi_{*}\left(\theta_{\Sigma}\right)=\bigoplus_{\chi \in \hat{G}} \mathcal{L}_{\chi}
$$

Le choix d'une racine primitive $n$-ième permet d'identifier $G$ et $\hat{G}$; par suite les faisceaux $\mathscr{L}_{\chi}$ conduisent à une identification de $\operatorname{Pic}_{C / S}[n]$ et $(\mathbb{Z} / n \mathbb{Z})^{2 g}$. Un avantage de cette définition, est qu'elle garde un sens comme on va le voir, si on spécialise $C$ en un point du bord de $\bar{M}_{g}$. Le nombre de composantes connexes de $\mathcal{M}_{g}(n)$ (c'est-à-dire le nombre de Nielsen), est l'indice $\left[\mathrm{GL}_{2 g}(\mathbb{Z} / n \mathbb{Z}): \operatorname{Sp}_{g}(\mathbb{Z} / n \mathbb{Z})\right]$, voir [30].

\subsection{Structures de niveau sur les courbes stables}

Lorsque $C$ est une courbe stable, nodale, le théorème 8.1.4 iv), suggère qu'une structure de niveau $G$ sur $C$, doit pouvoir se définir en termes de $G$-fibrés principaux de base $C$, mais maintenant dégénérés.

DÉFInition 8.2.1. - Soit $C$ une courbe stable de genre $g \geqslant 2$ définie sur $k$. On définit un $G$-revêtement principal (ou torseur) stable (ou dégénéré) $\pi: \Sigma \longrightarrow C$, comme étant un $G$-revêtement stable, à donnée de Hurwitz $\xi=0$.

Cela signifie (définition 4.1.4) que $\Sigma$ est une courbe stable et que l'action de $G$ sur $\Sigma$ est stable, c'est-à-dire libre en dehors des points doubles, et agissant stablement aux points doubles. Une $G$-pré-structure de niveau sur $C / S$, est la donnée d'une classe d'équivalence de $G$-revêtements principaux stables de base $C$ (4.1.4). D'une autre manière, si un $G$-fibré principal de base $C$ lisse est interprété comme un morphisme $C \longrightarrow B G$, de $C$ dans le champ classifiant de $G[\mathbf{3}],[\mathbf{1}]$, on voit que cette définition diffère de manière essentielle de la définition 8.1 .3 si $C$ dégénère en une courbe nodale stable.

Soit $f: C \longrightarrow S$ une courbe stable de genre $g \geqslant 2$. Pour tout $S$-schéma $T$, soit $F_{C / S}(T)$ l'ensemble des $G$-pré-structures de niveau sur $C \times_{S} T$. Le préfaisceau $F_{C / S}$ n'est bien sûr pas en général un faisceau sur $S_{f p p f}$, même si le centre $Z(G)$ est non trivial. Une des raisons est que le groupe des automorphismes d'un $G$-torseur peut contenir strictement $Z(G)$. On définit alors $\mathrm{Ni}_{C / S, G}$, le faisceau des $G$-structures de niveau sur $f: C \longrightarrow S$ comme étant le faisceau fppf associé au préfaisceau $F_{C / S}$. Une $G$-structure de niveau naïve sur $C / S$ est une section $\alpha \in \Gamma\left(S, \mathrm{Ni}_{C / S, G}\right)$.

Pour obtenir la bonne définition, on considère le champ

$$
\overline{\mathscr{H}}_{g}(G):=\overline{\mathscr{H}}_{h, G, 0} \text {. }
$$

Si le niveau $G^{\prime}$ domine le niveau $G$, ce qui signifie que $G$ est un quotient de $G^{\prime}$, il y a un morphisme naturel de champs $\overline{\mathscr{H}}_{g}\left(G^{\prime}\right) \longrightarrow \overline{\mathscr{H}}_{g}(G)$ et un morphisme analogue 
$\bar{M}_{g}\left(G^{\prime}\right) \longrightarrow \bar{M}_{g}(G)$ entre schémas modulaires. Le point iv) du théorème 8.1.4 suggère que la compactification naturelle de ${ }_{G} \mathcal{M}_{g}=\mathcal{M}_{g}(G)=\mathcal{H}_{g}(G) \square Z(G)$ est

$$
\overline{\mathcal{M}}_{g}(G)=\overline{\mathscr{H}}_{g}(G) \sqcap Z(G) \text {. }
$$

Cela est justifié par le résultat suivant :

ThÉORÈme 8.2.2. - Soit ${ }_{G} \overline{\mathcal{M}}_{g}$ la normalisation de $\overline{\mathcal{M}}_{g}$ dans ${ }_{G} \mathcal{M}_{g}=\mathcal{M}_{g}(G)$ (compactification de Deligne-Mumford). Il existe un morphisme propre, birationnel

$$
\overline{\mathcal{M}}_{g}(G):=\overline{\mathcal{H}}_{g}(G) \sqcap Z(G) \longrightarrow{ }_{G} \overline{\mathcal{M}}_{g} \text {. }
$$

Les deux champs ont des espaces grossiers de modules identiques. En particulier, si le champ de droite est représentable (par exemple si $G$ domine le niveau $(n), n \geqslant 3$ ), c'est l'espace grossier de modules de $\overline{\mathcal{M}}_{g}(G)$.

Démonstration. - Il est clair que le groupe des automorphismes de tout objet de $\overline{\mathcal{H}}_{g}(G)$ contient $Z(G)$, ce qui permet de former le 2 -quotient $\overline{\mathscr{H}}_{g}(G) \square Z(G)=\overline{\mathcal{M}}_{g}(G)$ (section 6.1.2). Ce champ est de Deligne-Mumford, lisse (proposition 6.1.7). Il contient comme sous-champ ouvert dense le champ ${ }_{G} \mathcal{M}_{g}=\mathcal{M}_{g}(G)$. Il en résulte, suite à la définition de la normalisation (Deligne [28], Laumon et Moret-Bailly [73]), qu'il existe bien un morphisme naturel

$$
\overline{\mathcal{H}}_{g}(G) \sqcap Z(G) \longrightarrow{ }_{G} \overline{\mathcal{M}}_{g}
$$

qui étend le morphisme ${ }_{G} \mathcal{M}_{g} \longrightarrow \mathcal{M}_{g}$. On peut préférer un argument plus direct, par exemple ([90], theorem 7.2.3). Ce morphisme est propre car les champs invoqués le sont. Il reste à prouver l'égalité des espaces grossiers de modules respectifs $M$ et $N$. Le morphisme entre champs induit un morphisme $M \longrightarrow N$ entre espaces grossiers, qui est fini et un isomorphisme sur un sous-schéma ouvert partout dense. Comme la propriété de normalité se transfère des champs aux espaces grossiers, alors $M$ et $N$ sont normaux, si bien que le morphisme précédent est un isomorphisme. La dernière assertion de représentabilité si le niveau domine un niveau $n \geqslant 3$ provient de Deligne [28], proposition 3.5.

Remarque 8.2.3. - Le morphisme $\overline{\mathcal{M}}_{g}(G)=\overline{\mathcal{H}}_{g}(G) \sqcap Z(G) \longrightarrow{ }_{G} \overline{\mathcal{M}}_{g}$ est un isomorphisme sur les sous-champs ouverts formés par les structures de niveau sur les courbes lisses de genre $g$. Noter que le champ de gauche est lisse, mais en général non représentable, et celui de droite est non lisse en général. Ces deux champs ont le même espace modulaire grossier $\bar{M}_{g}(G)$ (fin pour ${ }_{G} \overline{\mathcal{M}}_{g}$ sous les conditions précédentes).

Le champ $\bar{M}_{g}(G)$ est défini au-dessus de $\mathbb{Z}[1 /|G|]$. En conséquence immédiate de la définition 8.2.1, chaque point possède du champ possède une interprétation modulaire précise. Soit $\pi: \Sigma \longrightarrow C$ un revêtement principal (dégénéré) de base la courbe stable $C$, définissant une structure de niveau $G$ sur $C$. Le groupe des $G$-automorphismes du revêtement $\operatorname{Aut}_{G}(\Sigma)$ gouverne la structure locale de l'espace des modules formel en ce point. Sa détermination explicite, en particulier savoir s'il contient strictement ou non le centre de $G$, peut s'avérer difficile. Le fait que ce groupe 
puisse être différent de $Z(G)$, est le fait essentiel qui distingue le cas stable du cas lisse. On peut être un peu plus précis sur ce groupe, lorsque le niveau $G$ domine le niveau $(n), n \geqslant 3$, comme le montre la proposition suivante, conséquence facile du lemme de rigidité (par exemple : Brylinski [18], Deligne [28], van Geemen-Oort [50]) :

Proposition 8.2.4.- $\quad$ i) Soit $\pi: \Sigma \longrightarrow C$ un $G$-fibré principal de base la $S$-courbe stable C. Si $f \in \operatorname{Aut}_{G}(\Sigma)$, alors $f$ induit l'automorphisme identité de $C$, i.e. $\operatorname{Aut}_{G}(\Sigma) \subset \operatorname{Aut}_{C}(\Sigma)$,

ii) Soit $C / S$ une courbe stable. Soit un niveau $G$ d'ordre inversible dans $\theta_{S}$. Alors $C / S$ admet une $G$-struture de niveau naïve après extension fppf de $S$.

Démonstration. - Pour la première assertion, on se ramène tout de suite à $G=(\mathbb{Z} / n \mathbb{Z})^{2 g}$. On considère pour cela une surjection $G \longrightarrow \mathbb{Z} / n \mathbb{Z}$, dont on note $H$ le noyau. Le revêtement $\pi: \Sigma \longrightarrow C$ factorise en $\Sigma \longrightarrow \Sigma / H \longrightarrow C$. Un $G$-automorphisme de $\Sigma$ induit un $G / H$-automorphisme de la courbe quotient $\Sigma \longrightarrow \Sigma / H$, ce qui justifie la réduction annoncée. Le résultat se teste sur les fibres géométriques, ce qui permet de supposer aussi que $S$ est le spectre d'un corps algébriquement clos $k$. Le niveau étant maintenant le niveau $(n), n \geqslant 3$, notons $h$ l'automorphisme de $\pi: C \longrightarrow S$ induit par le passage au quotient de $f$; on a donc $\pi f=h \pi$. En particulier, $f$ définit un automorphisme de $\theta_{C}$-algèbre

$$
h^{*}\left(\pi_{*}\left(\theta_{\Sigma}\right)\right) \longrightarrow \pi_{*}\left(\theta_{\Sigma}\right)
$$

commutant à l'action de $G$, et donc un automorphisme qui fixe chaque facteur isotypique de $\pi_{*}\left(\theta_{C}\right)$. En particulier, cela entraîne que $h$ induit l'identité sur $\mathrm{Pic}^{0}(C)[n]$, voir la Proposition ci-dessous. Mais on sait que sous cette condition le

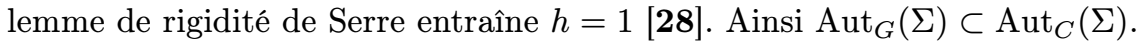

Prouvons le point ii). Le problème est local sur $S$. Soit un point géométrique $s \in S$. On montre d'abord que $C_{s}$ admet une $G$-structure de niveau. Soit une déformation $\mathscr{C} \longrightarrow \operatorname{Spec}(R)$ de $C_{s}$ de base un anneau de valuation discrète complet de corps résiduel $k$, de corps des fractions $K$, à fibre générique lisse. Après une extension finie séparable éventuelle de $K$, et remplacement de $R$ par le normalisé, on peut équiper la fibre générique $C_{K}$ d'une $G$-structure de niveau $\Sigma_{K} \longrightarrow C_{K}$. Par réduction stable, on peut même supposer que $\Sigma_{K}$ se prolonge en une $G$-courbe stable $\Sigma \longrightarrow \operatorname{Spec}(R)$. Il est alors clair que $\Sigma / G=\mathscr{C}$ (voir section 5.1.5). Pour conclure, on reprend l'argument utilisé pour prouver l'existence locale d'une clôture galoisienne (théorème 6.6.6). Soit une structure de niveau représentée par un $G$-torseur stable $\Sigma_{s} \longrightarrow C_{s}$. La déformation universelle de ce $G$-torseur existe sur une extension $R^{*} / R$ finie et plate de la base de la déformation universelle de $C_{s}$. Ce sont les points doubles de type NS qui sont responsables de la ramification de cette extension. Quitte à effectuer une extension étale de $S$ en $s$, on peut supposer que la déformation universelle est définie sur $S$. Il suffit alors d'effectuer le changement de base $\operatorname{par} \operatorname{Spec}\left(R^{*}\right) \longrightarrow \operatorname{Spec}(R)$ pour obtenir une structure de niveau (naïve). 
Des arguments différents, basés sur le théorème de de Jong-Pikaart [85], permettent de prouver qu'une structure de niveau existe après une extension finie fidèlement plate (Romagny [90], cor 7.2.4).

\subsection{Le niveau abélien $(n)$}

8.3.1. Groupes de décomposition et d'inertie. - On se limite dans cette section au niveau abélien $(n), n \geqslant 3$, donc $G=(\mathbb{Z} / n \mathbb{Z})^{2 g}$. Les résultats de la section 7.4 permettent de décrire avec précision la structure combinatoire du bord. Pour cela, nous reprenons les suites exactes (71), (72), (73) et les traduisons dans le cadre d'un $G$-fibré principal. Soit $\pi: \Sigma \longrightarrow C=\Sigma / G$ une structure de niveau $(n)$ sur la courbe stable $C$, définie sur le corps algébriquement clos $k$. Sous les présentes hypothèses, en notant $\tilde{C}=\sqcup_{i} \tilde{C}_{i}$ la normalisation de $C$ et $\Gamma$ le graphe dual, la suite exacte (71) donne une suite exacte

$$
1 \longrightarrow H^{1}(\Gamma) \otimes \mathbb{Z} / n \mathbb{Z} \longrightarrow \operatorname{Pic}(C)[n] \longrightarrow \prod_{i} \operatorname{Pic}\left(\tilde{C}_{i}\right)[n] \longrightarrow 1
$$

En particulier, cela conduit aux identifications

$$
\operatorname{Pic}(C)[n] \stackrel{\sim}{\longrightarrow} H_{e t}^{1}(C, \mathbb{Z} / n \mathbb{Z}) \stackrel{\sim}{\longrightarrow}(\mathbb{Z} / n \mathbb{Z})^{2 g-h(\Gamma)} .
$$

Notons que si $\Delta$ est le graphe modulaire associé à $\Sigma$, on a $\Gamma=\Delta / G$. On peut rendre plus explicites les groupes de décomposition et d'inertie.

Proposition 8.3.1. - Dans la situation ci-dessus, soient $D$ et I les groupes de décomposition et d'inertie (définition 7.2.8). Si $C_{i}$ est une composante irréductible de $C$, soient $D_{i}$ et $I_{i}$ les groupes de décomposition et d'inertie correspondants et $v_{i}$ la valence du sommet de $\Gamma$ associé. Alors, on a :

i) $G / D \stackrel{\sim}{\longrightarrow}(\mathbb{Z} / n \mathbb{Z})^{h(\Gamma)}$ et $G / I \stackrel{\sim}{\longrightarrow}(\mathbb{Z} / n \mathbb{Z})^{2 g-h(\Gamma) \stackrel{\sim}{\longrightarrow}} \operatorname{Pic}(C)[n]$.

ii) $I_{i}=I \cap G_{i}, G_{i} / I_{i} \stackrel{\sim}{\longrightarrow}(\mathbb{Z} / n \mathbb{Z})^{2 g_{i}}$ et $D \stackrel{\sim}{\longrightarrow}(\mathbb{Z} / n \mathbb{Z})^{2 g-h(\Gamma)}$.

iii) $I_{i} \stackrel{\sim}{\longrightarrow}(\mathbb{Z} / n \mathbb{Z})^{v_{i}-1}$ et $G_{i} \stackrel{\sim}{\longrightarrow}(\mathbb{Z} / n \mathbb{Z})^{2 g_{i}+v_{i}-1}$.

Démonstration. - Considérons le diagramme suivant à lignes exactes et à flèches verticales injectives:

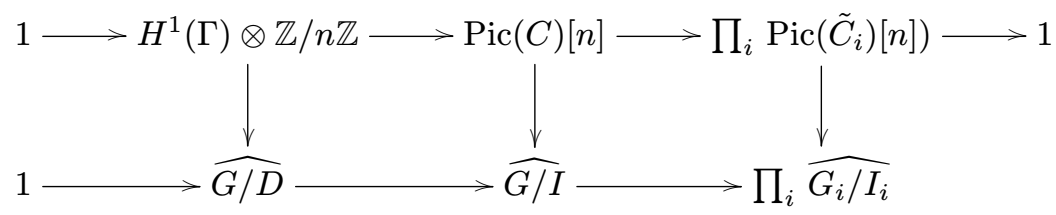

L'exactitude de la suite horizontale du bas est le contenu du théorème 7.3.1. On montre d'abord que la flèche verticale du milieu est une bijection. Cela se ramène à prouver que si $L \in \operatorname{Pic}(C)[n]$, alors $\pi^{*}(L) \cong \theta_{\Sigma}$ (voir la preuve du théorème 7.3.1). 
Soit $d$ l'ordre de $L$ et considérons le revêtement étale connexe $\tau: C^{*} \longrightarrow C$ de degré $d$ défini par $L$, c'est-à-dire tel que

$$
\tau_{*}\left(\theta_{C^{*}}\right)=\bigoplus_{i=0}^{d-1} L^{i} .
$$

Du fait que $L$ est d'ordre exact $d$, la courbe $C^{*}$ est connexe, et par ailleurs certainement stable. De plus, par construction $\tau^{*}(L) \cong \theta_{C^{*}}$. Considérons une déformation du $G$-revêtement $\pi: \Sigma \longrightarrow C$ de base $\operatorname{Spec}(R)$, où $R$ est un anneau de valuation discrète complet de corps résiduel $k$, à fibre générique lisse. Du fait que $C^{*} \longrightarrow C$ est étale, on sait qu'il existe une déformation $\chi^{*} \longrightarrow \chi$ de ce revêtement de base une quelconque base prescrite, en particulier $\chi$; on peut se référer par exemple à la proposition 5.1.2. Notons $\eta$ le point générique de $\operatorname{Spec}(R)$, et $Y_{\eta}$ (resp. $\left.X_{\eta}\right)$ les fibres génériques respectives. Alors le revêtement $Y_{\eta} \longrightarrow X_{\eta}$ induit au dessus de $\eta$ se factorise par $X_{\eta}^{*}$. Cela force $\pi: \mathcal{Y} \longrightarrow \chi$ à se factoriser par $X^{*}$, et au bout du compte $\pi: \Sigma \longrightarrow C$ se factorise par $C^{*}$, ce qui entraîne bien $\pi^{*}(L) \cong \theta_{\Sigma}$.

Prouvons maintenant les points i) à iii). Partant de de l'égalité $\widehat{G / I}=\operatorname{Pic}(C)[n]$ et des identifications connues

$$
H^{1}(\Gamma) \otimes \mathbb{Z} / n \mathbb{Z} \cong(\mathbb{Z} / n \mathbb{Z})^{h(\Gamma)} \quad \text { et } \quad \operatorname{Pic}\left(\tilde{C}_{i}\right)[n] \cong(\mathbb{Z} / n \mathbb{Z})^{2 g_{i}},
$$

le diagramme ci-dessus montre que les applications verticales sont des bijections, donc d'une part $\widehat{G / D} \cong(\mathbb{Z} / n \mathbb{Z})^{h(\Gamma)}$, et d'autre part $\widehat{G_{i} / I_{i}}=\operatorname{Pic}\left(\tilde{C}_{i}\right)[n]$ pour tout indice $i$. Par rapport à la suite exacte (73), on a maintenant la suite exacte

$$
1 \longrightarrow \widehat{G / D} \longrightarrow \widehat{G / I} \longrightarrow \prod_{i} \widehat{G_{i} / I_{i}} \longrightarrow 1 \text {. }
$$

Par dualité, on obtient une injection $\prod_{i} G_{i} / I_{i} \mapsto G / I$, donc $G_{i} \cap I=I_{i}$ pour tout indice $i$. Comme $G_{i} / I_{i} \cong(\mathbb{Z} / n \mathbb{Z})^{2 g-h(\Gamma)}$, on a finalement $I \cong(\mathbb{Z} / n \mathbb{Z})^{h(\Gamma)}$, et pour une raison identique $D \cong(\mathbb{Z} / n \mathbb{Z})^{h(\Gamma)}$.

Notons maintenant $v_{i}$ la valence du sommet d'indice $i$ du graphe $\Gamma$. Le groupe $I_{i}$ est engendré par $v_{i}$ éléments $\left\{\sigma_{i, \alpha}\right\},\left(\alpha=1, \ldots, v_{i}\right)$, dont l'ordre divise $n$, et soumis à une relation $\sum_{\alpha} \sigma_{i, \alpha}=0$. Dans le groupe $I$ qui est le sous-groupe engendré par les $I_{i}$, un décompte naif des générateurs conduit du fait que chaque $\sigma_{i, \alpha}$ apparaît deux fois, via une arête orientée et l'arête opposée, à une majoration de

$$
\sum_{i} v_{i}-A-(S-1)=h(\Gamma) .
$$

Les $S$ relations $\sum_{\alpha} \sigma_{i, \alpha}$ sont dépendantes, car liées par $\sum_{i}\left(\sum_{\alpha} \sigma_{i, \alpha}\right)=0$, ce qui explique la contribution $S-1$. Par ailleurs, on sait que $I \cong(\mathbb{Z} / n \mathbb{Z})^{h(\Gamma)}$, et on voit ainsi qu'entre les générateurs indiqués, il ne peut y avoir d'autres relations que les relations imposées par le graphe, les usuelles relations de courant. Cela conduit aux égalités $I_{i, \alpha}=\mathbb{Z} / n \mathbb{Z}$ et $I_{i}=(\mathbb{Z} / n \mathbb{Z})^{v_{i}-1}$, donc finalement à $G_{i} \cong(\mathbb{Z} / n \mathbb{Z})^{2 g_{i}+v_{i}-1}$ comme indiqué. 
8.3.2. Composantes irréductibles du bord. - La description des composantes irréductibles du bord de $\bar{M}_{g}(n)$ est aisée. La description générale donnée dans les sections 7.2 et 7.4 montre que les composantes irréductibles correspondent au choix d'un graphe modulaire $\Gamma$, qui est soit un segment, soit une boucle.

segment : dans ce cas, l'image de la composante dans $\bar{M}_{g}$ est isomorphe au produit $\overline{\mathcal{M}}_{g_{1}} \times \overline{\mathcal{M}}_{g_{2}},\left(g_{1}+g_{2}=g\right)$. Pour les groupes $I$ et $D$, on a visiblement

$$
I=1 \quad, \quad D=G=(\mathbb{Z} / n \mathbb{Z})^{2 g} .
$$

De manière plus précise, le groupe $G$ se décompose en $G=G_{1} \times G_{2}, G_{i} \cong(\mathbb{Z} / n \mathbb{Z})^{2 g_{i}}$ $(i=1,2)$. Il en résulte en particulier que la composante irréductible correspondante s'identifie à $\overline{\mathcal{M}}_{g_{1}, 1}(n) \times \overline{\mathcal{M}}_{g_{2}, 1}(n)$, espaces modulaires de structure de niveau $(n)$ avec un point marqué.

boucle : dans ce cas, on a (section 7.4$)$

$$
I=\mathbb{Z} / n \mathbb{Z}, \quad D=(\mathbb{Z} / n \mathbb{Z})^{2 g-1} .
$$

Avec les notations de cette section, l'élément $g_{0}$ est d'ordre $n$, et $G=D \times\left\langle g_{0}\right\rangle$. La composante correspondante du bord s'identifie avec l'espace modulaire classifiant les structures de niveau $(n)$ sur une courbe de genre $g-1$ avec une seule piqûre, c'està-dire essentiellement à un espace de Hurwitz défini par le groupe $G=(\mathbb{Z} / n \mathbb{Z})^{2 g}$, et une donnée de Hurwitz réduite à un seul élément d'ordre $n$. L'image d'une telle composante dans $\bar{M}_{g}$ est la strate notée $\Delta_{0}=\bar{M}_{g-1,2}$.

Il est possible d'étendre la description précédente des composantes de codimension un du bord à un groupe de niveau arbitraire, c'est-à-dire au champ $\bar{M}_{g}(G)$. Cela permet par exemple de retrouver de manière naturelle quelques résultats de BoggiPikaart [16]. Le niveau étant toujours le niveau abélien $(n), n \geqslant 3$, on obtient facilement en corollaire des méthodes précédentes la structure connue des sousgroupes d'inertie pour les points doubles [50]. Les hypothèses sont celles de la proposition 8.3.1 :

Proposition 8.3.2. - Soit $Q_{\alpha}$ un point double de $C$, et soit $H_{\alpha}$ le stabilisateur d'un quelconque point double de $\Sigma$ au dessus de $Q_{\alpha}$. Alors $H_{\alpha}=1$ si et seulement si $Q_{\alpha}$ déconnecte $C$, sinon $H_{\alpha}=\mathbb{Z} / n \mathbb{Z}$.

Démonstration. - Notons tout d'abord que le resultat est clair si $C$ n'a qu'un seul point double, comme il résulte de la preuve précédente. On va ramener le cas général à ce cas particulier par un argument de déformation. Supposons d'abord que le point double $Q=Q_{\alpha}$ ne déconnecte pas $C$. Dans ce cas la normalisation partielle $C^{*}$ de $C$ en $Q$ est une courbe stable de genre $g-1$ marquée par deux points $Q^{\prime}$ et $Q^{\prime \prime}$, et $C$ se déduit de $C^{*}$ par l'identification $Q^{\prime}=Q^{\prime \prime}$. Cette construction, le clutching morphism de Knudsen [68], s'étend à la déformation universelle de $\left(C^{*}, Q^{\prime}, Q^{\prime \prime}\right)$, et conduit à une déformation

$$
\mathscr{C}^{*} \longrightarrow \operatorname{Spec}\left(R^{*}\right)
$$


de $C$ dans laquelle le point double $Q$ s'étend. Comme le clutching morphism est un morphisme représentable, fini et non ramifié (loc. cit. cor. 3.9), on voit que cette déformation n'est pas autre chose que la déformation universelle de $C$ qui préserve le point double $Q$. Elle peut se décrire comme étant la restriction de la déformation universelle $\mathscr{C} \longrightarrow \operatorname{Spec}(R)$ de $C$ à l'hypersurface $t_{1}=0$, si $t_{1}$ désigne le paramètre de déformation de $Q$. On peut donc conclure que la fibre générique de cette déformation est une courbe avec un unique point double $Q$ qui reste du type boucle.

Le niveau étant toujours le niveau abélien $(n), n \geqslant 3$, l'interprétation modulaire des points du bord permet de décrire en des termes simples la ramification du morphisme $\overline{\mathcal{M}}_{g}(n) \longrightarrow \overline{\mathcal{M}}_{g}$, en d'autres termes sa structure logarithmique [77]. Fixons $C$ un point de $\bar{M}_{g}$ sur le corps algébriquement clos $k$; on suppose comme toujours que $n$ est inversible dans $k$. Soit $\mathrm{Ni}_{G}(C)$ l'ensemble des structures de niveau naïves $G=$ $(\mathbb{Z} / n \mathbb{Z})^{2 g}$ sur $C$ (section 8.2$)$. Il y a une action évidente $\operatorname{de} \operatorname{Out}(G)=\mathrm{GL}_{2 g}(\mathbb{Z} / n \mathbb{Z})$ sur $\mathrm{Ni}_{G}(C)$. Alors :

Lemme 8.3.3. - L'action de $\operatorname{Out}(G)=\mathrm{GL}_{2 g}(\mathbb{Z} / n \mathbb{Z})$ sur $\mathrm{Ni}_{G}(C)$ est transitive.

Démonstration. - On suppose d'abord que $S$ est le spectre d'un corps algébriquement clos $k$. Dans le cas lisse, le résultat est clair du fait qu'une structure de niveau naïve est identique à une vraie structure de niveau. Si maintenant $C$ est singulière, le corollaire précédant montre que les points doubles de $C$ qui sont les images des points doubles de $\Sigma$ avec une isotropie non triviale, ne dépendent en fait que de $C$, plus précisément du graphe $\Gamma$, et pas de la structure de niveau représentée par $\pi: \Sigma \longrightarrow C$. En ces points l'indice de ramification est égal à $n$. Soit maintenant $X$ la déformation universelle équivariante de $\Sigma$, de base $\operatorname{Spec}\left(k\left[\left[t_{1}, \ldots, t_{r}, \ldots, t_{3 g-3}\right]\right]\right)$, où $t_{\alpha}$ est le paramètre de déformation de l'orbite de points doubles au-dessus du point $Q_{\alpha}$ de $C$. La courbe quotient $Y=X / G$ se déduit de la déformation universelle $\mathscr{C}$ de $C$, ayant pour base $\operatorname{Spec}\left(k\left[\left[\tau_{1}, \ldots, \tau_{r}, \ldots, \tau_{3 g-3}\right]\right]\right)$, par le changement de base donné par $\tau_{\alpha}=t_{\alpha}^{e_{\alpha}}$ (théorème 5.1.5). Ce qui vient d'être dit montre que $Y$, à isomorphisme de déformation près, ne dépend pas du choix de la structure de niveau.

Soient maintenant deux structures de niveau $(n), \pi_{i}: \Sigma_{i} \longrightarrow C(i=1,2)$ sur la courbe $C$. Soient $X_{i}(i=1,2)$ les déformations universelles équivariantes respectives. On peut ainsi supposer que les déformations $X_{1} / G$ et $X_{2} / G$ de $C$ sont isomorphes, donc que $X_{1} / G \cong X_{2} / G \cong Y$ est une déformation donnée de $C$ de base $S=$ $\operatorname{Spec}\left(k\left[\left[\tau_{1}, \ldots, \tau_{r}, \ldots, \tau_{3 g-3}\right]\right]\right)$. On peut en outre, par une spécialisation convenable, la base étant ramenée à $S=\operatorname{Spec}(R)$, avec $R$ anneau de valuation discrète complet de corps résiduel $k$, supposer que les fibres génériques de $X_{i}(i=1,2)$ et de $Y$ sont lisses, et définissent des structures de niveau situées dans une même orbite de $\mathrm{GL}_{2 g}(\mathbb{Z} / n \mathbb{Z})$. De manière équivalente, il existe $\theta \in \mathrm{GL}_{2 g}(\mathbb{Z} / n \mathbb{Z})$ tel que le schéma $\operatorname{Isom}\left(X_{1}^{\theta}, X_{2}\right)$ classifiant les isomorphismes équivariants au dessus de $Y$, soit fini, non ramifié et surjectif. Il y a donc au moins une composante connexe de $\operatorname{Isom}_{\pi}\left(X_{1}^{\theta}, X_{2}\right)$ qui domine $S$ et qui alors étale sur $S$, donc isomorphe à $S$. On en déduit un $Y$-isomorphisme 
$X_{1}^{\theta} \stackrel{\sim}{\longrightarrow} X_{2}$, d'où finalement un isomorphisme $\Sigma_{1}^{\theta} \stackrel{\sim}{\longrightarrow} \Sigma_{2}$ au-dessus de $C$, d'où la conclusion.

Il a été observé que le morphisme $\mathcal{M}_{g}(G) \sqcap Z(G) \longrightarrow \mathcal{M}_{g}$ est un Out $(G)$-torseur. Il n'en n'est plus de même pour le morphisme $\overline{\mathcal{M}}_{g}(G) \sqcap Z(G) \longrightarrow \overline{\mathcal{M}}_{g}$. Au niveau des espaces grossiers de modules, le morphisme $\bar{M}_{g}(n) \longrightarrow \bar{M}_{g}$ est un revêtement galoisien de groupe de Galois $\mathrm{GL}_{2 g}(\mathbb{Z} / n \mathbb{Z})$. Pour préciser cela, soit un point $\xi$ de $\bar{M}_{g}(n)$ défini sur $k$, représenté par $\pi: \Sigma \longrightarrow C$. Le groupe d'inertie $I_{\xi}$ dans ce revêtement a la description suivante :

$$
I_{\xi}=\left\{\theta \in \mathrm{GL}_{2 g}(\mathbb{Z} / n \mathbb{Z}), \Sigma^{\theta} \cong \Sigma\right\} .
$$

Un élément de $I_{\xi}$ déterminé par $f: \Sigma \stackrel{\sim}{\longrightarrow} \Sigma^{\theta}$ définit donc un diagramme commutatif

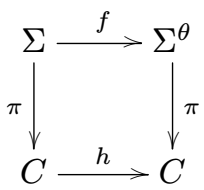

dans lequel l'automorphisme horizontal du bas $h: C \stackrel{\sim}{\longrightarrow} C$ est celui déduit de $f$ par passage au quotient. Notons $\widehat{I}_{\xi}$ le sous-groupe formé des couples $(f, \theta)$ comme ci-dessus. On a la suite exacte

$$
1 \longrightarrow \operatorname{Aut}_{G}(\Sigma) \longrightarrow \widehat{I}_{\xi} \longrightarrow I_{\xi} \longrightarrow 1 \text {. }
$$

Considérons le groupe $\operatorname{Aut}_{\pi}(\Sigma)$ des $C$-automorphismes (non équivariants a priori) de $\Sigma$ qui commutent avec $\pi$, et qui préservent globalement $G$. Du fait que $n \geqslant 3$, le lemme de rigidité implique que $\operatorname{Aut}_{G}(\Sigma) \subset \operatorname{Aut}_{\pi}(\Sigma)$. L'application $(f, \theta) \mapsto h$ est alors bien définie; il en résulte une suite exacte

$$
1 \longrightarrow \frac{\operatorname{Aut}_{\pi}(\Sigma)}{\operatorname{Aut}_{G}(\Sigma)} \longrightarrow I_{\xi} \longrightarrow \operatorname{Aut}(C) \longrightarrow 1
$$

Noter que la surjectivité à droite découle du lemme 8.9. Si $C$ est lisse, on a certainement $\operatorname{Aut}_{\pi}(\Sigma)=\operatorname{Aut}_{G}(\Sigma)=G$, et $I_{\xi}=\operatorname{Aut}(C)$. Dans le cas général, on a le résultat suivant :

Proposition 8.3.4. - Soit $m$ le nombre de points doubles de $C$, ou nombre d'arêtes de $\Gamma$, qui ne déconnectent pas $\Gamma$. Alors, on a un isomorphisme

$$
\frac{\operatorname{Aut}_{\pi}(\Sigma)}{G} \stackrel{\sim}{\longrightarrow}(\mathbb{Z} / n \mathbb{Z})^{m}
$$

Démonstration. - Soit $f \in \operatorname{Aut}_{\pi}(\Sigma)$, il existe $\theta \in \operatorname{Aut}(G)$ rendant $G$-équivariant l'isomorphisme $f: \Sigma \stackrel{\sim}{\longrightarrow} \Sigma^{\theta}$. Si $X$ est une déformation infinitésimale équivariante de $\Sigma$, et si $\imath: C \hookrightarrow X$ est le plongement équivariant correspondant, le couple $\left(X, \imath f^{-1}\right)$ définit donc une déformation notée $X_{f}$ de $\Sigma^{\theta}$. Si $\chi \longrightarrow \operatorname{Spec}(S)$ avec $S=k\left[\left[t_{1}, \ldots, t_{3 g-3}\right]\right]$ est la déformation universelle équivariante de $\Sigma$, alors $\chi^{\theta} \longrightarrow$ 
$\operatorname{Spec}(S)$ est celle de $\Sigma^{\theta}$. Il vient alors de $f$, un automorphisme $\hat{f} \operatorname{de} \operatorname{Spec}(S)$, qui conduit au diagramme cartésien



où $h$ induit $f$ sur les fibres au dessus de l'origine $0 \in S$. Comme $\pi f=\pi$, les déformations de $C$ obtenues par passage au quotient par $g$ sont équivalentes, ce qui force $\hat{f}$ à être l'identité $\operatorname{sur} \operatorname{Spec}(R)$ base de la déformation universelle de $C$. Ainsi $\hat{f}$ appartient au groupe de Galois du revêtement $\operatorname{Spec}(S) \longrightarrow \operatorname{Spec}(R)$, groupe identifié à $(\mathbb{Z} / n \mathbb{Z})^{m}$. Noter que si $\hat{f}=1$, alors par examen au point générique, on obtient en conséquence que $f$ est la translation par un élément de $G$.

Réciproquement, partons d'un élément $\hat{f}$ du groupe de Galois $(\mathbb{Z} / n \mathbb{Z})^{m}$, et notons $\chi_{\hat{f}}$ la déformation déduite du carré cartésien



Par passage au quotient par $G$, on obtient un isomorphisme $\chi_{\hat{f}} / G \stackrel{\sim}{\longrightarrow} \chi / G$. Par le lemme 8.3.3 on conclut qu'il y a au-dessus $\operatorname{de} \operatorname{Spec}(S)$ un isomorphisme équivariant $\chi_{\hat{f}} \stackrel{\sim}{\longrightarrow} \chi^{\theta}$ pour un $\theta$ convenable. Le diagramme cartésien qui en découle, analogue à $(87)$, assure que tout élément du groupe de Galois est dans l'image de $\operatorname{Aut}_{\pi}(\Sigma)$.

En général, pour un revêtement principal dégénéré et pour un niveau arbitraire dominant $(n), n \geqslant 3$, chacune des deux inclusions

$$
G \subset \operatorname{Aut}_{G}(\Sigma) \subset \operatorname{Aut}_{\pi}(\Sigma)
$$

peut être stricte. Notons que l'anneau local complété de $\bar{M}_{g}(G)$ au point défini par $\Sigma$ est $\hat{\theta}_{\Sigma} \cong k\left[\left[\tau_{1}, \ldots, \tau_{3 g-3}\right]\right]^{\text {Aut }_{G}(\Sigma)}$. La singularité éventuelle de cet anneau local provient donc du groupe $\operatorname{Aut}_{G}(\Sigma)$ et de son action sur l'espace tangent $H_{G}^{1}\left(\Sigma, \Theta_{\Sigma}\right)$. Comme application, donnons une preuve différente du résultat connu suivant (van Geemen-Oort [50]) :

Proposition 8.3.5. - Soit un revêtement principal dégénéré $\pi: \Sigma \longrightarrow C$ définissant une structure de niveau $(n), n \geqslant 3$. On suppose que $C$ a deux composantes irréductibles se coupant transversalement en deux points, alors on a

$$
\operatorname{Aut}_{\pi}(\Sigma) / G \stackrel{\sim}{\longrightarrow} \mathbb{Z} / n \mathbb{Z}
$$


Démonstration. - Par hypothèse, on a $C=C^{\prime} \cup C^{\prime \prime}$, avec $C^{\prime}$ (resp. $C^{\prime \prime}$ ) lisse de genre $g^{\prime} \geqslant 1\left(\operatorname{resp} g^{\prime \prime} \geqslant 1\right)$, et $g=g^{\prime}+g^{\prime \prime}+1$. Soit $C^{\prime} \cap C^{\prime \prime}=\left\{Q^{\prime}, Q^{\prime \prime}\right\}$. Comme $h(\Gamma)=1$, les stabilisateurs des points doubles de $\Sigma$, donc au dessus de $Q^{\prime}$ ou $Q^{\prime \prime}$, sont égaux, disons à $H \cong \mathbb{Z} / n \mathbb{Z}$. Soit $\Sigma^{\prime}$, resp. $\Sigma^{\prime \prime}$, une composante de $\Gamma$ qui relève $C^{\prime}$, resp. $C^{\prime \prime}$, et supposons que $P^{\prime} \in \Sigma^{\prime} \cap \Sigma^{\prime \prime}$ soit au dessus de $Q^{\prime}$. Si $f \in \operatorname{Aut}_{G}(\Sigma)$, on a $f\left(P^{\prime}\right) \in G P^{\prime}$, on peut se ramener en modifiant $f$ par un élément de $g \in G$ à $f\left(P^{\prime}\right)=P^{\prime}$. Alors $f\left(\Sigma^{\prime}\right)=\Sigma^{\prime}$ et $f\left(\Sigma^{\prime \prime}\right)=\Sigma^{\prime \prime}$. La restriction de $f$ à $\Sigma^{\prime}$ commute à l'action de $G^{\prime}$, donc est définie par un élément de $G^{\prime}$, du fait que $\Sigma^{\prime}$ est lisse. Par une nouvelle réduction on peut supposer de plus que $f \mid \Sigma^{\prime}=i d$. Alors on doit avoir $f \mid \Sigma^{\prime \prime} \in H \cong \mathbb{Z} / n \mathbb{Z}$. Il est facile de voir qu'inversement un automorphisme $f$ de $\Sigma$ défini par ces deux conditions centralise l'action de $G$. Le résultat en découle.

Si maintenant $P^{\prime \prime} \in \Sigma^{\prime \prime}$ est un point au dessus de $Q^{\prime \prime}$, il est clair que les actions de $f$ en les espaces tangents en ces deux points sont définies par des racines $n$-ième de l'unité opposées. Ainsi l'action de $\operatorname{Aut}_{G}(\Sigma) / G$ sur l'espace de la déformation équivariante universelle de $\Sigma$ est donnée par $\tau_{1} \mapsto \epsilon \tau_{1}, \tau_{2} \mapsto \epsilon^{-1} \tau_{2}, \tau_{\alpha} \mapsto \tau_{\alpha}(\alpha \geqslant 3)$. D'où vient le fait qu'en ce point $\bar{M}_{g}(n)$ a une singularité $A_{n-1} \times($ variété lisse de codimension 2).

Le calcul explicite du groupe $\operatorname{Aut}_{G}(\Sigma)$, généralisant le calcul de la proposition 8.3.5 est possible avec un niveau général, et sous une condition de généricité de $C$. La condition requise est celle qui pour $f \in \operatorname{Aut}_{G}(\Sigma)$ assure que l'automorphisme induit $h$ de la courbe $C$, pointée par les points de branchement, est égal à l'identité. De manière générale, après avoir fait le choix d'une orientation du graphe $\Gamma$, on a le résultat :

Proposition 8.3.6. - Soit $\operatorname{Aut}_{G, \pi}(\Sigma)=\left\{f \in \operatorname{Aut}_{G}(\Sigma), \pi f=\pi\right\}$. On a une suite exacte :

$$
1 \longrightarrow \operatorname{Aut}_{G, \pi}(\Sigma) \stackrel{j}{\longrightarrow} \prod_{v} G \stackrel{\partial}{\longrightarrow} \prod_{e} G /\left(\bigcap_{\omega \mapsto e} G_{\omega}\right)
$$

où e $(0)$ resp. e(1) désigne l'origine resp. l'extrémité de e, et $\partial\left(\left(\sigma_{v}\right)_{v}\right)=\left(\sigma_{e(1)}^{-1} \sigma_{e(0)}\right)_{e}$.

Démonstration. - Définissons l'application $j$. Par hypothèse l'automorphisme $h$ de $C$ induit par un élément $f \in \operatorname{Aut}_{G, \pi}(\Sigma)$ est supposé être l'identité. Dès lors pour tout sommet $v$ de $\Gamma$ la courbe $\Sigma_{v}=\pi^{-1}\left(C_{v}\right)$ est fixée par $f$, ce qui entraîne que l'automorphisme défini par restriction de $f$ à cette courbe coïncide avec la restriction d'un élément $\sigma_{v} \in G$. En effet, quitte à modifier $f$ par un élément de $G$, on peut supposer que $f$ fixe une composante irréductible $\Delta_{v}$ de $\Sigma_{v}$. Sur cette composante $f$ est alors donné par un élément de $G$, en fait du centre du stabilisateur de cette composante, donc est égal à cet élément partout. On définit alors l'injection $j$ par la collection $j(f)=\left(\sigma_{v}\right)_{v}$.

La condition de cohérence qu'on doit exiger d'une collection $\left(\sigma_{v}\right)$ pour qu'elle définisse un automorphisme de $\Sigma$ par recollement des $\sigma_{v}$ est que pour tout point double $Q$ de $C$, donc toute arête $e$ de $\Gamma$, et si $a$ et $b$ sont les extrémités de $e$, alors $\sigma_{a}(P)=\sigma_{b}(P)$ pour tout point double $P \in \Sigma$ d'image $Q$. Cette condition, qui équivaut à ce que $\sigma_{b}^{-1} \sigma_{a}$ fixe tout point double au dessus de $Q$, assure que les automorphismes 
$\sigma_{v} \operatorname{des} \Sigma_{v}$ se recollent en un $G$-automorphisme de $\Sigma$. Cette condition s'exprime bien $\operatorname{par} \partial\left(\left(\sigma_{v}\right)\right)=1$.

EXemple 8.3.7. - Les champs $\overline{\mathcal{M}}_{0}[n]$ et $\overline{\mathcal{M}}_{1}[n](n \geqslant 5)$.

On se place dans la catégorie des $\mathbb{Z}[1 / n]$-schémas. On va montrer que la théorie bien connue du champ classifiant les courbes elliptiques, éventuellement généralisées, munies d'un sous-groupe cyclique d'ordre $n$, respectivement d'un point d'ordre exactement $n$ (Deligne-Rapoport [31]), se retrouve très simplement dans le contexte des champs de Hurwitz, comme application des résultats de la présente section ${ }^{(2)}$.

On forme d'abord le champ $\overline{\mathscr{H}}_{n, 1}$ classifiant les revêtements étales d'une courbe de genre 1 avec un point marqué, i.e. une courbe elliptique. Si $\pi: C \longrightarrow E$ est un tel revêtement, il y a donc une action libre de $G=\mathbb{Z} / n \mathbb{Z}$ sur $E$ telle que $C / G \cong E$; en particulier le genre de $C$ est 1 . La courbe $C$ est marquée par les points de la fibre $\pi^{-1}(O)$, si $O \in E$ définit le marquage de $E$. En fait, on affine le marquage de $C$ en spécifiant un point noté encore $O$ dans $\pi^{-1}(O)$. En conséquence les isomorphismes doivent fixer ce point. Si $E$ (et alors $C$ ) est lisse, on voit que l'action de $G$ s'identifie à un plongement $G=\mathbb{Z} / n \mathbb{Z} \hookrightarrow C[n]$.

Soit maintenant un point géométrique du bord, donc au-dessus du point à l'infini de $\bar{M}_{1,1}$ de graphe dual une boucle. Les résultats de la section 7.2 montrent que le graphe dual $\Gamma$ de la courbe stable marquée $C$ est décrit par une donnée

$$
H \subset G_{0} \subset G, \quad g_{0} \in G-G_{0} .
$$

Le genre de $C$ étant 1 , cela force à avoir $H=G_{0}$, et $\Gamma$ est soit une courbe de genre 1 avec un point double, soit un polygone de Néron $\mathscr{P}_{l}$ à $l=[G: H] \geqslant 2$ côtés $([\mathbf{3 1}])$. Si $l=1$ c'est la courbe rationnelle avec un point double. Rappelons qu'on a par construction

$$
\mathscr{P}_{l}=\mathbb{P}^{1} \times \mathbb{Z} / l \mathbb{Z}
$$

Si on note $[x, i](0 \leqslant i \leqslant l-1)$ un point de la $i$-ième copie de $\mathbb{P}^{1}$, alors les identifications sont $[\infty, i]=[0, i+1]$. On suppose que le marquage est donné par le point $[1,0]$. Distinguons à l'intérieur du groupe $\operatorname{Aut}\left(\mathscr{P}_{l}\right)$ le sous-groupe $\operatorname{Aut}_{+}\left(\mathscr{P}_{l}\right)$ des « rotations », c'est-à-dire le sous-groupe formé par les automorphismes

$$
\varphi_{h, \alpha}([x, i])=\left[h_{i}(x), i+\alpha\right]
$$

où $h_{0}, \ldots, h_{i} \in G_{m}$, le groupe des homographies qui fixent 0 et $\infty$, et $\alpha \in \mathbb{Z} / l \mathbb{Z}$. Le groupe Aut ${ }_{+}\left(\mathscr{P}_{l}\right)$ est le produit en couronne $G_{m} \curlywedge(\mathbb{Z} / l \mathbb{Z})$. Il n'est pas difficile de voir qu'à conjugaison près (fixant le marquage), l'automorphisme $\sigma$ donné par l'action du générateur $1 \in \mathbb{Z} / n \mathbb{Z}$, peut-être réduit à la forme canonique suivante

$$
\sigma[x, i]=[\tau(x), i+\alpha], \quad \tau(x)=\zeta x, 1 \leqslant \alpha<l,(\alpha, l)=1
$$

(2) La notation de [31] est $\mathcal{M}_{\Gamma_{0}(n)}$, respectivement $\mathcal{M}_{\Gamma_{00}(n)}$. 
où $d=n / l$ et $\zeta$ est une racine primitive $d$-ième de l'unité. Partant de cette observation, et tenant compte du point formé d'une courbe de genre 1 avec un seul point double, il est facile de compter le nombre $s$ de pointes, et de retrouver la formule connue

$$
s=\frac{1}{2} \sum_{1 \leqslant l / n} \varphi(l) \varphi\left(\frac{n}{l}\right) .
$$

Il est facile et par ailleurs bien connu que, dès lors que $n \geqslant 5$, alors un point géométrique de $\overline{\mathcal{H}}_{1, n}$ a un groupe d'automorphisme trivial, de sorte que ce champ est représentable. Il y a une action naturelle de $(\mathbb{Z} / n \mathbb{Z})^{*}$ sur $\overline{\mathcal{H}}_{1, n}(2.2 .1)$, et il découle des définitions qu'on a

$$
\left[\overline{\mathcal{H}}_{1, n} /(\mathbb{Z} / n \mathbb{Z})^{*}\right]=\overline{\mathcal{H}}_{0, n} \text {. }
$$

Ce champ quotient n'est pas représentable. Il est facile cependant de compter à isomorphisme près le nombre de pointes, et de retrouver un résultat bien connu. Un calcul immédiat montre en effet que l'automorphisme $\sigma$ d'ordre $n$ est conjugué à $\sigma^{\beta}(\operatorname{avec}(\beta, n)=1)$ si et seulement si $\beta \equiv 1(\bmod n /(l, n / l))$. On en tire que le nombre de pointes de $\overline{\mathcal{H}}_{0, n}$ est

$$
\sum_{1 \leqslant l \mid n} \varphi((l, n / l)) .
$$

Il nous semble intéressant d'étudier dans cette optique le problème de modules posé par les revêtements de degré arbitraire d'une courbe de genre 1 par une courbe de genre 2, avec un unique point de branchement. On sait que ce problème conduit à une courbe modulaire usuelle (voir Kani [65]). 



\section{CHAPITRE 9}

\section{REVÊTEMENTS CYCLIQUES}

Le champ des revêtements cycliques admet une description particulière et finalement assez simple ${ }^{(1)}$.

$\mathrm{Si}$ on fait le choix pour la base de la droite $\mathbb{P}^{1}$, on donnera dans ce cas une description très concrète du sous-champ ouvert classifiant les revêtements non dégénérés, comme champ quotient, généralisant le résultat de Arsie-Vistoli [5]. La construction est parallèle à celle de Jarvis [59], [58], qui traite des courbes à spin.

\subsection{Revêtements cycliques versus racines d'un faisceau inversible}

9.1.1. Racines et quasi-racines d'un faisceau inversible. - On fixe un entier $r \geqslant 2$. Le concept de racine $r$-ième se définit en deux temps [59], [58] :

DÉFInItion 9.1.1. - Soit D une courbe préstable. Une quasi-racine r-ième d'un faisceau inversible $\kappa$ sur $D$ est la donnée d'un couple $(\mathcal{L}, \Phi)$ où $\mathcal{L}$ est un faisceau sans torsion de rang 1 , et $\Phi$ est un morphisme

$$
\Phi: \mathscr{L}^{\otimes r} \longrightarrow \kappa
$$

qui est un isomorphisme en tout point où $\mathcal{L}$ est libre. Une quasi-racine est une racine r-ième si de plus :

i) en un point singulier de $\mathcal{L}$, i.e. un point en lequel $\mathcal{L}$ n'est pas libre, le conoyau de $\Phi$ est de rang $r-1$.

ii) $r \operatorname{deg} \mathscr{L}=\operatorname{deg} \kappa$ où $\operatorname{deg} \mathscr{L}=\chi(\mathscr{L})-\chi\left(\theta_{C}\right)$.

Dans le contexte des revêtements cycliques, on sera amené à considérer les racines d'ordre $r$ d'un faisceau inversible $\theta_{D}\left(-\sum_{i} m_{i} Q_{i}\right)$. Dans ce cas, les conditions cidessus (et en particulier la condition iii) : $\operatorname{deg}(\mathscr{L})=2 g-2-\sum_{i} m_{i}$ ) étant imposées, Jarvis [59], [58] a montré que le champ classifiant les données $\left(D, \mathscr{L},\left\{Q_{i}\right\}\right)$, où $\mathscr{L}$ est une racine $r$-ième comme cela vient d'être défini, est un champ de Deligne-Mumford

(1) Bien que ce ne soit pas nécessaire pour la suite, il est instructif de comparer le champ des revêtements à groupe de Galois cyclique avec le champ des courbes à spin étudié en détail par Jarvis et d'autres [59], [58]. 
séparé et lisse au dessus de $\mathbb{Z}[1 / r]$. Un tel résultat exige qu'une définition convenable des familles de racines $r$-ièmes de faisceaux inversibles soit proposée. Comme on va le voir, cette définition est en fait très naturelle dans le cadre des champs de Hurwitz. Elle utilise au préalable la description par Faltings de la déformation verselle des modules sans torsion sur l'anneau local d'un point double (voir section 7.3.2 et [45], $[58])$.

Comme il est expliqué dans [58], la seule donnée d'une racine $r$-ième du faisceau $\omega_{D}\left(-\sum_{i=1}^{n} b_{i} Q_{i}\right)$, c'est-à-dire la version relative de la définition 9.1.1, définit un champ algébrique qui est singulier si $r$ n'est pas premier. Pour contourner ce défaut, et lorsque la courbe stable $D$ acquiert des points doubles, on doit rendre plus précise la structure de $\operatorname{Spin}$, en fixant la structure locale du faisceau $\mathcal{L}$ au voisinage d'un point double $P$. Soit $S$ la base, et $s \in S$ l'image de $P$. Posons $R=\hat{\theta}_{S, s}$, et soit $x, y: x y=\pi \in \mathcal{M}_{R}$ un système de coordonnées en $P$. On exige de que la structure locale de $\mathcal{L}$, c'est-à-dire après passage à l'anneau local complété (ou un voisinage étale), soit de la forme ${ }^{(2)}$ (voir section 7.3.2 pour les notations) :

$$
\hat{\mathscr{L}}_{Q} \cong E\left(\tau^{a}, \tau^{b}\right), \quad a+b=r, \quad \pi=\tau^{r} \quad\left(\tau \in \mathcal{M}_{R}\right) .
$$

9.1.2. Description des revêtements cycliques. - Sauf mention contraire, on fixe un corps de base algébriquement clos $k$ et les schémas sont des $k$-schémas. Soit une donnée de ramification $R=\sum_{i=1}^{s} b_{i}\left[H_{i}, \chi_{i}\right]$ relative au groupe $G=\mathbb{Z} / n \mathbb{Z}$, et soit $b=\sum_{i} b_{i}$. Fixons aussi une racine primitive $n$-ième de l'unité $\zeta_{n} \in k$. Si on choisit un générateur $\sigma$ de $G$, on sait que cela équivaut à spécifier l'ordre $e_{i}$ de $H_{i}$, ainsi que les entiers $k_{i}, 1 \leqslant k_{i}<e_{i}$ (l'holonomie locale) définissant les classes $\left[H_{i}, \chi_{i}\right]$ par $\chi_{i}\left(\sigma^{n / e_{i}}\right)=\zeta_{n}^{k_{i} n / e_{i}}$.

Soit un $G$-revêtement $\pi: C \longrightarrow D \cong C / G$, entre deux $k$-courbes $C$ et $D$ lisses. Il n'y a pas besoin de préciser pour le moment si les points de branchement sont piqués ou marqués. Un tel revêtement, à groupe de Galois cyclique, est totalement déterminé par la $\left(\theta_{D}, G\right)$-algèbre cohérente

$$
\pi_{*}\left(\theta_{C}\right)=\bigoplus_{j=0}^{n-1} \mathscr{L}_{j}
$$

où la décomposition du membre de droite est la décomposition facteurs isotypiques. Le faisceau $\mathscr{L}_{j}$ est le sous-faisceau propre de l'opérateur $\sigma$ de valeur propre $\zeta_{n}^{j}$. Dans la suite, on posera $\mathscr{L}_{1}=\mathscr{L}$. La multiplication dans $\pi_{*}\left(\theta_{C}\right)$ induit des morphismes $\mathscr{L}_{j} \otimes \mathscr{L}_{k} \longrightarrow \mathscr{L}_{j+k}$, les indices étant pris modulo $n$. Il en résulte en particulier, pour tout $j$ et $d^{\prime} \mid d$, des morphismes

$$
\Phi_{j}: \mathscr{L}^{\otimes j} \longrightarrow \mathscr{L}_{j}, \quad \Phi_{d^{\prime}, d}: \mathscr{L}_{d^{\prime}}^{\otimes d / d^{\prime}} \longrightarrow \mathscr{L}_{d} .
$$

(2) Ce sont les pure $n$ roots de [58]. Cette restriction est nécessaire pour que le foncteur des déformations infinitésimales du triplet $(D, \mathcal{L}, \Phi)$ soit formellement lisse (loc. cit. proposition 5.4.4). Cette condition est clarifiée dans [2]. 
Ces morphismes décrivent la structure d'algèbre sur $\pi_{*}\left(\theta_{C}\right)$. Le morphisme

$$
\Phi=\Phi_{n}: \mathscr{L}^{n} \longrightarrow \theta_{D}
$$

permet d'identifier la donnée de ramification du revêtement $\pi: C \longrightarrow D$. Limitonsnous pour débuter au cas lisse. Soit $D$ une courbe lisse définie sur $k$; rappelons en premier la description classique des revêtements cycliques de base $D$, en termes du triplet $(D, \mathscr{L}, \Phi)$ :

Proposition 9.1.2. - Si au point $Q_{i} \in D$ l'indice de ramification est $e_{i}$, l'action de $\sigma^{n / e_{i}}$ au dessus de $Q_{i}$ (l'holonomie locale) est décrite par $k_{i}, 1 \leqslant k_{i}<e_{i}$, où $\nu_{i}$ est tel que $1 \leqslant \nu_{i}<e_{i}, \nu_{i} k_{i} \equiv 1\left(e_{i}\right)$ et $m_{i}=n / e_{i}$, alors

$$
\operatorname{Div}(\Phi)=B, \text { c'est-à-dire } \Phi\left(\mathscr{L}^{n}\right)=\theta_{D}\left(-\sum_{i=1}^{b} m_{i} \nu_{i} Q_{i}\right) .
$$

Il y a un unique isomorphisme d'algèbres qui est l'identité sur $\mathscr{L}$ :

$$
\pi_{*}\left(\theta_{C}\right)=\bigoplus_{i=0}^{n-1} \mathcal{L}_{i} \stackrel{\sim}{\longrightarrow} \bigoplus_{i=0}^{n-1} \mathcal{L}^{\otimes i}\left(\left[\frac{i B}{n}\right]\right)
$$

Démonstration. - Le premier point est classique (voir par exemple Morrison et Pinkham [79]). Rappelons brièvement les arguments. Soient $Q$ un point de branchement, $\theta=\theta_{Q}$ l'anneau local de $D$ en $Q, v$ la valuation associée. Soit $\bar{\theta}=\bigcap_{P \longrightarrow Q} \theta_{P}$ la clôture intégrale de $\theta$ dans le corps des fonctions de $C$. Soit la décomposition en sous-espaces propres $\theta=\oplus_{i=0}^{n-1} \theta \xi_{i}$; posons $\xi:=\xi_{1}$. Notons que $\xi^{i}=h_{i} \xi_{i}$ avec $h_{i} \in \mathcal{Q}$. Soit par ailleurs $\xi_{i}^{n}=f_{i} \in \Theta$. Du fait de la normalité de $\bar{\theta}$, il est clair que $v\left(f_{i}\right)<n$. Posons

$$
v\left(f_{1}\right)=d=\frac{n}{e} \nu, \quad \text { avec } \quad e=\operatorname{pgcd}(n, d), 1 \leqslant \nu<e .
$$

Notons que $d>0$ car $Q$ est un point de branchement. Si $\pi \in \Theta$ est une uniformisante, et si $P$ est un point de $C$ au-dessus de $Q$, l'égalité $e v_{P}(\xi)=\nu v_{P}(\pi)$ dit que $e / v_{P}(\pi)$. Comme $\tau^{n / e}$ est une unité de $\theta$, où $\tau=\xi^{e} / \pi^{\nu}$, on voit que le nombre des points de $C$ au-dessus de $Q$ est au moins $n / e$. Il en découle que ce nombre est $n / e$, et que l'indice de ramification est $e$. On a par ailleurs $\xi^{i n}=h_{i}{ }^{n} \xi_{i}{ }^{n}$ donc $n v\left(h_{i}\right)+v\left(\xi_{i}^{n}\right)=n d$, et comme $v\left(\xi_{i}^{n}\right)<n$, on en tire $v\left(h_{i}\right)=[i d / n]$ et $v\left(\xi_{i}^{n}\right)=n\langle i d / n\rangle$. Il en résulte la description :

$$
\mathscr{L}_{i}=\mathscr{L}^{\otimes i}\left(\sum_{j=1}^{b}\left[\frac{i \nu_{j}}{e_{j}}\right] Q_{j}\right) \stackrel{\sim}{\longrightarrow} \mathscr{L}^{\otimes i}\left(\left[\frac{i B}{n}\right]\right) .
$$

De manière plus précise, il y a un unique isomorphisme qui est l'identité sur $\mathscr{L}^{\otimes i}$.

On peut conclure que le revêtement $\pi: C \longrightarrow D$ est de fait totalement déterminé par le couple $(\mathscr{L}, \Phi)$. Notons d'abord que le terme de droite dans (90) est une 
$\vartheta_{D}$-algèbre, la multiplication étant donnée par les applications naturelles

$$
\mathscr{L}^{i}\left[\frac{i B}{n}\right] \otimes \mathscr{L}^{j}\left[\frac{j B}{n}\right] \longrightarrow \mathscr{L}^{i+j}\left[\frac{(i+j) B}{n}\right],
$$

où l'indice $i+j$ est pris modulo $n$. Il est clair que l'unique isomorphisme qui est l'identité sur $\bigoplus_{i=0}^{n-1} \mathscr{L}^{i}$ est un isomorphisme d'algèbre.

La description (89) d'un revêtement cyclique entre courbes lisses s'étend facilement à une famille de courbes lisses. Il suffit de donner un sens au terme de droite dans (89). Supposons que dans la donnée de ramification choisie, les valeurs distinctes prises par les $n_{i}=\frac{n}{e_{i}} \nu_{i}$ sont $p_{1}<\cdots<p_{s}$. Il est commode de mettre le diviseur $B$ sous la forme $B=\sum_{i=1}^{s} p_{i} B_{i}$, les $B_{i}$ étant maintenant disjoints deux à deux. Posons $\operatorname{deg}\left(B_{i}\right)=k_{i}$ qui représente le nombre d'occurrence de l'holonomie $p_{i}$ (ou $k_{i}$ ), de sorte que $\sum_{i} k_{i} p_{i}=\sum_{j} n_{j}=n m$, l'entier $m$ étant défini par cette équation.

La base $S$ du revêtement est maintenant un $k$-schéma arbitraire. Pour tout $1<$ $p=\frac{n}{e} \nu$ avec $1 \leqslant \nu<e, \operatorname{pgcd}(e, \nu)=1$, soit $\Delta_{p} \subset C$ le sous-schéma des points de $C$ d'holonomie $p$. On sait (section 4) que si $p=p_{i}, \Delta_{p}$ est un diviseur de Cartier relatif étale de degré $k_{i} \frac{n}{e_{i}}$ sur $S$. Si $p=p_{i}$, on notera $\Delta_{p}=\Delta_{i}$. Le groupe quotient de $\mathbb{Z} / n \mathbb{Z}$ par le sous-groupe d'ordre $e_{i}$ agit librement sur $\Delta_{i}$. Le quotient $B_{i} \subset D$ de $\Delta_{i}$ par ce groupe est un diviseur de cartier relatif étale de degré $k_{i}$ sur $S$. On a alors :

Proposition 9.1.3. - Soit $\pi: C \longrightarrow D$ un revêtement cyclique entre courbes lisses de base $S$. Avec les notations précédentes, en particulier $\mathscr{L}:=\mathscr{L}_{1}$, on a $B=\sum_{i=1}^{s} p_{i} B_{i}$, et :

i) Il y a un unique isomorphisme $\mathscr{L}_{i} \stackrel{\sim}{\longrightarrow} \mathscr{L}^{\otimes i}\left[\frac{i B}{n}\right]$ qui est l'identité sur $\mathscr{L}^{\otimes i}$ et un unique isomorphisme d'algèbres

$$
E=\pi_{*}\left(\theta_{C}\right) \cong \bigoplus_{i=0}^{n-1} \mathcal{L}^{\otimes i}\left[\frac{i B}{n}\right]
$$

ii) le champ de Hurwitz $\mathscr{H}_{g, n, \xi}$ dont les sections sont les revêtements cycliques de degré $n$, de base une courbe lisse de genre $g$, et de donnée de ramification $\xi$, est isomorphe au champ dont les objets sont les triplets $(D, \mathcal{L}, \Phi)$, où $D$ est lisse de genre $g$, et $(\mathscr{L}, \Phi)$ est une racine d'ordre $n$ de $\Theta_{D}(-B)$, le diviseur $B=\sum_{j=1}^{s} p_{j} B_{j}$ étant comme prescrit par $\xi$.

Démonstration. - Du fait que $B=\sum_{j=1}^{s} p_{j} B_{j}$, avec $B_{j}$ étale de degré $k_{j}$ sur la base $S$, on peut poser

$$
\left[\frac{i B}{n}\right]=\sum_{j}\left[\frac{i p_{j} B_{j}}{n}\right] .
$$

L'assertion i) ainsi que la première partie de ii) se vérifient par un argument local analogue à celui utilisé dans la preuve (89). On peut d'une autre manière tout déduire de loc. cit. En effet, si la base $S$ est intègre, l'égalité de diviseurs de Cartier relatifs $\operatorname{Div}\left(\Phi_{i}\right)=\left[\frac{i B}{n}\right]$ découle du fait qu'il y a égalité sur toute fibre géométrique. Comme 
toutes ces constructions sont compatibles aux changements de base, le cas général se déduit de ce cas particulier par changement de base à partir de la déformation universelle d'une quelconque fibre. Noter que l'isomorphisme invoqué est unique. Pour finir la preuve de i), notons que le terme de gauche dans (91) possède une structure naturelle de $\theta_{D}$-algèbre, l'identification est alors claire.

Pour ii) on observera d'abord que la catégorie formée des triplets $(D, \mathcal{L}, \Phi)$ est un champ algébrique. L'assertion se résume donc à vérifier que le foncteur indiqué est un isomorphisme, donc à la fois un monomorphisme et un épimorphisme ([73], cor. (3.7.1)). Le fait que ce soit un monomorphisme signifie que pour deux revêtements $\pi: C \longrightarrow D$ et $\pi^{\prime}: C^{\prime} \longrightarrow D^{\prime}$ de base $S$, le morphisme

$$
\operatorname{Hom}\left(\pi, \pi^{\prime}\right) \longrightarrow \operatorname{Hom}\left((D, \mathscr{L}, \Phi),\left(D^{\prime}, \mathscr{L}^{\prime}, \Phi^{\prime}\right)\right)
$$

est bijectif, il est donc clair que cela découle de 2). Pour vérifier que ce foncteur est un épimorphisme, il suffit de noter que si on part d'un objet $(D / S, \mathcal{L}, \Phi)$ comme il est indiqué, alors $\mathscr{C}=\bigoplus_{i=0}^{n-1} \mathscr{L}^{\otimes i}\left[\frac{i B}{n}\right]$ a une structure naturelle de $\theta_{D}$-algèbre, il suffit alors de poser $C=\operatorname{Spec}_{\emptyset_{D}}(\mathscr{C})$.

\subsection{Revêtements cycliques stables}

\subsubsection{Revêtements cycliques stables et quasi-racines d'un faisceau} inversible. $-\mathrm{Si} \pi: C \longrightarrow D$ est un revêtement stable défini sur $k$, avec $D$ éventuellement singulière, on peut former de nouveau la décomposition

$$
\pi_{*}\left(\theta_{C}\right)=\bigoplus_{j=0}^{n-1} \mathscr{L}_{j}
$$

Le $\theta_{D}$-module $\pi_{*}\left(\theta_{C}\right)$ n'est plus en général localement libre de rang $n$, mais seulement sans torsion de rang $n$. Rappelons, dans le contexte des revêtements cycliques, la description précise des singularités des $\mathscr{L}_{j}$ (voir 7.3.2). Considérons un point double $P \in C$ de stabilisateur d'ordre $e \mid n$, et soit $Q$ le point double image dans $D$. Si $\sigma$ est un générateur fixé de $G$, on notera $\tau=\sigma^{n / e}$ le générateur distingué du stabilisateur $H=G_{P}$. La description locale au point $Q$ de la décomposition (92) est comme suit.

On rappelle qu'on a choisi une racine primitive $n$-ième de l'unité $\zeta_{n} \in k$, et on pose $\zeta_{e}=\left(\zeta_{n}\right)^{n / e}$. Choisissons des coordonnées locales $x, y$ le long des branches telles que

$$
\tau(x)=\zeta_{e}^{k} x \quad, \quad \tau(y)=\zeta_{e}^{e-k} y,
$$

conditions exprimant la stabilité de l'action. Notons par un tilde la normalisation $k[[x]] \times k[[y]]$ de $\hat{\theta}_{Q}$. Si un caractère de $H$ est identifié à sa valeur sur le générateur $\tau$, rappelons la décomposition en facteurs isotypiques (75) :

Lemme 9.2.1. - La décomposition en $H$-sous faisceaux propres de $\hat{\theta}_{P}$ est :

$$
\hat{\theta}_{P}=\hat{\theta}_{Q} \bigoplus\left(\bigoplus_{\alpha=1}^{e-1} \widetilde{\hat{\theta}_{Q}}\left(x^{\alpha}, y^{e-\alpha}\right)\right)
$$


En application des résultats généraux de la section 7.3 , on peut décrire le lieu singulier des faisceaux $\mathscr{L}_{j}$ (voir en particulier le corollaire 7.3.3) ${ }^{(3)}$ :

Proposition 9.2.2. - Soit $Q \in D$ un point double, et e l'ordre du sous-groupe d'isotropie en un point $P$ au dessus de $Q$. Le faisceau $\mathscr{L}_{j}$ est localement libre en $Q$ si et seulement si $j \equiv 0(\bmod e)$.

Posons $\mathscr{L}=\mathscr{L}_{1}$, et analysons maintenant les morphismes (88) induits par la multiplication, et plus particulièrement les morphismes $\Phi_{j}: \mathscr{L}^{\otimes j} \longrightarrow \mathscr{L}_{j}(1 \leqslant j \leqslant n)$.

Proposition 9.2.3. - Si $Q \in D$ est du type $N S$ (e $>1)$, il y a deux cas :

i) Le point $Q$ est une singularité de $\mathcal{L}_{j}$, alors la dimension de la fibre en $Q$ du conoyau de $\Phi_{j}$ est $j-1$, et l'idéal de $\theta_{Q}$ image de $\left(\Phi_{j}\right)_{Q}$ est non principal.

ii) Dans le cas contraire, si e|j, la dimension du conoyau de $\Phi_{j}$ en $Q$ est encore $j-1$, mais l'image de $\Phi_{j}$ est alors un idéal principal, i.e. libre de rang $1 . S i$ $j=n$, le morphisme $\Phi: \mathscr{L}^{\otimes n} \longrightarrow \Theta_{D}(-B)$, induit par la multiplication, fait de $\mathcal{L}$ une racine $n$-ième de $\theta_{D}(-B)$.

Démonstration. - Cela résulte d'un calcul élémentaire (voir section 7.3.2). Traitons le premier cas, soit $e \mid j$. Il résulte de la proposition 9.2.3, et avec des notations évidentes, qu'on peut prendre la « résolvante» $X_{j}=\sum_{i=1}^{n / e} \zeta_{e}^{-i j} \sigma^{i j}\left(x^{j \nu}, y^{e-j \nu}\right)$ comme

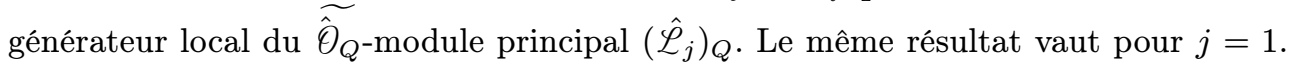
On voit alors immédiatement que l'image par $\Phi_{j}$ de $X_{1}^{\otimes j}$ est

$$
\left(\Phi_{j}\right)_{Q}\left(X_{1}^{\otimes j}\right)=\left(u^{\left[\frac{j \nu}{e}\right]}, v^{j-1-\left[\frac{j \nu}{e}\right]}\right) X_{j} .
$$

Du fait que la fibre en $Q$ de $\mathscr{L}^{\otimes j}$ modulo le sous-module de torsion n'est pas libre, car le point est NS, les deux membres de l'égalité (93) sont sans torsion de rang 1, non libres. Ainsi, le conoyau de $\Phi_{j}$ en $Q$ s'identifie à

$$
\frac{k[[u]] \times k[[v]]}{\left(u^{[j \nu / e]}, v^{j-1-[j \nu / e]}\right)}
$$

et la dimension de ce conoyau est donc $j-1$. Pour la dernière assertion, la seule chose à vérifier est l'égalité $n \operatorname{deg}(\mathscr{L})=-\operatorname{deg}(B)$. Comme cette égalité est invariante par déformation, de même que la formation de $\mathscr{L}$, il suffit de la vérifier dans le cas lisse, ce qui est clair.

Pour mesurer l'écart entre $\mathcal{L}^{\otimes n}$ et $\vartheta_{D}(-B)$ en présence de points doubles de type NS, il est utile de passer à la courbe semi-stable modifiée $\rho: \tilde{D} \longrightarrow D$, munie d'un faisceau inversible $\Theta(1)$, tel que $\rho_{*}(\theta(1))=\mathscr{L}$ (voir proposition 7.3 .9 et la construction qui précède). Il est naturel de se demander si le morphisme $\Phi$ (proposition 9.2.3) provient par la projection $\rho_{*}$ d'un morphisme analogue $\tilde{\Phi}: \theta_{\tilde{D}}(n) \longrightarrow \theta_{\tilde{D}}$. La réponse est donnée essentiellement par [58], proposition 3.1.5.

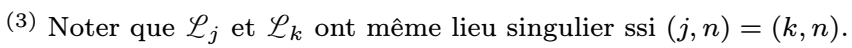


Proposition 9.2.4. - Sous les conditions précédentes, il existe un unique morphisme

$$
\tilde{\Phi}: \vartheta_{\tilde{D}}(n) \longrightarrow \vartheta_{\tilde{D}}(-B)
$$

tel que $\rho_{*}(\tilde{\Phi})=\Phi$.

Démonstration. - Soit $U$ l'ouvert image réciproque du complémentaire du lieu singulier de $\mathcal{L}$. Le morphisme $\rho$ est un isomorphisme sur $U$. Comme $U$ est schématiquement dense, le morphisme $\tilde{\Phi}$ s'il existe est unique. Il suffit de le construire au-dessus d'un voisinage d'un point singulier $Q$ d'image $s \in S$, voisinage appelé $D$ pour simplifier. On suppose donc $D=\operatorname{Spec}\left(\theta_{Q}\right)$ avec $\theta_{Q}=\theta_{s}[[x, y]] /\left(x y-\pi^{e}\right)$. De la description de $\tilde{D}$ comme sous-schéma de $\mathbb{P}^{1} \times D$ par les équations

$$
\xi y-q v=\xi p-x v=0,
$$

on note que $\tilde{D}=U_{1} \cup U_{2}$, avec

$$
U_{1}=\operatorname{Spec}\left(\hat{\theta}_{Q}[s] /(s y-q, s p-x)\right) \quad, \quad U_{2}=\operatorname{Spec}\left(\hat{\theta}_{Q}[t] /(y-q t, p-x t)\right) .
$$

Le cocycle qui définit $\Theta_{\tilde{D}}(n)$ est $s_{1,2}=t^{n} \in \Gamma\left(U_{1} \cap U_{2}, \theta_{\tilde{D}}\right)$. Il suffit de trouver des fonctions régulières $\phi_{i}$ sur $U_{i}(i=1,2)$ avec $\phi_{2}=t^{n} \phi_{1}$. Rappelons que $p=\pi^{\nu}$, $q=\pi^{e-\nu}$. Si $a=m \nu, b=m(e-\nu)$, alors $p^{b}=q^{a}$. Le choix naturel est de prendre $\phi_{1}=y^{a}$ et $\phi_{2}=x^{b}$. La vérification est immédiate.

Revenant à la situation générale, on notera que le faisceau inversible $\Theta(-n-B)$ est de la forme $\Theta(E)$ pour un certain diviseur de Cartier $E$, le diviseur des zéros de $\Phi$. Ce diviseur a pour support la réunion des diviseurs de Weil mis en évidence dans la proposition 7.3.9.

Avec les notations précédentes, à un point de type NS, on attache une paire d'entiers (non ordonnés) le symbole $(a, b)$, avec :

$$
a=m \nu \quad, \quad b=n-m \nu \quad, \quad m=\frac{n}{e} \quad, \quad a+b=n .
$$

On a $1 \leqslant \nu<e$, et $\operatorname{pgcd}(\nu, e)=1$. En particulier $\operatorname{pgcd}(a, n)=m$. Le symbole décrit l'action du stabilisateur d'un point $P \in C$ au dessus de $Q$, sur les branches formelles en $P$. Notons que l'image de $\Phi$ en $Q$ est l'idéal (non principal) $\left(u^{a}, v^{b}\right) \widetilde{\hat{\theta}}_{Q}$ de colongeur $n-1$ (proposition 9.1.1).

Soit maintenant $\pi: C \longrightarrow D=C / G$ une section du champ de Hurwitz $\overline{\mathcal{H}}_{g, n, \xi}$, au dessus de la base $S$. Dans la notation du champ, cela pour alléger, on omet le genre $g$ de $C$, ainsi que la donnée $\xi$. Notons $q$ (resp. $p$ ) le morphisme structural du $S$-schémas $C$ (resp. $D$ ). Soit de nouveau la décomposition en facteurs isotypiques :

$$
\pi_{*}\left(\theta_{C}\right)=\bigoplus_{j=0}^{n-1} \mathscr{L}_{j},
$$

les faisceaux $\mathscr{L}_{j}$ étant des faisceaux sans torsion de rang 1 (relatifs) sur $S$, i.e. plats sur $S$ et les fibres au-dessus d'un point géométrique étant sans torsion de rang 1. La structure locale de $\mathscr{L}=\mathscr{L}_{1}$ peut être précisée de la manière suivante : 
Proposition 9.2.5. - Soit $Q \in D_{s}$ un point de la fibre au-dessus du point géométrique $s \in S$. On suppose que $Q$ est un point singulier de $\mathcal{L}$, de symbole $(a, b)$; alors $\hat{\mathscr{L}}_{Q}$ est un module sans torsion de rang 1 au dessus de $\hat{\theta}_{s}$ de type $E\left(\tau^{a}, \tau^{b}\right)$.

Démonstration. - Il suffit d'appliquer la Proposition 7.3.7.

On prouve que le champ des revêtements cycliques de degré $n$ et de donnée de ramification fixée $\xi$ (noté $\overline{\mathcal{H}}_{g, n, \xi}$ ), est isomorphe au champ dont les objets sont les courbes stables marquées (ou piquées) $)^{(4)}\left(D, B=\sum_{j=1}^{s} p_{j} B_{j}\right.$ ) équipées d'une racine $n$-ième $\mathcal{L}$ du faisceau $\theta_{D}(-B), p_{i}=m_{i} \nu_{i}$.

ThÉORÈme 9.2.6. - Le foncteur $(\pi: C \longrightarrow D) \mapsto(D, \mathcal{L}, \Phi)$ établit un isomorphisme de $\overline{\mathcal{H}}_{g, n, \xi}$ sur le champ dont les objets sont les triplets $(D, \mathcal{L}, \Phi)$, où $D$ est une courbe stable marquée par $B=\sum_{i=1}^{s} B_{i}$ et $(\mathcal{L}, \Phi)$ est une racine d'ordre $n$ de $\emptyset_{D}\left(-\sum_{i=1}^{s} p_{i} B_{i}\right)$.

Démonstration. - Le fait que la catégorie fibrée en groupoïdes d'objets les triplets $(D, \mathscr{L}, \Phi)$ soit un champ algébrique séparé et lisse est une conséquence directe des résultats de Jarvis ([58], theorem 5.3.1). Essentiel est le résultat qui décrit la déformation universelle d'une racine d'ordre $n$ (loc. cit., et [59] theorem 2.3.2). Ce résultat dit que si $(D, \mathcal{L}, \Phi)$ est une courbe stable marquée par le diviseur $B_{1}+\cdots+B_{s}$, munie d'une racine d'ordre $n$ de $\Theta(-B)\left(B=\sum_{i} p_{i} B_{i}\right)$, la base de la déformation universelle est une algèbre de séries formelles $k\left[\left[\tau_{1}, \ldots, \tau_{b}, t_{b+1}, \ldots, t_{3 g-3+b}\right]\right]$, et le morphisme qui a une déformation de ce triplet associe la déformation correspondante de la courbe marquée $D$, s'identifie à

$$
\psi: \operatorname{Spec}\left(k\left[\left[\tau_{1}, \ldots, \tau_{b}, \tau_{b+1}, \ldots, \tau_{3 g-3+b}\right]\right]\right) \longrightarrow \operatorname{Spec}\left(k\left[\left[t_{1}, \ldots, t_{b}, \ldots, t_{3 g-3+b}\right]\right]\right)
$$

où $\psi^{*}\left(t_{j}\right)=\tau_{j}^{e_{i}}$ si $j \leqslant b$, et $\psi^{*}\left(t_{j}\right)=\tau_{j}$ si $b<j \leqslant 3 g-3+b$. Si $Q$ est un point singulier de $\mathscr{L}$ de signature $(a, b), a+b=n$, on pose $e=\frac{n}{\operatorname{pgcd}(a, b)}$. On notera que ce résultat dit que si le triplet $(D, \mathcal{L}, \Phi)$ de base $\operatorname{Spec}(k)$ dérive du revêtement $\pi$ : $C \longrightarrow D$, alors $\pi$ et $(D, \mathscr{L}, \Phi)$ ont « même » théorie des déformations (comparer avec le Théorème 5.5). Cela signifie que si $\pi: \mathscr{C} \longrightarrow \mathscr{D}$ est la déformation universelle du revêtement $\pi: C \longrightarrow D$, alors le triplet image $(\mathscr{D}, \mathscr{L}, \Phi)$ est la déformation universelle de $(D, \mathcal{L}, \Phi)$. Ceci étant, armé de cet argument, la preuve est analogue à celle de la proposition 9.2.3.

Montrons que le foncteur est un monomorphisme. Cela revient d'abord à prouver que si un $S$-automorphisme $f$ de $C$ induit l'identité sur $(D, \mathcal{L}, \Phi)$, alors $f=1$. Comme le groupe $\operatorname{Aut}(C / S)$ est fini non ramifié, il suffit de le voir sur les fibres, donc lorsque $S=\operatorname{Spec}(k)$. Il est clair que $f$ est l'identité en dehors des points doubles de $C$, dès lors $f=1$. On suppose maintenant avoir deux objets $\pi: C \longrightarrow D$ et $\pi: C^{\prime} \longrightarrow D$ avec

(4) La courbe $D$ est marquée par le diviseur $B=B_{1}+\cdots+B_{s}$, le diviseur relatif $B_{i}$ étant étale de degré $k_{i}$ sur la base $S$, et les $B_{i}$ étant disjoints deux à deux. Rappelons que $B_{i}$ est le lieu des points de branchement d'holonomie fixée $p_{i}$. 
une même image $(D, \mathcal{L}, \Phi)$, on prouve que les deux revêtements sont isomorphes. Du fait de l'unicité de l'isomorphisme, le problème est local sur $S$, on peut donc supposer que tout est défini au-dessus de la base de la déformation universelle, commune aux trois objets, disons de la fibre $\left(D_{s}, \mathscr{L}_{s}, \Phi_{s}\right)$. Cela permet de supposer que $S$ est lisse (connexe), et que les fibres génériques des courbes en présence sont lisses.

Soit le $S$-schéma $\nu: \operatorname{Isom}\left(\pi, \pi^{\prime}\right) \longrightarrow S$ formé des isomorphismes induisant l'identité $\operatorname{sur}(D, \mathcal{L}, \Phi)$. On sait que $\nu$ est fini non ramifié sur $S$. On sait par ailleurs que $\nu$ est un monomorphisme dominant, c'est donc un isomorphisme.

Il reste à voir que, localement sur $S$, tout triplet $(D, \mathcal{L}, \Phi)$ de base $S$ provient d'un revêtement. Il suffit de relever un triplet défini sur la base $S=$ Spec $k$, car l'identification des foncteurs de déformations permet alors de relever localement une famille arbitraire. Dans le cas ponctuel, on déforme le triplet $(D, \mathcal{L}, \Phi)$ à une base $\operatorname{Spec}(R)$, où $R$ est un anneau de valuation discrète complet de corps résiduel $k$, de fibre générique $D_{K}$ lisse. Quitte à épaissir $R$, on peut supposer que la fibre générique du triplet provient du revêtement $C_{K} \longrightarrow D_{K}$. Le modèle stable de ce revêtement (section 5.2) répond à la question.

9.2.2. Composantes irréductibles du bord. - La description des composantes irréductibles du bord de $\overline{\mathcal{H}}_{g, n, \xi}$ découle directement des résultats de la section 7.4. Dans cette description, on se limite à considérer une base dont le graphe dual est, soit un segment, soit une boucle.

Le segment : Il s'agit de décrire les diviseurs du champ $\overline{\mathcal{H}}_{g, n, \xi}$ déterminés par les graphes modulaires de Hurwitz $\Gamma$ qui recouvrent le segment. Décrire un revêtement stable $C \longrightarrow \Sigma$ de groupe $G=\mathbb{Z} / n \mathbb{Z}$ de base $\Sigma=\Sigma_{1} \cup \Sigma_{2}$, courbe avec deux composantes lisses de genres respectifs $1 \leqslant g_{1} \leqslant g_{2}, g_{1}+g_{2}=g$, un seul point double, et de donnée de ramification $\xi$, est équivalent à la donnée d'une racine $n$-ième du faisceau $\vartheta_{\Sigma}(-B)$, où $B$ est le diviseur de branchement. Il s'avère cependant beaucoup plus commode dans les descriptions qui suivent de conserver le point de vue des revêtements. Dans la suite, la base sera notée indifféremment $D$ ou $\Sigma$. Le diviseur de branchement sera noté

$$
B=\sum_{\alpha=1}^{b} m_{\alpha} \nu_{\alpha} Q_{\alpha}
$$

avec $m_{\alpha}=n / e_{\alpha}, 1 \leqslant \nu_{\alpha}<e_{\alpha}$, et $\operatorname{pgcd}\left(e_{\alpha}, \nu_{\alpha}\right)=1$. On notera que $\sum_{\alpha=1}^{b} m_{\alpha} \nu_{\alpha} \equiv 0$ $(\bmod n)$.

Le revêtement $C \longrightarrow \Sigma=\Sigma_{1} \cup \Sigma_{2}$ comme spécifié ci-dessus impose à $C$ d'avoir la topologie suivante (comparer avec la discussion générale de la section 7.4) :

$$
C=\operatorname{Ind}_{G_{1}}^{G} C_{1} \vee \operatorname{Ind}_{G_{2}}^{G} C_{2}
$$

pour deux courbes lisses $C_{1}$ et $C_{2}$ induisant des revêtements $C_{1} \longrightarrow \Sigma_{1}$ et $C_{2} \longrightarrow \Sigma_{2}$ de groupes respectifs $G_{1}$ et $G_{2}$, sous-groupes de $G$. Le stabilisateur d'un point double $\left.P \in C_{1} \cap C_{2}\right)$ de $C$ étant $H \subset G_{1} \cap G_{2}$, on pose $n_{i}=\left|G_{i}\right|(i=1,2)$ et $e=|H|$. Donc 
$e$ divise $n_{1}$ et $n_{2}$, et $\operatorname{ppcm}\left(n_{1}, n_{2}\right)=n$ du fait de la connexité de $C$. Il est aisé de voir que si le diviseur de branchement $B_{i}^{*}$ de $C_{i} \longrightarrow \Sigma_{i}$ est écrit sous la forme

$$
B_{i}^{*}=\sum_{\alpha=1}^{b_{i}} m_{\alpha}^{i} \nu_{\alpha}^{i} Q_{\alpha}^{i}+\frac{n_{i}}{e} \nu^{i} Q^{i}=B_{i}+\frac{n_{i}}{e} \nu^{i} Q^{i} \quad(i=1,2)
$$

avec $m_{\alpha}^{i}=n_{i} / e_{\alpha}^{i}$, les $Q_{\alpha}^{i}$ (resp. $\left.Q^{i}, i=1,2\right)$ étant les points qui après identification donnent le point double $Q_{\alpha}$ (resp. $Q$ ). Le diviseur de branchement du revêtement $\pi: C \longrightarrow \Sigma$ est en conséquence

$$
B=\frac{n}{n_{1}}\left(\sum_{\alpha=1}^{b_{1}} m_{\alpha}^{1} \nu_{\alpha}^{1} Q_{\alpha}^{1}\right)+\frac{n}{n_{2}}\left(\sum_{\alpha=1}^{b_{2}} m_{\alpha}^{2} \nu_{\alpha}^{2} Q_{\alpha}^{2}\right)=\frac{n}{n_{1}} B_{1}+\frac{n}{n_{2}} B_{2} .
$$

On doit avoir les congruences $\sum_{\alpha=1}^{b_{i}} m_{\alpha}^{i} \nu_{\alpha}^{i}+\frac{n_{i}}{e} \nu^{i} \equiv 0\left(n_{i}\right), i=1,2$ qui si les $B_{i}$ sont connus, déterminent de manière unique $e$ et les $\nu^{i}$. Par exemple $\frac{n_{i}}{e} \nu^{i}$ est le reste modulo $n_{i}$ de $\sum_{\alpha=1}^{b_{i}} m_{\alpha}^{i} \nu_{\alpha}^{i}$; notons que $\nu^{1}+\nu^{2}=e$. En d'autres termes, la signature du point double $Q$, obtenu par identification de $Q^{1}$ et $Q^{2}$ est donnée par (94) :

$$
a=\frac{n}{e} \nu^{1} \quad, \quad b=\frac{n e}{\nu^{2}} .
$$

On a $a+b=n$ et $n / e=\operatorname{pgcd}(a, b)$. La partition, notée dans la suite $\pi$, du diviseur de branchement (ou de la donnée de ramification) en

$$
\text { ( } \pi) B=\frac{n}{n_{1}} B_{1}+\frac{n}{n_{2}} B_{2}
$$

détermine tous les autres paramètres, c'est-à-dire $m^{1} \nu^{1}=\frac{n_{1}}{e^{1}} \nu^{1}$ et $m^{2} \nu^{2}=\frac{n_{2}}{e^{2}} \nu^{2}$, restes respectifs modulo $n_{i}$ de $\sum_{\alpha=1}^{b_{i}} m_{\alpha}^{i} \nu_{\alpha}^{i}(i=1,2)$. Rappelons que dans l'écriture précédente on a $1 \leqslant \nu^{i}<e^{i}$ et $\left(\nu^{i}, e^{i}\right)=1$. La congruence initiale

$$
\operatorname{deg}(B)=\frac{n}{n_{1}}\left(\sum_{\alpha=1}^{b_{1}} m_{\alpha}^{1} \nu_{\alpha}^{1}\right)+\frac{n}{n_{2}}\left(\sum_{\alpha=1}^{b_{2}} m_{\alpha}^{2} \nu_{\alpha}^{2}\right) \equiv 0 \quad(\bmod n)
$$

entraîne immédiatement $e^{1}=e^{2}$. Notons par ailleurs que la stabilité impose les conditions $2 g_{i}-2+b_{i} \geqslant 0(i=1,2)$. La signature $(a, b)$ du point double est de fait déterminée par les seuls supports de la partition $\pi$, i.e. la partition de l'ensemble points de branchements $[1, b]=I_{1} \sqcup I_{2}$. Nous noterons dans la suite $\delta_{g_{1}, g_{2}, \pi}$ ou $\delta_{g_{1}, g_{2}, B_{1}, B_{2}}$ la composante irréductible du bord correspondante à ces choix. Son image dans $\overline{\mathcal{M}}_{g^{\prime}, b}$ est la composante $\delta_{g_{1}, g_{2}, I_{1}, I_{2}}$. Noter que $\delta_{g_{1}, g_{2}, B_{1}, B_{2}}=\delta_{g_{2}, g_{1}, B_{2}, B_{1}}$.

La boucle : $\left(g^{\prime} \geqslant 1\right)$ On suppose que la base du revêtement est maintenant une courbe irréductible $\Sigma$, de genre géométrique $g^{\prime}-1$, avec un unique point double $Q$. Notons $\tilde{\Sigma}$ la normalisation de $\Sigma$ et soient $Q^{1}, Q^{2}$, les deux points de $\tilde{\Sigma}$ pré-images de $Q$. On sait que pour l'espace total $C$ du revêtement, il y a deux possibilités :

i) La courbe $C$ est irréductible, disons avec $d=n / e$ points doubles. Alors la normalisation $\tilde{C}$ est irréductible, et le revêtement cyclique $\tilde{C} \longrightarrow \tilde{\Sigma}$ est ramifié avec 
$b+2$ points de branchement, ceux provenant des points de branchement de $C \longrightarrow \Sigma$ et en outre $Q_{1}, Q_{2}$. Le diviseur de branchement est donné par

$$
\tilde{B}=B+m_{1} \nu_{1} Q^{1}+m_{2} \nu_{2} Q^{2},
$$

où $B$ est le diviseur de branchement de $\Sigma \longrightarrow C$. Notons que $m_{1} \nu_{1}+m_{2} \nu_{2}=n$, en fait $n / e=m_{1}=m_{2}$ et $a=m_{1} \nu_{1}, b=m_{2} \nu_{2}$ est la signature du point double $Q$. Nous noterons $\delta_{0, a, b}$, la composante décrite de cette manière.

ii) La courbe $C$ est réductible. Dans ce cas il y a dans $C$ une seule $G$-orbite de composantes irréductibles, fixons $C_{0}$ l'une d'entre elles. Il y a aussi une seule orbite de points doubles, soit $P \in C_{0}$ l'un de ces points. Notons $G_{0}$ le stabilisateur de $C_{0}$ et $H$ celui de $P$, d'ordres respectifs $n_{0}<n$ et $e$, et $e \mid n_{0}$. Soit $g_{0} \in G \backslash G_{0}$ un élément tel que $P \in C_{0} \cap g_{0}\left(C_{0}\right)$. On sait que $G_{0} \cup g_{0}$ engendre $G$ du fait de la connexité de $C$ (voir exemple 7.2.4), et que par ailleurs $H \subset G_{0} \cap g_{0} G_{0} g_{0}^{-1}=G_{0}$ puisque le groupe est abélien. Notons que l'élément $g_{0}$ est déterminé modulo $G_{0}$, et au changement $g_{0} \mapsto g_{0}^{-1}$ près. La courbe $C$ est alors obtenue par identification des couples de points $\left(g P, g g_{0}^{-1} P\right) \in \operatorname{Ind}_{G_{0}}^{G}\left(C_{0}\right)$. Le diviseur de branchement de $C_{0} \longrightarrow \Sigma_{0}$ est visiblement de la forme

$$
B_{0}+\frac{n_{0}}{e} \nu^{1} Q_{1}+\frac{n_{0}}{e} \nu^{2} Q_{2} \quad, \quad \nu^{1}+\nu^{2}=e
$$

avec nécessairement la factorisation de $B$ désignée de nouveau $\pi$ :

$$
(\pi): B=\frac{n}{n_{0}} B_{0} .
$$

La signature du point double $Q$ est $a=\frac{n}{n_{0}} a_{0}, b=\frac{n}{n_{0}} b_{0}$, avec $a_{0}=\frac{n_{0}}{e} \nu^{1}, b_{0}=\frac{n_{0}}{e} \nu^{2}$. Noter que ce choix n'est soumis à aucune contrainte, au sens où $e \mid n_{0}$ étant fixé, on choisit $\nu_{1}<e$, premier à $e$, alors $\nu_{2}$ s'en déduit du fait de l'égalité (95). En sens inverse, on a $e=n_{0} / \operatorname{pgcd}\left(n_{0}, a_{0}\right)$.

La notation retenue pour cette composante irréductible est $\delta_{0, \pi, a, b}$. Le cas i) correspond à $n_{0}=n$. La notation regroupe donc les deux cas.

\subsection{Revêtements cycliques de $\mathbb{P}^{1}$}

Lorsque la base est $\mathbb{P}^{1}$, on peut identifie le champ (ouvert) de Hurwitz à un champ quotient (comparer avec [5] qui se limite aux revêtements uniformes).

9.3.1. Quelques calculs de torseurs. - Il est connu que la classification des objets au dessus de la catégorie des $k$-schémas (où $k$ est un corps ou un anneau de base), localement pour la topologie étale ou la topologie fpqc, d'une marque donnée (avec la terminologie de Demazure-Gabriel [32], chap. III, $\S 5$, no. 1), est équivalente à la donnée du champ classifiant $B$, avec pour groupe structural $G$, le groupe des automorphismes de l'objet marque. Une définition plus précise est comme suit. Soit $\mathcal{M}$ un champ (algébrique) au dessus de $k$. Soit $X$ un $k$-schéma, et soit une section $P \in \mathcal{M}(X)$, la marque. Disons que $Q \in \mathcal{M}(U)$ est de marque $P$, s'il y a un morphisme 
$\alpha: U \longrightarrow X$, un recouvrement (étale) $U^{\prime} \longrightarrow U$, tels que $Q \times_{U} U^{\prime} \cong P \times_{X} U^{\prime}$, donc si $\operatorname{Isom}\left(Q, P \times_{X} U\right)\left(U^{\prime}\right) \neq \varnothing$.

On définit un champ $\mathcal{M}(P)$, le champ des objets de marque $P$, dont la catégorie des objets au-dessus de $U$ sont les couples $(Q, \alpha: U \longrightarrow X)$, avec la propriété de dessus, traduisant le fait que $Q$ est de marque $P$, les morphismes étant définis de manière évidente. Ce champ n'est qu'une présentation différente du champ classifiant $B(G / X)$, si $G=\operatorname{Aut}(P)$ est le groupe algébrique des automorphismes de $P$ ([73], $(2.4 .2))$.

Proposition 9.3.1. - Sous les hypothèses qui précèdent, on a un isomorphisme de champs

$$
\mathcal{M}(P) \stackrel{\sim}{\longrightarrow} B(G / X)
$$

où $G=\operatorname{Aut}_{k}(P)$ désigne le $X$-groupe algébrique des automorphismes de $P$. En particulier, si $G$ est lisse de type fini, le champ $\mathcal{M}(P)$ est algébrique.

Démonstration. - Rappelons brièvement la définition de l'isomorphisme $(c f[\mathbf{3 2}])$. $\mathrm{Si}$ $(Q, \alpha) \in \mathcal{M}(S)$ est une section de marque $P$ au-dessus de $S$, il est manifeste que le $S$-schéma $\operatorname{Isom}_{S}\left(P \times S, Q \times_{X} S\right)$ est de manière naturelle un $G$-fibré principal de base $S$, définissant le foncteur (96). En sens inverse, si $E \longrightarrow S$ est un torseur sous $\operatorname{Aut}_{X}(P) \times_{X} S$, le produit contracté

$$
E \times{ }^{\operatorname{Aut}(P) \times{ }_{X} S}\left(P \times_{X} S\right)
$$

fournit un objet de marque $P$ de base $S$. Ces deux constructions sont inverses l'une de l'autre. Pour le dire d'une autre manière, le champ $\mathcal{M}(P)$ est visiblement une gerbe sur $X$, qui est neutre car $P$ en est une section sur $X$. Le résultat découle alors de [73], lemme (3.21).

Par exemple, on peut prendre la marque $k^{n}$, avec $\mathcal{M}$ le champ des faisceaux localement libres de rang $n$ sur les $k$-schémas, voir [73], théorème (4.6.2.1). Alors le principe qui vient d'être rappelé dit que ce champ est isomorphe à $B \mathrm{GL}(n)$. Plus significatif pour la suite est le cas du groupe projectif linéaire $\operatorname{PGL}(n)=\operatorname{Aut} \mathbb{P}^{n}$. Le champ classifiant $B$ PGL $(n)$ est isomorphe au champ dont les objets sont les fibrations en $P^{n}$ au dessus d'un $k$-schéma. Le cas utile pour la suite est un mélange de ces exemples. Fixons un faisceau localement libre $V$ de $\operatorname{rang} r \geqslant 1$ sur $P^{1}$, donc en vertu d'un théorème de Grothendieck, de la forme $V=\theta\left(n_{1}\right) \oplus \cdots \oplus \theta\left(n_{r}\right)$, avec $n_{1} \leqslant \cdots \leqslant n_{r}$. Soit la catégorie fibrée en groupoïdes $\mathscr{P}$, dont les objets au-dessus de $S$ les couples $(D \longrightarrow S, E)$, avec $D \longrightarrow S$ une fibration en $\mathbb{P}^{1}$, et $E$ un fibré vectoriel sur $D$ de type $V$ le long des fibres, c'est-à-dire, localement sur $S$, de la forme $\bigoplus_{i=1}^{r} \mathscr{L}_{i}$, où $\mathscr{L}_{i}$ est inversible de degré relatif $n_{i}$. Noter que cela a un sens car, localement pour la topologie étale, $D=\mathbb{P}_{S}^{1}$. L'identification des objets est un exercice aisé :

Lemme 9.3.2. - La catégorie fibrée en groupoïdes $\mathscr{D}$ est un champ algébrique. La marque des objets de $\mathscr{D}$ est $\left(\mathbb{P}^{1}, V\right)$. 
Le groupe des automorphismes $G$ du modèle, est le groupe qui linéarise universellement le fibré $V$. Ce groupe s'insère donc dans une suite exacte

$$
1 \longrightarrow H=\operatorname{Aut}(V) \longrightarrow G \longrightarrow \operatorname{PGL}(1) \longrightarrow 1 \text {. }
$$

Démonstration. - Le premier point se résume essentiellement à montrer que si $(D, E)$ et $\left(D^{\prime}, E^{\prime}\right)$ sont deux objets de $\mathscr{P}$ au-dessus du schéma $S$, alors le faisceau sur $S_{e t}$ :

$$
(T \longrightarrow S) \mapsto \operatorname{Isom}_{T}\left(\left(D_{T}, E_{T}\right),\left(D_{T}^{\prime}, E_{T}^{\prime}\right)\right)
$$

est représentable par un $S$-schéma de type fini. On peut procéder en deux étapes. Soit d'abord le foncteur $\operatorname{Isom}_{S}\left(D, D^{\prime}\right)$ qui est représenté un torseur $I$ sous le groupe $\operatorname{PGL}(1)=\operatorname{Aut}\left(\mathbb{P}^{1}\right)$. Si $\Phi: D \times_{S} \mathscr{~} \stackrel{\sim}{\longrightarrow} D^{\prime} \times_{S} \mathscr{J}$ est l'isomorphisme universel, on est ramené visiblement à prouver que le foncteur défini sur la catégorie des $\mathscr{I}$-schémas

$$
(U \longrightarrow \mathcal{I}) \mapsto \operatorname{Isom}_{U}\left(E_{U}, \Phi^{*}\left(E^{\prime}\right)_{U}\right)
$$

est représentable, en fait représenté par un schéma affine sur I. C'est un fait bien connu. Identifions maintenant la marque des objets de $\mathscr{P}$. Il est clair que toute fibration en $\mathbb{P}^{1}$ de base $S$ est localement pour la topologie étale de la forme $\mathbb{P}_{S}^{1}$. Supposons donc $D=\mathbb{P}_{S}^{1}$. Ceci étant, le module localement libre $E$ étant de type $V$, quitte à localiser si nécessaire, est de la forme $E=\vartheta_{\mathbb{P}_{S}^{1}}\left(n_{1}\right) \oplus \cdots \oplus \vartheta_{\mathbb{P}_{S}^{1}}\left(n_{r}\right)=p^{*}(V)$, où $p: \mathbb{P}_{S}^{1} \longrightarrow \mathbb{P}_{k}^{1}$ est le morphisme de changement de base. Ce qui montre que la marque est bien $\left(\mathbb{P}^{1}, V\right)$. Pour décrire le groupe des automorphismes de l'objet marque, rappelons que si un groupe algébrique $G$ agit sur une variété $X$, l'action étant $\mu: G \times X \longrightarrow X$, une $G$-linéarisation d'un faisceau cohérent $F$, est un isomorphisme

$$
\Phi: p_{2}^{*}(F) \stackrel{\sim}{\longrightarrow} \mu^{*}(F)
$$

vérifiant une relation de cocycle bien connue (voir par exemple [81] ainsi que la section 6.1). En général, un faisceau $F$ localement libre de rang $n$ sur $X$ n'est pas $G$-linéarisable, cependant il le devient si le groupe $G$ est agrandi convenablement. De manière précise, l'argument rappelé en début de preuve montre qu'il existe un groupe algébrique $\tilde{G}$, et un morphisme de groupes $\tilde{G} \longrightarrow G$, tel que les $G$-linéarisations de $F$ correspondent de manière bijective aux sections de $\tilde{G} \longrightarrow G$. En fait, on applique la construction du début au couple de faisceaux $\left(\mu^{*}(F), p_{2}^{*}(F)\right)$, $\mu$ et $p_{2}$ étant respectivement l'action et la projection $G \times X \longrightarrow X$, de sorte que

$$
\tilde{G}=\operatorname{Isom}\left(p_{2}^{*}(F), \mu^{*}(F)\right) .
$$

D'une manière simplifiée, les points de $\tilde{G}$ sont les couples $(g, \phi)$, avec $g \in G$, et $\phi: F \stackrel{\sim}{\longrightarrow} g^{*}(F)$. La loi de composition est

$$
(g, \phi)(h, \psi)=\left(g h, h^{*}(\phi) \psi\right) .
$$

Si on a $g^{*}(F) \cong F$ pour tout $g \in G$, alors ce groupe s'insère dans une extension

$$
1 \longrightarrow \operatorname{Aut}(F) \longrightarrow \tilde{G} \longrightarrow G \longrightarrow 1 \text {. }
$$

Appliqué à la situation présente, cela donne la seconde partie du lemme. 
Noter que l'extension (98) n'est en général pas scindée du fait que $\Theta(n)$ est PGL(1)-linéarisable si et seulement si $n$ est pair; il n'est que GL(2)-linéarisable. Soit $\alpha_{g}: \theta(1) \stackrel{\sim}{\longrightarrow} g^{*}(\theta(1))$ la linéarisation tautologique de $\theta(1)$; elle induit une linéarisation de $V$,

$$
\bigoplus_{i=1}^{r} \alpha_{g}^{\otimes n_{i}}: V \stackrel{\sim}{\longrightarrow} g^{*}(V) \quad(g \in \mathrm{GL}(2)) .
$$

Noter que $\lambda 1_{2}$ agit diagonalement sur $V$ par la matrice diagonale $\operatorname{diag}\left(\lambda^{n_{1}}, \ldots, \lambda^{n_{r}}\right)$. On notera cependant que la linéarisation tautologique de $V$ sous GL(2) se descend dans tous les cas en une linéarisation sous $\mathrm{GL}(2) / \mu_{d}$, si $d$ est le pgcd des $n_{i}$. Le groupe $H$ est aisé à décrire. Supposons la partition $\left(n_{i}\right)$ de $r=\sum_{i=1}^{r} n_{i}$, prenant $p$ valeurs distinctes $k_{1}<\cdots<k_{p}$, où $k_{i}$ apparaît $\alpha_{i}$ fois. Ainsi $V$ a une filtration canonique

$$
0 \neq F^{p} \subset \cdots \subset F^{1}=E, \quad F^{j} / F^{j+1}=\vartheta_{\mathbb{P}_{S}^{1}}\left(k_{j}\right)^{\oplus\left(\alpha_{j}\right)} .
$$

En fait, si $V=\bigoplus_{j=1}^{p} \vartheta_{\mathbb{P}^{1}}\left(k_{j}\right)^{\oplus\left(\alpha_{j}\right)}$, on a $F^{j}=\bigoplus_{m=j}^{p} \vartheta_{\mathbb{P}^{1}}\left(k_{m}\right)^{\oplus\left(\alpha_{m}\right)}$. En particulier un automorphisme de $E$ respecte la filtration $F^{\bullet}$. Il est alors immédiat que $H=\operatorname{Aut}(E)$ est le produit semi-direct

$$
H=U \rtimes \prod_{j=1}^{p} \mathrm{GL}\left(\alpha_{i}\right)
$$

d'un groupe unipotent $U$ par un produit de groupes linéaires. Dans certains cas, $G$ est en fait un produit semi-direct. Supposons par exemple $\alpha_{1}=\ldots \alpha_{p}=1$. Soit une relation de Bezout $1=\sum_{j} m_{j} \frac{k_{j}}{d}$. Considérons le caractère de $H$ donné par $\chi: H \longrightarrow \prod_{j=1}^{p} \operatorname{GL}\left(\alpha_{i}\right) \stackrel{\psi}{\longrightarrow} G_{m}$ avec $\psi\left(\left(g_{j}\right)\right)=\prod_{j} \operatorname{det}\left(g_{j}\right)^{m_{j}}$, et soit $K$ le noyau de $\chi$. On a la remarque évidente :

LEMME 9.3.3. - Sous les conditions indiquées, le groupe $H$ est le produit semi-direct $H=K \rtimes \mathrm{GL}(2) / \mu_{d}$. Si de plus d est pair, la suite (97) est scindée.

Démonstration. - Notons $\alpha: \mathrm{GL}(2) \longrightarrow G$ la section induite par la linéarisation canonique. Alors les éléments de $K$ qui sont aussi dans l'image de $\alpha$, sont les $\operatorname{diag}\left(\lambda^{n_{1}}, \ldots, \lambda^{n_{r}}\right)$, avec $\lambda^{\sum m_{i} n_{i}}=\lambda^{d}=1$. Cette intersection est donc réduite à l'identité. Le reste est clair. Pour le point deux, notons que si les $n_{i}$ sont tous pairs, alors on obtient une section de $G \longrightarrow \operatorname{PGL}(1)$ par factorisation de $g \in \mathrm{GL}(2) \mapsto \oplus_{i} \alpha_{g}^{\otimes n_{i}} \operatorname{det}(g)^{-n_{i} / 2}$.

\subsubsection{Interlude : Géométrie du discriminant des formes binaires. -}

L'espace affine des formes binaires admet une stratification naturelle dont la géométrie est intimement liée à celle des schémas de Hurwitz classifiant les revêtements cycliques de $\mathbb{P}^{1}$. Soit $N$ un entier fixé dans toute cette section, et soit $\mu=n_{1} \leqslant n_{2} \leqslant \cdots \leqslant n_{r}$ une partition (fixée) de poids $N$. Une forme binaire (non nulle)

$$
a_{0} X^{N}+a_{1} X^{N-1} Y+\cdots+a_{N} Y^{N}
$$


est représentée par le point $\left(a_{0}, \ldots, a_{N}\right) \in \mathbb{A}_{*}^{N+1}$. On notera $X_{\mu}=X_{\left(n_{1}, \ldots, n_{r}\right)}$ la sous-variété (localement fermée) de l'espace affine $\mathbb{A}^{N+1}$ des formes binaires de degré $N$, formée de celles qui sont le produit de $r$ facteurs linéaires non proportionnels, de multiplicités respectives $n_{1} \leqslant n_{2} \leqslant \cdots \leqslant n_{r}$. On parlera de $\mu$ comme du type combinatoire de la forme binaire. Il est facile de décrire $X_{\mu}$ comme un cône au dessus d'un produit d'espaces projectifs. On écrira préférablement la partition $\mu$ sous la forme duale $\mu=m_{1}^{k_{1}} \ldots m_{s}^{k_{s}}$, signifiant que la fonction $i \mapsto n_{i}$ prend les valeurs distinctes $m_{1}<\cdots<m_{s}$, respectivement $k_{1}, \ldots, k_{s}$ fois. Une forme binaire de type $\mu$ peut alors être écrite sous la forme plus rigide

$$
F=\prod_{i=1}^{s} f_{i}^{m_{i}} \quad, \quad\left(f_{i}, f_{j}\right)=1 \text { si } i \neq j \quad, \quad \operatorname{deg}\left(f_{i}\right)=k_{i}
$$

où $f_{i}$ est sans facteur multiple. La décomposition est génériquement bien définie à l'action du groupe diagonalisable près

$$
T_{\mu}=\left\{\lambda=\left(\lambda_{1}, \ldots, \lambda_{s}\right), \prod_{i=1}^{s} \lambda_{i}^{m_{i}}=1\right\} .
$$

Pour décrire avec plus de précision la stratification de $\mathbb{A}_{*}^{N+1}$, de strates les sousschémas $X_{\mu}$, rappelons (voir par exemple [66]) quelques propriétés du morphisme de Viète. Soit $Z_{N}=\left(\mathbb{A}_{*}^{2}\right)^{N}$ la variété des $2 N$-uples

$$
\left(u_{1}, v_{1}, \ldots, u_{N}, v_{N}\right) \text { tels que }\left(u_{i}, v_{i}\right) \neq(0,0) \text { pour } 1 \leqslant i \leqslant N .
$$

Il y a sur $Z_{N}$ une action évidente d'une part du groupe $G_{m}^{N}$, et d'autre part du groupe symétrique $\mathfrak{S}_{N}$. Il en résulte une action du produit semi-direct $G_{m}^{N} \rtimes \mathfrak{S}_{N}$ $\operatorname{sur}\left(\mathbb{A}_{*}^{2}\right)^{N}$, telle que $Z_{N} / G_{m}^{N} \rtimes \mathfrak{S}_{N}=\left(\mathbb{P}^{1}\right)^{N} / \mathfrak{S}_{N}=\mathbb{P}^{N}$. Soit le sous-groupe $T_{N}=$ $\left\{\left(t_{1}, \ldots, t_{N}\right) \in G_{m}^{N} ; \prod_{i} t_{i}=1\right\}$. Les choses s'expriment mieux en termes de cônes au dessus d'une variété. Rappelons que si $X$ est une variété normale, et si $\mathcal{L}$ est un faisceau inversible ample sur $X$, alors l'algèbre $R=\bigoplus_{d \geqslant 0} \Gamma\left(X, \mathscr{L}^{\otimes d}\right)$ est de type fini, normale. La variété affine $\operatorname{Spec}(R)$ est le cône de base $X$ défini par $\mathscr{L}$. Si $X$ est lisse, le sommet est le seul point singulier, point singulier conique, et il est immédiat que le fibré vectoriel $V(\mathscr{L}) \longrightarrow X$, induit une résolution de la singularité conique, via le morphisme naturel $V(\mathscr{L}) \longrightarrow \operatorname{Spec}(R)$. Le diviseur exceptionnel est la section nulle de la fibration, et son complémentaire est isomorphe au cône épointé $\operatorname{Spec}(R)-0$. Notons par ailleurs que $(\operatorname{Spec}(R)-0) / G_{m}=X$, et $\operatorname{Pic}(\operatorname{Spec}(R)-0)=\operatorname{Pic}(X) /[\mathscr{L}]$. Ainsi la variété notée $\mathbb{A}_{*}^{N+1}$ des formes binaires de degré $N$ peut être identifiée avec le cône épointé de base $\mathbb{P}^{N}$, relatif à $\Theta(1)$. Rappelons que le morphisme de Viète (relations coefficients-racines) $v: Z_{N}=\left(\mathbb{A}_{*}^{2}\right)^{N} \longrightarrow \mathbb{A}_{*}^{N+1}$ est défini comme suit :

$$
\left(u_{1}, v_{1}, \ldots, u_{N}, v_{N}\right) \mapsto \prod_{i=1}^{N}\left(u_{i} X-v_{i} Y\right)=a_{0} X^{N}+\cdots+a_{N} Y^{N}
$$

Ce morphisme permet de justifier l'identification classique suivante : 
LEMme 9.3.4. - Le quotient $X_{N}=Z_{N} / T_{N}$ est le cône épointé de base $\left(\mathbb{P}^{1}\right)^{N}$ défini par le faisceau inversible $\Theta(1, \ldots, 1)$, et de plus $Z_{N} / T_{N} \rtimes \mathfrak{S}_{N}=\mathbb{A}_{*}^{N+1}$.

Pour décrire $X_{\mu}$, on forme d'abord le plongement diagonal de $\left(\mathbb{A}_{*}^{2}\right)^{r}$ dans $\left(\mathbb{A}_{*}^{2}\right)^{N}$ décrit par

$$
\left(u_{1}, v_{1}, \ldots, u_{r}, v_{r}\right) \mapsto(\underbrace{u_{1}, v_{1}, \ldots, u_{1}, v_{1}}_{n_{1}}, u_{2}, v_{2}, \ldots, \underbrace{u_{r}, v_{r}, \ldots, u_{r}, v_{r}}_{n_{r}})
$$

On note que l'image de (100) est la sous-variété des points fixes de $\mathfrak{S}_{n_{1}} \times \cdots \times \mathfrak{S}_{n_{r}}$. Le sous-groupe de $T_{N} \rtimes \mathfrak{S}_{N}$ qui agit sur cette image est le normalisateur de $\mathfrak{S}_{n_{1}} \times$ $\cdots \times \mathfrak{S}_{n_{r}}$, donc est le groupe $T_{\mu} \rtimes \operatorname{Norm}_{\mathfrak{S}_{N}}\left(\mathfrak{S}_{n_{1}} \times \cdots \times \mathfrak{S}_{n_{r}}\right)$, avec $T_{\mu}$ donné par

$$
T_{\mu}=T_{N}^{\mathfrak{S}_{n_{1}} \times \cdots \times \mathfrak{S}_{n_{r}}}=\left\{\left(t_{1}, \ldots t_{1}, \ldots, \ldots, t_{r}, \ldots, t_{r}\right)\right\}, t_{1}^{n_{1}} \ldots t_{r}^{n_{r}}=1 .
$$

Noter que cette action est n'set libre que génériquement, pas partout. On a utilisé en passant le lemme évident suivant :

LEMme 9.3.5. - Soit un produit semi direct $G=H \rtimes K$, et soit $\Delta \subset K$ un sousgroupe de $K$. Le normalisateur de $\Delta$ dans $G$ est $H^{\Delta} \rtimes N_{K}(\Delta)$.

Le groupe diagonalisable $T_{\mu}$ n'est connexe que si $d=\operatorname{pgcd}\left(n_{1}, \ldots, n_{r}\right)=1$, sinon $T_{\mu} / T_{\mu}^{\circ}=\mathbb{Z} / d \mathbb{Z}$. Noter qu'à cela correspond une action libre du groupe diagonalisable $T_{\mu} \cong \operatorname{ker}\left(G_{m}^{r} \longrightarrow G_{m}\right),\left(t_{1}, \ldots, t_{r}\right) \mapsto t_{1}^{n_{1}} \ldots t_{r}^{n_{r}}$ sur $\left(\mathbb{A}_{*}^{2}\right)^{r}$ qui s'exprime par

$$
\left(t_{1}, \ldots, t_{r}\right) \cdot\left(u_{1}, v_{1}, \ldots, u_{r}, v_{r}\right)=\left(t_{1} u_{1}, t_{1} v_{1}, \ldots, t_{r} u_{r}, t_{r} v_{r}\right)
$$

et qui rend le plongement (100) équivariant. Il en découle une action résiduelle du normalisateur $N_{\mu}:=\operatorname{Norm}_{\mathfrak{S}_{N}}\left(\mathfrak{S}_{n_{1}} \times \cdots \times \mathfrak{S}_{n_{r}}\right)$ sur $\left.Z_{\mu}=\left(\mathbb{A}_{*}^{2}\right)^{r}\right) / T_{\mu}$. Rappelons que les valeurs distinctes prises par les $n_{i}$ sont $m_{i}=n_{k_{1}+\cdots+k_{i}}, m_{1}<\cdots<m_{s}$. On trouve pour le normalisateur $N_{\mu}$ le produit en couronne $N_{\mu}=\left(\mathfrak{S}_{m_{1}} \prec \mathfrak{S}_{k_{1}}\right) \times \cdots \times\left(\mathfrak{S}_{m_{s}} \prec \mathfrak{S}_{k_{s}}\right)$. En résumé, on a le fait connu suivant :

Proposition 9.3.6. - i) Le quotient $Z_{\mu}=\left(\mathbb{A}_{*}^{2}\right)^{r} / T_{\mu}$ s'identifie au cône épointé au dessus de $\left(\mathbb{P}^{1}\right)^{r}$ relatif $\grave{a} \mathscr{L}=\Theta\left(n_{1}, \ldots, n_{r}\right)$.

ii) Le quotient $C_{\mu}=Z_{\mu} / N_{\mu}$ s'identifie au cône épointé au-dessus de $\mathbb{P}^{k_{1}} \times \cdots \times \mathbb{P}^{k_{s}}$ défini par le faisceau inversible $\Theta\left(m_{1}, \ldots, m_{s}\right)$. Le morphisme $C_{\mu} \longrightarrow \mathbb{A}_{*}^{N+1}$ est fini birationnel sur son image, il définit la normalisation de la strate fermée $\overline{X_{\mu}}$.

iii) La variété $X_{\mu}$ est isomorphe au complémentaire dans $C_{\mu}=Z_{\mu} / N_{\mu}$ de l'hypersurface $\Delta\left(f_{1} \ldots f_{s}\right)=0(\Delta(\cdot)$ étant le discriminant usuel).

Démonstration. - Pour i), on note que les fonctions invariantes sous $T_{\mu}$ sont les fonctions polynomiales $f\left(u_{1}, v_{1}, \ldots, u_{r}, v_{r}\right)$ telles que pour $t \in T_{\mu}$ :

$$
f\left(t_{1} u_{1}, t_{1} v_{1}, \ldots, t_{r} u_{r}, t_{r} v_{r}\right)=f\left(u_{1}, v_{1}, \ldots, u_{r}, v_{r}\right)
$$

si $f$ est multihomogène de multidegré $\left(\alpha_{1}, \ldots, \alpha_{r}\right)$, le poids de $f$ est $t \mapsto t_{1}^{\alpha_{1}} \ldots t_{r}^{\alpha_{r}}$, donc on doit avoir $\left(\alpha_{1}, \ldots, \alpha_{r}\right)=e\left(n_{1}, \ldots, n_{r}\right)$ pour un $e \geqslant 1$. L'assertion i) en découle. D'une autre manière, on peut noter que la projectivisation du plongement (100), est le plongement polydiagonal $\left(\mathbb{P}^{1}\right)^{r} \mapsto\left(\mathbb{P}^{1}\right)^{N}$ et que la restriction de 
$\Theta(1, \ldots, 1)$ est $\Theta\left(n_{1}, \ldots, n_{r}\right)$. L'assertion découle alors du lemme 9.3.4.

Pour ii), on note que l'action du groupe fini $N_{\mu}$, se réduit à l'action de $\mathfrak{S}_{k_{1}} \times$ $\cdots \times \mathfrak{S}_{k_{s}}$, ce qui conduit au fait que les points de $Z_{\mu} / N_{\mu}$ ont pour coordonnées (multihomogènes), les coefficients des produits partiels

$$
f_{1}(X, Y)=\prod_{1}^{k_{1}}\left(u_{i} X-v_{i} Y\right)=a_{0}^{1} X^{k_{1}}+\cdots a_{k_{1}}^{1} Y^{k_{1}}, \ldots
$$

On peut identifier un point de ce quotient à un $s$-uple de formes $\left[f_{1}, \ldots, f_{s}\right]$, de degrés respectifs $k_{1}, \ldots, k_{s}$. L'identification étant

$$
\left[f_{1}, \ldots, f_{s}\right]=\left[g_{1}, \ldots, g_{s}\right] \Longleftrightarrow g_{i}=\lambda_{i} f_{i} \quad\left(\lambda_{i} \in k^{*}\right), \prod_{i=1}^{s} \lambda_{i}^{m_{i}}=1 .
$$

Comme dans la description précédente, on retrouve bien la description des points du cône de base $\mathbb{P}^{k_{1}} \times \cdots \times \mathbb{P}^{k_{s}}$ relatif au faisceau $\Theta\left(m_{1}, \ldots, m_{s}\right)$. D'une autre manière, le quotient $Z_{\mu} / N_{\mu}$ est isomorphe à $\mathbb{A}_{*}^{k_{1}+1} \times \cdots \times \mathbb{A}_{*}^{k_{s}+1} / T^{\prime}$, le tore $T^{\prime}$ étant le noyau du morphisme $G_{m}^{s} \longrightarrow G_{m}$ qui envoie $\left(\lambda_{1}, \ldots, \lambda_{s}\right)$ sur $\prod_{i=1}^{s} \lambda_{i}^{m_{i}}$; l'identification s'obtient en effectuant le quotient partiel de $Z_{\mu}$ par le sous-tore de $T_{\mu}$ donné par les équations $\prod_{i=1}^{k_{1}} t_{i}=1, \ldots, \prod_{i=1}^{k_{s}} t_{i}=1$. Le morphisme $Z_{\mu} / N_{\mu} \longrightarrow \mathbb{A}_{*}^{N+1}$ n'est autre que $\left(f_{1}, \ldots, f_{s}\right) \mapsto f_{1}^{m_{1}} \ldots f_{s}^{m_{s}}$. Tout est plus clair sur le diagramme

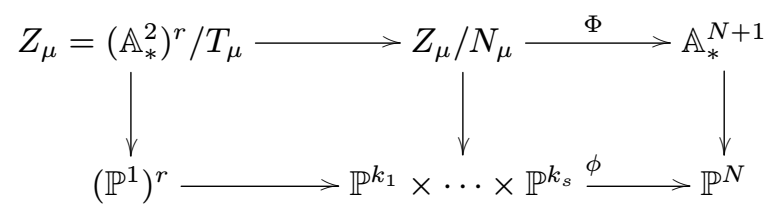

Les flèches $\Phi, \phi$ sont celles explicitées ci-dessus lors de la preuve, c'est-à-dire

$$
\phi\left(D_{1}, \ldots, D_{s}\right)=\sum_{i=1}^{s} m_{i} D_{i}, \Phi\left(\left[f_{1}, \ldots, f_{s}\right]\right)=\left[f_{1}^{m_{1}} \ldots f_{s}^{m_{s}}\right] .
$$

Les relations d'incidence entre les strates $X_{\mu} \subset \mathbb{A}_{*}^{N+1}$ se décrivent facilement au moyen d'un ordre entre partitions de poids donné $N$ (voir [66]) :

$$
\eta=\left(\eta_{1} \leqslant \cdots \leqslant \eta_{l}\right) \leqslant \mu=\left(n_{1} \leqslant \cdots \leqslant n_{r}\right) \Longleftrightarrow \eta_{j}=\sum_{i \in I_{j}} n_{i}
$$

où les $I_{j}$ forment une partition de $[1, r]$. L'adhérence de la strate $X_{\mu}$ est $\overline{X_{\mu}}=$ $\bigcup_{\eta \leqslant \mu} \quad X_{\eta}$. Le morphisme $C_{\mu}=Z_{\mu} / N_{\mu} \longrightarrow \mathbb{A}_{*}^{N=1}$ a pour image $\overline{X_{\mu}}$, est la désingularisation de $\overline{X_{\mu}}$. Après projectivisation, ce morphisme se réduit à $\mathbb{P}^{k_{1}} \times \cdots \times \mathbb{P}^{k_{s}} \longrightarrow \mathbb{P}^{N}$ qui est le composé d'un plongement de Veronese, suivi d'une projection linéaire; ce morphisme est la normalisation de son image. 
EXEMPle 9.3.7. - Le cas $\mu=(1, \ldots, 1,2)$, soit $k_{1}=N-2, k_{2}=1, m_{1}=1$, $m_{2}=2$, correspond à l'hypersurface discriminant (privée de l'origine), qui a pour normalisation le cône épointé de base $\mathbb{P}^{N-2} \times \mathbb{P}^{1}$, relativement à $\Theta(1,2)$. Le morphisme de normalisation est donné par $\left[f_{1}, f_{2}\right] \mapsto F=f_{1} f_{2}^{2}$. Si $f_{1}$ a un facteur linéaire multiple, on peut l'échanger avec $f_{2}$, ce qui montre que ce morphisme n'est pas bijectif sur l'image. Regardons comme exemple le cas des formes quartiques (99), correspondant à $N=4$. Le discriminant $\Delta$ caractérise les formes avec un facteur linéaire double, donc $\mu=(1,1,2)$. Pour $\mu=(1,3), \bar{X}_{1,3}$ est le cône épointé audessus de $\mathbb{P}^{1} \times \mathbb{P}^{1}$ défini par $\mathscr{L}=\Theta(1,3)$. La théorie classique donne des équations $P(F)=Q(F)=0$ pour $\bar{X}_{1,3}$, où $P$ et $Q$ sont les covariants

$$
\begin{aligned}
& P=\frac{1}{6}\left(a_{2}^{2}-3 a_{1} a_{3}+12 a_{0} a_{4}\right) \\
& Q=a_{0} a_{2} a_{4}-\frac{3}{8} a_{0} a_{3}^{2}-\frac{3}{8} a_{1}^{2} a_{4}+18 a_{1} a_{2} a_{3}-\frac{1}{36} a_{2}^{3} .
\end{aligned}
$$

Dans le cas $\mu=(2,2)$, donc $m_{1}=2, s=1, k_{1}=2, X_{2,2}$ représente les formes quartiques carré d'une forme quadratique; dans ce cas aussi on obtient des équations en écrivant que la forme hessienne $H(F)$ est proportionnelle à $F$. Enfin si $\mu=(4)$ qui est le cas des formes avec un seul facteur linéaire, les équations de $X_{4}$, la strate fermée, se résument à $H(F)=0$.

9.3.3. Le champ des revêtements cycliques de la droite projective. - On code la ramification d'un revêtement cyclique de degré $n$ sous la forme d'une suite de diviseurs $e_{i}>1$ de $n$, avec $1 \leqslant i \leqslant r$, et pour tout $i$, un entier $1 \leqslant \eta_{i}<e_{i}$, tel que $\operatorname{pgcd}\left(e_{i}, \eta_{i}\right)=1$. On posera $n_{i}=\frac{n}{e_{i}} \eta_{i}$. On suppose que $n_{1} \leqslant \cdots \leqslant n_{r}$, définissant ainsi une partition $\mu$ de poids $N=n m=\sum_{i} n_{i}$.

Soit $\pi: C \longrightarrow D$ un revêtement cyclique à ramification fixée de type $\mu$, de base $S$, les fibres géométriques de $D \longrightarrow S$ étant isomorphes à $\mathbb{P}^{1}$. On sait (proposition 9.1.3) que le revêtement $\pi: C \longrightarrow D$ peut être reconstruit en partant de la base $D$, équipée de la structure additionnelle $(\mathscr{L}, \Phi)$. Il sera préférable dans la suite de noter la partition $\mu$ comme une suite strictement croissante avec répétitions, donc de considérer les entiers $m_{j}(1 \leqslant j \leqslant s)$ distincts définis par la partition $n_{1} \leqslant \cdots \leqslant n_{r}$. Cela permet d'écrire le diviseur $B$ tel que $\operatorname{Div}(\Phi)=B$ sous la forme $B=\sum_{i=1}^{s} m_{i} B_{i}$, les diviseurs $B_{i}$ étant maintenant disjoints deux à deux. $\operatorname{Posons} \operatorname{deg}\left(B_{i}\right)=k_{i}$, de sorte que $\sum_{i} k_{i} m_{i}=\sum_{j} n_{j}=n m$, l'entier $m$ étant défini par cette équation.

Revenons à la variété lisse $X_{\mu}$ partie ouverte du cône $Z_{\mu} / N_{\mu}$. Le groupe GL(2) agit de manière naturelle sur l'espace vectoriel des formes binaires de degré donné, i.e. $g . F(X, Y)=F\left(g^{-1} X, g^{-1} Y\right)$, mais cette action ne se descend pas en général en une action de PGL(1). L'entier $m$ étant comme introduit au dessus, on notera cependant qu'il y a une action du groupe $\mathrm{GL}(2) / \mu_{m}$ sur le cône épointé $Z_{\mu} / N_{\mu}$, de manière explicite

$$
g\left[f_{1}, \ldots, f_{s}\right]=\left[g \cdot f_{1}, \ldots, g \cdot f_{s}\right]
$$


Cette définition est cohérente car $\sum_{i=1}^{s} k_{i} m_{i}=n m$. De manière équivalente, le faisceau $\Theta\left(m_{1}, \ldots, m_{s}\right)$ sur $\mathbb{P}^{k_{1}} \times \cdots \times \mathbb{P}^{k_{s}}$ est canoniquement GL(2)/ $\mu_{m}$-linéarisé. La description qu'on a en vue du champ $\mathcal{H}_{n, \mu}$ des revêtements cycliques de degré $n$ de $\mathbb{P}^{1}$, à ramification de type $\mu$, et qui dans le cas des courbes généralise [5], est :

THÉORÈme 9.3.8. - Si les points de branchement sont marqués (non ordonnés), on a un isomorphisme de champs

$$
\mathscr{H}_{n, \mu}=\left[X_{\mu} /\left(\mathrm{GL}(2) / \mu_{m}\right)\right] .
$$

Si les points de branchement sont piqués (ordonnés), on note $Y_{\mu}$ le complémentaire dans $Z_{\mu}$ de la grosse diagonale. Alors, il existe une action du groupe multiplicatif $\mathbb{G}_{m}$ sur $Y_{\mu}$ telle que

$$
\mathcal{H}_{n, \mu}=\left[Y_{\mu} / G_{m}\right] \text {. }
$$

L'action de $\mathbb{G}_{m}$ sur $Y_{\mu}$ sera précisée dans la preuve.

Démonstration. - On définit un morphisme $\mathcal{H}_{n, \mu} \longrightarrow\left[X_{\mu} /\left(\mathrm{GL}(2) / \mu_{m}\right)\right]$, qui après vérification, sera un isomorphisme. Soit un revêtement $p: C \longrightarrow D$, de base $S$. La courbe $q: D \longrightarrow S$ est un fibré en coniques, et le sous-faisceau propre $\mathscr{L} \subset \pi_{*}\left(\theta_{C}\right)$ relatif à la valeur propre $\zeta_{n}$ est un fibré en droites sur $D$ de degré $-m$ le long des fibres. Par ailleurs, la multiplication définit un morphisme injectif $\Phi: \mathscr{L}^{\otimes n} \longrightarrow \vartheta_{D}$, le diviseur $B$ de $\Phi$ ayant par définition un type combinatoire fixé le long des fibres. Ceci se traduit par

$$
B=\operatorname{Div}(\Phi)=\sum_{1<e \mid n, 1 \leqslant \eta<e} \frac{n}{e} \eta B_{e, \eta} .
$$

D'une part, si l'on pose $V=\Theta(-m)$, on récupère un torseur sous $G=\mathrm{GL}(2) / \mu_{m}$ :

$$
P=\operatorname{Isom}_{S}\left(\left(\mathbb{P}_{S}^{1}, V_{S}\right),(D, L)\right) .
$$

D'autre part, du fait de la forme du diviseur de la section $\Phi$, cette section définit un morphisme $P \longrightarrow X_{\mu} \subset \mathbb{A}^{N}$, qui est par construction clairement $G$-équivariant. Cette construction définit un foncteur $\mathcal{H}_{n, \mu} \longrightarrow\left[X_{\mu} / G\right]$. Pour prouver que ce foncteur est un isomorphisme, il suffit de voir que c'est simultanément un monomorphisme et un épimorphisme [73]. En fait, il est possible d'exhiber un foncteur quasi-inverse, comme la preuve ci-dessous le suggère. Pour tout schéma $S$, on doit s'assurer que la restriction de ce foncteur à la catégorie des sections au-dessus de $S$ est pleinement fidèle. On est ramené à voir que tout $S$-isomorphisme de revêtements $f: C \stackrel{\sim}{\longrightarrow} C^{\prime}$ correspond de manière bijective à un isomorphisme des objets images par le foncteur considéré. Notons $h: D \stackrel{\sim}{\longrightarrow} D^{\prime}$ le $S$-isomorphisme entre les courbes quotients. Il définit d'une part un isomorphisme entre les $G$-torseurs associés $P \stackrel{\sim}{\longrightarrow} P^{\prime}$, et un isomorphisme $\mathbb{Z} / n \mathbb{Z}$-équivariant

$$
\psi: \mathcal{E}=\oplus_{i=0}^{n-1} \mathscr{L}_{i} \stackrel{\sim}{\longrightarrow} h^{*}\left(\mathcal{E}^{\prime}=\oplus_{i=0}^{n-1} \mathcal{L}_{i}^{\prime}\right) .
$$

Notons $\psi_{i}: \mathscr{L}_{i} \stackrel{\sim}{\longrightarrow} h^{*}\left(\mathcal{L}_{i}^{\prime}\right)$ sa restriction à $\mathscr{L}_{i}$. Rappelons la relation $\Phi^{\prime} . \psi_{1}^{\otimes n}=\Phi$; il s'agit donc de voir que l'isomorphisme initial $f$ peut être reconstitué de manière unique en partant de la donnée $\left(h, \psi_{1}\right)$. C'est exactement le contenu de la proposition 9.1.3. 
Dans le cas des points de branchement ordonnés la donnée qui se substitue à $\left(\mathbb{P}^{1}, \Theta(-m)\right)$ est $\left(\mathbb{P}^{1},\left(Q_{1}, \ldots, Q_{b}\right), \Theta(-m)\right)$, le groupe $G$ est donc

$$
G=\mathbb{G}_{m}=\operatorname{Aut}(\theta(-m)) \text {. }
$$

Il est évident que cela revient dans la preuve précédente à remplacer $X_{\mu}$ par $Y_{\mu}$, dont les points correspondent à la donnée de $b$ points distints $Q_{1}, \ldots, Q_{b}$ de $\mathbb{P}^{1}$, et d'une forme binaire $f$ de diviseur des zéros $\operatorname{Div}(f)=\sum_{j} n_{j} Q_{j}$. L'action de $G_{m}$ dérive de l'action

$$
\lambda\left[\left(u_{1}, v_{1}\right), \ldots,\left(u_{b}, v_{b}\right)\right]=\left[\lambda^{1 / m}\left(u_{1}, v_{1}\right), \ldots, \lambda^{1 / m}\left(u_{b}, v_{b}\right)\right]
$$

Le reste est clair.

Par exemple, si $n=3$, c'est-à-dire pour les revêtements galoisiens triples de la droite projective, le diviseur $B$ est de la forme $B=B_{1}+2 B_{2}$, avec $B_{1}$ et $B_{2}$ disjoints et sans multiplicités; on a entre les degrés respectifs la relation $k_{1}+2 k_{2}=3 \mathrm{~m}$. On a en fonction de la prescription générale :

$$
\operatorname{Div}\left(\Phi_{2}\right)=\left[\frac{2 B}{3}\right]=2 B_{1}+B_{2} .
$$

On voit que $X_{\mu}$ est dans ce cas un ouvert du cône de base $\mathbb{P}^{k_{1}} \times \mathbb{P}^{k_{2}}$ défini par $\Theta(1,2)$. Dans [5], Arsie et Vistoli ont traité le cas générique $\mu=\left(1^{N}\right)$; les revêtements correspondants sont appelés par eux uniformes. Dans cette situation, on a $s=1$, et $X_{\left(1^{N}\right)}$ est simplement le cône des formes de degré $N$ sans racine multiple. 


\section{CHAPITRE 10}

\section{CLASSES TAUTOLOGIQUES}

La position du champ de Hurwitz comme correspondance entre champs de courbes marquées (ou piquées) :

$$
\overline{\mathcal{M}}_{g, r} \stackrel{\imath}{\longleftarrow} \overline{\mathcal{H}}_{g, G, \xi} \stackrel{\delta}{\longrightarrow} \overline{\mathcal{M}}_{g^{\prime}, b}
$$

suggère l'existence sur ce champ de fibrés tautologiques liés au caractère galoisien des revêtements. Les plus naturels résultent de la décomposition du fibré de Hodge en facteurs isotypiques. L'essentiel de cette section porte sur l'étude de ces facteurs. Il est important pour les applications d'avoir des informations sur les classes de Chern de ces fibrés. En suivant Mumford, des résultats dans ce sens sont obtenus pour un groupe cyclique par application du théorème de Riemann-Roch (10.4). Noter que si le champ de Hurwitz est de dimension 1, cela redonne le calcul du degré de ces fibrés en droites de [43]. Dans la sous-section 10.3 on prouve une relation de Riemann-Hurwitz d'ordre supérieur reliant les classes kappa de Morita-MillerMumford (théorème 10.3.4). Enfin on étend la formule de Cornalba-Harris qui traite du champ des courbes hyperelliptiques au cas cyclique arbitraire. On peut aussi déduire sans difficulté de nos résultats les relations dans le groupe de Picard de [70].

\subsection{Fibré de Hodge}

10.1.1. G-fibrés vectoriels sur le champ de Hurwitz. - Le groupe $G$ agit sur $\overline{\mathcal{H}}_{g, G, \xi}$ (définition 6.1.1) par conjugaison de l'action. Précisément, si $\pi: C \longrightarrow D$ est une section de $\overline{\mathcal{H}}_{g, G, \xi}$ au-dessus de $S$, un élément $s \in G$ change la seule action de $G$ sur $C$ en $(g, x) \mapsto s^{-1} g s x$. Soit ${ }^{s} C$ la courbe $C$ munie ce l'action tordue par $s$. Utilisons la notation $\overline{\mathscr{H}}_{g, G, \xi}^{a d j}$ pour signifier que cette action de $G$, l'action adjointe, est prise en compte. Par construction, l'automorphisme $s: C \stackrel{\sim}{\longrightarrow} C$ devient un $G$-isomorphisme

$$
s: C \stackrel{\sim}{\longrightarrow}{ }^{s} C .
$$

La collection de ces isomorphismes fait de l'identité id : $\overline{\mathcal{H}}_{g, G, \xi} \stackrel{\sim}{\longrightarrow} \overline{\mathcal{H}}_{g, G, \xi}^{a d j}$ un isomorphisme de $G$-champs, l'action sur le terme de droite étant l'action (strictement) triviale (définition 6.1.2). En conclusion, l'action adjointe de $G$ sur $\overline{\mathscr{H}}_{g, G, \xi}$ est triviale du point de vue des champs. On sait alors (proposition 6.1.6) qu'on peut choisir un 
atlas $U \rightarrow \overline{\mathscr{H}}_{g, G, \xi}$ qui est $G$-équivariant, où l'action de $G$ sur $U$ triviale (noter qu'un tel atlas est contruit explicitement dans la section 6.3).

Soit $F$ un $G$-faisceau cohérent (resp. localement libre de rang fini) sur $\overline{\mathcal{H}}_{g, G, \xi}$ (voir [90], example 4.3), où l'action de $G$ est l'action triviale au sens strict. De la sorte, pour tout $g \in G$, et tout $G$-revêtement $\pi: C \longrightarrow D$ de base $S$, on a d'une part un faisceau cohérent $F(\pi)$ sur $S$, et d'autre part une $G$-linéarisation de $F(\pi)$. Pour tout morphisme $f: T \longrightarrow S$, l'isomorphisme

$$
F\left(f^{*}(\pi)\right) \stackrel{\sim}{\longrightarrow} f^{*}(F(\pi))
$$

(avec des notations évidentes) commute aux $G$-linéarisations. Il s'ensuit que si $F$ est un faisceau localement libre, la décomposition en facteurs isotypiques

$$
F(\pi)=\bigoplus_{\chi \in \hat{G}} F(\pi)_{\chi} \otimes V_{\chi}
$$

est préservée par l'isomorphisme (10.1.1). Les faisceaux $F(\pi)_{\chi}$ s'organisent en un faisceau $F_{\chi}$ sur $\overline{\mathcal{H}}_{g, G, \xi}$, et on a la décomposition

$$
F=\bigoplus_{\chi \in \hat{G}} F_{\chi} \otimes V_{\chi}
$$

La conséquence pour l'anneau de $K$-théorie $\mathrm{K}_{G}^{\bullet}\left(\overline{\mathcal{H}}_{g, G, \xi}\right)$, construit à partir de la catégorie des $G$-faisceaux cohérents, est :

Proposition 10.1.1. - On a:

$$
\mathrm{K}_{G}^{\bullet}\left(\overline{\mathcal{H}}_{g, G, \xi}\right) \stackrel{\sim}{\longrightarrow} \mathrm{K}^{\bullet}\left(\overline{\mathscr{H}}_{g, G, \xi}\right) \otimes R(G) .
$$

Un procédé pour obtenir des $G$-faisceaux sur $\overline{\mathcal{H}}_{g, G, \xi}$ est le suivant. Soit le morphisme représentable d'oubli de l'action de $G$ :

$$
\imath: \overline{\mathcal{H}}_{g, G, \xi} \longrightarrow \overline{\mathcal{M}}_{g,(r)}
$$

où, comme dans ce qui précède, $r$ désigne le nombre de points de ramification, et $b$ le nombre de points de branchement, nombres tous deux contenus dans la donnée de Hurwitz. Si $\pi: C \longrightarrow D$ est un revêtement définissant une section de $\mathcal{H}_{g, G, \xi}$, la base $D$ est considéré comme piquée par $b$ points, ces points contenant les points de branchement. Comme il résulte de la définition, la courbe $C$ est marquée par les préimages des points de branchement. Le marquage de ces points par paquets, indiqué par le $r$ entre parenthèses, se déduisant de la numérotation des points de branchement (définition 6.2.3). Il y a une variante immédiate dans laquelle $\overline{\mathcal{M}}_{g, r}$ remplace $\overline{\mathcal{M}}_{g,(r)}$.

Lemme 10.1.2. - Soit $F$ un faisceau cohérent (resp. un fibré vectoriel) sur $\bar{M}_{g, r}$. Le faisceau $\imath^{*}(F)$ sur $\overline{\mathcal{H}}_{g, G, \xi}$ est un $G$-faisceau cohérent (resp. un G-fibré vectoriel), 
relativement à l'action triviale de G. Dans le cas d'un fibré vectoriel, il se décompose en la somme directe de ses composants isotypiques :

$$
\imath^{*}(F)=\bigoplus_{\chi \in \widehat{G}} F_{\chi} \otimes V_{\chi},
$$

où les $F_{\chi}$ sont des faisceaux localement libres sur $\overline{\mathscr{H}}_{g, G, \xi}$ et la somme directe est indexée par les représentations irréductibles de $G$.

Démonstration. - Si $F$ est un faisceau sur $\bar{M}_{g, r}$, donc défini par une collection de faisceaux cohérents $F(q)=F(C \stackrel{q}{\longrightarrow} S)$, on a pour tout morphisme de $\overline{\mathcal{M}}_{g, r}$, i.e. pour tout carré cartésien



la donnée d'un isomorphisme $\phi_{f, h}: F(q) \stackrel{\sim}{\longrightarrow} h^{*}\left(F\left(q^{\prime}\right)\right)$, les $\phi_{f, h}$ étant assujettis à satisfaire à la relation de cocycle

$$
\phi_{f^{\prime} f, h^{\prime} h}=h^{*}\left(\phi_{f^{\prime}, h^{\prime}}\right) \cdot \phi_{f, h} .
$$

Si $q: C \longrightarrow S$ provient d'un $G$-revêtement $\pi: C \longrightarrow D$, alors tout élément $g \in G$ définit un automorphisme de $C \longrightarrow S$ dans $\bar{M}_{g, r}$, mais pas dans le champ de Hurwitz, sauf si $g$ est dans le centre. Il en résulte un isomorphisme $\phi(g): F(q) \stackrel{\sim}{\longrightarrow} F(q)$ qui $\mathrm{du}$ fait de la relation de cocycle représente une $G$-linéarisation sur le faisceau image réciproque $\imath^{*}(F)$ défini par $\imath^{*}(F)(\pi)=F(q)$. Si de plus $F$ est localement libre, la décomposition (101) suit.

Un $G$-fibré vectoriel sur le champ de Hurwitz, par exemple comme obtenu dans le lemme 10.1.2, admet en conséquence une décomposition en facteurs isotypiques (101). Soient $F$ et $H$ deux $G$-fibrés vectoriels sur le champ de Hurwitz. Nous dirons qu'ils sont disjoints si une même représentation irréductible $V_{\chi}$ n'apparaît pas simultanément dans $F$ et dans $H$. Notons le fait évident suivant :

LEMME 10.1.3. - Supposons avoir une suite exacte de G-fibrés vectoriels et de $G$-morphismes $0 \longrightarrow E \longrightarrow F \longrightarrow H \longrightarrow 0$. Si $E$ et $H$ sont disjoints, alors $F=E \oplus H$.

Démonstration. - L'hypothèse signifie que pour une représentation irréductible $V_{\chi}$ donnée, on a soit $E_{\chi}=F_{\chi}$, soit $H_{\chi}=F_{\chi}$. Le résultat en découle. 
10.1.2. Décomposition du fibré de Hodge. - Rappelons (voir [55]) que le fibré de Hodge $\mathbb{E}_{g^{\prime}, b}$ (resp. $\left.\mathbb{E}_{g, r}\right)$ sur $\overline{\mathcal{M}}_{g^{\prime}, b}\left(\operatorname{resp} . \overline{\mathcal{M}}_{g, r}\right.$ ) est le faisceau dont les sections sur l'objet $p: D \longrightarrow S$ (resp. $q: C \longrightarrow S$ ), courbe stable marquée de genre $g^{\prime}$ (resp. $g$ ), est le faisceau localement libre de rang $g^{\prime}$ (resp. $g$ ) sur $S$, i.e.

$$
\mathbb{E}(p)=p_{*}\left(\omega_{D / S}^{1}\right)
$$

La dualité de Serre montre que ce faisceau est dual du faisceau $\mathrm{R}^{1} q_{*}\left(\theta_{D}\right)$, et d'autre part que le morphisme trace permet une identification $R^{1} q_{*}\left(\omega_{D / S}\right)=\theta_{S}$. La classe de Hodge $\lambda \in \operatorname{Pic}\left(\overline{\mathcal{M}}_{g^{\prime}, b}\right)$ est par définition $\lambda=\operatorname{det}(\mathbb{E})=c_{1}(\mathbb{E})$; on définit plus généralement $\lambda_{i}=c_{i}(\mathbb{E})$, voir la section 10.3 pour les conventions et références sur l'anneau de Chow. Pour éviter toute confusion, on notera dans la suite $\mathbb{E}_{b s}$ (resp. $\lambda_{b s}$ ) le fibré de Hodge sur la base.

Soit un revêtement galoisien stable $\pi: C \longrightarrow D$ de base $S$ et de groupe $G, p$ : $D \longrightarrow S$ et $q: C \longrightarrow S$ les morphismes structuraux. Sous ces conditions, les faisceaux $q_{*} \omega_{C / S}$ et $\mathrm{R}^{1} q_{*}\left(\theta_{C}\right)$ sont des $G$-faisceaux pour l'action triviale de $G$, localement libres, et en dualité. Le faisceau localement libre de rang $g=g_{C}$ (genre de $C$ ) $q_{*} \omega_{C / S}$ définit un $G$-faisceau sur le champ $\overline{\mathcal{H}}_{g, n}^{\xi}$ que nous noterons dans la suite $\mathbb{E}_{\text {tot }}$ (c'est le fibré de Hurwitz-Hodge de Jarvis-Kimura [64]). Ce fibré vectoriel est exactement l'image réciproque du fibré de Hodge sur $\overline{\mathcal{M}}_{g, r}$. Le fibré de Hodge sur $\overline{\mathcal{M}}_{g^{\prime}, b}$ étant noté $\mathbb{E}_{b s}$, on veut comparer $\mathbb{E}_{\text {tot }}$ et $\delta^{*}\left(\mathbb{E}_{b s}\right)$. Soit la décomposition en facteurs isotypiques

$$
\mathbb{E}_{\mathrm{tot}}=\bigoplus_{v \in \hat{G}} \mathbb{E}_{v} \otimes V_{v}
$$

La fibre de cette décomposition au point $C \longrightarrow D$ est simplement la décomposition en facteurs irréductibles de $H^{0}\left(C, \omega_{C}\right)$. La formule de Chevalley-Weil (6) permet de préciser le rang de chaque facteur, la donnée de ramification $\xi=\sum_{i=1}^{b}\left[H_{i}, \chi_{i}\right]$ étant toujours fixée.

Proposition 10.1.4. - Considérons la décomposition du fibré de Hodge en facteurs isotypiques :

$$
\mathbb{E}_{\mathrm{tot}}=\bigoplus_{v \in \hat{G}} \mathbb{E}_{v} \otimes V_{v}
$$

On a $\mathbb{E}_{1}=\delta^{*}\left(\mathbb{E}_{b s}\right)$. Si $v \neq 1$, le rang de $\mathbb{E}_{v}$ est donné par la formule de ChevalleyWeil :

$$
r g\left(\mathbb{E}_{v}\right)=\operatorname{dim}(v)\left(g^{\prime}-1+\sum_{i}\left(1-\frac{1}{e_{i}}\right)\right)-\sum_{i} \sum_{l=1}^{e_{i}-1}\left(\frac{e_{i}-l}{e_{i}}\right)\left(v^{\vee}, \chi_{i}^{l-1}\right)_{H_{i}}
$$


Si $G$ est cyclique(1) d'ordre $n,(102)$ se spécialise en :

$$
r g\left(\mathbb{E}_{v}\right)=g^{\prime}-1+\sum_{i=1}^{b}\left\langle\frac{-v \nu_{i}}{e_{i}}\right\rangle
$$

où $\langle\cdot\rangle$ désigne la partie fractionnaire.

Démonstration. - Il suffit de se placer sur $S=\operatorname{Spec}(k)$. Si $\pi: C \longrightarrow \Sigma$ est un revêtement du type indiqué défini sur $k(|G| \neq 0 \in k)$, il suffit, pour avoir l'expression (102), d'expliciter pour tout $v$ le rang du facteur isotypique $H^{0}\left(C, \theta_{C}\right)_{v}$, ou de manière équivalente, de trouver la trace de Lefschetz (section 3.2) :

$$
L_{G}\left(\Omega_{C}\right)=\left[H^{0}\left(C, \Omega_{C}\right)\right]-\left[H^{1}\left(C, \Omega_{C}\right)\right] \in R(G) .
$$

Si $\pi_{*}\left(\Omega_{C}\right)=\bigoplus_{v \in \hat{G}} E_{v} \otimes V_{v}$, cette dernière s'exprime par $L_{G}\left(\Omega_{C}\right)=\sum_{v} \chi\left(E_{v}\right)\left[V_{v}\right] \in$ $R(G)$. Cette expression montre qu'il suffit de trouver le degré de $E_{v}$ et d'appliquer alors le théorème de Riemann-Roch comme dans loc.cit. Le degré s'obtient facilement au moyen de la suite exacte

$$
0 \longrightarrow \pi^{*}\left(E_{v}\right) \longrightarrow \Omega_{C} \otimes V_{v}^{\vee} \longrightarrow Q_{v} \longrightarrow 0
$$

où $Q_{v}$ est concentré sur les orbites de points de ramification, plus précisément $Q_{v}=$ $\bigoplus_{i} \operatorname{Ind}_{H_{i}}^{G}\left(\left(Q_{v}\right)_{P_{i}}\right)$ où $P_{i}$ est un quelconque point d'une l'orbite de type $\left[H_{i}, \chi_{i}\right]$. De cette observation, on tire facilement

$$
\operatorname{deg}\left(E_{v}\right)=\frac{\operatorname{dim}(v)\left(2 g_{C}-2\right)}{|G|}-\sum_{i=1}^{b} \sum_{l=1}^{e_{i}-1} \frac{e_{i}-l}{e_{i}}\left(v^{\vee}, \chi_{i}^{l-1}\right)_{H_{i}} .
$$

La formule de Riemann-Hurwitz donne $\frac{2 g-2}{|G|}=2 g^{\prime}-2+\sum_{i=1}^{b}\left(1-\frac{1}{e_{i}}\right)$, d'où par substitution dans la formule de Riemann-Roch :

$$
\chi\left(E_{v}\right)=\operatorname{dim}(v)\left(g^{\prime}-1+\sum_{i}\left(1-\frac{1}{e_{i}}\right)\right)-\sum_{i} \sum_{l=1}^{e_{i}-1}\left(\frac{e_{i}-l}{e_{i}}\right)\left(v^{\vee}, \chi_{i}^{l-1}\right)_{H_{i}} .
$$

Si maintenant $G$ est cyclique d'ordre $n$ engendré par $\sigma$, alors pour tout entier $0 \leqslant$ $v<n, v$ désignant la représentation de caractère $\chi_{v}(\sigma)=\zeta_{n}^{v}$, on a $\left(\chi_{v}{ }^{\vee}, \chi_{i}^{l-1}\right)_{H_{i}}=0$ sauf si $l-1$ est le reste modulo $e_{i}$ de $-v \nu_{i}$. D'où le résultat.

Pour confirmer la validité de l'expression (102), vérifions que $\sum_{v} \operatorname{dim}(v) r g\left(\mathbb{E}_{v}\right)=$ $g_{C}=g$. La somme sur les caractères irréductibles de la partie qui suit le signe moins,

(1) Par exemple si $v=n-1$, on trouve pour le rang de la composante correspondante du fibré de Hodge $g^{\prime}-1+\sum_{i=1}^{b} \frac{\nu_{i}}{e_{i}}=g^{\prime}-1+\frac{\sum_{i} m_{i} \nu_{i}}{n}$. 
donne

$$
\begin{aligned}
\sum_{i, l} \frac{e_{i}-l}{e_{i}}\left(\sum_{v}(\operatorname{dim} v) v^{\vee}, \chi_{i}^{l-1}\right)_{H_{i}} & =\sum_{i, l}\left(\chi_{\mathbb{C}[G]}, \chi_{i}^{l-1}\right)_{H_{i}} \\
& =\sum_{i, l}\left(\frac{e_{i}-l}{e_{i}}\right) \frac{|G|}{e_{i}} \\
& =\# G \sum_{i} \frac{e_{i}\left(e_{i}-1\right)}{2} .
\end{aligned}
$$

La première somme se simplifie du fait de $\sum_{v} \operatorname{dim}(v)^{2}=\# G$; les choses étant mises ensemble, la formule suit.

ExEMPLE 10.1.5. - Soit le cas $n=3$, les revêtements sont alors des revêtements triples galoisiens de genre $g$ de $\mathbb{P}^{1}$. Dans ce cas une donnée de ramification équivaut à une partition des points de branchement $[1, g+2]=\Lambda_{1} \sqcup \Lambda_{2}$, avec $\left|\Lambda_{1}\right| \equiv\left|\Lambda_{2}\right|$ $(\bmod 3)$. Le fibré de Hodge est une somme directe de deux facteurs $\mathbb{E}=\mathbb{E}_{1} \oplus \mathbb{E}_{2}$ où

$$
r g\left(\mathbb{E}_{1}\right)=\frac{\left|\Lambda_{1}\right|+2\left|\Lambda_{2}\right|}{3}-1, r g\left(\mathbb{E}_{2}\right)=\frac{2\left|\Lambda_{1}\right|+\left|\Lambda_{2}\right|}{3}-1 .
$$

Il y a une construction duale des facteurs isotypiques $\mathbb{E}_{v}$. Soit un revêtement galoisien stable $\pi: C \longrightarrow D$ de groupe $G$ au-dessus de la base $S$. Le faisceau $\pi_{*}\left(\theta_{C}\right)$ est un $\left(\theta_{D}, G\right)$-module sans torsion de rang $|G|$, plat sur $S$ et de formation compatible à tout changement de base. Il se décompose donc en facteurs isotypiques $\pi_{*}\left(\theta_{C}\right)=\bigoplus_{v \in \hat{G}} \mathscr{L}_{v} \otimes V_{v}$. Il est immédiat de voir que $\mathscr{L}_{1}=\pi_{*}\left(\theta_{C}\right)^{G}=\theta_{D}$, et que le rang de $\mathscr{L}_{v}$ est égal à $\operatorname{dim}(v)$. Le $\theta_{D}$-module $\mathscr{L}_{v}$ est un module plat et sans torsion relativement à $S$.

Proposition 10.1.6. - On a $p_{*}\left(\Theta_{C}\right)=\Theta_{S}$, et si $v \neq 1$, on a $p_{*}\left(\mathscr{L}_{v}\right)=0$ et

$$
\mathbb{E}_{v^{\vee}} \cong R^{1} p_{*}\left(\mathscr{L}_{v}\right)^{\vee} \cong R^{1} q_{*}\left(e v^{*}([v])\right.
$$

Démonstration. - Tout est clair, sauf peut-être le dernier point. Notons que, par dualité de Grothendieck-Serre, on a un isomorphisme canonique $q_{*}\left(\omega_{C / S}\right) \cong$ $R^{1} q_{*}\left(\theta_{C}\right)^{\vee}$ qui est un $G$-isomorphisme. Par ailleurs

$$
R^{1} q_{*}\left(\theta_{C}\right)=R^{1} p_{*} \pi_{*}\left(\theta_{C}\right)=\bigoplus_{v \in \hat{G}} R^{1} p_{*}\left(\mathscr{L}_{v} \otimes V_{v}\right)=\bigoplus_{v \in \hat{G}} R^{1} p_{*}\left(\mathscr{L}_{v}\right) \otimes V_{v} .
$$

Par identification des facteurs isotypiques, on obtient le résultat. Le dernier point résulte de la définition de l'évaluation (50). 
On peut moduler cette construction en se reportant à la section 6.5.2, en particulier en considérant le carré 2-commutatif



Une section sur $S$ du champ $\bar{D}$ est un revêtement $\pi: C \longrightarrow D$ de base $S$, muni d'une

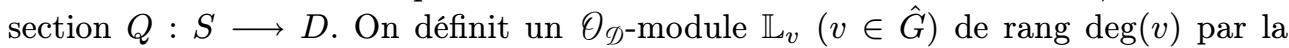
prescription suivante :

$$
\mathbb{L}_{v}(\pi)=Q^{*}\left(\mathscr{L}_{v}\right) .
$$

Invoquant le morphisme $\psi: \overline{\mathscr{C}}_{g, G, \xi} \longrightarrow \overline{\mathscr{D}}=\overline{\mathscr{H}}_{g, G, \xi} \times \overline{\mathcal{M}}_{g^{\prime}, b} \overline{\mathscr{C}}_{g^{\prime}, b}$ (proposition 6.5.4), on voit que $\psi_{*}\left(\theta_{\bar{C}_{g, r}}\right)=\bigoplus_{v \in \operatorname{Irrep}(G)} \mathbb{L}_{v} \otimes V_{v}$. Avec cette définition, il est aisé de voir que $\mathbb{E}^{\vee}{ }_{v^{\vee}}=R^{1} \pi_{*}\left(\mathbb{L}_{v}\right)=-\pi_{!}\left(\mathbb{L}_{v}\right)$. Sous ces hypothèses, posons pour tout $v \in \hat{G}$ :

$$
\lambda_{v}=\operatorname{det}\left(\mathbb{E}_{v}\right) \in \operatorname{Pic}\left(\overline{\mathscr{H}}_{g, G, \xi}\right) .
$$

On notera par la même lettre la première classe de Chern de $\mathbb{E}_{v}$. Avec ces notations, on a $\lambda_{1}=\delta^{*}\left(\lambda_{b s}\right)$ et $\lambda=\lambda_{\text {tot }}=\bigotimes_{v \in \hat{G}} \lambda_{v}^{\otimes \operatorname{deg}(v)}$.

REMARQUE 10.1.7. - Mumford a fait remarquer que le fibré $\mathbb{E}$ possède une propriété spécifique du fait de la dégénérescence de la suite spectrale de Hodge pour une courbe préstable $\pi: C \longrightarrow D$, en fait la $G$-courbe universelle dans le cadre qui nous occupe. Soit $V=\mathrm{R}^{1} \pi_{*}\left(\Omega_{C / D}\right)$ le premier faisceau d'hypercohomologie du complexe de De Rham relatif $\Omega^{\bullet}{ }_{C / D}=\theta_{C} \stackrel{d}{\longrightarrow} \omega_{C / D}$. On a la suite exacte (voir [80], [17]) :

$$
0 \longrightarrow \mathbb{E} \longrightarrow V \longrightarrow \mathbb{E}^{\vee} \longrightarrow 0 \text {. }
$$

Il est clair que dans la situation équivariante présente, le groupe $G$ agit sur les trois termes de cette extension. Pour tout caractère irréductible $v \in \hat{G}$, il vient donc une extension

$$
0 \longrightarrow \mathbb{E}_{v} \longrightarrow V_{v} \longrightarrow\left(\mathbb{E}^{\vee}\right)_{v} \longrightarrow 0
$$

avec $\left(\mathbb{E}^{\vee}\right)_{v}=\left(\mathbb{E}_{v^{\vee}}\right)^{\vee}$. Cette suite exacte implique, comme dans le cas $G=1$, des relations entre les classes de Chern des $\mathbb{E}_{v}([\mathbf{8 0}])$, que nous préciserons dans une section suivante.

Rappelons la définition des importantes classes $\psi$. On définit un faisceau inversible noté $\mathscr{L}_{i} \in \operatorname{Pic}\left(\overline{\mathcal{M}}_{g, n}\right)$ par

$$
\mathscr{L}_{i}(q: D \longrightarrow S)=\sigma_{i}^{*}\left(\omega_{D / S}\right)
$$

en notant $\sigma_{i}$ la $i$-ème section. Pour éviter toute confusion avec les faisceaux introduits ci-dessus, on notera par une même lettre $\psi_{i}$, le faisceau inversible $\mathscr{L}_{i}$, et sa première classe de Chern $c_{1}\left(\mathscr{L}_{i}\right)$. La même lettre désignera aussi pour simplifier à la fois $\psi_{i} \in \operatorname{Pic}\left(\overline{\mathcal{M}}_{g^{\prime}, b}\right)$, ainsi que son image réciproque $\delta^{*}\left(\psi_{i}\right)$ dans la groupe $\operatorname{Pic}\left(\overline{\mathcal{H}}_{g, G, \xi}\right)$. Rappelons que la structure du groupe $\operatorname{Pic}\left(\overline{\mathcal{M}}_{g, n}\right)\left(\operatorname{resp} . \operatorname{Pic}\left(\mathcal{M}_{g, n}\right)\right)$ est connue, voir 
Harris-Morrison [55], Manin [76]. Si $g \neq 1,2$, ces groupes sont libres de rang fini(2). Considérons le morphisme de groupes

$$
\delta^{*}: \operatorname{Pic}\left(\overline{\mathcal{H}}_{g, G, \xi}\right) \longrightarrow \operatorname{Pic}\left(\overline{\mathcal{M}}_{g^{\prime}, b}\right) .
$$

Si le groupe de droite est libre (de rang fini), par exemple si $g^{\prime}=0$, il n'est pas difficile de montrer que ce morphisme est injectif (voir par exemple Jarvis [59]). Naturellement, le groupe $\operatorname{Pic}\left(\overline{\mathcal{H}}_{g, G, \xi}\right)$ n'est discret que dans des cas exceptionnels, essentiellement $g^{\prime}=0$ et $G$ abélien. Dans ce dernier cas, compte tenu du fait général qui dit que le groupe de Picard d'un champ de Deligne-Mumford séparé ne diffère du groupe de Picard de son espace de modules grossier que par la torsion, on obtient $\operatorname{Pic}\left(\overline{\mathscr{H}}_{g, G, \xi}\right) \otimes \mathbb{Q} \cong \operatorname{Pic}\left(\bar{H}_{g, G, \xi}\right) \otimes \mathbb{Q}$, de sorte que

$$
\operatorname{Pic}\left(\overline{\mathcal{H}}_{g, G, \xi}\right) \otimes \mathbb{Q} \cong \operatorname{Pic}\left(\overline{\mathcal{M}}_{0, b}\right) \otimes \mathbb{Q} \text {. }
$$

La torsion, qui fait la différence entre $\operatorname{Pic}\left(\overline{\mathcal{H}}_{g, G, \xi}\right)$ et $\operatorname{Pic}\left(\overline{\mathcal{M}}_{0, b}\right)$, est non triviale en général. Ces groupes ont un même rang égal à $2^{b-1}-\left(\begin{array}{l}b \\ 2\end{array}\right)-1$, voir [76]. Dans ce cas, la partie de torsion de $\operatorname{Pic}\left(\overline{\mathcal{H}}_{g, G, \xi}\right)$ contient des informations intéressantes (voir Arsie-Vistoli [5]).

\subsection{Les fibrés en droites $\psi_{i, \chi}$ et $\mu_{i, v}$}

On va décrire de manière exhaustive une collection de fibrés tautologiques qui dérivent des fibres au-dessus de $Q_{1}, \ldots, Q_{b}$, les points marqués de la base. Du fait de leur construction, ces fibrés contiennent beaucoup d'information sur la ramification. Rappelons que les points de branchement véritables sont inclus dans les $Q_{i}$. Notons $\sigma_{i}: S \longrightarrow D$ le morphisme définissant $Q_{i}$. Il est clair que le $\left(\theta_{S}, G\right)$-faisceau $\theta_{\pi^{-1}\left(Q_{i}\right)}$ est localement libre de rang $|G|$. Tenant compte de la décomposition en facteurs isotypiques $\vartheta_{\pi^{-1}\left(Q_{i}\right)} \cong \bigoplus_{v \in \widehat{G}} \sigma_{i}^{*}\left(\mathscr{L}_{v}\right) \otimes V_{v}$, on pose

$$
\mathscr{L}_{i, v}(\pi)=\sigma_{i}^{*}\left(\mathscr{L}_{v}\right)
$$

Cette construction définit un fibré vectoriel $\mathscr{L}_{i, v}$ sur $\overline{\mathcal{H}}_{g, G, \xi} \operatorname{de} \operatorname{rang} \operatorname{deg}(v)$. Si $Q_{i}$ est un point de branchement d'holonomie $\left[H_{i}, \chi_{i}\right]$, il est possible de dévisser les fibrés $\mathscr{L}_{i, v}$ en somme directe de sous-fibrés portant une part de l'information sur la ramification. Fixons un représentant $\left(H_{i}, \chi_{i}\right)$ de l'holonomie en le point de branchement d'indice $i$. Soit alors le diviseur de Cartier relatif $\Delta_{i}$, fini et étale sur $S$, lieu des points de la fibre $\pi^{-1}\left(Q_{i}\right)$ d'holonomie exactement $\left(H_{i}, \chi_{i}\right)$. Il est clair que $\Delta_{i} \longrightarrow S$ est un torseur de groupe $C_{G}\left(H_{i}\right) / H_{i}$, le diviseur $\pi^{-1}\left(Q_{i}\right)$ ayant pour expression

$$
\pi^{-1}\left(Q_{i}\right)=e_{i}\left(\operatorname{Ind}_{C_{G}\left(H_{i}\right)}^{G} \Delta_{i}\right) \quad\left(e_{i}=\left|H_{i}\right|\right) .
$$

(2) Si $g \geqslant 3$, les faisceaux inversibles $\lambda$, et $\psi_{i}(1 \leqslant i \leqslant n)$ forment une base $\operatorname{de} \operatorname{Pic}\left(\mathcal{M}_{g, n}\right)$; si on ajoute à ces faisceaux inversibles les faisceaux $\delta$ associés aux composantes du bord, on obtient une base de $\operatorname{Pic}\left(\overline{\mathcal{M}}_{g, n}\right)$, voir loc. cit. 
En particulier $\theta_{\pi^{-1}\left(Q_{i}\right)}=\operatorname{Ind}_{C_{G}\left(H_{i}\right)}^{G}\left(\theta_{e_{i} \Delta_{i}}\right)$. On est ainsi ramené à préciser la structure de $\left(\theta_{S}, G\right)$-module de $\operatorname{Ind}_{C_{G}\left(H_{i}\right)}^{G}\left(\theta_{e_{i} \Delta_{i}}\right)$, c'est-à-dire la structure du $\left(\theta_{S}, C_{G}\left(H_{i}\right)\right)$-module $\theta_{e_{i} \Delta_{i}}$. Pour $0 \leqslant k<e_{i}$, soit la suite exacte de $\left(\vartheta_{S}, C_{G}\left(H_{i}\right)\right)$-modules

$$
0 \longrightarrow \theta_{\Delta_{i}}\left(-k \Delta_{i}\right) \longrightarrow \theta_{(k+1) \Delta_{i}} \longrightarrow \vartheta_{k \Delta_{i}} \longrightarrow 0 .
$$

Soit $\chi$ un caractère irréductible de $C_{G}\left(H_{i}\right)$ avec $\rho_{\chi}: C_{G}\left(H_{i}\right) \longrightarrow \mathrm{GL}\left(W_{\chi}\right)$ la représentation associée. Si $h \in H_{i}$, du fait que $H_{i}$ est dans le centre de $C_{G}\left(H_{i}\right)$, la restriction de $\rho_{\chi}(h)$ à $H_{i}$ est une homothétie $\rho_{\chi}(h)=\chi_{i}^{j}(h)$, pour un certain $j \in\left[0, e_{i}-1\right.$ [. Nous parlerons de $\chi_{i}^{j}$ comme du poids de la restriction de $\chi$ à $H_{i}$. Le groupe $H_{i}$ agit sur le faisceau conormal $\theta_{\Delta_{i}}\left(-\Delta_{i}\right)$ au moyen du caractère $\chi_{i}$. Il est immédiat de déduire de la suite exacte (107) que les deux faisceaux localement libres $\theta_{\Delta_{i}}\left(-k \Delta_{i}\right)$ et $\theta_{k \Delta_{i}}$ sont disjoints, et donc si on les voit comme $\left(\theta_{S}, C_{G}\left(H_{i}\right)\right)$-modules on a :

$$
\theta_{(k+1) \Delta_{i}} \cong \theta_{\Delta_{i}}\left(-k \Delta_{i}\right) \oplus \theta_{k \Delta_{i}} .
$$

L'action de $H_{i}$ sur $\vartheta_{\Delta_{i}}\left(-k \Delta_{i}\right)$ est donnée par la caractère $\chi_{i}^{k}$, il en résulte que toute fibre de $\theta_{\Delta_{i}}\left(-k \Delta_{i}\right)$ s'identifie à la représentation $\operatorname{Ind}_{H_{i}}^{C_{G}\left(H_{i}\right)}\left(\chi_{i}^{k}\right)$. Il s'ensuit la décomposition

$$
\vartheta_{\Delta_{i}}\left(-k \Delta_{i}\right)=\bigoplus_{\chi \in \widehat{C_{G}\left(H_{i}\right)}, \chi_{\mid H_{i}}=\chi_{i}{ }^{k}} E_{i, \chi} \otimes W_{\chi},
$$

la somme portant sur les caractères irréductibles de $C_{G}\left(H_{i}\right)$ de restriction $\chi_{i}^{k}$ à $H_{i}$. En mettant bout à bout les décompositions (108), on en déduit aisément la décomposition

$$
\vartheta_{k \Delta_{i}}=\bigoplus_{\chi \in \widehat{C_{G}\left(H_{i}\right)}} E_{i, \chi} \otimes W_{\chi}
$$

où maintenant la somme directe porte sur les caractères irréductibles de $C_{G}\left(H_{i}\right)$ dont la restriction à $H_{i}$ est le caractère $\chi_{i}{ }^{\alpha}$, avec $0 \leqslant \alpha \leqslant k-1$. Finalement, on a la décomposition

$$
\vartheta_{e_{i} \Delta_{i}}=\bigoplus_{k=0}^{e_{i}-1} \theta_{\Delta_{i}}\left(-k \Delta_{i}\right)=\bigoplus_{\chi \in \widehat{C_{G}\left(H_{i}\right)}} E_{i, \chi} \otimes W_{\chi} .
$$

Cette construction définit pour tout $\chi \in \widehat{C_{G}\left(H_{i}\right)}$ un fibré $E_{i, \chi}$ de $\operatorname{rang} \operatorname{deg}(\chi)$. Explicitons les relations qui relient les fibrés $\mathscr{L}_{i, v}$ et les $E_{j, \chi}$.

Proposition 10.2.1. - Avec les notations précédentes, le faisceau $E_{i, \chi}$ est un fibré vectoriel de rang $\operatorname{deg}(\chi)\left(\chi \in \widehat{C_{G}\left(H_{i}\right)}\right)$. Le fibré vectoriel $\mathcal{L}_{i, v}(106)$, qui a pour rang $\operatorname{deg} v$, se décompose en

$$
\mathscr{L}_{i, v}=\bigoplus_{\chi \in \widehat{C_{G}\left(H_{i}\right)}} E_{i, \chi}^{n_{v, \chi}} \text { où } n_{v, \chi}=\left(\chi,\left.v\right|_{C_{G}\left(H_{i}\right)}\right) .
$$


Démonstration. - Dans l'anneau des représentations de $G$, soit la décomposition en facteurs irréductibles

$$
\operatorname{Ind}_{C_{G}\left(H_{i}\right)}^{G}\left(W_{\chi}\right)=\bigoplus_{v \in \hat{G}} V_{v}^{n_{v, \chi}}
$$

avec $n_{v, \chi}=\left\langle\operatorname{Ind}_{C_{G}\left(H_{i}\right)}^{G}\left(W_{\chi}\right), V_{v}\right\rangle_{\mid G}=\left\langle W_{\chi}, V_{v \mid C_{G}\left(H_{i}\right)}\right\rangle_{\mid C_{G}\left(H_{i}\right)}$. Par insertion de cette relation dans (109), et induction, en regroupant les termes on obtient exactement (110).

Les déterminants respectifs $\operatorname{det}\left(\mathscr{L}_{i, v}\right)$ et $\operatorname{det}\left(E_{i, \chi}\right)$ seront notés $\mu_{i, v}$ et $\psi_{i, \chi}$, ceci pour tous les $v \in \hat{G}, \chi \in \widehat{C_{G}\left(H_{i}\right)}$. On notera que si $\chi$ est un caractère de $C_{G}\left(H_{i}\right) / H_{i}$, on a $\mathscr{L}_{i, v}=e v_{Q_{i}}^{*}(v)$.

Dans la suite, on notera par la même lettre un faisceau inversible et sa première classe de Chern, par exemple $\mu_{i, v}$ et $\psi_{i, \chi}$. On remarquera que les fibrés vectoriels donnés par la décomposition $\Theta_{\Delta_{i}}\left(-\Delta_{i}\right)=\bigoplus_{\left.\chi \in \widehat{C_{G}\left(H_{i}\right.}\right), \chi_{\mid H_{i}}=\chi_{i}} E_{i, \chi} \otimes W_{\chi}$ se substituent aux images réciproques des $\mathscr{L}_{P} \in \operatorname{Pic}\left(\overline{\mathcal{M}}_{g, r}\right)$, qui ne sont pas définies car les points de ramification ne sont pas numérotés ${ }^{(3)}$. Cependant, la relation

$$
\bigoplus_{P \in \Delta_{i}} \mathscr{L}_{P}=\underset{\chi \in \widehat{C_{G}\left(H_{i}\right)}, \chi_{\mid H_{i}}=\chi_{i}}{\bigoplus} E_{i, \chi} \otimes W_{\chi}
$$

a un sens.

\subsection{Relations de Riemann-Hurwitz d'ordre supérieur}

10.3.1. Calculs dans l'anneau de Chow. - Avant d'effectuer des calculs dans l'anneau de Chow du champ de Hurwitz, fixons quelques conventions. Si $\mathcal{M}$ est un champ de Deligne-Mumford de dimension pure $n=\operatorname{dim} \mathcal{M}$, on notera $A^{\bullet}(\mathcal{M})$ l'anneau de Chow de $\mathcal{M}$ à coefficients rationnels. On retient les notations de Mumford [80], voir aussi Vistoli [97], en particulier l'identification $A^{k}(\mathcal{M})=A_{n-k}(\mathcal{M})$ (loc. cit.). Si $Z$ est un sous-champ fermé intègre de $\mathcal{M}$ de dimension $k$, on notera $[Z] \in A_{k}(\mathcal{M})$ le cycle correspondant, et $[Z]_{Q}$ la classe fondamentale de $Z$. Si $e(Z)$ est l'ordre du groupe des automorphismes d'un point générique de $Z$, alors [80],

$$
[Z]_{Q}=e(Z)^{-1}[Z] \text {. }
$$

L'identité de $A^{\bullet}(\mathcal{M})$ est $[\mathcal{M}]_{Q}$. Si $M$ est l'espace des modules grossiers, le morphisme $\mathcal{M} M$ induit un isomorphisme $A^{\bullet}\left(\mathcal{M} \stackrel{\sim}{\longrightarrow} A^{\bullet}(M)\right.$ [97]. L'exemple fondamental pour ce qui suit est $\mathcal{M}=\overline{\mathcal{M}}_{g, n}$. L'anneau $A^{\bullet}(\mathcal{M})$ contient une batterie de classes tautologiques, dont nous rappelons la définition ainsi que les relations fondamentales les concernant.

Soit la courbe universelle

$$
q: \overline{\mathscr{C}}_{g, n}=\bar{M}_{g, n+1} \longrightarrow \bar{M}_{g, n} .
$$

(3) Dans [64], un $G$-revêtement $\pi: C \longrightarrow D$ est marqué par des points $P_{i} \in \pi^{-1}\left(Q_{i}\right)$ 
Le morphisme $q$ est le morphisme noté usuellement $\pi_{n+1}$, oubli du $n+1$-ième point. Posons $K=c_{1}\left(\omega_{q}\right)$ et soit $D_{i}$ (ou $D_{i, n+1}$ ) l'image de la section universelle d'indice $i$ $(1 \leqslant i \leqslant n)$. Outre les classes kappa de Mumford-Morita-Miller [80], il est commode d'utiliser les classes $\tilde{\kappa}_{a}=q_{*}\left(K^{a+1}\right) \in A^{\bullet}\left(\overline{\mathcal{M}}_{g, n}\right)$ de Mumford, et les classes $\kappa_{a}$ modifiées de Arbarello-Cornalba [4]. Ces dernières sont définies par

$$
\kappa_{a}=q_{*}\left(c_{1}\left(\omega_{q}\left(\sum D_{i}\right)\right)^{a+1}\right) .
$$

Ces classes vérifient des relations importantes que nous rappelons. Les classes $\kappa_{a}$ et $\tilde{\kappa}_{a}$ sont reliées (voir [4]) par

$$
\kappa_{a}=\tilde{\kappa}_{a}+\sum_{i=1}^{n} \psi_{i}^{a} .
$$

Elles satisfont, relativement au morphisme $\pi_{n}: \overline{\mathcal{M}}_{g, n} \longrightarrow \overline{\mathcal{M}}_{g, n-1}$ d'oubli du point marqué d'indice $n$, à la relation simple

$$
\kappa_{l}=\pi_{n}^{*}\left(\kappa_{l}\right)+\psi_{n}^{l} .
$$

Rappelons que $D_{i, n+1}$ est le diviseur dont les points sont obtenus en collant une «bulle», i.e. un $\mathbb{P}^{1}$ marqué par $\{0,1, \infty\}$ au point d'indice $i$, soit $i=\infty$. Les classes $\psi_{i}$ satisfont, relativement à $\pi_{n+1}$, à la relation importante (lemme de comparaison) :

$$
\psi_{i}=\pi_{n+1}^{*}\left(\psi_{i}\right)+D_{i, n+1}(1 \leqslant i \leqslant n) .
$$

Revenons au champ $\overline{\mathscr{H}}_{g, G, \xi}$, source du revêtement universel $q: \overline{\mathscr{C}}_{g, G, \xi} \longrightarrow \overline{\mathscr{H}}_{g, G, \xi}$, et à $\omega=\omega_{q}$. On définit des classes $\kappa_{l}$ (resp. $\left.\tilde{\kappa}_{l}\right)$ dans $A^{l}\left(\overline{\mathcal{H}}_{g, G, \xi}\right) \otimes \mathbb{Q}$. Pour les distinguer des précédentes, on notera $\kappa_{l}^{\prime}$ (resp. $\left.\tilde{\kappa}_{l}^{\prime}\right)$ les classes dans $A^{l}\left(\overline{\mathcal{M}}_{g^{\prime}, b}\right) \otimes \mathbb{Q}$ de même nom qui vivent sur la base, c'est-à-dire dans $A^{l}\left(\overline{\mathcal{M}}_{g^{\prime}, b}\right)$. Du fait du carré cartésien (48), on voit que les classes $\kappa_{l}$ et $\tilde{\kappa}_{l}$ proviennent des classes de même nom de $\bar{M}_{g,(r)}$. Le morphisme $\imath: \overline{\mathcal{H}}_{g, G, \xi} \longrightarrow \bar{M}_{g,(r)}$ étant une immersion locale régulière (proposition 6.5 .2 ), on notera que le morphisme de Gysin $\imath^{!}$est défini au niveau des cycles. Au niveau de la première classe de Chern, et pour prendre en compte les éléments de torsion du groupe de Picard, il est utile d'utiliser le formalisme du produit d'intersection de Deligne (voir Deligne [29], Beilinson-Manin [10], Jarvis [59]).

Rappelons que cette construction associe à une courbe préstable $q: C \longrightarrow S$, et à deux faisceaux inversibles $\mathcal{L}, \mathcal{M}$ sur $C$, un faisceau inversible $\langle\mathcal{L}, \mathcal{M}\rangle \in \operatorname{Pic}(S)$. Cette construction est bi-fonctorielle pour les isomorphismes entre faisceaux inversibles, symétrique au sens où $\langle\mathcal{L}, \mathcal{M}\rangle=\langle\mathcal{M}, \mathscr{L}\rangle$, et bi-multiplicative au sens suivant :

$$
\left\langle\mathscr{L}_{1} \otimes \mathscr{L}_{2}, \mathcal{M}\right\rangle \simeq\left\langle\mathscr{L}_{1}, \mathcal{M}\right\rangle \otimes\left\langle\mathscr{L}_{2}, \mathcal{M}\right\rangle \quad, \quad\left\langle\mathscr{L}, \mathcal{M}^{-1}\right\rangle \simeq\langle\mathscr{L}, \mathcal{M}\rangle^{-1} .
$$

La formation de ces faisceaux et isomorphismes commute aux changements de base arbitraires. Il est connu ([10], [29]) qu'on a un isomorphisme canonique comme suit :

Proposition 10.3.1. - On a

$$
\langle\mathscr{L}, \mathcal{M}\rangle=\operatorname{det} R q_{*}(\mathscr{L} \otimes \mathcal{M}) \otimes\left(\operatorname{det} R q_{*} \mathscr{L}\right)^{-1} \otimes\left(\operatorname{det} R q_{*} \mathcal{M}\right)^{-1} \otimes \operatorname{det} R q_{*} \theta_{C} .
$$


Noter que le déterminant de la cohomologie $\operatorname{det} R q_{*}(\cdot)$ dans (112) se réduit à $\operatorname{det} R^{0} q_{*}(\cdot) \otimes\left(\operatorname{det} R^{1} q_{*}(\cdot)\right)^{-1}$, voir [69]. Si $\omega=\omega_{C / S}$ est le faisceau dualisant relatif, l'identité suivante (en notation additive) est satisfaite (théorème de Deligne-RiemannRoch ([29], [59]) :

$$
2 \operatorname{det} R q_{*} \mathscr{L}=\langle\mathscr{L}, \mathscr{L}\rangle-\langle\mathscr{L}, \omega\rangle+2 \operatorname{det} R q_{*} \omega
$$

Si $D$ est un diviseur de Cartier relatif et $\mathcal{M}=\theta_{C}(D)$, de la définition et de la suite exacte $0 \longrightarrow \theta(-D) \longrightarrow \vartheta \longrightarrow \theta_{D} \longrightarrow 0$ découle immédiatement la relation (voir [10]) :

$$
\langle\mathcal{L}, \mathcal{M}\rangle=\operatorname{det}_{\vartheta_{S}}\left(q_{*}\left(\mathscr{L} \otimes \theta_{D}\right)\right) \otimes\left(\operatorname{det}_{\vartheta_{S}} q_{*}\left(\theta_{D}\right)\right)^{-1}
$$

Dans ce contexte, la classe $\kappa=\kappa_{1}$ est représentée par le faisceau inversible défini par le produit d'intersection $\kappa_{1}=\langle\omega, \omega\rangle$. Débutons par une remarque préliminaire.

LEMme 10.3.2. - Soit $q: C \stackrel{\pi}{\longrightarrow} D \stackrel{p}{\longrightarrow} S$ un revêtement de degré $n$ entre $S$-courbes lisses. Pour tout faisceau inversible $\mathcal{L} \in \operatorname{Pic}(D)$, on a

$$
\left\langle\pi^{*}(\mathscr{L}), \pi^{*}(\mathscr{L})\right\rangle=n\langle\mathscr{L}, \mathscr{L}\rangle .
$$

Démonstration. - Du fait que $\pi$ est fini, on a $R q_{*}(\cdot)=R p_{*} \pi_{*}(\cdot)$. Par suite, pour tout faisceau inversible $\mathcal{M}$ sur $D$, on a $R q_{*}\left(\pi^{*}(\mathcal{M})=R p_{*}\left(\mathcal{M} \otimes \pi_{*}\left(\theta_{C}\right)\right)\right.$. Les hypothèses de lissité assurent que $\pi$ est plat, de sorte que $E=\pi_{*}\left(\theta_{C}\right)$ est localement libre de rang $n$. Le problème se réduit à vérifier qu'il y a pour tout fibré vectoriel $E$ de rang $n$, et tous faisceaux inversibles $\mathcal{L}, \mathcal{M}$ sur $D$, un isomorphisme canonique :

$n\langle\mathcal{L}, \mathcal{M}\rangle=\operatorname{det} R p_{*}(\mathcal{L} \otimes \mathcal{M} \otimes E)-\operatorname{det} R p_{*}(\mathscr{L} \otimes E)-\operatorname{det} R p_{*}(\mathcal{M} \otimes E)+\operatorname{det} R p_{*}(E)$.

En suivant les arguments de ([29], 9.5), on peut se ramener étale-localement au cas où $E$ admet une filtration par des sous-fibrés à quotients successifs de rang 1 . Supposons que $E$ admette une filtration $0=E_{0} \subset E_{1} \subset \cdots \subset E_{n}=E$, où $E_{j}$ est localement libre de rang $j$, et $\mathscr{L}_{j}=E_{j} / E_{j-1}$ est localement libre de rang 1 . On obtient, en notation additive :

$$
\begin{aligned}
\left\langle\pi^{*}(\mathscr{L}), \pi^{*}(\mathcal{M})\right\rangle= & \sum_{j} \operatorname{det} R p_{*}\left(\mathscr{L} \otimes \mathcal{M} \otimes \mathscr{L}_{j}\right)-\sum_{j} \operatorname{det} R p_{*}\left(\mathscr{L} \otimes \mathscr{L}_{j}\right) \\
& -\sum_{j} \operatorname{det} R p_{*}\left(\mathcal{M} \otimes \mathscr{L}_{j}\right)+\sum_{j} \operatorname{det} R p_{*}\left(\mathscr{L}_{j}\right) .
\end{aligned}
$$

Compte tenu du fait que les $\mathscr{L}_{j}$ sont de rang 1 , ceci peut s'écrire $\sum_{j}\langle\mathcal{L} \otimes$ $\left.\mathscr{L}_{j}, \mathcal{M}\right\rangle-\sum_{j}\left\langle\mathcal{M}, \mathscr{L}_{j}\right\rangle$. La conclusion découle dans ce cas de la bilinéarité du produit d'intersection. 
10.3.2. Relations de Riemann-Hurwitz d'ordre supérieur. - Soit de nouveau la $G$-courbe universelle $\pi: \overline{\mathscr{C}}_{g, G, \xi} \longrightarrow \overline{\mathscr{H}}_{g, G, \xi}$ (section 6.5.2). Elle s'insère dans un carré 2-commutatif

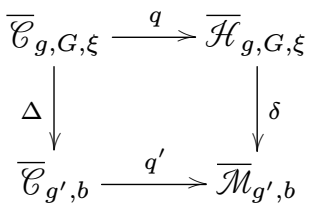

Notons $\omega=\omega_{\overline{\mathscr{C}}_{g, G, \xi} / \overline{\mathscr{H}}_{g, G, \xi}} \in \operatorname{Pic}\left(\overline{\mathscr{C}}_{g, G, \xi}\right)$ le faisceau inversible dualisant relatif du morphisme $q$. Ce faisceau peut être vu sur un atlas. Soit

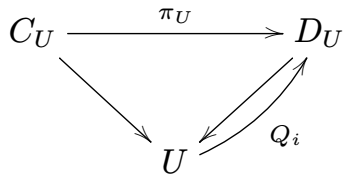

un $G$-revêtement de base $U$ définissant un atlas de $\overline{\mathcal{H}}_{g, G, \xi}$. Le revêtement obtenu par changement de base $p_{2}: \tilde{C}_{U}=C_{U} \times_{U} C_{U} \longrightarrow C_{U}$, équipé de la section diagonale (il n'est pas nécessaire de stabiliser), fait de $C_{U}$ un atlas de $\mathscr{C}_{g, G, \xi}$. L'incarnation de $\omega$ sur l'atlas $C_{U} \longrightarrow \overline{\mathscr{C}}_{g, G, \xi}$ est $\omega_{C_{U} / U}$. En notant $\omega^{\prime}$ le faisceau $\omega_{\bar{C}_{g^{\prime}, b}} / \bar{M}_{g^{\prime}, b}$, on a $\Delta^{*}\left(\omega^{\prime}\right)\left(C_{U}\right)=\pi_{U}^{*}\left(\omega_{D_{U} / U}\right)$.

Pour tout indice $i$ avec $1 \leqslant i \leqslant b$, soit $R_{i} \subset \overline{\mathscr{C}}_{g, G, \xi}$ la partie du diviseur de ramification au dessus de la section $Q_{i}$. La réalisation de $R_{i}$ au niveau de l'atlas $C_{U} \longrightarrow \overline{\mathscr{C}}_{g, G, \xi}$ est claire, c'est le diviseur de ramification de même nom dans $C_{U}$. Rappelons que ce dernier est étale de degré $\left[G: H_{i}\right]$ sur $C_{U}$, de sorte que $R_{i}$ est étale de degré $\left[G: H_{i}\right]$ sur $\overline{\mathcal{H}}_{g, G, \xi}$. Le diviseur de ramification complet $\mathfrak{R} \subset \overline{\mathscr{C}}_{g, G, \xi}$ a pour expression

$$
\mathfrak{R}=\sum_{i}\left(e_{i}-1\right) R_{i}
$$

Notons $B_{i} \subset \overline{\mathscr{C}}_{g^{\prime}, b}$ le diviseur image de la section $Q_{i}$. La formule de ramification classique, une fois transcrite au niveau des champs, a pour forme :

Proposition 10.3.3. - i) Pour tout $1 \leqslant i \leqslant b$, on a l'égalité de diviseurs de Cartier $\Delta^{*}\left(B_{i}\right)=e_{i} R_{i}$.

ii) Dans le groupe de Picard de $\overline{\mathscr{C}}_{g, G, \xi}$, on a la formule de ramification :

$$
\omega=\Delta^{*}\left(\omega^{\prime}\right) \otimes\left(\otimes_{i=1}^{b} \theta\left(R_{i}\right)^{\otimes e_{i}-1}\right) .
$$

Démonstration. - Pour le premier point, notons le diagramme commutatif

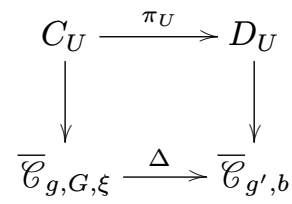


dans lequel les flèches verticales sont représentées respectivement par $p_{2}: \tilde{C}_{U} \longrightarrow C_{U}$ et $D_{U} \times_{U} D_{U} \longrightarrow D_{U}$ muni de la section diagonale. Pour identifier $\Delta^{*}\left(B_{i}\right)$, il suffit d'examiner l'image réciproque dans $C_{U}$, qui est $\pi_{U}^{*}\left(B_{i}\right)=e_{i} R_{i}$.

ii) Le faisceau $\Delta^{*}\left(\omega^{\prime}\right)$ a pour description relativement à l'atlas $C_{U}$ l'image réciproque $\pi_{U}^{*}\left(\omega_{D_{U} / U}\right)$. De la sorte, la relation (113) n'est que la simple traduction de la formule de ramification ordinaire appliquée à $\pi_{U}: C_{U} \longrightarrow D_{U}$, jointe à la description du diviseur de ramification (Proposition 6.6.13).

Soit $\tau_{i}: R_{i} \hookrightarrow \overline{\mathscr{C}}_{g, G, \xi}$ l'inclusion. On peut énoncer le résultat principal de cette section, un analogue supérieur de la formule de Riemann-Hurwitz, relation qui exhibe une propriété remarquable ${ }^{(4)}$ des classes kappa ${ }^{(5)}$. On rappelle que les coefficients dans l'anneau de Chow sont rationnels. Noter que les classes $\kappa_{l}$ sont définies au niveau de $\overline{\mathcal{H}}_{g, G, \xi}$, le marquage étant assuré par le diviseur de ramification.

ThÉorème 10.3.4. - Pour tout $l \geqslant 0$, on a la relation de Riemann-Hurwitz dans $A^{l}\left(\overline{\mathscr{H}}_{g, G, \xi}\right):$

$$
\tilde{\kappa}_{l}=|G| \delta^{*}\left(\tilde{\kappa}_{l}^{\prime}\right)+\sum_{i}(-1)^{l+1}\left(1-e_{i}^{l+1}\right)\left(q_{\left.\right|_{R_{i}}}\right)_{*}\left(c_{1}\left(N_{R_{i}}\right)^{l}\right)
$$

ou, plus simplement, $\kappa_{l}=|G| \delta^{*}\left(\kappa_{l}^{\prime}\right)$.

Démonstration. - De la relation de Riemann-Hurwitz (113), on tire en prenant la première classe de Chern des deux membres

$$
K=\Delta^{*}\left(K^{\prime}\right)+\sum_{i}\left(e_{i}-1\right) c_{1}\left(\Theta\left(R_{i}\right)\right) .
$$

En particulier, en élevant à la puissance $l+1$ des deux membres de cette égalité, et tenant compte aussi du fait que $R_{i}$ et $R_{j}$ sont disjoints si $i \neq j$ :

$$
K^{l+1}=\Delta^{*}\left({K^{\prime}}^{l+1}\right)+\sum_{i} \sum_{j=1}^{l+1}\left(\begin{array}{c}
l+1 \\
j
\end{array}\right)\left(e_{i}-1\right)^{j} \Delta^{*}\left({K^{\prime}}^{l+1-j}\right) c_{1}\left(\theta\left(R_{i}\right)\right)^{j} .
$$

D'une autre manière, en insérant pour $1 \leqslant j \leqslant l+1$, l'égalité

$$
\Delta\left(K^{\prime}\right)^{l+1-j}=\left(K-\sum_{i}\left(e_{i}-1\right) c_{1}\left(\Theta\left(R_{i}\right)\right)\right)^{l+1-j}
$$

on obtient

$$
\Delta^{*}\left(K^{\prime l+1-j}\right) c_{1}\left(\Theta\left(R_{i}\right)\right)^{j}=\sum_{k=0}^{l+1-j}\left(\begin{array}{c}
l+1-j \\
k
\end{array}\right)\left(1-e_{i}\right)^{k} K^{l+1-j-k} c_{1}\left(\Theta\left(R_{i}\right)\right)^{k+j}
$$

(4) Un résultat analogue a été formulé récemment et de manière indépendante par Jarvis-Kimura [64], Theorem 3.12.

(5) Le résultat est énoncé pour les revêtements galoisiens, mais il est facile de valider la preuve dans le cas non galoisien. Ce résultat montre que le cadre des champs de Hurwitz permet de voir des choses invisibles sinon. 
On a $K^{l+1-j-k} c_{1}\left(\Theta\left(R_{i}\right)\right)^{k+j}=\tau_{i *}\left(c_{1}\left(\omega_{q \mid R_{i}}\right)^{l+1-j-k} c_{1}\left(\Theta_{R_{i}}\left(R_{i}\right)\right)^{k+j-1}\right)$, ce qui, compte tenu du fait que $\omega_{q \mid R_{i}} \cong \vartheta_{R_{i}}\left(-R_{i}\right)$, donne finalement l'expression

$$
\Delta^{*}\left({K^{\prime}}^{l+1-j}\right) c_{1}\left(\Theta\left(R_{i}\right)\right)^{j}=\sum_{k=0}^{l+1-j}\left(\begin{array}{c}
l+1-j \\
k
\end{array}\right)\left(1-e_{i}\right)^{k}(-1)^{l+1-j-k} \tau_{i_{*}} c_{1}\left(\Theta_{R_{i}}\left(R_{i}\right)\right)^{l} .
$$

Le coefficient qui précède $\tau_{i *} c_{1}\left(\vartheta_{R_{i}}\left(R_{i}\right)\right)^{l}$ dans l'expression de dessus est

$$
(-1)^{l+1-j} \sum_{k=0}^{l+1-j}\left(\begin{array}{c}
l+1-j \\
k
\end{array}\right)\left(e_{i}-1\right)^{k}=(-1)^{l+1-j} e_{i}^{l+1-j} .
$$

En reportant cela dans l'expression (115), on obtient que le coefficient devant l'expression $\tau_{i, !} c_{1}\left(\theta_{R_{i}}\left(R_{i}\right)\right)^{l}$ est

$$
\sum_{j=1}^{l+1}\left(\begin{array}{c}
l+1 \\
j
\end{array}\right)\left(e_{i}-1\right)^{j}\left(-e_{i}\right)^{l+1-j}=(-1)^{l+1}-\left(-e_{i}\right)^{l+1} .
$$

Appliquons $q_{*}$ aux deux membres, de sorte qu'il suffit de prouver que $q_{*}\left(\Delta^{*}\left(K^{\prime l+1}\right)\right)=$ $|G| \delta^{*}\left(\tilde{\kappa}_{l}\right)$. Soit le morphisme fini $\psi: \overline{\mathscr{C}}_{g, G, \xi} \longrightarrow \overline{\mathcal{H}}_{g, G, \xi} \times \bar{M}_{g^{\prime}, b} \overline{\mathscr{C}}_{g^{\prime}, b}$. Si $p_{1}, p_{2}$ sont les projections du produit cartésien sur les deux facteurs, on note que

$$
q_{*} \psi^{*} p_{2}^{*}\left(K^{\prime l+1}\right)=p_{1 *} \psi_{*} \psi^{*} p_{2}{ }^{*}\left(K^{\prime l+1}\right)
$$

Mais, par la formule de projection, jointe au fait que $\psi$ est fini de degré $|G|$, on a $\psi_{*} \psi^{*}(\cdot)=|G|(\cdot)$ (voir [80], Proposition 3.8). Par ailleurs, du fait de la platitude des morphismes, on a la propriété de changement de base ([97], Lemma 3.9) :

$$
p_{1 *} p_{2}^{*}\left(K^{\prime l+1}\right)=\delta^{*}\left(\tilde{\kappa}_{l}^{\prime}\right) .
$$

La relation (114) en découle.

Voyons maintenant comment on passe de cette relation à la forme compacte qui relie les classes $\kappa_{l}$ et $\kappa_{l}^{\prime}$. Notons d'abord que, par définition, on a

$$
\kappa_{l}=\tilde{\kappa}_{l}+\sum_{i}\left(q_{\mid R_{i}}\right)_{*}\left(c_{1}\left(N_{\mid R_{i}}^{\vee}\right)^{l}\right)
$$

Comme $\Delta^{*}\left(N_{B_{i}}^{\vee}\right)=\left(N_{R_{i}}^{\vee}\right)^{e_{i}}$, on a

$$
\begin{aligned}
e_{i}^{l}\left(q_{\mid R_{i}}\right)_{*}\left(c_{1}\left(N_{\mid R_{i}}^{\vee}\right)^{l}\right) & =\left(q_{\mid R_{i}}\right)_{*}\left(c_{1}\left(\Delta^{*}\left(N_{\mid B_{i}}^{\vee}\right)\right)^{l}\right) \\
& =\left(q_{R_{i}}\right)_{*} q_{R_{i}}^{*}\left(\delta^{*}\left(\psi_{i}^{l}\right)\right)=m_{i} \delta^{*}\left(\psi_{i}^{l}\right) \quad\left(m_{i}=\frac{|G|}{e_{i}}\right)
\end{aligned}
$$

d'où finalement la relation

$$
\kappa_{l}=|G| \delta^{*}\left(\tilde{\kappa}_{l}^{\prime}\right)+\sum_{i} e_{i}^{l+1} \frac{m_{i}}{e_{i}^{l}} \delta^{*}\left(\psi_{i}\right)^{l}=|G| \delta^{*}\left(\kappa_{l}^{\prime}\right) .
$$


Si $l=0$, la relation (114) se réduit à la formule de Riemann-Hurwitz usuelle

$$
2 g-2=|G|\left(2 g^{\prime}-2\right)+\sum_{i}\left(1-\frac{1}{e_{i}}\right)
$$

soit $2 g-2+r=|G|\left(2 g^{\prime}-2+b\right)$. On sait en effet que $\tilde{\kappa}_{0}=(2 g-2)\left[\overline{\mathcal{H}}_{g, G, \xi}\right]_{Q}$, $\tilde{\kappa}_{0}^{\prime}=\left(2 g^{\prime}-2\right)\left[\overline{\mathcal{M}}_{g^{\prime}, b}\right]_{Q}$ (voir $\left.[\mathbf{8 0}]\right)$, et comme $R_{i} \longrightarrow \overline{\mathcal{H}}_{g, G, \xi}$ est fini de degré $\left|\frac{G}{H_{i}}\right|$, la somme se réduit à $\sum_{i}\left(e_{i}-1\right) \frac{|G|}{e_{i}}\left[\overline{\mathcal{H}}_{g, G, \xi}\right]_{Q}$, de sorte que la relation de RiemannHurwitz usuelle en découle.

Si $l=1$, il est possible de donner une preuve différente de (114), exploitant à la place l'égalité $\tilde{\kappa}_{1}=\langle\omega, \omega\rangle$, et utilisant la bimultiplicativité du produit d'intersection de Deligne. Il faut évaluer $\langle\omega, \omega\rangle$ de deux manières. La première est simplement la formule de Mumford pour la courbe $C_{U} \longrightarrow U$, et la seconde revient à substituer à $\omega$ le second membre de la relation de Riemann-Hurwitz (113). Limitons-nous au cas $G$ cyclique, le cas d'un groupe $G$ arbitraire pouvant se traiter de manière analogue, avec quelques modifications. Un problème survient cependant du fait qu'on ne peut appliquer directement le lemme 10.3.2 à $\operatorname{Pic}\left(\overline{\mathcal{H}}_{g, G, \xi}\right)$, du fait de la non platitude de $\Delta$, i.e. de $\pi_{*}\left(\theta_{C}\right)$, si $C$ est singulière. Pour valider le lemme, il faut définir le produit d'intersection $\langle\mathcal{L}, \mathcal{M}\rangle$ si l'un des deux faisceaux inversibles $\mathcal{L}$ ou $\mathcal{M}$ dégénère en un faisceau sans torsion de rang 1 sur la $S$-courbe $C$.

Grâce à la proposition 7.3.9, si $\mathscr{L}$ est sans torsion de rang 1 , on sait qu'il y a une désingularisation $\rho: \tilde{C} \longrightarrow C$ de $\mathscr{L}$ avec un faisceau inversible canonique $\theta(1)$ tel que $\mathcal{L}=\rho_{*}(\theta(1))$. On a $R \rho_{*}(\theta(1))=\mathcal{L}$. Si $\tilde{q}=q \rho: \tilde{C} \longrightarrow S$ est le morphisme composé, on note que $R q_{*}(\mathscr{L})=R \tilde{q}_{*}(\Theta(1))$ est un complexe parfait, de sorte que $\operatorname{det}\left(R q_{*}(\Theta(1))\right.$ a un sens, ce qui permet de définir $\langle\mathscr{L}, \mathcal{M}\rangle$ comme étant

$$
\langle\mathcal{L}, \mathcal{M}\rangle=\left\langle\Theta(1), \rho^{*}(\mathcal{M})\right\rangle
$$

Avec ces conventions, si $\mathscr{P} \in \operatorname{Pic}(C)$, pour calculer le produit d'intersection $\langle\mathscr{L} \otimes \mathscr{P}, \mathcal{M}\rangle$, il suffit de noter que $\mathscr{L} \otimes \mathscr{P}=\rho_{*}\left(\mathscr{\theta}(1) \otimes \rho^{*}(\mathscr{P})\right.$. Dès lors, la relation d'additivité

$$
\langle\mathscr{L} \otimes \mathscr{P}, \mathcal{M}\rangle=\langle\mathscr{L}, \mathcal{M}\rangle+\langle\mathscr{P}, \mathcal{M}\rangle
$$

est clairement vérifiée. De cette remarque découle la validité du lemme 10.3.2 pour un revêtement stable. On remarquera que si $G$ est cyclique d'ordre $n$, en utilisant la notation $\mathscr{L}_{j}$ pour les fibrés introduits en (105), on peut justifier comme dans la section 10.3.1 la relation

$$
\left\langle\omega^{\prime}, \omega^{\prime}\right\rangle=\operatorname{det} R q_{*}\left(\omega^{\prime 2} \otimes \mathscr{L}_{j}\right)-2 \operatorname{det} R q_{*}\left(\omega^{\prime} \otimes \mathscr{L}_{j}\right)+\operatorname{det} R q_{*}\left(\mathscr{L}_{j}\right) .
$$

10.3.3. Faisceaux inversibles associés aux composantes du bord. - Dans cette section, on se limite pour des raisons de simplicité à un groupe $G$ cyclique, même si pour l'essentiel cette restriction est superflue.

Fixons les notations en ce qui concerne les composantes irréductibles du bord de $\overline{\mathcal{M}}_{g, n}$. Si $0 \leqslant i \leqslant[g / 2]$ et $A \subset[1, n]$, on note $\Delta_{i, A}$ la composante irréductible de $\partial \overline{\mathcal{M}}_{g, n}$ qui correspond (par le graphe dual) à un segment, l'un des sommets étant pondéré par $i$ et marqué par les indices appartenant à $A$, l'autre sommet pondéré par $g-i$ 
et marqué par le complémentaire $B$ de $A$. Si $i=g / 2$, alors $\Delta_{g / 2, A}=\Delta_{g / 2, B}$, et si $i=0$, la stabilité impose $|A| \geqslant 2$. La composante associée à la boucle est notée $\Delta_{0}$. Ces composantes sont des diviseurs de Cartier, du fait de la lissité de $\bar{M}_{g, n}$. Elles définissent des faisceaux inversibles qui seront seront notés $\theta\left(\Delta_{i, A}\right)$, ou simplement $\Delta_{i, A}$. Pour éviter toute confusion, si $\Delta$ est un diviseur de Cartier effectif dans l'un des champs considérés, on notera $[\Delta] \in A^{1}$ le cycle (ou classe de cycle) associé.

Rappelons que le bord $\overline{\mathscr{H}}_{g, G, \xi}-\mathscr{H}_{g, G, \xi}$ admet une stratification (section 7.4) compatible avec le morphisme $\delta: \overline{\mathcal{H}}_{g, G, \xi} \longrightarrow \overline{\mathcal{M}}_{g^{\prime}, b}$. Les strates, qui ne sont pas en général les composantes irréductibles, sont indexées par les graphes modulaires de Hurwitz de type $(g, n)$ à une seule orbite d'arêtes.

À un tel graphe $\Gamma$ est associé un diviseur de Cartier $\Delta_{\Gamma}$ contenu dans le bord du champ de Hurwitz, et comme le champ de Hurwitz est lisse, un faisceau inversible $\vartheta\left(\Delta_{\Gamma}\right)$. Si $G$ est cyclique, ce qui simplifie la combinatoire du bord, on va mettre en évidence quelques relations universelles simples, analogues à celles observées par Jarvis pour les courbes à spin, qui relient les faisceaux inversibles $\theta(\Delta)$ (les notations sont celles de la section 9.2.2). L'argument général s'applique à un groupe de Galois arbitraire, mais avec des notations plus compliquées. Comme on suppose le groupe $G$ cyclique, rappelons qu'avec cette hypothèse les graphes de Hurwitz sont indexés par des partitions $\pi$ du diviseur de branchement, et qu'une telle partition induit une partition $(A, B)$ de l'ensemble des points de branchement. Si $n=p$ est premier, il y a une unique partition $\pi$ subordonnée à $(A, B)$. La notation retenue pour le diviseur correspondant est $\Delta_{\pi}$ et $\Theta\left(\Delta_{\pi}\right)$ désigne le faisceau inversible associé. On note $e_{\pi}$ l'indice d'inertie d'un point double d'une courbe générique dans $\Delta_{\pi}$, et on utilisera la notation $m_{\pi}=|G| / e_{\pi}$.

Dans la proposition suivante, il est cependant inutile de supposer $G$ cyclique.

Proposition 10.3.5. - Dans le groupe $\operatorname{Pic}\left(\overline{\mathscr{H}}_{g, G, \xi}\right)$, on a les relations

$$
\begin{array}{ll}
\text { i) } & \delta^{*}\left(\theta\left(\Delta_{i, A, B}\right)\right)=\bigotimes_{\pi} \theta\left(\Delta_{\pi}\right)^{\otimes e_{\pi}} \\
\text { ii) } & \delta^{*}\left(\theta\left(\Delta_{0}\right)\right)=\bigotimes_{\pi} \theta\left(\Delta_{0, a, b, \pi}\right)^{\otimes e_{\pi}}
\end{array}
$$

où le produit tensoriel porte sur les partitions $\pi$ subordonnées à $(A, B)$ (voir 9.2.2).

Démonstration. - Soit un point $\pi: C \longrightarrow D$ (générique) dans une composante où la base $D$ est supposée avoir un seul point double, dans le cas i) deux composantes irréductibles, une seule dans le cas ii). On sait d'autre part que la déformation équivariante universelle de $C$ a pour base une algèbre de séries formelles $\operatorname{Spec}\left(k\left[\left[\tau_{1}, \ldots, \tau_{N}\right]\right]\right)$, où $N=3 g-3+b$, et $\tau=\tau_{1}$ représente le paramètre de l'orbite des points doubles. Soit la base $\operatorname{Spec}\left(k\left[\left[t_{1}, \ldots, t_{N}\right]\right]\right)$ de la déformation universelle de $(D, Q), t=t_{1}$ étant le paramètre de $Q$, avec les relations $t=\tau^{e}, t_{j}=\tau_{j}$ si $j \geqslant 2$. $\mathrm{Du}$ fait que les équations locales des diviseurs irréductibles mentionnés dans i) sont dans ces coordonnées locales, respectivement $t=0$ et $\tau=0$, la relation en découle. La preuve de ii) est analogue. 
On peut aussi étudier le comportement du bord de $\overline{\mathscr{H}}_{g, n, \xi}$ relativement à l'immersion locale non ramifiée $\imath: \overline{\mathscr{H}}_{g, n, \xi} \longrightarrow \overline{\mathcal{M}}_{g, r}$. Rappelons que seules les orbites de points de ramification sont indexées, et non les points eux-mêmes.

Proposition 10.3.6. - Soit $\Delta$ le diviseur du bord de $\bar{M}_{g, r}$, alors

$$
\imath^{*}(\Delta)=\sum_{\pi} m_{\pi} \Delta_{\pi} \quad \text { ò̀ } \quad m_{\pi}=\frac{|G|}{e_{\pi}} .
$$

Démonstration. - Soit $\pi: C \longrightarrow D$ un revêtement correspondant à un point général de $\Delta_{\pi}$, de sorte que le graphe modulaire de $D$ est un segment, ou une boucle. Il y a donc dans $C$ une unique orbite de points doubles, de cardinal $m=m_{\pi}$. Dans la déformation universelle non équivariante de $C$, soient $t_{1}, \ldots, t_{m} \in R$ les paramètres de déformation de ces points. Si $t_{i}$ est le paramètre de $P_{i}$, on sait que le stabilisateur $H$ de $P_{1}$ laisse invariant $t_{1}$, et qu'on a $t_{i}=g^{*}\left(t_{1}\right)$ si $g\left(P_{1}\right)=P_{i}$. L'équation du bord $\Delta$ dans la carte $\operatorname{Spec}(R)$ est $t_{1} \cdots t_{m}=0$. On utilise la carte locale correspondante pour le point $C \longrightarrow D$ dans $\overline{\mathscr{H}}_{g, n, \xi}$, qui est $\operatorname{Spec}\left(R_{G}\right)$, où $R_{G}=R / J$, et $J$ est l'idéal engendré par les $g(a)-a), a \in R, g \in G$. On obtient que l'équation locale de $\imath^{*}(\Delta)$ en $C \longrightarrow D$ est $\prod_{i=1}^{m} t_{i}=u^{m} \epsilon$ où $u$ est l'image de $t_{1}$, et $\epsilon$ est inversible. Comme $u=0$ est l'équation locale au point considéré du diviseur $\Delta_{\pi}$, la conclusion suit.

Soit de nouveau le revêtement universel $\overline{\mathscr{C}}_{g, G, \xi} \longrightarrow \overline{\mathcal{H}}_{g, G, \xi}$, la base de ce revêtement étant $\overline{\mathscr{D}}_{g, G, \xi}=\overline{\mathscr{C}}_{g^{\prime}, b} \times \overline{\mathscr{M}}_{g^{\prime}, b} \overline{\mathscr{H}}_{g, G, \xi}$. Rappelons que $G$ est cyclique avec $n=|G|$. On abrège les notations en supprimant les indices, ainsi que le surlignage, donc $\mathcal{H}=\overline{\mathcal{H}}_{g, n}$, $\mathscr{C}=\overline{\mathscr{C}}_{g, n}$ et $\mathscr{D}=\overline{\mathscr{D}}_{g, n}$. Le champ $\mathscr{D}$ classifie les revêtements $C \longrightarrow D \longrightarrow S$ munis d'une section additionnelle $Q: S \longrightarrow D$. On a en conséquence les sections universelles

$$
Q_{i}: \mathcal{H} \longrightarrow \mathscr{D}, \quad Q_{i}(C \longrightarrow D)=\left(C \longrightarrow D, Q_{i}\right) .
$$

Elles proviennent par changement de base des sections de même nom de la courbe $\overline{\mathscr{C}}_{g^{\prime}, b} \longrightarrow \overline{\mathcal{M}}_{g^{\prime}, b}$. Dans la suite $G$ est identifié à $\mathbb{Z} / n \mathbb{Z}$, la classe de 1 définissant un automorphisme $\sigma$. En conservant les notations antérieures, soit $\mathscr{L}=\mathscr{L}_{1}$ le faisceau sans torsion de rang 1 sur $\bar{D}$ de lieu singulier la partie NS du diviseur de branchement. La structure locale de $\mathscr{L}$ en un point singulier est décrite dans la proposition 7.3.7. Rappelons qu'on a une désingularisation $\tilde{\mathscr{D}}=\operatorname{Proj}(\operatorname{Sym}(\mathscr{L})) \stackrel{\rho}{\longrightarrow} \mathscr{D}$ de $\mathscr{L}$, conduisant à un diagramme

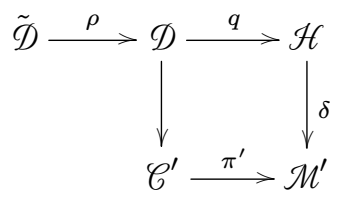

Le diviseur de branchement $\mathscr{B} \subset \mathscr{D}$ du revêtement universel est le sous-champ fermé de sections les $\left(C \longrightarrow D, Q \in\left\{Q_{i}\right\}\right)$. Notant $Q_{i}$ l'image de la section de même nom, 
on a

$$
\mathscr{B}=\sum_{i=1}^{b} m_{i} \eta_{i} Q_{i}
$$

Le diviseur $\mathcal{B}$ est disjoint du lieu singulier de $\mathcal{L}$, on peut alors le voir comme un diviseur de $\tilde{\mathscr{D}}$. Soit le morphisme introduit dans la proposition 9.2 .4 :

$$
\tilde{\Phi}: \theta_{\tilde{D}}(n) \longrightarrow \theta_{\tilde{D}}(-\mathcal{B}) .
$$

On peut mesurer l'écart qui est concentré sur le bord entre d'une part $\theta_{\tilde{D}}(n)$ et d'autre part $\Theta(\mathcal{B})$. Posons $\Xi=\theta_{\tilde{D}}(-n-\mathcal{B})$. Notons aussi $E_{\pi}$ le diviseur de Weil de $\tilde{\mathscr{D}}$ qui a pour support la partie horizontale du lieu exceptionnel, celle au-dessus de $\Delta_{\pi}$ (proposition 7.3.8). Le résultat suivant sera largement utilisé dans la suite :

Proposition 10.3.7. - Le diviseur des zéros de $\tilde{\Phi}$ est

$$
\Xi=\operatorname{Div}(\tilde{\Phi})=\theta_{\tilde{D}}\left(\sum_{\pi=\mathrm{NS}} \frac{a b}{m} E_{\pi}\right),
$$

et on $a\langle\Xi, \tilde{\omega}\rangle=\theta$.

Démonstration. - On se place dans la situation $S=U$, où $U$ désigne un atlas (ou une carte locale) de $\mathcal{H}$ autour d'un revêtement donné muni d'un point double de type NS de la base. Noter que le terme de gauche ne fait intervenir que les composantes NS du bord. On a noté qu'à chaque composante $\Delta_{\pi}$ de type NS, on peut associer dans $\tilde{D}$ un diviseur de Weil lisse $E_{\pi}$, tel que $E_{\pi} \longrightarrow \Delta_{\pi}$ est un fibré en $\mathbb{P}^{1}$, et que si $(a, b)$ est la signature correspondante à $\pi$, alors d'une part $\frac{a b}{m} E_{\pi}$ est Cartier, et d'autre part $\Xi=\theta\left(\sum_{\pi=\mathrm{NS}} \frac{a b}{m} E_{\pi}\right)$.

Pour valider cette égalité, on se place dans la situation locale de dessus, de sorte que le diviseur $\Xi=\operatorname{Div}(\tilde{\Phi})$ a pour équation locale $\phi_{1}=0$ sur $U_{1}$, resp. $\Phi_{2}=0$ sur $U_{2}$. Une équation locale du diviseur $E$ en dehors des points $z_{0}, z_{\infty}$ est $\tau=0$, comme $\phi_{1}=y^{a}=\tau^{a b / m}$, la relation est démontrée.

La seconde relation découle du fait que $\tilde{\omega}=\rho^{*}(\omega)$, d'où vient en particulier que $\tilde{\omega}$ est trivial sur chaque diviseur exceptionnel.

L'identification de $\tilde{\mathscr{D}}$ avec $\operatorname{Proj}(\operatorname{Sym}(\mathscr{L}))$ montre qu'un point de $\widetilde{D}$ est représenté par un triplet $(C \longrightarrow D, Q, \xi)$ dans lequel $Q$ est un point de $D$, et $\xi$ un point de $\mathbb{P}(\mathscr{L}) \otimes k(Q)$. Comme $\operatorname{dim}(\mathbb{P}(\mathscr{L}) \otimes k(Q))=1$ sauf si $Q$ est un point double NS, la dimension étant alors 2 , on voit que les deux points singuliers $z_{0}, z_{\infty}$ correspondent aux deux directions dans le plan $\mathscr{L} \otimes k(Q)$ données par les fibres des faisceaux inversibles, désignés ci-après par $\mu$, le long des deux branches. Cela sera précisé dans la section suivante. 


\subsection{Appliquer le théorème de Riemann-Roch}

10.4.1. Mise en place du théorème de Grothendieck-Riemann-Roch (GRR). - De nombreux travaux récents, faisant suite au travail bien connu de Mumford [80], ont confirmé l'utilité d'avoir de l'attention pour les classes de Chern des fibrés $\mathbb{E}_{v}$ (voir par exemple [17]). Dans loc. cit., Mumford montre qu'une utilisation judicieuse du théorème de Grothendieck-Riemann-Roch (GRR) permet d'exprimer le caractère de Chern du fibré de Hodge sur $\bar{M}_{g, n}$ sous la forme compacte

$$
\operatorname{ch}(\mathbb{E})-1=\sum_{d \geqslant 0} \frac{B_{d+1}}{(d+1) !} \kappa_{d}-\sum_{i=1}^{n} \frac{B_{d+1}}{(d+1) !} \psi_{i}^{d}+\frac{1}{2} \frac{B_{d+1}}{(d+1) !} j_{*}\left(\sum_{i+j=d-1}(-\psi)^{i} \hat{\psi}^{j}\right)
$$

où $B_{d}$ désigne le $d$-ième nombre de Bernoulli. Les notations complètes seront précisées ci-dessous. Dans cette section, on souhaite obtenir une relation similaire portant maintenant sur les facteurs isotypiques $\mathbb{E}_{v}$ du fibré de Hodge. Dans ce but, en suivant Mumford [80], Faber-Pandharipande [44], Chiodo [22], Coates-Givental [23], on est conduit à appliquer le théorème GRR au faisceau inversible $\theta(1)$, en fait au complexe parfait $R q_{*}\left(\mathscr{L}_{v}\right)$, cela relativement à la courbe semi-stable

$$
\tilde{q}: \tilde{D} \longrightarrow \mathcal{H} .
$$

Notre objectif est d'obtenir des relations entre les classes de Chern d'un quelconque facteur isotypique $\mathbb{E}_{v}$ du fibré de Hodge d'une part, et les classes $\psi$ et $\kappa$ d'autre part.

D'un point de vue technique, il faut noter que les champs $\mathscr{D}$ et $\widetilde{D}$ ne sont pas lisses, mais seulement normaux avec des singularités de type $\mathrm{A}_{l}$. Heureusement, la théorie de Fulton-MacPherson admet une extension aux champs de Deligne-Mumford, comme prouvé par Vistoli [97]. Le morphisme $\tilde{q}$ est localement d'intersection complète (l.c.i.) dans le sens de Fulton, ce qui autorise à appliquer dans le présent contexte des champs de Deligne-Mumford, le théorème GRR, prouvé par Fulton-MacPherson dans le cadre des schémas sur un corps. Sur la manière d'appliquer le théorème de Grothendieck-Riemann-Roch dans notre contexte, on suit de près l'argumentation de Coates-Givental [23]. Rappelons que, mis à part $\mathscr{D}$ et $\widetilde{D}$, les champs qui interviennent sont lisses et équidimensionnels.

Pour ce qui concerne les règles de calcul dans l'anneau de Chow, rappelons que les classes de Chern d'un fibré vectoriel sont des éléments de $A^{\bullet}$, et par cap produit elles agissent sur l'anneau de Chow des cycles. La relation point de départ de notre calcul est donc le théorème GRR :

$$
\operatorname{ch}\left(\mathrm{R} \tilde{q}_{!}(\theta(1))[\mathcal{H}]=\mathrm{R} \tilde{q}_{*}\left(\operatorname{ch}(\Theta(1)) \cdot \operatorname{Td}^{\vee}\left(\Omega_{\tilde{q}}^{1}\right)\right)[\mathcal{H}]\right.
$$

avec pour objectif la relation suivante, les notations utilisées étant précisées cidessous : 
THÉORÈme 10.4.1. - On a la relation suivante :

$$
\begin{aligned}
& \operatorname{ch}\left(\mathrm{R} \tilde{q}_{!}(\theta(1))[\mathcal{H}]\right. \\
& =\sum_{d \geqslant 0} \frac{B_{d+1}}{n(d+1) !} \kappa_{d}-\sum_{i} \frac{B_{d+1}\left(\frac{\eta_{i}}{e_{i}}\right)}{(d+1) !} \psi_{i}^{d}+\sum_{\pi, d \geqslant 1} \frac{e B_{d+1}\left(\frac{\eta_{0}}{e}\right)}{(d+1) !}\left(\sum_{a+b=d-1}\left(-\psi_{0}\right)^{a} \psi_{\infty}^{b}\right)[\mathcal{H}] .
\end{aligned}
$$

La preuve résultera d'une suite de calculs indépendants, regroupés en lemmes. On se concentre en premier sur le faisceau $\Omega_{\tilde{q}}^{1}$. Rappelons les notations utilisées ci-dessus (proposition 7.3.8), en particulier $\& \subset \mathscr{D}$ est le lieu singulier de $\mathscr{D}$, c'est-à-dire le lieu $\operatorname{des}(C \longrightarrow D, Q)$, où $Q$ est un point double de $D$. On a $\phi=\phi_{\mathrm{NS}} \sqcup \phi_{\mathrm{RR}}$. On note $\tilde{\phi}$ l'image inverse de $\phi$ qui représente le lieu exceptionnel. On a vu que $\tilde{\phi}$ est formé d'une partie horizontale $\mathcal{E}$, et d'une partie de type II, qu'on note $\mathcal{Z}$ (proposition 4.1.8). Enfin $Z \subset \widetilde{D}$ qui est le lieu singulier de $\widetilde{D}$, est union de deux parties, l'une qui correspond au type NS, l'autre au type RR. Cette dernière identique à $\phi_{\mathrm{RR}}$ (Proposition 7.3.8). On a une décomposition de ces sous-champs en des sommes disjointes indexées par les partitions $\pi$, i.e par les composantes du bord. En particulier $E_{\pi}$ désignera le cycle associé à la partie horizontale $\mathcal{E}_{\pi}$.

Soit $\imath: Z \subset \widetilde{D}$ le morphisme de plongement du lieu singulier. Les lemmes qui suivent sont des extensions immédiates d'énoncés connus dans le contexte des espaces de modules de courbes, ou d'applications.

LEMme 10.4.2. - On a l'égalité $\omega_{\tilde{q}} \otimes \theta_{Z}=\theta_{Z}$, et la suite exacte :

$$
0 \longrightarrow \Omega_{\tilde{q}} \longrightarrow \omega_{\tilde{q}} \longrightarrow \imath_{*}\left(\theta_{Z}\right) \longrightarrow 0
$$

Démonstration. - Localement sur une base $S$, un atlas si on veut, le morphisme $\tilde{q}$ est de la forme

$$
C \stackrel{\imath}{\hookrightarrow} M \stackrel{\tilde{q}}{\longrightarrow} S
$$

avec $\imath$ un plongement de $C$ dans une variété affine lisse d'équation $f=0$. Soit $I$ l'idéal de $C$ dans $\theta_{M}$. On a d'une part

$$
\omega_{\tilde{q}}=\operatorname{Hom}\left(I / I^{2}, \wedge^{2} \Omega_{M} \otimes \theta_{C}\right),
$$

et d'autre part, la fibre générique de $\tilde{q}$ étant lisse, on a la suite exacte

$$
0 \longrightarrow I / I^{2} \stackrel{i}{\longrightarrow} \Omega_{M} \otimes \theta_{C} \stackrel{p}{\longrightarrow} \Omega_{C} \longrightarrow 0
$$

qui conduit à une injection

$$
\mu: \Omega_{C / S} \hookrightarrow \omega_{\tilde{q}} \quad, \quad \mu(p(\xi))(\bar{a})=i(a) \wedge \xi .
$$

Comme dans notre situation $C \longrightarrow S$ est une courbe semi-stable à fibre générique lisse, on peut quitte à localiser pour la topologie étale, supposer que $S=\operatorname{Spec}(R)$, avec si on veut $R$ normal, et même lisse, et $f=x y-\pi$ avec $\pi \in \mathcal{M}_{R}$ non diviseur de zéro dans $R$, et même $\pi=t^{m}$ pour un élément $t$ membre d'un système de paramètres de $R$. On a $i(\bar{f})=\overline{y d x+x d y}$, et

$$
i(\bar{f}) \wedge(p(\xi=\overline{A d x+B d y}))=\overline{(B y-A x)} d x \wedge d y .
$$


En particulier, $B y-A x=0$ dans $\theta=R[[x, y]] /(x y-\pi)$ équivaut à $x / B$, et $y / A$, donc $\xi=0$. Pour décrire le conoyau de $\mu$, soit $\eta \in \omega$, donc $\eta(\bar{f})=\bar{h} d x \wedge d y, \bar{h} \in \emptyset$. Alors $\eta$ est dans l'image de $\mu$ si et seulement si $h \in(x, y)$. Cela confirme d'une part la suite exacte (120), et d'autre part que $\Omega_{\tilde{q}}=\mathcal{J}_{\text {sing }} \omega_{\tilde{q}}$. En conséquence $\omega_{\tilde{q}} \otimes \theta_{Z}=\theta_{Z}$.

Noter que $\omega_{\tilde{q}}$ vient de $\mathscr{C}^{\prime}$, via le morphisme $\Delta^{\prime}: \widetilde{D} \longrightarrow \mathscr{C}^{\prime}$, i.e. $\omega_{\tilde{q}}=\Delta^{\prime *}\left(\omega_{\pi^{\prime}}\right)$. Dans la partie droite de l'égalité (119), le terme $\operatorname{ch} \theta(1)=e^{c_{1}(\theta(1))}$ est facile à expliciter. Tenant compte de (117) et (118), on a

$$
c_{1}(\theta(1))=-\frac{1}{n}\left(\sum_{i=1}^{b} m_{i} \eta_{i} Q_{i}+\sum_{\pi=\mathrm{NS}} \frac{\alpha \beta}{e} E_{\pi}\right)=-\left(\sum_{i} \frac{\eta_{i}}{e_{i}} Q_{i}+\sum_{\pi=\mathrm{NS}} \frac{\alpha \beta}{e} E_{\pi}\right),
$$

où $(a, b)=m(\alpha, \beta)$ désigne la signature. Dans la suite, si $D$ est un diviseur de Cartier dans un champ, on notera par une même lettre la classe de Chern du faisceau inversible $\vartheta(D)$, avec la classe $[D]$ du cycle correspondant. Du fait qu'à la fin on évalue tout sur la classe fondamentale, cela ne devrait pas créer de confusion. La suite (120) implique l'égalité

$$
\operatorname{Td}^{\vee}\left(\Omega_{\tilde{q}}\right)=\operatorname{Td}^{\vee}(\omega) \operatorname{Td}^{\vee}\left(-\imath_{*}\left(\theta_{Z}\right)\right)
$$

avec $\operatorname{Td}^{\vee}(\omega)=\frac{K}{e^{K}-1}$ (on supprime les indices). Soit le terme de droite de GRR (119). Du fait que les $Q_{i}$ et les $E_{\pi}$ sont disjoints, on a

$$
\begin{aligned}
\operatorname{ch}(\theta(1)) & =\exp \left(\sum_{i} \frac{\eta_{i}}{e_{i}}\left(-Q_{i}\right)+\sum_{\pi=\mathrm{NS}} \frac{\alpha \beta}{e}\left(-E_{\pi}\right)\right) \\
& =\exp \left(\sum_{i} \frac{\eta_{i}}{e_{i}}\left(-Q_{i}\right)\right)+\exp \left(\sum_{\pi=\mathrm{NS}} \frac{\alpha \beta}{e}\left(-E_{\pi}\right)\right)-1 .
\end{aligned}
$$

On a observé ci-dessus que l'intersection de $K$ avec les cycles $E_{\pi}$ est nulle, et que par ailleurs les $Q_{i}$ sont disjoints du lieu singulier, de la sorte, en gardant en mémoire la notation abrégée $a=a(\pi), b=b(\pi), e=e(\pi), m=m(\pi)=\frac{n}{e(\pi)}$, on a l'égalité :

$$
\frac{K}{e^{K}-1}\left(\exp \left(-\sum_{\pi=\mathrm{NS}} \frac{\alpha \beta}{e} E_{\pi}\right)-1\right)=\left(\exp \left(-\sum_{\pi=\mathrm{NS}} \frac{\alpha \beta}{e} E_{\pi}\right)-1\right) .
$$

Finalement, l'expression $\operatorname{ch}(\theta(1)) \operatorname{Td}^{\vee}(\omega) \operatorname{Td}^{\vee}\left(-\imath_{*}\left(\theta_{Z}\right)\right)$ se réduit à une somme de deux termes, à savoir pour le premier :

$$
\left(\exp \left(-\sum_{\pi=\mathrm{NS}} \frac{\alpha \beta}{e} E_{\pi}\right)-1\right) \operatorname{Td}^{\vee}\left(-\imath_{*}\left(\theta_{Z}\right)\right)
$$

et pour le second :

$$
\frac{K}{e^{K}-1} \exp \left(\sum_{i} \frac{\eta_{i}}{e_{i}}\left(-Q_{i}\right)\right) \operatorname{Td}^{\vee}\left(-\imath_{*}\left(\theta_{Z}\right)\right)
$$


qui se simplifie en

$$
\frac{K}{e^{K}-1}\left(\exp \left(\sum_{i} \frac{\eta_{i}}{e_{i}}\left(-Q_{i}\right)\right)+\operatorname{Td}^{\vee}\left(-\imath_{*}\left(\theta_{Z}\right)\right)-1\right) .
$$

Comme $K$ a une intersection triviale avec tout cycle contenu dans le lieu singulier, cette dernière expression se réduit à

$$
\frac{K}{e^{K}-1} \exp \left(\sum_{i} \frac{\eta_{i}}{e_{i}}\left(-Q_{i}\right)\right)+\operatorname{Td}^{\vee}\left(-\imath_{*}\left(\theta_{Z}\right)\right)-1 .
$$

Après simplification, rappelant que $\eta_{i}$ désigne l'holonomie en $Q_{i}$, la somme des deux résultats (122) et (123) devient :

$$
\begin{aligned}
& \left(\exp \left(-\sum_{\pi=\mathrm{NS}} \frac{\alpha \beta}{e} E_{\pi}\right)-1\right) \operatorname{Td}^{\vee}\left(-\imath_{*}\left(\theta_{Z}\right)\right) \\
& +\frac{K}{e^{K}-1} \exp \left(\sum_{i} \frac{\eta_{i}}{e_{i}}\left(-Q_{i}\right)\right)+\mathrm{Td}^{\vee}\left(-\imath_{*}\left(\theta_{Z}\right)\right)-1
\end{aligned}
$$

$$
=\left(\exp \left(-\sum_{\pi=\mathrm{NS}} \frac{\alpha \beta}{e} E_{\pi}\right)\right) \operatorname{Td}^{\vee}\left(-\imath_{*}\left(\theta_{Z}\right)\right)+\frac{K}{e^{K}-1} \exp \left(\sum_{i} \frac{\eta_{i}}{e_{i}}\left(-Q_{i}\right)\right)-1 .
$$

Le terme 1 disparaît dans $\tilde{q}_{*}$, on peut donc le négliger. Rappelons la fonction génératrice des nombres de Bernoulli :

$$
\frac{x}{e^{x}-1}=\sum_{n \geqslant 0} \frac{B_{n}}{n !} x^{n}
$$

et aussi la définition des polynômes de Bernoulli :

$$
B_{n}(x)=\sum_{p=0}^{n}\left(\begin{array}{l}
n \\
p
\end{array}\right) B_{p} x^{n-p} .
$$

On a $B_{0}=1, B_{1}=-1 / 2$ et $B_{r}=0$ si $r>1$ impair. Cela permet d'écrire le terme de gauche de (124):

$$
\frac{K}{e^{K}-1} \exp \left(\sum_{i} \frac{\eta_{i}}{e_{i}}\left(-Q_{i}\right)\right)=\left(\sum_{d \geqslant-1} \frac{B_{d+1}}{(d+1) !} K^{d+1}\right)\left(\sum_{l \geqslant 0} \frac{1}{l !}\left(\frac{\eta_{i}}{e_{i}}\right)^{l}\left(-Q_{i}\right)^{l}\right) .
$$

Par application de $\tilde{q}_{*}$, les termes de degré 0 ont une contribution nulle, de sorte que le produit ci-dessus devient

$$
\frac{K}{e^{K}-1} \exp \left(\sum_{i} \frac{\eta_{i}}{e_{i}}\left(-Q_{i}\right)\right)=\left(\sum_{d \geqslant-1} \frac{B_{d+1}}{(d+1) !} K^{d+1}\right)\left(1+\sum_{l \geqslant 0} \frac{1}{(l+1) !}\left(\frac{\eta_{i}}{e_{i}}\right)^{l+1}\left(-Q_{i}\right)^{l+1}\right)
$$


ce qui conduit à l'expression

$$
\sum_{d \geqslant 0} \frac{B_{d+1}}{(d+1) !} K^{d+1}+\sum_{i} \sum_{l, r \geqslant 0} \frac{B_{r}}{r !(l+1) !}\left(\frac{\eta_{i}}{e_{i}}\right)^{l+1} K^{r}\left(-Q_{i}\right)^{l+1} .
$$

La relation $K^{h}\left(-Q_{i}\right)^{l+1}=-K^{h+l} Q_{i}$ ramène cette expression à

$$
\sum_{d \geqslant 0} \frac{B_{d+1}}{(d+1) !} K^{d+1}-\sum_{i} \sum_{l, r \geqslant 0} \frac{B_{r}}{r !(l+1) !}\left(\frac{\eta_{i}}{e_{i}}\right)^{l+1} K^{r+l} Q_{i} .
$$

On isole les termes de degré $d+1$ qui contribuent par application de $\tilde{q}_{*}$ à $\mathrm{ch}_{d}$, et on trouve :

$$
\begin{gathered}
\frac{B_{d+1}}{(d+1) !} K^{d+1}-\sum_{i}\left(\sum_{l, r \geqslant 0, r+l=d} \frac{B_{r}}{r !(l+1) !}\left(\frac{\eta_{i}}{e_{i}}\right)^{l+1}\right) K^{r+l} Q_{i} \\
=\frac{B_{d+1}}{(d+1) !} K^{d+1}-\sum_{i} \frac{B_{d+1}\left(\frac{\eta_{i}}{e_{i}}\right)}{(d+1) !} K^{d} Q_{i} .
\end{gathered}
$$

Soient les classes $\kappa_{l} \in A^{\bullet}(\overline{\mathcal{M}})$ qui, rappelons-le, vérifient $\kappa_{l}=\pi_{*}\left(K^{l+1}\right)+\sum_{i} \psi_{i}^{l}(111)$. On sait aussi que les classes $\kappa_{l}$ définies sur $\mathcal{H}$, et celles définies sur $\overline{\mathcal{M}}^{\prime}$, sont reliées par la relation de Riemann-Hurwitz d'ordre supérieur $\kappa_{l}=n \delta^{*}\left(\kappa_{l}^{\prime}\right)(114)$. Alors, en appliquant $\tilde{q}_{*}$ à l'expression (125), on obtient pour le premier terme

$$
\tilde{q}_{*}\left(K^{d+1}\right)=q_{*}\left(\Delta^{*}\left(K^{\prime}\right)^{d+1}\right)=\delta^{*}\left(K^{\prime d+1}\right)=\delta^{*} \pi_{*}^{\prime}\left(\tilde{\kappa}_{d}^{\prime}\right)=\delta^{*}\left(\kappa^{\prime}-\sum_{i} \psi_{i}^{d}\right)
$$

et pour l'autre terme figurant dans la somme :

$$
\tilde{q}^{*}\left(K^{d} Q_{i}\right)=q_{*}\left(\delta^{*}\left(K^{\prime}\right)^{d} \Delta^{*}\left(Q_{i}\right)\right)=\delta^{*}\left(\psi_{i}^{d}\right) .
$$

On trouve finalement comme résultat l'expression

$$
\frac{B_{d+1}}{n(d+1) !} \kappa_{d}-\sum_{i} \frac{B_{d+1}\left(\frac{\eta_{i}}{e_{i}}\right)}{(d+1) !} \psi_{i}^{d}
$$

évaluée sur le cycle fondamental $[\mathcal{H}]$. L'expression (126) met en évidence la contribution de la partie ouverte de $\mathcal{H}$ à la formule (119). Il reste à élucider la contribution du bord.

10.4.2. Termes de bord. - On se concentre maintenant sur l'expression qui fait intervenir le bord de $\overline{\mathscr{H}}_{g, n, \tilde{\xi}_{1}}$ :

$$
\tilde{q}_{*}\left(\exp \left(-\sum_{\pi=\mathrm{NS}} \frac{\alpha \beta}{e} E_{\pi}\right) \operatorname{Td}^{\vee}\left(-\imath_{*}\left(\theta_{Z}\right)\right)\right) .
$$

L'abus d'écriture signalé se poursuit. Le lieu singulier $Z$ de $\widetilde{D}$ (resp. $\varnothing$ de $\mathscr{D}$ ) se décompose en $\mathcal{Z}=Z_{\mathrm{NS}} \sqcup \mathcal{Z}_{\mathrm{RR}}$ (resp. $\oint_{\mathrm{NS}} \sqcup \oint_{\mathrm{RR}}$ ). La partie significative est $\mathcal{Z}_{\mathrm{NS}}$, qui se décompose en une somme disjointe (proposition 7.3.8) :

$$
Z_{\mathrm{NS}}=\underset{\pi=\mathrm{NS}}{\sqcup} Z_{\pi}, \quad Z_{\pi}=Z_{\pi, 0} \sqcup Z_{\pi, \infty}
$$


Le premier travail est de préciser le comportement du faisceau $\mathcal{L}$ de rang 1 , relativement au lieu singulier. Considérons dans ce but le champ $\overline{\mathcal{H}}_{g, G, \xi}^{\prime}$ (où $g$ est le genre de $D$ ) classifiant les revêtements $C \longrightarrow D$ stables marqués, avec sur $C$ le choix d'un point $P$ dans la dernière orbite (singulière) numérotée . L'opération de recollement (clutching) de deux revêtements le long de l'orbite du point sélectionné $P_{1}$ sur $C_{1}$, et $P_{2}$ sur $C_{2}$, définit un morphisme

$$
j: \overline{\mathcal{H}}_{g_{1}, n_{1}, \tilde{\xi}_{1}}^{\prime} \times \overline{\mathcal{H}}_{g_{2}, n_{2}, \tilde{\xi}_{2}}^{\prime} \longrightarrow \phi_{\pi} \subset \mathcal{D} .
$$

De manière précise, ce morphisme applique la section donnée par un couple de revêtements $C_{\alpha} \longrightarrow D_{\alpha}(\alpha=1,2)$, plus un point sélectionné $P_{\alpha}$ dans l'orbite singulière de rang $b_{\alpha}+1$, le revêtement obtenu par recollement de $C_{1}$ et $C_{2}$ le long des sections $g P_{1}$ et $g P_{2}$, ceci pour tout $g \in G / H$. On suppose dans cette opération que les holonomies en $P_{1}$ et $P_{2}$ sont opposées. La base du résultat est $D=D_{1} \cup D_{2}$, courbe obtenue par recollement le long des sections images $Q_{1}$ et $Q_{2}$. Dans le cas $(\mathrm{R})$, on a $H=1$. Ceci étant, on souhaite prouver que $j$ se relève en un morphisme

$$
\tilde{j}: \overline{\mathcal{H}}_{g_{1}, n_{1}, \tilde{\xi}_{1}}^{\prime} \times \overline{\mathcal{H}}_{g_{2}, n_{2}, \tilde{\xi}_{2}}^{\prime} \longrightarrow Z_{\pi} \subset \widetilde{\mathscr{D}} \text {. }
$$

Pour $\alpha=1,2$, utilisant des notations allégées, il y a un morphisme évident

$$
j_{\alpha}: \overline{\mathcal{H}}_{g_{\alpha}, \tilde{\xi}_{\alpha}}^{\prime} \longrightarrow \mathscr{D}_{g_{\alpha}, \tilde{\xi}_{\alpha}}
$$

qui applique une paire $\left(C_{\alpha} \longrightarrow D_{\alpha}, P_{\alpha}\right)$, formée d'un revêtement et d'un point sélectionné dans la dernière orbite, sur $\left(D_{\alpha}, Q_{\alpha}\right)$ où $Q_{\alpha}$ est l'image de $P_{\alpha}$. Notons $\mathscr{L}_{\alpha}$ le faisceau localement libre de rang 1 tautologique sur $\mathscr{D}_{g_{\alpha}, \tilde{\xi}_{\alpha}}$ introduit dans la section 10.2. Comme $Q_{\alpha}$ n'est pas un point double, l'image de $j_{\alpha}$ est disjointe du lieu singulier de $\mathscr{D}_{\alpha}$, de la sorte $j_{\alpha}^{*}\left(\mathscr{L}_{\alpha}\right)$ est un faisceau inversible sur $\overline{\mathcal{H}}_{g_{\alpha}}^{\prime}, \tilde{\xi}_{\alpha}$ que nous noterons encore $\mathcal{L}_{\alpha}$, ou abusivement $\mu_{\alpha}$ (notation de la section 10.2). Noter qu'il est relié au faisceau cotangent au point $Q_{\alpha} \in D_{\alpha}$ par

$$
\mathscr{L}_{1}^{\otimes n_{1}}=\psi_{1}^{m_{1} \eta_{1}} \quad, \quad \mathscr{L}_{2}^{\otimes n_{2}}=\psi_{2}^{m_{2} \eta_{2}} .
$$

On retient à l'occasion la notation $\eta_{1}=\eta, \eta_{2}=e-\eta$. Dans cette construction, le revêtement $C \longrightarrow D=D_{1} \cup D_{2}$ obtenu par recollement de $\operatorname{Ind}_{G_{1}}^{G}\left(C_{1}\right) \longrightarrow D_{1}$ et $\operatorname{Ind}_{G_{2}}^{G} \longrightarrow D_{2} \longrightarrow D$ le long de l'orbite $G P_{1}$ (resp. $G P_{2}$ ) a une section $Q: S \longrightarrow D$ constituée de points doubles. Ce qui explique la structure particulière de l'image réciproque de $\mathscr{L}$ :

LEMME 10.4.3. - L'image réciproque $j^{*}(\mathscr{L})$ est un faisceau localement libre de rang 2 :

$$
\left.j^{*}(\mathscr{L})=p_{1}^{*}\left(\mathscr{L}_{1}\right) \oplus p_{2}^{*}\left(\mathscr{L}_{2}\right)\right)
$$

Démonstration. - Cela découle du fait que, si on décrit la situation au point $P=$ $\left(P_{1}, P_{2}\right)$ d'image $Q$, après complétion et choix d'une racine $e$-ième de l'unité $\zeta_{e}=$ $\left(\zeta_{n}\right)^{n / e}$, on a $\hat{\theta}_{P}=k[[x, y]] /(x y)$, l'action de $H$ stabilisateur de $P$ étant $x \mapsto \zeta^{k} x$, $y \mapsto \zeta^{-k} y$. Alors $\hat{\theta}_{Q}=k[[X, Y]] /(X Y)$ où $X=x^{e}, Y=y^{e}$. Si $\eta k \equiv 1(\bmod e)$, de 
sorte qu'on a $\mathscr{L}_{Q}=x^{\eta} \emptyset_{Q}+y^{e-\eta} \bigoplus_{Q}$, module sans torsion de rang 1 . Par passage à la fibre en $Q$, on voit alors que

$$
\mathscr{L}_{Q} \otimes k=x^{\eta} k[[X]] \otimes k \oplus y^{e-\eta} k[[Y]] \otimes k=\mathscr{L}_{P_{1}} \otimes k \oplus \mathscr{L}_{P_{2}} \otimes k \cong k^{2} .
$$

Ceci justifie la relation (128). Pour une preuve en familles, on peut se limiter à une base $S=\operatorname{Spec}(R)$. Alors $D=\operatorname{Spec}(R[[X, Y]] /(X Y))$ et $\mathscr{L}_{Q}=x^{\eta} \vartheta_{Q} \oplus y^{e-\eta} \theta_{Q}$. Ainsi en posant $\xi=x^{\eta}, v=y^{e-\eta}(\operatorname{voir}(77)$ et proposition 7.3.7), on a

$$
\mathscr{L}_{Q} \otimes_{\vartheta_{Q}} R=x^{\eta} R \oplus y^{e-\eta} R=\left(\mathscr{L}_{1}\right)_{Q_{1}} \oplus\left(\mathscr{L}_{2}\right)_{Q_{2}} .
$$

La conclusion est mieux décrite par le diagramme 2-cartésien de la figure 1, dans lequel $\mathbb{P}\left(j^{*}(\mathscr{L})\right)=\mathbb{P}\left(p_{1}^{*}\left(\mathscr{L}_{1}\right) \oplus p_{2}^{*}\left(\mathscr{L}_{2}\right)\right)$ est un fibré en $\mathbb{P}^{1}$ d'image

$$
Z_{\pi}=Z_{\pi, 0} \cup E_{\pi} \cup Z_{\pi, \infty} \subset \widetilde{\mathscr{D}} \text {. }
$$

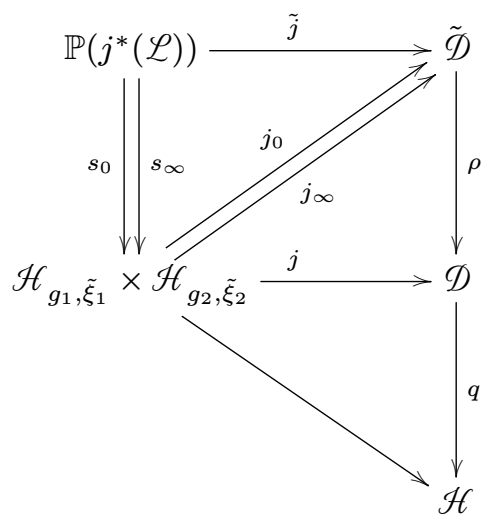

Figure 1. Préimage du faisceau $\mathscr{L}$

Les deux sections canoniques $s_{0}, s_{\infty}$ de la fibration en $\mathbb{P}^{1}$ donnent les deux relèvements $j_{0}, j_{\infty}$ de $j$, d'images $Z_{0}, Z_{\infty}$. Le faisceau inversible $\mathscr{L}_{1}$ n'est autre que le faisceau inversible qui a pour fibre en $\left(C_{1} \longrightarrow D_{1}, P_{1}\right), Q_{1}^{*}\left(\mathscr{L}_{1}\right)$ noté aussi $\mu_{0}$; même chose pour $\mathscr{L}_{2}$ et $\mu_{\infty}$. Ces faisceaux inversibles sont reliés aux faisceaux inversibles $\psi_{0}$ et $\psi_{\infty}\left(\right.$ les $\psi_{ \pm}$de Mumford [80]) par les relations :

$$
n_{1} \mu_{0}=\frac{n_{1}}{e} \eta_{1} \psi_{0}, n_{2} \mu_{\infty}=\frac{n_{2}}{e} \eta_{2} \psi_{\infty}
$$

où la notation est additive et on a conservé la notation $\eta=\eta_{1}, e-\eta=\eta_{2}$. En particulier, en substituant la notation $0, \infty$ à 1,2 :

$$
n \mu_{0}=m \eta_{0} \psi_{0} \quad, \quad n \mu_{\infty}=m \eta_{\infty} \psi_{\infty} .
$$


Les notations signifient que le diviseur de branchement se décompose en $B=\frac{n}{n_{1}} B_{1}+$ $\frac{n}{n_{2}} B_{2}$ avec

$$
B_{1}=\sum_{i=1}^{b_{1}} m_{i}^{1} \eta_{i}^{1} Q_{i}^{1} \quad, \quad B_{2}=\sum_{j=1}^{b_{2}} m_{j}^{2} \eta_{j}^{2} Q_{j}^{2}
$$

avec les congruences habituelles

$$
\sum_{i=1}^{b_{1}} m_{i}^{1} \eta_{i}^{1}+\frac{n_{1}}{e} \eta_{1} \equiv 0 \quad\left(\bmod n_{1}\right) \quad, \quad \sum_{j=1}^{b_{2}} m_{j}^{2} \eta_{j}^{2}+\frac{n_{2}}{e} \eta_{2} \equiv 0 \quad\left(\bmod n_{2}\right) .
$$

L'étape suivante revient à identifier le faisceau conormal le long du lieu singulier $Z \subset \widetilde{\mathscr{D}}$. Comme $\widetilde{D} \longrightarrow \mathcal{H}$ est une courbe nodale, $Z$ est localement une intersection complète de codimension 2 dans $\widetilde{D}$. Au-dessus d'un point $(C \longrightarrow D, Q)$, où $Q$ est un point double NS, la fibre de $\rho$ est

$$
\operatorname{Proj}(\mathscr{L} \otimes k(Q))=\mathscr{L}_{1} \otimes k\left(Q_{1}\right) \oplus \mathscr{L}_{2} \otimes k\left(Q_{2}\right)
$$

si les branches (ordonnées) en $Q$ sont $D_{1}=\{Y=0\}, D_{2}=\{X=0\}$, et si les origines sont $Q_{1} \in D_{1}, Q_{2} \in D_{2}$. On utilisera de manière interchangeable la notation $D_{1}=D_{0}, D_{2}=D_{\infty}$ pour assurer une certaine cohérence avec les points $z_{0}, z_{\infty}$, et donc $\mu_{1}=\mu_{0}, \mu_{2}=\mu_{\infty}$.

On suppose avoir $\mathscr{D}=\operatorname{Spec}(A)$ avec $A=k[[X, Y]] /(X Y)$, et

$$
\widetilde{D}=\operatorname{Proj}(A[\xi, v] /(Y \xi, X v)) .
$$

Cela correspond comme précédemment à $\mathscr{L}=A \xi+A v$, et $\mathscr{L} \otimes k=\mathscr{L}_{1} \otimes k \oplus \mathscr{L}_{2} \otimes k=$ $k \xi \oplus k v$. Rappelons qu'on pose $\xi=x^{\eta}, v=y^{e-\eta}$, de sorte que $Y \xi=X v=0$, et qu'on utilise de manière alternative la notation $\eta=\eta_{1}, e-\eta=\eta_{2}$ pour signifier qu'on a numéroté les deux branches au point double $Q$, de 1 à 2 (ou $\{0, \infty\}$ ). D'une autre manière,

$$
\widetilde{D}=\operatorname{Proj}(k[\xi, v] /(Y \xi, X v))=D_{1} \cup E \cup D_{2} \quad\left(E=\mathbb{P}^{1}\right)
$$

avec les deux points $z_{0}=D_{1} \cap E, z_{\infty}=D_{2} \cap E \in Z$. Précisons les notations. Le point $z_{\infty}=(0: 1)$ correspond à la projection $\mathscr{L}_{1} \otimes k\left(Q_{1}\right) \oplus \mathscr{L}_{2} \otimes k\left(Q_{2}\right) \longrightarrow \mathscr{L}_{2} \otimes k\left(Q_{2}\right)$, et $z_{0}=(1: 0)$ à la seconde projection. On a le lemme élémentaire :

LEMme 10.4.4. - Soit $\pi: P=\mathbb{P}\left(\mathscr{L}_{1} \oplus \mathscr{L}_{2}\right) \longrightarrow S$ une fibration en droites projectives, avec $\mathscr{L}_{1}, \mathscr{L}_{2}$ des faisceaux inversibles sur $S$. Soit le point $z_{0}=[1: 0] \in P$ (resp. $z_{\infty}=[0: 1]$ ) correspondant à la projection $\mathscr{L}_{1} \oplus \mathscr{L}_{2} \longrightarrow \mathscr{L}_{1}$ (resp. la projection $\left.\mathscr{L}_{1} \oplus \mathscr{L}_{2} \longrightarrow \mathscr{L}_{2}\right)$, et notons $D_{0} \subset P$ (resp. $D_{\infty}$ ) l'image. Alors le faisceau conormal $N_{D_{0}}^{\vee}=z_{0}^{*}\left(\Theta\left(-D_{0}\right)\right)$ est $\mathscr{L}_{2} \otimes \mathscr{L}_{1}^{-1}$ (resp. $N_{D_{\infty}}^{\vee}=z_{\infty}^{*}\left(\theta\left(-D_{\infty}\right)\right)=\mathscr{L}_{1} \otimes \mathscr{L}_{2}^{-1}$.

On est en position de pouvoir décrire le faisceau conormal à $Z_{\pi} \subset \widetilde{D}$ lorsque $\pi$ est de type NS, le cas d'une composante de type RR se traitant de manière standard. Oubliant provisoirement l'indice $\pi$, la composante $Z_{\pi}$ de type NS se décompose en

$$
\mathcal{Z}=Z_{0} \sqcup Z_{\infty}
$$


où les deux composantes ont pour image par $\rho$ la composante $\phi_{\pi} \subset \mathscr{D}$. On a vu que pour chacune de ces deux composantes, le faisceau conormal est la somme directe des faisceaux cotangents en les origines des branches des composantes du point double. Ainsi $Q_{1} \in D_{1}$ la branche de paramètre $x$, et $z_{0} \in E=\mathbb{P}^{1}$ pour la première, et $Q_{2} \in D_{2}$ et $z_{\infty} \in E$ pour la seconde. De la description précédente il est facile d'en déduire le faisceau conormal le long de $Z_{0}$ (resp. $Z_{\infty}$ ). On trouve

$$
\mathcal{N}_{Z_{0}}=j_{0, *}\left(\psi_{0} \oplus\left(\mathscr{L}_{2} \otimes \mathscr{L}_{1}^{-1}\right)\right) \quad, \quad \mathcal{N}_{Z_{\infty}}=j_{\infty, *}\left(\psi_{\infty} \oplus\left(\mathscr{L}_{1} \otimes \mathscr{L}_{2}^{-1}\right)\right)
$$

ou d'une autre manière

$$
\mathcal{N}_{Z_{0}}=j_{0, *}\left(\psi_{0} \oplus\left(\mu_{\infty} \otimes \mu_{0}^{-1}\right)\right) \quad, \quad \mathcal{N}_{Z_{\infty}}=j_{\infty, *}\left(\psi_{\infty} \oplus\left(\mu_{0} \otimes \mu_{\infty}^{-1}\right)\right) .
$$

Dans (130), les faisceaux inversibles sont vus sur $\mathcal{H}_{g_{1}, n_{1}, \tilde{\xi}_{1}}^{\prime} \times \mathcal{H}_{g_{2}, n_{2}, \tilde{\xi}_{2}}^{\prime}$. Ce sont les images inverses des faisceaux de même nom sur chaque facteur. Concentrons nous sur la contribution de la partie singulière de l'intersection (127), c'est-à-dire le terme

$$
\vartheta_{\widetilde{D}}\left(\sum_{\pi=\mathrm{NS}} \frac{a b}{m} E_{\pi}\right)
$$

avec $a b / m=m \eta_{0} \eta_{\infty}$. Supprimons provisoirement l'indice $\pi$, et évaluons l'image inverse $j_{0}^{*}(E)$ (resp. $j_{\infty}^{*}(E)$ ). Du fait que le diviseur de branchement $B$ est disjoint du lieu singulier,

$$
j_{0}^{*}(\theta(-n))=j_{0}^{*}\left(\Theta\left(m \eta_{0} \eta_{\infty} E\right)\right)=s_{0}^{*} \tilde{j}^{*}(\theta(-n))=\mu_{0}^{-n}
$$

et de même $j_{\infty}^{*}(\theta(-n))=\mu_{\infty}^{-n}$. Ainsi, par comparaison avec (129), on obtient la relation entre classes de Chern.

LEMME 10.4.5. - On a les relations

$$
\eta_{\infty} j_{0}^{*}(-E)=\psi_{0} \quad \text { et } \quad \eta_{0} j_{\infty}^{*}(-E)=\psi_{\infty} .
$$

Revenons maintenant à l'expression (127), c'est-à-dire

$$
\tilde{q}_{*}\left(\exp \left(-\sum_{\pi=\mathrm{NS}} \frac{\eta_{0} \eta_{\infty}}{e} E_{\pi}\right) \mathrm{Td}^{\vee}\left(-\imath_{*}\left(\theta_{Z}\right)\right)\right) .
$$

LEMME 10.4.6. - Le morphisme fini surjectif $j: \mathcal{H}_{g_{1}, \tilde{\xi}_{1}}^{\prime} \times \mathcal{H}_{g_{2}, \tilde{\xi}_{2}}^{\prime} \longrightarrow \tilde{\phi}_{\pi}$ est de degré $n / e^{2}$.

Démonstration. - Le groupe $G$ est abélien, les éléments de $G$ sont des automorphismes pour un revêtement générique $(C \longrightarrow D) \in Z_{\pi}$. On voit immédiatement que le degré grossier de $j$, c'est-à-dire au niveau des espaces grossiers de modules est un. L'isotropie générique d'un point de $\mathcal{H}_{g_{1}, \tilde{\xi}_{1}}^{\prime} \times \mathcal{H}_{g_{2}, \tilde{\xi}_{2}}^{\prime}$ est le groupe $H^{2}$, donc d'ordre $e^{2}$. Le résultat suit.

Pour évaluer le terme de droite du produit de l'intérieur des parenthèses, on utilise l'expression de Mumford, sous la forme utilisée Coates-Givental [23]. Soient $i_{0}: Z_{0} \hookrightarrow$ $\tilde{D}$ et $i_{\infty}: Z_{\infty} \hookrightarrow \tilde{D}$ les immersions régulières de codimension 2 , et $N_{0}$ le faisceau conormal (localement libre de rang 2). Rappelons qu'on travaille à $\pi$ fixé. 
Lemme 10.4.7. - Il y a une série formelle en deux variables $P(U, V)$ telle que

$$
\operatorname{Td}^{\vee}\left(-\left[\theta_{Z}\right]\right)-1=i_{0, *} P\left(c_{1}\left(N_{0}\right), c_{2}\left(N_{0}\right)\right)+i_{\infty, *} P\left(c_{1}\left(N_{\infty}\right), c_{2}\left(N_{\infty}\right)\right) .
$$

Démonstration. - C'est la relation de Mumford qui découle du théorème de GRR appliqué à l'immersion régulière $i_{0}$, respectivement $i_{\infty}$.

Pour exploiter l'expression (132) notons que, les coefficients étant rationnels, on a $A_{\bullet}\left(\mathcal{Z}_{0}\right)=\left(A_{\bullet}\left(\tilde{Z}_{0}\right)_{r e d}\right)$, et $\left[\tilde{Z}_{0}\right]=\eta_{\infty}\left[\left(Z_{0}\right)_{r e d}\right]$. On a un résultat analogue pour la composante $Z_{\infty}$. En particulier,

$$
\begin{aligned}
\left(i_{0, *} P\left(c_{1}\left(N_{0}\right), c_{2}\left(N_{0}\right)\right)\right)[\tilde{\mathscr{D}}] & =i_{0, *}\left(P\left(c_{1}\left(N_{0}\right), c_{2}\left(N_{0}\right)\right) \cdot i_{0}^{!}[\tilde{\mathscr{D}}]\right) \\
& =\eta_{\infty} i_{0, *}\left(P\left(c_{1}\left(N_{0}\right), c_{2}\left(N_{0}\right)\right)\left[\left(\mathcal{Z}_{0}\right)_{r e d}\right]\right) \\
& =\eta_{\infty}\left(P\left(c_{1}\left(N_{0}\right), c_{2}\left(N_{0}\right)\right) \cdot j_{0, *}\left[\mathcal{H}_{g_{1}, \tilde{\xi}_{1}}^{\prime} \times \mathcal{H}_{g_{2}, \tilde{\xi}_{2}}^{\prime}\right]\right) \\
& =\eta_{\infty} j_{0, *}\left(j_{0}^{*}\left(P\left(c_{1}\left(N_{0}\right), c_{2}\left(N_{0}\right)\right)\right) \cdot\left[\mathcal{H}_{g_{1}, \tilde{\xi}_{1}}^{\prime} \times \mathcal{H}_{g_{2}, \tilde{\xi}_{2}}^{\prime}\right]\right) .
\end{aligned}
$$

Le calcul de Mumford ramène finalement l'expression (133) à

$1+\frac{1}{2} \sum_{k \geqslant 1} \frac{B_{k+1}}{(k+1) !}\left(\sum_{\pi=\mathrm{NS}} \eta_{\infty} j_{0, *}^{\pi}\left[\frac{\psi_{0}^{k}+\left(\frac{\eta_{\infty} \psi_{\infty}-\eta_{0} \psi_{0}}{e}\right)^{k}}{\frac{\eta_{\infty}}{e}\left(\psi_{0}+\psi_{\infty}\right)}\right]+\eta_{0} j_{\infty, *}^{\pi}\left[\frac{\psi_{\infty}^{k}+\left(\frac{\eta_{0} \psi_{0}-\eta_{\infty} \psi_{\infty}}{e}\right)^{k}}{\frac{\eta_{0}}{e}\left(\psi_{0}+\psi_{\infty}\right)}\right]\right)$.

Noter que $\psi_{0}+\frac{\eta_{\infty} \psi_{\infty}-\eta_{0} \psi_{0}}{e}=\frac{\eta_{\infty}}{e}\left(\psi_{0}+\psi_{\infty}\right)$, et que $B_{k}=0$ si $k>1$ impair.

Les termes relatifs à des indices $\pi$ distincts ont une intersection nulle, dès lors l'exponentielle dans le produit (127) se simplifie en

$$
1+\sum_{\pi=\mathrm{NS}} \sum_{h \geqslant 1}\left(\frac{\eta_{0} \eta_{\infty}}{e}\right)^{h} \frac{\left(-E_{\pi}\right)^{h}}{h !} .
$$

De la sorte, on peut se concentrer sur les seuls termes à $\pi$ fixé. Cela permet d'alléger les formules en omettant la référence à $\pi$ dans les expressions qui suivent. Le produit d'intersection de (132) et de (134) se traite avec la formule de projection qui permet de tout ramener sur $\mathcal{H}_{g_{1}, \tilde{\xi}_{1}}^{\prime} \times \mathcal{H}_{g_{2}, \tilde{\xi}_{2}}^{\prime}$. On utilise la relation

$$
(-E)^{h} j_{0, *}(\cdot)=j_{0, *}\left(\left(\frac{\psi_{0}}{\eta_{\infty}}\right)^{h}(\cdot)\right) .
$$

Tenant compte de la formule de projection et des relations (131), l'image par $\tilde{q}_{*}$ du produit de (127) et de (133) se décompose en trois termes, d'une part l'image par $\imath_{*}$ de

$$
\frac{1}{2} \sum_{d \geqslant 1} \frac{1}{(d+1) !} \sum_{k+h=d} \eta_{\infty} B_{k+1}\left(\begin{array}{l}
d+1 \\
k+1
\end{array}\right)\left(\frac{\eta_{0} \psi_{0}}{e}\right)^{h}\left[\frac{\psi_{0}^{k}+\left(\frac{\eta_{\infty} \psi_{\infty}-\eta_{0} \psi_{0}}{e}\right)^{k}}{\frac{\eta_{\infty}}{e}\left(\psi_{0}+\psi_{\infty}\right)}\right]
$$

et d'autre part l'expression symétrique, le second crochet

$$
\frac{1}{2} \sum_{d \geqslant 1} \frac{1}{(d+1) !} \sum_{k+h=d} \eta_{0} B_{k+1}\left(\begin{array}{l}
d+1 \\
k+1
\end{array}\right)\left(\frac{\eta_{\infty} \psi_{\infty}}{e}\right)^{h}\left[\frac{\psi_{\infty}^{k}+\left(\frac{\eta_{0} \psi_{0}-\eta_{\infty} \psi_{\infty}}{e}\right)^{k}}{\frac{\eta_{0}}{e}\left(\psi_{0}+\psi_{\infty}\right)}\right] .
$$


Dans ces deux expressions, les sommations portent sur $k \geqslant 1$ et $h \geqslant 0$ tels que $h+k=d \geqslant 1$. Il reste à traiter dans (127) le produit de l'exponentielle avec le terme constant 1 de celui de droite, soit

$$
\tilde{q}_{*}\left(\sum_{\pi=\mathrm{NS}} \sum_{h \geqslant 1}\left(\frac{\eta_{0} \eta_{\infty}}{e}\right)^{h} \frac{\left(-E_{\pi}\right)^{h}}{h !}\right)
$$

c'est-à-dire à évaluer le terme $\tilde{q}_{*}\left(-E_{\pi}\right)^{h}$, pour $h \geqslant 1$ et $\pi$ fixés. On va utiliser le diagramme de la figure 1 (page 194) pour revenir sur la fibration en $\mathbb{P}^{1}$. On utilise dans cette évaluation le fait que $\theta(n)=\Theta\left(-\frac{a b}{m} E+\star\right)$, où $(\star)$ regroupe des diviseurs disjoints de $E=E_{\pi}=\tilde{j}_{*}\left(\left[\mathbb{P}\left(\mathscr{L}_{1} \oplus \mathscr{L}_{2}\right)\right]\right)$. Dans l'anneau de Chow, on a ainsi les égalités

$$
\begin{aligned}
(-E)^{h}=-c_{1}\left(\theta_{E}(-E)\right)^{h-1} & =-\left(\frac{e}{\eta_{0} \eta_{\infty}}\right)^{h-1} c_{1}\left(\theta_{E}(1)\right)^{h-1} \\
& =-\left(\frac{e}{\eta_{0} \eta_{\infty}}\right)^{h-1} \tilde{j}_{*}\left(c_{1}(\theta(1))^{h-1} .\right.
\end{aligned}
$$

Soit le fibré en $\mathbb{P}^{1}$ :

$$
p: \mathbb{P}^{1}\left(\mathscr{L}_{1} \oplus \mathscr{L}_{2}\right) \longrightarrow \mathcal{H}_{g_{1}, \tilde{\xi}_{1}}^{\prime} \times \mathcal{H}_{g_{2}, \tilde{\xi}_{2}}^{\prime},
$$

avec ses sections $s_{1}$ (resp. $\left.s_{2}\right)$ d'image $D_{1}\left(\right.$ resp. $\left.D_{2}\right)$. Notons que par construction $\tilde{j}^{*}(\Theta(1))=\Theta(1)$, alors tenant compte de la relation $\Theta(1)=\Theta\left(D_{1}\right) \otimes p^{*}\left(\mathcal{L}_{2}\right)$, on a

$$
\left.\left.p_{*}\left(c_{1}(\Theta(1))\right)^{h}\right)=\sum_{j=0}^{h}\left(\begin{array}{l}
h \\
j
\end{array}\right) p_{*}\left(c_{1}\left(\Theta\left(D_{1}\right)\right)\right)^{j} p^{*}\left(c_{1}\left(\mathscr{L}_{2}\right)^{k-j}\right)\right) .
$$

D'une part, le terme avec $j=0$ a une contribution nulle, et d'autre part en appliquant la formule de projection, cette expression se réduit à

$$
\begin{aligned}
\left.p_{*}\left(c_{1}(\Theta(1))\right)^{h}\right) & =\sum_{j=1}^{h}\left(\begin{array}{l}
h \\
j
\end{array}\right) p_{*}\left(c_{1}\left(\Theta\left(D_{1}\right)\right)^{j}\right) c_{1}\left(\mathscr{L}_{2}\right)^{h-j} \\
& =\sum_{j=1}^{h}\left(\begin{array}{l}
h \\
j
\end{array}\right) c_{1}\left(\Theta_{D_{1}}\left(D_{1}\right)\right)^{j-1} c_{1}\left(\mathscr{L}_{2}\right)^{h-j} .
\end{aligned}
$$

Mais $\vartheta_{D_{1}}\left(D_{1}\right)=\mathscr{L}_{1} \otimes \mathscr{L}_{2}^{-1}$, de sorte que le terme de droite de (138) se transforme en

$$
\sum_{j=1}^{h}\left(\begin{array}{l}
h \\
j
\end{array}\right)\left(c_{1}\left(\mathscr{L}_{1}\right)-c_{1}\left(\mathscr{L}_{2}\right)\right)^{j-1} c_{1}\left(\mathscr{L}_{2}\right)^{h-j}
$$

expression qu'on peut écrire de manière formelle

$$
\frac{\sum_{j=1}^{h}\left(\begin{array}{c}
h \\
j
\end{array}\right)\left(c_{1}\left(\mathscr{L}_{1}\right)-c_{1}\left(\mathscr{L}_{2}\right)\right)^{j} c_{1}\left(\mathscr{L}_{2}\right)^{h-j}}{c_{1}\left(\mathscr{L}_{1}\right)-c_{1}\left(\mathscr{L}_{2}\right)}=\frac{\left(c_{1}\left(\mathscr{L}_{1}\right)\right)^{h}-\left(c_{1}\left(\mathscr{L}_{2}\right)\right)^{h}}{c_{1}\left(\mathscr{L}_{1}\right)-c_{1}\left(\mathscr{L}_{2}\right)}
$$


et qui se réduit ainsi à $\sum_{\alpha=0}^{h-1} c_{1}\left(\mathscr{L}_{1}\right)^{\alpha} c_{1}\left(\mathscr{L}_{2}\right)^{h-1-\alpha}$. Finalement, le résultat du calcul conduit à

$$
\begin{aligned}
\tilde{q}_{*}\left(\sum_{h \geqslant 1}\left(\frac{\eta_{0} \eta_{\infty}}{e}\right)^{h} \frac{(-E)^{h}}{h !}\right) & =\imath_{*}\left(\sum_{h \geqslant 1}\left(\frac{\eta_{0} \eta_{\infty}}{e}\right)^{h} \frac{1}{h !}\left(-\left(\frac{e}{\eta_{0} \eta_{\infty}}\right)^{h-1} p_{*}\left(c_{1}(\theta(1))^{h-1}\right)\right)\right) \\
& =-\imath_{*}\left(\sum_{h \geqslant 1, \alpha<h-1} \frac{\eta_{0} \eta_{\infty}}{e} \frac{1}{h !} c_{1}\left(\mathcal{L}_{1}\right)^{\alpha} c_{1}\left(\mathscr{L}_{2}\right)^{h-2-\alpha}\right) \\
& =-\sum_{h \geqslant 2} \frac{\eta_{0} \eta_{\infty}}{e h !} \sum_{0 \leqslant \alpha \leqslant h-2}\left(\frac{\eta_{0} \psi_{0}}{e}\right)^{\alpha}\left(\frac{\eta_{\infty} \psi_{\infty}}{e}\right)^{h-2-\alpha} .
\end{aligned}
$$

Tenant compte du facteur $1 / 2$ qui corrige le fait que les branches sont numérotées, la contribution en degré $d+1$ est alors :

$$
\frac{-1}{2}\left(\frac{\eta_{0} \eta_{\infty}}{e}\right) \frac{1}{(d+1) !} \sum_{0 \leqslant \alpha \leqslant d-1}\left(\frac{\eta_{0} \psi_{0}}{e}\right)^{\alpha}\left(\frac{\eta_{\infty} \psi_{\infty}}{e}\right)^{d-1-\alpha}
$$

Il ne reste plus qu'à mettre ensemble les pièces du puzzle, donc effectuer la somme des expressions (135), plus l'expression symétrique (136), avec (137). On peut se limiter aux seuls termes homogènes de degré $d-1$ en $\psi_{0}, \psi_{\infty}$. On doit multiplier les expressions (135), (136) et (139) par $\psi_{0}+\psi_{\infty}$, ce qui, en laissant de côté le coefficient $1 /(2(d+1)$ !), donne pour la partie homogène de degré $d$ :

$$
\begin{aligned}
& \sum_{k+h=d, k \geqslant 1} \eta_{\infty} B_{k+1}\left(\begin{array}{l}
d+1 \\
k+1
\end{array}\right)\left(\frac{\eta_{0}}{e}\right)^{h}\left[\frac{\psi_{0}^{d}+\psi_{0}^{h}\left(\frac{\eta_{\infty} \psi_{\infty}-\eta_{0} \psi_{0}}{e}\right)^{k}}{\frac{\eta_{\infty}}{e}}\right] \\
& +\sum_{k+h=d, k \geqslant 1} \eta_{0} B_{k+1}\left(\begin{array}{l}
d+1 \\
k+1
\end{array}\right)\left(\frac{\eta_{\infty}}{e}\right)^{h}\left[\frac{\psi_{\infty}^{d}+\psi_{\infty}^{h}\left(\frac{\eta_{0} \psi_{0}-\eta_{\infty} \psi_{\infty}}{e}\right)^{k}}{\frac{\eta_{0}}{e}}\right] \\
& -\left(\frac{\eta_{0} \eta_{\infty}}{e}\right)\left(\psi_{0}+\psi_{\infty}\right) \sum_{0 \leqslant \alpha \leqslant d-1}\left(\frac{\eta_{0} \psi_{0}}{e}\right)^{\alpha}\left(\frac{\eta_{\infty} \psi_{\infty}}{e}\right)^{d-1-\alpha} .
\end{aligned}
$$

Isolons le coefficient du monôme $\psi_{0}^{a} \psi_{\infty}^{b}$. Supposons d'abord $a, b \geqslant 1$. Le coefficient qui tient compte de la contribution des trois expressions est :

$$
\begin{gathered}
-e \sum_{k+h=d, k \geqslant b}(-1)^{b} B_{k+1}\left(\begin{array}{l}
d+1 \\
k+1
\end{array}\right)\left(\begin{array}{l}
k \\
b
\end{array}\right)\left(\frac{\eta_{0}}{e}\right)^{a}\left(\frac{\eta_{\infty}}{e}\right)^{b} \\
-e \sum_{l+h=d, l \geqslant a}(-1)^{a} B_{l+1}\left(\begin{array}{l}
d+1 \\
l+1
\end{array}\right)\left(\begin{array}{l}
l \\
a
\end{array}\right)\left(\frac{\eta_{0}}{e}\right)^{a}\left(\frac{\eta_{\infty}}{e}\right)^{b}-e\left(\frac{\eta_{0}}{e}\right)^{a}\left(\frac{\eta_{\infty}}{e}\right)^{b} .
\end{gathered}
$$

LEMME 10.4.8. - Sous les conditions précédentes $(a+b=d, a, b \geqslant 1)$, on $a$ :

$$
-e \sum_{k+h=d, k \geqslant b}(-1)^{b} B_{k+1}\left(\begin{array}{l}
d+1 \\
k+1
\end{array}\right)\left(\begin{array}{l}
k \\
b
\end{array}\right)-e \sum_{l+h=d, l \geqslant a}(-1)^{a} B_{l+1}\left(\begin{array}{l}
d+1 \\
l+1
\end{array}\right)\left(\begin{array}{l}
l \\
a
\end{array}\right)-e=0 .
$$


Démonstration. - voir Chiodo [22].

Il reste à identifier les termes extrêmes. Le coefficient de $\psi^{d}$ est facile à isoler. Rappelant que $B_{1}=-1 / 2$, on trouve

$$
\left.e\left(B_{d+1}\left(\frac{\eta_{0}}{e}\right)-\frac{\eta_{0}}{e}\right)^{d+1}-\left(B_{d+1}(1)-1+\frac{d+1}{2}\right)\left(\frac{\eta_{0}}{e}\right)^{d}\right)-\eta_{\infty}\left(\frac{\eta_{0}}{e}\right)^{d} .
$$

On peut supposer $d$ impair, donc $B_{d+1}(1)=B_{d+1}$, de sorte qu'après simplification, on obtient à côté du terme $e B_{d+1}\left(\frac{\eta_{0}}{e}\right)$

$$
=-e\left(\frac{\eta_{0}}{e}\right)^{d+1}+e\left(\frac{\eta_{0}}{e}\right)^{d}-\eta_{\infty}\left(\frac{\eta_{0}}{e}\right)^{d}=\left(\frac{\eta_{0}}{e}\right)^{d}\left(e-\eta_{0}\right)-\eta_{\infty}\left(\frac{\eta_{0}}{e}\right)^{d}=0 .
$$

On a une expression analogue pour le terme symétrique. En resumé, on a obtenu pour la somme des trois expressions

$$
e\left(\frac{B_{d+1}\left(\frac{\eta_{0}}{e}\right) \psi_{0}^{d}+B_{d+1}\left(\frac{\eta_{\infty}}{e}\right) \psi_{\infty}^{d}}{\psi_{0}+\psi_{\infty}}\right)=e\left(\frac{B_{d+1}\left(\frac{\eta_{0}}{e}\right)\left(\psi_{0}^{d}+\psi_{\infty}^{d}\right)}{\psi_{0}+\psi_{\infty}}\right)
$$

et comme on peut supposer $d$ impair, l'expression se réduit à

$$
e B_{d+1}\left(\frac{\eta_{0}}{e}\right) \sum_{a+b=d-1}\left(-\psi_{0}\right)^{a} \psi_{\infty}^{b}
$$

Les différentes contributions à la formule de Riemann-Roch, une fois réunies, conduisent au résultat final, soit :

$$
\begin{gathered}
\operatorname{ch}\left(\mathrm{R} \tilde{q}_{!}(\Theta(1))[\mathcal{H}]=\sum_{d \geqslant 0} \frac{B_{d+1}}{n(d+1) !} \kappa_{d}\right. \\
-\sum_{i} \frac{B_{d+1}\left(\frac{\eta_{i}}{e_{i}}\right)}{(d+1) !} \psi_{i}^{d}+\sum_{\pi, d \geqslant 1} \frac{e}{(d+1) !} B_{d+1}\left(\frac{\eta_{0}}{e}\right)\left(\sum_{a+b=d-1}\left(-\psi_{0}\right)^{a} \psi_{\infty}^{b}\right)[\mathcal{H}] .
\end{gathered}
$$

Parallèlement à la relation (140), il est utile de noter que la propriété d'annulation de Mumford $\mathrm{ch}_{2 k}(\mathbb{E})=0(k=1,2, \ldots)([\mathbf{8 0}])$ est vérifiée par les facteurs isotypiques $\mathbb{E}_{v}$ comme noté dans ([17], prop. 3.2) :

Proposition 10.4.9. - Pour tout facteur isotypique $\mathbb{E}_{v}(v \in \hat{G})$, on a la relation

$$
\operatorname{ch}\left(\mathbb{E}_{v}\right)+\operatorname{ch}\left(\left(\mathbb{E}_{v}\right)^{\vee}\right)=0 .
$$

Démonstration. - Pour les détails, on se reportera à ([17]). La théorie de Hodge montre que le fibré $V$, le terme du milieu de l'extension 104, est muni de la connexion de Gauss-Manin, régulière au-dessus de $\mathscr{H}_{g, G, \xi}$, et à pôles logarithmiques le long du bord, qui est un diviseur à croisements normaux. La propriété de nilpotence de la partie polaire implique que les classes de Chern de $V$ sont triviales. Dans la situation équivariante l'extension (104) se décompose selon les caractères de $G$ en une somme directe d'extensions

$$
0 \longrightarrow \mathbb{E}_{v} \longrightarrow V_{v} \longrightarrow\left(\mathbb{E}_{v}\right)^{\vee} \longrightarrow 0
$$


L'argument de Mumford rappelé ci-dessus s'applique tel quel aux facteurs isotypiques $\mathrm{du}$ fait que la connexion préserve ces facteurs. La suite exacte de dessus donne alors la relation $c\left(\mathbb{E}_{v} \oplus\left(\mathbb{E}_{v}\right)^{\vee}\right)=0$, d'où vient la relation (141).

\subsection{La relation de Cornalba-Harris revisitée}

10.5.1. Les faisceaux inversibles $\psi$ et $\mu$ dans le cas cyclique. - Dans cette section, le groupe $G$ est cyclique. On va voir que les relations entre les faisceaux inversibles $\psi, \mu$ introduits dans la section 10.2 peuvent être précisées. Soit $G=\mathbb{Z} / n \mathbb{Z}$, la classe de 1 correspondant à l'automorphisme noté $\sigma$. Pour tout $j \in \mathbb{Z}$, soit $\chi_{j}$ le caractère tel que $\chi_{j}(\sigma)=\zeta_{n}^{j}$. L'holonomie en un point de branchement $Q_{i}$ est définie par l'entier $k_{i}, 1 \leqslant k_{i}<e_{i}, \operatorname{pgcd}\left(k_{i}, e_{i}\right)=1$. Soit $1 \leqslant \nu_{i}<e_{i}$ tel que $k_{i} \nu_{i} \equiv 1$ $\left(\bmod e_{i}\right)$.

Rappelons que $\mu_{i, \alpha}=\psi_{i, \alpha}$ (proposition 10.2.1).

Proposition 10.5.1. - Soit $\alpha=l e_{i}+k<n$, avec $0 \leqslant k<e_{i}$. Alors :

$$
\psi_{i, \alpha}=\psi_{i, k} \otimes \psi_{i, e_{i}}^{\otimes l} \quad, \quad \psi_{i, e_{i}}^{\otimes m_{i}}=0 \quad\left(\psi_{0}=0\right) \quad \text { et } \quad \psi_{i, \alpha}=\psi_{i, 1}^{\alpha} \psi_{i}^{-\left[\frac{\alpha \nu_{i}}{e_{i}}\right]} .
$$

Le morphisme d'évaluation ev $v_{i}^{*}: \operatorname{Pic}\left(B\left(C_{G}\left(H_{i}\right) / H_{i}\right)\right) \longrightarrow \operatorname{Pic}\left(\overline{\mathscr{H}}_{g, n, \xi}\right)$ est injectif.

Démonstration. - Le morphisme $\pi: \Delta_{i} \longrightarrow S$ est un $G / H_{i}$-torseur, il en découle immédiatement que si $\alpha=l e_{i}, \psi_{i, \alpha}=\psi_{i, e_{i}}^{\otimes l}$, et $\psi_{i, e_{i}}^{\otimes m_{i}}=0$. En considérant la décomposition du faisceau $\pi_{*}\left(\theta_{\Delta_{i}}\left(-k \Delta_{i}\right)\right.$ ) (108), on voit facilement que les facteurs indexés par les $j \equiv k k_{i}\left(\bmod e_{i}\right)$ sont $\psi_{j+l e_{i}}=\psi_{i, j} \otimes \psi_{i, e_{i}}^{\otimes l}$. On peut justifier ces relations d'une autre manière, en observant qu'au voisinage de la section $Q_{i}$ on a $\mathscr{L}_{j}=\mathscr{L}^{\otimes j}\left[\frac{j \nu_{i}}{e_{i}} Q_{i}\right]$. Par image réciproque, on en tire immédiatement, pour tout indice $\alpha$, la relation $\psi_{i, \alpha}=\psi_{i, 1}^{\alpha} \psi_{i}^{-\left[\frac{\alpha \nu_{i}}{e_{i}}\right]}$.

Prouvons le dernier point. On se limite à $g^{\prime}=0$, qui est le seul cas considéré dans la suite. Il suffit de prouver que $\psi_{i, e_{i}}$ est d'ordre $m_{i}$. On est ramené à exhiber un revêtement $\pi: C \longrightarrow D$ entre courbes lisses, de base $\operatorname{Spec}(k(\Delta))$, où $\Delta$ une courbe projective lisse, tel que le revêtement galoisien étale $\Delta_{i} \longrightarrow \operatorname{Spec}(\Delta)$ soit connexe, avec la ramification indiquée. Choisissons la courbe $\Delta$ de genre $h$ tel que $2 h \geqslant b$. Comme les $e_{i}$ sont premiers à la caractéristique $p$ si $p>0$, cela ne pose pas de problème si $\Delta$ est générique. On prouve l'assertion pour l'indice $i=1$. On peut certainement trouver $\mathscr{L}_{1}, \ldots, \mathscr{L}_{b} \in \operatorname{Pic}(\Delta)$ d'ordres respectifs $e_{1}, \ldots, e_{b}$, et tels que la somme $\sum_{i=1}^{b} \mathbb{Z} \mathscr{L}_{i}$ soit directe. Du fait que $g^{\prime}=0$, on a $\operatorname{ppcm}\left(e_{1}, \ldots, e_{b}\right)=n$.

Soit un diviseur $D_{i}$ tel que $\mathcal{L}_{i}=\Theta\left(D_{i}\right)$. Il y a une fonction rationnelle $\phi_{i} \in k(\Delta)^{*}$ telle que $e_{i} D_{i}=\operatorname{Div}\left(\phi_{i}\right)$. Si $D=\sum_{i=1}^{b} \nu_{i} D_{i}$, on a $n D=\operatorname{Div}\left(\prod_{i=1}^{b} \phi_{i}^{m_{i} \nu_{i}}\right)$, et la classe de $D$, c'est-à-dire $\otimes_{i} \mathcal{L}_{i}^{\nu_{i}}$, est d'ordre $n$. On observe aussi que la fonction rationnelle $\psi=\prod_{i=2}^{b} \phi_{i}^{m_{i} \nu_{i}}$ est d'ordre $m_{1}$ dans $k\left(\Delta^{*}\right) / k\left(\Delta^{*}\right)^{m_{1}}$. En effet, si pour un diviseur strict $d$ de $m_{1}$, on a $\psi^{\frac{m_{1}}{d}}=\gamma^{m_{1}}$, alors on peut supposer que $\psi=\gamma^{d}$. Dès lors 
$\prod_{i=1}^{b} \phi_{i}^{m_{i} \nu_{i}}$ serait une puissance $d$-ième avec $d / m$, ce qui à exclure car $D$ est d'ordre $n$. Considérons alors le corps de fonctions d'une variable $E=k(\Delta)(t, \xi)$ où

$$
\xi^{n}=\left(t-\phi_{1}\right)^{m_{1} \nu_{1}} \prod_{i=2}^{b}\left(t-\phi_{1}+\phi_{i}\right)^{m_{i} \nu_{i}} .
$$

On va voir qu'il est de degré $n$ sur $k(\Delta)(t)$, et qu'il y a une seule place $\wp$ au-dessus de $t=\phi_{1}$, d'indice de ramification $e=e_{1}$ et de degré résiduel $m_{1}$. Soit $v_{\wp}$ la valuation normalisée en $\wp$. On a $n v_{\wp}(\xi)=m_{1} \nu_{1} e$ donc $e$ est un multiple de $e_{1}$. Il suffit pour conclure de voir que le degré résiduel est au moins égal à $m_{1}$. Comme $\left(\xi^{e_{1}} /\left(t-\phi_{1}\right)^{\nu_{1}}\right)^{m_{1}}=\prod_{i=2}^{b}\left(t-\phi_{1}+\phi_{i}\right)^{m_{i} \nu_{i}}$, dans le corps résiduel $k(\wp)$ la classe de $u=\xi^{e_{1}} /\left(t-\phi_{1}\right)^{\nu_{1}}$ vérifie $\bar{u}^{m_{1}}=\prod_{i=2}^{b} \phi_{i}^{m_{i} \nu_{i}}=\psi$. Il en découle que son degré est $\geqslant m_{1}$, donc égal à $m_{1}$. Le résultat en découle.

Dans la suite, le groupe étant toujours cyclique, on posera $\mu_{i}=\psi_{i, 1}$, qui est le faisceau tel que $\mu_{i}(\pi)=\sigma_{i}^{*}(\mathscr{L})$. On remarque que $p_{*}(\mathscr{L})=0$, plus généralement $p_{*}\left(\mathscr{L}_{j}\right)=0$ si $1 \leqslant j<n$, de sorte que $p_{!}\left(\mathscr{L}_{j}\right)=-\left[\mathrm{R}^{1} p_{*}\left(\mathscr{L}_{j}\right)\right]$. Les classes $\mu_{i}, \psi_{i}$ et $\psi_{i, j}$ sont reliées par des relations universelles simples :

Proposition 10.5.2. - On a dans $\operatorname{Pic}\left(\overline{\mathcal{H}}_{g, n, \xi}\right)$ les relations :

$$
\psi_{i, j}=j \mu_{i}-\left[\frac{j \nu_{i}}{e_{i}}\right] \psi_{i} \quad ; \quad n \mu_{i}=m_{i} \nu_{i} \psi_{i} \quad ; \quad n \mu_{i, k_{i}}=m_{i} \psi_{i} \quad ; \quad m_{i} \psi_{i, e_{i}}=0 .
$$

Démonstration. - Notons $Q_{i}$ l'image de la section $\sigma_{i}$, de sorte que le faisceau conormal à $Q_{i}$ est $\theta_{Q_{i}}\left(-Q_{i}\right) \cong \Omega_{D / S}^{1} \otimes \theta_{Q_{i}}$. Comme pour $i \neq j$, les sections $\sigma_{i}$ et $\sigma_{j}$ sont disjointes, il vient

$$
\sigma_{i}^{*}\left(\theta\left(-Q_{j}\right)\right)= \begin{cases}\psi_{i} & \text { si } i=j, \\ 0 & \text { si } i \neq j .\end{cases}
$$

Du fait que $\mathcal{L}$ est une racine $n$-ième de $\theta_{D}\left(-\sum_{j=1}^{b} m_{j} \nu_{j} Q_{j}\right)$, par image réciproque, il vient $n \sigma_{i}^{*}(\mathscr{L}) \cong m_{i} \nu_{i} \psi_{i}$ (le groupe de Picard étant noté additivement). De là vient la relation

$$
\sigma_{i}^{*}\left(\mathscr{L}_{j}\right)=j \sigma_{i}^{*}(\mathscr{L})-\left[\frac{j \nu_{i}}{e_{i}}\right] \psi_{i}
$$

c'est-à-dire $\psi_{i, j}=j \mu_{i}-\left[\frac{j \nu_{i}}{e_{i}}\right] \psi_{i}$ comme indiqué. On a alors $\nu_{i} \mu_{i, k_{i}}=\nu_{i} k_{i}-\nu_{i}\left[\frac{k_{i} \nu_{i}}{e_{i}}\right] \psi_{i}=$ $\mu_{i}$. En multipliant les deux membres par $m_{i}$, la relation $n \mu_{i, k_{i}}=m_{i} \psi_{i}$ suit. La dernière relation découle de la proposition 10.5.1.

10.5.2. Relation de Cornalba-Harris. - Le théorème de Deligne-Riemann-Roch permet de mettre en évidence des relations simples entre les classes $\lambda_{j}(1 \leqslant j \leqslant n-1)$ et les classes $\psi_{i, \alpha}$, et finalement les classes $\lambda_{i}$ et $\psi_{j}$ seules. Le raisonnement s'inspire de ([59], Theorem 3.3.4). Il est cependant préférable de travailler dans $\operatorname{Pic}\left(\overline{\mathscr{H}}_{g, G, \xi}\right) \otimes \mathbb{Q}$ et d'obtenir des relations qui incluent des termes de bord.

Rappelons que pour $1 \leqslant j \leqslant n-1$, on a (proposition 10.1.6) :

$$
\lambda_{n-j}=\operatorname{det}\left(\mathbb{E}_{n-j}\right)=\left(\operatorname{det} R^{1} p_{*}\left(\mathscr{L}_{j}\right)\right)^{-1}=\operatorname{det} R p_{*}\left(\mathscr{L}_{j}\right) .
$$


En évaluant d'une autre manière le produit d'intersection

$$
\left\langle\theta_{\tilde{D}}(1), \Xi\right\rangle=\left\langle\theta_{\tilde{D}}, \theta_{\tilde{D}}(-n-\mathscr{B})\right\rangle
$$

on va mettre en évidence des relations importantes entre les faisceaux inversibles $\lambda_{i}$, $\mu_{j}$ et $\psi_{k}$. Dans le cas du champ des courbes hyperelliptiques de genre $g$ ces relations se réduisent essentiellement à la relation de Cornalba-Harris ([55]).

ThÉorème 10.5.3. - Soit $1 \leqslant j<n,(j, n)=1$. Dans le groupe $\operatorname{Pic}\left(\overline{\mathcal{H}}_{g, n, \xi}\right) \otimes \mathbb{Q}$, on $a$ :

$$
\sum_{\pi=\mathrm{NS}} \frac{a(j) b(j)}{m} \theta\left(\Delta_{\pi}\right)=
$$

$$
2 n\left(\lambda^{\prime}-\lambda_{n-j}\right)-\sum_{\alpha=1}^{b} j n\left\langle\frac{j m_{\alpha} \nu_{\alpha}}{n}\right\rangle \mu_{\alpha}+\sum_{\alpha=1}^{b} n\left\langle\frac{j m_{\alpha} \nu_{\alpha}}{n}\right\rangle\left(1+\left[\frac{j m_{\alpha} \nu_{\alpha}}{n}\right]\right) \psi_{\alpha} .
$$

Démonstration. - Il suffit de prouver le résultat pour $j=1$, le cas $j$ premier à $n$ s'en déduisant par des modifications évidentes. Evaluons d'abord le produit d'intersection (142). On a

$$
\begin{aligned}
\left\langle\theta_{\tilde{D}}(1), \theta_{\tilde{D}}(-n-\mathscr{B})\right\rangle & =-n\left\langle\theta_{\tilde{D}}(1), \theta_{\tilde{D}}(1)\right\rangle-\left\langle\theta_{\tilde{D}}(1), \theta_{\tilde{D}}(\mathcal{B})\right\rangle \\
& =-n\left\langle\theta_{\tilde{D}}(1), \theta_{\tilde{D}}(1)\right\rangle-\sum_{\alpha} m_{\alpha} \nu_{\alpha} \psi_{\alpha} .
\end{aligned}
$$

Par le théorème de Deligne-Riemann-Roch, on obtient

$$
n\left\langle\theta_{\tilde{D}}(1), \theta_{\tilde{D}}(1)\right\rangle=2 n \operatorname{det} R r_{*}(\mathscr{L})+\left\langle\theta_{\tilde{D}}(n+\mathscr{B}), \tilde{\omega}\right\rangle-\left\langle\theta_{\tilde{D}}(\mathscr{B}), \tilde{\omega}\right\rangle-2 n \operatorname{det} R r_{*} \tilde{\omega} .
$$

Comme $\left\langle\theta_{\tilde{D}}(n+\mathcal{B}), \tilde{\omega}\right\rangle=\Theta$, il vient d'abord $\left\langle\theta_{\tilde{D}}(\mathcal{B}), \tilde{\omega}\right\rangle=\sum_{\alpha} m_{\alpha} \nu_{\alpha} \psi_{\alpha}$ et finalement

$$
\operatorname{det} \mathrm{R} r_{*}(\tilde{\omega})=\operatorname{det} \mathrm{R} p_{*}(\omega)=\lambda^{\prime} \text {. }
$$

Tenant compte de la relation $n \mu_{i}=m_{i} \nu_{i} \psi_{i}$, et après multiplication par $n$, les relations (143) par restriction à $\mathscr{H}_{g, n, \xi}$ donnent pour tout $1 \leqslant j \leqslant n-1$ les relations

$$
2 n \lambda_{n-j}-2 n \lambda^{\prime}=-\sum_{\alpha=1}^{b} j n\left\langle\frac{j m_{\alpha} \nu_{\alpha}}{n}\right\rangle \mu_{\alpha}+\sum_{\alpha=1}^{b} n\left\langle\frac{j m_{\alpha} \nu_{\alpha}}{n}\right\rangle\left(1+\left[\frac{j m_{\alpha} \nu_{\alpha}}{n}\right]\right) \psi_{\alpha}
$$

On se limite ${ }^{(6)}$ maintenant à $g^{\prime}=0$, et dans la suite à $n=p$ premier, c'est-à-dire que l'on considère des revêtements de $\mathbb{P}^{1}$. Une application de la relation (143) conduit immédiatement au résultat principal de [43]. Dans cette application le degré $n$ est arbitraire, le champ de Hurwitz est une courbe, on a donc $b=4$. On évalue le degré de $\lambda_{n-j}$, on trouve en notant $r \in\{1,2,3\}$ le nombre des $i, 1 \leqslant i \leqslant 4$ tels que $a_{i}+a_{4}=1$ (comparer avec loc.cit, theorem 1)

$$
\operatorname{deg}\left(\lambda_{n-j}\right)=\frac{1}{n}\left((r-1) t_{4}-\sum_{i=1}^{3} \sup \left(0, t_{4}+t_{i}-1\right)\right)
$$

(6) cette restriction dictée par les applications en vues est en fait inutile, les résultats sont vrais en toute généralité 
Par sommation des relations (143), on peut exprimer $\lambda$ comme combinaison linéaire des classes $\psi_{\alpha}$ et $\delta_{\pi}$. La relation obtenue permet d'étendre la relation de CornalbaHarris [55] qui correspond à $p=2$.

LEMme 10.5.4. - Dans le groupe $\operatorname{Pic}\left(\overline{\mathcal{H}}_{g, p}\right)$, on a la relation :

$$
p^{2}\left(\frac{p^{2}-1}{6}\right) \sum_{\pi=\mathrm{NS}} \delta_{\pi}=-2 p^{2} \lambda+p\left(\frac{p^{2}-1}{6}\right) \sum_{\alpha} \psi_{\alpha} .
$$

Démonstration. - On effectue la somme sur $j$ des relations (143), et on multiplie par $p$ les deux membres. On a $\lambda^{\prime}=0$ de sorte que la somme des termes de gauche dans (143) se réduit à évaluer la somme $\sum_{j=1}^{p-1} a(j) b(j)$. Rappelons que $a(j)=p\left\langle\frac{j a}{p}\right\rangle$, et $b(j)=p-a(j)$. La somme est visiblement

$$
p\left(\sum_{r=1}^{p-1} r-\sum_{r=1}^{p-1} r^{2}\right)=\frac{p\left(p^{2}-1\right)}{6} .
$$

Pour le terme de droite, notons que $\psi_{\alpha}=\nu_{\alpha} \mu_{\alpha}$. Donc après multiplication par $p$, la somme des termes de droite se ramène facilement à

$$
\sum_{\alpha} \psi_{\alpha}\left(\sum_{j} p^{2}\left\langle\frac{j \nu_{\alpha}}{p}\right\rangle-\left(p\left\langle\frac{j \nu_{\alpha}}{p}\right\rangle\right)^{2}\right)=\frac{p\left(p^{2}-1\right)}{6} \sum_{\alpha} \psi_{\alpha} .
$$

Les relations (143) et (145) deviennent particulièrement simples dans le cas hyperelliptique. Soit $\overline{\mathscr{H}}_{g}$ le champ des courbes hyperelliptiques de genre $g \geqslant 1$, les points de Weierstrass étant numérotés de 1 à $2 g+2$. La position remarquable de $\overline{\mathcal{H}}_{g}$ est résumée par le diagramme

$$
\overline{\mathcal{M}}_{g, 2 g+2} \stackrel{\imath}{\longleftarrow} \overline{\mathcal{H}}_{g} \stackrel{\delta}{\longrightarrow} \overline{\mathcal{M}}_{0,2 g+2}
$$

Un principe général, valable pour un champ de Hurwitz avec $g^{\prime}=0$, est que les relations de Riemann-Hurwitz supérieures jointes aux relations (10.5.3) ramènent en principe un calcul dans le champ $\overline{\mathscr{H}}_{g}$ à un calcul dans $\overline{\mathcal{M}}_{0,2 g+2}$.

Illustrons ce principe en montrant comment retrouver la relation de CornalbaHarris (voir [55]) qui exprime, dans le lieu hyperelliptique, la classe $\lambda$ comme combinaison linéaire des composantes du bord.

LEMME 10.5.5. - Dans le groupe $\operatorname{Pic}\left(\overline{\mathcal{H}}_{g}\right)$, on a la relation :

$$
8(2 g+1) \lambda=4 \sum_{\pi=\mathrm{RR}} \alpha(g+1-\alpha) \Delta_{\pi}^{\mathrm{RR}}+8 \sum_{\pi=\mathrm{NS}} \beta(g-\beta) \Delta_{\pi}^{\mathrm{NS}} .
$$

Démonstration. - Pour $p=2$, la relation (145) devient $2 \sum_{\pi=\mathrm{NS}} \Delta_{\pi}=-8 \lambda+$ $\sum_{\alpha=1}^{2 g+2} \psi_{\alpha}$. En multipliant les deux membres par $2 g+1$, on peut alors invoquer la relation connue ci-après entre les classes $\psi$ et les composantes du bord dans $\bar{M}_{0, n}$ :

$$
2(n-1) \sum_{\alpha=1}^{n} \psi_{\alpha}=\sum_{(I, J),|I|=j} j(n-j)\left[\Delta^{\prime}{ }_{I, J}\right] .
$$


Dans cette relation, les partitions sont ordonnées. On ramène cette relation dans $\operatorname{Pic}\left(\overline{\mathscr{H}}_{g}\right)$ par application de $\delta^{*}$, tenant compte de la proposition 10.3.5, qui dit que $\delta^{*}\left(\Delta_{\pi}^{\prime}\right)$ vaut $\Delta_{\pi}$ dans le cas RR, et $2 \Delta_{\pi}$ dans le cas NS. Après arrangement, cela conduit à

$8(2 g+1) \lambda=4 \sum_{\pi=\mathrm{RR}} \alpha(g+1-\alpha) \Delta_{\pi}^{\mathrm{RR}}+2 \sum_{\pi=\mathrm{NS}} 2[(2 \beta+1)(2 g+1-2 \beta)-(2 g+1)] \Delta_{\pi}^{\mathrm{NS}}$, expression qui après simplification du coefficient entre crochets est exactement le résultat annoncé.

Soit $\bar{H}_{g}$ le lieu des courbes hyperelliptiques, que l'on peut exprimer comme le champ quotient $\left[\overline{\mathcal{H}}_{g} / \mathfrak{S}_{2 g+2}\right]$ où $\mathfrak{S}_{2 g+2}$ désigne le groupe symétrique, muni du morphisme d'oubli de l'involution $j: \bar{H}_{g} \longrightarrow \overline{\mathcal{M}}_{g}$. Noter que $j$ est seulement un plongement au-dessus de $H_{g}$, ainsi qu'aux points génériques des composantes du bord. Comme on va le voir, la relation (146) poussée dans $A^{1}\left(\bar{H}_{g}\right)$ est exactement la relation de Cornalba-Harris [55].

THÉORÈME 10.5.6. - Dans $A^{1}\left(\bar{H}_{g}\right)$, on a la relation

$$
(4 g+2) \lambda=\frac{g}{2}\left[\Delta_{1}^{\mathrm{RR}}\right]_{Q}+\sum_{\alpha=2}^{\left[\frac{g+1}{2}\right]} \alpha(g+1-\alpha)\left[\Delta_{\alpha}^{\mathrm{RR}}\right]_{Q}+2 \sum_{\beta=1}^{\left[\frac{g}{2}\right]} \beta(g-\beta)\left[\Delta_{\beta}^{\mathrm{NS}}\right]_{Q}
$$

Démonstration. - Soit $\imath: \overline{\mathscr{H}}_{g} \longrightarrow \bar{H}_{g}$ le morphisme quotient par $\mathfrak{S}_{2 g+2}$. Noter que le fibré de Hodge sur $\overline{\mathscr{H}}_{g}$ est l'image réciproque du fibré de même nom sur $\bar{H}_{g}$. Prenons la première classe de Chern des deux membres de la relation (146), on obtient

$$
\begin{gathered}
8(2 g+1) \lambda=4 \sum_{\pi=\mathrm{RR}} \alpha(g+1-\alpha)\left[\Delta_{\pi}^{\mathrm{RR}}\right]_{Q}+8 \sum_{\pi=\mathrm{NS}} \beta(g-\beta)\left[\Delta_{\pi}^{\mathrm{NS}}\right]_{Q} \\
=4 \sum_{\pi=\mathrm{RR}} \frac{\alpha(g+1-\alpha)}{2}\left[\Delta_{\pi}^{\mathrm{RR}}\right]+8 \sum_{\pi=\mathrm{NS}} \frac{\beta(g-\beta)}{4}\left[\Delta_{\pi}^{\mathrm{NS}}\right] .
\end{gathered}
$$

On prend en compte le fait que dans le cas RR, le groupe des automorphismes d'un point général d'une composante est d'ordre 2, par contre il est d'ordre 4 dans le cas NS. Si $\pi$ (partition non ordonnée) est subordonnée à la partition $(I, J)$ de $[1,2 g+2]$, posons $j=|I| \leqslant|J|, j=2 \alpha$ dans le cas pair, et $j=2 \beta+1$ si impair, il vient par application de $\imath_{*}$

$$
4 \sum_{\pi=\mathrm{RR}} \frac{\alpha(g+1-\alpha)}{2}\left(\begin{array}{c}
2 g+2) !(16 g+8) \lambda= \\
j
\end{array}\right) i_{*}\left[\Delta_{\pi}^{\mathrm{RR}}\right]+8 \sum_{\pi=\mathrm{NS}} \frac{\beta(g-\beta)}{4}\left(\begin{array}{c}
2 g+2 \\
j
\end{array}\right) i_{*}\left[\Delta_{\pi}^{\mathrm{NS}}\right] .
$$

Il est par ailleurs clair que le degré de $\Delta_{\pi}^{\mathrm{RR}}\left(\operatorname{resp} . \Delta_{\pi}^{\mathrm{NS}}\right)$ sur son image $\Delta_{j}^{\mathrm{RR}}$ (resp. $\left.\Delta_{j}^{\mathrm{NS}}\right)$ est $j !(2 g+2-j)$ !, sauf si $j=2$, car dans ce cas une courbe hyperelliptique $C \in \Delta_{\pi}^{\mathrm{RR}}$ a une composante rationnelle, munie d'une involution. Les deux points fixes de l'involution sont les points marqués. Les points d'intersection $P^{\prime}, P^{\prime \prime}$ avec l'autre composante, de genre $g-1$ sont échangés par l'involution. Il y a une seconde involution 
qui a pour point fixe $P^{\prime}, P^{\prime \prime}$ et qui commute avec la première. Donc l'échange des deux points marqués $P^{\prime}, P^{\prime \prime}$ conduit à une courbe isomorphe. Il vient finalement en rappelant que le cas RR correspond à $j$ pair

$$
\begin{gathered}
(2 g+2) !(16 g+8) \lambda=4 g \frac{(2 g+2) !}{4}\left[\Delta_{1}^{\mathrm{RR}}\right]+ \\
4 \sum_{\alpha=2}^{\left[\frac{g+1}{2}\right]} \frac{\alpha(g+1-\alpha)}{2}(2 g+2) !\left[\Delta_{\alpha}^{\mathrm{RR}}\right]+8 \sum_{\beta=1}^{\left[\frac{g}{2}\right]} \frac{\beta(g-\beta)}{4}(2 g+2) !\left[\Delta_{\beta}^{\mathrm{NS}}\right] .
\end{gathered}
$$

En revenant aux classes fondamentales des diverses composantes, on trouve pour le membre de droite :

$4 g \frac{(2 g+2) !}{2}\left[\Delta_{1}^{\mathrm{RR}}{ }_{Q}\right]+4 \sum_{\alpha=2}^{\left[\frac{g+1}{2}\right]} \alpha(g+1-\alpha)(2 g+2) !\left[\Delta_{\alpha}^{\mathrm{RR}}\right]_{Q}+8 \sum_{\beta=1}^{\left[\frac{g}{2}\right]} \beta(g-\beta)(2 g+2) !\left[\Delta_{\beta}^{\mathrm{NS}}\right]_{Q}$.

Finalement, en simplifiant par $8(2 g+2)$ !, on obtient la relation de Cornalba-Harris :

$$
(8 g+4) \lambda=g\left[\Delta_{1}^{\mathrm{RR}}\right]_{Q}+\sum_{\alpha=2}^{\left[\frac{g+1}{2}\right]} 2 \alpha(g+1-\alpha)\left[\Delta_{\alpha}^{\mathrm{RR}}\right]_{Q}+4 \sum_{\beta=1}^{\left[\frac{g}{2}\right]} \beta(g-\beta)\left[\Delta_{\beta}^{\mathrm{NS}}\right]_{Q}
$$

10.5.3. Intégrales de Hodge-Hurwitz hyperelliptiques. - Le contexte est identique à celui de la section précédente, dont on conserve les notations, cependant on se restreint de plus au champ des courbes hyperelliptiques $\overline{\mathscr{H}}_{g}$. Le principe de correspondance invoqué dans ce chapitre est appliqué de nouveau ${ }^{(7)}$. On va exploiter le fait que des calculs d'intersection avec le lieu hyperelliptique $\bar{H}_{g} \subset \bar{M}_{g}$ une fois transportés sur le champ hyperelliptique se ramènent à des calculs dans $\overline{\mathcal{M}}_{0,2 g+2}$. Cela montre que l'objet naturel pour de tels calculs est $\overline{\mathcal{H}}_{g}$ plutôt que $\bar{H}_{g}$. On notera $\overline{\mathcal{H}}_{g}^{1}$ le champ des courbes hyperelliptiques avec un unique point de Weierstrass marqué. La méthode utilisée est une amplification du calcul de Faber-Pandharipande ([44]). Elle conduit très simplement à l'évaluation d'intégrales

$$
\int_{\overline{\mathcal{H}}_{g}^{1}} \kappa_{1} \mu_{1}^{2 g-2}=\frac{(2 g-1)^{2}}{2^{2 g}(2 g+1) !} \quad \text { et } \quad \int_{\overline{\mathcal{H}}_{g}^{1}} \mu_{1}^{2 g-1}=\frac{1}{2^{2 g}(2 g+1) !} .
$$

Rappelons que le faisceau inversible $\mu_{i} \in \operatorname{Pic}\left(\overline{\mathscr{H}}_{g}\right)$ (notation désignant aussi sa classe de Chern) est l'image réciproque du faisceau $\mathscr{L}_{i} \in \operatorname{Pic}\left(\overline{\mathcal{M}}_{g, 2 g+2}\right)$ (section 10.2). On conserve la notation $\mu_{i}$ pour éviter toute confusion avec les $\psi_{i}$ qui proviennent du bas. A. J. Bene a obtenu des résultats analogues, mais par une approche combinatoire basée sur une cellulation de $\overline{\mathcal{H}}_{g}$ au moyen des graphes épais, et le schéma d'intégration de Penner [11]. À la différence de notre méthode, il travaille avec les classes combinatoires $W_{a}$ de Witten, et étudie l'intersection de ces classes avec le lieu hyperelliptique.

Rappelons que, par convention, dans le champ $\overline{\mathcal{H}}_{g}$ les points de Weierstrass sont marqués de 1 à $2 g+2$. Comme indiqué ci-dessus $\overline{\mathscr{H}}_{g}^{1}$ désigne le champ dont les objets

(7) Dans [64] on trouvera des applications de même nature, mais à des objets différents. 
sont les courbes hyperelliptiques stables marquées par un seul point de Weierstrass. Plus généralement on peut considérer le champ $\overline{\mathcal{H}}_{g}^{j}$ formé des courbes hyperelliptique stables avec $j(1 \leqslant j \leqslant 2 g+2)$ points de Weierstrass marqués. Il est aisé de justifier son existence en adaptant les arguments de la section 6.2. Noter que si $j \leqslant 2 g$ il est possible que sur une courbe hyperelliptique stable appartenant à $\overline{\mathscr{H}}_{g}^{j}$ deux points de Weierstrass libres s'effondrent. Par oubli du marquage par le point d'indice $j$ on obtient un morphisme $\pi_{j}: \overline{\mathscr{H}}_{g}^{j} \longrightarrow \overline{\mathcal{H}}_{g}^{j-1}$ de degré $2 g+3-j$. On notera finalement $\bar{H}_{g} \subset \overline{\mathcal{M}}_{g}$ l'image de $\overline{\mathcal{H}}_{g}$ dans $\overline{\mathcal{M}}_{g}$, i.e le lieu hyperelliptique. On a une factorisation de $\imath: \overline{\mathcal{H}}_{g} \longrightarrow \bar{M}_{g}$, qui est l'oubli du marquage par les points de Weierstrass, sous la forme

$$
\overline{\mathcal{H}}_{g} \stackrel{\pi}{\longrightarrow} \overline{\mathcal{H}}_{g}^{1} \longrightarrow \bar{H}_{g} \subset \overline{\mathcal{M}}_{g}
$$

Le morphisme $\pi$ est l'oubli du marquage par les points de numéros $2 g+2, \ldots, 2$ dans l'ordre indiqué, c'est-à-dire la composition $\pi_{2} \cdots \pi_{2 g+2}$, son degré est $(2 g+1)$ !. Pour ramener le calcul à un calcul dans $A^{\bullet}\left(\overline{\mathcal{H}}_{g}\right)$ à un calcul dans $A^{\bullet}\left(\overline{\mathcal{M}}_{0,2 g+2}\right)$, on note que

$$
\int_{\overline{\mathcal{H}}_{g}^{1}} \xi=\frac{1}{(2 g+1) !} \int_{\overline{\mathcal{H}}_{g}} \pi^{*}(\xi) .
$$

Il faut expliciter l'image réciproque par $\pi$ des classes $\mu_{1}$ et $\kappa_{a}$. Par utilisation itérée de la relation générale $\pi_{n+1}^{*}\left(\psi_{1}\right)=\psi_{1}-\left[D_{1, n+1}\right]$ on obtient, avec nos notations

$$
\pi_{j}^{*}\left(\mu_{1}\right)=\mu_{1}-\left[D_{1, j}\right] \cap\left[\overline{\mathcal{H}}_{g}^{j}\right]
$$

(l'indice $j$ signifie que $j$ points de Weierstrass sont marqués). Comme $D_{1, j}$ est le lieu des courbes obtenues en collant une «bulle» (c'est-à-dire un $\mathbb{P}^{1}$ ) contenant les points de Weierstrass de numéros 1 et $j$, à une courbe de genre $g$ marquée par $j-1$ points, l'intersection avec le lieu hyperelliptique est vide. On obtient donc que $\pi^{*}\left(\mu_{1}\right)=\mu_{1}$, ce qui nous autorise à noter cette classe $\mu_{1}$ sans précision supplémentaire. Le même argument montre que pour la classe $\kappa_{a}$ on a

$$
\pi^{*}\left(\kappa_{a}\right)=\kappa_{a}-\sum_{\alpha=2}^{2 g+2} \mu_{\alpha}^{a}
$$

Traitons en premier la seconde intégrale. Elle se ramène au calcul de $\int_{\overline{\mathcal{H}}_{g}} \mu_{1}^{2 g-1}$. Vu que $2 \mu_{1}=\delta^{*}\left(\psi_{1}\right)$, elle s'obtient immédiatement en partant du résultat bien connu

$$
\int_{\bar{M}_{0, n}} \psi_{1}^{\alpha_{1}} \cdots \psi_{n}^{\alpha_{n}}=\frac{(n-3) !}{\alpha_{1} ! \cdots \alpha_{n} !} \quad\left(\alpha_{1}+\cdots+\alpha_{n}=n-3\right) .
$$

En tenant compte du fait que le degré de $\delta$ est $\frac{1}{2}$, on obtient

$$
\int_{\overline{\mathcal{H}}_{g}^{1}} \mu_{1}^{2 g-1}=\frac{1}{2^{2 g}} \int_{\overline{\mathcal{M}}_{0,2 g+2}} \psi_{1}^{2 g-1}=\frac{1}{2^{2 g}(2 g+1) !}
$$

Pour la première intégrale, on a un résultat plus compliqué : 
ThÉORÈme 10.5.7. - Avec les notations précédentes, si $0 \leqslant a \leqslant 2 g-1$, on a

$$
\int_{\overline{\mathcal{H}}_{g}^{1}} \kappa_{a} \mu_{1}^{2 g-1-a}=\frac{1}{2^{2 g-1-a}(2 g+1) !}\left(\left(\begin{array}{c}
2 g \\
a+1
\end{array}\right)-\frac{2 g+1}{2^{a+1}}\left(\begin{array}{c}
2 g-1 \\
a
\end{array}\right)\right) .
$$

Démonstration. - En utilisant la relation (147) on voit que l'intégrale (148) se ramène à une intégrale sur le champ $\overline{\mathscr{H}}_{g}$ classifiant les courbes hyperelliptiques avec points de Weierstrass marqués

$$
(2 g+1) ! \int_{\overline{\mathcal{H}}_{g}^{1}} \kappa_{a} \mu_{1}^{2 g-1-a}=\int_{\overline{\mathcal{H}}_{g}}\left(\kappa_{a}-\sum_{2}^{2 g+2} \mu_{\alpha}^{a}\right) \mu_{1}^{2 g-1-a}=\int_{\overline{\mathcal{H}}_{g}} \kappa_{a} \mu_{1}^{2 g-1-a}-\sum_{2}^{2 g+2} \int_{\overline{\mathcal{H}}_{g}} \mu_{\alpha}^{a} \mu_{1}^{2 g-1-a} .
$$

Tenant compte de la relation de Riemann-Hurwitz à l'ordre $a$, qui dans le cas hyperelliptique s'écrit sous la forme simple $\kappa_{a}=2 \delta^{*}\left(\kappa_{a}^{\prime}\right)$, et de la formule de projection, il vient

$$
\int_{\overline{\mathcal{H}}_{g}} \kappa_{a} \mu_{1}^{2 g-1-a} \frac{1}{2^{2 g-2-a}} \int_{\overline{\mathcal{H}}_{g}} \delta^{*}\left(\kappa_{a}^{\prime} \psi_{1}^{2 g-1-a}\right)=\frac{1}{2^{2 g-2-a}} \int_{\overline{\mathcal{M}}_{0,2 g+2}} \kappa_{a}^{\prime} \psi_{1}^{2 g-1-a} \delta_{*}[1]
$$

Mais $\delta_{*}[1]=\delta_{*}\left([\overline{\mathscr{H}}]_{Q}\right)=\frac{1}{2} \delta_{*}\left(\left[\overline{\mathcal{H}}_{g}\right]\right)=\frac{1}{2}\left[\bar{M}_{0,2 g+2}\right]$, de sorte que l'intégrale de droite se réduit à

$$
\frac{1}{2^{2 g-1-a}} \int_{\bar{M}_{0,2 g+2}} \kappa_{a}^{\prime} \psi_{1}^{2 g-1-a}
$$

De la même manière, on obtient

$$
\int_{\overline{\mathcal{H}}_{g}} \mu_{\alpha}^{a} \mu_{1}^{2 g-1-a}=\frac{1}{2^{2 g-1}} \int_{\bar{M}_{0,2 g+2}} \psi_{\alpha}^{a} \psi_{1}^{2 g-1-a}=\frac{1}{2^{2 g}}\left(\begin{array}{c}
2 g-1 \\
a
\end{array}\right) .
$$

Pour conclure, notons le résultat élémentaire suivant, conséquence facile de l'« équation des cordes» (que l'on trouve dans Knudsen [68] où elle ne porte pas de nom) :

Lemme 10.5.8. - Pour $4 \leqslant a+3 \leqslant n$, soit $\tau_{a, n}=\int_{\bar{M}_{0, n}} \kappa_{a} \psi_{1}^{n-3-a}$. Alors, on a

$$
\tau_{a, n}=\left(\begin{array}{c}
n-2 \\
a+1
\end{array}\right)
$$

Démonstration. - On procède par récurrence sur $n$. Soit $\pi_{n+1}: \bar{M}_{0, n+1} \longrightarrow \bar{M}_{0, n}$ le morphisme d'oubli du point $x_{n+1}$. On a $\pi_{n+1}^{*}\left(\kappa_{a}\right)=\kappa_{a}-\psi_{n+1}^{a}$, donc

$$
\pi_{n+1}^{*}\left(\kappa_{a}\right) \psi_{1}^{n-a-2}=\kappa_{a} \psi_{1}^{n-a-2}-\psi_{1}^{n-a-2} \psi_{n+1}^{a} .
$$

En intégrant, et par la formule de projection, on obtient

$$
\int_{\overline{\mathcal{M}}_{0, n+1}} \pi_{n+1}^{*}\left(\kappa_{a}\right) \psi_{1}^{n-a-2}=\tau_{a, n+1}-\int_{\overline{\mathcal{M}}_{0, n+1}} \psi_{1}^{n-a-2} \psi_{n+1}^{a}=\int_{\overline{\mathcal{M}}_{0, n}} \kappa_{a} \pi_{n+1, *}\left(\psi_{1}^{n-a-2}\right) .
$$

Mais par utilisation de l'équation des cordes, on trouve

$$
\pi_{n *}\left(\psi_{1}^{a_{1}} \cdots \psi_{n-1}^{a_{n-1}}\right)=\sum_{j, a_{j}>0} \psi_{1}^{a_{1}} \cdots \psi_{j}^{a_{j}-1} \cdots \psi_{n-1}^{a_{n-1}}
$$


en particulier $\pi_{n+1, *}\left(\psi_{1}^{n-a-2}\right)=\psi_{1}^{n-a-3}$. Finalement, si $a+3 \leqslant n$, on obtient la relation $\tau_{a, n+1}=\tau_{a, n}+\left(\begin{array}{c}n-2 \\ a\end{array}\right)$, et donc $\tau_{a, n}=\left(\begin{array}{c}n-3 \\ a\end{array}\right)+\left(\begin{array}{c}n-4 \\ a\end{array}\right)+\cdots+\left(\begin{array}{l}a \\ a\end{array}\right)=\left(\begin{array}{c}n-2 \\ a+1\end{array}\right)$.

Pour conclure la preuve de (148), les résultats qui précèdent donnent après division par le facteur $(2 g+1)$ ! :

$$
\int_{\overline{\mathcal{H}}_{g}^{1}} \kappa_{a} \mu_{1}^{2 g-1-a}=\frac{1}{(2 g+1) !}\left(\frac{1}{2^{2 g-1-a}}\left(\begin{array}{c}
2 g \\
a+1
\end{array}\right)-\frac{2 g+1}{2^{2 g}}\left(\begin{array}{c}
2 g-1 \\
a
\end{array}\right)\right)
$$

qui est exactement l'expression cherchée.

Pour $a=0$, le calcul précédent i.e (148) a pour conséquence la relation suivante (Faber-Pandharipande [44], prop. 4) :

$$
1+\sum_{g \geqslant 0} t^{2 g} \int_{\bar{M}_{g, 1}}\left(\sum_{i=1}^{g}(-1)^{i+1}\left(2^{g+1-i}-1\right) \psi_{1}^{g-i} \lambda_{i-1}\right) \psi_{1}^{2 g-1}=\frac{\sin (t / 2)}{t / 2} .
$$

Pour $a=1$, A. J. Bene [11] obtient de manière analogue, via la relation de Mumford qui décrit la classe fondamentale $\left[\mathcal{H}_{g}\right]$, une relation (plus compliquée) reliant certaines intégrales de Hodge

$$
\int_{\bar{M}_{g, 1}}\left(\sum_{i=1}^{g}(-1)^{i+1}\left(2^{g+1-i}-1\right) \psi_{1}^{g-i} \lambda_{i-1}\right) \kappa_{1} \psi_{1}^{2 g-2}=\frac{14 g^{2}-11 g+3}{3.2^{2 g}(2 g+1) !} .
$$

On peut s'interroger sur la validité d'une relation analogue pour $a \geqslant 2$.

Il est possible de traiter, par les méthodes analogues aux précédentes, des calculs d'autres intégrales du type Hodge sur le champ hyperelliptique.

EXEMPLE 10.5.9. - Soit $a=2 g-1$, un calcul similaire au précédent donne l'intégrale de $\kappa_{2 g-1}$ évaluée sur le lieu hyperelliptique $\bar{H}_{g}$, on trouve

$$
\int_{\bar{H}_{g}} \kappa_{2 g-1}=\frac{1}{(2 g+2) !}-\frac{1}{2^{2 g}(2 g+1) !} .
$$

Si $g=2$, c'est le calcul de Mumford ([80]) qui donne

$$
\int_{\bar{M}_{2}} \kappa_{3}=\frac{1}{1152} \text {. }
$$





\section{INDEX}

Action stable, 44, 63

Champ de Hurwitz (cas galoisien), 82

Champ de Hurwitz (cas non galoisien), 96

Clôture galoisienne

d'un revêtement, 15

d'une famille, 94

Classes de Nielsen, 25

Classes $\kappa, 179$

Classes $\psi, 175$

Courbe marquée, 21, 42

Courbe piquée, 43

Courbe préstable, courbe nodale, 41

Courbe stable, 41

det (déterminant d'un complexe parfait), 42

Discriminant, 32, 90, 103

Div (opération Div de Knudsen-Mumford), 31

Diviseur de branchement, 15, 31

Diviseur de ramification, 15, 31, 47

Donnée de Hurwitz, 19

Donnée de ramification, 19

Équivalence de revêtements, 16

Espace de Teichmüller, 26

Faisceau sans torsion, 121

Fibré de Hodge, 172

Formule de Chevalley-Weil, 35

Formule de Riemann-Hurwitz, 16

$G$-champ, 76

$G$-courbe, 16
$G$-équivalence, 17

$G$-revêtement, 16

$G$-type, 18

Graphe modulaire de Hurwitz, 109

Graphe modulaire, graphe dual, 107

Grothendieck-Riemann-Roch, 188

Holonomie, 18

Mapping class group, 24

Marquage, 21, 42

Monodromie

Groupe de, 15

Type de, 95

Nombre de Nielsen, 25

Orbite marquée, 21

Orbite spéciale, non spéciale, 16

Piquage, 43

Point marqué, voir courbe marquée

Points de type I, II, III, 44

Représentations de Hurwitz, 16, 33

Revêtement simple, 26, 35

Revêtement, 15

Revêtement galoisien, 15

Rigidification, 80

Strates du bord, 125

Structure de niveau

sur une courbe lisse, 133

sur une courbe stable, 136

Structure de niveau $(n), 133,135,139$ 



\section{BIBLIOGRAPHIE}

[1] D. Abramovich, A. Corti \& A. Vistoli - « Twisted bundles and admissible covers », Comm. Algebra 31 (2003), p. 3547-3618.

[2] D. Abramovich \& T. J. Jarvis - « Moduli of twisted spin curves », Proc. Amer. Math. Soc. 131 (2003), p. 685-699.

[3] D. Abramovich \& A. Vistoli - « Compactifying the space of stable maps », J. Amer. Math. Soc. 15 (2002), p. 27-75.

[4] E. Arbarello \& M. Cornalba - «Combinatorial and algebro-geometric cohomology classes on the moduli spaces of curves », J. Algebraic Geom. 5 (1996), p. 705-749.

[5] A. Arsie \& A. Vistoli - « Stacks of cyclic covers of projective spaces », Compos. Math. 140 (2004), p. 647-666.

[6] M. Asada, M. Matsumoto \& T. Oda - « Local monodromy on the fundamental groups of algebraic curves along a degenerate stable curve», J. Pure Appl. Algebra 103 (1995), p. 235-283.

[7] H. BASs - « Covering theory for graphs of groups », J. Pure Appl. Algebra 89 (1993), p. 3-47.

[8] A. Beauville - « Prym varieties and the Schottky problem », Invent. Math. 41 (1977), p. 149-196.

[9] S. BECKMANN - « Ramified primes in the field of moduli of branched coverings of curves », J. Algebra 125 (1989), p. 236-255.

[10] A. A. BeǏlinson \& Y. I. MAnin - « The Mumford form and the Polyakov measure in string theory », Comm. Math. Phys. 107 (1986), p. 359-376.

[11] A. J. BEnE - «Combinatorial classes, hyperelliptic loci, and Hodge integrals », prépublication arXiv:math.GT/0610603.

[12] D. J. Benson - Representations and cohomology. I, Cambridge Studies in Advanced Math., vol. 30, Cambridge Univ. Press, 1991. 
[13] J. Bertin - «Compactification des schémas de Hurwitz », C. R. Acad. Sci. Paris Sér. I Math. 322 (1996), p. 1063-1066.

[14] J. Bertin \& S. Maugeais - « Déformations équivariantes des courbes semistables », Ann. Inst. Fourier (Grenoble) 55 (2005), p. 1905-1941.

[15] J. BERTin \& A. MÉzARD - «Déformations formelles des revêtements sauvagement ramifiés de courbes algébriques », Invent. Math. 141 (2000), p. 195238.

[16] M. Boggi \& M. PikaArT - « Galois covers of moduli of curves », Compositio Math. 120 (2000), p. 171-191.

[17] J. Bryan, T. Graber \& R. Pandharipande - « The orbifold quantum cohomology of $\mathbb{C}^{2} / \mathbb{Z}_{3}$ and Hurwitz-Hodge integrals », J. Algebraic Geom. 17 (2008), p. 1-28.

[18] J.-L. BRYLinski - « Propriétés de ramification à l'infini du groupe modulaire de Teichmüller», Ann. Sci. École Norm. Sup. 12 (1979), p. 295-333.

[19] A. Cadoret \& A. Tamagawa - «Stratification of Hurwitz spaces by closed modular subvarieties », Pure Appl. Math. Q. 5 (2009), p. 227-253.

[20] D. Chen - « Covers of the projective line and the moduli space of quadratic differentials », prépublication arXiv:mathAG/1005.3120.

[21] C. Chevalley \& A. Weil - « Über das Verhalten der Integrale erster Gattung bei Automorphismen des Funktionenkörpers », Abh. Math. Sem. Hamburg Univ. 10 (1934), p. 358-361.

[22] A. Chiodo - « Towards an enumerative geometry of the moduli space of twisted curves and $r$ th roots », Compos. Math. 144 (2008), p. 1461-1496.

[23] T. Coates \& A. Givental - «Quantum Riemann-Roch, Lefschetz and Serre », Ann. of Math. 165 (2007), p. 15-53.

[24] K. Coombes \& D. Harbater - « Hurwitz families and arithmetic Galois groups », Duke Math. J. 52 (1985), p. 821-839.

[25] A. F. Costa \& S. M. NATANZON - « Topological classification of $\mathbb{Z}_{p}^{m}$ actions on surfaces », Michigan Math. J. 50 (2002), p. 451-460.

[26] P. DÈBES - « Arithmétique et espaces de modules de revêtements », in Number theory in progress, Vol. 1 (Zakopane-Kościelisko, 1997), de Gruyter, 1999, p. 75102 .

[27] P. DĖBes \& M. EMsAlem - « Harbater-Mumford components and towers of moduli spaces », J. Inst. Math. Jussieu 5 (2006), p. 351-371. 
[28] P. Deligne - «Le lemme de Gabber », Astérisque 127 (1985), p. 131-150.

[29] _ _ «e déterminant de la cohomologie », in Current trends in arithmetical algebraic geometry (Arcata, Calif., 1985), Contemp. Math., vol. 67, Amer. Math. Soc., 1987, p. 93-177.

[30] P. Deligne \& D. Mumford - «The irreducibility of the space of curves of given genus », Publ. Math. I.H.É.S. 36 (1969), p. 75-109.

[31] P. Deligne \& M. Rapoport - « Les schémas de modules de courbes elliptiques », in Modular functions of one variable, II (Proc. Internat. Summer School, Univ. Antwerp, Antwerp, 1972), Lecture Notes in Math., vol. 349, Springer, 1973, p. 143-316.

[32] M. Demazure \& P. Gabriel - Groupes algébriques, North-Holland, 1970.

[33] S. P. Diaz - « On the Natanzon-Turaev compactification of the Hurwitz space», Proc. Amer. Math. Soc. 130 (2002), p. 613-618.

[34] S. P. Diaz \& D. Edidin - « Towards the homology of Hurwitz spaces », J. Differential Geom. 43 (1996), p. 66-98.

[35] R. DijkgraAF - «Mirror symmetry and elliptic curves », in The moduli space of curves (Texel Island, 1994), Progr. Math., vol. 129, Birkhäuser, 1995, p. 149-163.

[36] C. J. EARLE - «On the moduli of closed Riemann surfaces with symmetries », in Advances in the theory of riemann surfaces (Proc. Conf., Stony Brook, N.Y., 1969), Princeton Univ. Press, 1971, p. 119-130. Ann. of Math. Studies, No. 66.

[37] B. Edixhoven - « Néron models and tame ramification », Compositio Math. 81 (1992), p. 291-306.

[38] A. L. Edmonds - « Surface symmetry. I», Michigan Math. J. 29 (1982), p. 171183.

[39] T. EKEDAhL - « Boundary behaviour of Hurwitz schemes », in The moduli space of curves (Texel Island, 1994), Progr. Math., vol. 129, Birkhäuser, 1995, p. 173198.

[40] T. Ekedahl, S. Lando, M. Shapiro \& A. Vainshtein - « Hurwitz numbers and intersections on moduli spaces of curves », Invent. Math. 146 (2001), p. 297327.

[41] G. Ellingsrud \& K. LøNSTED - « An equivariant Lefschetz formula for finite reductive groups », Math. Ann. 251 (1980), p. 253-261.

[42] M. Emsalem - «Familles de revêtements de la droite projective », Bull. Soc. Math. France 123 (1995), p. 47-85. 
[43] A. Eskin, M. Kontsevich \& A. Zorich - « Lyapunov spectrum of square-tiled cyclic covers », J. Mod. Dyn. 5 (2011), p. 319-353.

[44] C. Faber \& R. Pandharipande - « Hodge integrals and Gromov-Witten theory », Invent. Math. 139 (2000), p. 173-199.

[45] G. Faltings - «Moduli-stacks for bundles on semistable curves », Math. Ann. 304 (1996), p. 489-515.

[46] M. D. FrIEd - «Fields of definition of function fields and Hurwitz familiesgroups as Galois groups », Comm. Algebra 5 (1977), p. 17-82.

[47] _ _ « Introduction to modular towers : generalizing dihedral group-modular curve connections », in Recent developments in the inverse Galois problem (Seattle, WA, 1993), Contemp. Math., vol. 186, Amer. Math. Soc., 1995, p. 111-171.

[48] M. D. Fried \& H. VölKLeIN - « The inverse Galois problem and rational points on moduli spaces», Math. Ann. 290 (1991), p. 771-800.

[49] W. Fulton - «Hurwitz schemes and irreducibility of moduli of algebraic curves », Ann. of Math. 90 (1969), p. 542-575.

[50] B. van Geemen \& F. OORT - «A compactification of a fine moduli space of curves », in Resolution of singularities (Obergurgl, 1997), Progr. Math., vol. 181, Birkhäuser, 2000, p. 285-298.

[51] D. GieseKer - Lectures on moduli of curves, Tata Institute of Fundamental Research Lectures on Mathematics and Physics, vol. 69, Published for the Tata Institute of Fundamental Research, Bombay, 1982.

[52] T. Graber \& R. VAKIL - « Hodge integrals and Hurwitz numbers via virtual localization », Compositio Math. 135 (2003), p. 25-36.

[53] A. Grothendieck (éd.) - Revêtements étales et groupe fondamental, Lecture Notes in Math., vol. 224, Springer, 1971, Séminaire de Géométrie Algébrique du Bois Marie 1960-1961 (SGA 1).

[54] J. Harris, T. Graber \& J. Starr - «A note on Hurwitz schemes of covers of a positive genus curve », prépublication arXiv:math.AG/0205056.

[55] J. HARris \& I. MorRISON - Moduli of curves, Graduate Texts in Math., vol. 187, Springer, 1998.

[56] J. Harris \& D. Mumford - « On the Kodaira dimension of the moduli space of curves », Invent. Math. 67 (1982), p. 23-88.

[57] R. Hartshorne - Residues and duality, Lecture Notes in Math., vol. 20, Springer, 1966, Lecture notes of a seminar on the work of A. Grothendieck, given at Harvard 1963/64. 
[58] T. J. JARVIS - « Torsion-free sheaves and moduli of generalized spin curves », Compositio Math. 110 (1998), p. 291-333.

[59] « « Geometry of the moduli of higher spin curves », Internat. J. Math. 11 (2000), p. 637-663.

[60] « « The Picard group of the moduli of higher spin curves », New York J. Math. 7 (2001), p. 23-47.

[61] T. J. Jarvis, R. Kaufmann \& T. Kimura - «Pointed admissible $G$-covers and $G$-equivariant cohomological field theories », Compos. Math. 141 (2005), p. 926978.

[62] _ « Stringy K-theory and the Chern character », Invent. Math. 168 (2007), p. $23-81$.

[63] T. J. JARVis \& T. KimURA - « Orbifold quantum cohomology of the classifying space of a finite group », in Orbifolds in mathematics and physics (Madison, WI, 2001), Contemp. Math., vol. 310, Amer. Math. Soc., 2002, p. 123-134.

[64] _ « A representation-valued relative Riemann-Hurwitz theorem and the Hurwitz-Hodge bundle », prépublication arXiv:math.AG/08102488.

[65] E. KAnI - «Hurwitz spaces of genus 2 covers of an elliptic curve», Collect. Math. 54 (2003), p. 1-51.

[66] G. KATZ - « How tangents solve algebraic equations, or a remarkable geometry of discriminant varieties », Expo. Math. 21 (2003), p. 219-261.

[67] N. M. Katz \& B. MazUR - Arithmetic moduli of elliptic curves, Annals of Math. Studies, vol. 108, Princeton Univ. Press, 1985.

[68] F. F. KNUdSEn - « The projectivity of the moduli space of stable curves. II. The stacks $M_{g, n} »$, Math. Scand. 52 (1983), p. 161-199.

[69] F. F. Knudsen \& D. Mumford - « The projectivity of the moduli space of stable curves. I. Preliminaries on "det" and "Div" », Math. Scand. 39 (1976), p. 19-55.

[70] A. Kokotov, D. Korotkin \& P. Zograf - « Isomonodromic tau function on the space of admissible covers », Adv. Math. 227 (2011), p. 586-600.

[71] M. Kontsevich - « Enumeration of rational curves via torus actions », in The moduli space of curves (Texel Island, 1994), Progr. Math., vol. 129, Birkhäuser, 1995 , p. 335-368.

[72] S. LANDO - « Ramified coverings of the two-dimensional sphere and intersection theory in spaces of meromorphic functions on algebraic curves », Uspekhi Mat. Nauk 57 (2002), p. 29-98. 
[73] G. Laumon \& L. Moret-Bailly - Champs algébriques, Ergebnisse Math. Grenzg., vol. 39, Springer, 2000.

[74] E. Looijenga - «Smooth Deligne-Mumford compactifications by means of Prym level structures », J. Algebraic Geom. 3 (1994), p. 283-293.

[75] _ _ « Correspondences between moduli spaces of curves », in Moduli of curves and abelian varieties, Aspects Math., E33, Vieweg, 1999, p. 131-150.

[76] Y. I. MANIN - Frobenius manifolds, quantum cohomology, and moduli spaces, American Mathematical Society Colloquium Publications, vol. 47, Amer. Math. Soc., 1999.

[77] S. MochizUKi - « The geometry of the compactification of the Hurwitz scheme», Publ. Res. Inst. Math. Sci. 31 (1995), p. 355-441.

[78] L. Moret-Bailly - « Construction de revêtements de courbes pointées », J. Algebra 240 (2001), p. 505-534.

[79] I. Morrison \& H. Pinkham - «Galois Weierstrass points and Hurwitz characters », Ann. of Math. 124 (1986), p. 591-625.

[80] D. Mumford - « Towards an enumerative geometry of the moduli space of curves $»$, in Arithmetic and geometry, Vol. II, Progr. Math., vol. 36, Birkhäuser, 1983, p. 271-328.

[81] D. Mumford, J. Fogarty \& F. KIRWAN - Geometric invariant theory, Ergebnisse Math. Grenzg., vol. 34, Springer, 1994.

[82] S. M. NATAnzon - «Moduli of Riemann surfaces, Hurwitz-type spaces, and their superanalogues », Uspekhi Mat. Nauk 54 (1999), p. 61-116.

[83] J. NiELSEN - «Die Struktur periodischer Transformationen von Flächen », Mat.-Fys. Medd. Danske Vid. Selsk. 15 (1937), p. 1-77; traduction anglaise : in Collected Papers 2, Birkhäuser (1986).

[84] A. Okounkov \& R. Pandharipande - «Gromov-Witten theory, Hurwitz numbers, and matrix models », in Algebraic geometry-Seattle 2005. Part 1, Proc. Sympos. Pure Math., vol. 80, Amer. Math. Soc., 2009, p. 325-414.

[85] M. PikAart \& A. J. DE Jong - « Moduli of curves with non-abelian level structure », in The moduli space of curves (Texel Island, 1994), Progr. Math., vol. 129, Birkhäuser, 1995, p. 483-509.

[86] M. RaYnAud - «p-groupes et réduction semi-stable des courbes », in The Grothendieck Festschrift, Vol. III, Progr. Math., vol. 88, Birkhäuser, 1990, p. 179197. 
[87] _ « Spécialisation des revêtements en caractéristique $p>0$ », Ann. Sci. École Norm. Sup. 32 (1999), p. 87-126.

[88] M. Romagny - «Sur quelques aspects des champs de revêtements de courbes algébriques », thèse de doctorat, Université Grenoble 1, 2002.

[89] _ « The stack of Potts curves and its fibre at a prime of wild ramification », J. Algebra 274 (2004), p. 772-803.

[90] _ « Group actions on stacks and applications », Michigan Math. J. 53 (2005), p. 209-236.

[91] M. SAÏDI - «Revêtements modérés et groupe fondamental de graphe de groupes », Compositio Math. 107 (1997), p. 319-338.

[92] J-P. SERRE - Représentations linéaires des groupes finis, 5e éd., Hermann, 1988.

[93] _ Topics in Galois theory, second éd., Research Notes in Math., vol. 1, A K Peters Ltd., 2008.

[94] C. S. SeShADRI - « Geometric reductivity over arbitrary base », Advances in Math. 26 (1977), p. 225-274.

[95] V. P. SNaith - Explicit Brauer induction, Cambridge Studies in Advanced Math., vol. 40, Cambridge Univ. Press, 1994.

[96] H.-H. TSEnG - «Orbifold quantum Riemann-Roch, Lefschetz and Serre », Geom. Topol. 14 (2010), p. 1-81.

[97] A. Vistoli - « Intersection theory on algebraic stacks and on their moduli spaces », Invent. Math. 97 (1989), p. 613-670.

[98] B. WAJNRYB - «Orbits of Hurwitz action for coverings of a sphere with two special fibers », Indag. Math. (N.S.) 7 (1996), p. 549-558.

[99] S. WEWERS - « Construction of Hurwitz spaces », Thèse, Universität DuisburgEssen, 1998.

[100] _ _ «eformation of tame admissible covers of curves », in Aspects of Galois theory (Gainesville, FL, 1996), London Math. Soc. Lecture Note Ser., vol. 256, Cambridge Univ. Press, 1999, p. 239-282. 